\title{
USE OF SYSTEM IDENTIFICATION TECHNIQUES FOR IMPROVING AIRFRAME FINITE ELEMENT MODELS USING TEST DATA
}

\section{Final Report}

Submitted to

NASA Langley Research Center

Contract NAG-1-1007

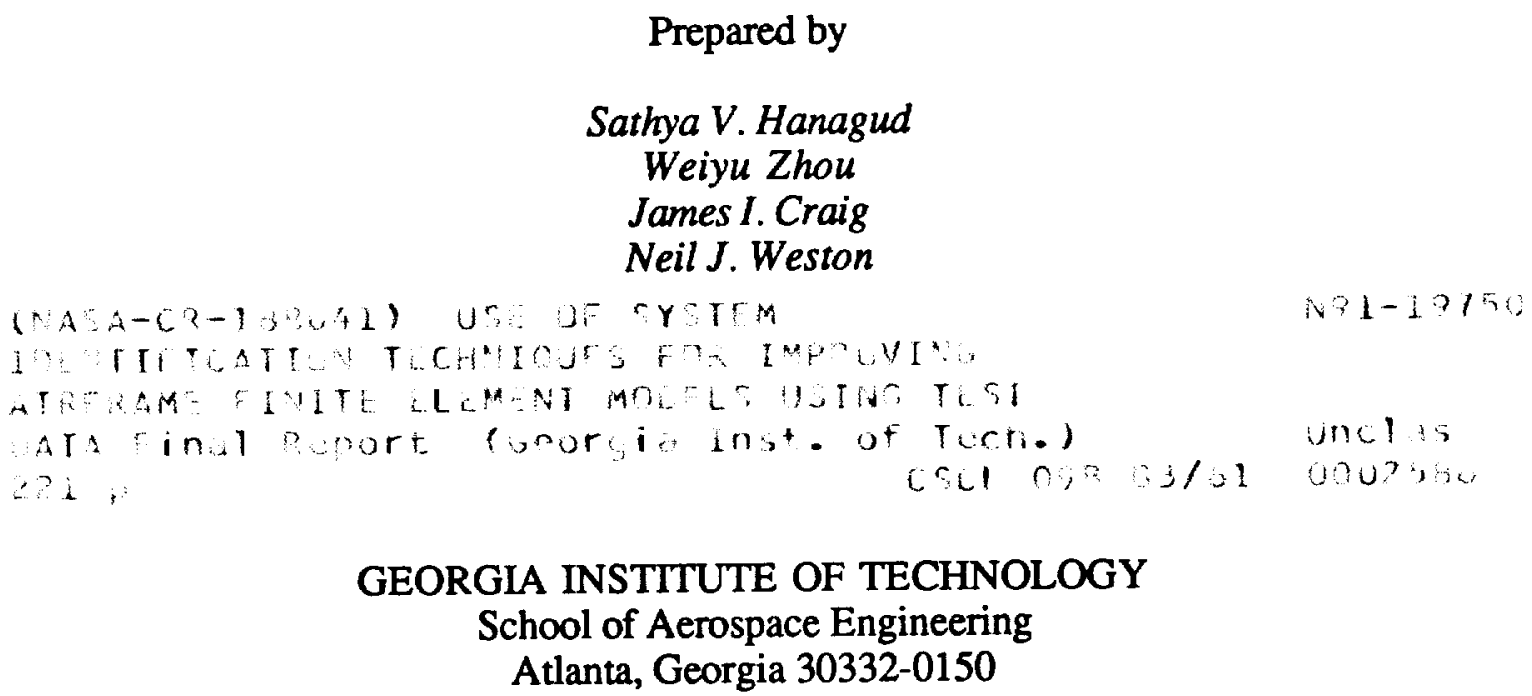

Prepared by

Sathya V. Hanagud

Weiyu Zhou

James I. Craig

Neil J. Weston

\section{GEORGIA INSTITUTE OF TECHNOLOGY}

School of Aerospace Engineering

Atlanta, Georgia 30332-0150 



\section{SUMMARY}

A method for using system identification techniques to improve airframe finite element models using test data has been developed and demonstrated. The method uses linear sensitivity matrices to relate changes in selected physical parameters to changes in the total system matrices. The values for these physical parameters were determined using constrained optimization with singular value decomposition. The method was confirmed using both simple and complex finite element models for which pseudo-experimental data was synthesized directly from the finite element model. The method was then applied to a real airframe model which incorporated all of the complexities and details of a large finite element model and for which extensive test data was available. The method was shown to work, and the differences between the identified model and the measured results were considered satisfactory. 



\section{TABLE OF CONTENTS}

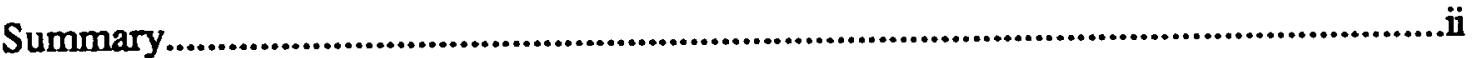

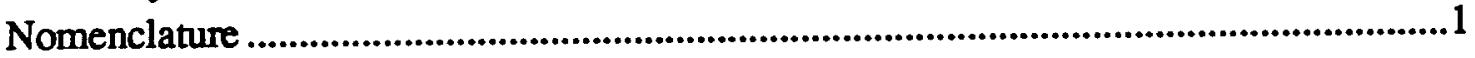

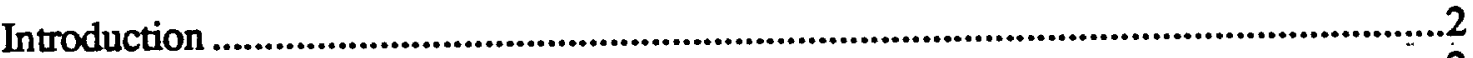

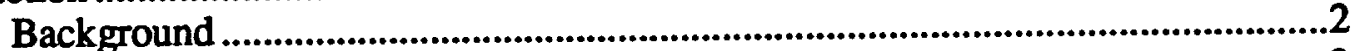

Review of Previous Pertinent Work ...............................................................2

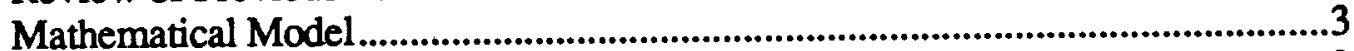

Basic Equations .................................................................................

Identification Procedure........................................................................5

Applications $\ldots$

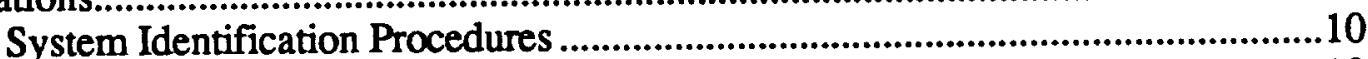

Simple Numerical Example .....................................................................10

Application to AH-1G Model........................................................................13

Results Using Simulated Test Data ..............................................................14

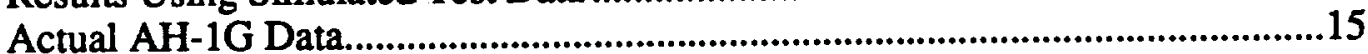

Conclusions and Recommendations.....................................................................18

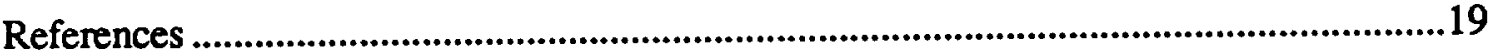

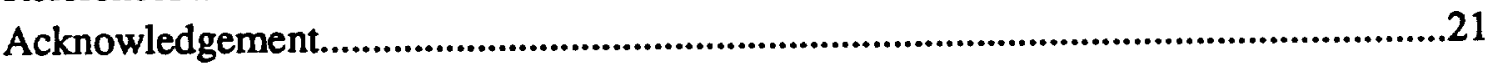

Appendix A: System Identification Program Listing

Appendix B: $\quad$ MSC/NASTRAN Input for Final AH-1G System Identification Run

Appendix C: MSC/NASTRAN Output from Final AH-1G System Identification Run 



\section{NOMENCLATURE}

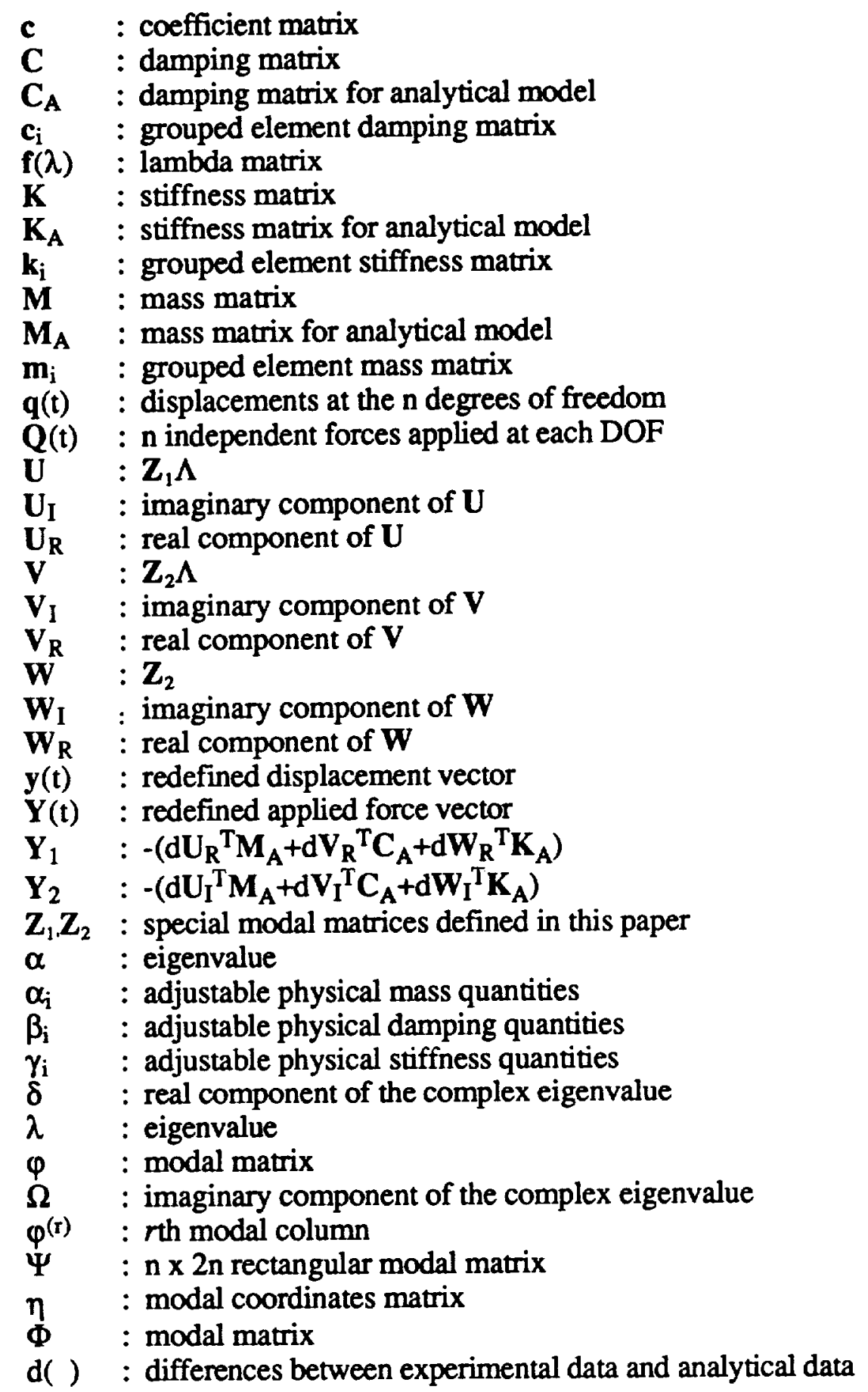





\section{INTRODUCTION}

\section{Background}

The vast bulk of the work reported to date on identification of structural dynamic systems has focused on identifying mathematical models that reproduce test results, but little consideration has been given to the physical basis for the identified system equations. Typically, the identification procedures make systematic adjustments to the system equation, commonly to the stiffness and/or mass matrices but also to the damping matrix, so that the identified eigenvalues and eigenvectors reproduce as closely as possible the results measured in tests. The result of this process is almost inevitably identified mass, stiffness and damping matrices that are fully populated, that is, which have nonzero values for almost all elements. Such matrices, while capable of producing plausible eigenvalues and eigenvectors, can nonetheless be physically implausible in the sense that the large numbers of nonzero elements throughout the system matrices implies direct connectivity among the degrees of freedom that does not exist physically.

Identified mathematical models that are based on physically implausible system matrices may be quite acceptable if the objective of the study is to develop a simulation model. However, such results for analysis purposes are generally unsatisfactory because it is difficult or impossible to relate specific features of the physical system to the analysis results. This problem is particularly troublesome when the objective of the identification of a system model from experimental measurements is an accurate system model that, in turn, will be used to make modifications to or improvements in the original physical system. Such an example might be the modification of an existing aircraft structure to accommodate a new mission. In this case it would be desirable to formulate a structural model for the present structure, verify its accuracy against experimental measurements, and then use it as the basis for the modifications. When the verification process yields identified system matrices that are mathematically acceptable but physically implausible, the resulting model may be useless as the basis for future structural modifications.

The objective of the present work was to develop a method for identifying physically plausible finite element system models of airframe structures from test data. The assumed models were based on linear elastic behavior with general (nonproportional) damping. Physical plausibility of the identified system matrices was insured by restricting the identification process to designated physical parameters only and not simply to the elements of the system matrices themselves. For example, in a large finite element model the identified parameters might be restricted to the moduli for each of the different materials used in the structure. In the case of damping, a restricted set of damping values might be assigned to finite elements based on the material type and on the fabrication processes used. In this case, different damping values might be associated with riveted, bolted and bonded elements.

The method itself is developed first, and several approaches are outlined for computing the identified parameter values. The method is applied first to a simple structure for which the "measured" response is actually synthesized from an assumed model. Both stiffness and damping parameter values are accurately identified. The true test, however, is the application to a full-scale airframe structure. In this case, a NASTRAN model and actual measured modal parameters formed the basis for the identification of a restricted set of physically plausible stiffness and damping parameters.

\section{Review of Previous Pertinent Work}

Airframes are generally modelled using powerful finite element analysis packages such as NASTRAN that airc capable of representing quite detailed aspects of the structural system. The accuracy of such models is determined by comparing the analytical results with flight or ground vibration test results. In the case of helicopter airframes, several recent efforts have focused on the correlation of NASTRAN model data with ground vibration test data ${ }^{1-3}$. The conclusions reached in these studies suggest that in cases where there is some degree of correlation, the model frequencies compare favorably with test frequencies, but generally only in the low frequency range 

below about $15 \mathrm{~Hz}^{1-2}$. The frequency response functions at selected locations also compare reasonably well in this range. Outside this range the comparisons are generally unsatisfactory, and the eigenvectors do not usually compare favorably in either range.

Although there have been numerous contributions to the literature in the area of the identification of structural dynamic systems ${ }^{4-25}$, the majority of reported methods are based on simply adjusting the elements of one or more of the $\mathbf{K}, \mathbf{M}$, and $\mathbf{C}$ matrices. While this approach is capable of yielding a system matrix whose eigenvalues and eigenvectors suitably match measured results, the methods generally lose all physical interpretability inherent in the original $\mathbf{K}$, $\mathbf{M}$ and $\mathbf{C}$ matrices by not maintaining relationships among elements dictated by the model topology. These difficulties are compounded for large-scale models with thousands of degrees of freedom.

Kuo and Wada ${ }^{25}$ used nonlinear sensitivity coefficients (NSC) in the identification procedure. Their sensitivity coefficients are between the system parameters and eigenvalues. In the present work the interest is in the change of system matrices as a function of physical variables of the structure. A different type of sensitivity coefficient between system matrices and physical variables has therefore been developed.

The most significant achievement in the present work ${ }^{30}$ is to preserve the physical interpretability of the $\mathbf{M}, \mathbf{C}, \mathbf{K}$ matrices so that the identification can provide evidence of possible sources of erroneous modeling and point to specific regions of the model that are unduly sensitive and need additional consideration in modeling. The identification procedure developed in this paper is capable of adjusting physical quantities such as boundary conditions, moments of inertia, stiffnesses, damping or other selected physical parameters.

\section{Mathematical_Model}

\section{Basic Equations}

Any linearly elastic structural system with $\mathrm{n}$ discrete degrees of freedom and with general viscous damping (either proportional or nonproportional) can be represented by $\mathrm{n}$ coupled ordinary differential equations that can be wrilten in the following form ${ }^{27}$ :

$$
\mathbf{M q \ddot { q }}(\mathrm{t})+\mathbf{C} \mathbf{q}(\mathrm{t})+\mathbf{K q}(\mathrm{t})=\mathbf{Q}(\mathrm{t})
$$

where $\mathbf{M}, \mathbf{C}$, and $\mathbf{K}$ are symmetric $\mathrm{n} \times \mathrm{n}$ inertia, damping, and stiffness matrices, respectively. In this formulation, $\mathbf{q}(t)$ are the displacements at the $n$ degrees of freedom and $Q(t)$ are the $n$ independent forces applied at each degree of freedom.

In the case of undamped or proportionally damped systems, there are $\mathrm{n}$ complex conjugate pairs of eigenvalues and $n$ distinct modes which are orthogonal with respect to $\mathbf{M}$ and $\mathbf{K}$. Using a transformation matrix of the form:

$$
\mathbf{q}(\mathrm{t})=\Psi \eta(\mathrm{t})
$$

will allow decomposition of the original system equations (Eq. 1) into $n$ decoupled equations that are straightforward to solve.

This transformation cannot be applied to the general nonproportionally damped problem in the same manner because for this case there are $2 n$ complex modes, $\varphi^{(r)}$, and consequently $2 n$ modal coordinates, $\eta_{r}(t)$, but there are only $n$ physical coordinates, $q_{i}(t)$.

One can overcome this difficulty by writing Eq. (1) as a set of $2 n$ ordinary differential equations in the form:

$$
\left[\begin{array}{cc}
0 & \mathbf{M} \\
\mathbf{M} & \mathbf{C}
\end{array}\right]\left\{\begin{array}{c}
\tilde{\mathbf{q}}(\mathrm{t}) \\
\mathbf{q}(\mathrm{t})
\end{array}\right\}+\left[\begin{array}{cc}
-\mathbf{M} & \mathbf{0} \\
\mathbf{0} & \mathbf{K}
\end{array}\right]\left\{\begin{array}{l}
\mathbf{q}(t) \\
\mathbf{q}(t)
\end{array}\right\}=\left\{\begin{array}{c}
0 \\
\mathbf{Q}(t)
\end{array}\right\} \text {. }
$$

If one then defines: $y(t)=\left\{\begin{array}{l}\ddot{q}(t) \\ \dot{q}(t)\end{array}\right\}$ and $Y(t)=\left\{\begin{array}{c}0 \\ Q(t)\end{array}\right\}$, the above equations can be written as a set of $2 n$ first order ordinary differential equations: 



$$
\left[\begin{array}{cc}
0 & M \\
M & C
\end{array}\right] y(t)+\left[\begin{array}{cc}
-M & 0 \\
0 & K
\end{array}\right] y(t)=Y(t) .
$$

This formulation has the advantage that the modes obtained from the solution of the homogeneous equations, obtained by letting $\mathbf{Y}(\mathrm{t})=0$ in Eqs. (3), are orthogonal, and hence can be used in conjunction with the expansion theorem to obtain the solution of the nonhomogeneous problem. The solution of the homogeneous equations is obtained by assuming as before a solution in the form:

$$
y(t)=\Phi e^{\alpha t}
$$

where $\Phi$ represents the spatial component of the solution and is a vector consisting of $2 n$ constant elements. The corresponding eigenvalue problem can be written as:

$$
\alpha\left[\begin{array}{cc}
\mathbf{0} & \mathbf{M} \\
\mathbf{M} & \mathbf{C}
\end{array}\right] \Phi+\left[\begin{array}{cc}
-\mathbf{M} & \mathbf{0} \\
\mathbf{0} & \mathbf{K}
\end{array}\right] \Phi=\left\{\begin{array}{l}
0 \\
0
\end{array}\right\}
$$

The solution of the eigenvalue problem yields $2 \mathrm{n}$ eigenvalues, $\alpha_{\mathfrak{l}}$, and $2 \mathrm{n}$ eigenvectors

$$
\Phi_{\mathrm{I}}^{\mathrm{T}}=\left\{\begin{array}{c}
\alpha_{\mathrm{T}} \phi_{\mathrm{T}}^{\mathrm{T}} \\
\phi_{\mathrm{T}}^{\mathrm{T}}
\end{array}\right\}, \quad \mathrm{r}=1,2, \ldots 2 \mathrm{n} .
$$

Equations (5) and (6) provide the solution to Eq. (1), but in order to simplify the computational work, it is convenient to formally separate these complex equations into real and imaginary pairs. Following the approach introduced by Cheng ${ }^{10}$, the real and imaginary components of the eigenvalues and eigenvectors are defined, respectively, as:

$$
\begin{aligned}
& \lambda_{\mathrm{r}}=\delta_{\mathrm{r}}+\mathrm{j} \Omega_{\mathrm{r}} \\
& \Phi_{\mathrm{r}}=\Phi_{\mathrm{R}_{\mathrm{r}}}+\mathrm{j} \Phi_{\mathrm{I}_{\mathrm{r}}}
\end{aligned}
$$

and in addition the following modal matrices are defined:

$$
\begin{aligned}
\mathbf{z}_{1}= & \left(\operatorname{Re}\left(\alpha_{1} \phi_{1}\right), \operatorname{Im}\left(\alpha_{1} \phi_{1}\right), \operatorname{Re}\left(\alpha_{2} \phi_{2}\right), \operatorname{Im}\left(\alpha_{2} \phi_{2}\right), . .\right. \\
& \left.\operatorname{Re}\left(\alpha_{n} \phi_{n}\right), \operatorname{Im}\left(\alpha_{n} \phi_{n}\right)\right) \\
\mathbf{z}_{2}= & \left(\operatorname{Re}\left(\phi_{1}\right), \operatorname{Im}\left(\phi_{1}\right), \operatorname{Re}\left(\phi_{2}\right), \operatorname{Im}\left(\phi_{2}\right), \ldots\right. \\
& \left.\operatorname{Re}\left(\phi_{n}\right), \operatorname{Im}\left(\phi_{n}\right)\right)
\end{aligned}
$$

Then the new system equation, Eq. (5), can now be rewritten with purely real terms in the form:

$$
\Lambda\left[\begin{array}{cc}
\mathbf{0} & \mathbf{M} \\
\mathbf{M} & \mathbf{C}
\end{array}\right]\left\{\begin{array}{l}
\mathbf{z}_{1} \\
\mathbf{Z}_{2}
\end{array}\right\}+\left[\begin{array}{cc}
-\mathbf{M} & \mathbf{0} \\
\mathbf{0} & \mathbf{K}
\end{array}\right]\left\{\begin{array}{l}
\mathbf{Z}_{1} \\
\mathbf{z}_{2}
\end{array}\right\}=\left\{\begin{array}{l}
\mathbf{0} \\
\mathbf{0}
\end{array}\right\}
$$

where the eigenvector matrix, $\Lambda$, is a block diagonal matrix with blocks

$$
\Lambda_{\mathrm{r}}=\left[\begin{array}{cc}
\delta_{\mathrm{r}} & \Omega_{\mathrm{r}} \\
-\Omega_{\mathrm{r}} & \delta_{\mathrm{r}}
\end{array}\right]
$$

along the diagonal and zeros elsewhere. Equation (9) can be further simplified by the introduction of $\mathbf{U}, \mathbf{V}$, and $\mathbf{W}$ as follows:

$$
\mathrm{MU}+\mathrm{CV}+\mathrm{KW}=\mathbf{0}
$$

where $U=Z_{1} \Lambda, V=Z_{2} \Lambda$ and $W=Z_{2}$ or explicitly:

$$
\begin{gathered}
\mathrm{U}=\left(\operatorname{Re}\left(\alpha_{1}{ }^{2} \phi_{1}\right), \operatorname{Im}\left(\alpha_{1}{ }^{2} \phi_{1}\right), \operatorname{Re}\left(\alpha_{2}{ }^{2} \phi_{2}\right), \operatorname{Im}\left(\alpha_{2}{ }^{2} \phi_{2}\right),\right. \\
\left.\ldots \operatorname{Re}\left(\alpha_{n}^{2} \phi_{n}\right), \operatorname{Im}\left(\alpha_{n}{ }^{2} \phi_{n}\right)\right) \\
V=\left(\operatorname{Re}\left(\alpha_{1} \phi_{1}\right), \operatorname{Im}\left(\alpha_{1} \phi_{1}\right), \operatorname{Re}\left(\alpha_{2} \phi_{2}\right), \operatorname{Im}\left(\alpha_{2} \phi_{2}\right), \ldots\right.
\end{gathered}
$$


- 


$$
\begin{aligned}
& \left.\operatorname{Re}\left(\alpha_{n} \phi_{n}\right), \operatorname{Im}\left(\alpha_{n} \phi_{n}\right)\right) \\
& \mathbf{W}=\left(\operatorname{Re}\left(\phi_{1}\right), \operatorname{Im}\left(\phi_{1}\right), \operatorname{Re}\left(\phi_{2}\right), \operatorname{Im}\left(\phi_{2}\right), \ldots \operatorname{Re}\left(\phi_{n}\right),\right. \\
& \left.\operatorname{Im}\left(\phi_{n}\right)\right)
\end{aligned}
$$

Finally, Eq. (11) can be separated into explicit real and imaginary equations in the form of the following two equations.

$$
\begin{aligned}
& M U_{R}+C V_{R}+K W_{R}=0 \\
& M U_{I}+C V_{I}+K W_{I}=0
\end{aligned}
$$

These equations are same as Eqs. (5), but they do not include complex variables. For the identification procedure, it is much easier to use these equations than to use Eqs. (5) directly.

\section{Identification Procedure}

To begin, suppose that the mass, damping and stiffness matrices for the initial analytical model are given by $\mathbf{M}_{A} \mathbf{C}_{A}$ and $\mathbf{K}_{\mathrm{A}}$, respectively, and the identified mass, damping and stiffness matrices are given by $\mathbf{M}, \mathbf{C}$ and $\mathbf{K}$. In a similar manner, the eigenvectors and eigenvalues for the analytical model are given by $U_{A}, V_{A}$ and $W_{A}$, while $U_{E}, V_{E}$ and $W_{E}$ are the eigenvectors and eigenvalues determined from test data. From these definitions it follows that the relationship between the identified model (based on the test data) and the analytical model can be written as:

$$
\begin{array}{ll}
\mathbf{M}=\mathbf{M}_{\mathrm{A}}+\mathrm{d} \mathbf{M}, \quad \mathbf{C}=\mathbf{C}_{\mathrm{A}}+\mathrm{dC}, \quad \mathbf{K}=\mathrm{K}_{\mathrm{A}}+\mathrm{dK} \\
\mathbf{U}_{\mathrm{E}}=\mathbf{U}_{\mathrm{A}}+\mathrm{d} \mathbf{U}, \quad \mathbf{V}_{\mathrm{E}}=\mathbf{V}_{\mathrm{A}}+\mathrm{d} \mathbf{V}, \quad \mathbf{W}_{\mathrm{E}}=\mathbf{W}_{\mathrm{A}}+\mathrm{dW}
\end{array}
$$

where $d \mathbf{M}, d C, d K, d U, d V$ and $d W$ are the changes. The identified model satisfies equations (13) and (14), so substituting equations (15) and (16) into equation (13) and (14), yields:

$$
\begin{aligned}
& d U_{R}^{T} M_{A}+d V_{R}{ }^{T} C_{A}+d W_{R}^{T} K_{A}= \\
& -\left(U_{E R}{ }^{T}, V_{E R}^{T}, W_{E R}{ }^{T}\right)(d M, d C, d K)^{T} \\
& d U_{I}^{T} M_{A}+d V_{I}^{T} C_{A}+d W_{I}^{T} K_{A}= \\
& -\left(U_{E I}{ }^{T}, V_{E I}^{T}, W_{E I}^{T}\right)(d M, d C, d K)^{T}
\end{aligned}
$$

These equations can be combined into the following form:

$$
\left[\begin{array}{lll}
U_{E R}{ }^{T} & V_{E R}^{T} & W_{E R}^{T} \\
U_{E I}^{T} & V_{E I}^{T} & W_{E I}^{T}
\end{array}\right]\left\{\begin{array}{l}
d M \\
d C \\
d K
\end{array}\right\}=\left\{\begin{array}{l}
Y_{1} \\
Y_{2}
\end{array}\right\},
$$

where

$$
\begin{aligned}
& Y_{1}=-\left(d U_{R}^{T} M_{A}+d V_{R}^{T} C_{A}+d W_{R}^{T} K_{A}\right) \\
& Y_{2}=-\left(d U_{I}^{T} M_{A}+d V_{I}^{T} C_{A}+d W_{I}^{T} K_{A}\right) .
\end{aligned}
$$

The right side of these equations is known, since $\mathbf{M}_{\mathrm{A}}, \mathbf{C}_{\mathrm{A}}$, and $\mathrm{K}_{\mathrm{A}}$ are given by the analytical model and $d U_{R}{ }^{T}, d V_{R}^{T}, d W_{R}^{T}, d U_{I}^{T}, d V_{I}^{T}$, and $d W_{I}^{T}$, which are the differences of the eigenvalues and eigenvectors between the analytical model and the experimental data, are known. Finally, the matrix

$$
\left[\begin{array}{llll}
U_{E R}{ }^{T} & V_{E R}{ }^{T} & W_{E R}{ }^{T} \\
U_{E I} T^{T} & V_{E I}{ }^{T} & W_{E I}{ }^{T}
\end{array}\right]
$$

contains only experiment data.

The solution to these equations are the changes of $\mathrm{dM}, \mathrm{dC}$ and $\mathrm{dK}$. Because of matrix symmetry, the number of unknowns in Eq. (19) is $3 n(n+1) / 2$. The number of equations depends on the number of known experimental modes. Suppose this number is $m$, then the number of 

equations are $m \times n$. If the number of the equations is larger than or equal to the number of unknowns and the rank of this matrix is equal to $3 n(n+1) / 2$, normal least square methods can be used to solve these equations. Otherwise, singular value decomposition, or constrained optimization can be used to solve Eq. (19) for the changes $\mathrm{dM}, \mathrm{dC}$ and $\mathrm{dK}$, and these results can then be substituted into Eq. (15) to determine the identified $M, C$ and $K$ matrices. It should be noted that this approach is capable of handling nonproportional damping and underdetermined problems in which fewer modes are measured than are computed from the analytical model.

At this stage the usual identification procedure can be performed. The values of $\mathbf{M}, \mathbf{C}$ and $\mathbf{K}$ can be put into the system equation, Eq. (1), and the experimental data can then be reproduced. However the identified $\mathbf{M}, \mathbf{C}$ and $\mathbf{K}$ cannot be related to particular physical quantities in the actual airframe, because the changes occur throughout the entire $\mathbf{M}, \mathbf{C}$ and $\mathbf{K}$ matrices. In order to preserve the physical interpretability of the identified system, it is necessary to develop a relationship between $\mathrm{dM}, \mathrm{dC}$ and $\mathrm{dK}$ and adjustable physical quantities such as boundary conditions, moments of inertia, stiffnesses or other selected physical parameters. To this end, assume that each of the system matrices can be decomposed into the form:

$$
M=\sum_{i=1}^{N_{m}} m_{i} \alpha_{i}, \quad C=\sum_{i=1}^{N_{c}} c_{i} \beta_{i} \text {, and } \quad K=\sum_{i=1}^{N_{k}} k_{i} \gamma_{i}
$$

where $\alpha_{i}, \beta_{i}$ and $\gamma_{i}$ are adjustable physical quantities and $m_{i}, c_{i}$ and $k_{i}$ are grouped element matrices with common physical quantities.

For example, in the finite element model of actual airframe, there is an $\mathrm{e}_{\mathrm{j}}$-th element, (see Fig. 1). The portion of the stiffness matrix that describes bending in the $x z$ plane of an element, assumed to be a principal plane (Fig. 2), in NASTRAN, is given by

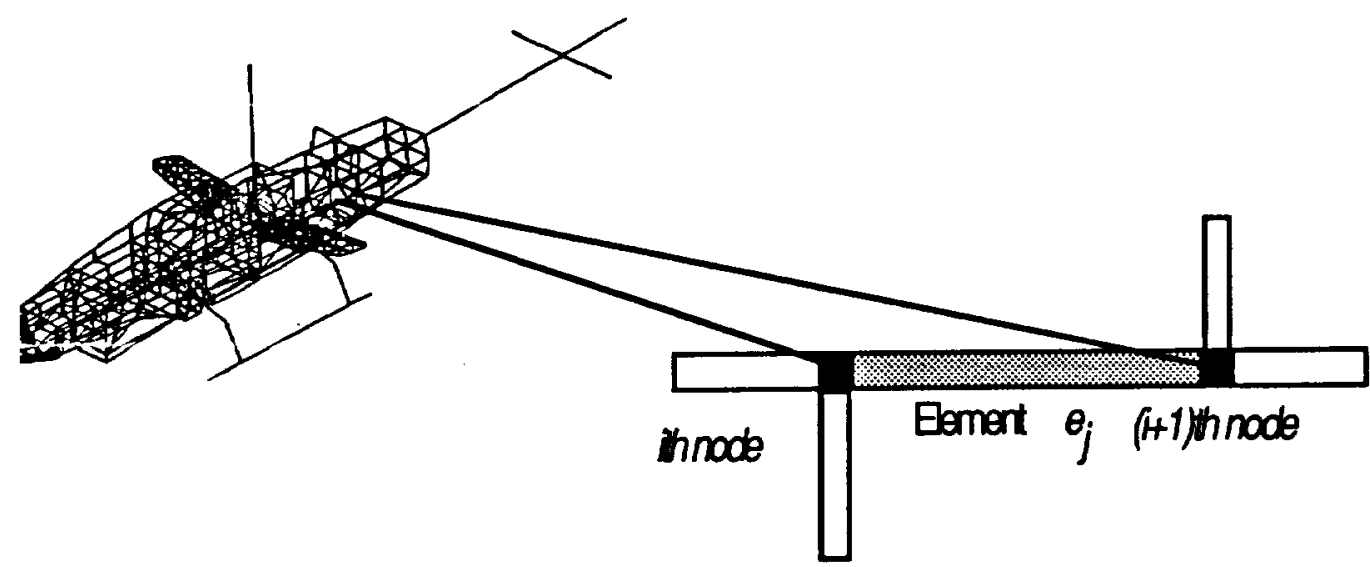

Fig. 1 Typical Bar Element, $\mathbf{e}_{j}$, in Airframe Model 



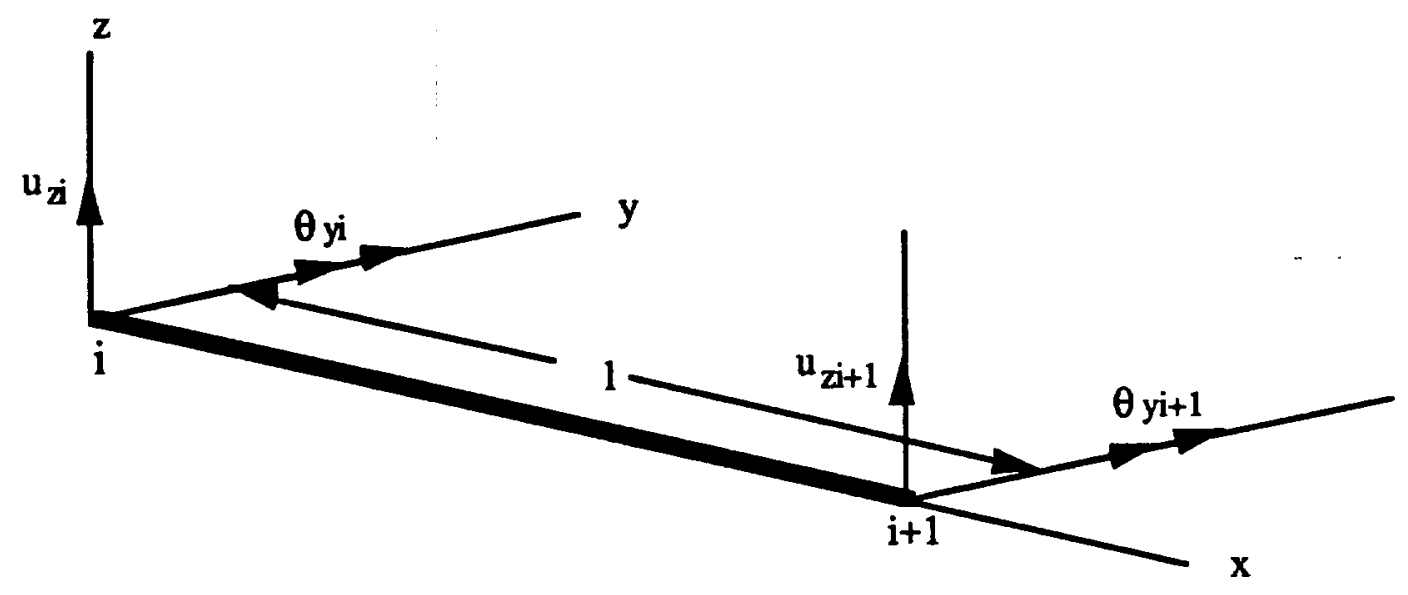

Fig. 2. Degrees of Freedom for Bending in the xz Plane

$$
\left\{\begin{array}{c}
\mathrm{F}_{\mathrm{zi}} \\
\mathrm{M}_{\mathrm{zi}} \\
\mathrm{F}_{\mathrm{zi}+1} \\
\mathrm{M}_{\mathrm{zi}+1}
\end{array}\right\}=\left[\begin{array}{cccc}
\mathrm{R} & -\frac{1}{2} \mathrm{R} & -\mathrm{R} & -\frac{1}{2} \mathrm{R} \\
\frac{12}{4} \mathrm{R}+\frac{E I_{y}}{1} & \frac{1}{2} \mathrm{R} & \frac{1^{2}}{2} \mathrm{R}-\frac{E I_{y}}{1} \\
& \mathrm{sym} & \mathrm{R} & \frac{1}{2} \mathrm{R} \\
& & & \frac{12}{4} \mathrm{R}+\frac{E I_{y}}{1}
\end{array}\right]\left\{\begin{array}{c}
u_{z i} \\
\theta_{z i} \\
u_{z i+1} \\
\theta_{z i+1}
\end{array}\right\}
$$

where $R=\left(\frac{1}{\mathrm{k}_{\mathrm{z}} \mathrm{AG}}+\frac{1^{3}}{12 \mathrm{EI}}\right)^{-1}$. If the modulus of elasticity, $\mathrm{E}$, is taken here as an adjustable physical quantity, $\gamma_{\mathrm{k}}$, then

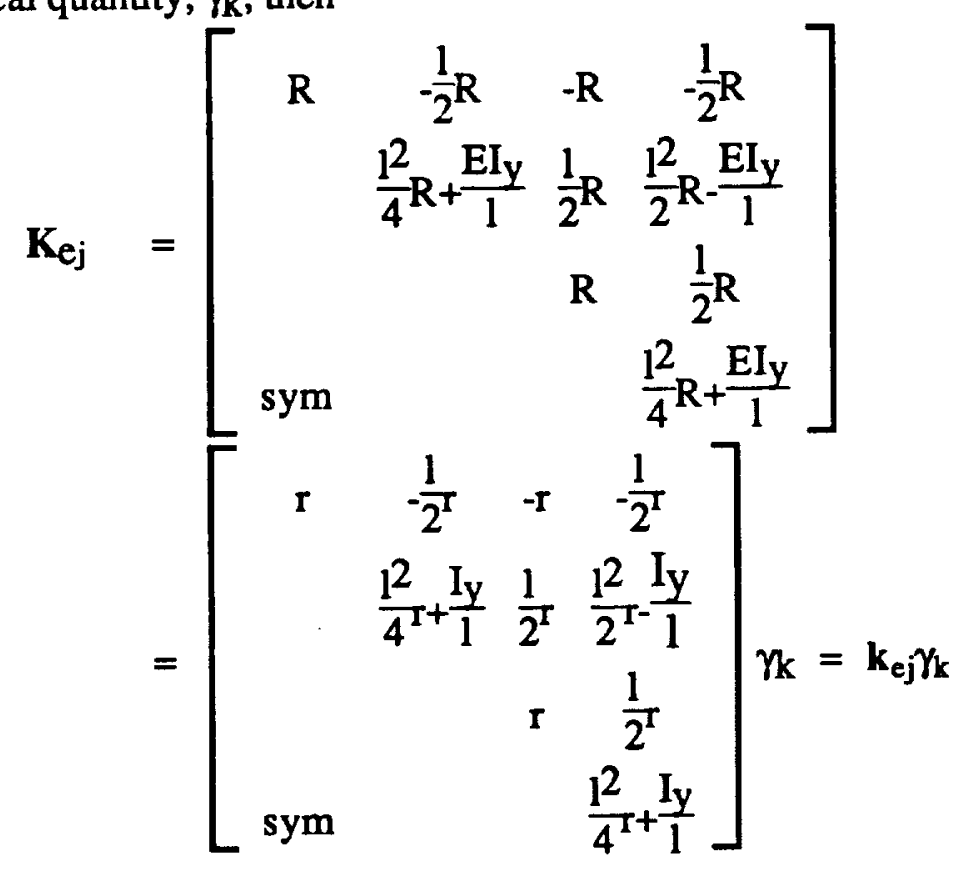



where $\gamma_{\mathrm{k}}=\mathrm{E}$ and $\mathrm{r}=\left(\frac{2(1+v) 1}{\mathrm{k}_{\mathrm{z}} \mathrm{A}}+\frac{1^{3}}{12 \mathrm{I}_{\mathrm{y}}}\right)^{-1}$. Suppose there are $\mathrm{n}$ elements which have the same $\mathrm{E}$ so that it is possible to express the stiffness as:

$$
\mathbf{k} \mathbf{k}=\sum_{e=1}^{n} \mathbf{k}_{\mathrm{ej}}
$$

When the modulus changes from $E$ to $E+d E$, the corresponding change in $\gamma_{k}$ is to $\gamma_{k}+d \gamma_{k}$. Considering all different $\gamma_{k}, K$ changes from $K$ to $K+d K$ where

$$
\mathrm{dK}=\sum_{\mathbf{k}=1}^{\mathbf{N}_{\mathbf{k}}} \mathbf{k}_{\mathbf{k}} \mathrm{d} \gamma_{\mathbf{k}}
$$

Similar procedures can be generalized to include the damping, other stiffness parameters, and mass.

$$
\begin{aligned}
& d \mathbf{M}=\sum_{i=1}^{N_{m}} \frac{\partial M}{\partial \alpha_{i}} d \alpha_{i}=\sum_{i=1}^{N_{m}} m_{i} d \alpha_{i} \\
& \mathrm{dC}=\sum_{\mathrm{i}=1}^{\mathrm{N}_{\mathrm{f}}} \frac{\partial \mathrm{C}}{\partial \beta_{\mathrm{i}}} \mathrm{d} \beta_{\mathrm{i}}=\sum_{\mathrm{i}=1}^{\mathrm{N}_{\mathrm{C}}} \mathrm{c}_{\mathrm{i}} \mathrm{d} \beta_{\mathrm{i}} \\
& \mathrm{d} \mathbf{K}=\sum_{\mathbf{i}=1}^{\mathbf{N}_{\mathrm{k}}} \frac{\partial \mathbf{K}}{\partial \gamma_{\mathrm{i}}} \mathrm{d} \gamma_{\mathrm{i}}=\sum_{\mathrm{i}=1}^{\mathbf{N}_{\mathrm{k}}} \mathbf{k}_{\mathrm{i}} \mathrm{d} \gamma_{\mathrm{i}}
\end{aligned}
$$

Substituting these into Eq. (19) yields a set of linear algebra equations with unknowns d $\alpha_{i}, d \beta_{i}$ and $d \gamma_{i}$ :

$$
\left[\begin{array}{llll}
U_{R} T \frac{\partial M}{\partial \alpha_{1}} \ldots & V_{R} T \frac{\partial C}{\partial \beta_{1}} \ldots W_{R} T \frac{\partial K}{\partial \gamma_{1}} \ldots \\
U_{I} T \frac{\partial M}{\partial \alpha_{1}} \ldots & V_{I} T \frac{\partial C}{\partial \beta_{1}} \ldots W_{I} \frac{\partial K}{\partial \gamma_{1}} \ldots
\end{array}\right]\left\{\begin{array}{c}
d \alpha_{1} \\
\cdot \\
\cdot \\
d \dot{N}_{m} \\
d \beta_{1} \\
\cdot \\
\cdot \\
d \beta_{N_{c}} \\
d \gamma_{1} \\
\cdot \\
\cdot \\
\cdot \\
d \gamma_{N_{k}}
\end{array}\right\}=\left\{\begin{array}{l}
Y_{1} \\
Y_{2}
\end{array}\right\}
$$

The number of unknowns in this equation is much less than the number of unknowns in Eq. (19), and also all the unknowns in this equation have physical meaning in the real structure.

However, neither Eq. (19) or Eq. (25) can be solved directly since the numbers of unknowns and equations are not equal in most of the cases. There exists a number of techniques for dealing with sets of equations that are under or over-determined or with matrices that are either singular or else poorly conditioned. The singular value decomposition, or SVD method ${ }^{26}$, is one of the most 

powerful ways to handle these problems. In the present study it was employed to compute solutions to Eq's. (19) and (25) which are highly under-determined for most practical situations. In this case the SVD method provides a least square type of solution to the problem.

In most cases, the selected physical parameters must also be restricted to positive values in order to make sense physically. However, the identification procedure outlined above cannot guarantee that the identified values will all be positive. This is of particular concern when the parameters are proportional to mass, an elastic modulus or a damping coefficient, all of which must be positive for the systems typically considered. Using a constrained optimization method, this problem can be eliminated. The present problem can be posed as one of minimizing

$$
f=d \alpha_{1}+d \alpha_{2}+\cdot \cdot+d \alpha_{N_{m}}+d \beta_{1}+. .+d \beta N_{c}+d \gamma_{1}+. .+d \gamma_{k}
$$

subject to the constraints

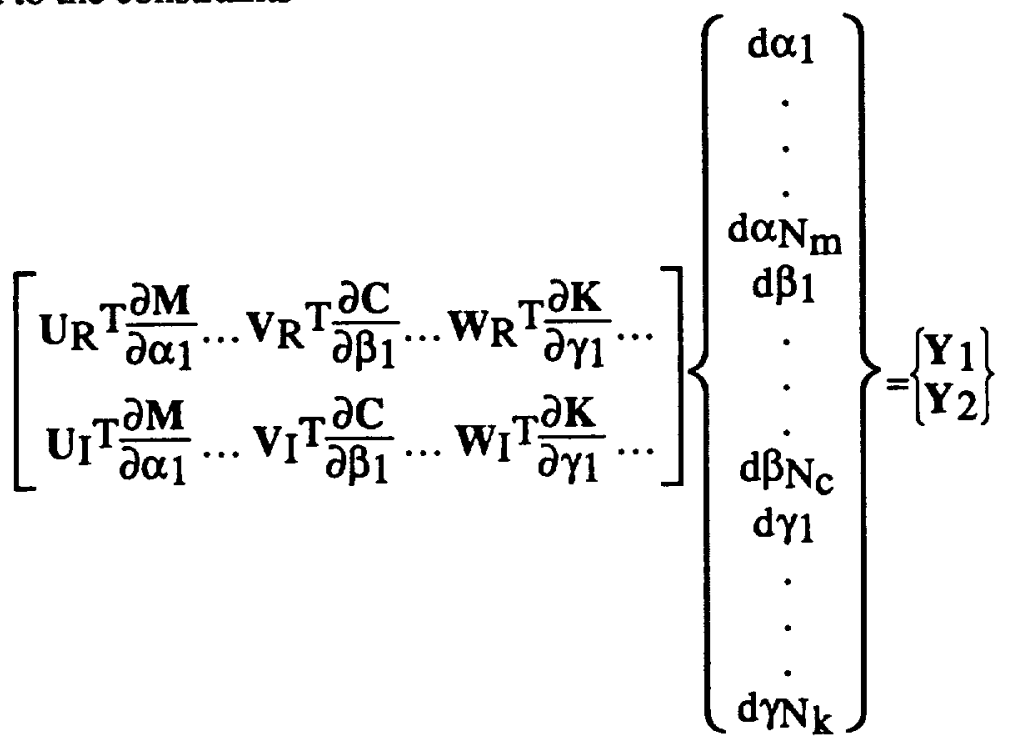

and

$$
d \alpha_{1} \geq 0, d \alpha_{2} \geq 0, . d \alpha_{N_{m}} \geq 0, d \beta_{1} \geq 0, . d \beta_{N_{c}} \geq 0, d \gamma_{1} \geq 0, . . d \gamma_{N_{k}} \geq 0
$$

The feasible solution $\left(d \alpha_{1}, d \alpha_{2}, \ldots, d \alpha_{N_{m}}, d \beta_{1}, \ldots, d \beta_{N_{c}}, d \gamma_{1}, \ldots, d \gamma_{N_{k}}\right)$ to this problem yields the identified selected physical parameters. 



\section{APPLICATIONS}

\section{Svstem_Identification _Procedures}

The method described above was applied to several practical examples. For these cases, the analytical finite element model for the structures was assumed correct and was developed using the NASTRAN finite element analysis package ${ }^{28}$. Then, values for selected physical parameters in the model were identified on the basis of measured experimental data (eigenvalues and eigenvectors) so that the analytical model more accurately represented the real structure. The assumption for this procedure was that the identification process could be applied in an iterative fashion by making successive small modifications to the selected physical parameters until satisfactory agreement with experimental results was obtained. For the $\mathrm{i}$-th iteration, there are the following relationships:

$$
\begin{aligned}
& \mathbf{M}^{\mathrm{i}}=\mathbf{M}^{\mathrm{i}-1}+\mathrm{d} \mathbf{M}, \mathbf{C}^{\mathrm{i}}=\mathbf{C}^{\mathrm{i}-1}+\mathrm{dC}, \mathbf{K}^{\mathrm{i}}=\mathbf{K}^{\mathrm{i}-1}+\mathrm{dK} \\
& \mathbf{U}^{\mathrm{i}}=\mathbf{U}^{\mathrm{i}-1}+\mathrm{d} \mathbf{U}, \mathbf{V}^{\mathrm{i}}=\mathbf{V}^{\mathrm{i}-1}+\mathrm{dV}, \mathbf{W}^{\mathrm{i}}=\mathbf{W}^{\mathrm{i}-1}+\mathrm{dW},
\end{aligned}
$$

Substitute these into equation (25), we can obtain

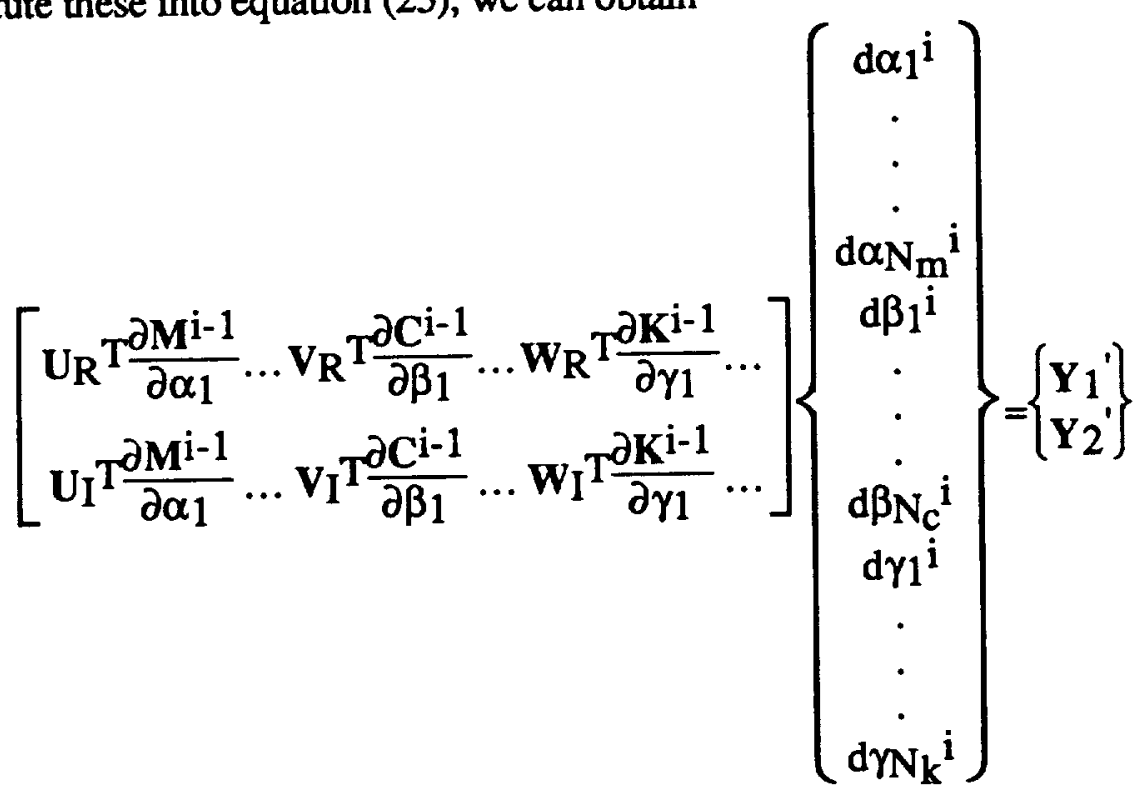

where

$$
\begin{aligned}
& \mathbf{Y}_{1}{ }^{\prime}=-\left(d U_{R^{T}}^{T} M^{i-1}+d V_{R}^{T} C^{i-1}+d W_{R}^{T} K^{i-1}\right) \\
& Y_{2}{ }^{\prime}=-\left(d U_{I}^{T} M^{i-1}+d V_{I}^{T} C^{i-1}+d W_{I}^{T} K^{i-1}\right)
\end{aligned}
$$

The convergence criteria was formulated as follows:

(1) Check the physical parameter differences $d \alpha_{1}, d \alpha_{2}, \ldots d \alpha \alpha_{m}, d \beta_{1}, \ldots d \beta_{N c}, d \gamma_{1}, \ldots, d \gamma_{k}$ either manually or programmatically. If these physical parameter differences are smaller than a tolerance value, the identified physical parameters are obtained.

(2) Check the differences of the experimental eigenvalues and the i-th iteration analytical results which are obtained after running NASTRAN. If the differences are smaller than a tolerance value, the identified system is obtained.

\section{Simple Numerical Example}

In order to verify the proposed approach, the identification procedure developed above was applied first to a very simple finite element model with only a few degrees of freedom. It is a simple variable cross section straight rod with fixed ends, and it contains all the desired parameters 

to be identified such as mass, stiffness and damping. It was modeled using 9 rod elements with lumped masses at each node as shown in Fig. 3, and representative values were assumed for all elements and mass properties. For the purpose of defining the damping, the elements were segregated into 4 groups and a different damping coefficient was specified for each group.

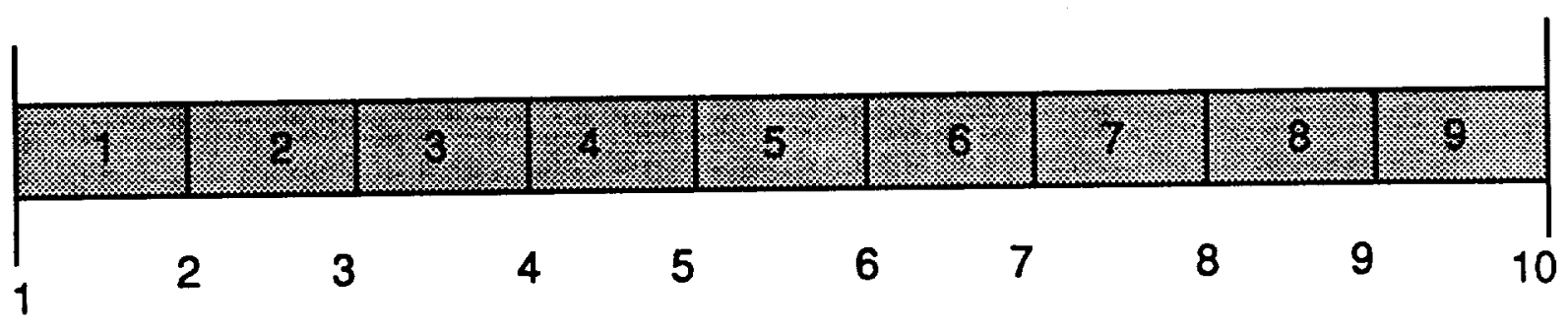

Figure 3. Simple Numerical Example

The assumed physical properties were defined to be typical of an aluminum rod. The length was $228.6 \mathrm{~mm}\left(9^{\prime \prime}\right)$ and the modulus $\mathrm{E}=68.9 \mathrm{GPa}$ (10e6 psi). The concentrated masses at each node were those given in Table 1 . This model was then employed to generate eigenvalues and eigenvectors which were used to represent "measured" test results. In all the cases presented in this report, the calculations were done in US customary units and the results converted to SI units. As a result, some of the percentage figures may be slightly in error due to numerical roundoff in the conversion of units.

TABLE 1.

ASSUMED PHYSICAL PROPERTIES FOR SIMPLE NUMERICAL EXAMPLE

\begin{tabular}{|c|l|l|l|}
\hline Index & $\begin{array}{c}\text { Mass at } \\
\text { Node } \\
(\mathrm{kg})\end{array}$ & $\begin{array}{c}\text { Element } \\
\text { Stiffness } \\
(\mathrm{MN} / \mathrm{m})\end{array}$ & $\begin{array}{c}\text { Element } \\
\text { Damping } \\
\text { Coefficient } \\
(\mathrm{kN} \mathrm{s} / \mathrm{m})\end{array}$ \\
\hline 1 & 21.89 & 367.7 & 28.34 \\
\hline 2 & 22.76 & 369.5 & 28.34 \\
\hline 3 & 23.64 & 371.3 & 28.34 \\
\hline 4 & 24.51 & 373.0 & 27.58 \\
\hline 5 & 25.39 & 374.8 & 27.58 \\
\hline 6 & 26.26 & 376.5 & 27.14 \\
\hline 7 & 27.14 & 378.3 & 27.14 \\
\hline 8 & 28.01 & 380.0 & 26.27 \\
\hline 9 & 28.89 & 381.8 & 26.27 \\
\hline 10 & 29.76 & & \\
\hline & & & \\
\hline
\end{tabular}

The objective of the identification was to determine the physical parameters such as damping constants ( $c$ or $\zeta$ ) and the cross section area for each rod element. There are three different cases to start to consider with this system. In the first case, the mass and the stiffness matrices were assumed to be accurate, and four damping parameters were identified assuming zero as initial analytical values of the damping matrix. The identified damping parameters are listed in Table 2 . 
$-$ 
TABLE 2.

CASE I: IDENTIFYING THE DAMPING PARAMETERS

\begin{tabular}{|c|c|c|c|c|}
\hline $\begin{array}{c}\text { Damping } \\
\text { Parameter }\end{array}$ & $\begin{array}{c}\text { Exact } \\
\text { Value }\end{array}$ & $\begin{array}{c}\text { Initial } \\
\text { Value }\end{array}$ & $\begin{array}{c}\text { Identified } \\
\text { Value }\end{array}$ & $\begin{array}{c}\text { Error } \\
(\%)\end{array}$ \\
\hline c1 & 28.34 & 0. & 28.3420 & -0.0029 \\
\hline c2 & 28.34 & 0. & 28.3420 & -0.0029 \\
\hline c3 & 28.34 & 0. & 28.3420 & -0.0029 \\
\hline c4 & 27.58 & 0. & 27.5805 & -0.0022 \\
\hline c5 & 27.58 & 0. & 27.5805 & -0.0022 \\
\hline c6 & 27.14 & 0. & 27.1431 & -0.0006 \\
\hline c7 & 27.14 & 0. & 27.1431 & -0.0006 \\
\hline c8 & 26.27 & 0. & 26.2676 & -0.0005 \\
\hline c9 & 26.27 & 0. & 26.2676 & -0.0005 \\
\hline
\end{tabular}

The second case was to identify the stiffness parameters assuming accurate values of mass and damping parameters which were the same for all elements $\mathbf{k}_{\mathbf{i}}=376.5 \mathrm{MN} / \mathrm{m}$. The identified stiffness parameters are listed below.

TABLE 3.

CASE II: IDENTIFYING STIFFNESS PARAMETERS

\begin{tabular}{|c|c|c|c|c|}
\hline $\begin{array}{c}\text { Stiffness } \\
\text { Parameter }\end{array}$ & $\begin{array}{c}\text { Exact } \\
(\mathrm{MN} / \mathrm{m})\end{array}$ & $\begin{array}{c}\text { Initial } \\
(\mathrm{MN} / \mathrm{m})\end{array}$ & $\begin{array}{c}\text { Identified } \\
(\mathrm{MN} / \mathrm{m})\end{array}$ & $\begin{array}{c}\text { Error } \\
(\%)\end{array}$ \\
\hline $\mathbf{k} 1$ & 367.7 & 376.5 & 367.7 & 0.0000 \\
\hline $\mathbf{k} 2$ & 369.5 & 376.5 & 369.5 & -0.0005 \\
\hline $\mathbf{k} 3$ & 371.3 & 376.5 & 371.3 & 0.0000 \\
\hline $\mathbf{k} 4$ & 373.0 & 376.5 & 373.0 & 0.0000 \\
\hline $\mathbf{k} 5$ & 374.8 & 376.5 & 374.8 & 0.0000 \\
\hline $\mathbf{k} 6$ & 376.5 & 376.5 & 376.5 & 0.0000 \\
\hline $\mathbf{k} 7$ & 378.3 & 376.5 & 378.3 & 0.0000 \\
\hline $\mathbf{k} 8$ & 380.0 & 376.5 & 380.0 & 0.0000 \\
\hline $\mathbf{k} 9$ & 381.8 & 376.5 & 381.8 & 0.0000 \\
\hline
\end{tabular}

In the third case, both the damping and the stiffness parameters were identified under the assumption of accurate mass value alone. The elements of the initial damping matrix were assumed to be zero, and the stiffness parameters were assumed to be the same for all elements $\left(k_{i}=376.5 \mathrm{MN} / \mathrm{m}\right)$. The identified damping and stiffness parameters are listed in Tables 4 and 5 . 

TABLE 4.

CASE III(A): DAMPING PARAMETERS

\begin{tabular}{|c|c|c|c|c|}
\hline $\begin{array}{l}\text { Damping } \\
\text { Parameter }\end{array}$ & Exact & Initial & Identified & $\begin{array}{c}\text { Error } \\
(\%)\end{array}$ \\
\hline c1 & 28.34 & 0. & 28.34 & 0.0000 \\
\hline c2 & 28.34 & 0. & 28.34 & 0.0000 \\
\hline c3 & 28.34 & 0. & 28.34 & 0.0000 \\
\hline c4 & 27.58 & 0. & 27.5812 & 0.0004 \\
\hline c5 & 27.58 & 0. & 27.5812 & 0.0004 \\
\hline c6 & 27.14 & 0. & 27.1437 & 0.0015 \\
\hline c7 & 27.14 & 0. & 27.1437 & 0.0015 \\
\hline c8 & 26.27 & 0. & 26.2679 & 0.0008 \\
\hline$c 9$ & 26.27 & 0. & 26.2679 & 0.0008 \\
\hline
\end{tabular}

TABLE 5. CASE III(B): STIFFNESS PARAMETERS

\begin{tabular}{|c|c|c|c|c|}
\hline $\begin{array}{c}\text { Stiffness } \\
\text { Parameter }\end{array}$ & $\begin{array}{c}\text { Exact } \\
(\mathrm{MN} / \mathrm{m}\end{array}$ & $\begin{array}{c}\text { Initial } \\
(\mathrm{MN} / \mathrm{m})\end{array}$ & $\begin{array}{c}\text { Identified } \\
(\mathrm{MN} / \mathrm{m})\end{array}$ & $\begin{array}{c}\text { Error } \\
(\%)\end{array}$ \\
\hline $\mathbf{k} 1$ & 367.7 & 376.5 & 367.700 & 0.0000 \\
\hline $\mathbf{k} 2$ & 369.5 & 376.5 & 369.492 & -0.0019 \\
\hline $\mathbf{k} 3$ & 371.3 & 376.5 & 371.285 & 0.0094 \\
\hline $\mathbf{k} 4$ & 373.0 & 376.5 & 373.044 & 0.0112 \\
\hline $\mathbf{k} 5$ & 374.8 & 376.5 & 374.763 & 0.0028 \\
\hline $\mathbf{k} 6$ & 376.5 & 376.5 & 376.521 & 0.0047 \\
\hline $\mathbf{k} 7$ & 378.3 & 376.5 & 378.290 & 0.0093 \\
\hline $\mathbf{k} 8$ & 380.0 & 376.5 & 379.964 & -0.0111 \\
\hline $\mathbf{k} 9$ & 381.8 & 376.5 & 381.908 & 0.0394 \\
\hline
\end{tabular}

All the results were obtained after only one iteration. For these simple cases the method accurately identified the selected physical parameter values (damping and cross section areas).

\section{Application to AH-1G Model}

A NASTRAN finite element model (FEM) for the AH-1G helicopter airframe has existed for a long time and was originally developed by Bell Helicopter Textron Inc. It is basically composed of two parts, one is stiffness modeling for idealizing the structures and the other is weight modeling for distributing weights to grid points. There are 4405 different elements with a total of 2764 degrees of freedom in the basic full model. A reduced model, based on Guyan reduction, contains only a total of 63 physical degrees of freedom.

Normally, the input and output data files from NASTRAN dynamic analyses are specially formatted and are quite large for a large finite element model such as the full AH-1G model. For convenience and accuracy, the present system identification programs were designed to automatically read NASTRAN output files and create NASTRAN input data deck files. At each step in the iterative identification procedure, the new modified physical parameters were put into the NASTRAN model bulk data in order to generate the required analytical results, such as eigenvalues, eigenvectors and other parameters, for the next iteration.

The mass, stiffness and damping matrices defined with respect to the internal degrees of freedom are not normal NASTRAN output data. However, such results can be developed by 

- 
using appropriate Direct Matrix Abstraction Programming (DMAP) utilities so that the identification program can automatically get this NASTRAN output data (see Appendix B).

\section{Results Using Simulated Test Data}

The NASTRAN model of an AH-1G airframe includes 4405 different elements with a total of 2764 degrees of freedom. In order to make sure that the identification procedure was appropriate to a such big model, the use simulation has been chosen to begin with. For this identification, the mass and stiffness properties of the analytical model were considered to be accurate, and nonproportional damping properties were identified. The physical damping parameters were associated with 8 distinctly different types of materials and structural fabrication techniques used in the airframe (e.g., aluminum, steel, riveted, welded, bolted, etc.) and one of these damping values was associated with each of the model elements using Eq. (11).

For this case, the test data were synthesized from the original NASTRAN model assuming small values for the extension and rotation viscous damping coefficients $(\mathrm{kN}-\mathrm{s} / \mathrm{m}$ and $\mathrm{N}-\mathrm{s} / \mathrm{rad}$ units):

\section{TABLE 6. ASSUMED INITIAL PHYSICAL DAMPING VALUES}

\begin{tabular}{|c|c|}
\hline Extension & Rotation \\
\hline$C_{1}=5.253$ & $C_{5}=93.4$ \\
\hline$C_{2}=8.756$ & $C_{6}=155.7$ \\
\hline$C_{3}=1.751$ & $C_{7}=31.14$ \\
\hline$C_{4}=1.226$ & $C_{8}=21.80$ \\
\hline
\end{tabular}

The synthesized data included 24 modes of which 6 were rigid body modes, and the frequency range was from 0.0 to $30.2 \mathrm{~Hz}$. The dimension of the mass, stiffness and damping matrices was $2764 \times 2764$. The initial values of the physical damping parameters for the analytical NASTRAN model were taken to be zero, and the results for the identified values are shown below:

\section{TABLE 7. IDENTIFIED PHYSICAL DAMPING PARAMETERS}

\begin{tabular}{|c|c|c|}
\hline Parameter & Initial & Identified \\
\hline $\mathrm{C}_{1}$ & 5.253 & 5.429 \\
\hline $\mathrm{C}_{2}$ & 8.756 & 10.490 \\
\hline $\mathrm{C}_{3}$ & 1.751 & 1.746 \\
\hline $\mathrm{C}_{4}$ & 1.226 & 6.069 \\
\hline $\mathrm{C}_{5}$ & 93.4 & 55.91 \\
\hline $\mathrm{C}_{6}$ & 155.7 & 160.35 \\
\hline $\mathrm{C}_{7}$ & 31.14 & 65.96 \\
\hline $\mathrm{C}_{8}$ & 21.80 & 55.91 \\
\hline
\end{tabular}

The error in the identified damping parameters as a function of the number of matrix elements for each of the 8 damping types is shown in Fig. 4. The error for those element types with more than 100 elements present is quite low, but it is much larger for those types with only a few elements present in the complete finite element model. The largest error was associated with what appeared to be elastomeric materials. 



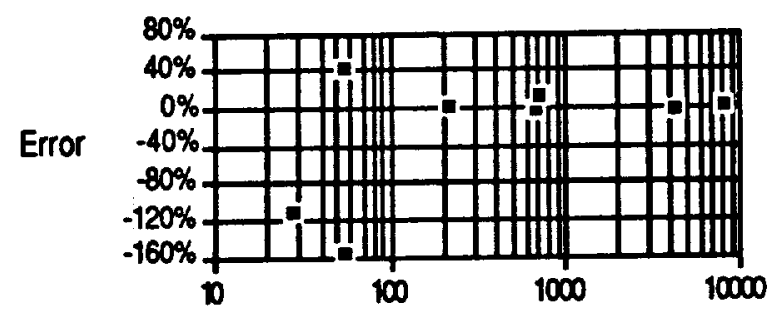

Number of Matrix Elements for Each Parameter

Fig. 4. Error in Damping Estimate as a Function of Number of Matrix Elements in Model

This simulation confirms the identification procedure for a complicated but yet well-defined example. If the assumptions such as nonproportional damping are correct for the airframe and if the experimental data are of high quality, the physical damping parameters can be identified from the test data.

\section{Actual AH-1G Data}

Actual test data for an AH-1G aiframe were provided by Bell Helicopter Textron Inc. based on ground vibration tests and included both resonance dwell and FRF (frequency response function) data. The experimental data were available for 8 different configurations of the $A H-1 G$ that were tested. The principal difference between the tests concerned the degree of complexity of the actual airframe tested. At one extreme, the bare airframe without most attachments was tested while at the other extreme the complete airframe with all attached mechanical components was tested. The test data from the most complex airframe configuration (with all difficult components present) showed the poorest agreement with the corresponding analytical model, while the data from the simplest test airframe showed the best agreement.

For this study, the test data from the most complex airframe configuration was used. Only the FRF data were employed, and the complex eigenvalues and eigenvectors for some 7 triaxial modes were obtained from the FRF data by using the TDAS ${ }^{\infty}$ curvefitting program ${ }^{29}$. The experimental data were provided as FRFs in TDAS universal file format, and the results generated by TDAS were complex eigenvalues and eigenvectors.

Before use in the identification program, the eigenvectors were normalized. Two options were used to normalize both experimental and analytical eigenvectors. One was to normalize the eigenvectors to the same point, and the other was to normalize based on the minimum deviation between the analytical and experimental eigenvectors.

The full finite element model for AH-1G airframe, as mentioned in the previous section, has a total of 2764 degrees of freedom which is very large for the identification procedure. In order to keep the problem tractable, a Guyan reduction was used in the present application to reduce the analytical model to a total of 63 degrees of freedom, which corresponded to the 23 distinct locations on the airframe at which experimental measurement were made. The error due to the reduction in degrees of freedom from 2764 to 63 is shown in Table 8.

(2) TDAS (Test Data Analysis) is a part of I-DEAS which is a computer-aided engineering product of Structural Dynamical Research Corporation (SDRC). 

TABLE 8.

EIGENVALUES (FREQUENCY) (WTTHOUT ANY DAMPING)

\begin{tabular}{|c|c|c|c|c|}
\hline Test & $\begin{array}{c}\text { Full } \\
\text { Model }\end{array}$ & Error (\%) & $\begin{array}{c}\text { Reduced } \\
\text { Model }\end{array}$ & $\begin{array}{c}\text { Error } \\
(\%)\end{array}$ \\
\hline 7.2475 & 7.6734 & 5.877 & 7.6932 & 6.150 \\
\hline 8.0458 & 8.3467 & 3.740 & 8.4026 & 4.435 \\
\hline 15.9539 & 14.6722 & -8.034 & 15.825 & -0.810 \\
\hline 17.2174 & 17.3701 & 0.887 & 17.784 & 3.294 \\
\hline 23.7396 & 20.7955 & -12.392 & 22.881 & -3.606 \\
\hline 24.6675 & 25.7955 & 4.573 & 28.238 & 14.475 \\
\hline 32.6848 & 31.7526 & -2.852 & 33.786 & 3.369 \\
\hline
\end{tabular}

Initially, both the full and the reduced models were used as analytical models. Using the actual experimental data, the physical parameters in the analytical models were obtained using the iterative procedure outlined earlier. The initial results for both the full model and the reduced model included several negative identified damping parameters which were obtained using the singular value decomposition method when either zero or positive initial guess values were assumed for the analytical model. Physically of course, the damping parameters should be greater than zero, but mathematically, the identification procedure is oblivious to this constraint. The constrained optimization procedure outlined earlier was therefore used in order to overcome this problem. In addition, the reduced model was used in most of the identification cases, expect when otherwise stated, because of the small error and big savings in computational time.

The complete system identification was carried out in two steps. The first step was to identify the stiffness, and for this process the initial damping values were assumed to be zero. The second step was to use the stiffness values obtained from the first step to identify the damping values.

This was done under the assumption that the greatest change in natural frequency can be obtained by changing the stiffness parameter, while changes in the damping parameters will only fine-tune the eigenvalues but will obtain accurate modal damping estimates for the structure.

At the first step, four stiffness parameters associated with elastic moduli for four principal materials used in the airframe were selected to be identified. After two iterations, the differences between the identified and the initial moduli and the analytical and experimental eigenvalues were those shown in the following tables:

TABLE 9.

IDENTIFIED MODULUS VALUES

\begin{tabular}{|c|c|c|c|c|c|}
\hline & $\begin{array}{c}\text { Initial } \\
(\mathrm{GPa})\end{array}$ & $\begin{array}{c}\text { After } \\
\text { first } \\
\text { iteration } \\
(\mathrm{GPa})\end{array}$ & $\begin{array}{c}\text { Change } \\
\text { from initial } \\
\text { value (\%) }\end{array}$ & $\begin{array}{c}\text { After } \\
\text { second } \\
\text { iteration } \\
(\mathrm{GPa})\end{array}$ & $\begin{array}{c}\text { Change } \\
\text { from initial } \\
\text { value(\%) }\end{array}$ \\
\hline mat.-1 & 22.1 & 21.7 & -1.81 & 3.937 & 23.03 \\
\hline mat.-2 & 72.4 & 72.5 & 0.19 & 9.417 & -10.41 \\
\hline mat.-3 & 200.0 & 190.5 & -4.75 & 28.435 & -1.95 \\
\hline mat.-4 & 120.7 & 112.2 & -7.01 & 19.396 & 10.83 \\
\hline
\end{tabular}



TABLE 10.

IDENTIFIED EIGENVALUES (FREQUENCY)

\begin{tabular}{|c|c|c|c|c|c|c|}
\hline Test & Original & $\begin{array}{c}\text { Error } \\
(\%)\end{array}$ & $\begin{array}{c}\text { After } \\
\text { first } \\
\text { iteration }\end{array}$ & $\begin{array}{c}\text { Error } \\
(\%)\end{array}$ & $\begin{array}{c}\text { After } \\
\text { second } \\
\text { iteration }\end{array}$ & $\begin{array}{c}\text { Error } \\
(\%)\end{array}$ \\
\hline 7.247 & 7.693 & 6.15 & 7.686 & 6.05 & 7.426 & 2.47 \\
\hline 8.046 & 8.403 & 4.43 & 8.394 & 4.33 & 8.064 & 0.22 \\
\hline 15.95 & 15.82 & -0.81 & 15.795 & -0.99 & 15.302 & -4.09 \\
\hline 17.22 & 17.78 & 3.29 & 17.762 & 3.16 & 17.180 & -0.22 \\
\hline 23.74 & 22.88 & -3.61 & 22.899 & -3.53 & 21.819 & -8.08 \\
\hline 24.67 & 28.24 & 14.5 & 28.195 & 14.3 & 27.544 & 11.6 \\
\hline 32.68 & 33.79 & 3.37 & 33.805 & 3.43 & 32.481 & -0.62 \\
\hline
\end{tabular}

As the second step, the damping parameters were identified for the previously identified stiffness conditions. Initial estimates for the damping parameters were developed by assuming a nominal damping ratio, $\zeta=5 \%$. For the extensional elements, it was therefore assumed that the initial viscous damping values would be $\mathrm{CE}=17.5$ for all extensional viscous damping, and that for the rotational elements (assuming the cross section area to be a circle) it would be $c_{R}=222$ for all rotational damping.

After one iteration, the results shown in Table 11 were obtained.

TABLE 11.

FINAL RESULTS FOR AH-1G MODEL

\begin{tabular}{|l|c|c|c|c|c|}
\hline \multicolumn{1}{|c|}{ Mode } & \multicolumn{2}{|c|}{ Test } & $\begin{array}{c}\text { NASTRAN } \\
\text { (original) }\end{array}$ & \multicolumn{2}{c|}{ NASTRAN (final) } \\
\hline & $\omega_{\mathrm{n}(\mathrm{Hz})}$ & $\zeta(\%)$ & $\omega_{\mathrm{n}}(\mathrm{Hz})$ & $\omega_{\mathrm{n}}(\mathrm{Hz})$ & $\zeta(\%)$ \\
\hline First Lat Bending & 7.247 & 2.19 & 7.693 & 7.425 & 3.00 \\
\hline First Vert Bending & 8.046 & 1.56 & 8.403 & 8.057 & 4.55 \\
\hline Second Lat Bending & 15.954 & 3.05 & 15.82 & 15.41 & 1.70 \\
\hline Second Vert Bending & 17.217 & 1.02 & 17.78 & 17.12 & 7.48 \\
\hline Fuselage Torsion & 23.737 & 1.70 & 22.88 & 21.83 & 0.24 \\
\hline Third Vert Bending & 24.667 & 1.31 & 28.24 & 27.702 & 6.25 \\
\hline Third Lat Bending & 32.685 & 1.95 & 33.74 & 32.498 & 0.97 \\
\hline
\end{tabular}


- - 


\section{CONCLUSIONS AND RECOMMENDATIONS}

A structural dynamic system identification procedure that is capable of identifying physical parameter changes has been developed. The changes in physical parameters of the system can therefore be related to observed experimental data. In the examples considered, physical parameters, such as the damping constant of a material that will result in a nonproportionally damped system, the modulus of elasticity of a material, and the dynamic stiffness of a beam element have been identified by using the experimentally obtained frequency response functions, modes and eigenvalues.

Following the validation of the developed procedures by using synthesized data on a small model, the method was applied to a large-scale NASTRAN finite element model of a helicopter airframe. Both synthesized data and observed experimentally identified modal data were used. Again, modulus of elasticity, stiffness and damping constants were the parameters considered for the four representative materials used in the airframe. With the exception of one material that had been used to construct a very small number of components, other material constants were identified reasonably accurately where synthesized data were used. When experimental modal data were used, the modal parameters calculated from the identified model did not yield the experimentally observed modes only in cases where the initial a priori finite element model output and the experimental model output differed considerably. When experimental output and the $a$ priori model output were reasonably close, the results of the identification were satisfactory.

Even though the method was shown to work and the difference between the identified model and the experimental observations were considered satisfactory in some cases, there are some other cases that need improvement to make the procedure applicable to a structural dynamic design process:

(1) While the numerical processes were improved and refined, no similar improvements in the quality of the test data could be realized. One result of this problem was that it was relatively difficult to match measured eigenvalues and eigenvectors with corresponding analytical values. Quite often, the measured and initial eigenvalues matched closely while the eigenvectors differed considerably, and the identified eigenvectors were not significantly closer in agreement. For this reason it is necessary to consider other experimental data, such as the AH-1G dwell data, which have been acquired by other means.

(2) In cases where selected portions of experimental data and a priori analytical data differ significantly while a large amount of experimental and analytical data are close together, it is necessary to minimize first the large errors by using $\mathrm{H}_{\infty}$ type of identification before using the least square analysis with singular value decomposition.

(3) It is important that a larger group of identifiable parameters be considered.

(4) It is necessary that we examine the convergence and accuracy of the complete process.

(5) We have used linear sensitivity coefficients. Accuracy and convergence may require nonlinear sensitivity coefficients.

(6) The real damping in a structural dynamic system may not be linear viscous damping with a nonproportional behavior. It is necessary to include other types of damping mechanisms.

(7) As pointed out by Bell's DAMVIBS conclusions ${ }^{1}$, nonlinearity is important in considering selected components of the airframe.

(8) We should also examine the experimental parameter estimation processes used to determine modal parameters used as inputs to the identification process. 



\section{REFERENCES}

1. DAMVIBS Report, Bell.Helicopter Textron, Inc., 1988.

2. DAMVIBS Report, Sikorsky Aircraft Division, United Technology Corp., 1988.

3. Dompka, R. V., "Modeling Difficult Components of the AH-1G Helicopter Airframe," Proceedings, AHS Annual Forum, 1988.

4. Ibañez, $\dot{P}$., "Review of Analytical and Experimental Techniques for Improving Structural Dynamic Models," Welding Research Council Bulletin No. 249, 1979.

5. Ibrahim, S. R. and Mikulcik, E. C., "A Method for the Direct Identification of Vibration Parameters from the Free Response," Shock and Vibration Bulletin, Vol. 47, Pt. 4, 1977, pp. 183-198.

6. Kubrusly, C. S., "Distributed Parameter System Identification: A Survey," International Journal of Control, Vol. 26, No. 4, 1977, pp. 509-535.

7. Distefano, N. and Rath, A., "System Identification in Nonlinear Structural Seismic Dynamics," Computer Methods in Applied Mechanics and Engineering, Vol. 5, 1975, pp. 353-372.

8. Hanagud, S., Meyyappa, M. and Craig, J. I., "Method of Multiple Scales and Identification of Nonlinear Structural Dynamic Systems," AIAA Journal, Vol. 23, No. 5, 1985, pp. 802807.

9. Caravani, P., Watson, M. L. and Thomson, W. T., "Recursive Least-Square Time Domain Identification for Structural Parameters," Journal of Applied Mechanics, Vol. 44, No. 1, 1977, pp. 135-140.

10. S. Hanagud, M. Meyyappa, Y.P Cheng and J. I. Craig, "Identification of Structural Dynamic Systems with Nonproportional Damping," AIAA Journal, Vol. 24, No. 10, pp. 1880-1882, November 1986.

11. Caravani, P. and Thomson, W. T., "Identification of Damping Coefficients in Multidimensional Linear Systems," Journal of Applied Mechanics, Vol. 41, No. 2, 1974, pp. 379-382.

12. Beliveau, J. G., "Identification of Viscous Damping in Structures from Modal Information," Journal of Applied Mechanics, Vol. 43, No. 2, 1976, pp. 335-339.

13. Chen, J. C. and Garba, J. A., "Analytical Model Improvements Using Perturbation Techniques," AIAA Journal, Vol. 18, No. 6, pp. 684-690.

14. Collins, J. D., Hart, G. C., Hasselman, T. K. and Kennedy, B., "Statistical Identification of Structures," AIAA Journal, Vol. 12, No. 2, 1974, pp. 185-190.

15. Torkamani, M. A. M. and Hart, G. C., "Earthquake Engineering: Parameter Identification," Preprint 2499, ASCE National Structural Engineering Convention, New Orleans, LA, 1975.

16. Meyyappa, M. and Craig, J. I., "Highrise Building Identification Using Transient Testing," Proceedings, 8th World Conference on Earthquake Engineering, San Francisco, Vol. 6, 1984, pp. 79-86.

17. Baruch, M., "Optimal Correction of Mass and Stiffness Matrices Using Measured Modes," AIAA Journal, Vol. 20, No. 11, 1982, pp. 1623-1626.

18. Baruch, M., "Methods of Reference Basis for Identification of Linear Dynamic Structures," AIAA Journal, Vol. 22, No. 4, 1984, pp. 561-564.

19. Berman, A., "Mass Matrix Correction Using an Incomplete Set of Measured Modes," AIAA Journal, Vol. 17, No. 10, 1979, pp. 1147-1148.

20. Berman, A. and Nagy, E. J., "Improvement of a Large Analytical Model Using Test Data," AIAA Journal, Vol. 21, No. 18, 1983, pp. 1168-1173. 

21. Kabe, A. M., "Stiffness Matrix Adjustment Using Mode Data," AIAA Journal, Vol. 23, No. 9, 1985, pp. 1431-1436.

22. Berman, A. and Flannelly, W. G., "Theory of Incomplete Models of Dynamic Structures" AIAA Journal, Vol. 9, No. 8, 1971, pp. 1481-1487.

23. Hanagud, S., Meyyappa, M., Cheng, Y. P., and Craig, J. I., "Rotorcraft Structural Dynamic Design Modifications," Presented at the 10th European Rotorcraft Forum, The Hague, Netherlands, 1984.

24. Hanagud, S., Meyyappa, M., Sarkar, S., and Craig, J. I., "A Coupled Rotor/Airframe Vibration Model with Higher Harmonic Control Effects," 42nd AHS Forum, Washington, D. C..1986.

25. Kuo, C. P. and Wada, B. K., "Nonlinear Sensitivity Coefficients in System Identification," AIAA Journal, Nov. 1988.

26. W. H. Press, B. P. Flannery, S. A. Teukolsky, and W. T. Vetterling, "Numerical Recipes -The Art of Scientific Computing", Cambridge University Press, 1987.

27. Leonard Meirovitch, "Analytical Methods in Vibrations", MacMillan Company, 1967.

28. "NASTRAN User's Manual", MacNeal-Schwendler Corporation, November 1988.

29. "I-DEAS-Antegrated Design Engineering Analysis Software", SDRC, 1989.

30. Hanagud, S. V., Zhou, W., Craig, J. I. and N. J. Weston, "Use of System Identification Techniques for Improving Airframe Finite Element Models Using Test Data," 32nd Structures, Structural Dynamics and Materials Conference, Baltimore, MD, April 8-10, 1991, AIAA Paper 91-1260. 
- 


\section{ACKNOWLEDGEMENT}

The work described in this report was sponsored primarily by the NASA Langley Research Center under contract NAG-1-1007. Additional support was provided by the US Army Research Office under contract DAAG-29-82-K0094 (Center for Excellence in Rotary Wing Aircraft Technology) and by Georgia Tech. The authors gratefully acknowledge the support provided for this work. 



\section{APPENDIX A}

\section{SYSTEM IDENTIFICATION PROGRAM LISTING}

The following pages contain a listing of the current version of the program used to carry out the structural system identification described in this report. The program is written in the CDC version of Fortran 77 and was run on a CDC Cyber 180-990 running under the NOS/VE operating system. The program requires the use of the IMSL Scientific Library (Version 11) in order to carry out the singular value decomposition and the constrained optimization procedures.

The program was used with Version 66C of MSC/NASTRAN which was also run on the same computer system and was used to solve the structural dynamic eigenvalue problems. MSC/NASTRAN was used to run the initial eigenvalue problem and all subsequent iterative solutions. As a result, the program listing also includes the necessary I/O calls needed to operate directly with MSCNASTRAN input and output data files. The program also requires the experimentally determined eigenvalues and eigenvectors to be present is separate input files for each eigenvalue.

\section{A. Summary of Parameters Used by the Program}

\begin{tabular}{|c|c|}
\hline Parameter & Dimension \\
\hline LAMBDR, LAMBDI & MD \\
\hline LAMBDTR, LAMBDTI & MD \\
\hline WAR, WAI & $\mathrm{ND} \times \mathrm{MD}$ \\
\hline WTR, WTI & $\mathrm{ND} \times \mathrm{MD}$ \\
\hline VTR,VTI & $\mathrm{ND} \times \mathrm{MD}$ \\
\hline DUR, DUI & $\mathrm{ND} \times \mathrm{MD}$ \\
\hline DVR, DVI & $\mathrm{ND} \times \mathrm{MD}$ \\
\hline DWR, DWI & ND $\times M D$ \\
\hline MASS & NV \\
\hline STIFF & NV \\
\hline DAMP & NV \\
\hline $\mathrm{DC}, \mathrm{D}^{\mathrm{k}}$ & NV \\
\hline DK & NV \\
\hline COEFF & NM $\times$ ID \\
\hline $\mathrm{Y}$ & NM \\
\hline WK & ID2 \\
\hline BETA & ID \\
\hline GAMMA & ID \\
\hline MODES & $22 \times 2$ \\
\hline NTESTk $(k=1,2,5)$ & $20 \times 4$ \\
\hline NT & 7 \\
\hline $\begin{array}{l}\text { IRTYPE } \\
\text { simple }\end{array}$ & 12 \\
\hline $\begin{array}{l}\text { BL,BU } \\
\text { general }\end{array}$ & 12 \\
\hline A & $12 \times 12$ \\
\hline C & 12 \\
\hline
\end{tabular}

\section{Definition}

real and imaginary parts of analytical eigenvalues $\lambda_{\mathrm{A}}$ real and imaginary parts of test eigenvalues $\lambda$ real and imaginary parts of analytical eigenvectors $W_{A}$ real and imaginary parts of test eigenvectors $\mathrm{W}$ real and imaginary parts of $V_{A}$ real and imaginary parts of $U-U_{A}$ real and imaginary parts of $\mathrm{V}-\mathrm{V}_{\mathrm{A}}$ real and imaginary parts of $W-W_{A}$ mass matrix

stiffness matrix

damping matrix

ci matrix

$\mathbf{k}_{\text {i matrix }}$ coefficient matrix

vector of the right hand side of the equations work vector

damping parameters

stiffness parameters

test eigenvector

test measurement location definitions

numbers of test eigenvectors corresponding to the eigenvectors of NASTRAN model

vector indicating the type of constraints exclusive of bounds, where IRTYPE( ()$=0,1,2,3$ indicates .EQ., .LE., .GE., and range constraints respectively vectors containing the lower and upper limits of the constraints matrix containing the coefficients of the constraints vector containing the coefficients of the objective function 
$-\ldots$ 
OBJ

XIB, XUB

12

XSOL, DSOL

12

ND

N

MD

NMODES

ID

NUNK

NNK

NNC

NMR

NMT

LP value of the objective function

vectors containing the lower and upper bounds on the variables

vectors containing the primal and the dual solutions

order of the system, default $=63$

order of the system, (input)

modes used in the identification, default $=25$

modes used in the identification, (input)

number of physical parameters, default $=12$

number of unknowns to be identified (input)

number of stiffness unknowns

number of damping unknowns

number of rigid body motion modes

number of test modes

choice of solving techniques

$L P=0$, singular value decomposition

$\mathrm{LP}=1$, constrained optimization

\section{B. Definition of Input and Output Files}

File Name

TEST1, TEST2, TEST5

GUYAN_DAMP_F06

GUYAN_KCOUT

GUYAN_ELECDAT

GUYAN_ELEKDAT

TEST_EIGENV1, ..., TEST_EIGENV7 TEST_EIGENVAL

\section{Definition}

files containing 3 different test measurement location definitions

NASTRAN output data file including analytical mass, stiffness, damping matrices, eigenvalues and eigenvectors output file including results

NASTRAN output data file including the grouped element matrices $\mathbf{c}_{\mathbf{i}}$

NASTRAN output data file including the grouped element matrices $\mathbf{k}_{\mathbf{i}}$

files containing the test eigenvectors

file containing the test eigenvalues 



\section{Program Organization}

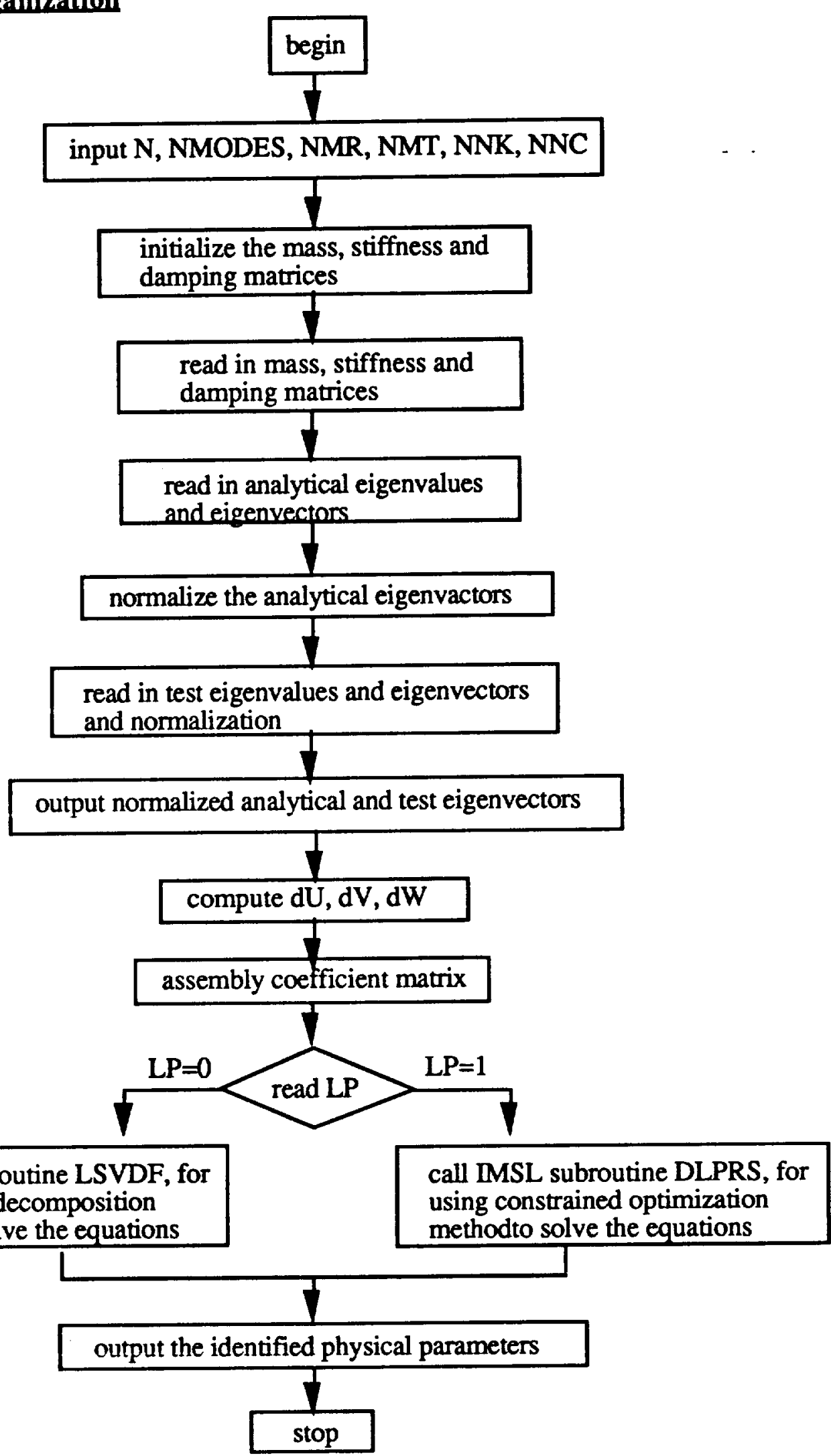

call IMSL subroutine LSVDF, for using singular decomposition technique to solve the equations

call IMSL subroutine DLPRS, for using constrained optimization methodto solve the equations 

This program is to identify the stiffness or damping parameters by using AHIG NASTRAN finite element models. The solution can be accomplished using singular value decomposition or constrained optimization.

Written by Weiyu Zhou, March, 1990.

\section{PROGRAM DATAPP (INP, OUTP, TAPE5 $=$ INP, TAPE 6 $=0$ UTP $)$}

PARAMETER $(N D=63, M D=25, I D=12$,

$*$

REAL LAMBDR (MD), LAMBDI (MD), LAMBDTR (MD), LAMBDTI (MD),

* WTR (ND, MD) ,WT I (ND, MD) ,WAR (ND, MD), WAI (ND, MD) ,

* DUR (ND, MD), DUI (ND, MD), DVR (ND,MD), DVI (ND, MD).

* DWR (ND, MD) , DWI (ND, MD), VTR (ND, MD) , VTI (ND, MD).

* MASS (NV), STIFF (NV), DAMP (NV), DC (NV), DK (NV),

* COEFF (NM, ID), Y (NM), WK (ID2), BETA (ID) , GAMMA (ID)

REAL CC (8), CK (4), DA (6), MODES $(22,2)$

INTEGER NTEST I $(20,4)$, NTEST2 $(20,4)$, NTEST5 $(22,4)$, NT (7) , IRTYPE (12)

REAL BL (12), BU (12),C (12),A (12,12),OBJ

REAL XLB (12), XUB (12), XSOL (12), DSOL (12)

DOUBLE PRECISION R,FI,RJ,FIJ,A , A2

CHARACTER *132 IDATA

CHARACTER $* 66$ MATID

COMPLEX SQU, SQUT, $C 1, C 2, C 3, C 4$

EQUIVALENCE (DC (1), DK (1))
C

$\mathrm{C}$

C

OPEN NASTRAN OUTPUT DATA FILE AND OTHER FILES

OPEN (UN IT $=7, F \mid L E=$ ' TEST 1 ', STATUS $=$ ' OLD')

OPEN (UN IT $=8, F \mid L E=$ ' TEST2 ', STATUS $=$ ' OLD')

OPEN (UN IT $=9, F \mid L E=$ ' TEST5', STATUS $=$ ' OLD')

OPEN $(U N|T=10, F| L E=$ ' GUYAN_DAMP FO6', FORM=' FORMATTED', IOSTAT $=1$ OS)

OPEN (UN IT $=12, F \mid L E=$ ' GUYAN KCOUT'', STATUS= ' UNKNOWN')

OPEN (UN IT $=14, F \mid L E=$ ' GUYAN ELECDAT', STATUS = ' OLD')

OPEN (UN IT $=15, F \mid L E=$ ' GUYAND ELEKDAT', STATUS = ' OLD')

OPEN (UNIT $=21, F \mid L E=$ ' TEST_ETGENVI', STATUS $=$ ' OLD')

OPEN (UN I T $=22, F I L E=$ ' TEST E I GENV2 ', STATUS $=$ ' OLD')

OPEN (UNIT $=23, F \mid L E=$ ' TEST ${ }^{-}$E IGENV 3 ', STATUS $=$ ' OLD')

OPEN (UNI $T=24, F \mid L E=$ ' TEST EIGENV4', STATUS $=$ ' OLD')

OPEN (UNIT $=25, F I L E=$ ' TEST EIGENV5' , STATUS $=$ ' OLD')

OPEN (UN I T $=26, F \mid L E=$ ' TEST E I GENV6 ' , STATUS $=$ ' OLD')

OPEN (UN I T $=27, F I L E=$ ' TEST EIGENV7' , STATUS $=$ ' OLD')

OPEN (UNIT $=28, F I L E=$ 'TEST ${ }^{-}$EIGENVAL' ', STATUS $=$' OLD')

IF (IOS.NE.0) WRITE (12,' ("i ERROR IN OPENING F06 FILE"/)')

$\operatorname{READ}(5, *) N$

WRITE $(12, '(1 X$, "ORDER OF THE SYSTEM - ", 14)')N

$\operatorname{READ}(5, *)$ NMODES

WRITE $(12, '(1 X, " N O$. OF MODES USED IN IDENTIFICATION ", 14) ') NMODES

$\operatorname{READ}(5, *)$ NMR

WRITE (12,' (1X,"NO. OF MODES OF RIGID BODY MOTION ", 14)') NMR

$\operatorname{READ}(5, *)$ NMT

WRITE $(12,1(1 X, " N O$. OF MODES OF TOTAL READ IN 1,14$) 1)$ NMT

$\operatorname{READ}(5, *)$ NNK

$\operatorname{READ}(5, *)$ NNC

NUNK $=$ NNC+NNK 

WRITE $(12, i(1 X, " N O$. OF UNKNOWNS TO BE IDENTIFIED ", 14)') NUNK

$\operatorname{READ}(5, *)(N T(1), 1=1,7)$

WRITE $(12, '(1 X, "$ TEST E IGEN NUMBER: $" /, 715) 1)($ NT $(1), 1=1,7)$

NELEM $=N *(N+1) / 2$

NCOEF F $=$ NUNK

$N C=N M O D E S * N$

$N C 2=N M O D E S * N * 2$

C

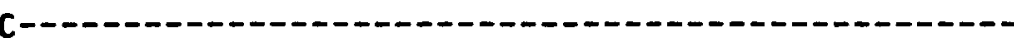

C READ IN THE MASS, STIFFNESS, AND DAMPING MATRICES

C

C

DO $1 \quad 1=1$, NELEM

$\operatorname{MASS}(1)=0.0$

$\operatorname{DAMP}(1)=0.0$

STIFF (1) $=0.0$

CONTINUE

C

c

C READ IN THE UPPER TRIANGULAR ELEMENTS

C

$\operatorname{READ}(5, *) N N$

DO $2 \quad||=1,3$

$3 \operatorname{READ}(10, '(A)$ ') IDATA

IF (IDATA (51:62). NE. 'INTERMEDIATE') GOTO 3

IF (IDATA (75:76) .EQ. 'MA') NU=7

IF (IDATA (75:76) .EQ.' $\left.K A^{\prime}\right) N U=8$

IF (IDATA (75:76) .EQ. 'BA') NU=9

IF (NN.NE. O) THEN

IF (NU.EQ. 7) WRITE (12, ' ("

MASS MATRIX") ')

IF (NU.EQ.8) WRITE (12, ' ("

STIFFNESS MATRIX") ')

IF (NU.EQ.9) WRITE (12, ' ("

DAMPING MATRIX") ')

\section{ENDIF}

$\operatorname{READ}(10,1$ (A) ') IDATA

$4 \operatorname{READ}\left(10,1(16, \operatorname{E2} 1.6,5 E 19.6,19)^{\prime}, \operatorname{ERR}=5\right) \mid 1,(D A(i), 1=1,6), 12$ IF.(I I.EQ.0) THEN

$\operatorname{READ}(10, '(A 66,17)$ ', ERR=5) MATID, i 3

GOTO 4

ENDIF

GOTO 7

$5 \quad$ DO $6 \quad 1=1,4$

6 READ (10,' (A)') IDATA

GOTO 4

7 DO $8 \mathrm{~K}=1,6$

IF (DA (K).EQ.0.0) GOTO 8

IF $((11+K-1) \cdot$ LT. I3) GOTO 8

IF (NN.NE .0) WRITE $(12, '(2 \mid 6, E 19.6) ') \mid 3,11+K-1$, DA (K)

$J 1=13$

$\mathrm{J} 2=\mid 1+K-1$

IF (NU.EQ.7) MASS $((\mathrm{Jl}-1) * N+J 2-(\mathrm{J} 1-1) * J 1 / 2)=D A(K)$

IF (NU.EQ.8) STIFF $((\mathrm{Jl}-1) * \mathrm{~N}+\mathrm{J} 2-(\mathrm{J} 1-1) * \mathrm{~J} 1 / 2)=\mathrm{DA}(\mathrm{K})$

8 IF (NU.EQ

IF (12.NE.63.OR.13.NE.63) GOTO 4

2 CONTINUE

C

C READ IN ANALYTICAL EIGENVALUES AND EIGENVECTORS

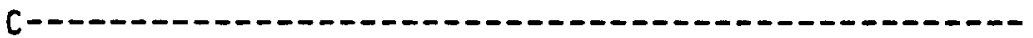

C

10 READ (10,' (A)') IDATA

IF (IDATA (18:21).NE.'ROOT') GOTO 10

$\operatorname{READ}\left(10,{ }^{\prime}(A)\right.$ ') IDATA

$J=0$

$11 \quad J=J+1$ 



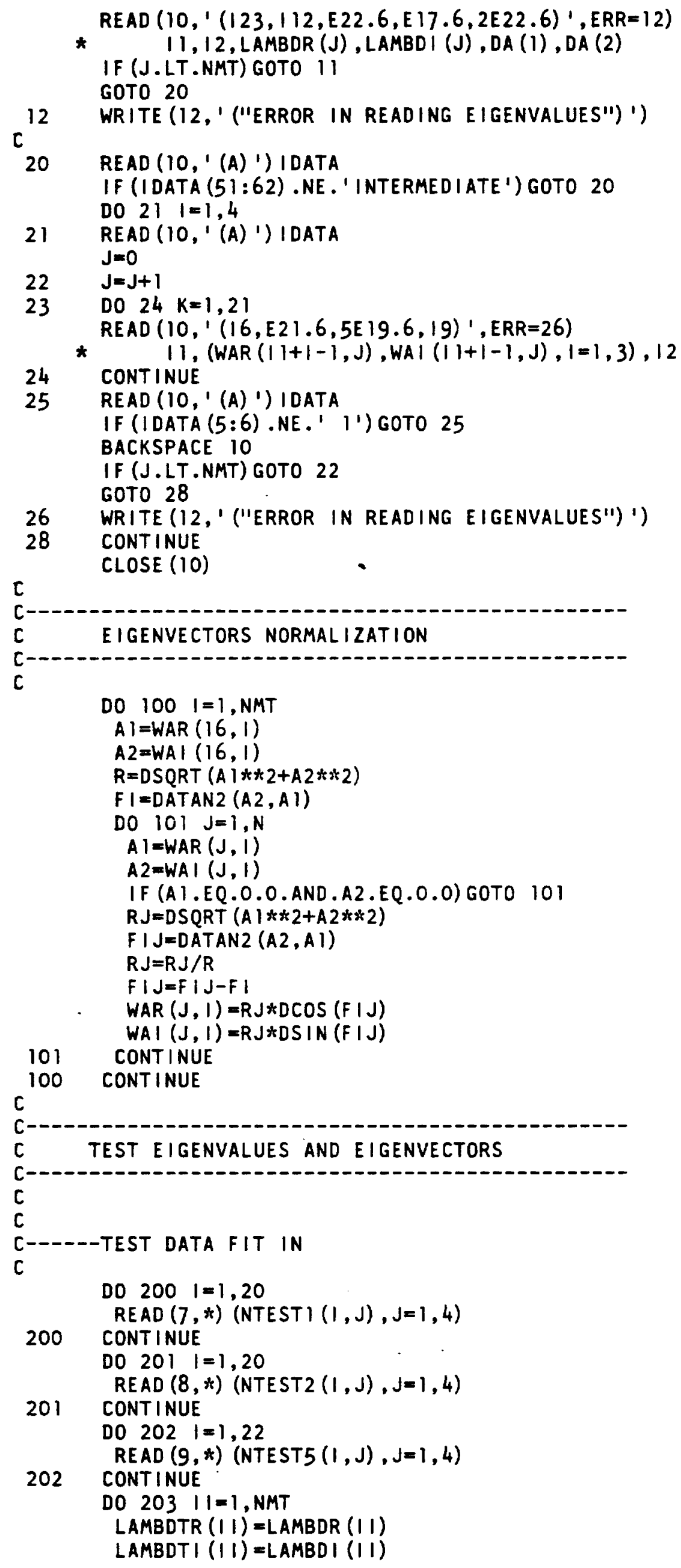


- 


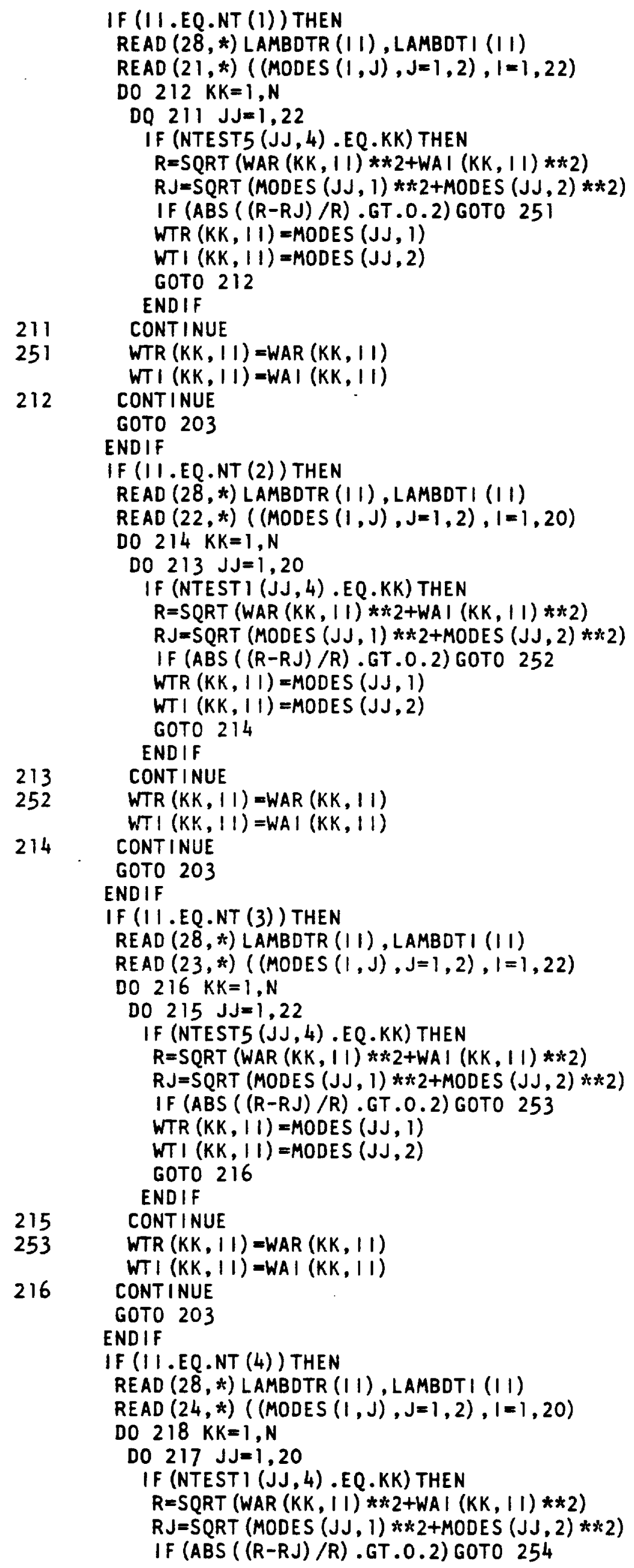





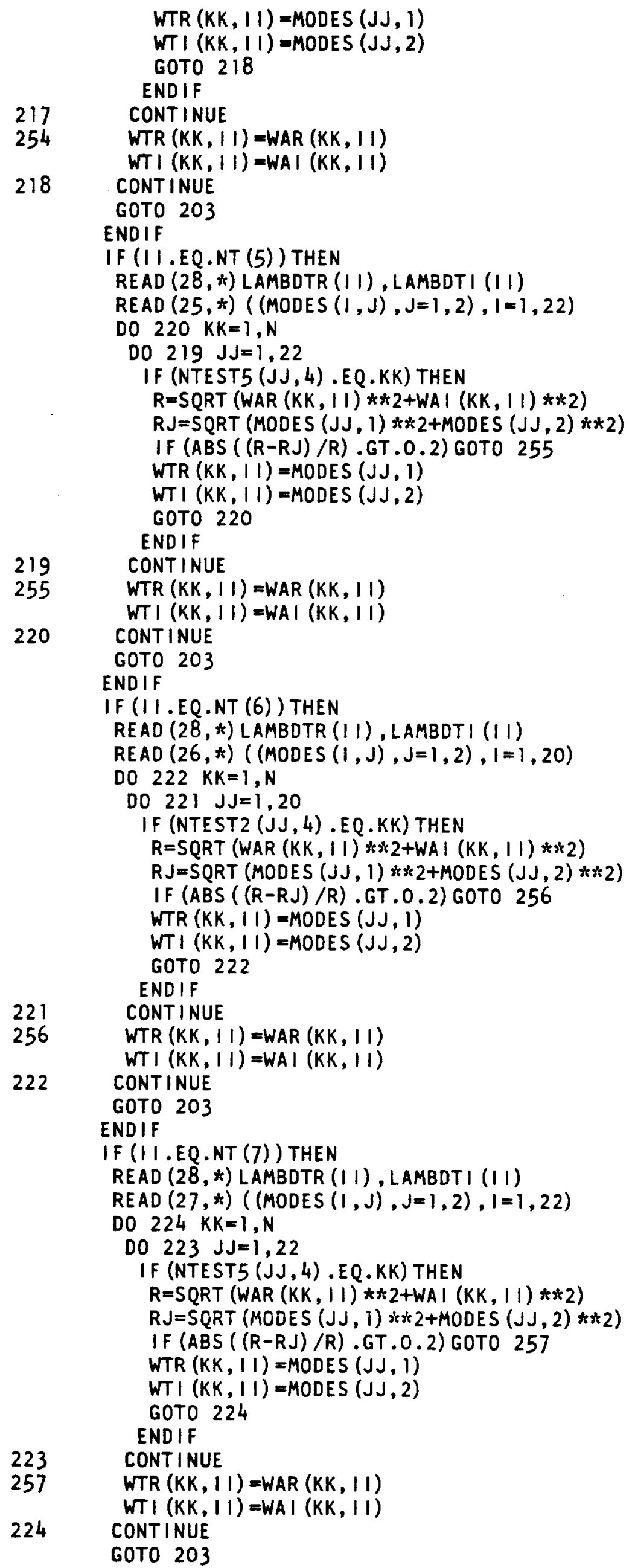





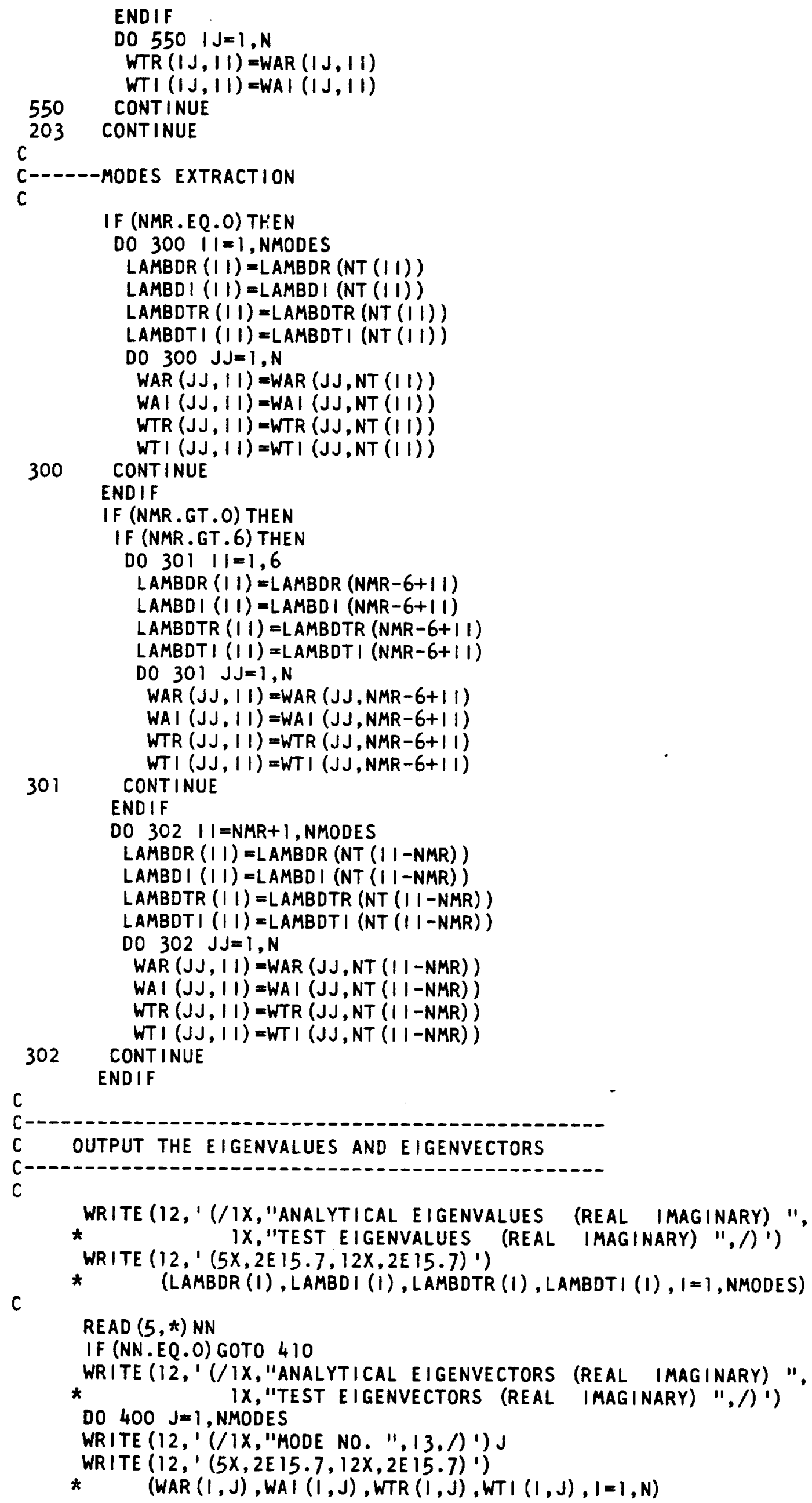





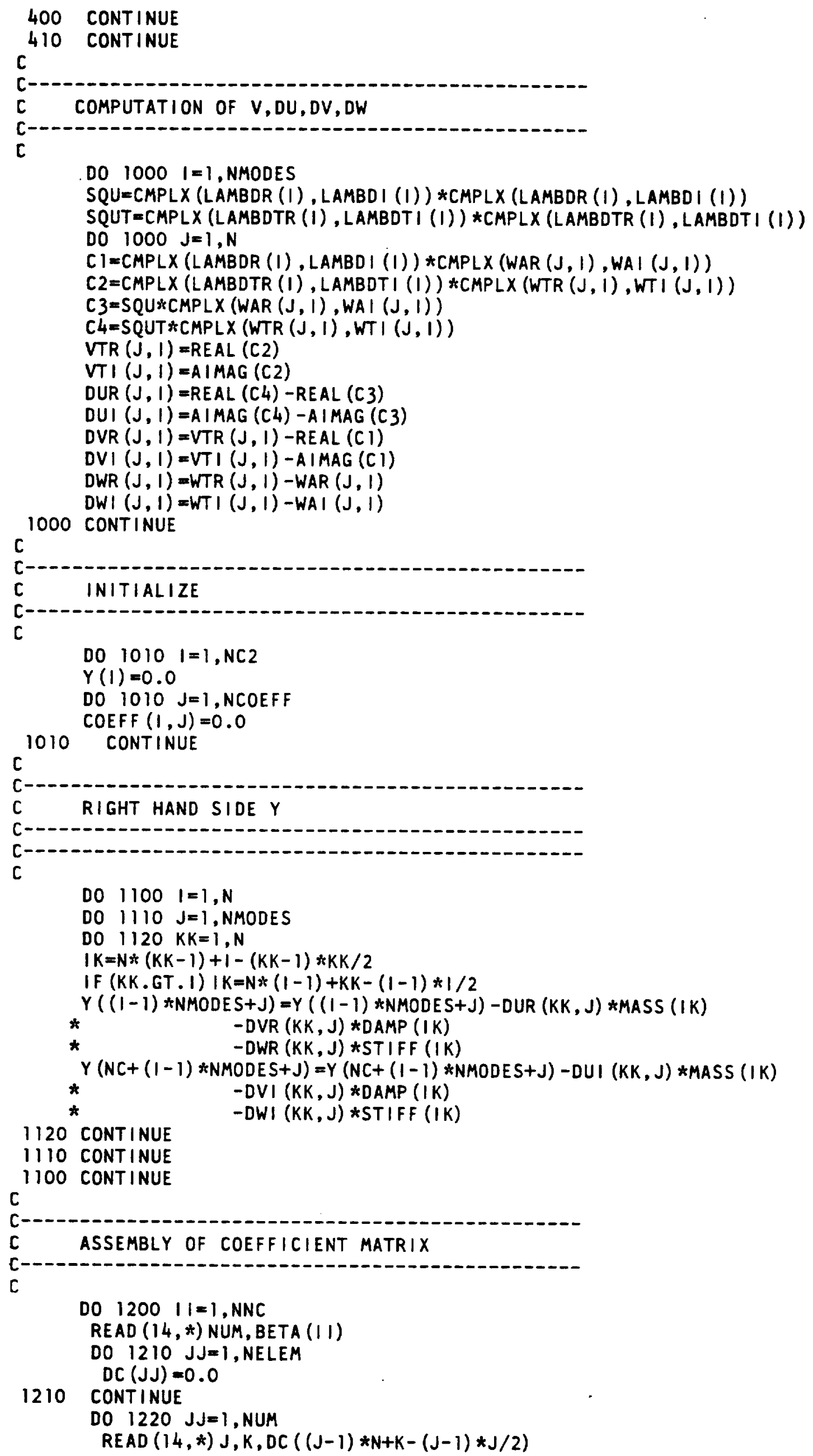



DO $1230 \quad \mid=1, N$

DO $1230 \mathrm{~J}=1$, NMODES

DO $1240 \quad K K=1, N$

$I K=N *(K K-1)+1-(K K-1) * K K / 2$

IF (KK.GT. I) IK=N*(1-1)+KK-(1-1)*1/2

$\operatorname{COEFF}((1-1) * N M O D E S+J, 11)=\operatorname{COEFF}((1-1) * N M O D E S+J, 11)$

$\begin{array}{ll} & +V T R(K K, J) * D C(1 K) \\ & \text { COEFF }(N C+(1-1) * N M O D E S+J, 11)=C O E F F(N C+(1-1) * N M O D E S+J, 11) \\ & +V T I(K K, J) * D C(I K)\end{array}$

1240 CONTINUE

1230 CONTINUE

1200 CONTINUE

DO $1201 \quad \mid 1=1$, NNK

$\operatorname{READ}(15, *)$ NUM, GAMMA $(11)$

DO $1202 \mathrm{JJ}=1$, NELEM

$D K(J J)=0.0$

1202 CONTINUE

DO $1203 \mathrm{JJ}=1$, NUM

1203 CONTINUE

$\operatorname{READ}(15, *) J, K, D K((J-1) * N+K-(J-1) * J / 2)$

DO $1204 \quad \mathrm{I}=1, \mathrm{~N}$

DO $1204 \mathrm{~J}=1$, NMODES

DO $1205 K K=1, N$

$1 K=N *(K K-1)+1-(K K-1) * K K / 2$

I F (KK.GT.1) IK=N* $(1-1)+K K-(1-1) * 1 / 2$

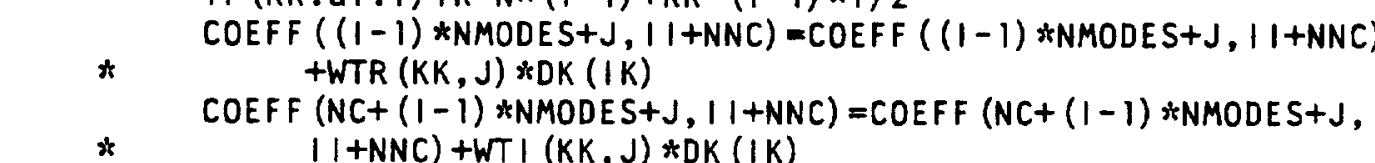

1205 CONTINUE

1204 CONTINUE

1201 CONTINUE

DO $1300 \quad 1=1$, NCOEFF

$B L(1)=0.0$

DO $1310 \mathrm{~K}=1, \mathrm{NC2}$

$1310 \quad B L(1)=B L(1)+\operatorname{COEFF}(K, 1) * Y(K)$

1300 CONTINUE

DO $1330 \quad I=1, N$ COEFF

DO $1320 \mathrm{~J}=1$, NCOEFF

$A(1, J)=0.0$

DO $1320 \mathrm{~K}=1, \mathrm{NC2}$

$1320 \quad A(I, J)=A(I, J)+\operatorname{COEFF}(K, I) * \operatorname{COEFF}(K, J)$

c

CONT INUE

c-

CHOICE OF SVD OR LP (LP=O, SINGULAR VALUE DECOMPOSITION

$L P=1$, CONSTRAINED OPTIMIZATION)

C

C

$\operatorname{READ}(5, *) L P$

IF (LP.EQ. I) GOTO 1401

C

SINGULAR VALUE DECOMPOSITION SOLUTION

C-

c

$\operatorname{CALL} \operatorname{LSVDF}(A, 12, N C O E F F, N C O E F F, B L, 12,1, B U, W K$, IER)

C

CALL LSVDF (A, IA, M, N, B, IB, NB, S, WK, IER)

REMARKS I. LSVDF COMPUTES THE SINGULAR VALUE DECOMPOSITION OF

A REAL M BY N MATRIX

$A=U * Q * V * *(T)$ WHERE

$U$ IS AN $M$ BY $M$ ORTHOGONAL MATRIX,

$\checkmark$ IS AN $N$ BY $N$ ORTHOGONAL MATRIX, AND 



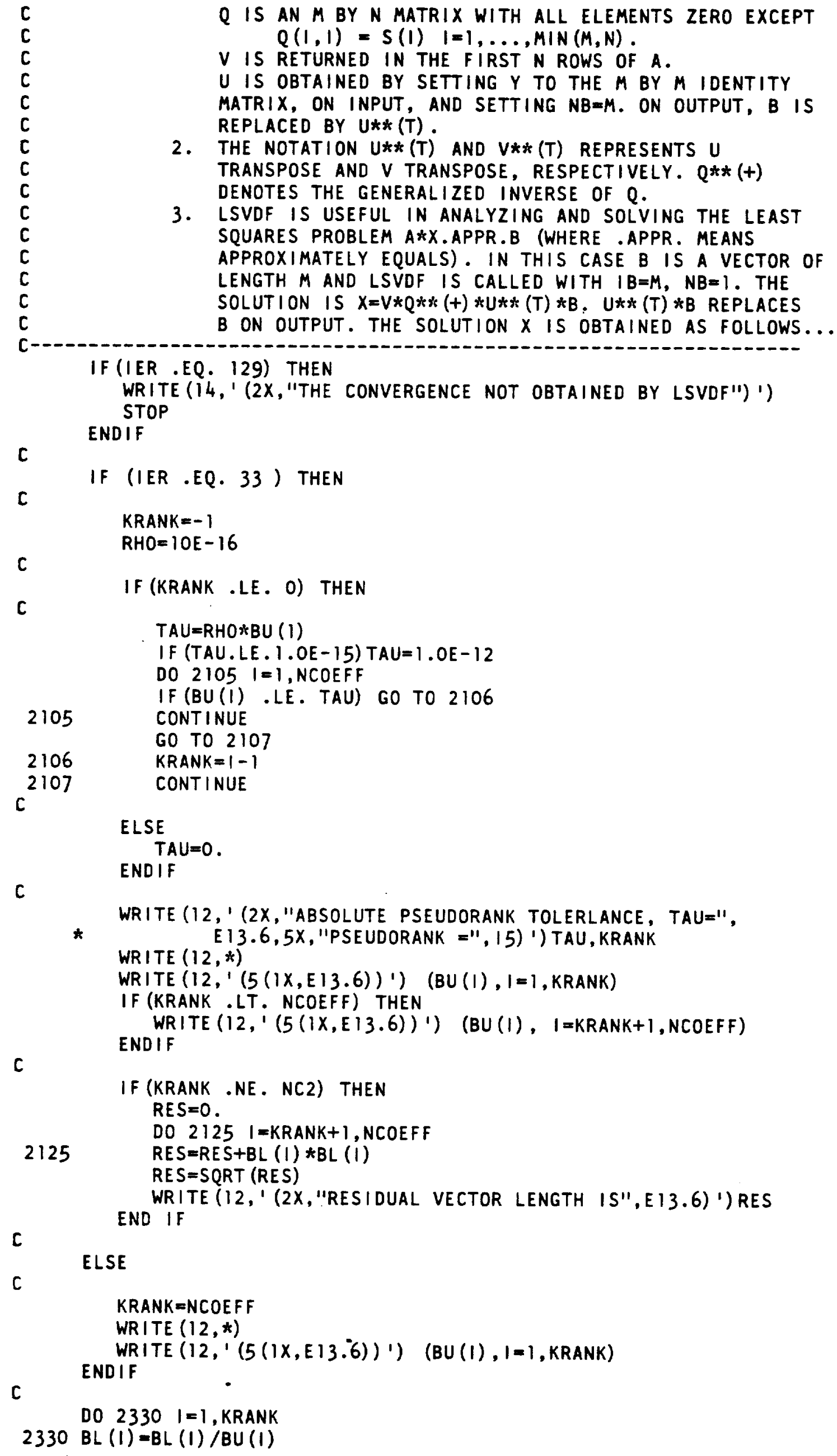





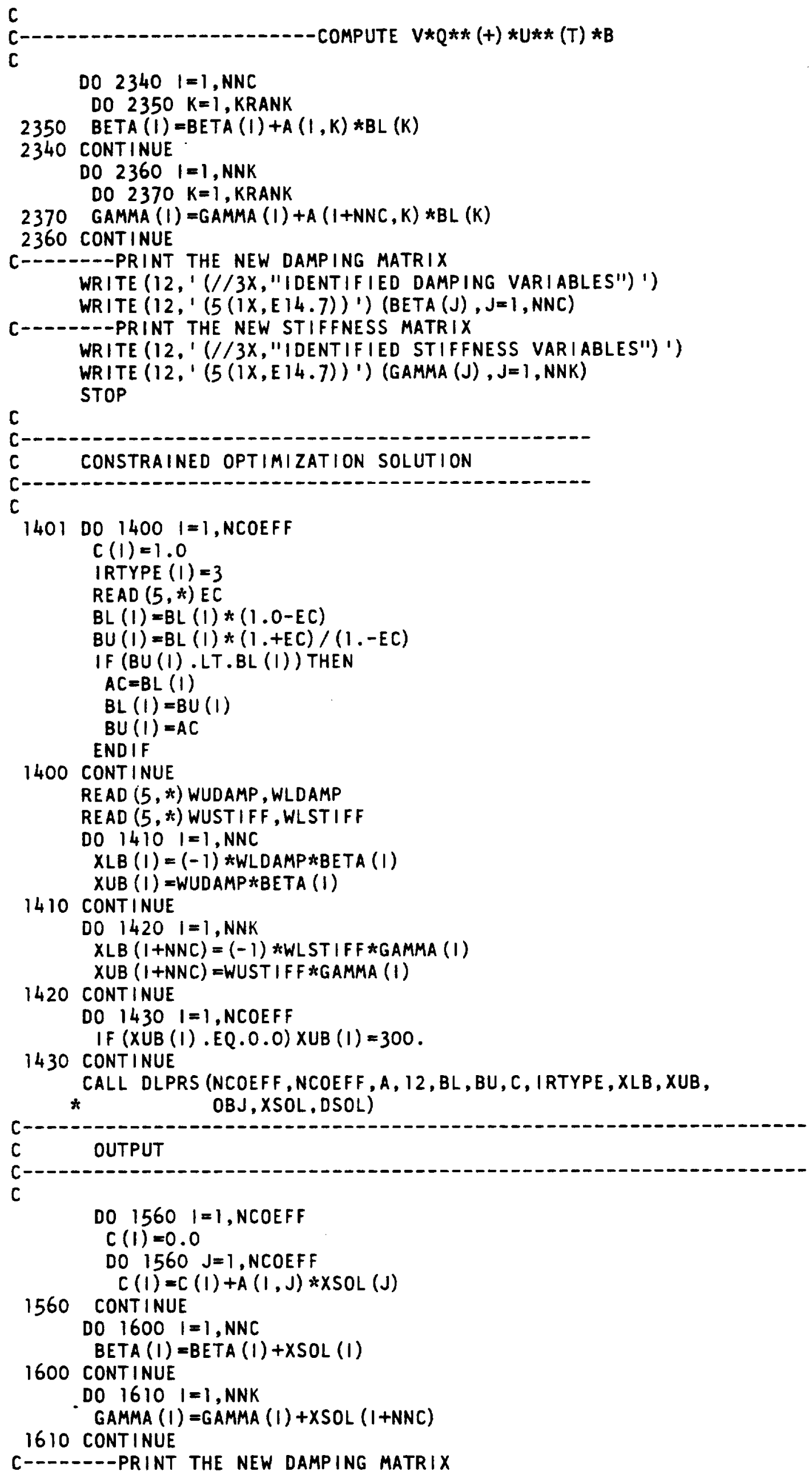



WRITE $(12,1(/ / 3 X, "$ IDENTIFIED DAMPING VARIABLES") ')

WRITE $(12, '(5(1 X, E 14.7)) ')$ (BETA (J), J $=1, N N C)$

C--.--PRINT THE NEW STIFFNESS MATRIX

WRITE $(12,1(/ / 3 X, "$ IDENTIFIED STIFFNESS VARIABLES") ')

WRITE $(12, '(5(1 X, E 14.7)) ')$ (GAMMA (J) , J = I, NNK)

STOP

END 



\section{APPENDIX B \\ MSC/NASTRAN INPUT FOR FINAL AH-1G SYSTEM IDENTIFICATION RUN}

The following pages include the listings of the input file for the final MSCANASTRAN runs used to compute the structural eigenvalues and eigenvectors for the identified AH-1G structural model. 

SOL 280

5he following a caras are adoed by weiyu zhou for the output of

the mass, stiffness and demping matrices and eigenvectors

ALTER 396

MATPRT KAA//

ALTER 342

MATPRT MAQ//

MATPRT BAA /

ALTER 454

MATPRT UAV/I,

$\$$

TITLE =AH-1C THREE-OIMENSIONAL BUILTLP OYNAMICS MODEL W/ CONTROLS MODELED

SUBTITLE D DIFFICULT COMPONENTS GVT CONFIGURATION HI (FULL IIP)

LABEL $=T H T S$ VERSI
MAX: INES $=500000$

s The following card is added by welyu znou for sorted output

ECHO=SORTIEIGC, ASET, ASET I, PARAM, MATI, PVISE

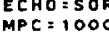

CMETHOD $=999$

SOYNRED $=998$

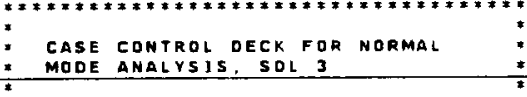

S.....................

The following cards are added by weiyu zhou for the output of

the eigenvectors

SET 1 :1001 THEU 1007 EXCEPT $1002,1005,1012,1013$

1017 THRU 1028 EXCEPT 1019,1020,1027,2511,2572,2649,2697, 19777

DTPUT

ISPLACEMENT ( SORT I, REAL) = 1

SPCFORCE = AL

ESE : AL

BECIN BULK

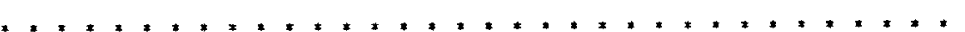

RIGIOELEMENTS UPOATE $11 / 30 / 84$ (R. DOMPKA-RESEARCHI

RIGIO ELEMENTS I REEZ INPUT TO REPLACE MPE EOUATIONS WHICH WERE

MODE $1002-7033,7037,8533,8537$ T

NODE 15212-19603.14823.

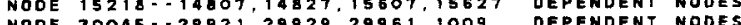

NODE $3004529921,2992929661,1009$ DEPENDENT NODES

BOTH METHODS ARE EOUIVALENT UUT RICID ELEMENTS AVOID PROBLEMS

IN FUTURE USES OF THE MODEL OY ALLOWIME MOVEMENT OF ATTACHMENT
GRID POINTS WITHOUT RECALCULATION OF MPC EOUATION COEFFICIENTS

$* * * * * * * * * * * * * * * * * * * * * * * * * * * * * * * * * * * * * * *$

GRID POINT DATA

GRID POINT DO

NOSE SUBASSEMBLY

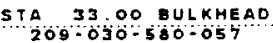

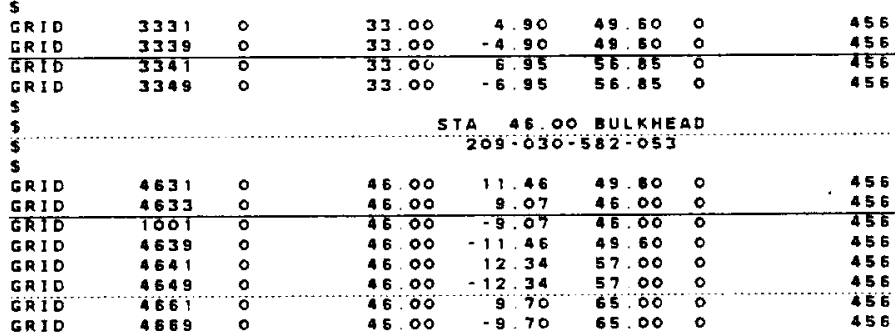

GRID
5

STA 61.25 BULKHEAD

$209-030-101=001$
$309-030-510-007$

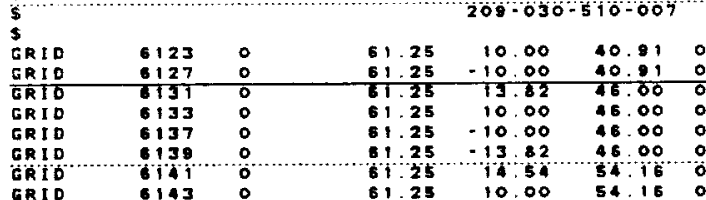




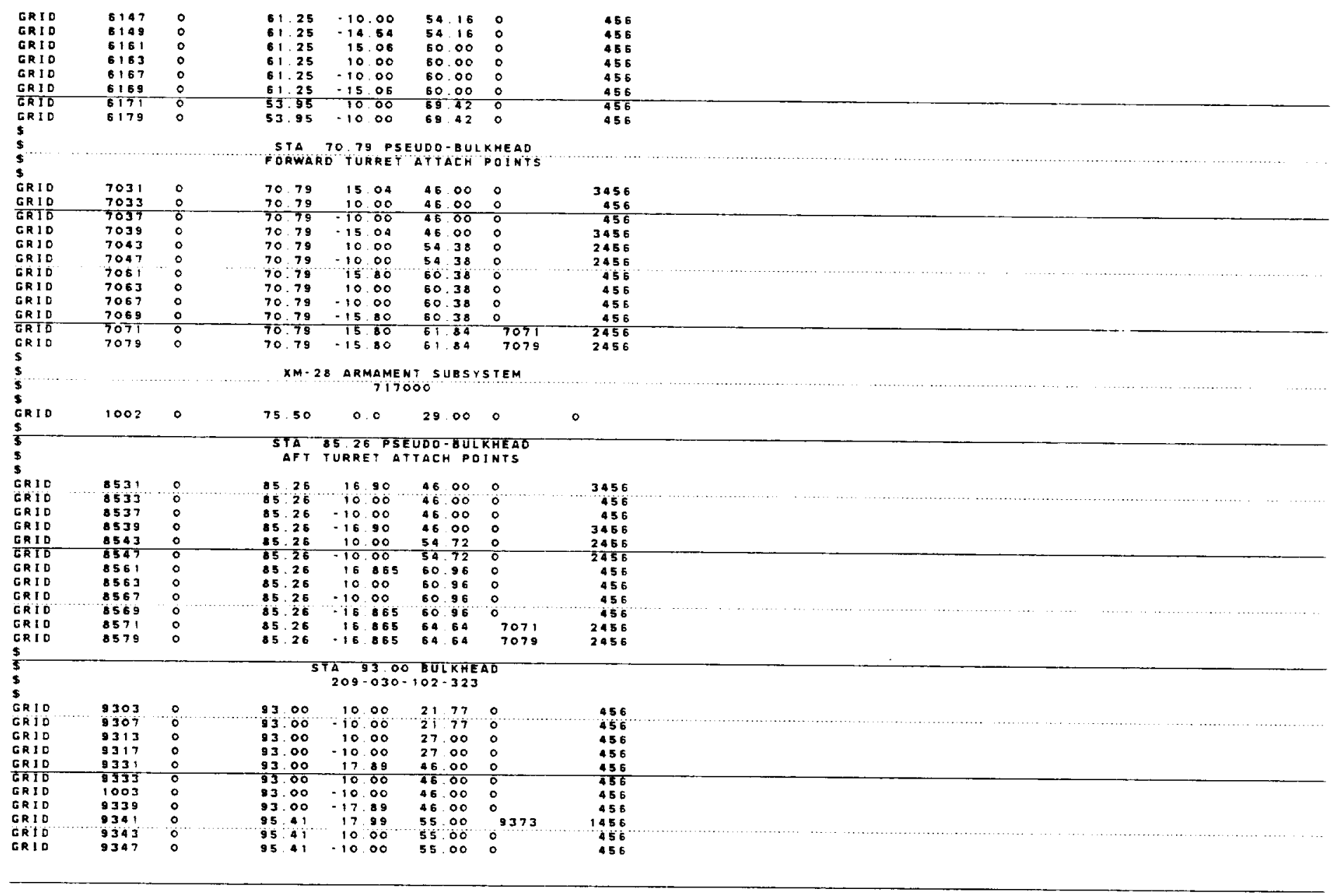

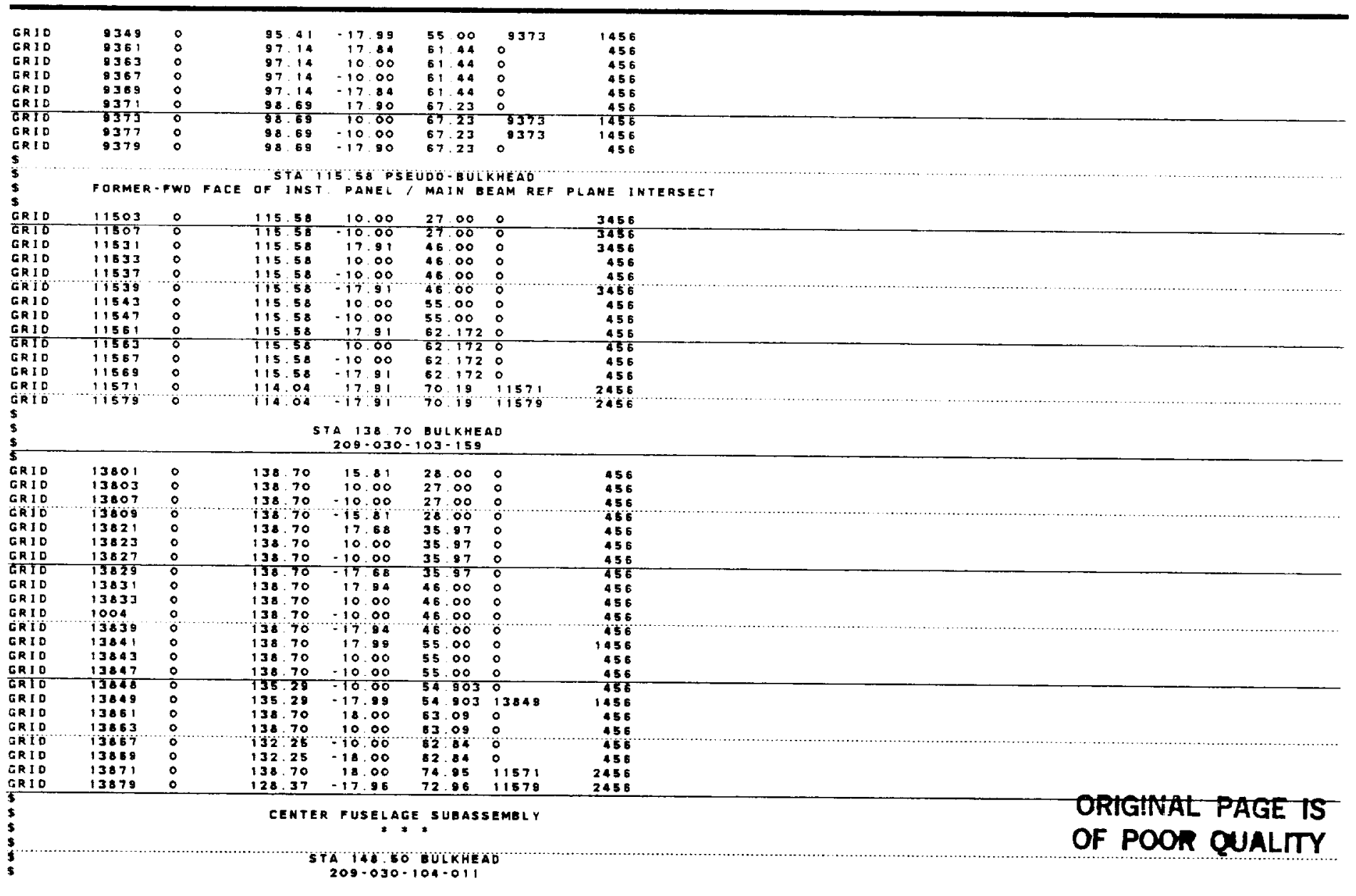




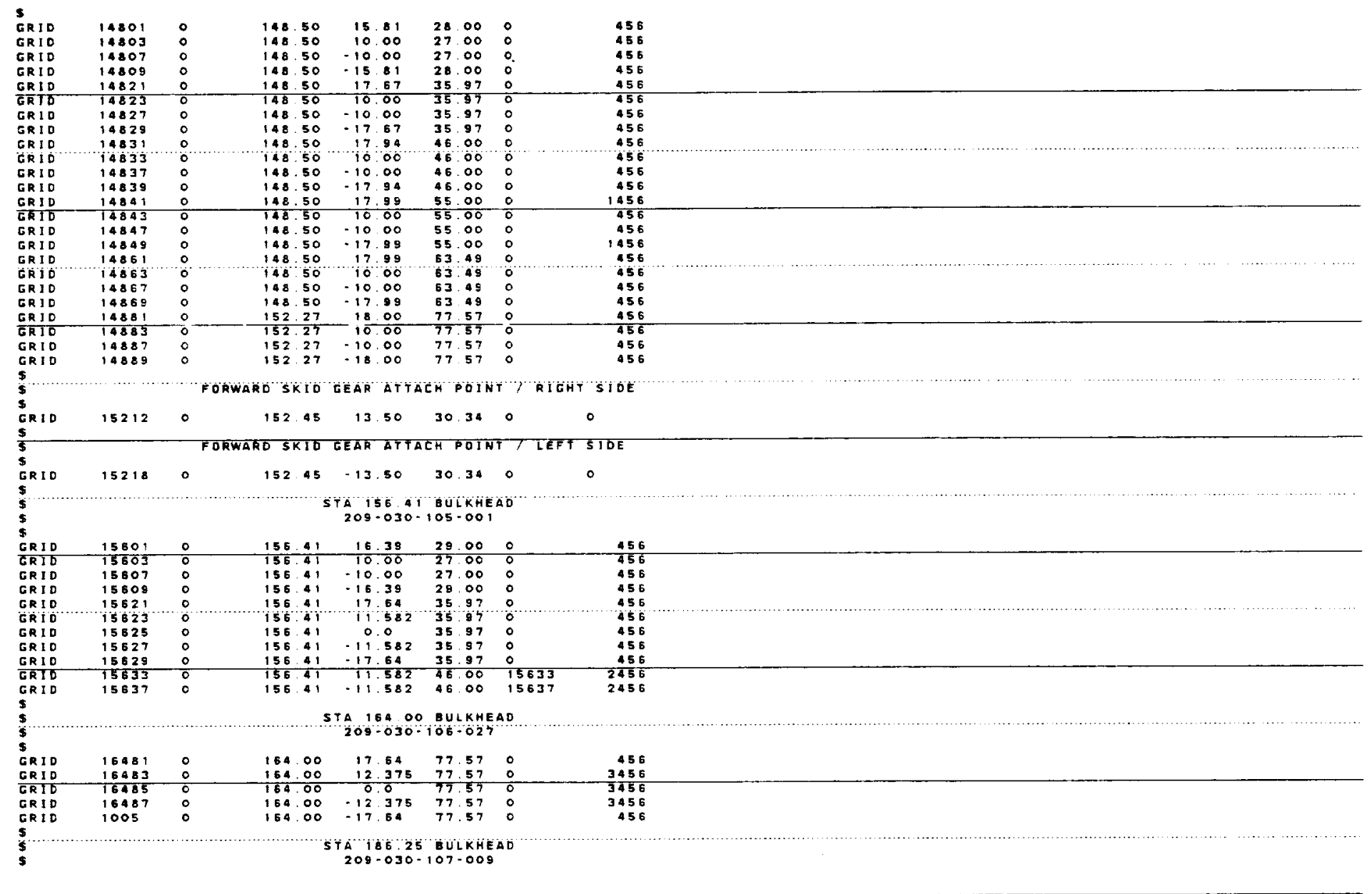

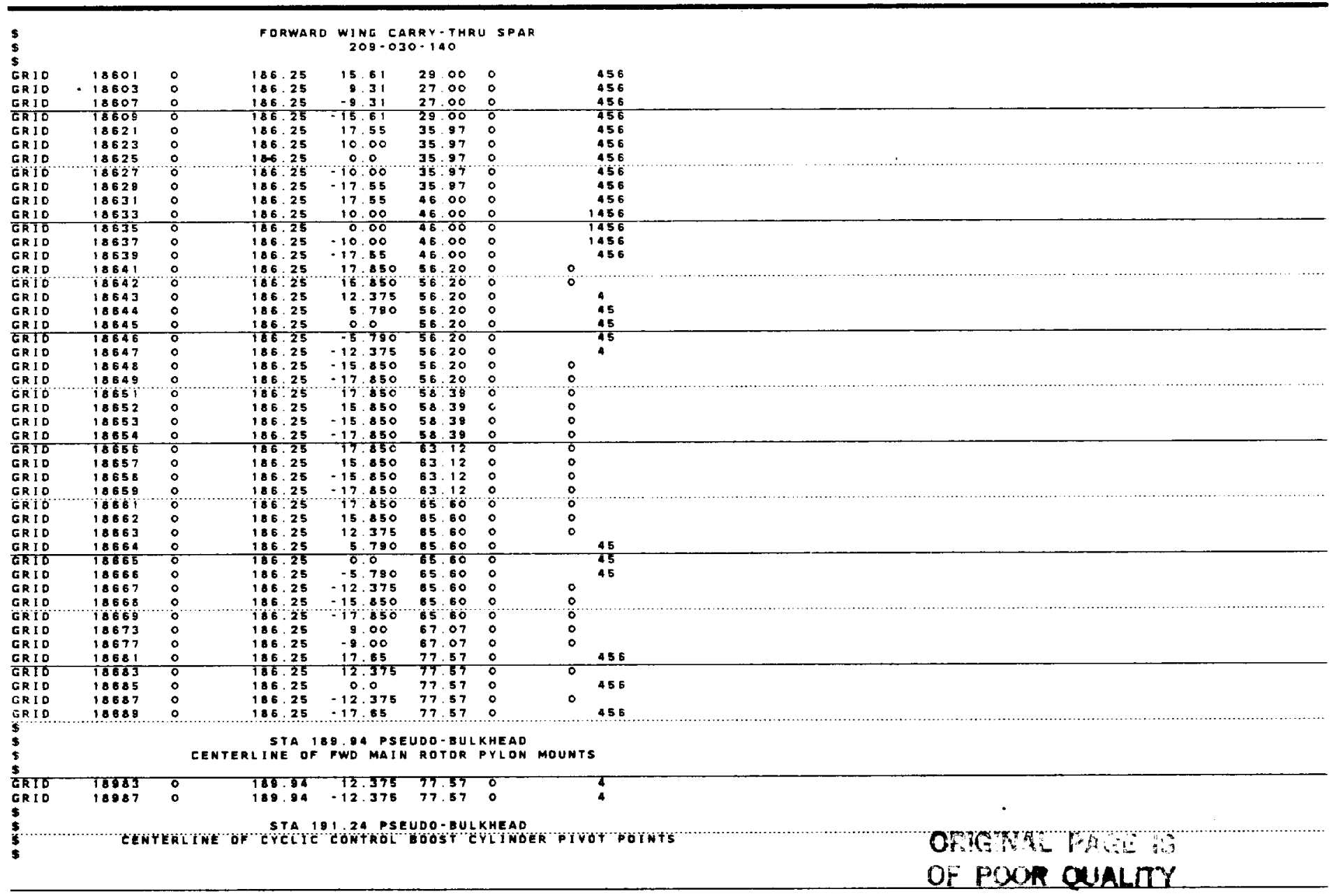




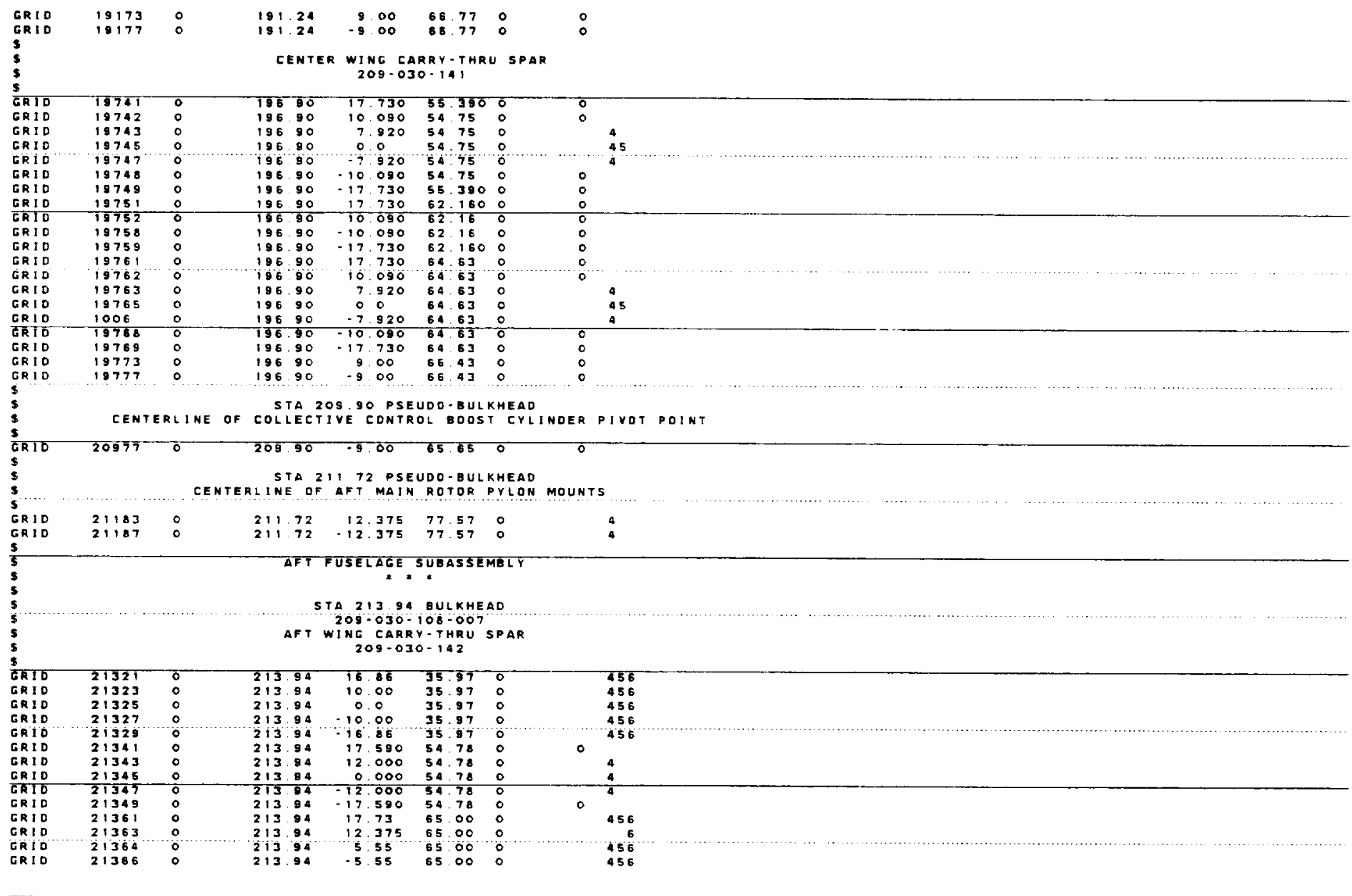

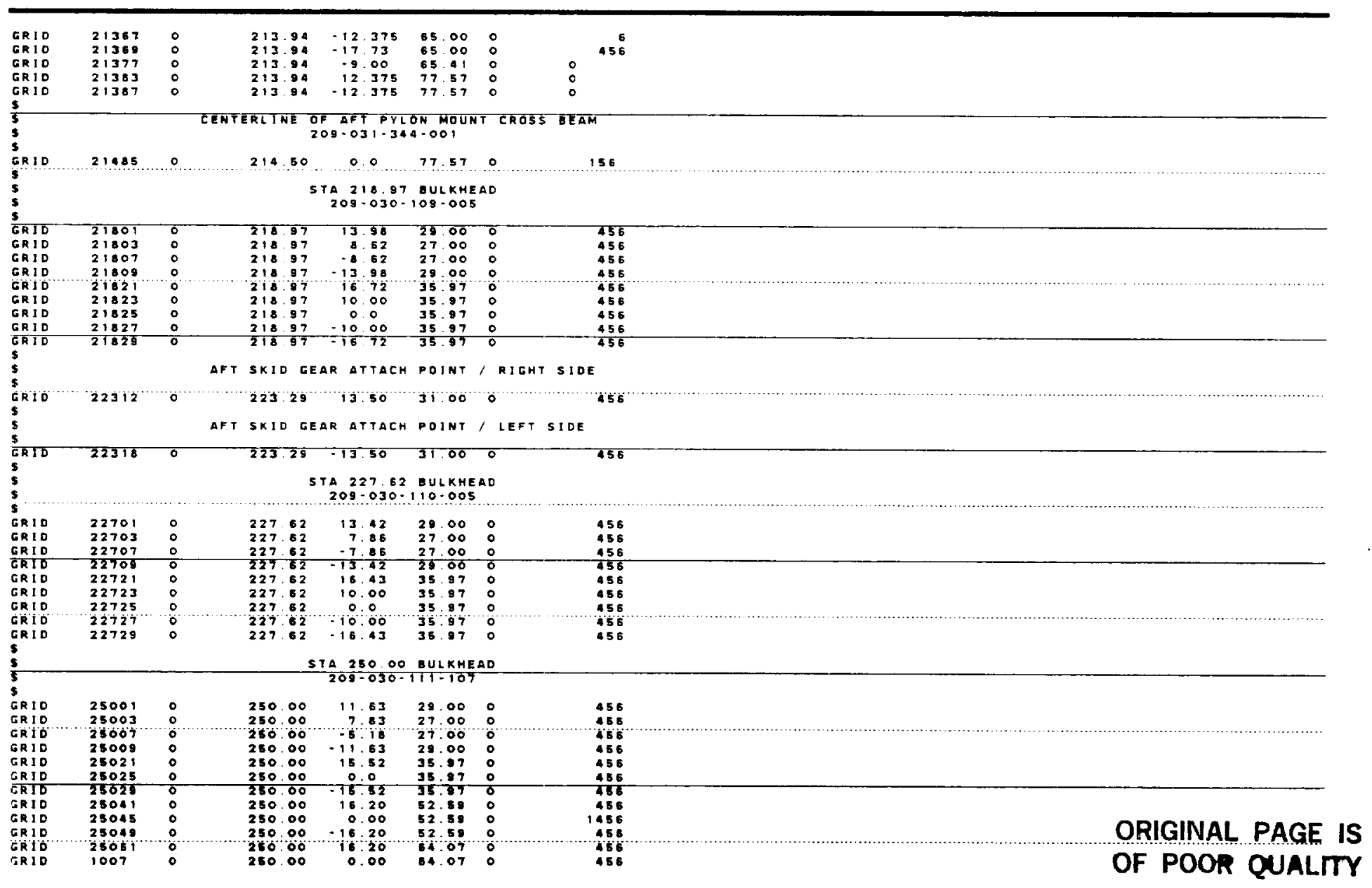




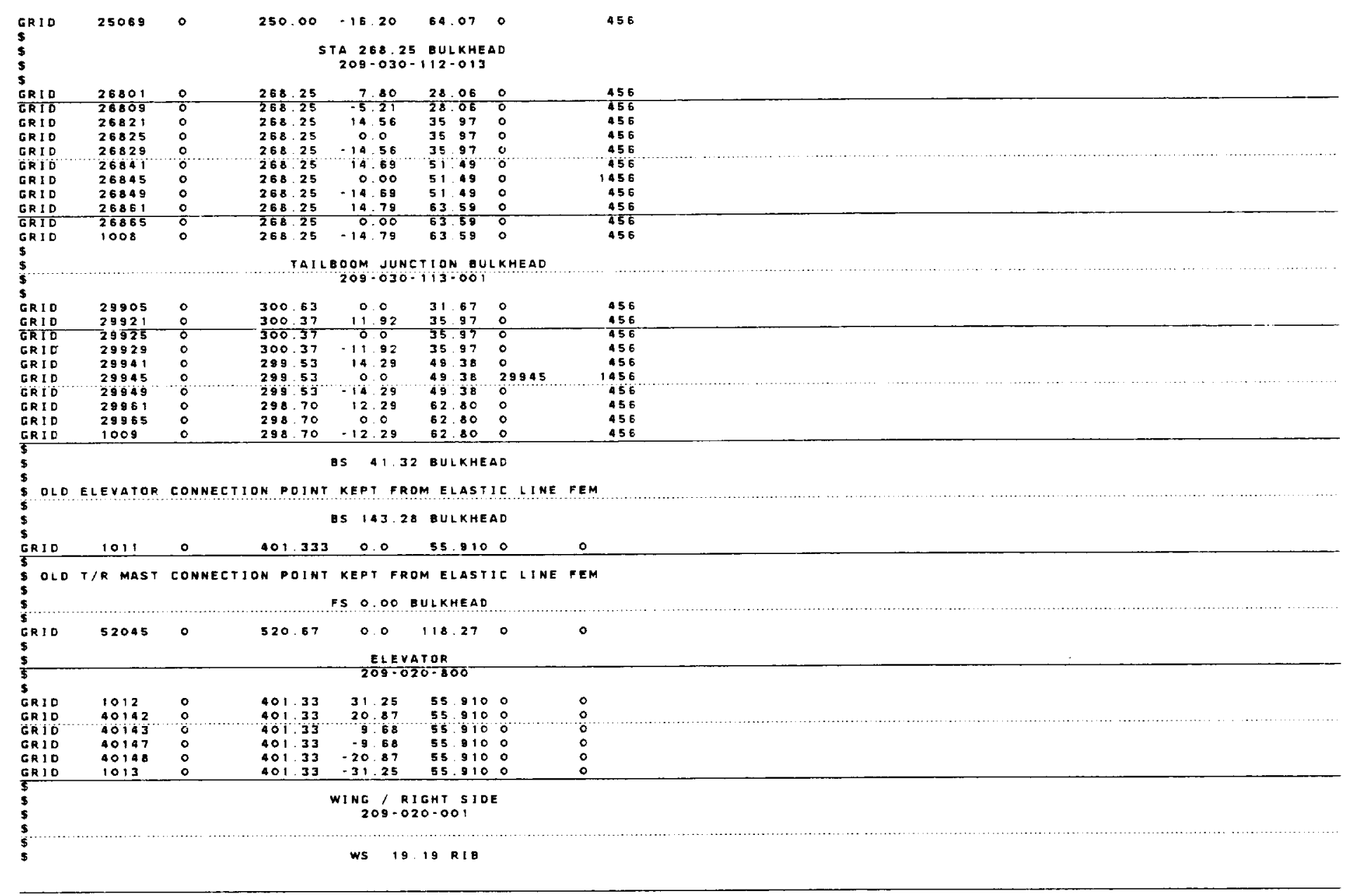

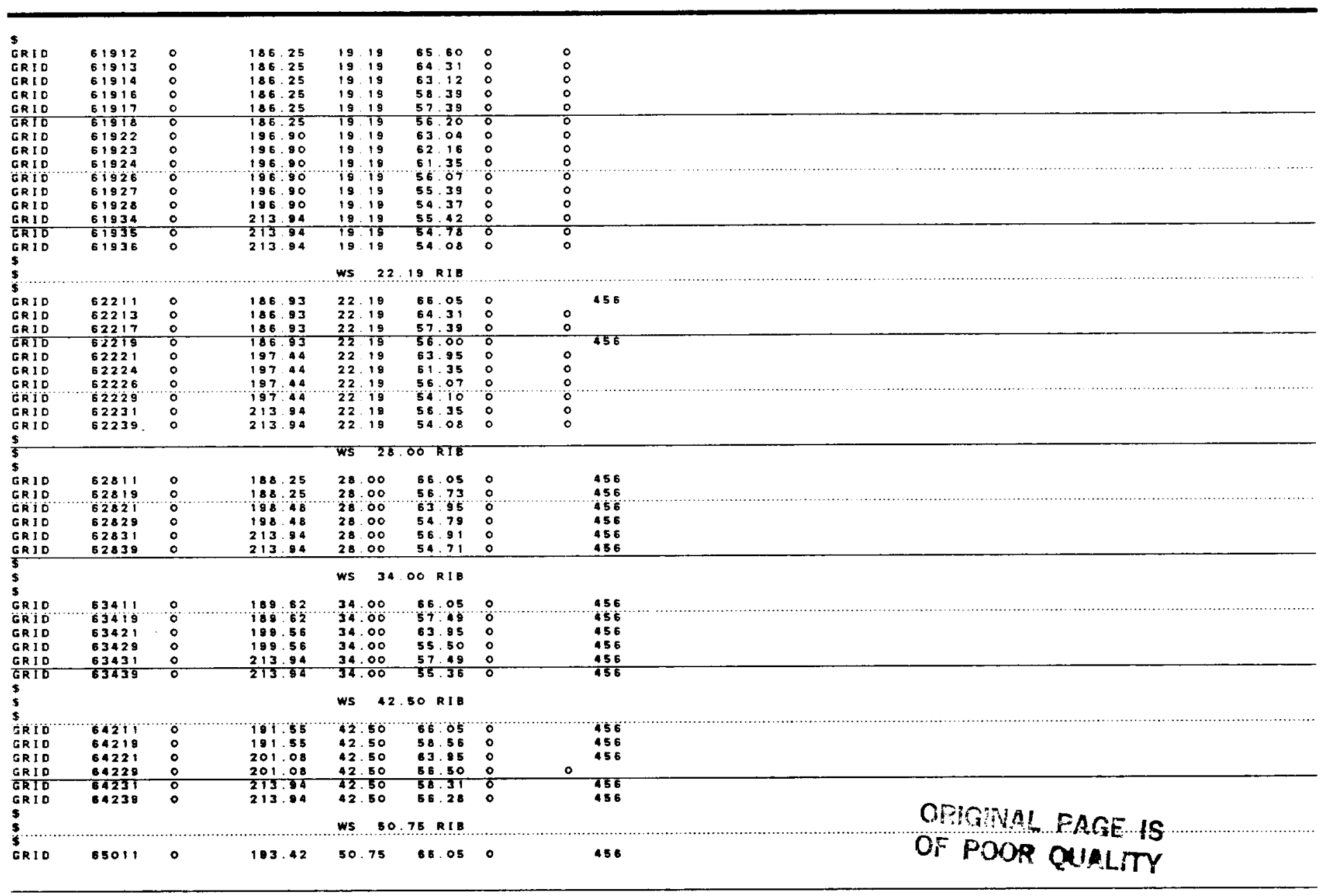




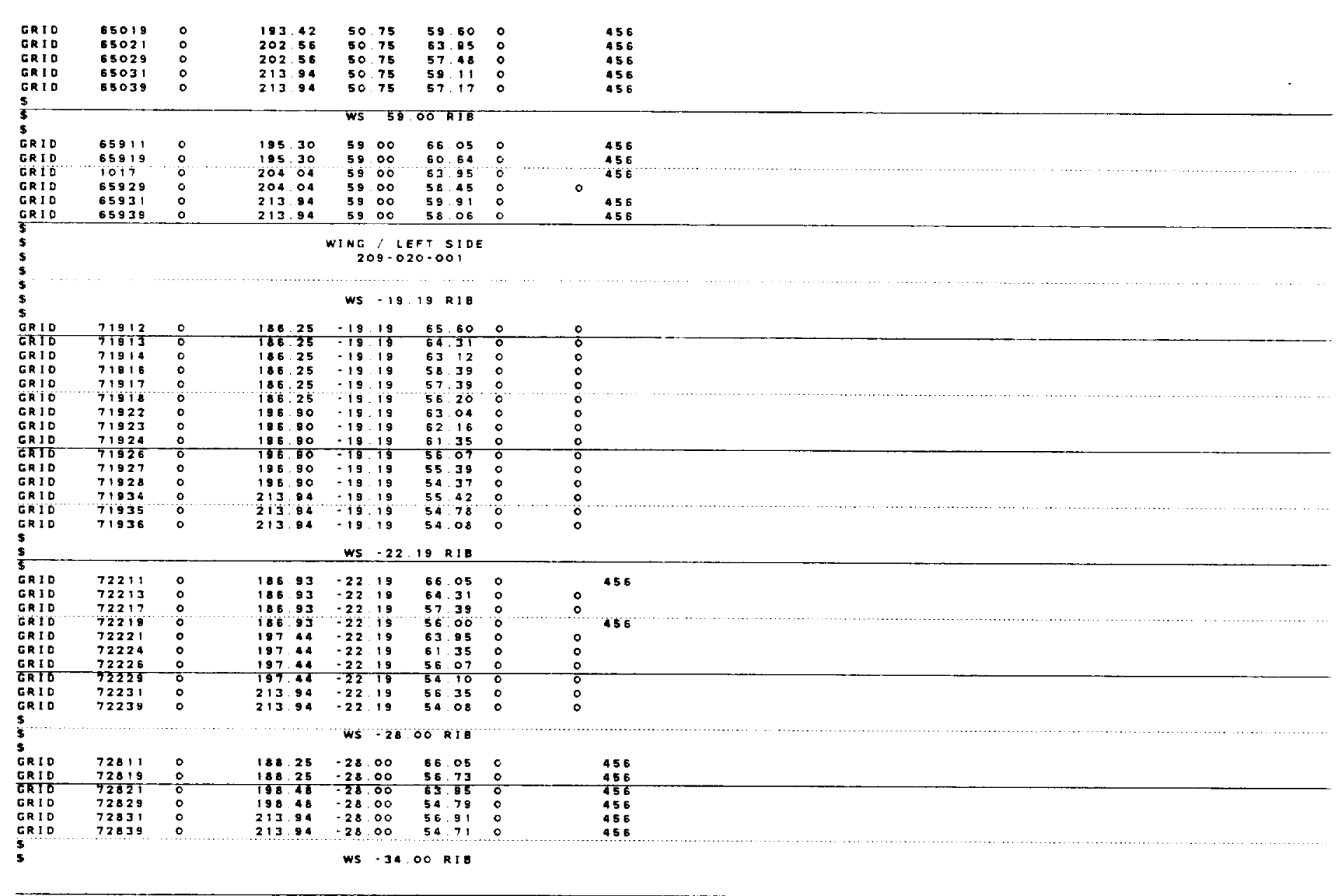

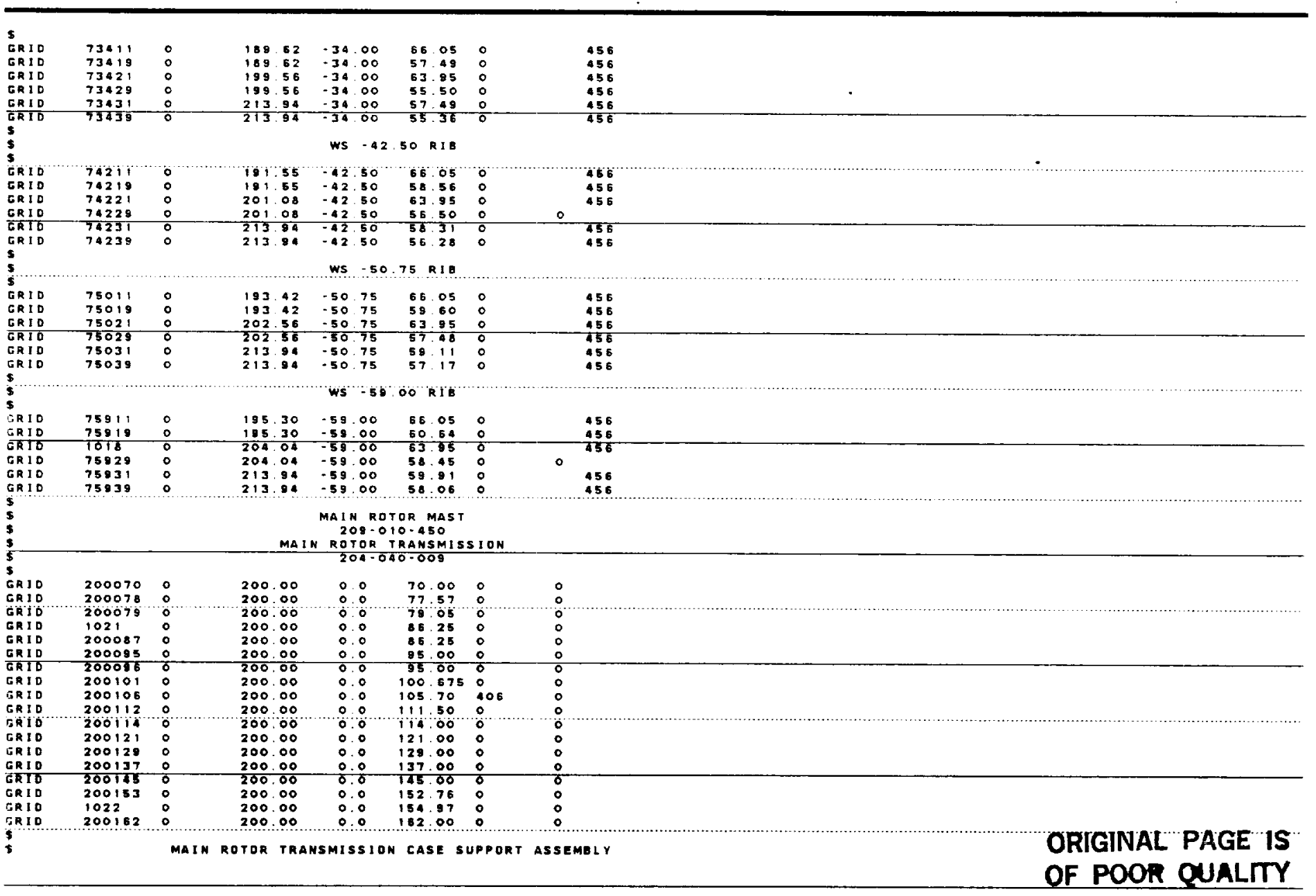




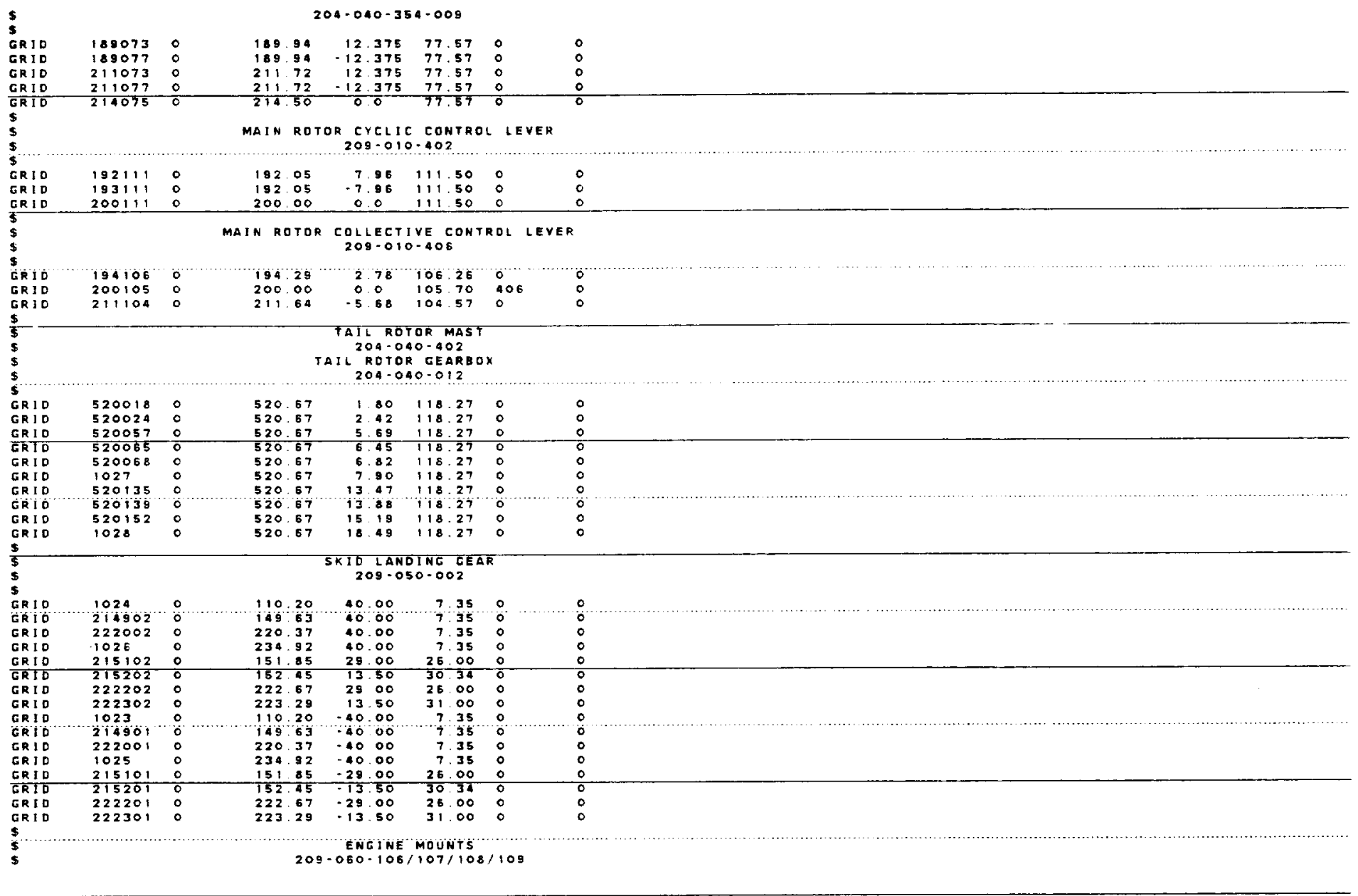

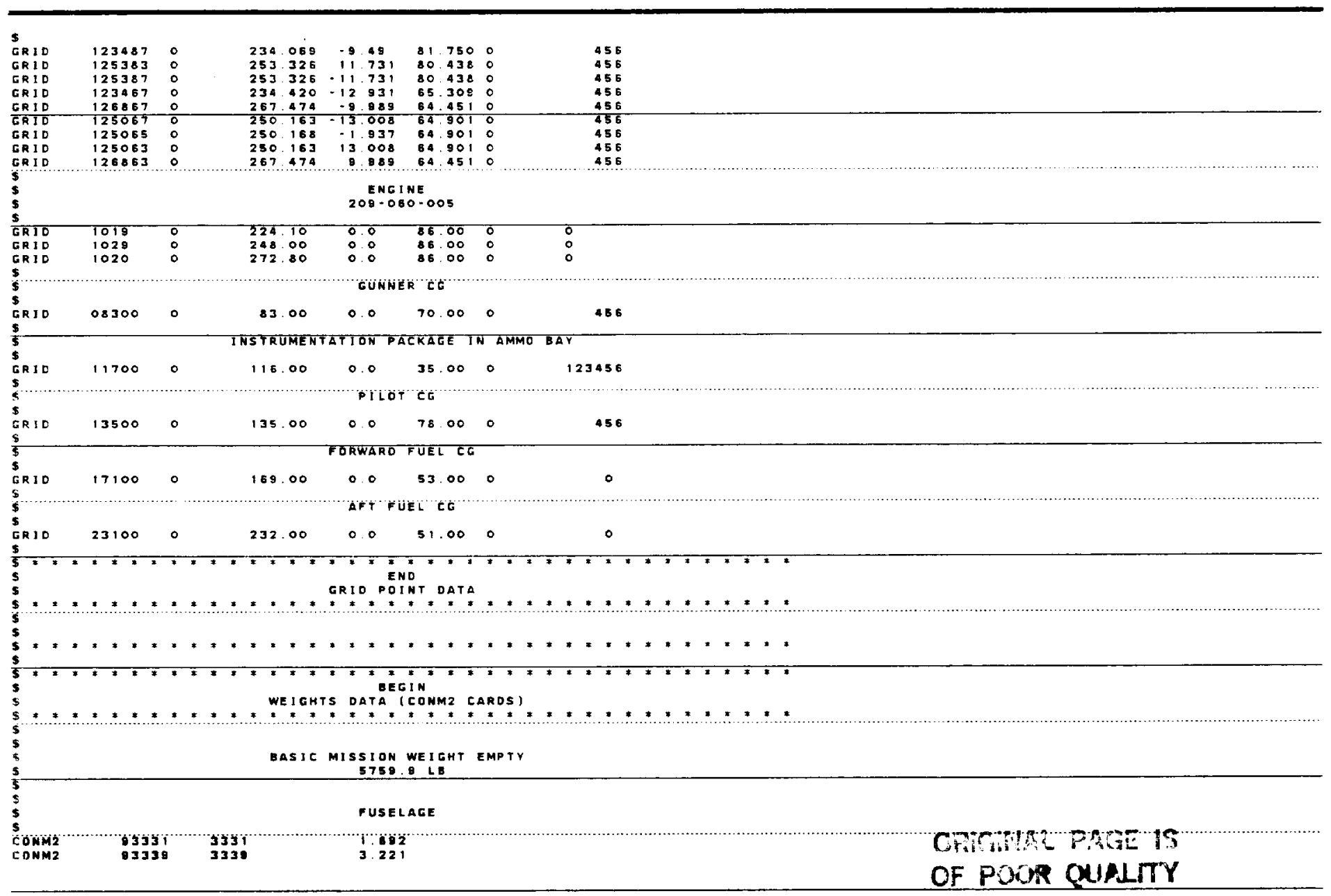




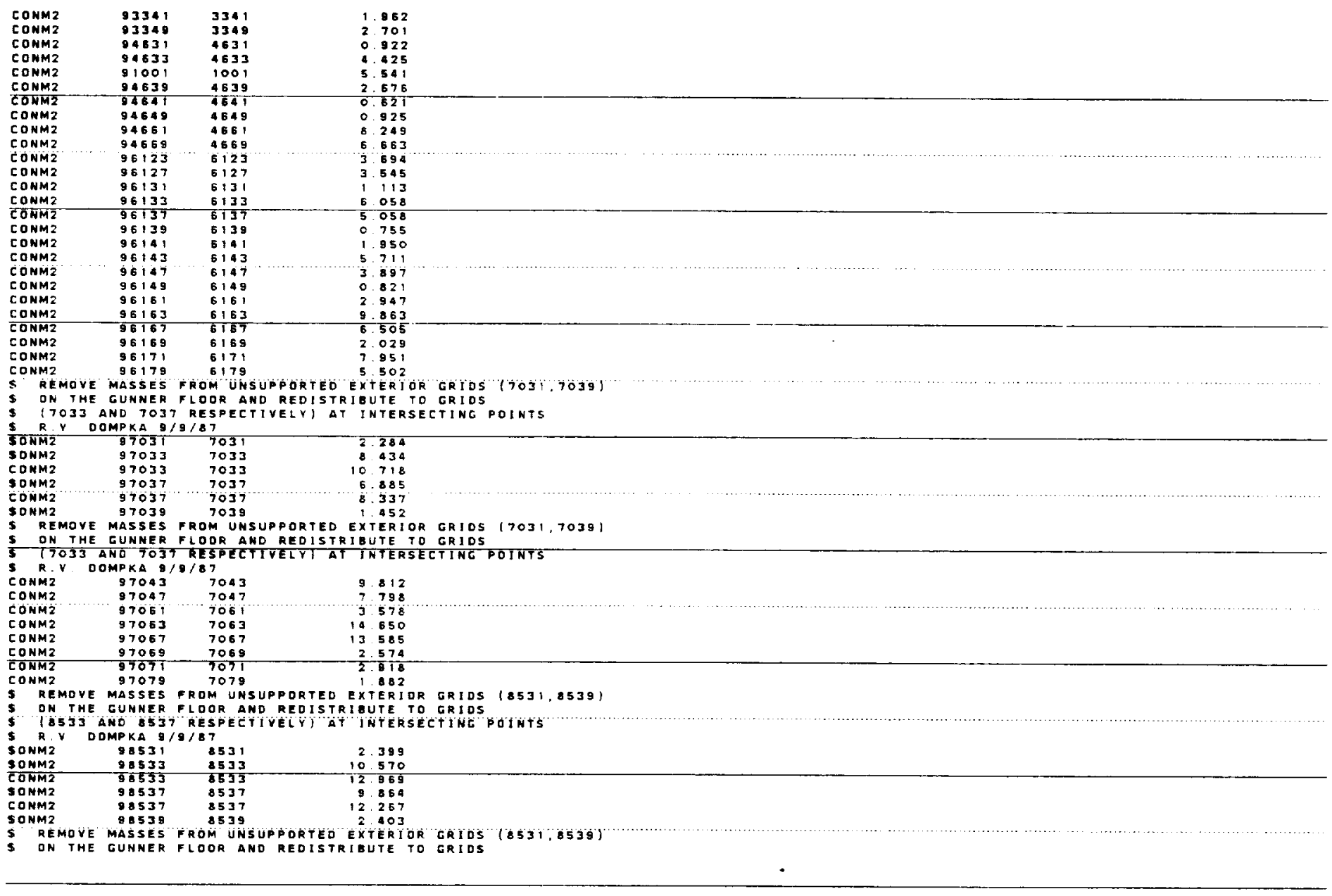

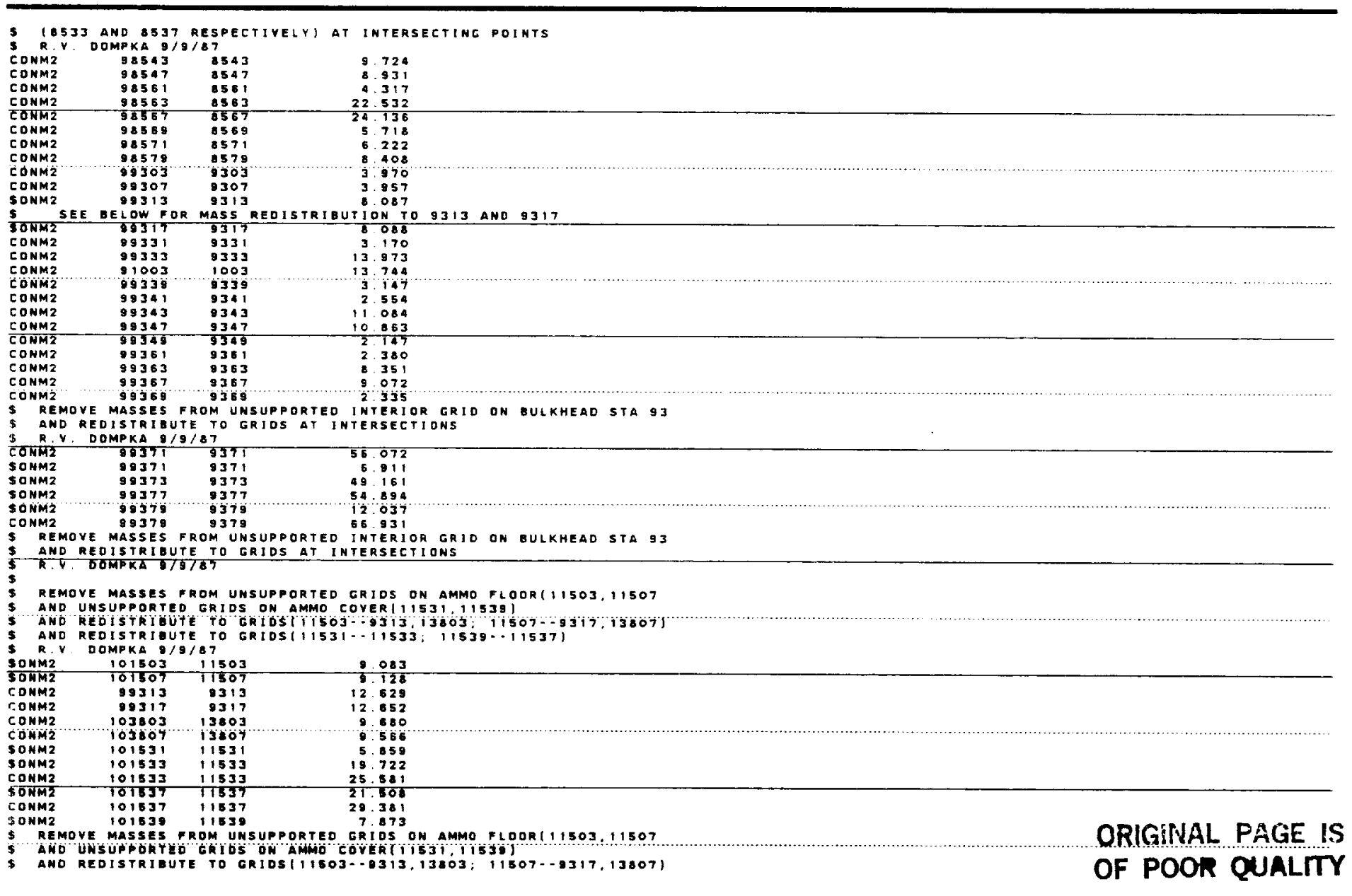




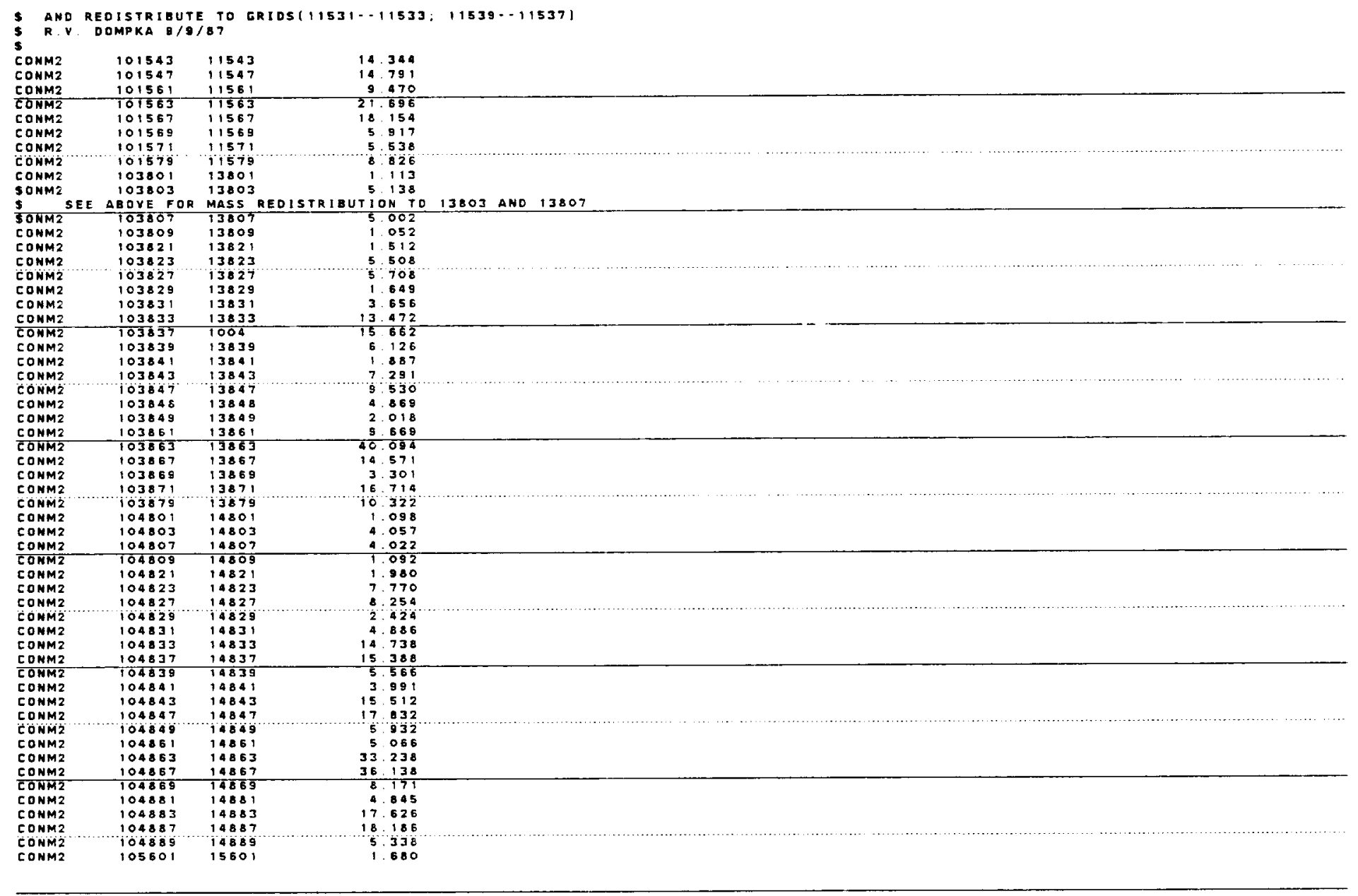

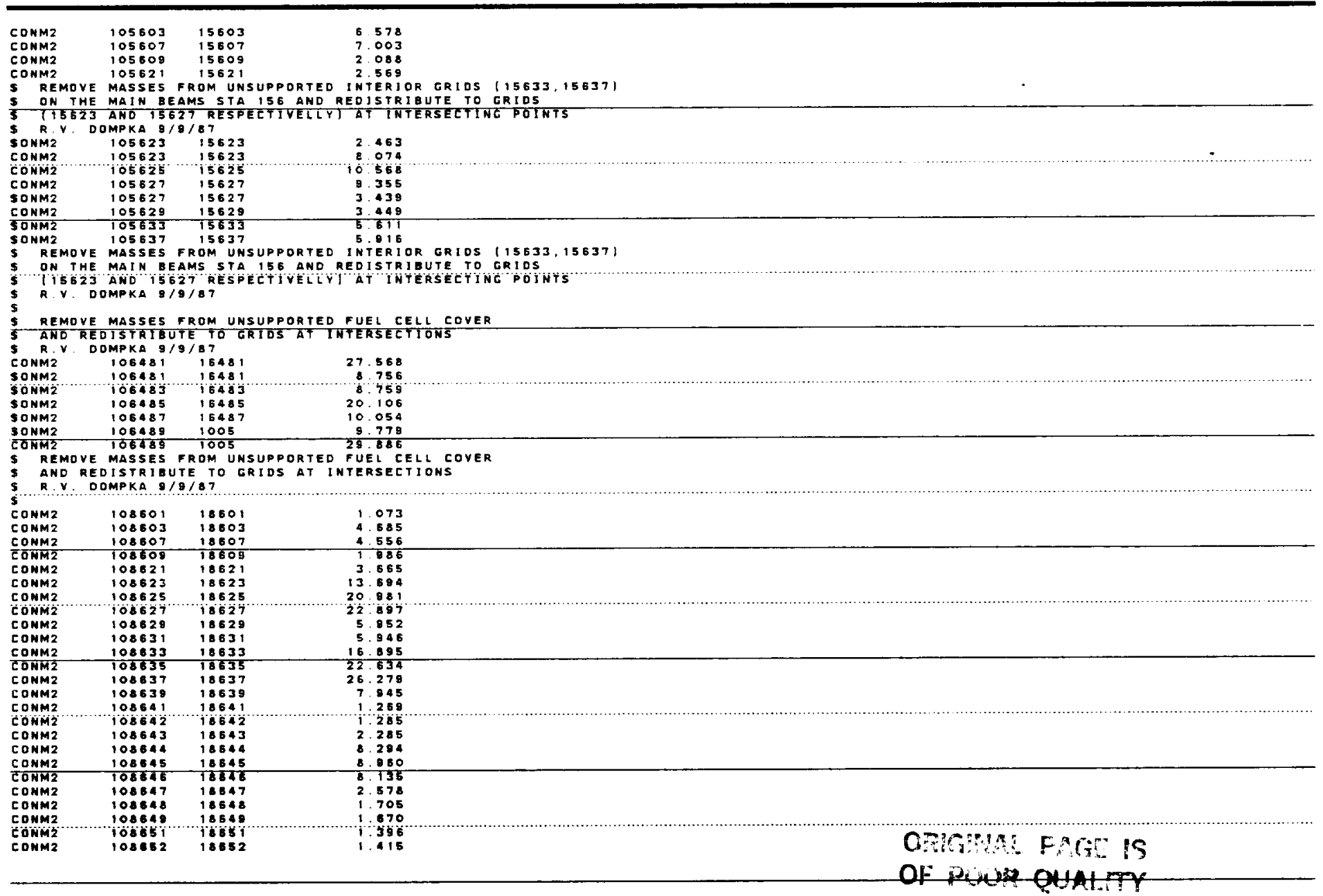




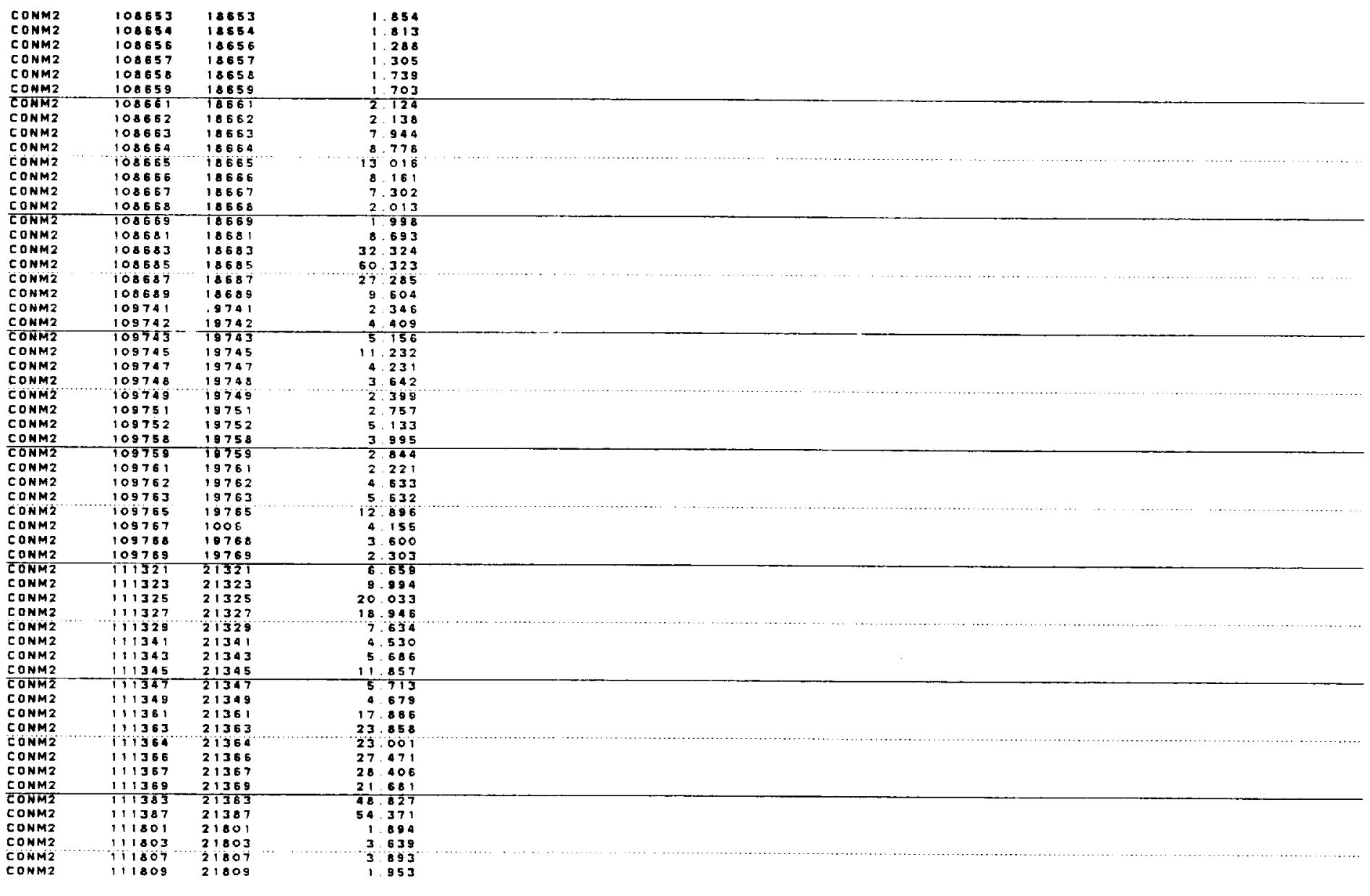

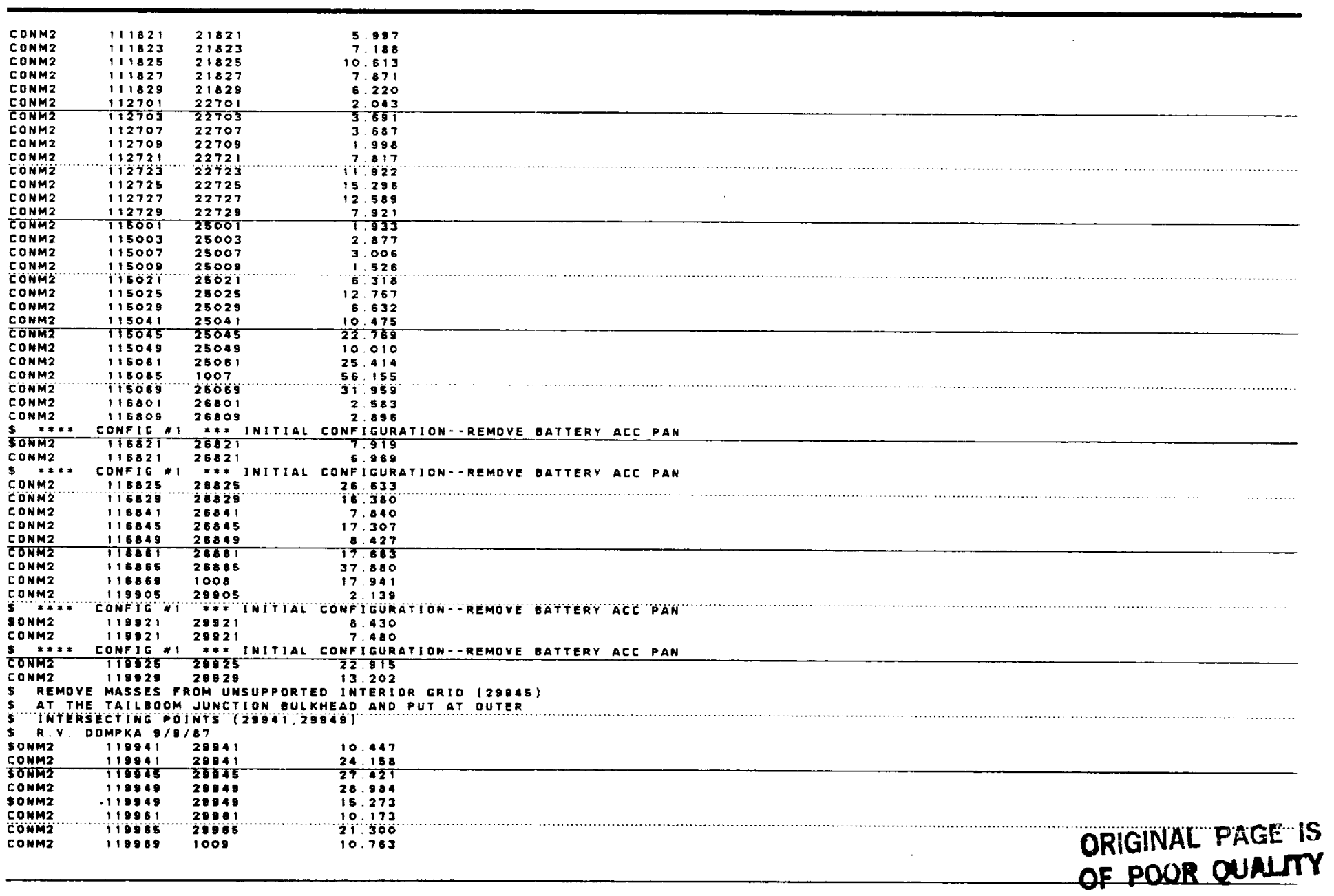


\$ REMOVE MASSES FROM UNSUPPORTED INTERIDR GRID (299A5)
S AT THE TAILBOOM JUNCTION BULKHEAD AND PUT AT OUTER INTERSECTINE POINTS (29941.29949)

R. $Y$ DOMPKA $9 / 9 / 87$

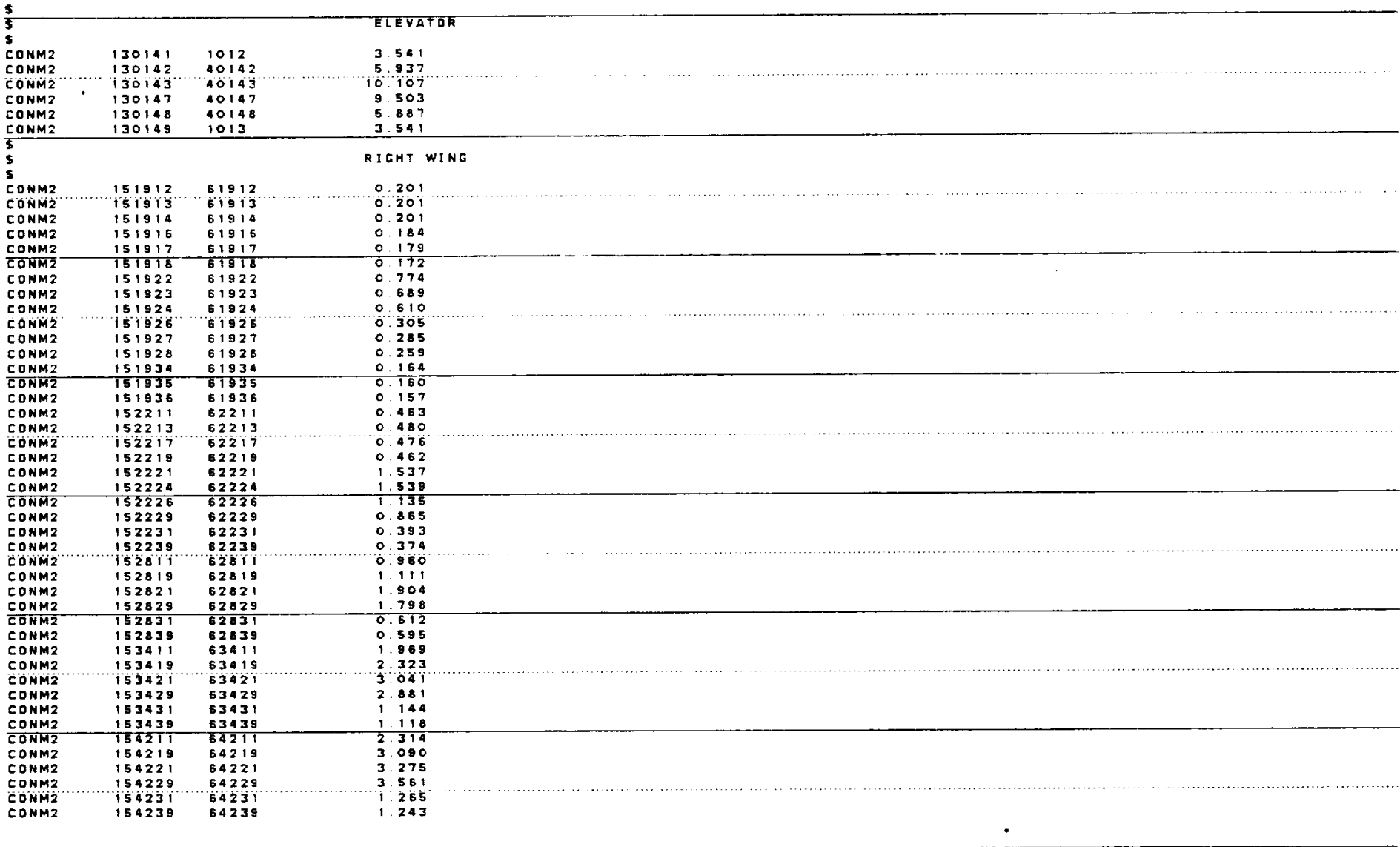

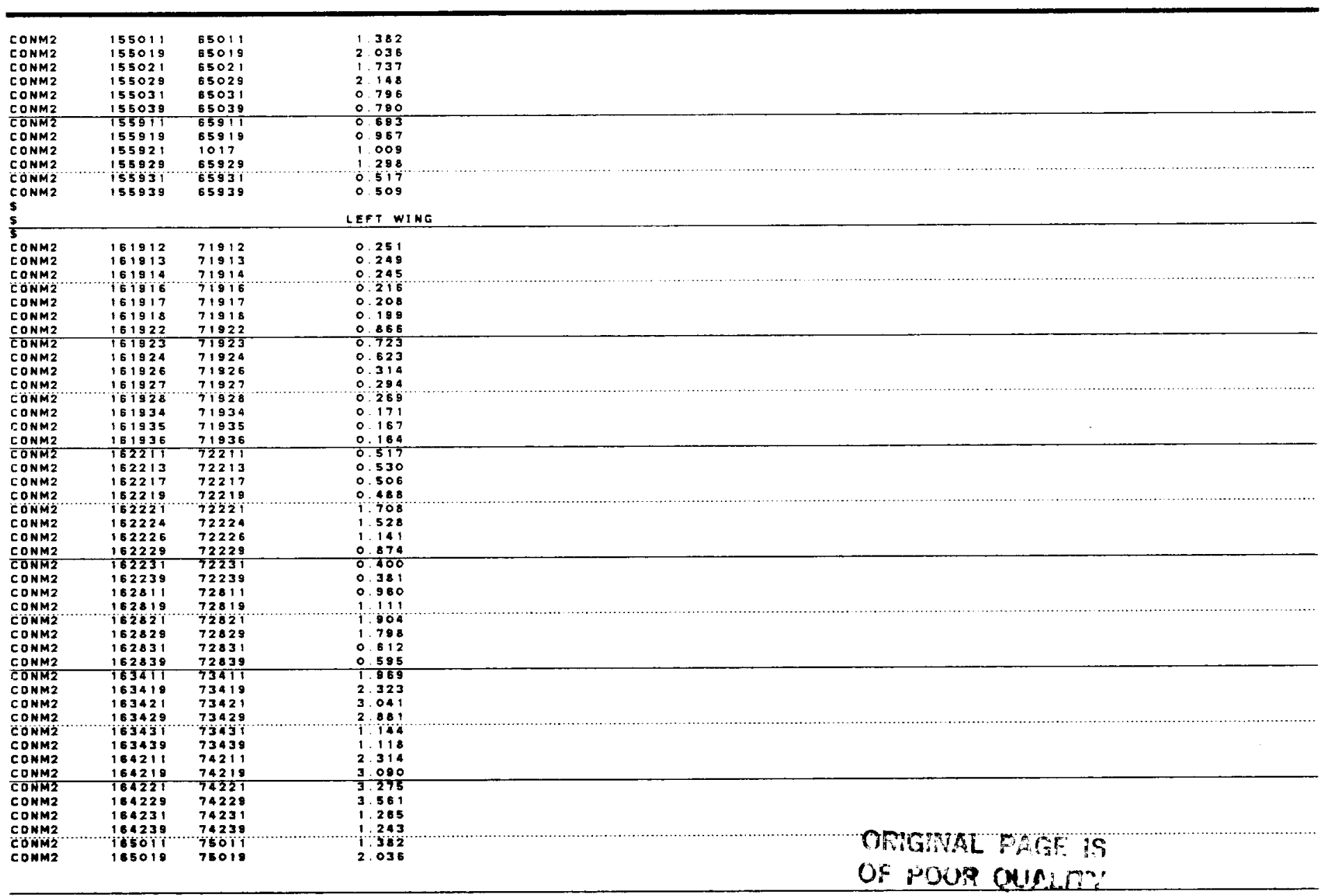




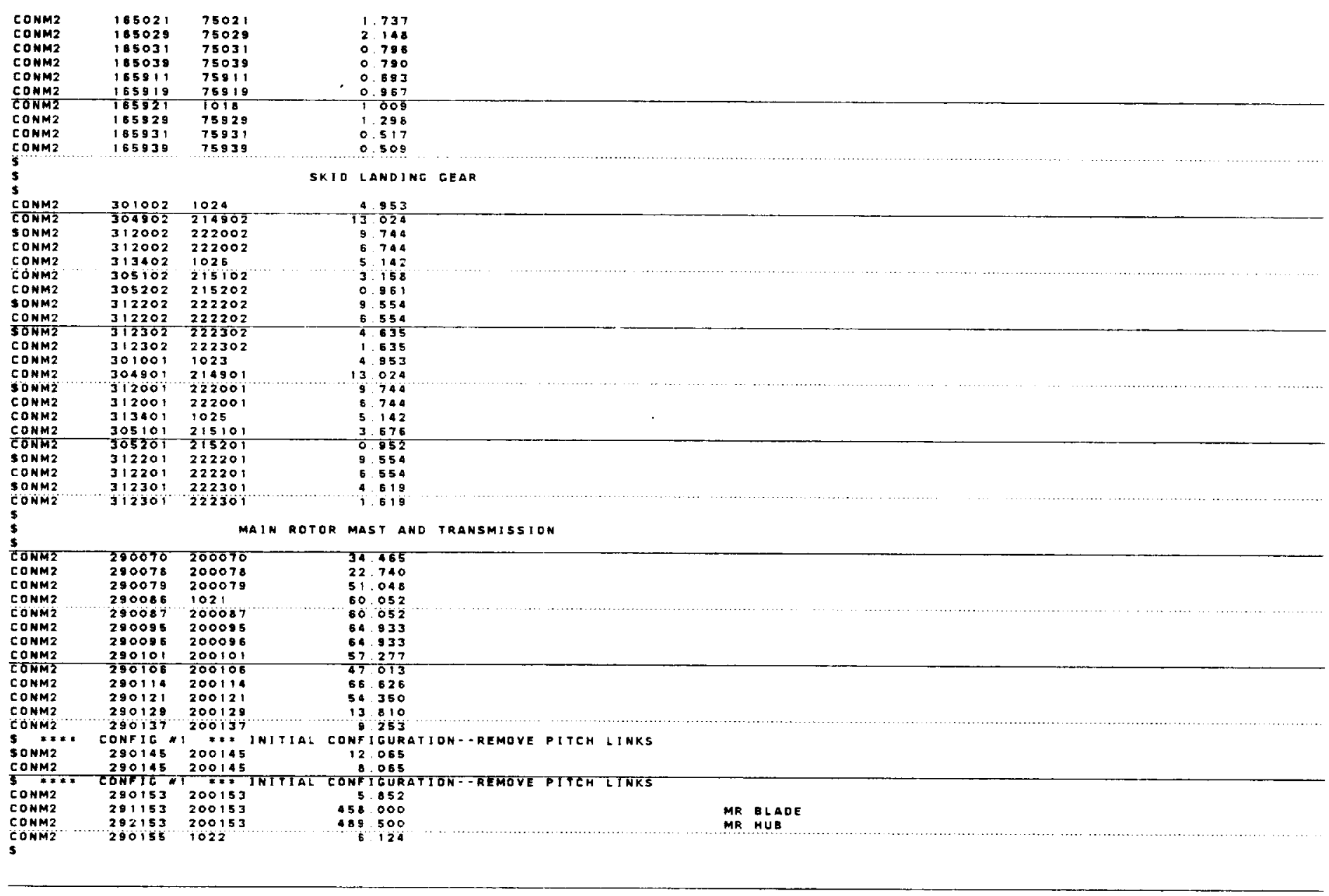

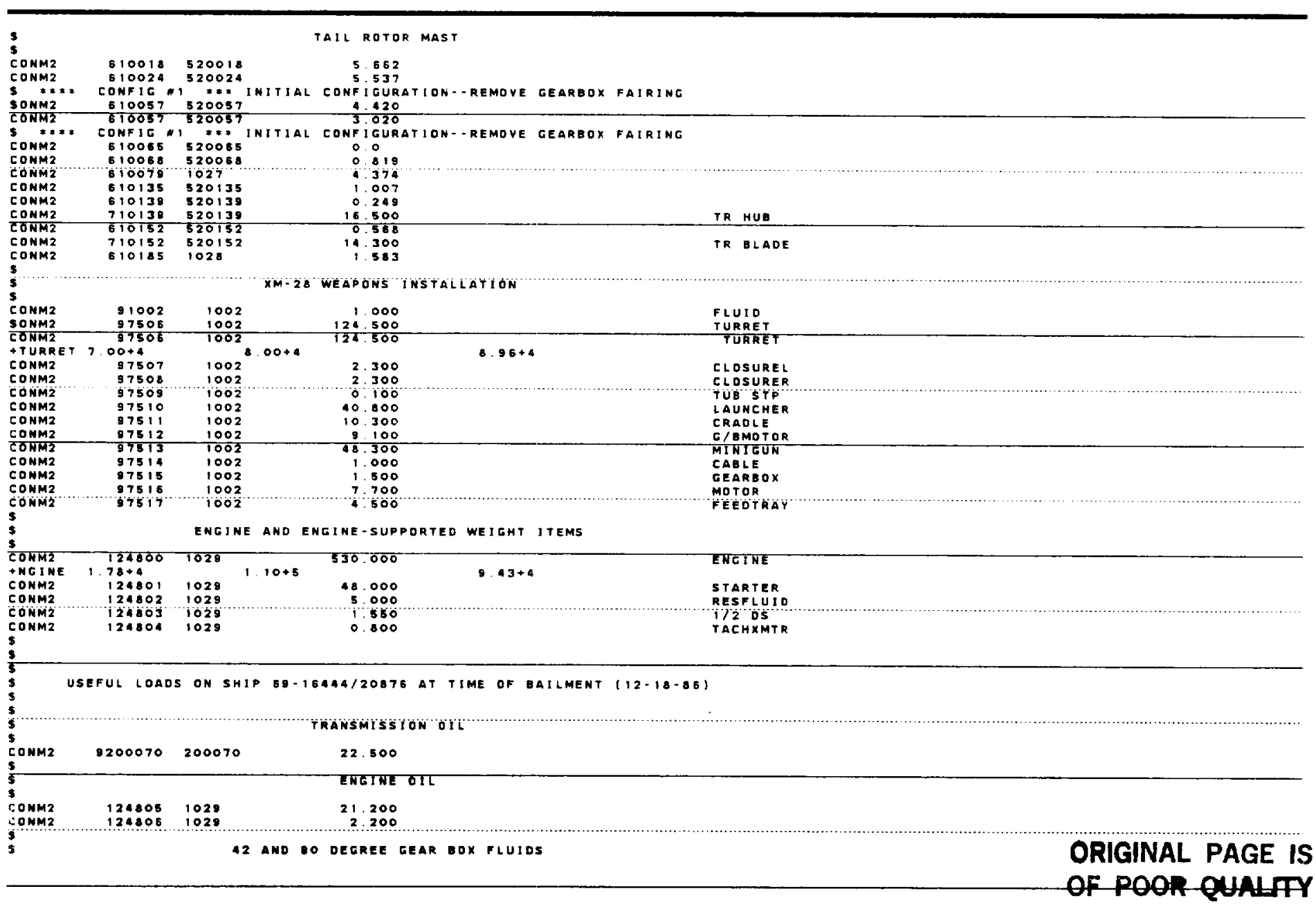




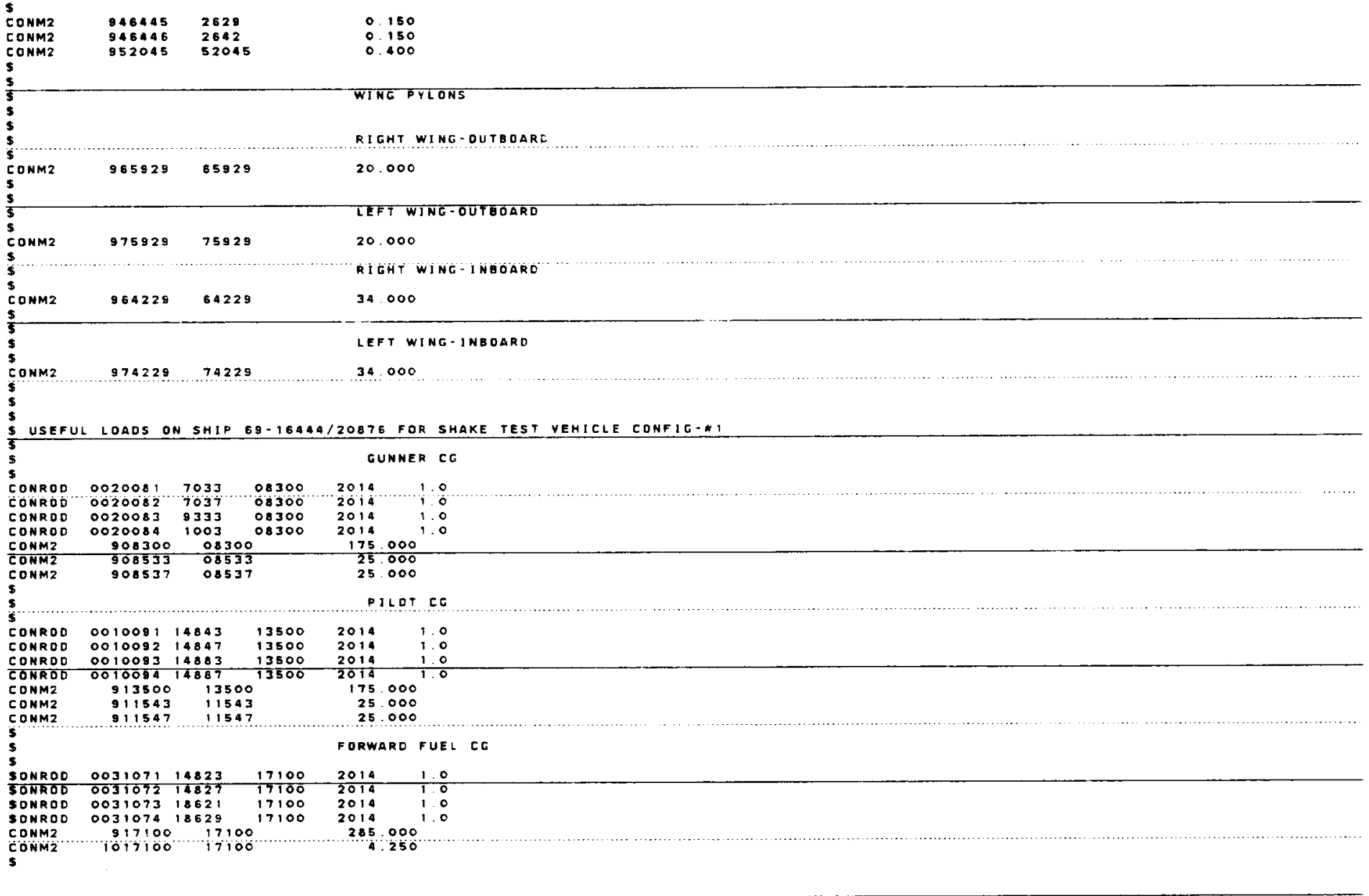

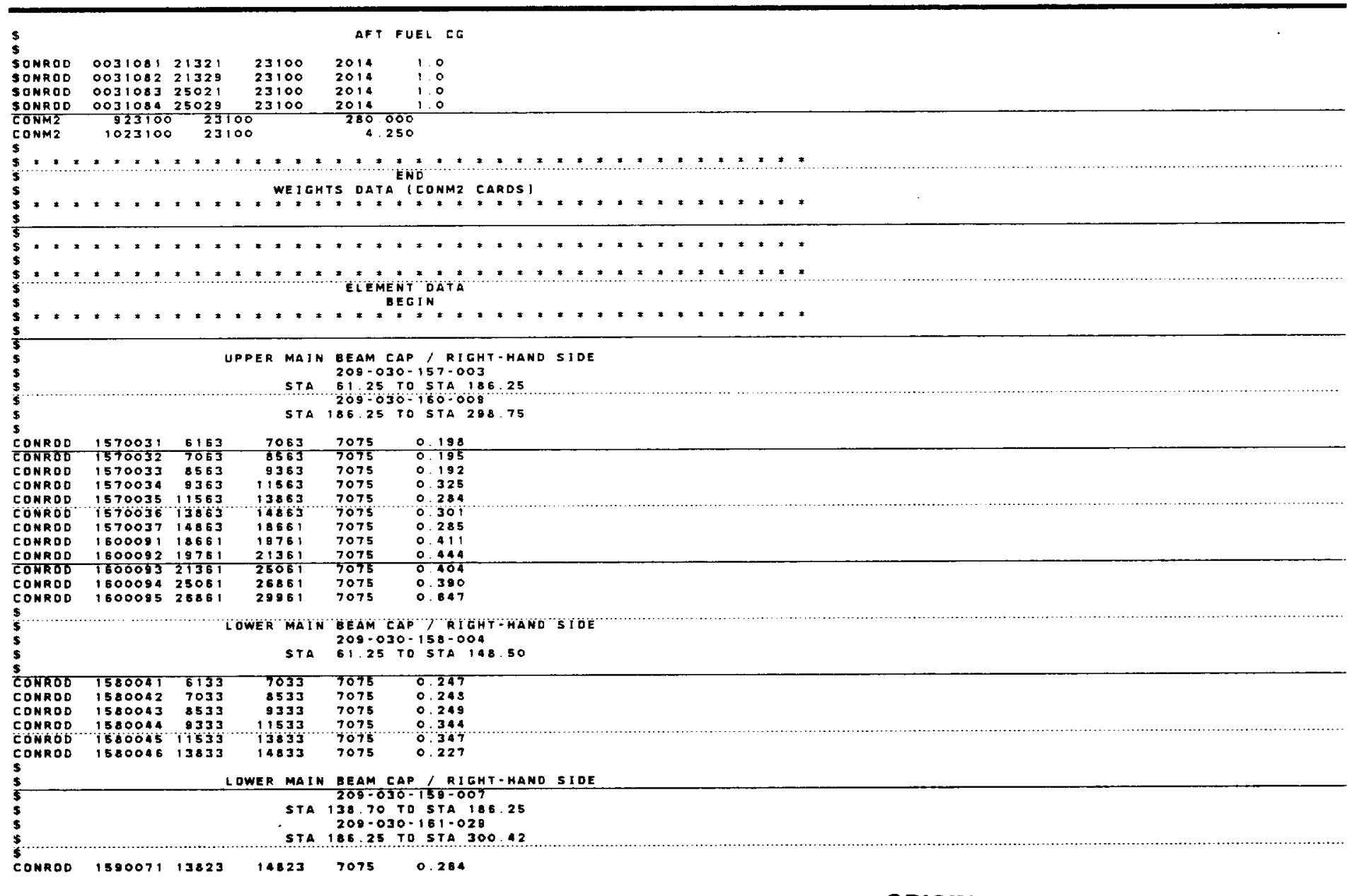




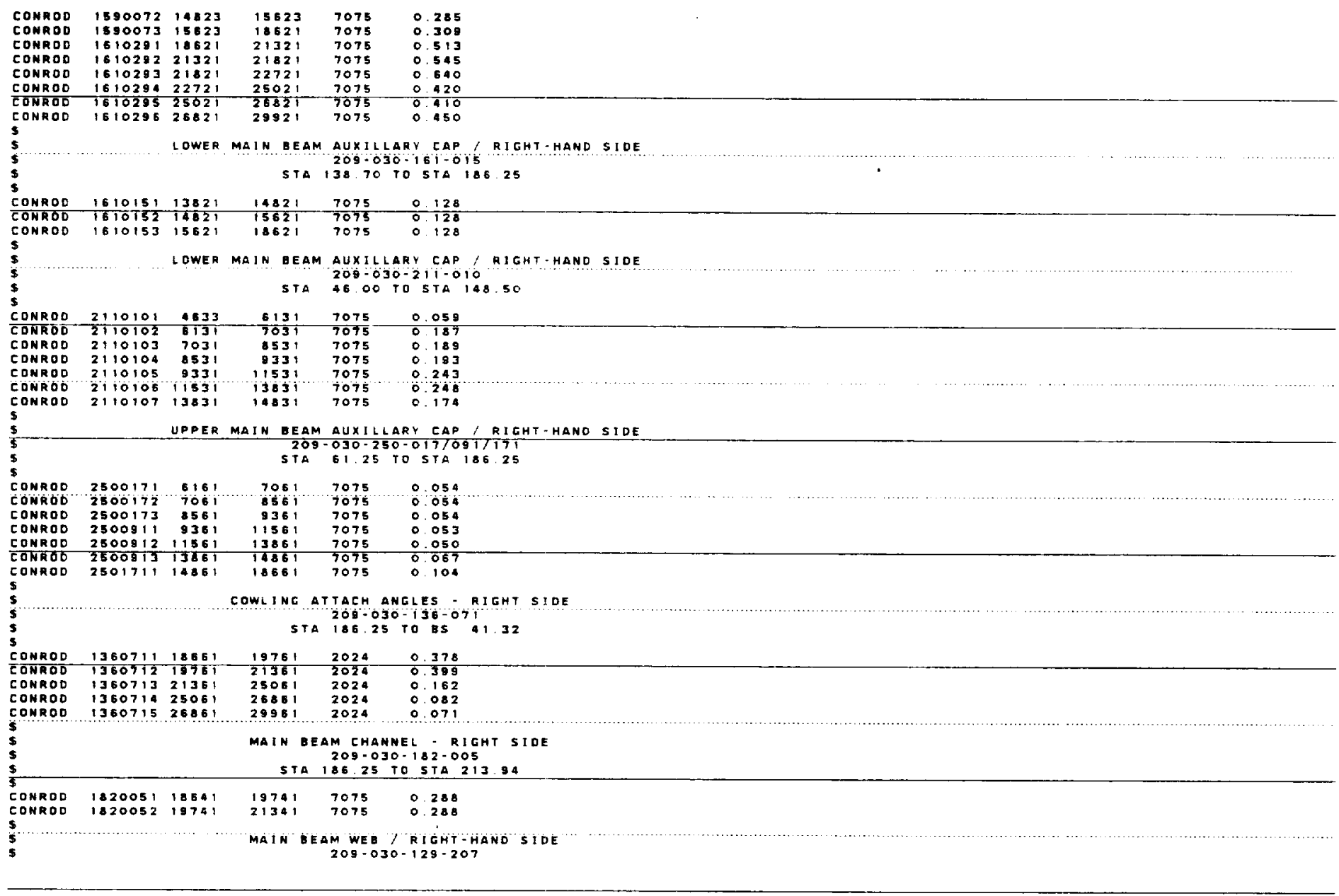

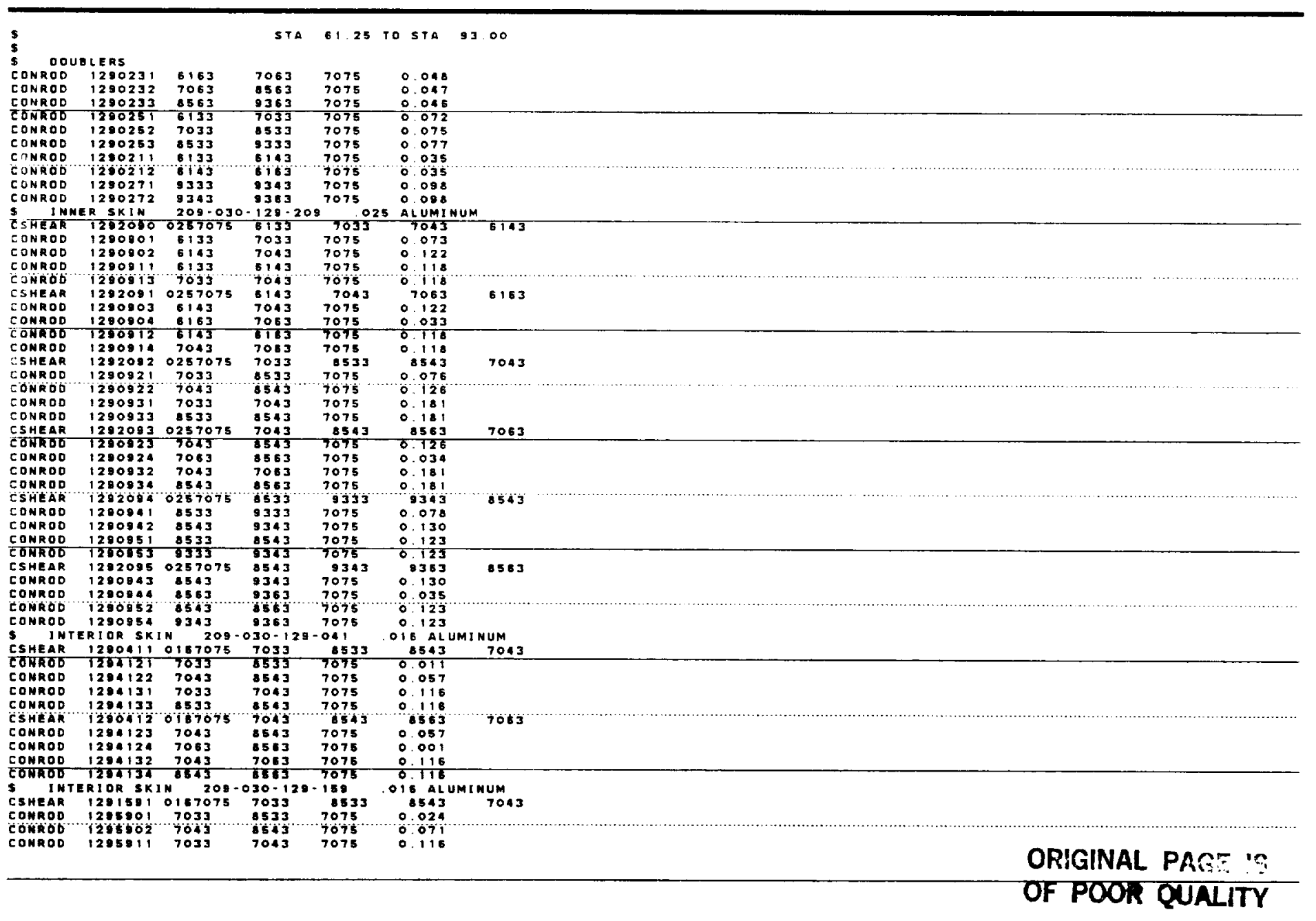




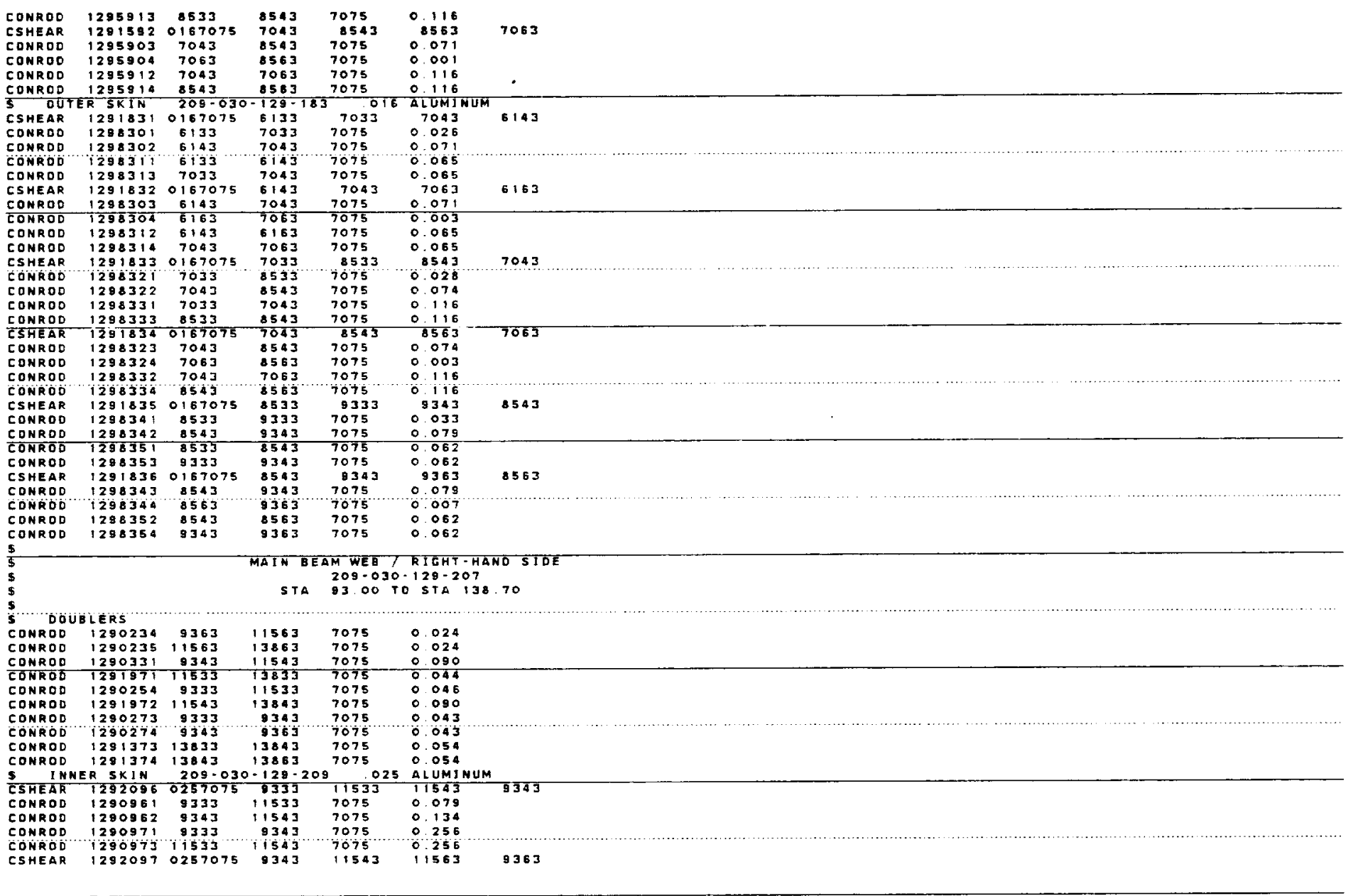

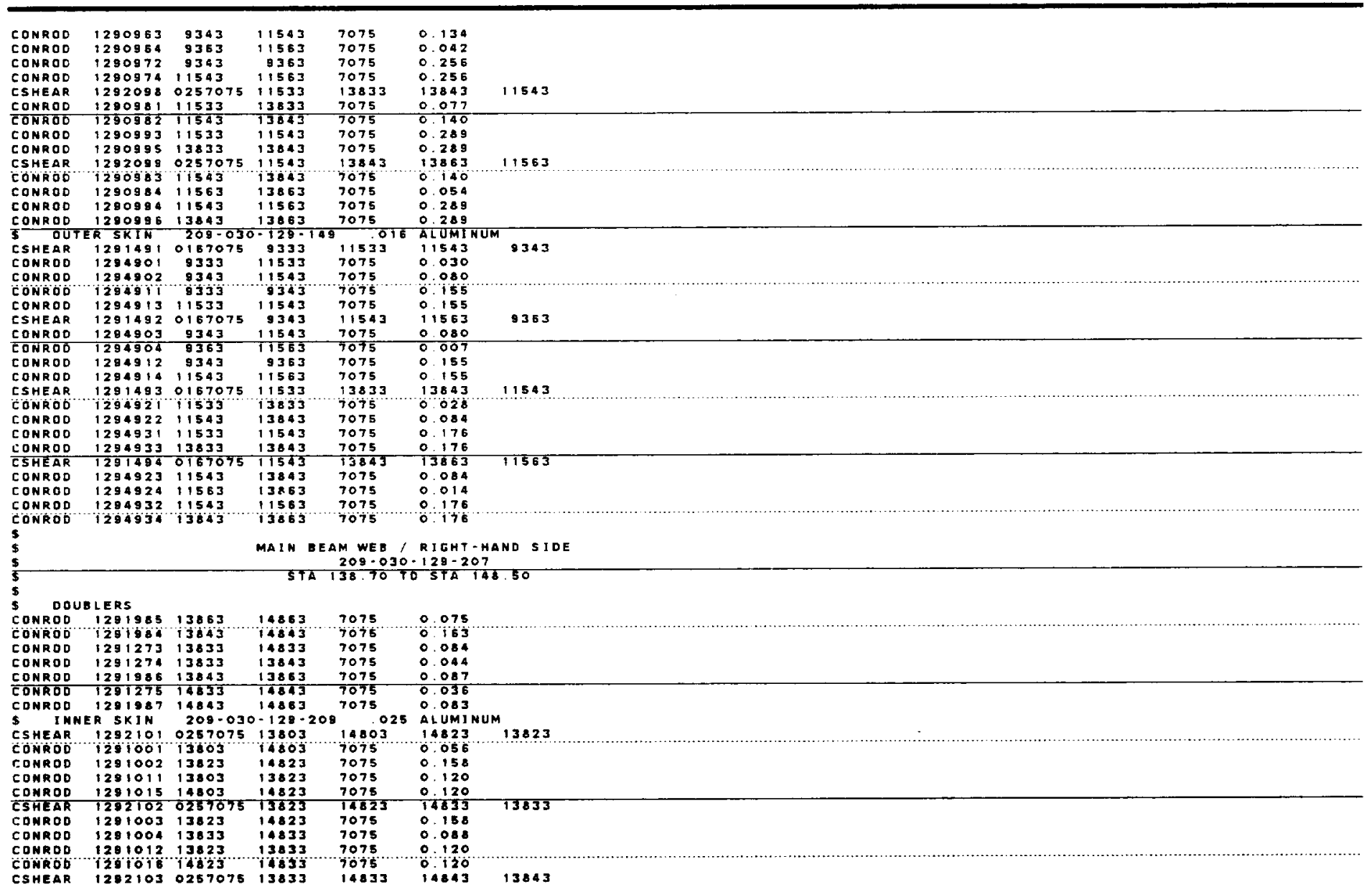




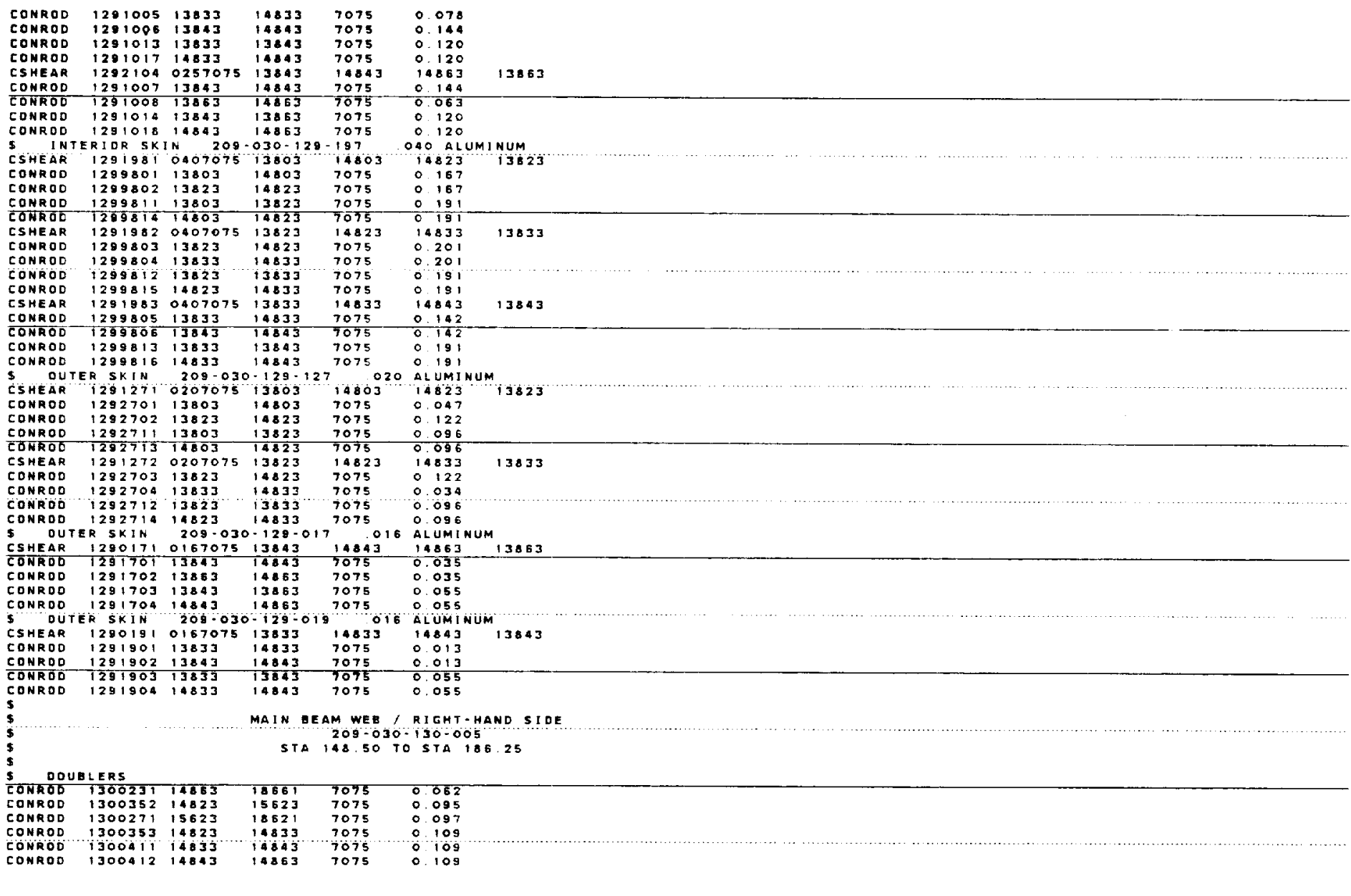

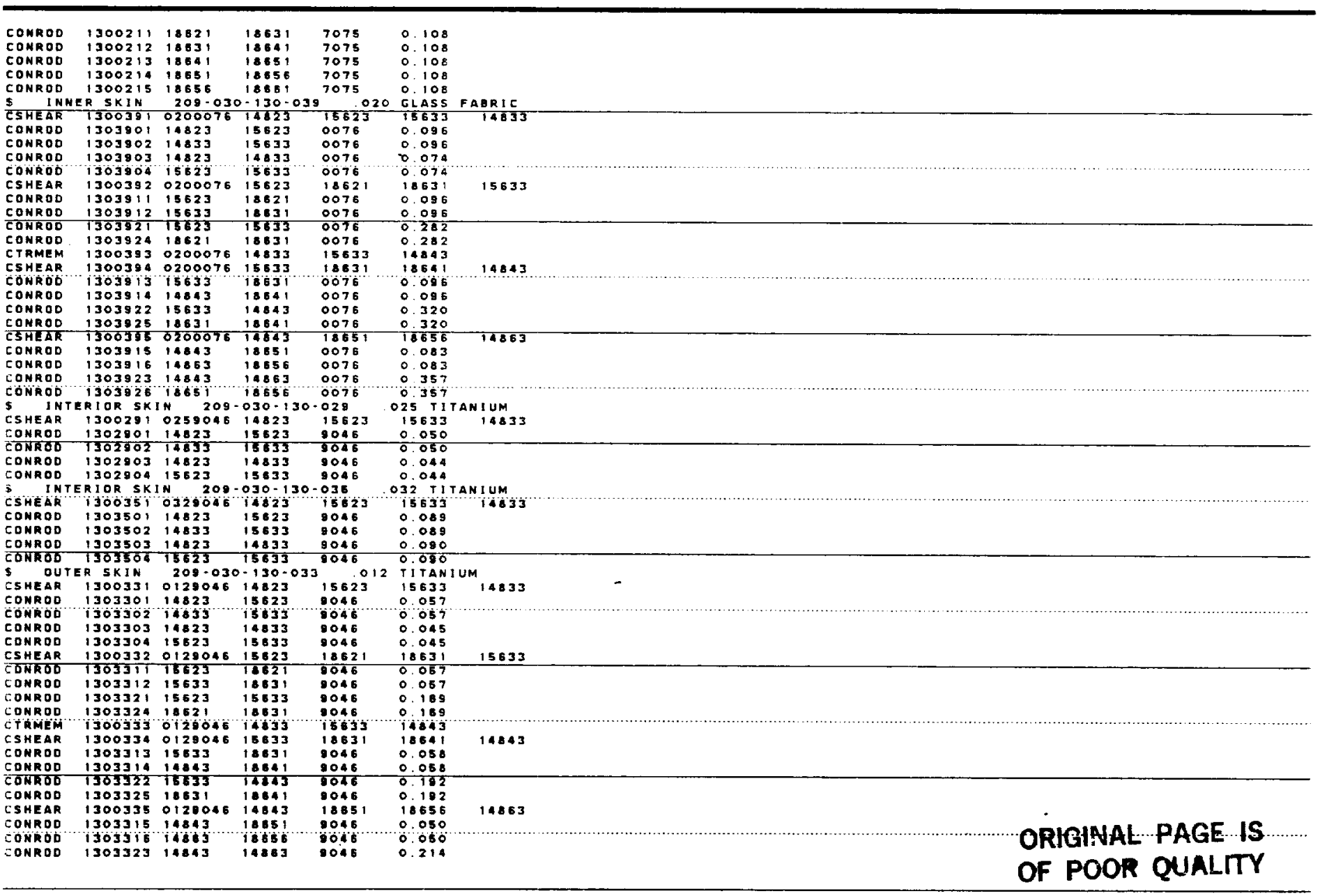




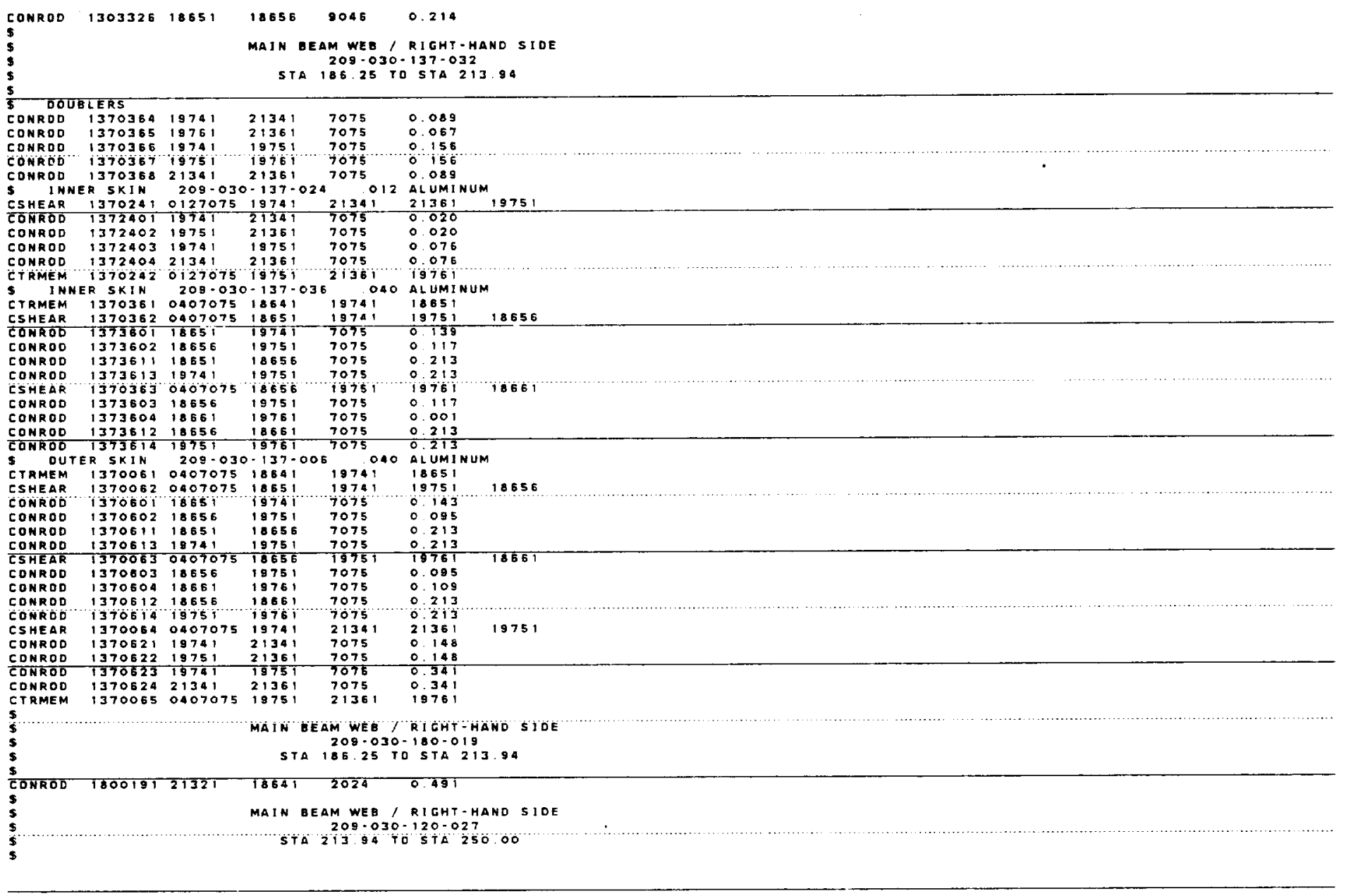

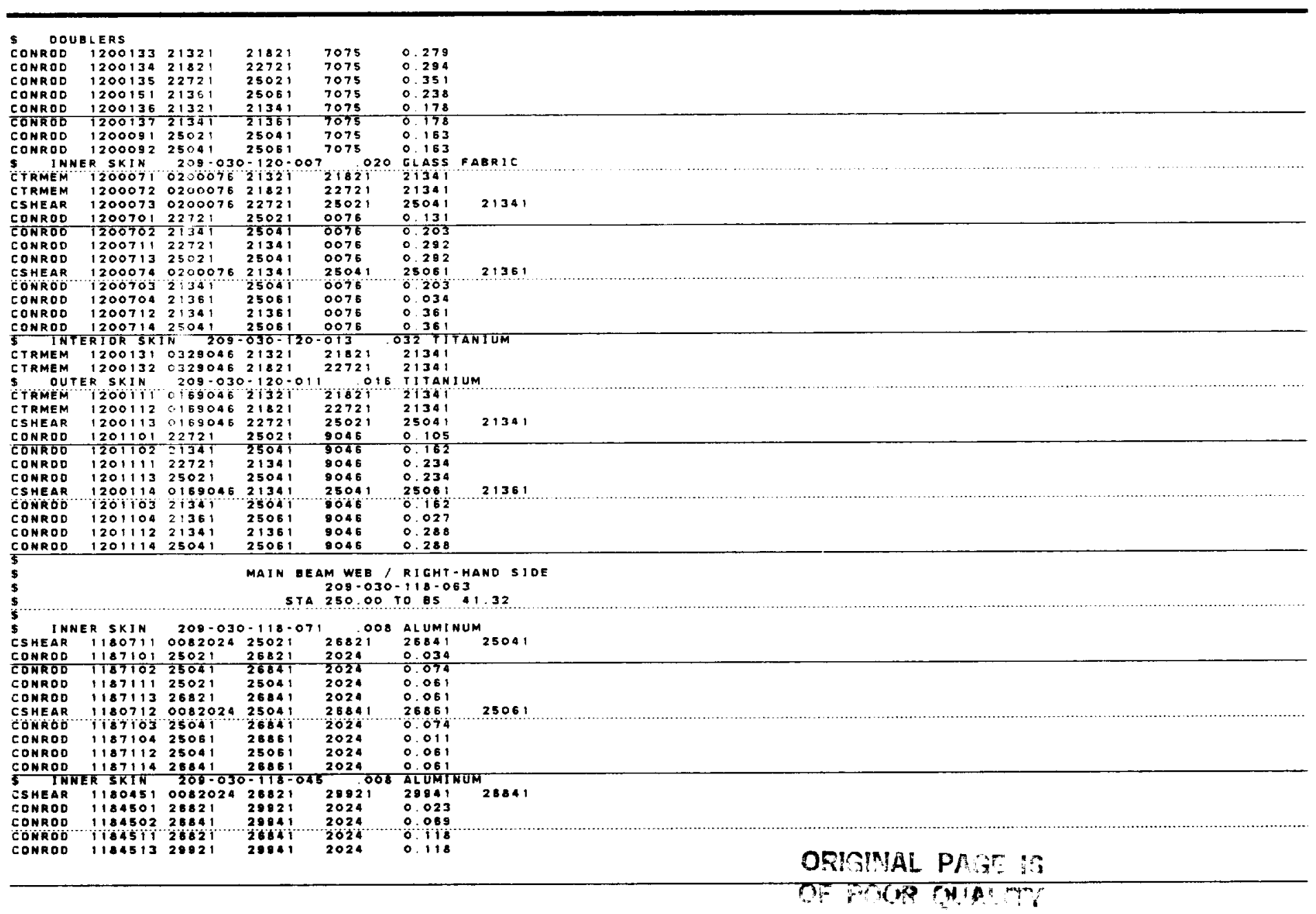




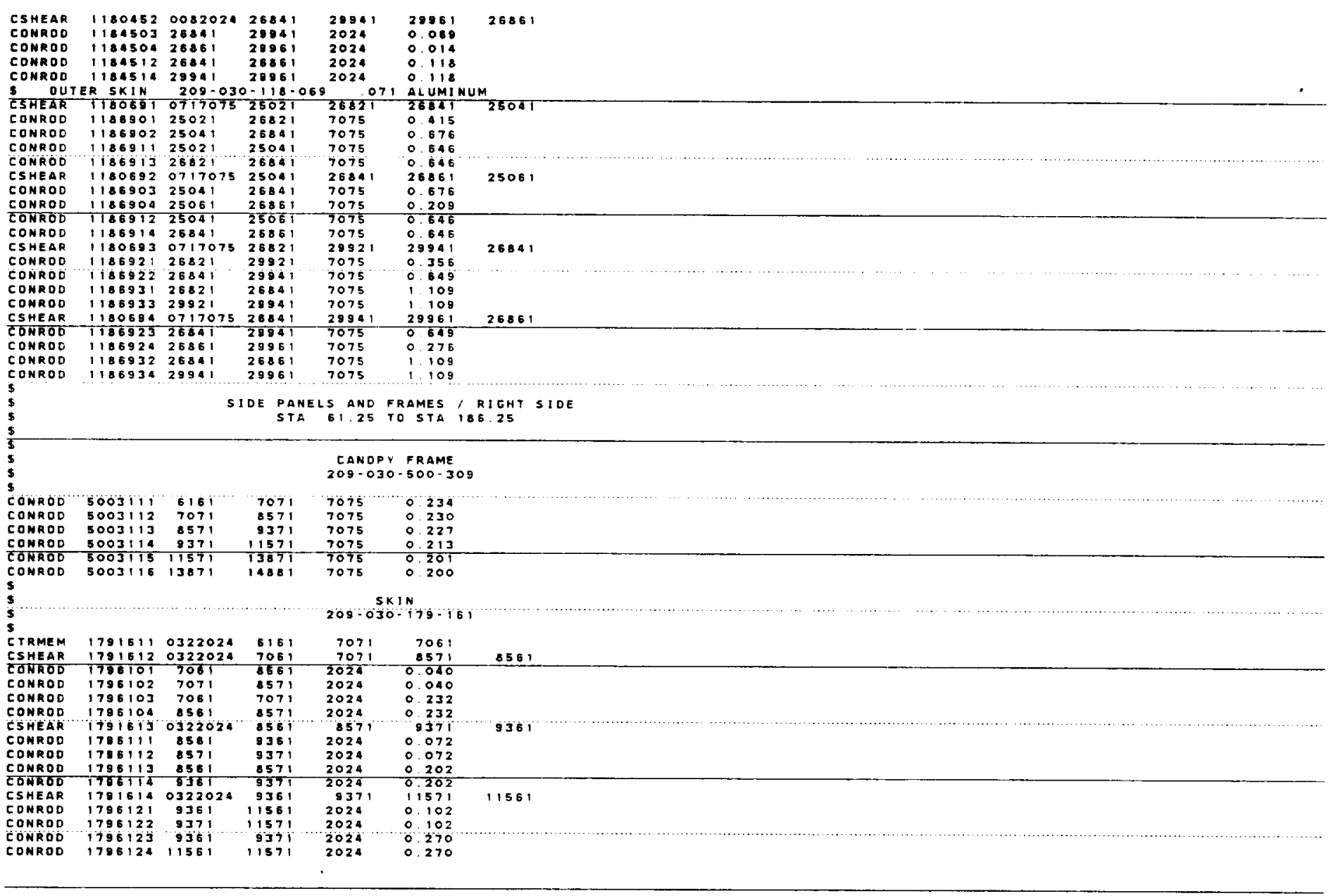

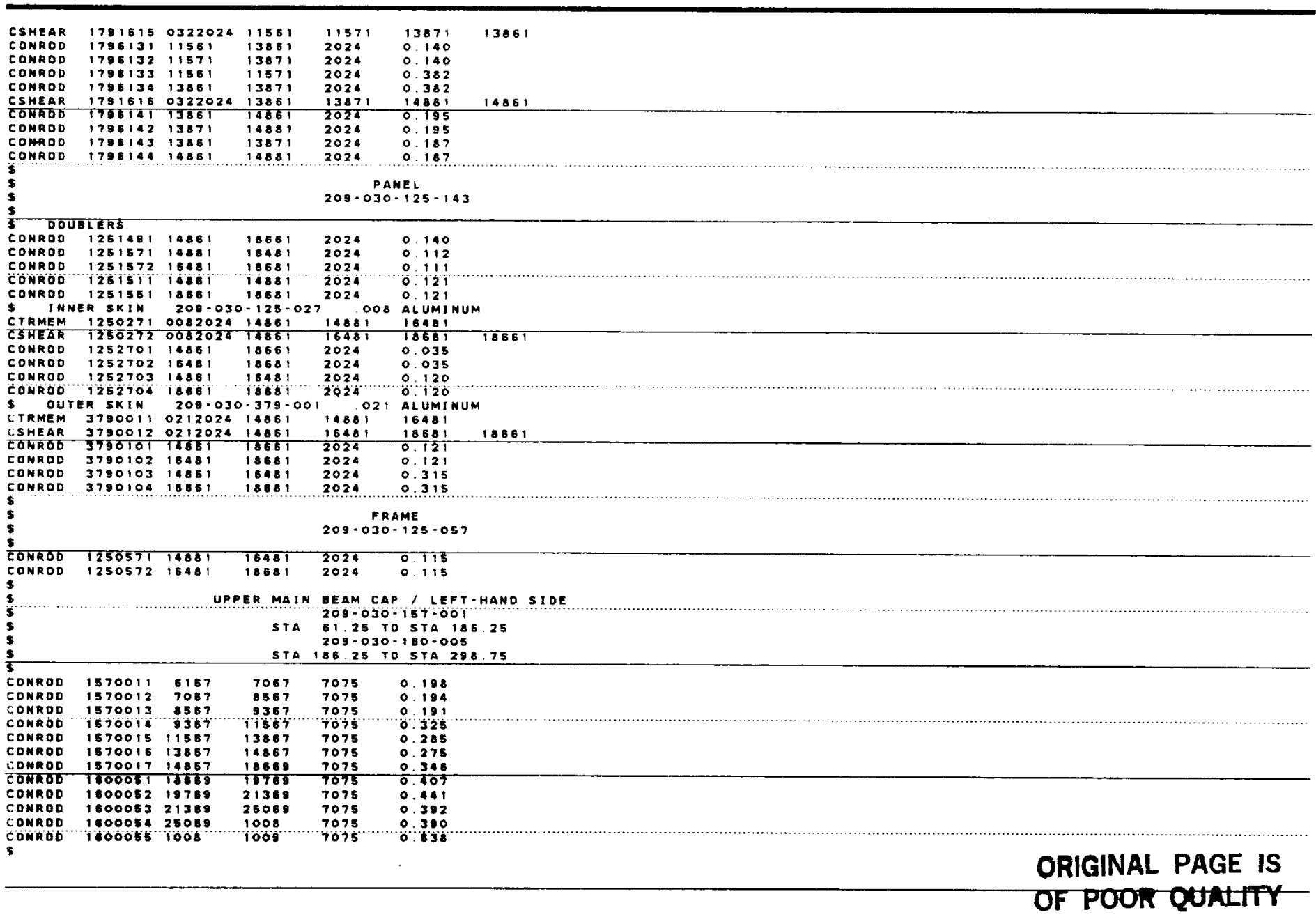




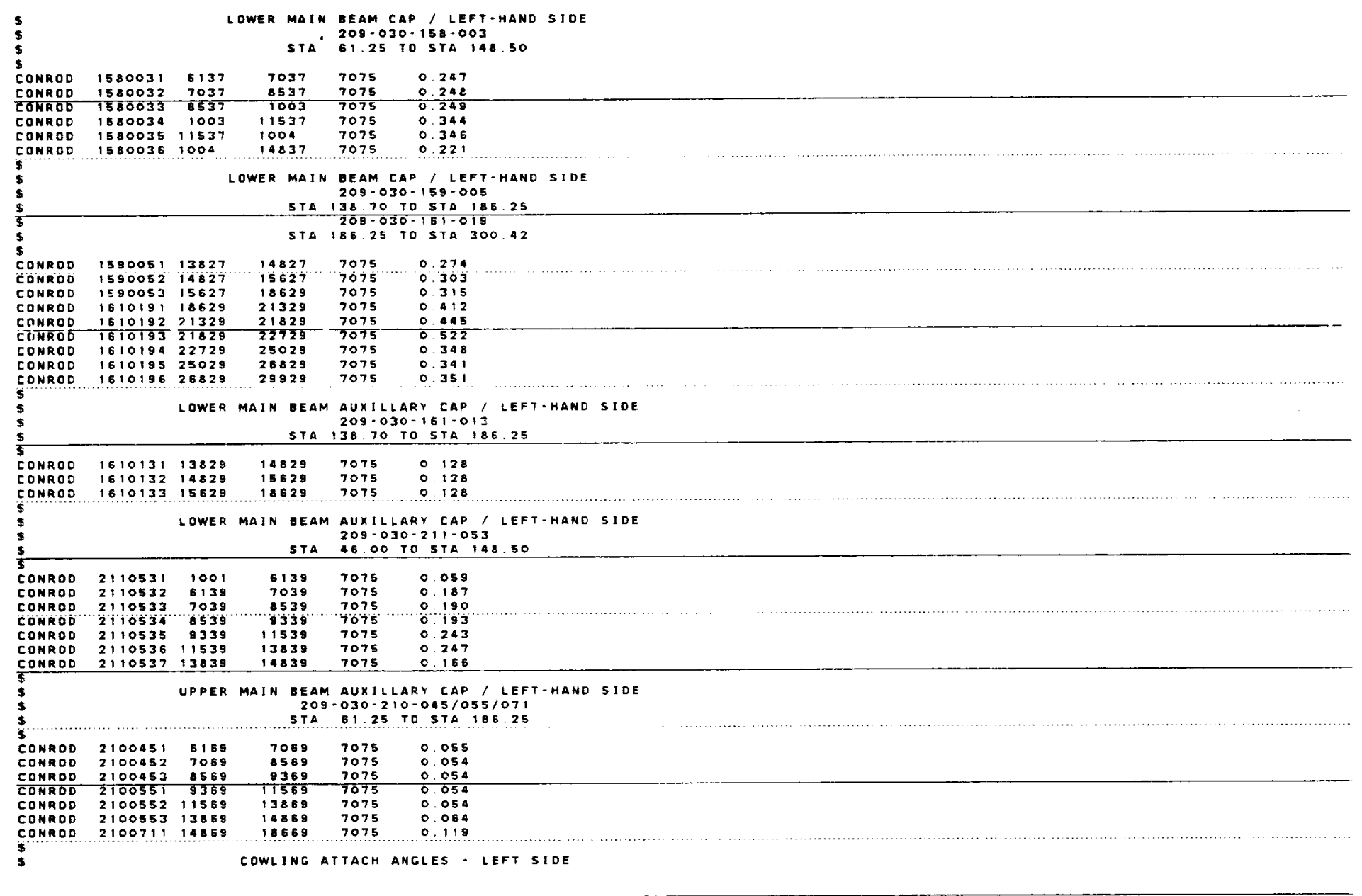

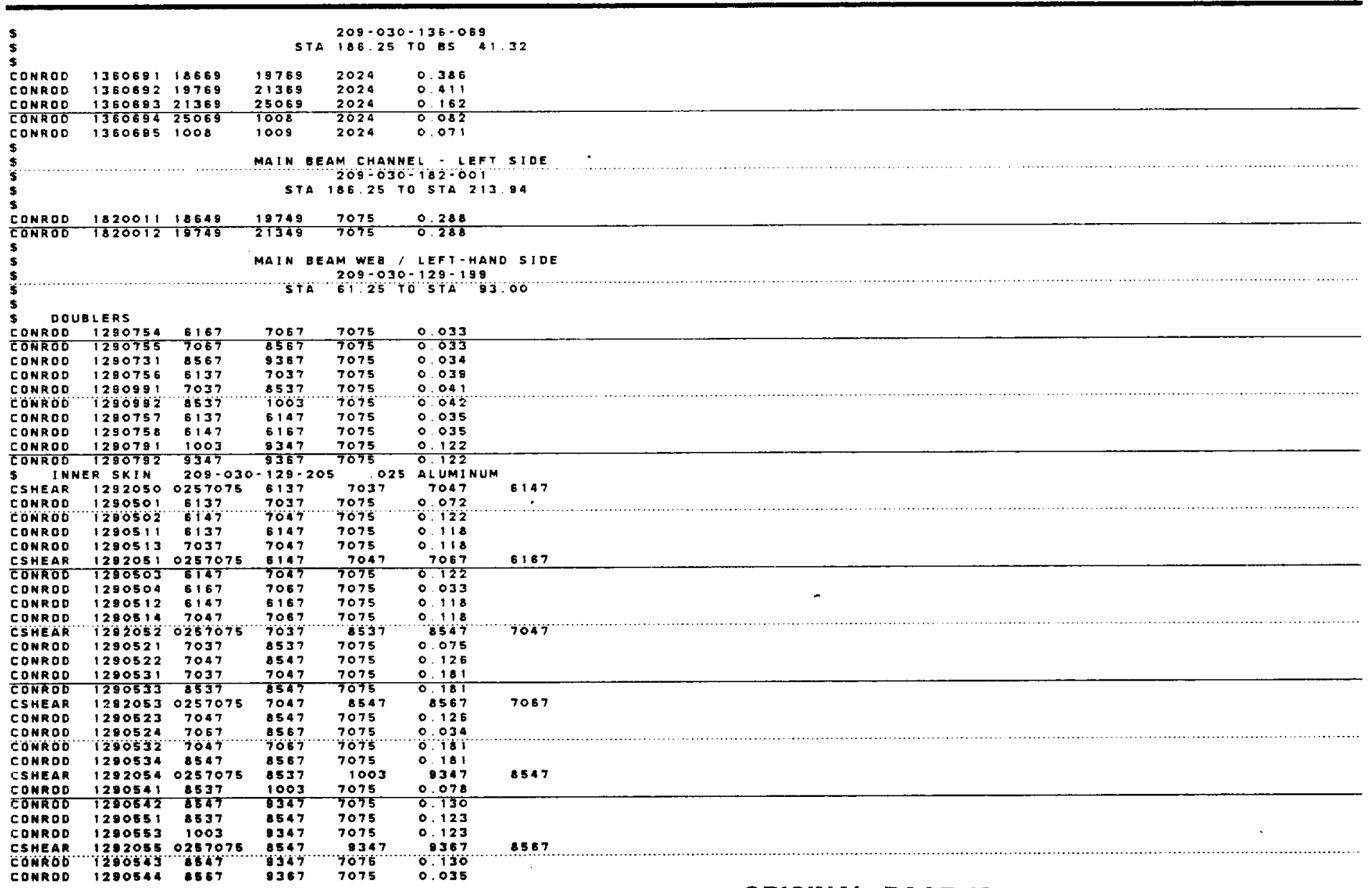




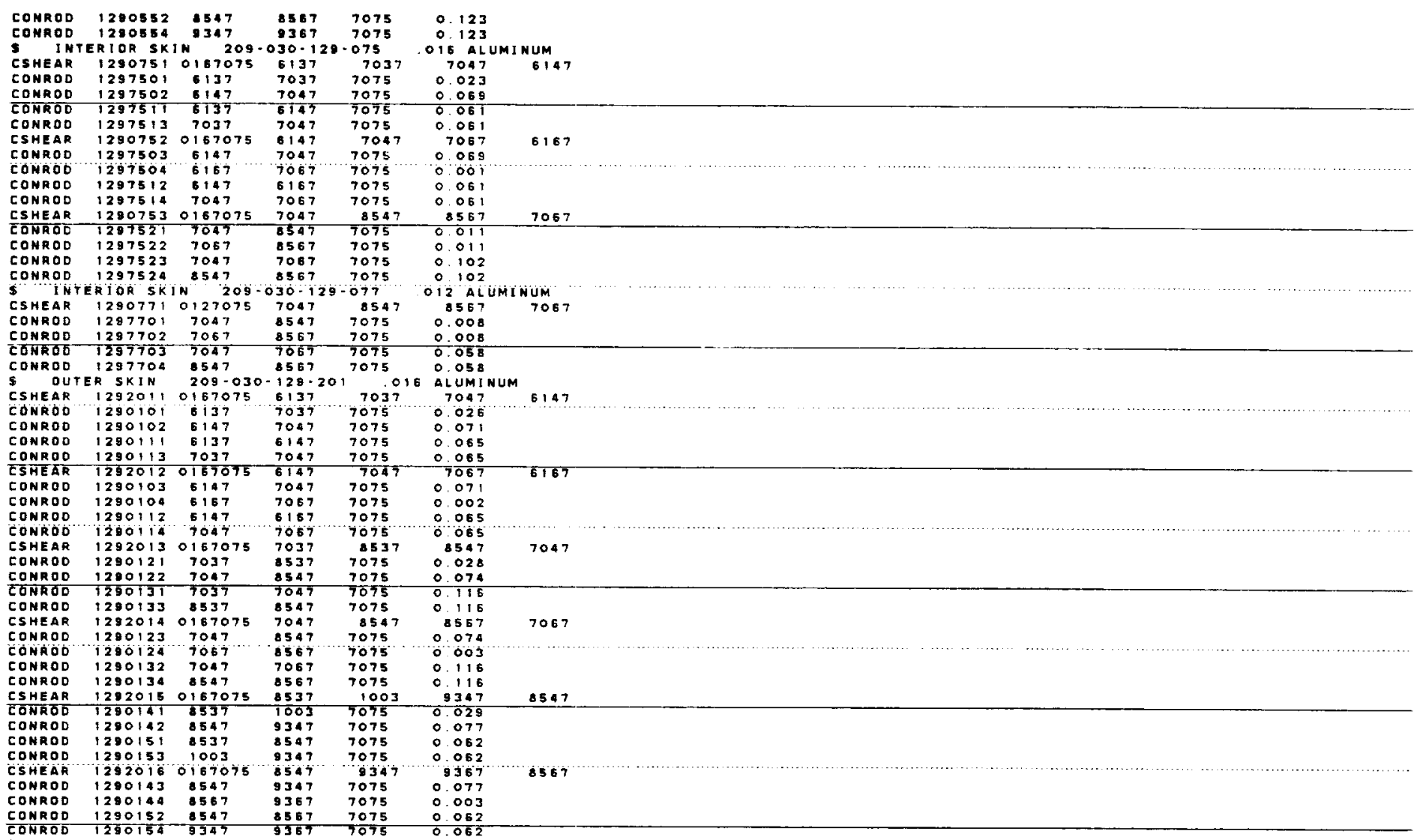

\begin{tabular}{lll} 
CONROD & 1290152 & 8547 \\
\hline CONROD & 1290154 & -9349
\end{tabular}

main beam we , LEFT-HANo SIDE

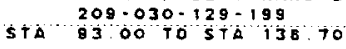

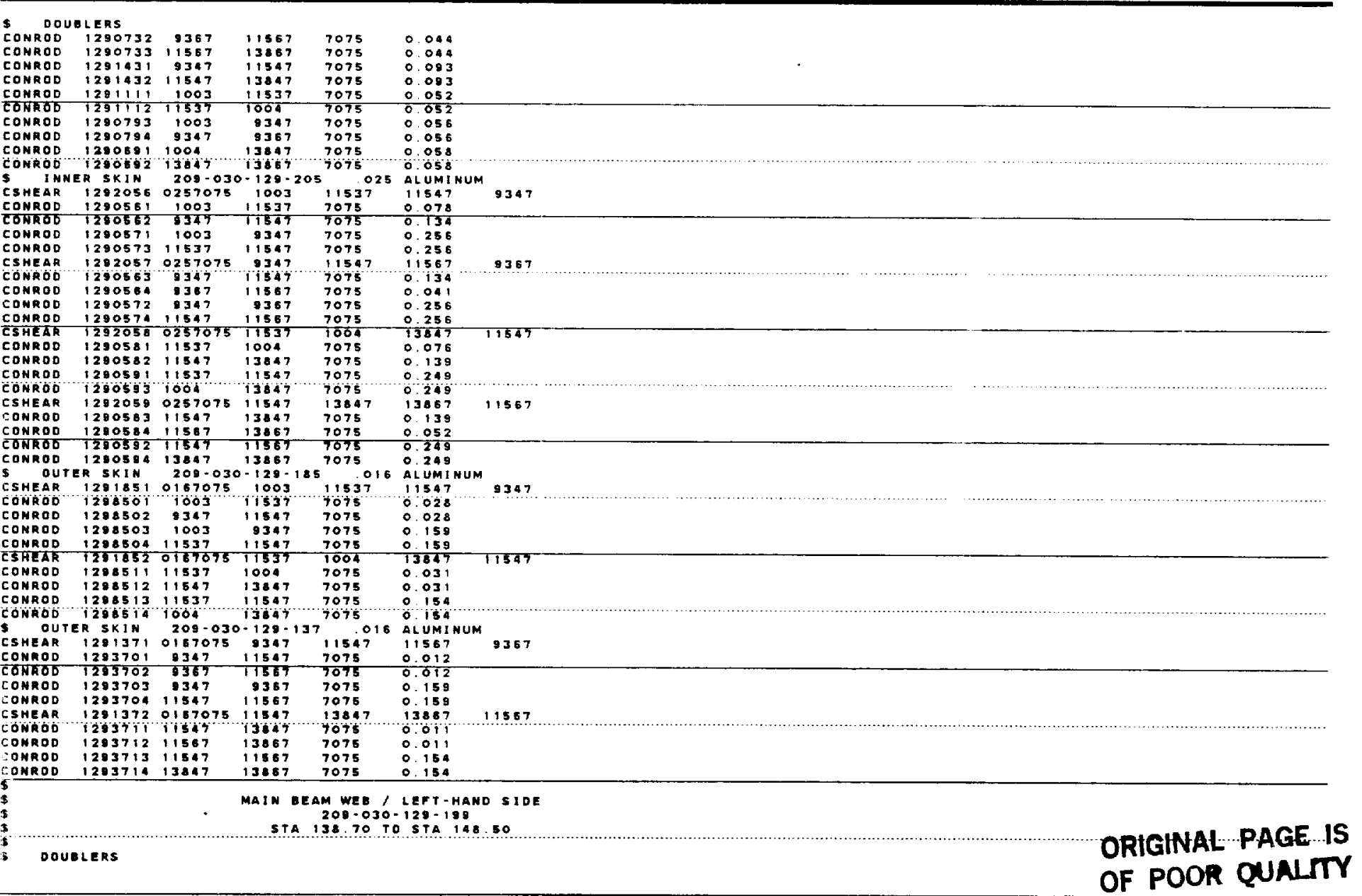




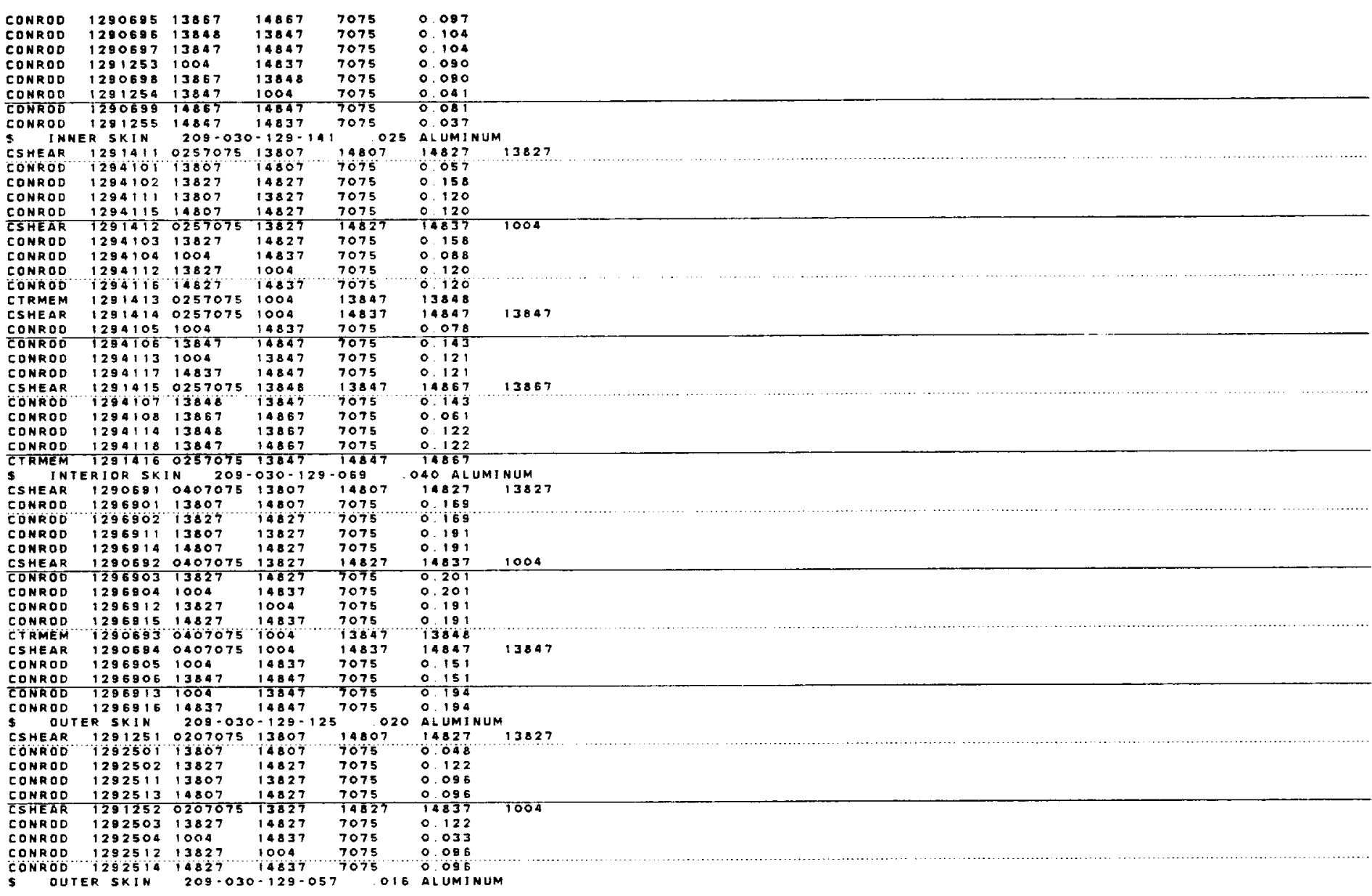

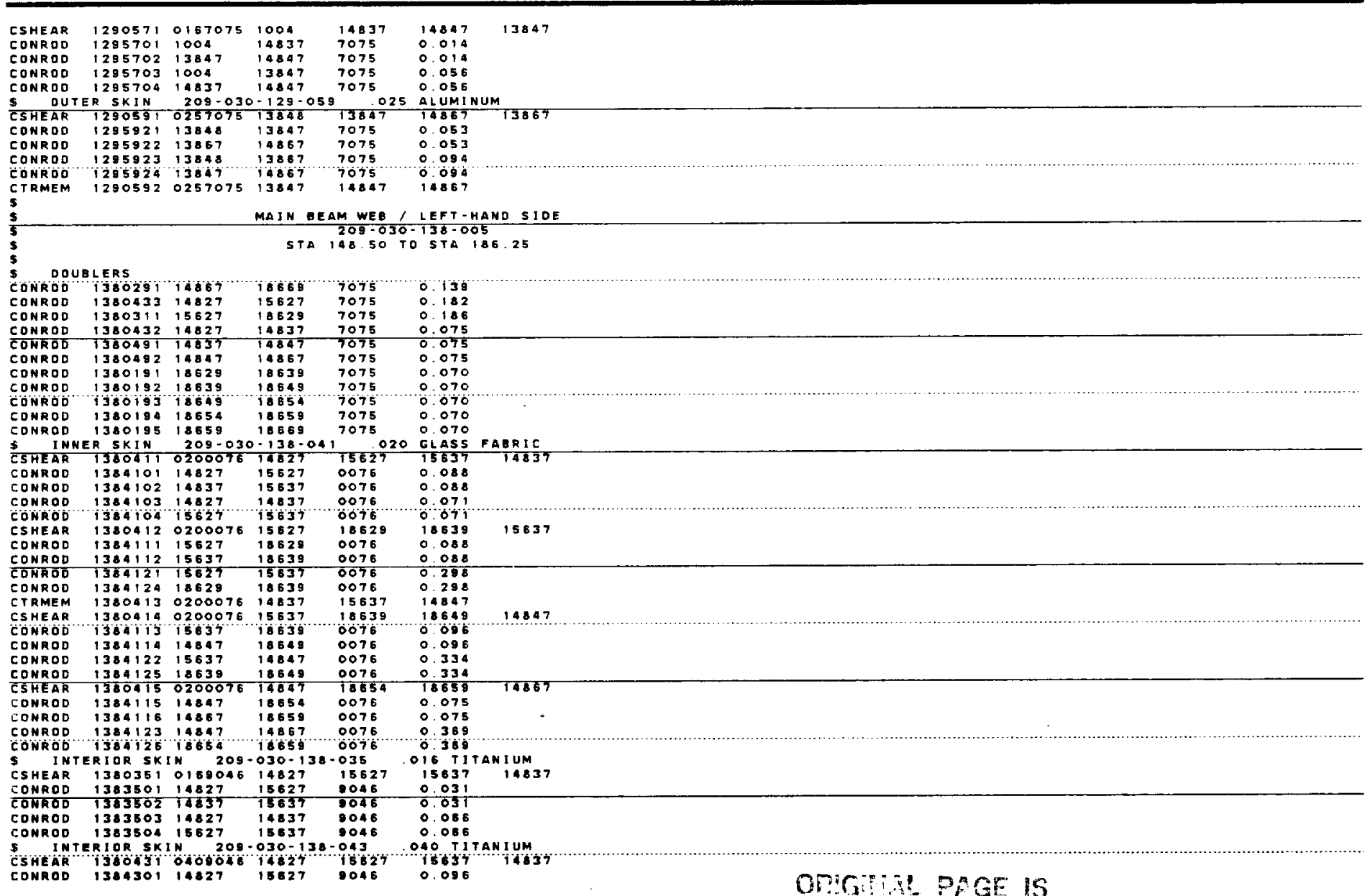




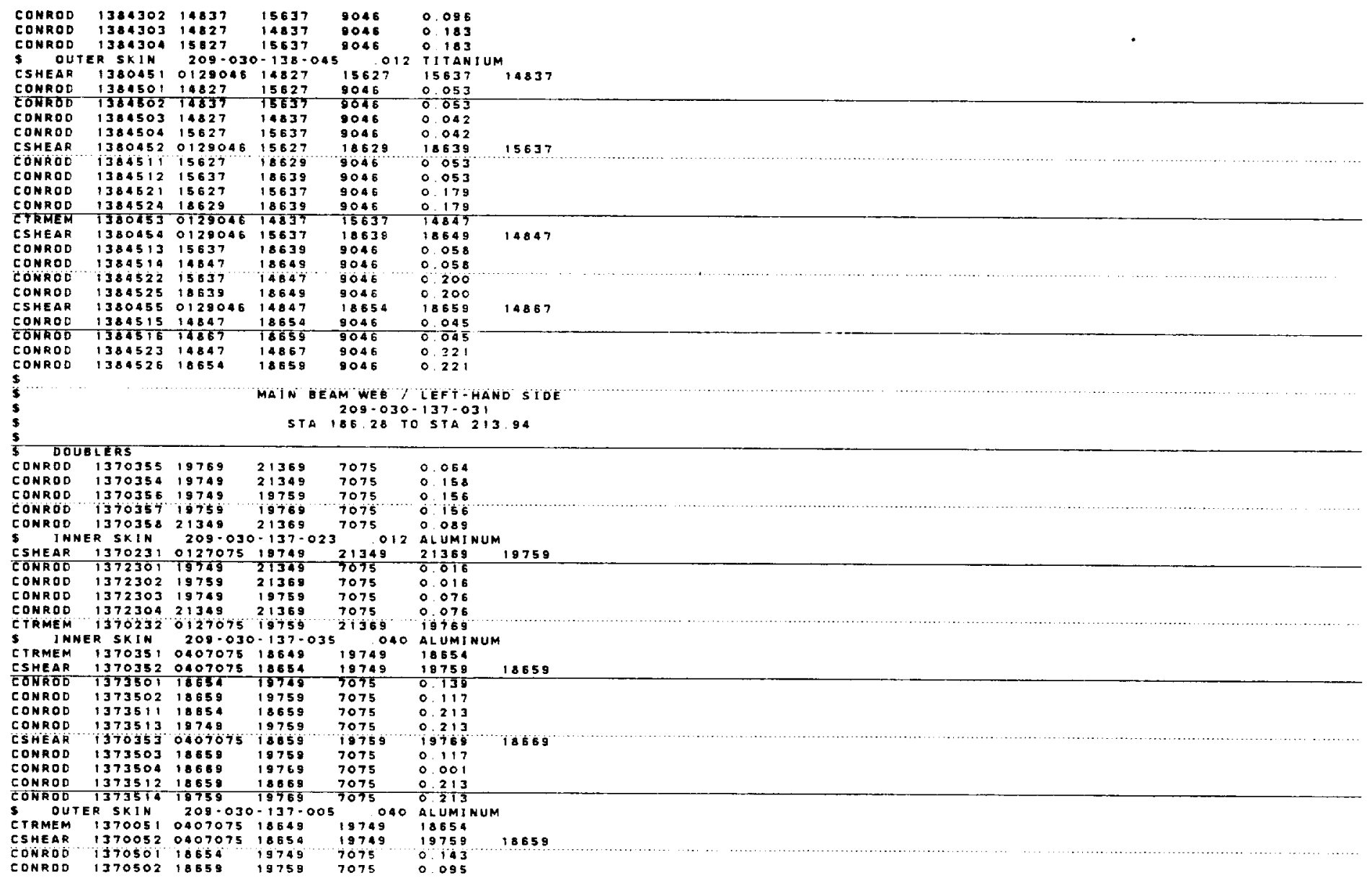

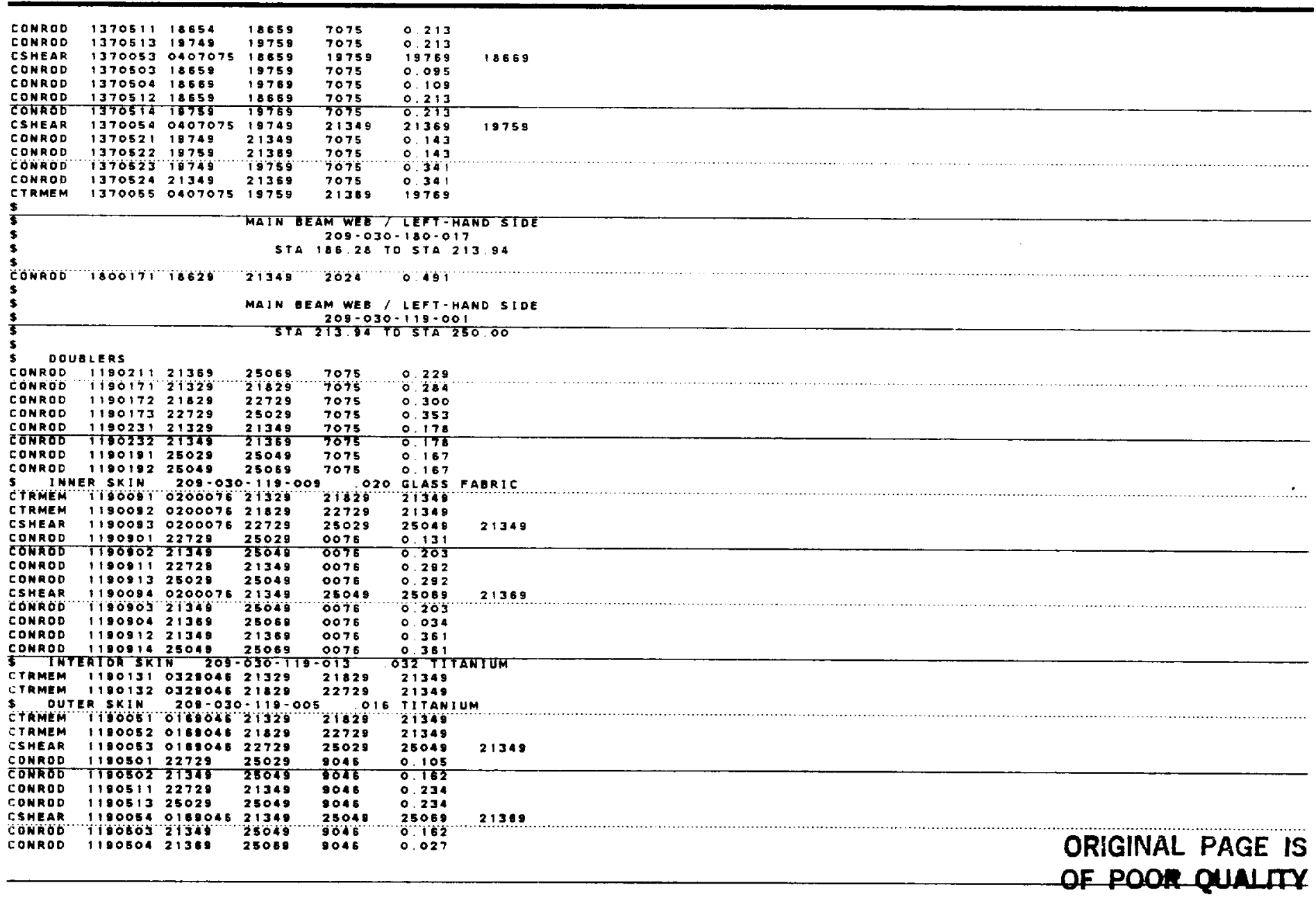




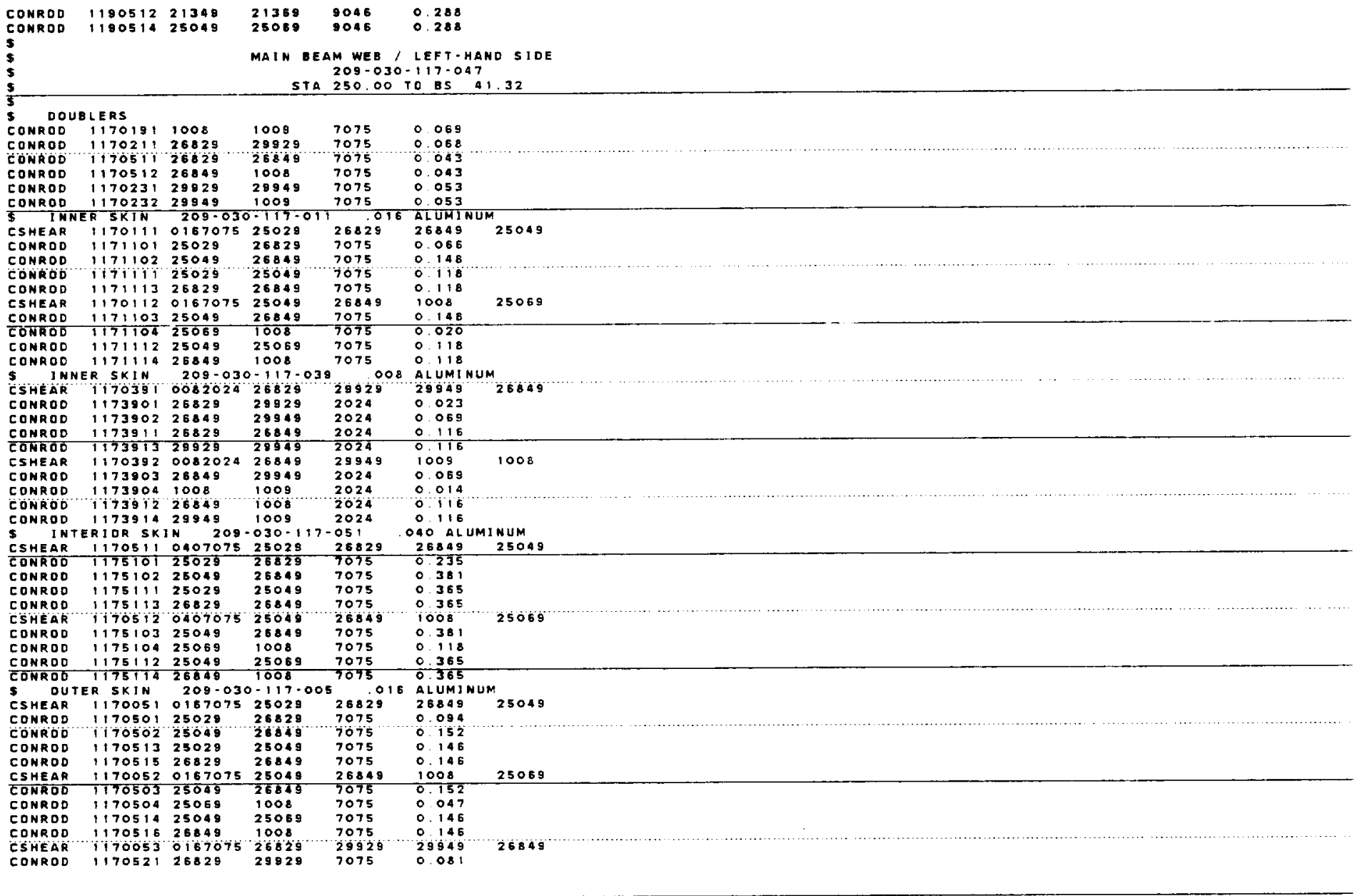

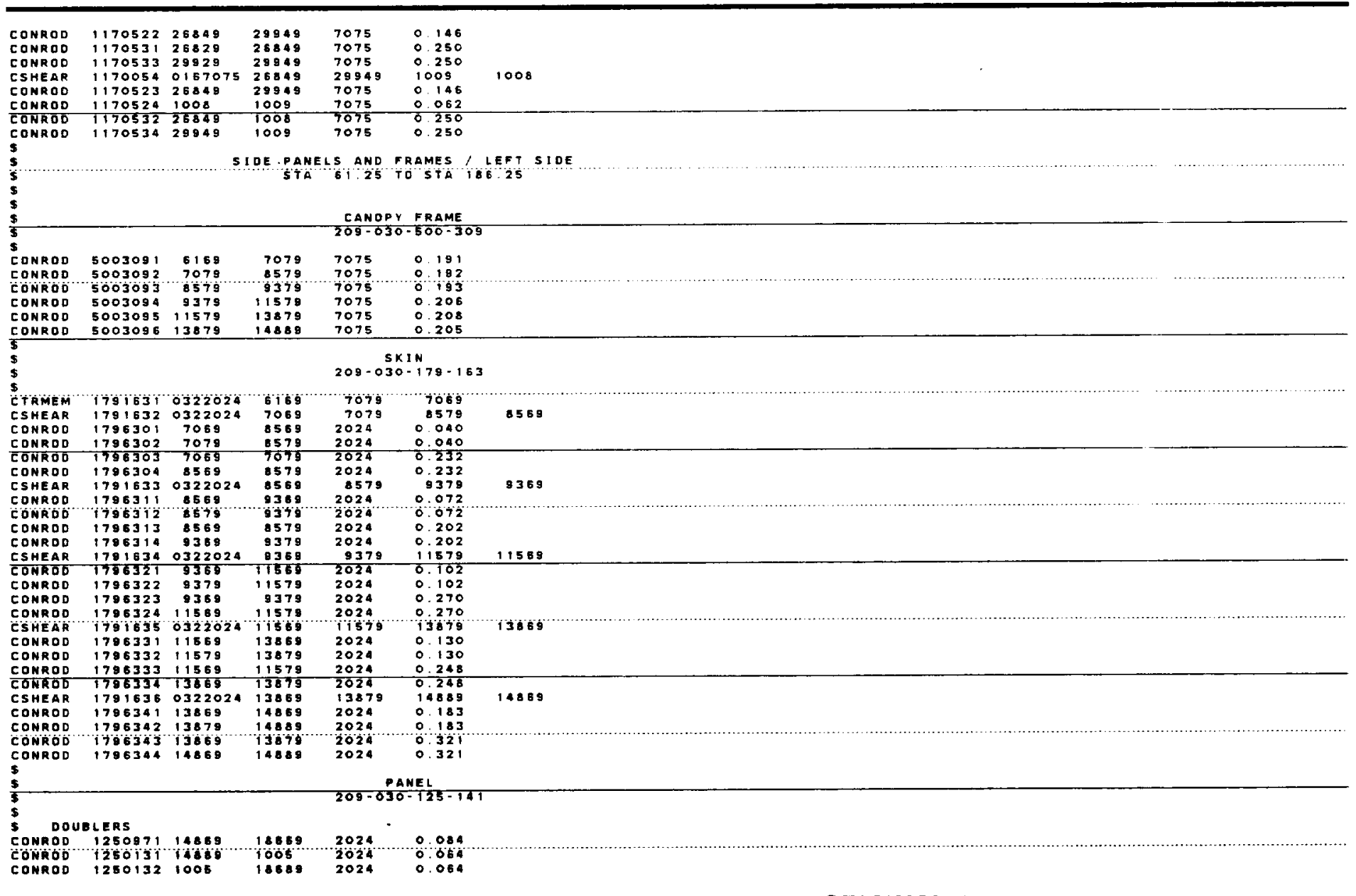




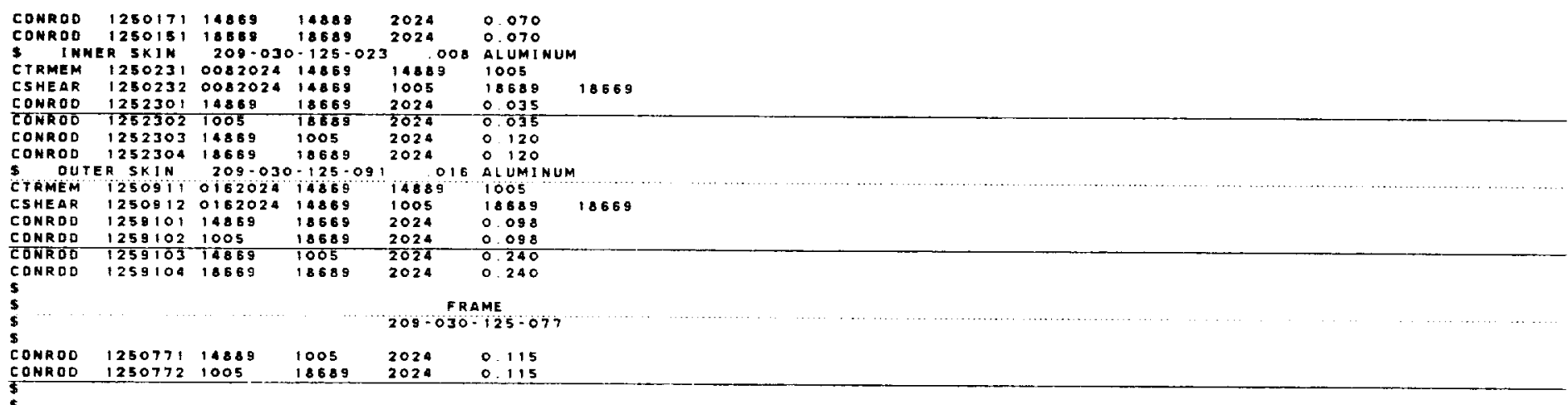

$\$$

$E L E V A T O R$
20902000

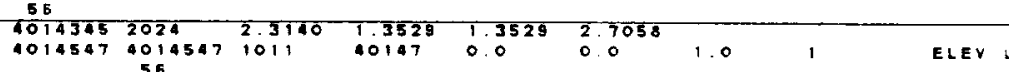

$10145472024 \quad 23140 \quad 13529 \quad 3529 \quad 27058 \ldots \ldots \ldots \ldots \ldots \ldots$

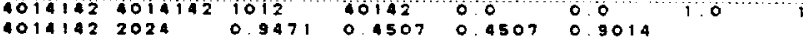

10142434014243014200143000700140

$10142432024 \quad 16745 \quad 0.8938 \quad 0.0938 \quad 17875$

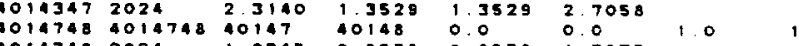

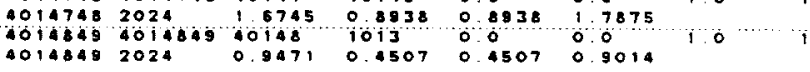

MAIN ROTOR PYION SUPPORT STRUCTURE

PYLON DEAM - RIGHT SIDE

$209-030-121-001$

STA 18625 TOSTE 2138

$\begin{array}{llllll}1210811 & 1210611 & 18583 & 18283 & 0.0 & 0.0 \\ 1210811 & 7075 & 0.543 & 0.3418 & 2.0812 & 0.0 \\ 1210612 & 1210812 & 18983 & 21183 & 0.0 & 0.0 \\ 12106127075 & 0.843 & 0.3418 & 2.0812 & 0.0 \\ 1210613 & 1210613 & 2183 & 21383 & 0.0 & 0.0 \\ 1210613 & 7075 & 0.643 & 0.3418 & 2.0812 & 0.0\end{array}$

FWO PYLON POST - RIGMT SIDE

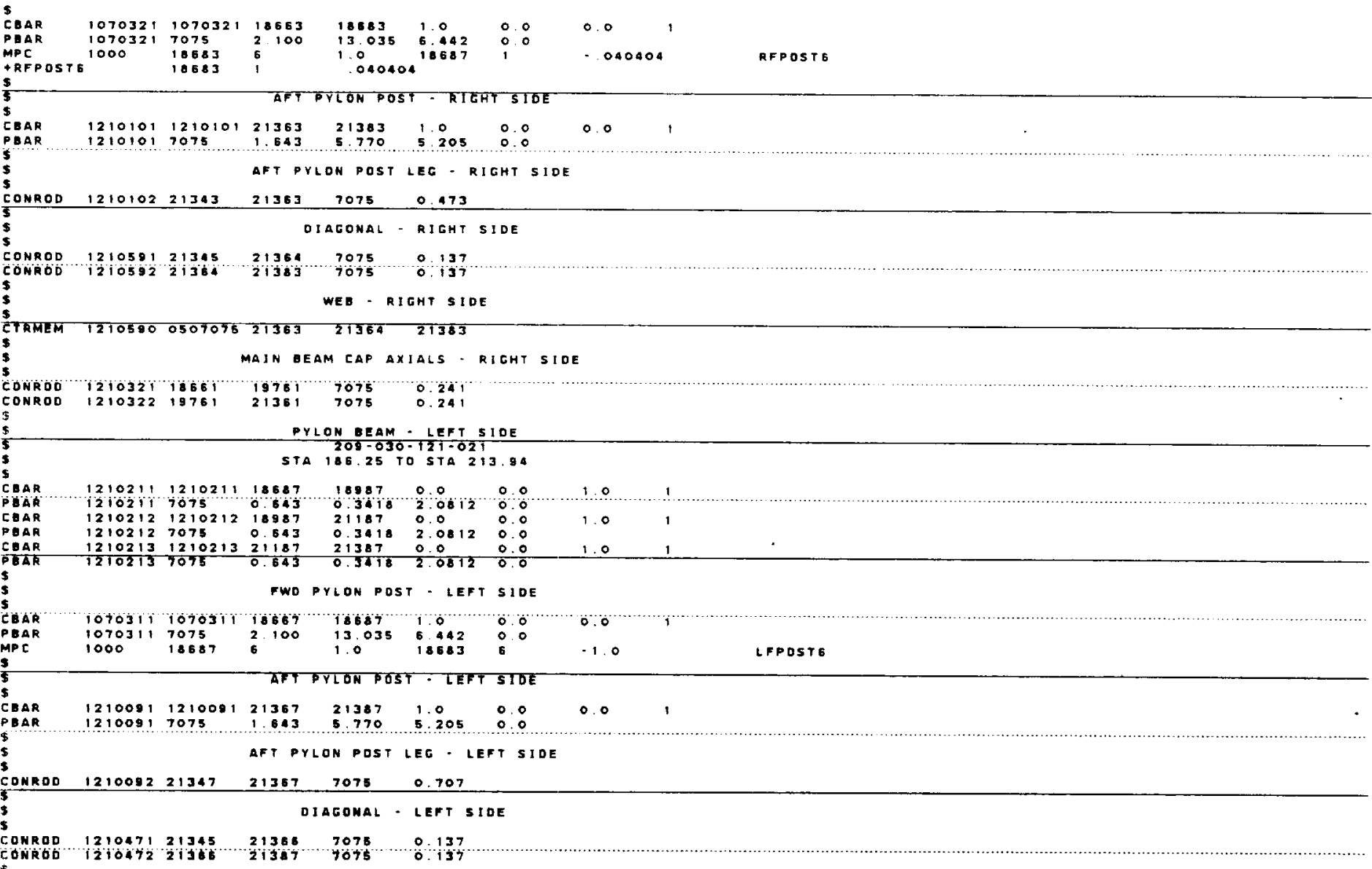




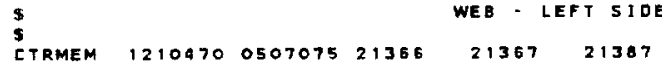

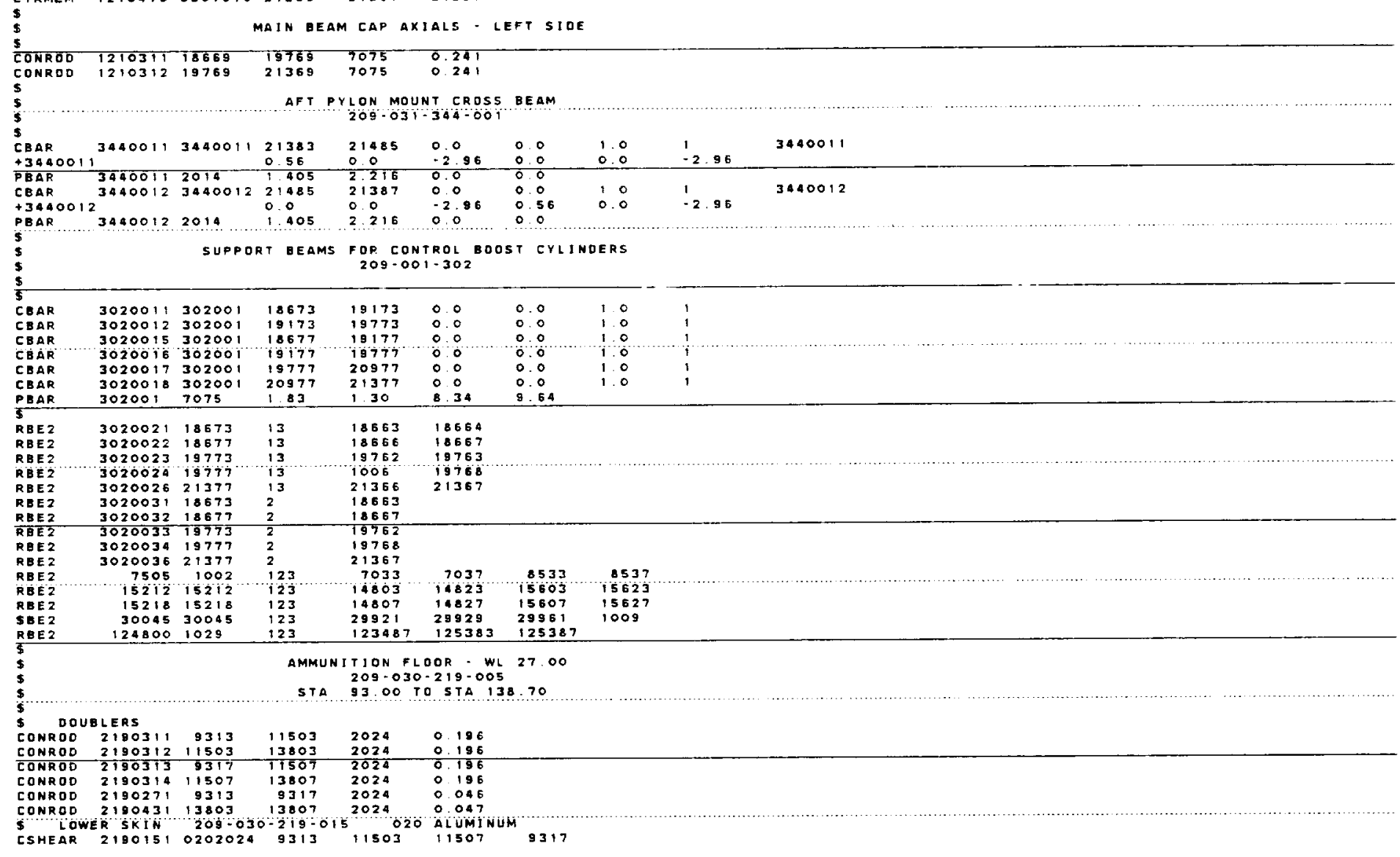

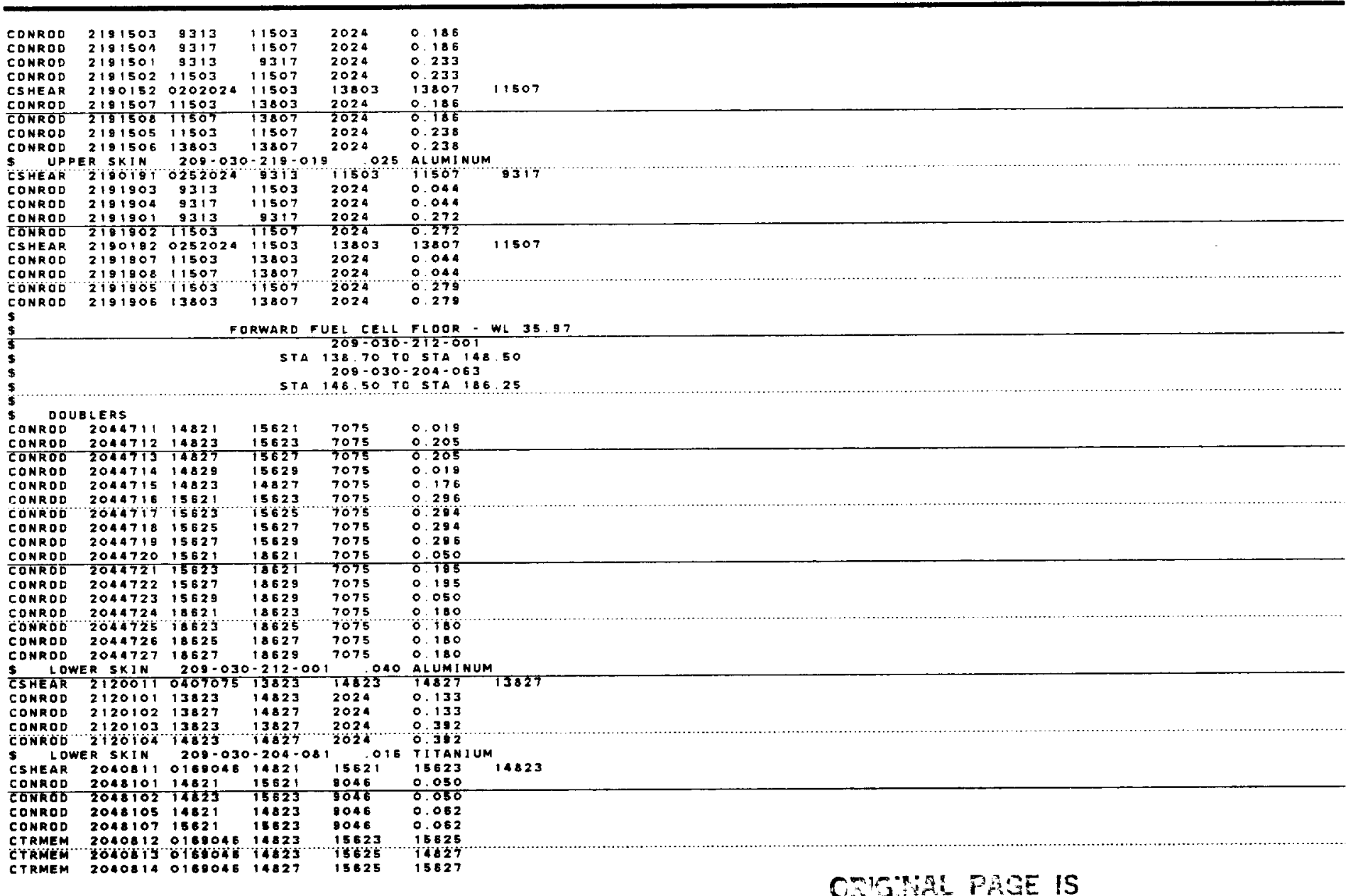




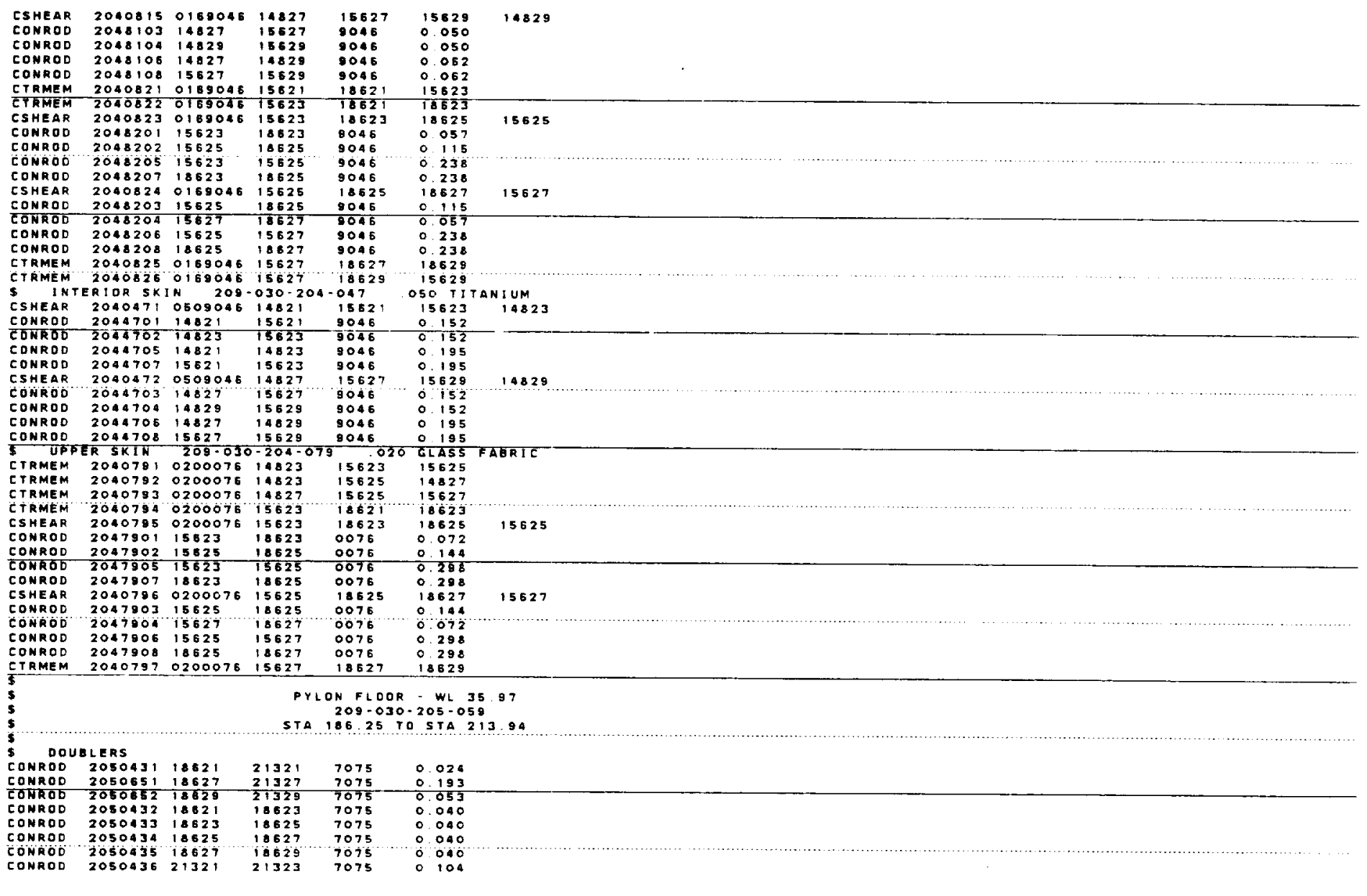

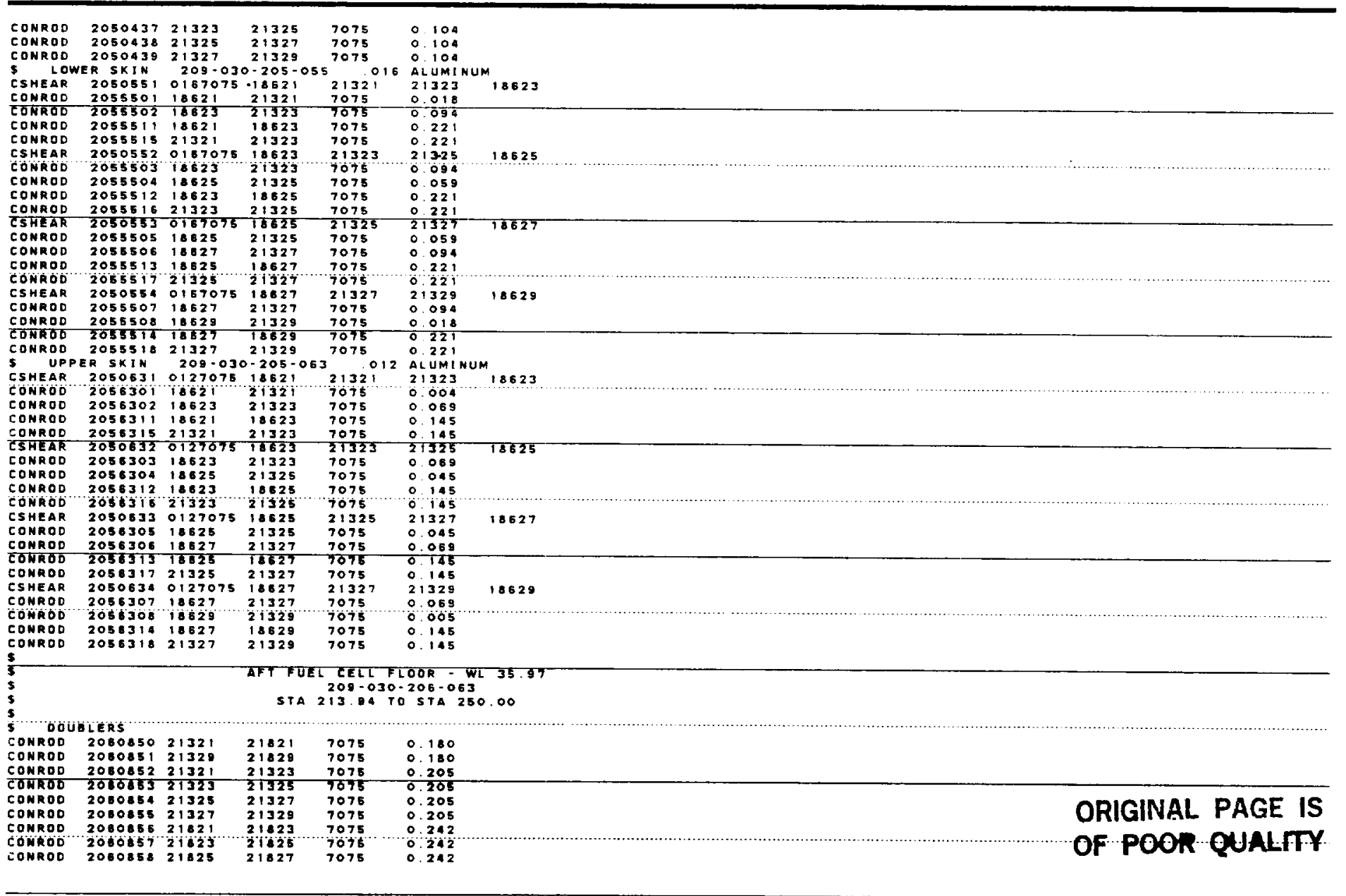




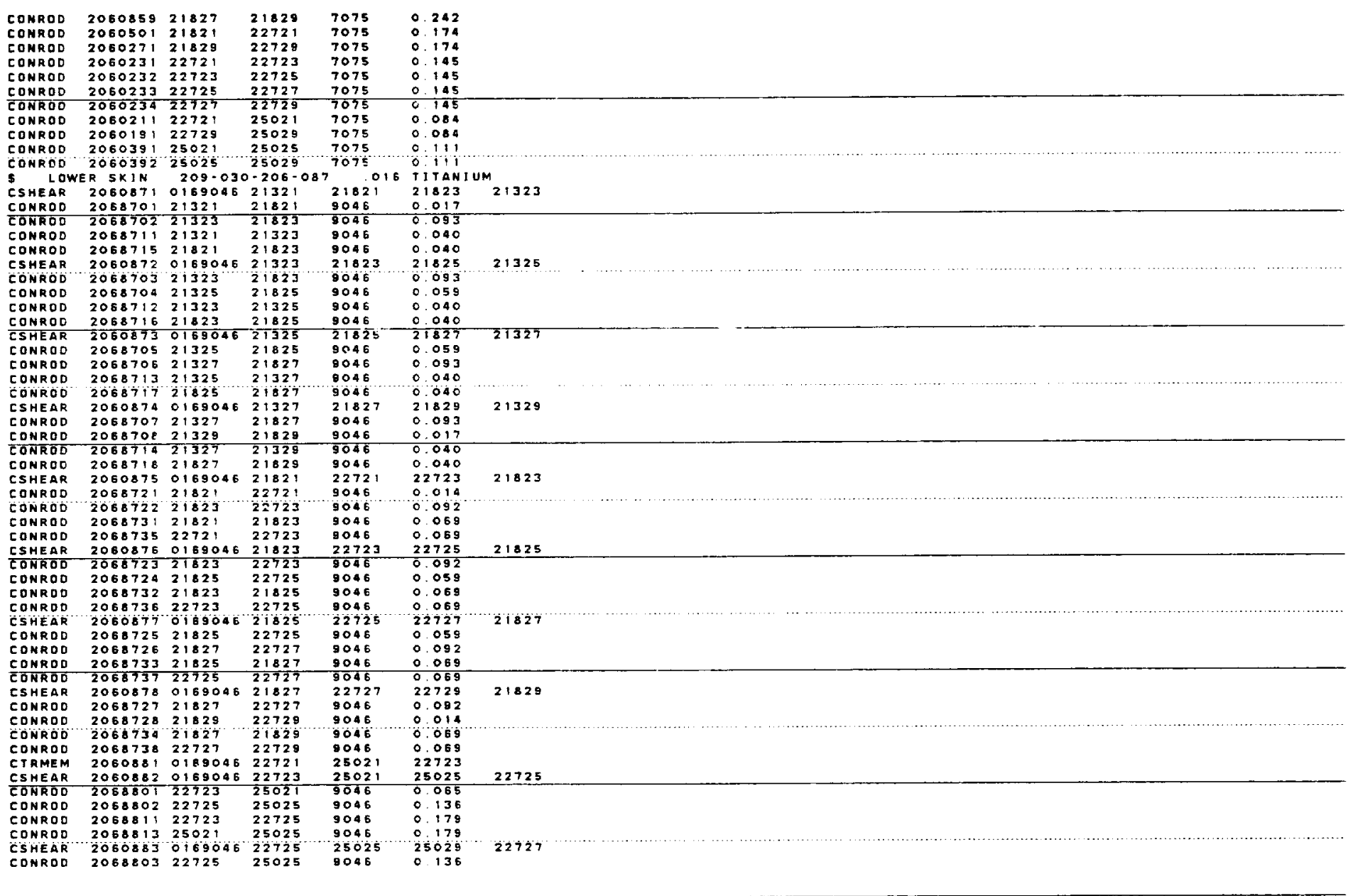

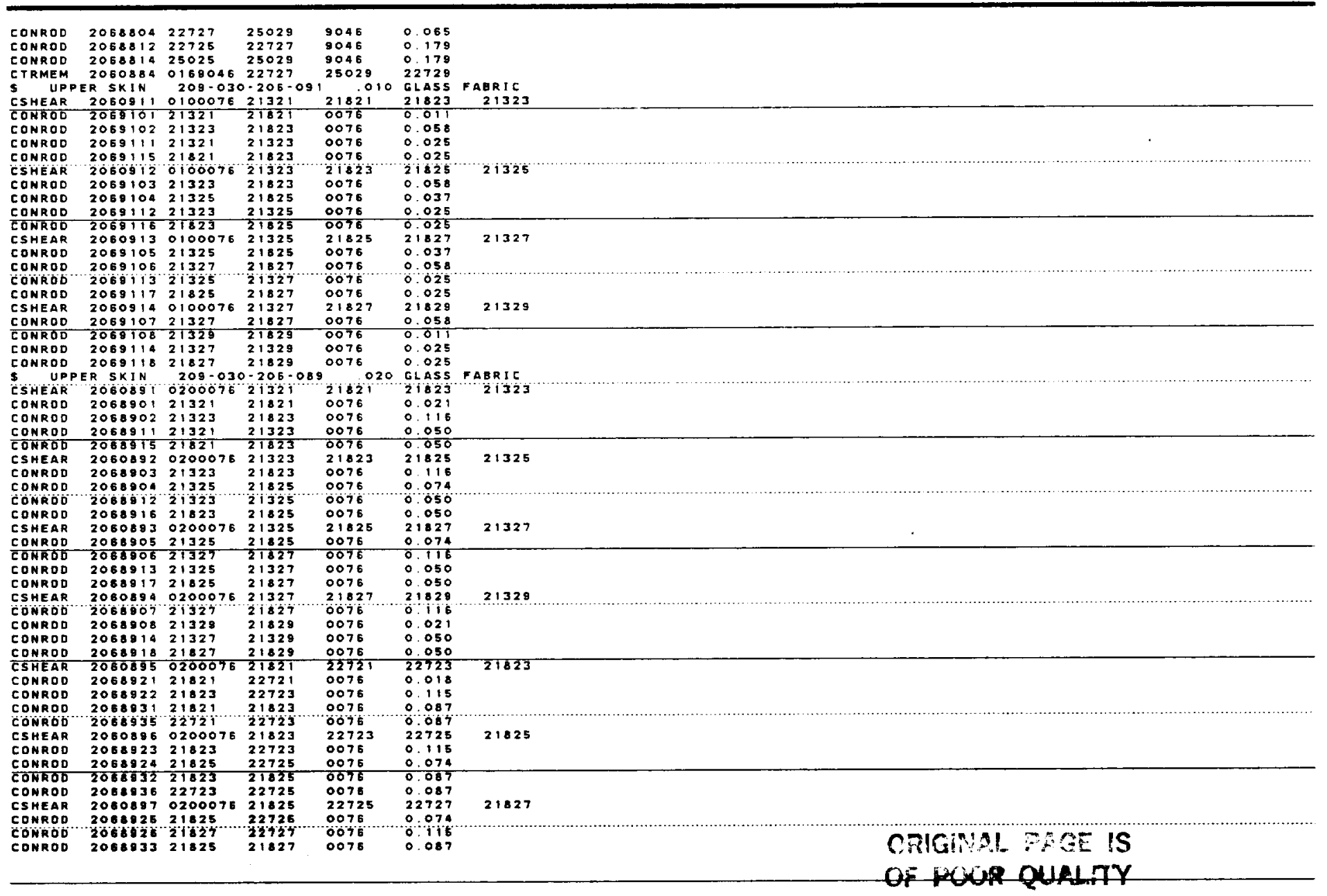




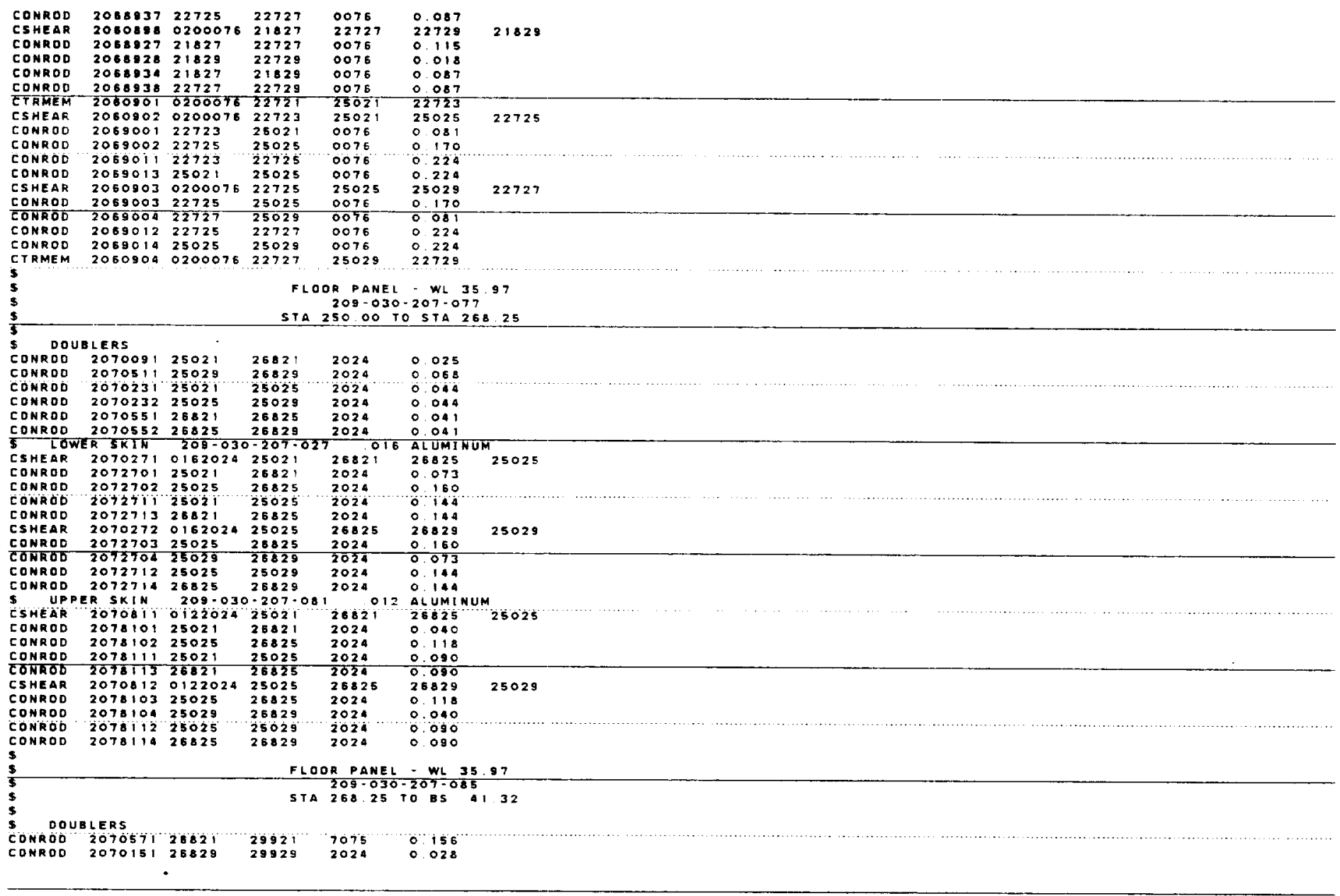

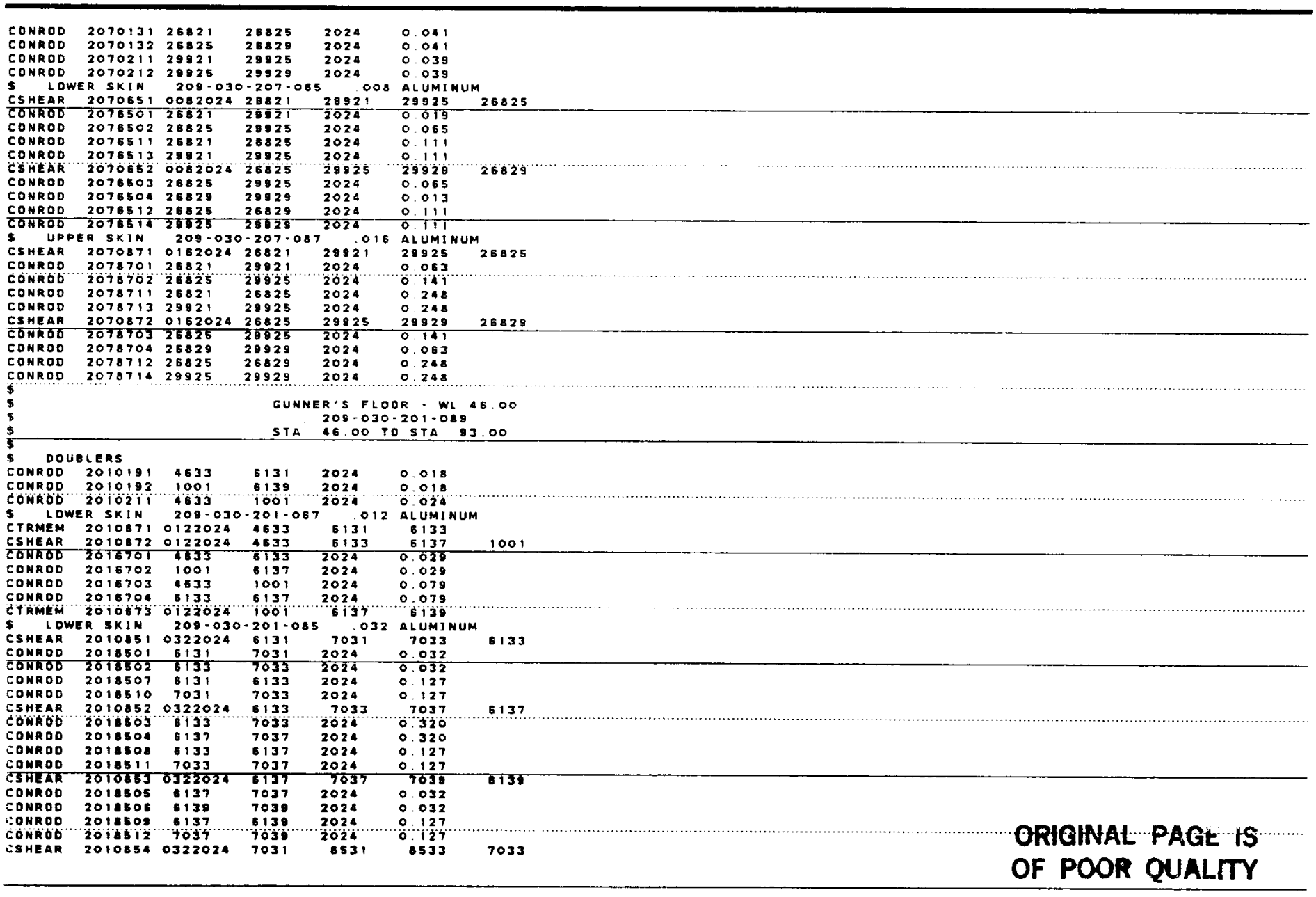




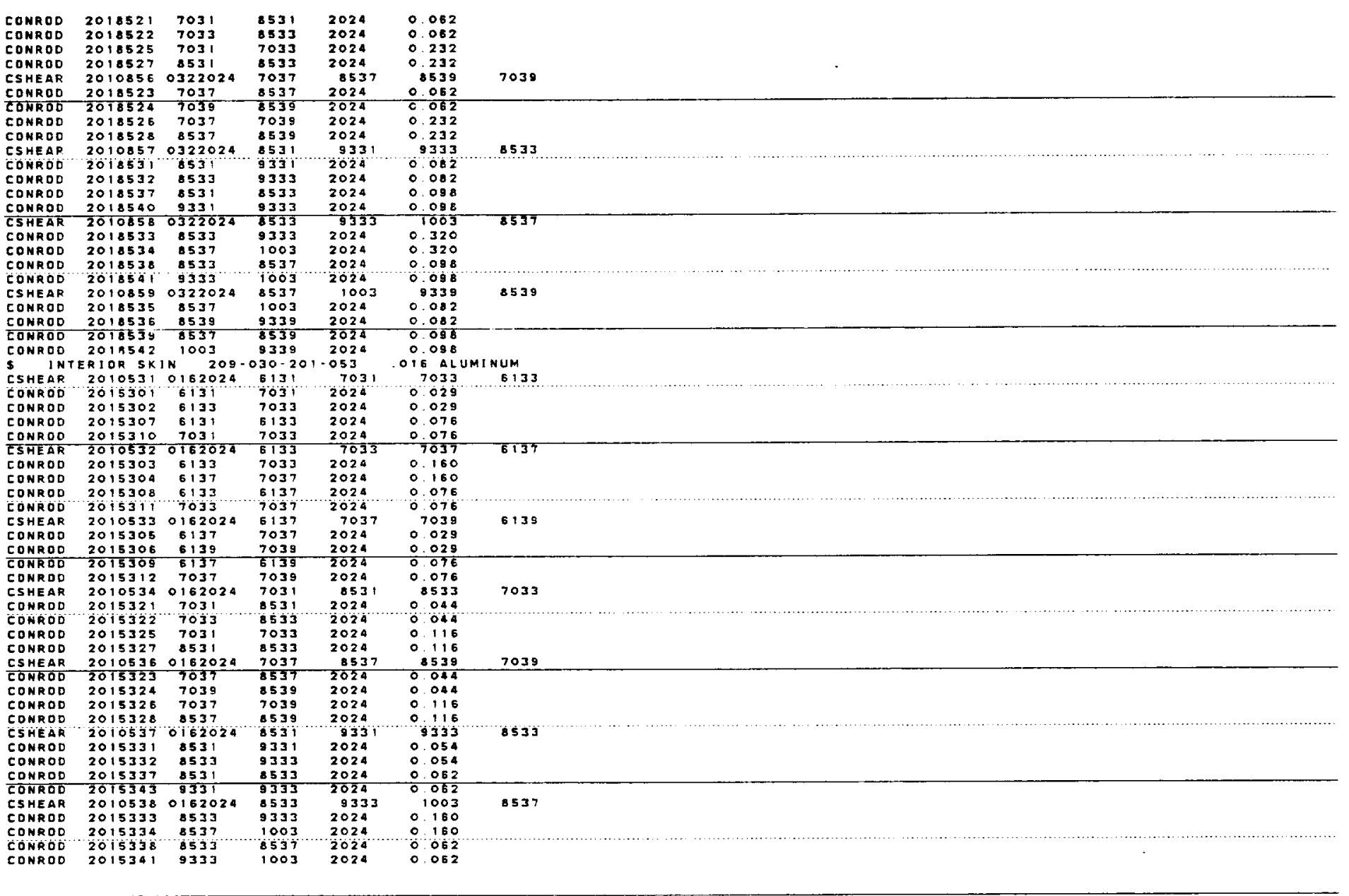

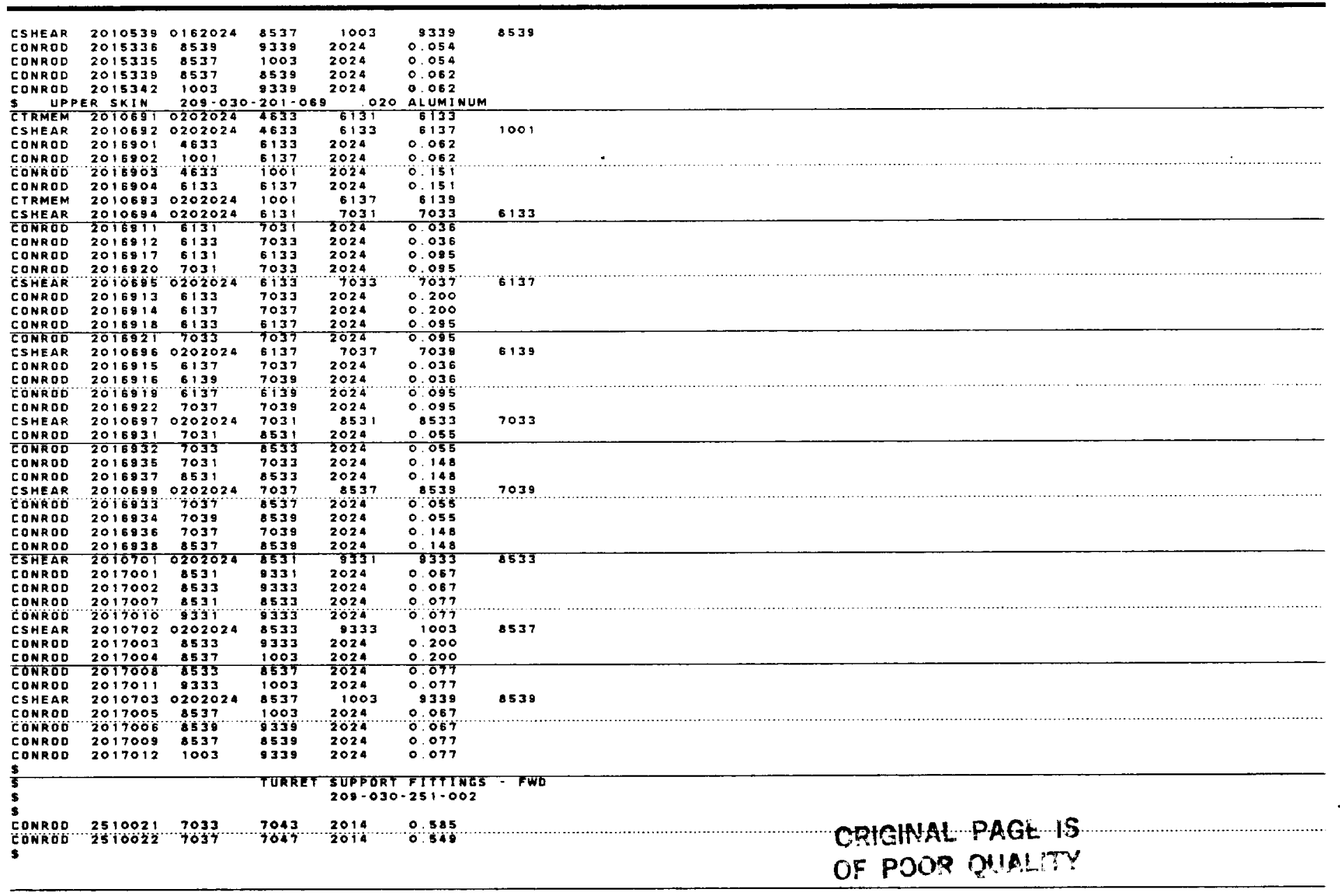




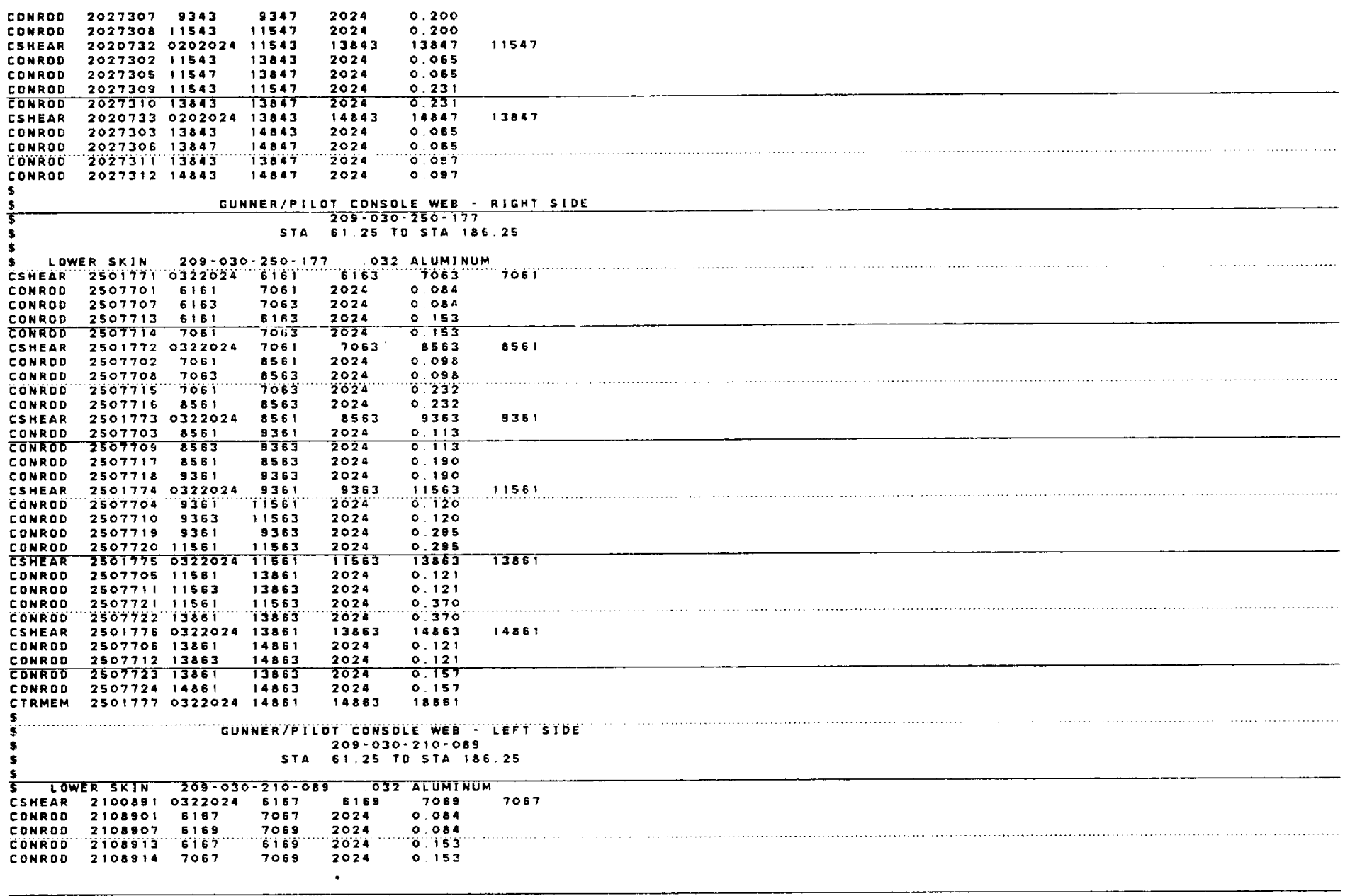

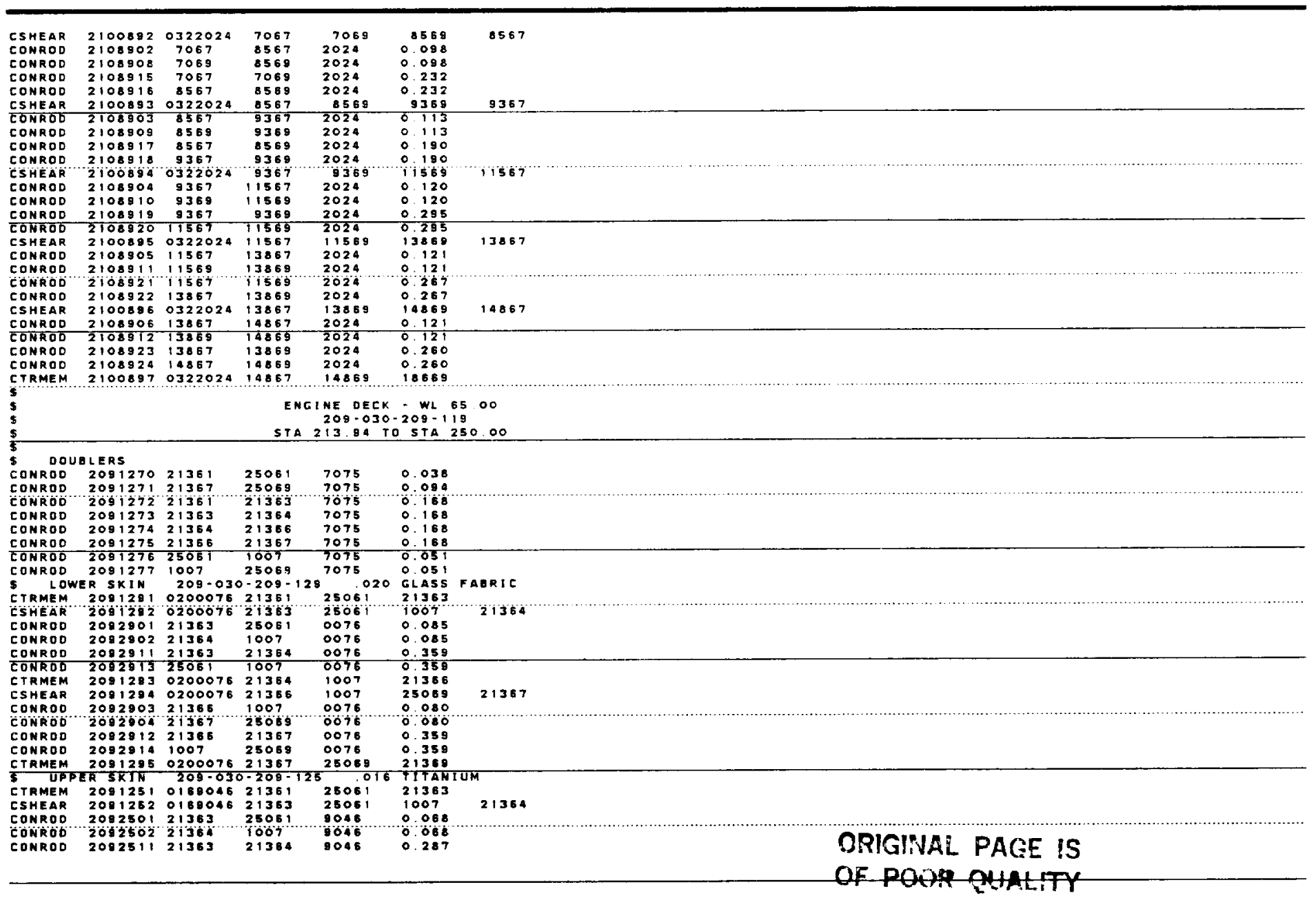




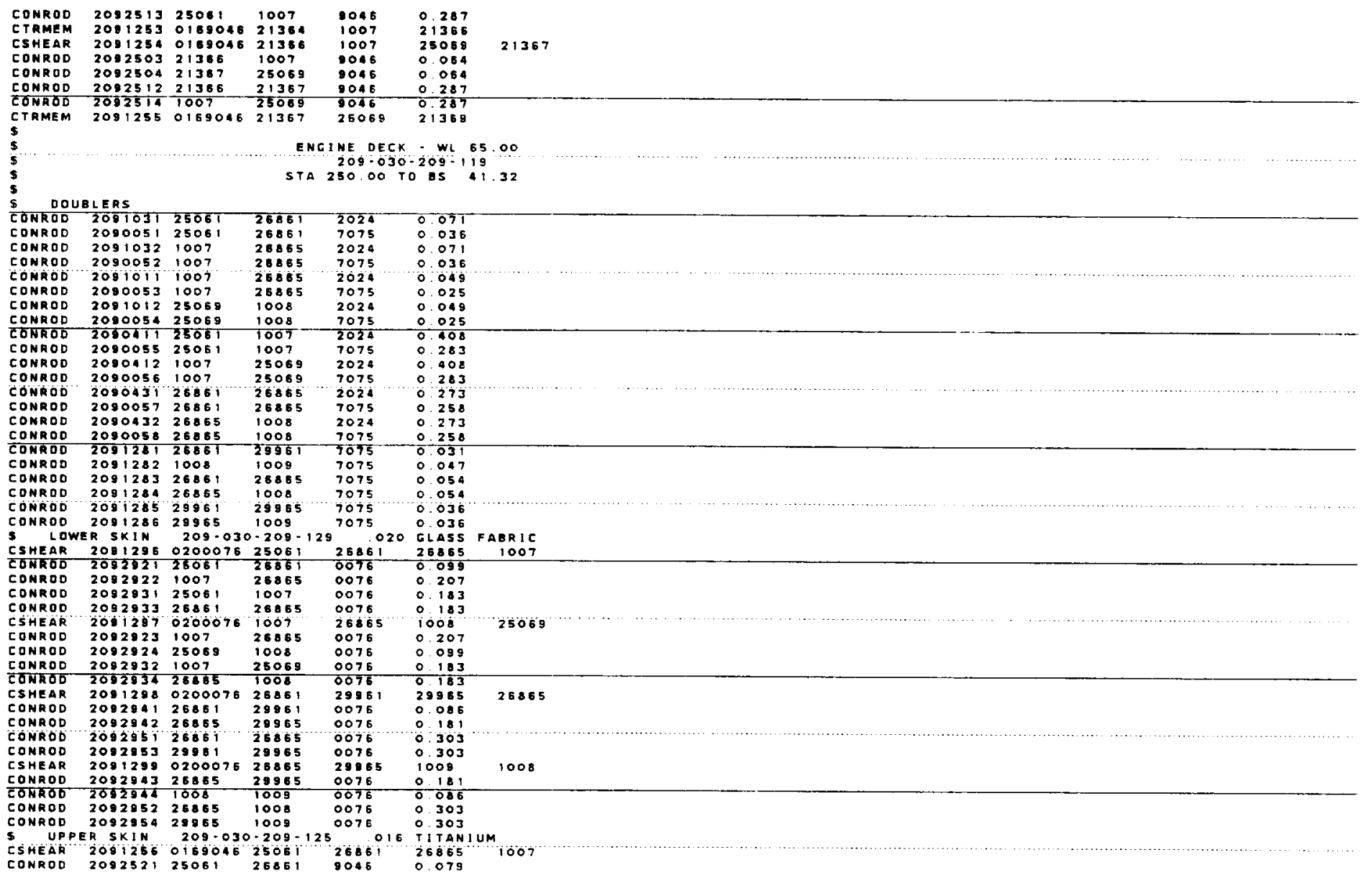

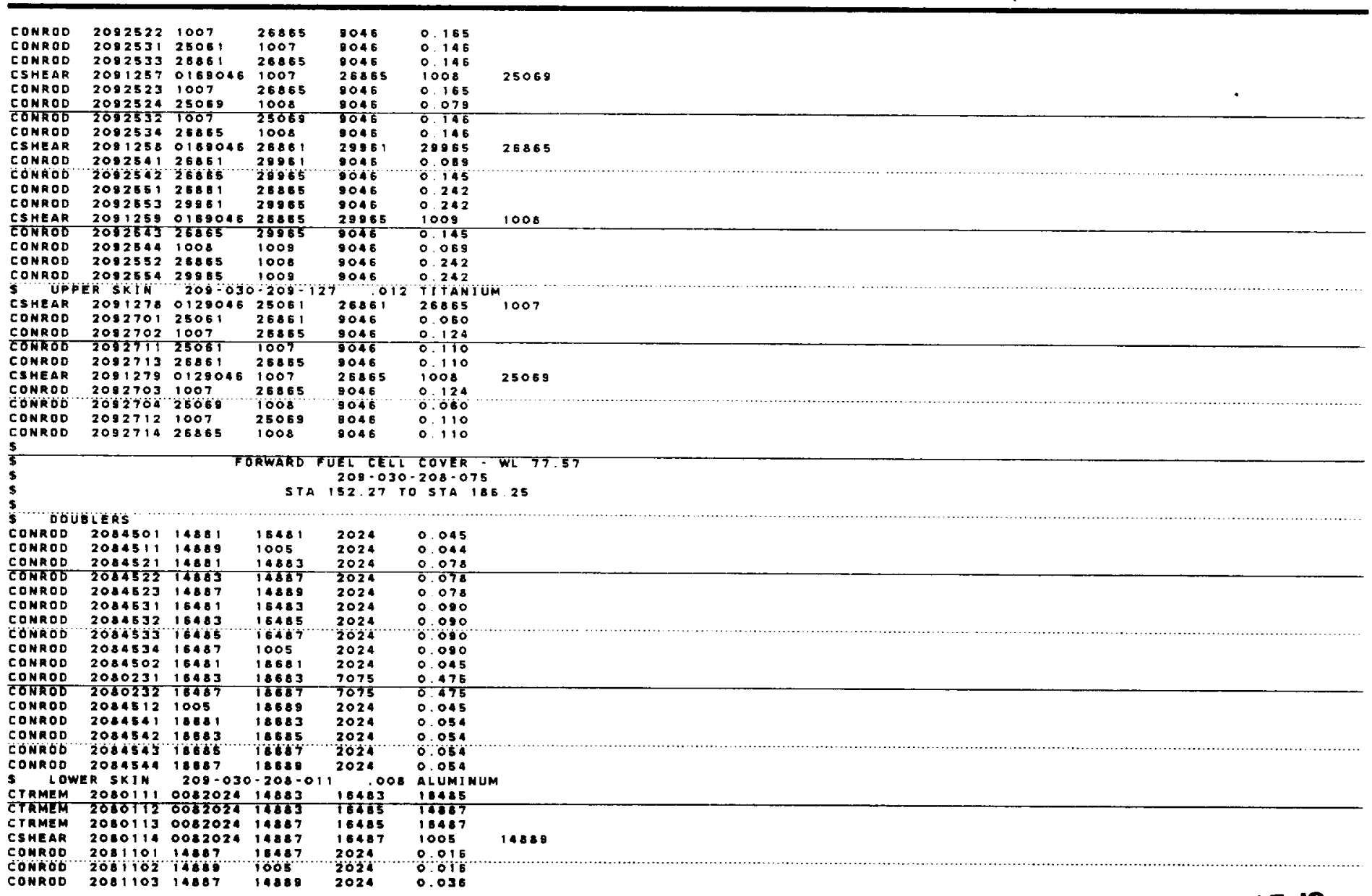




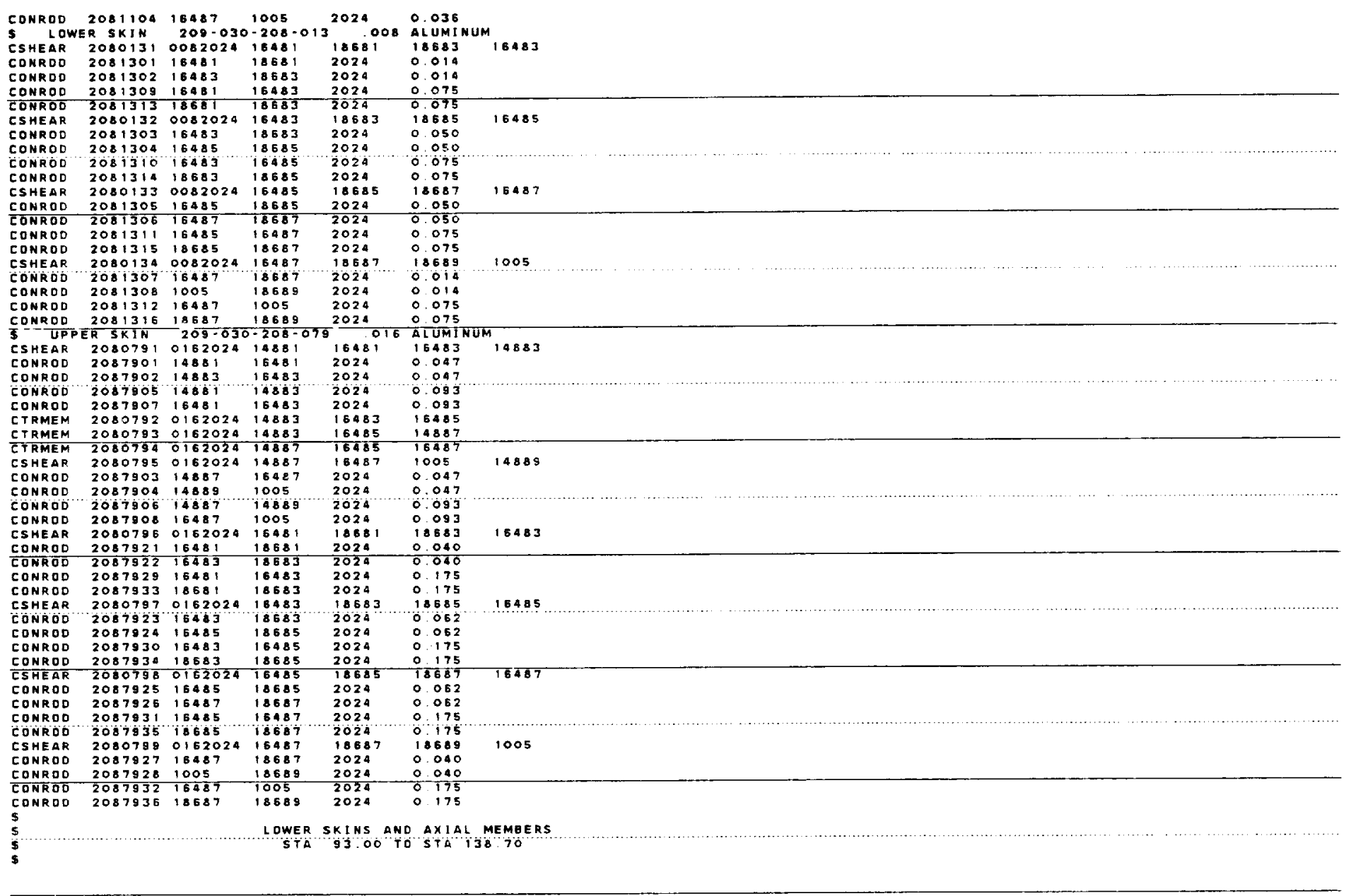

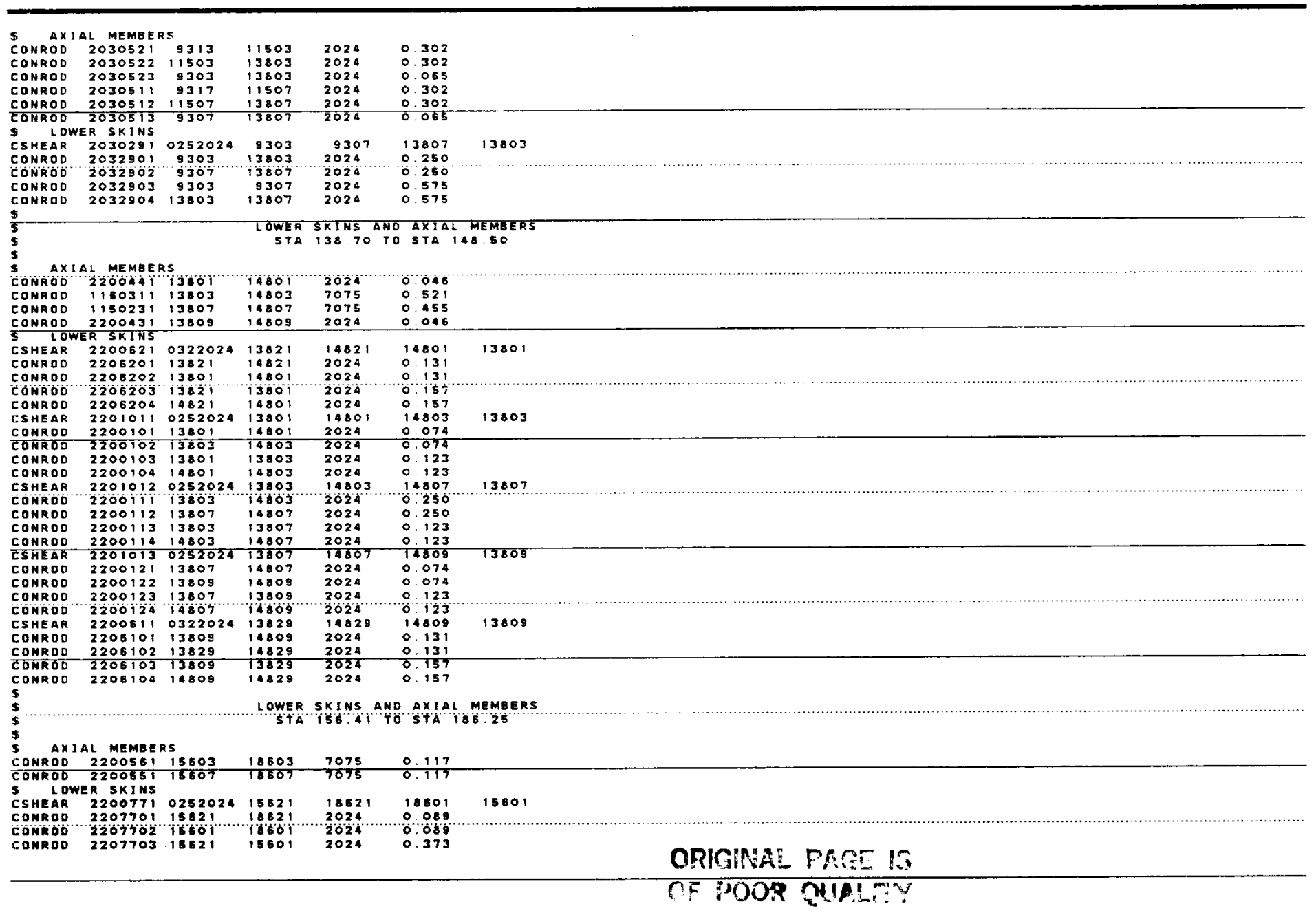




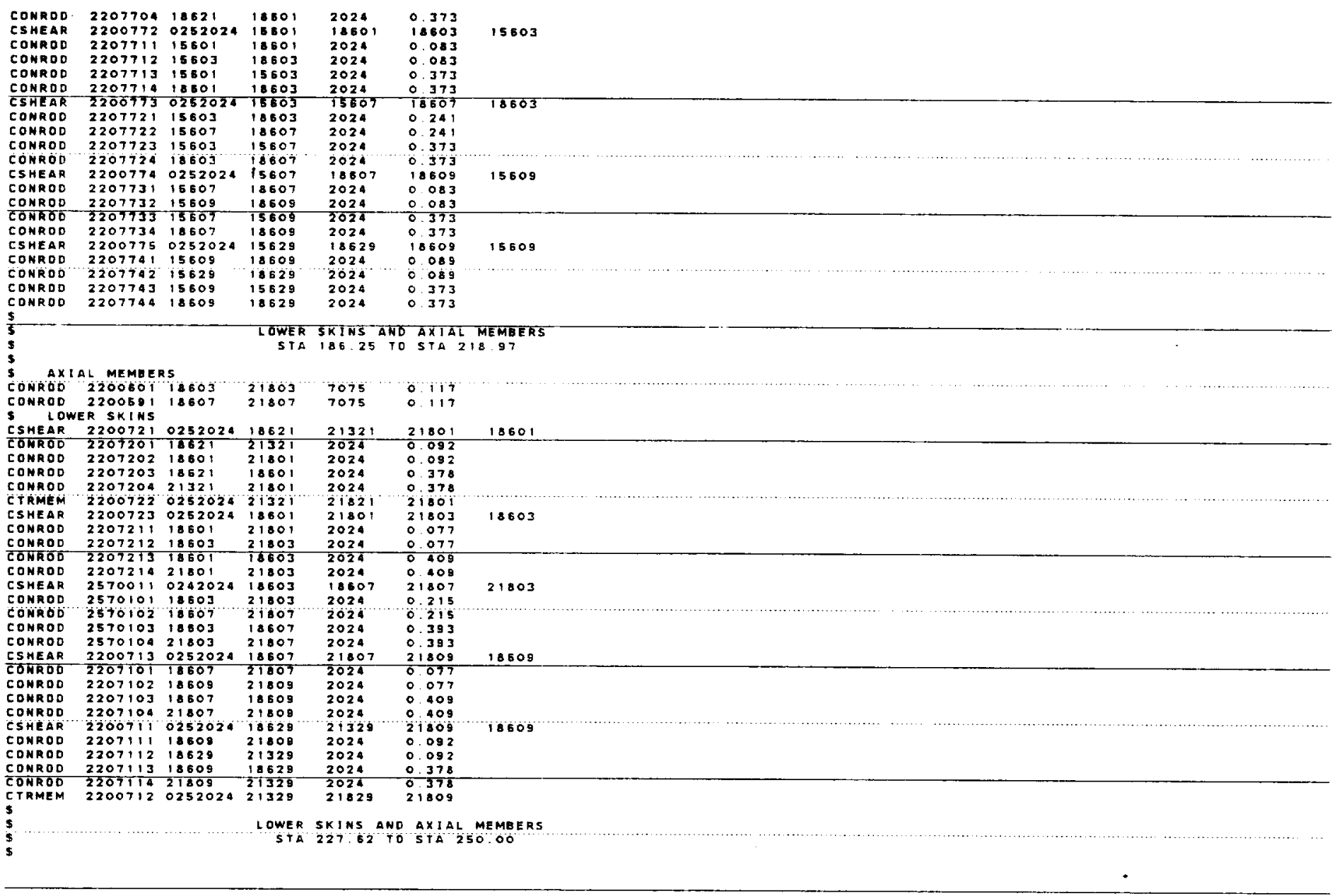

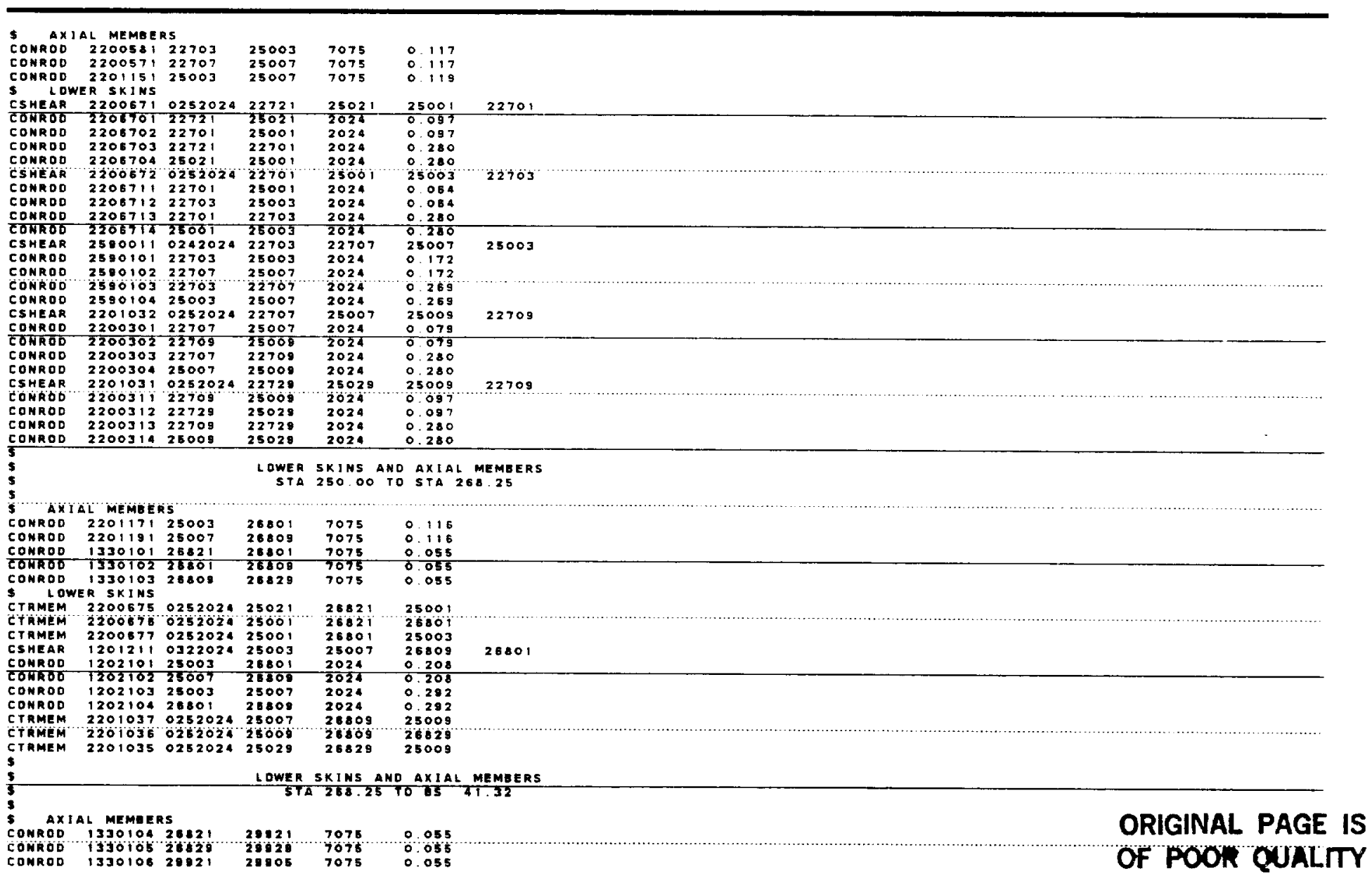




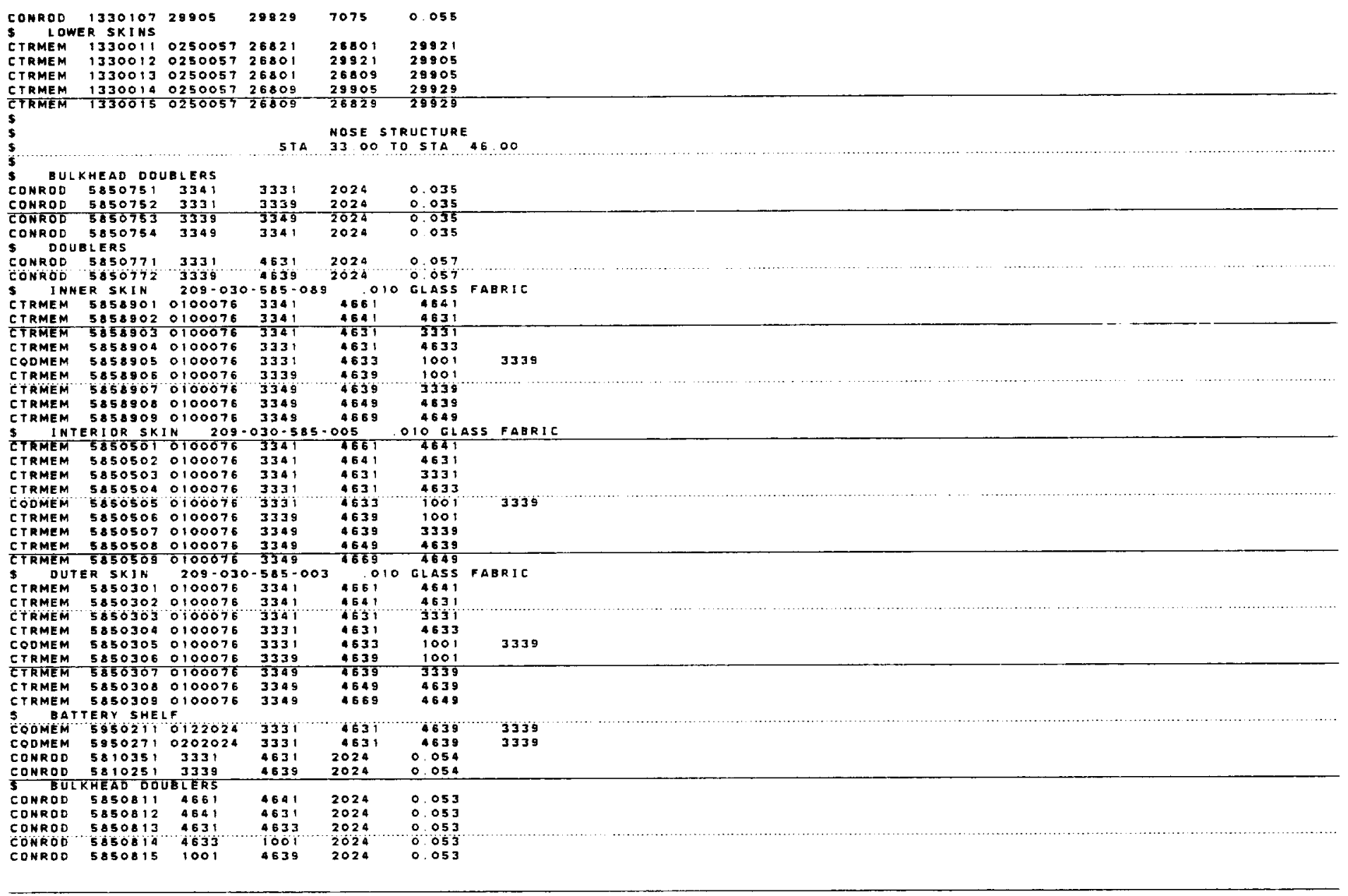

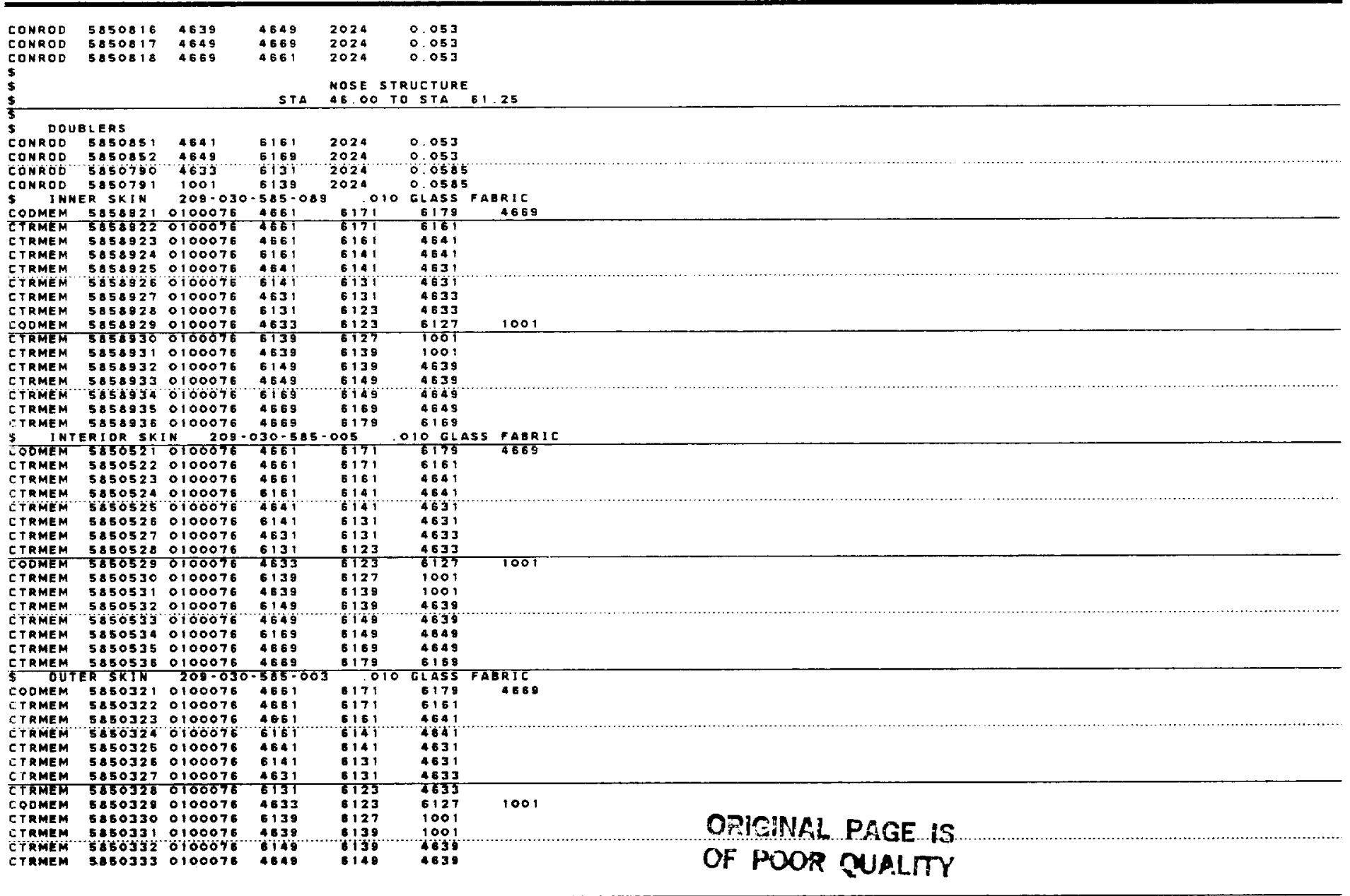




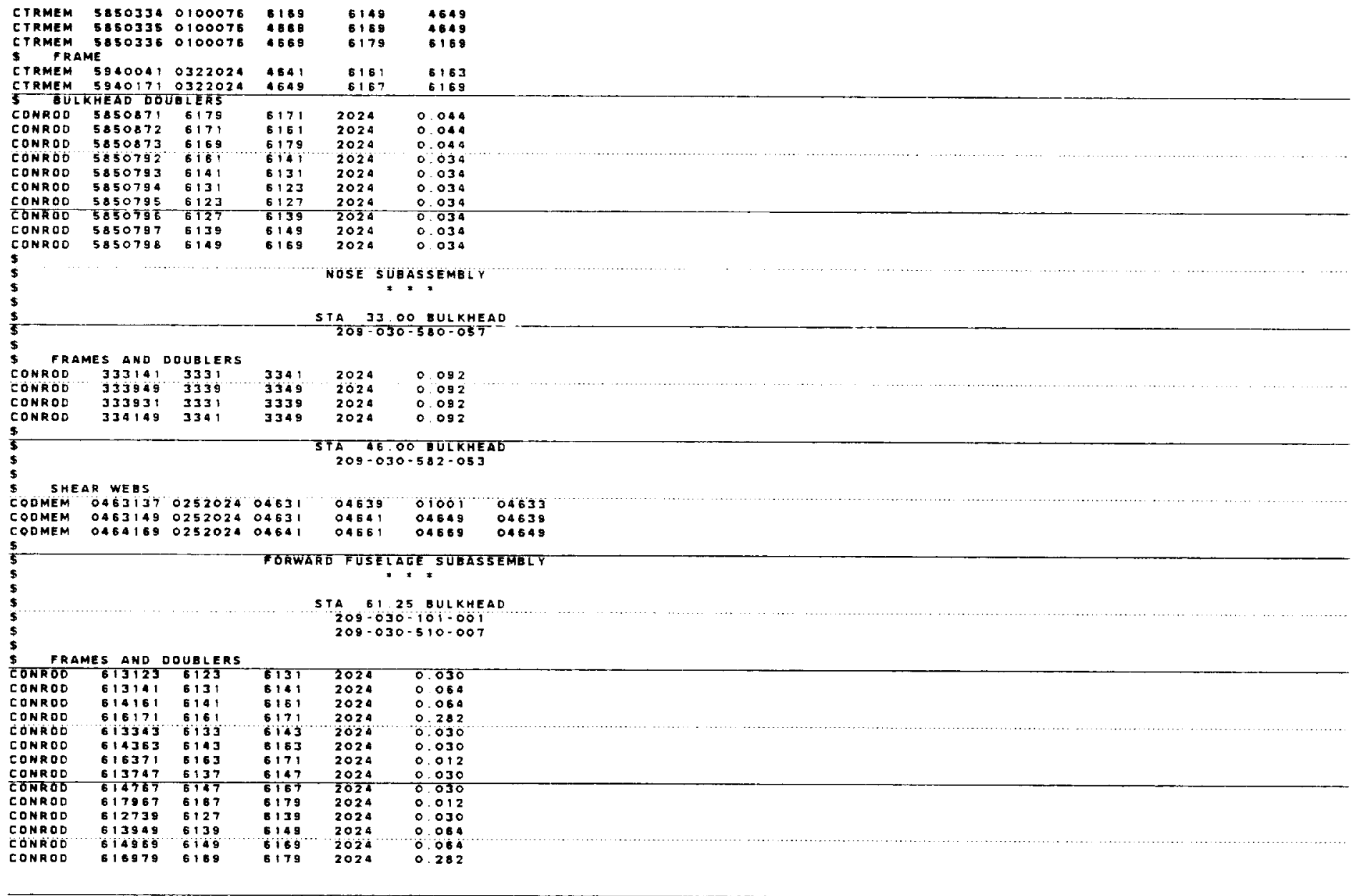

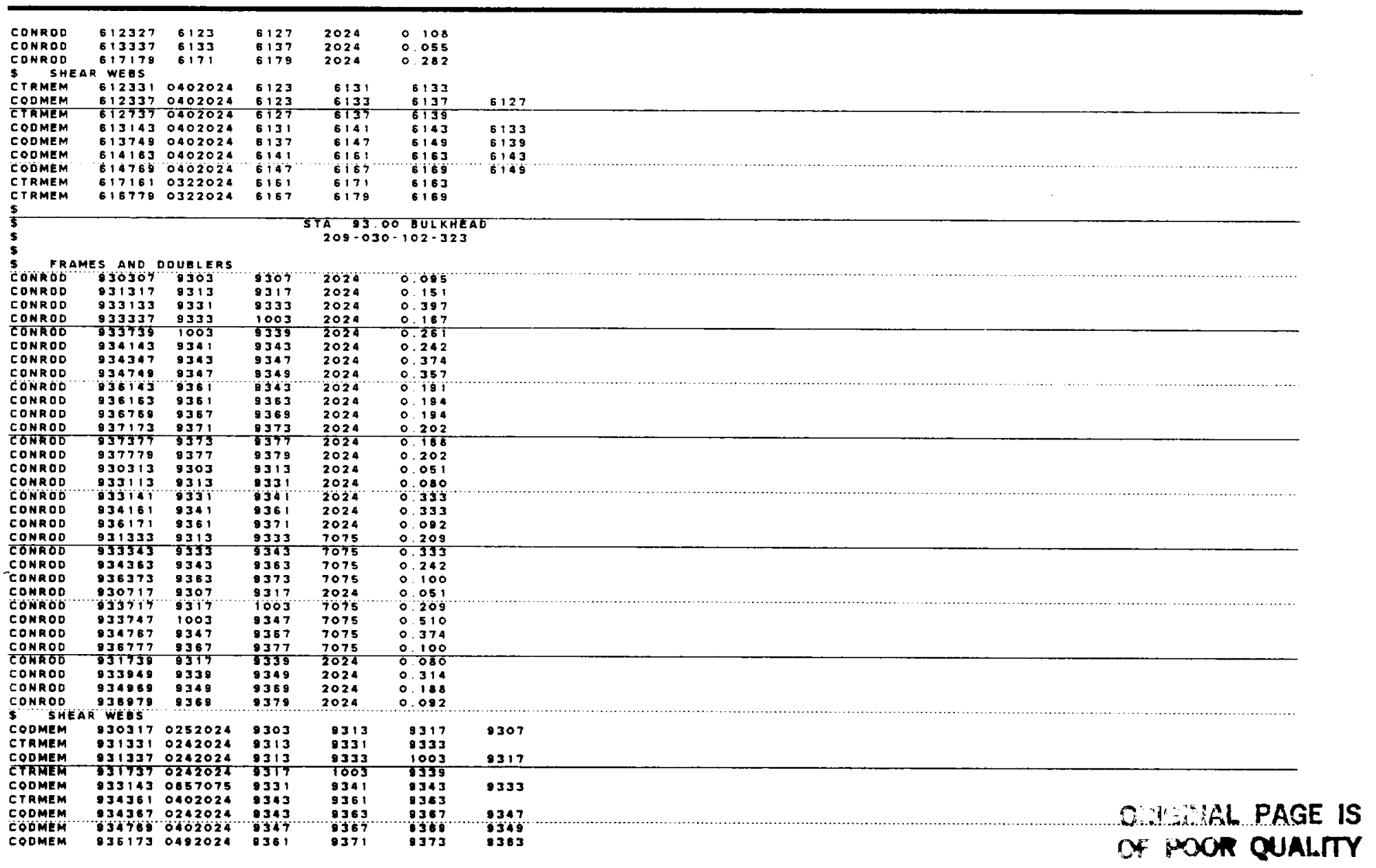




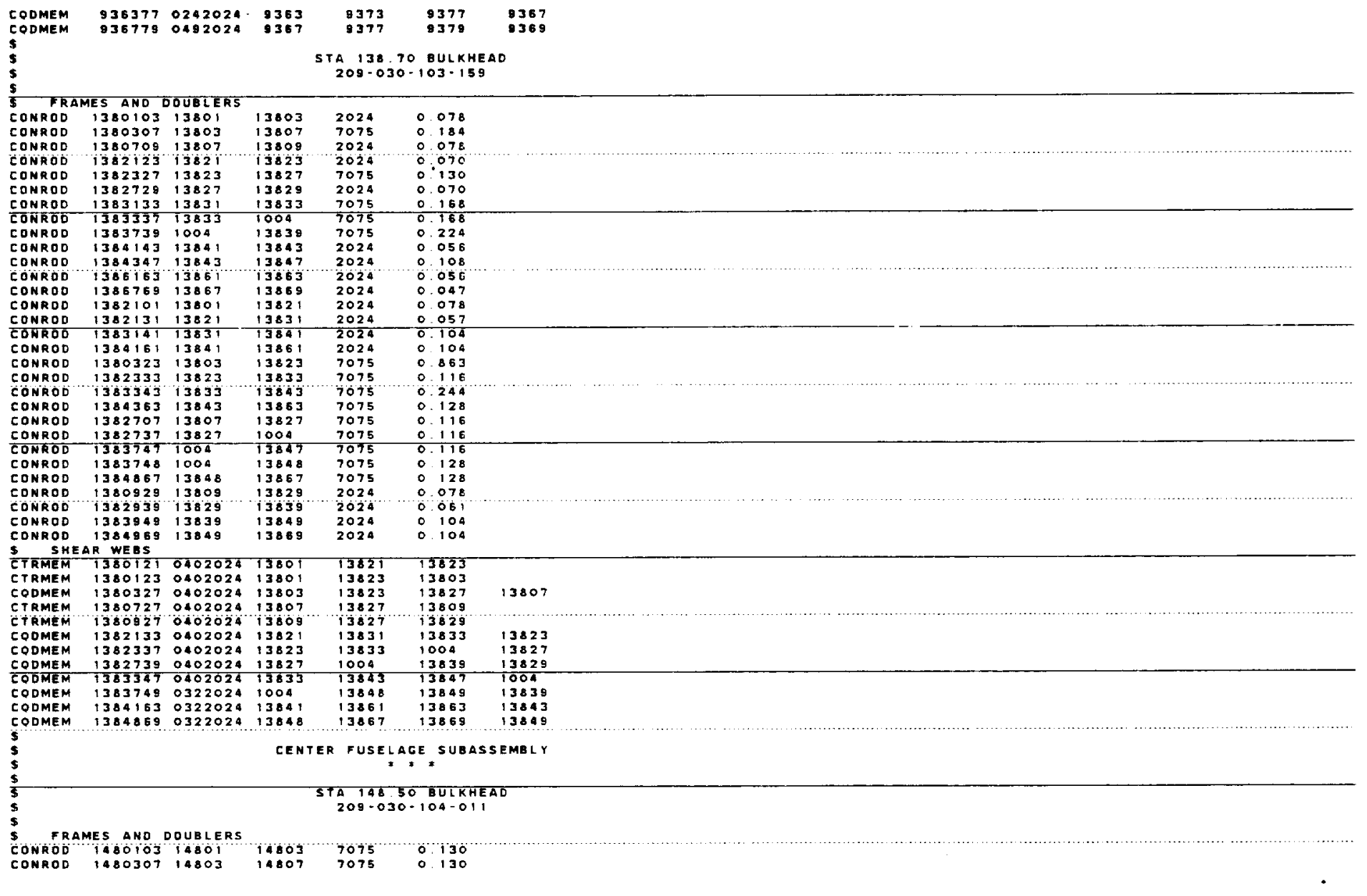

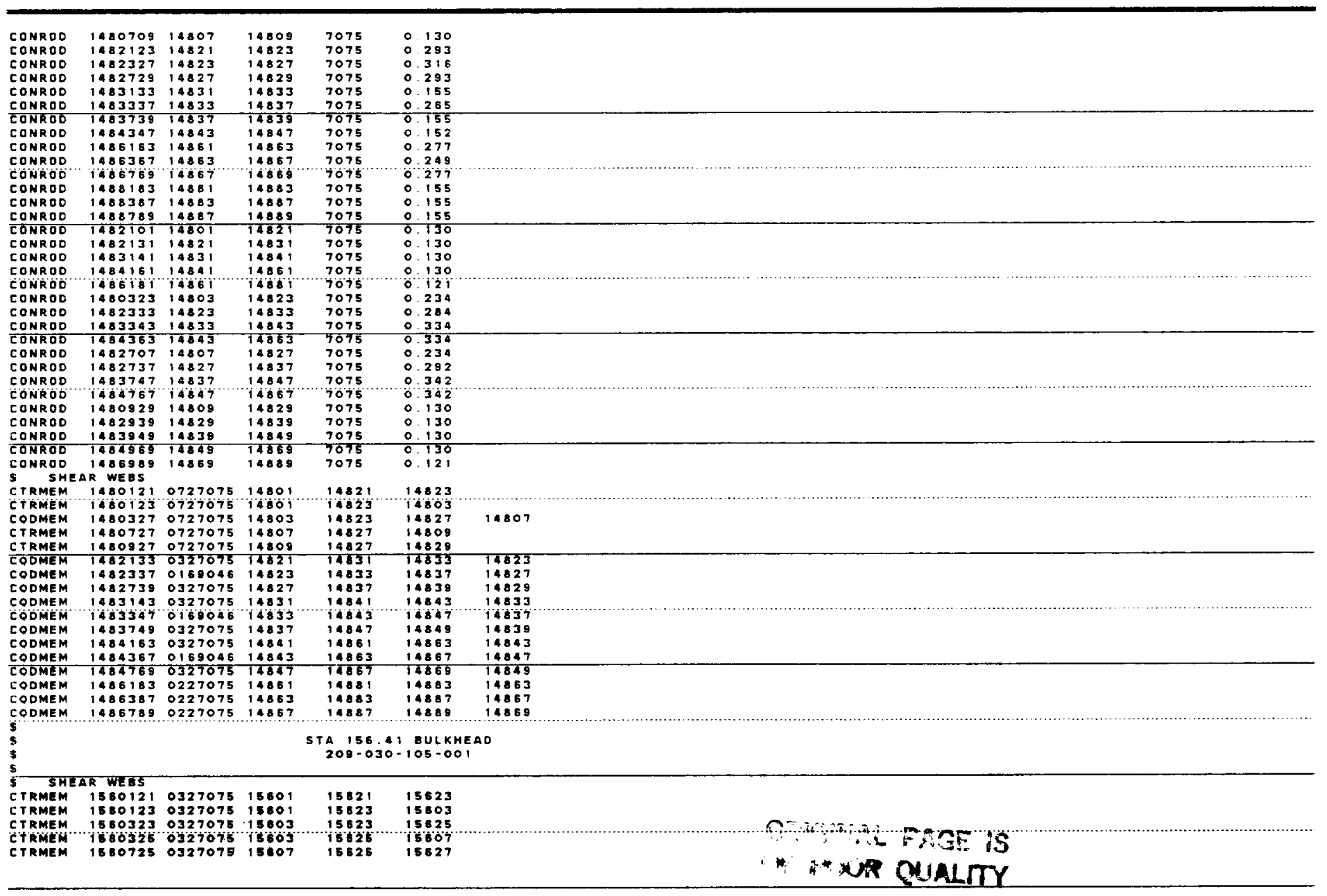




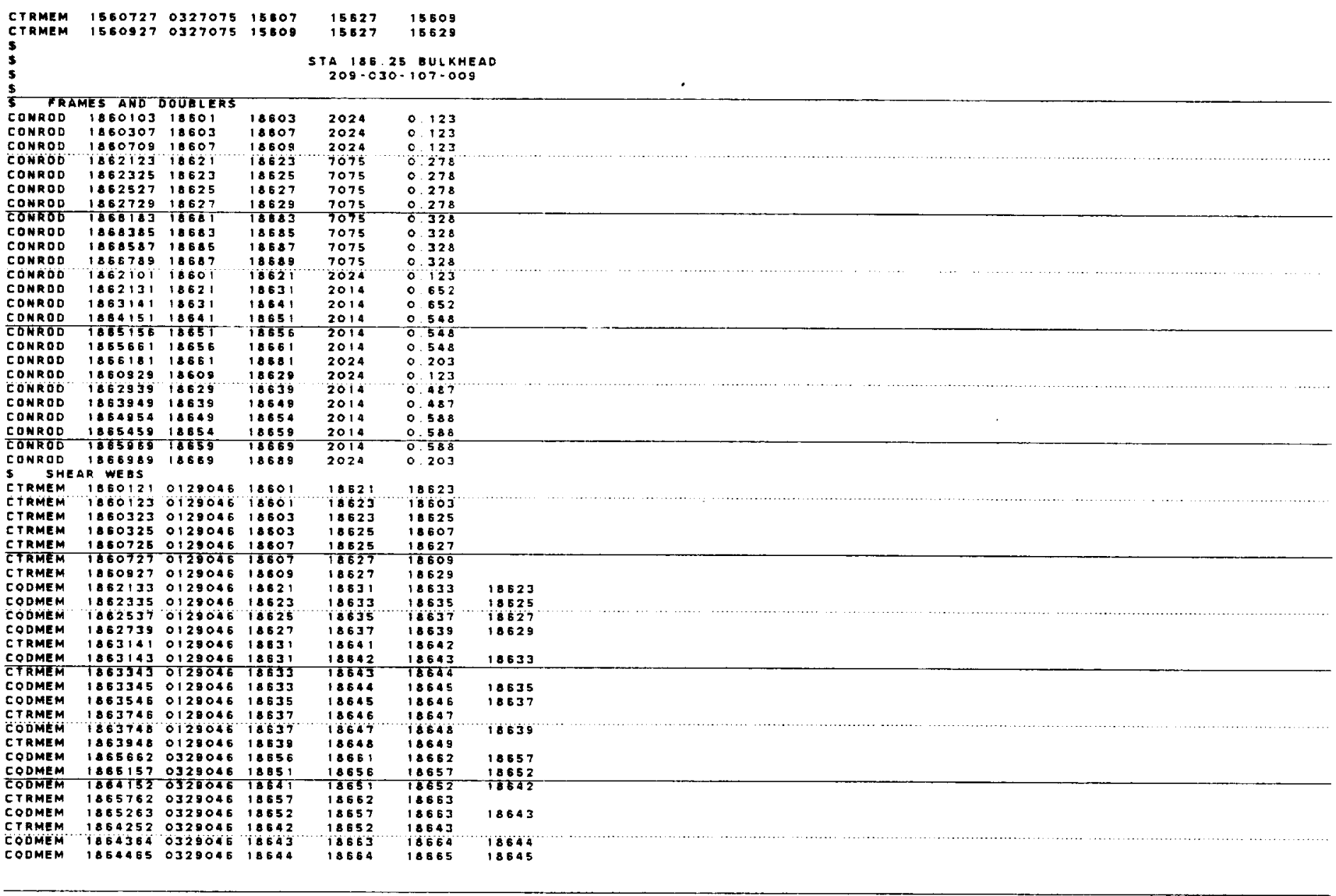

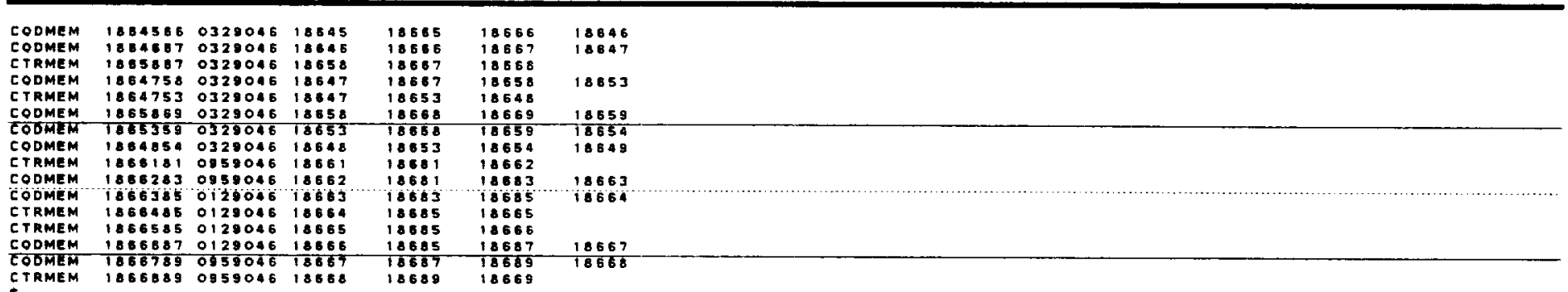

FORWARD WING CARRY-THAU SPAR

FRAMES and DoUELERS

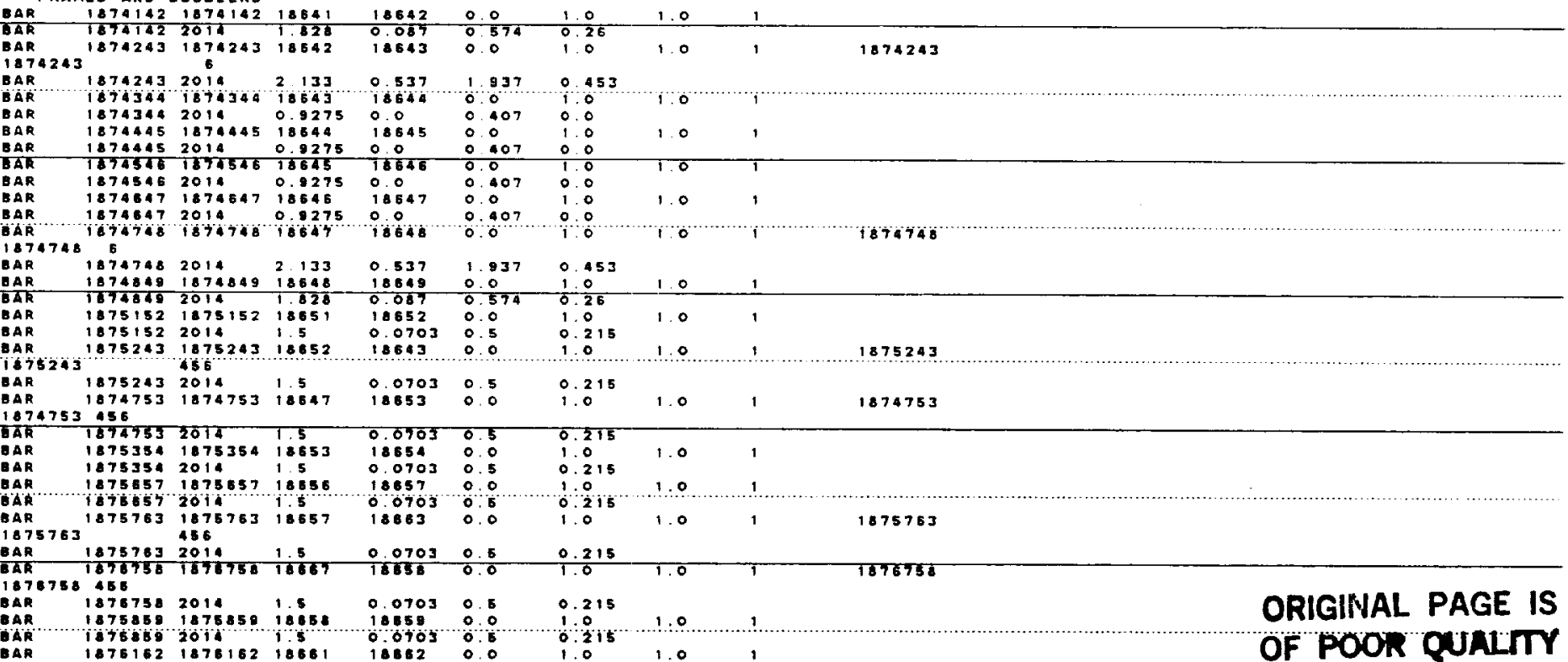




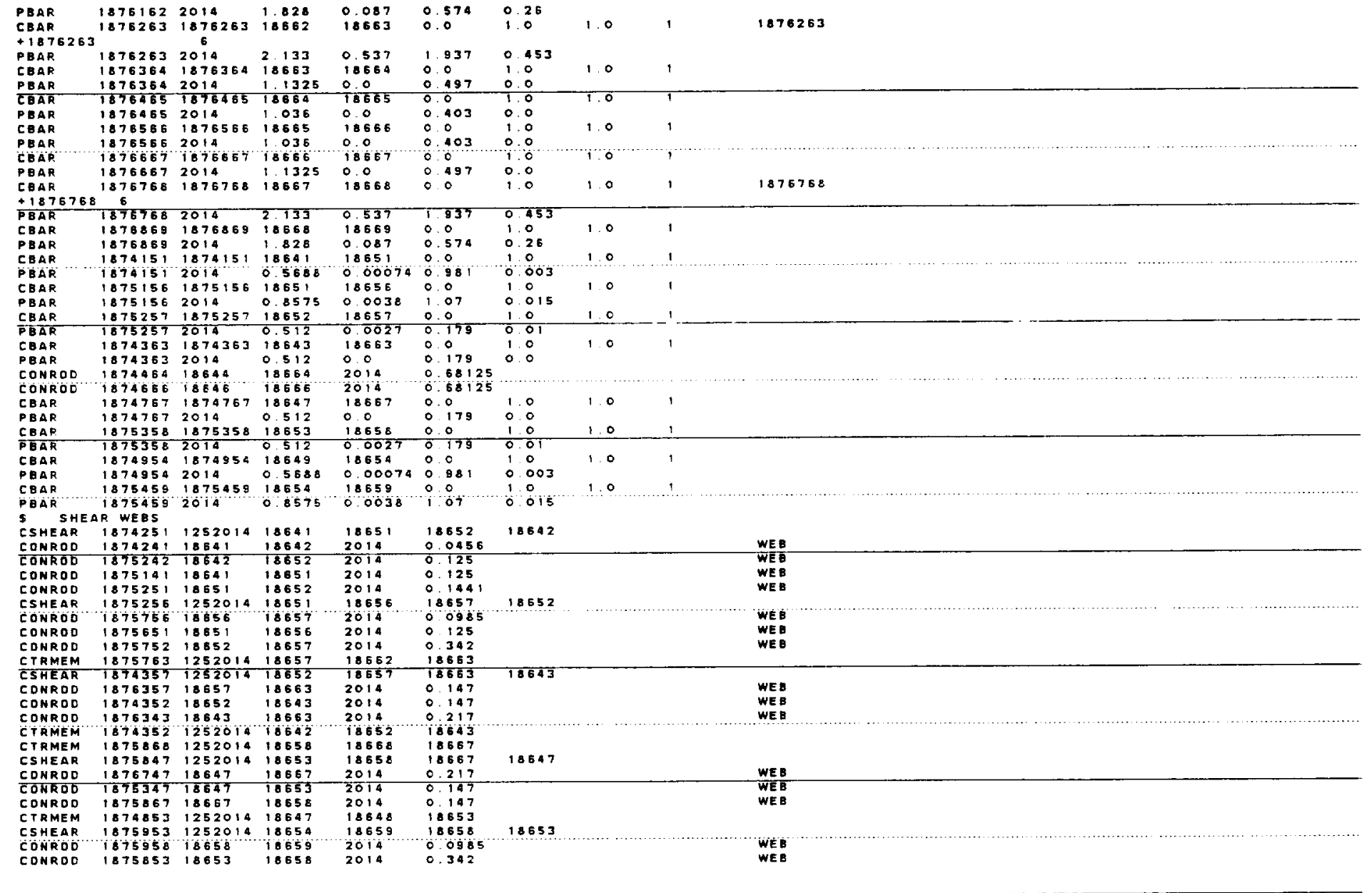

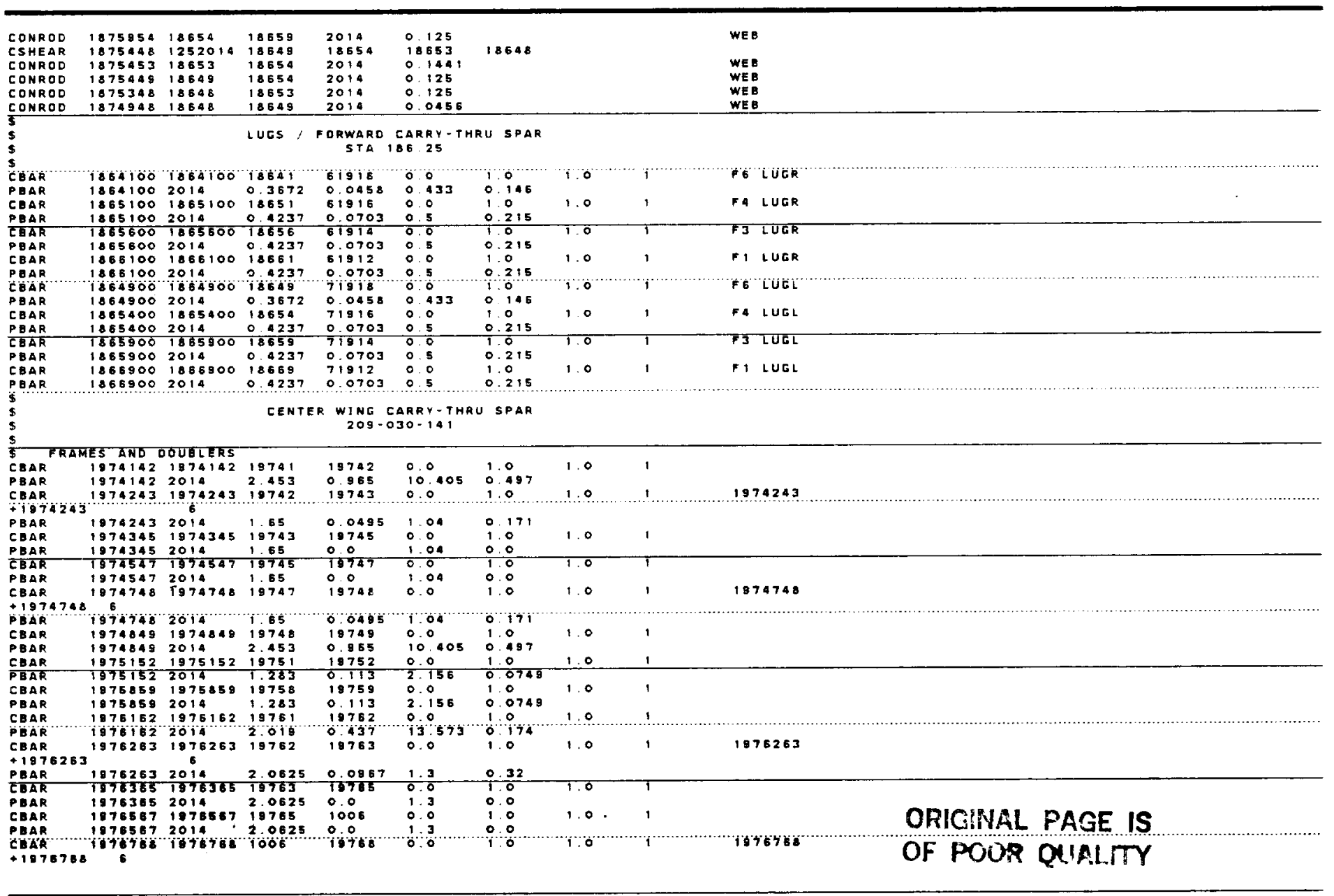




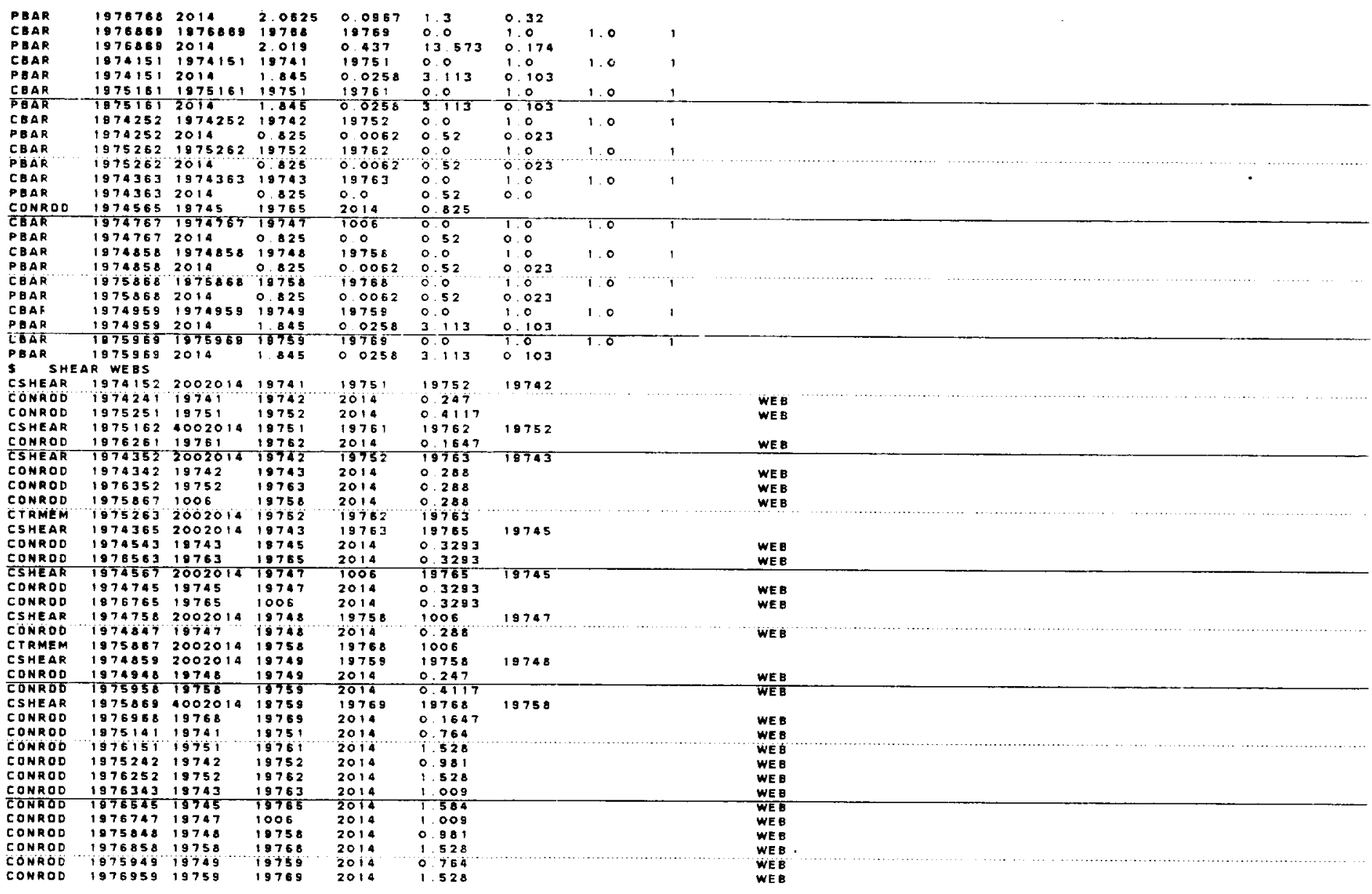

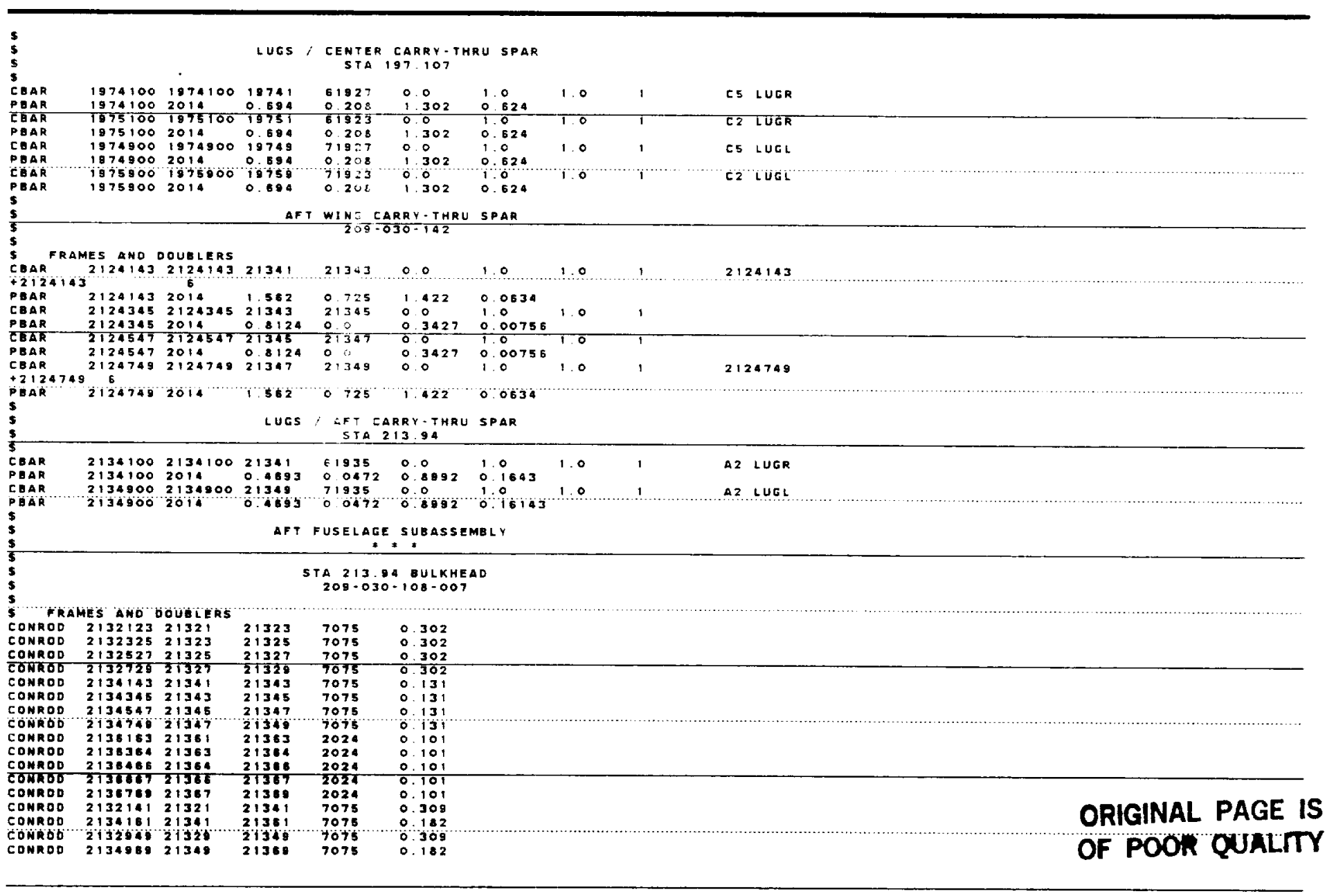


5 SHEAR WEBS

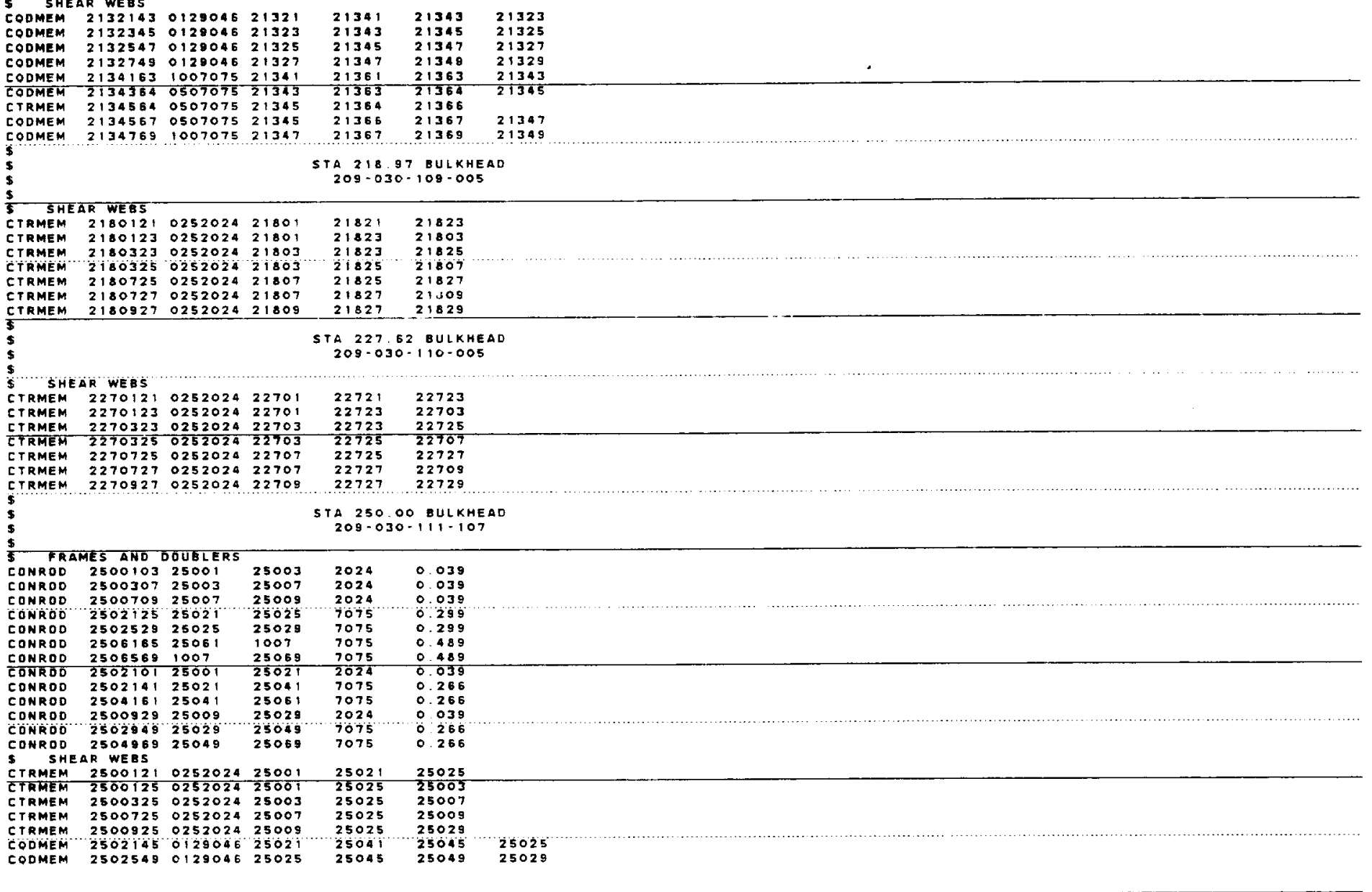

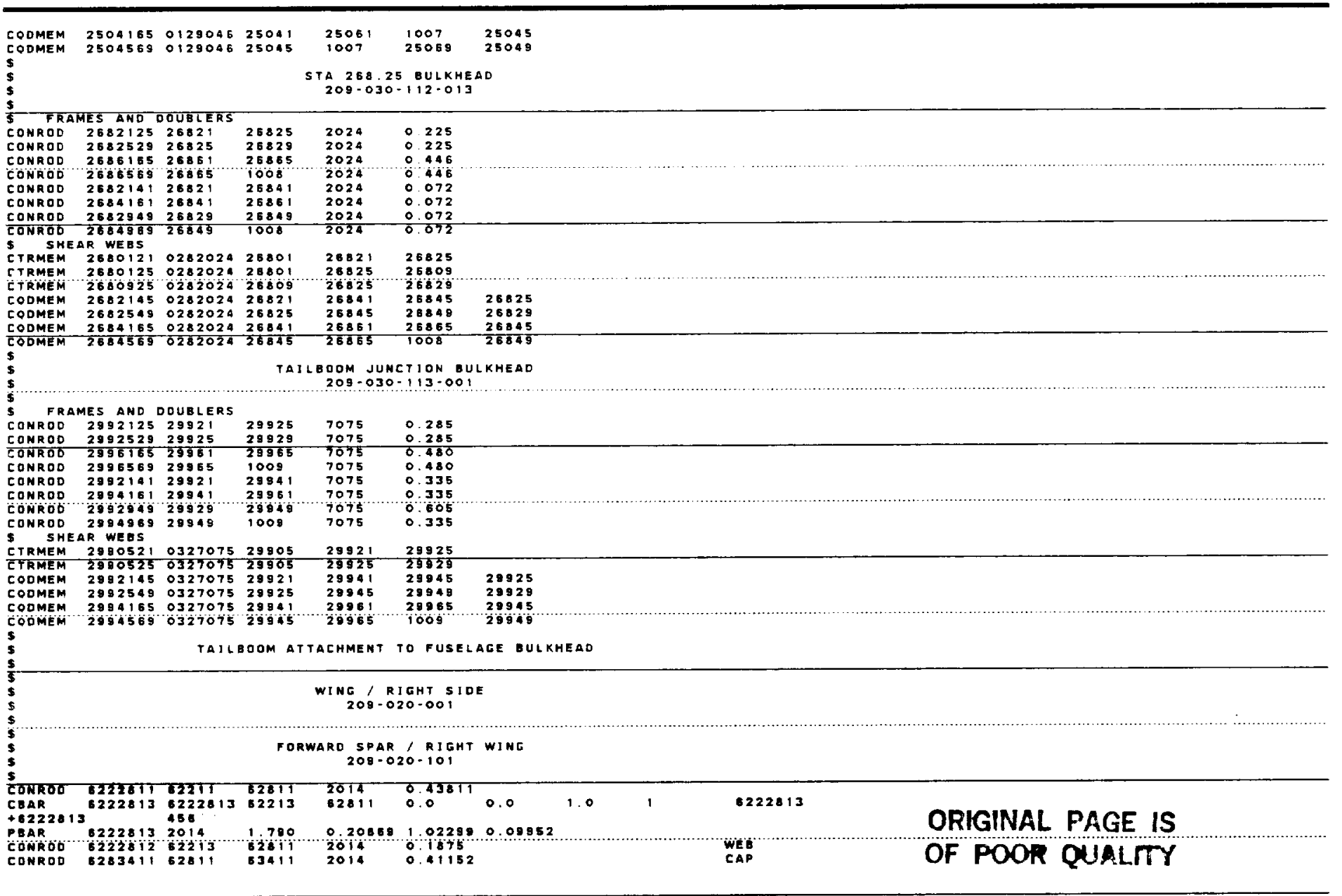




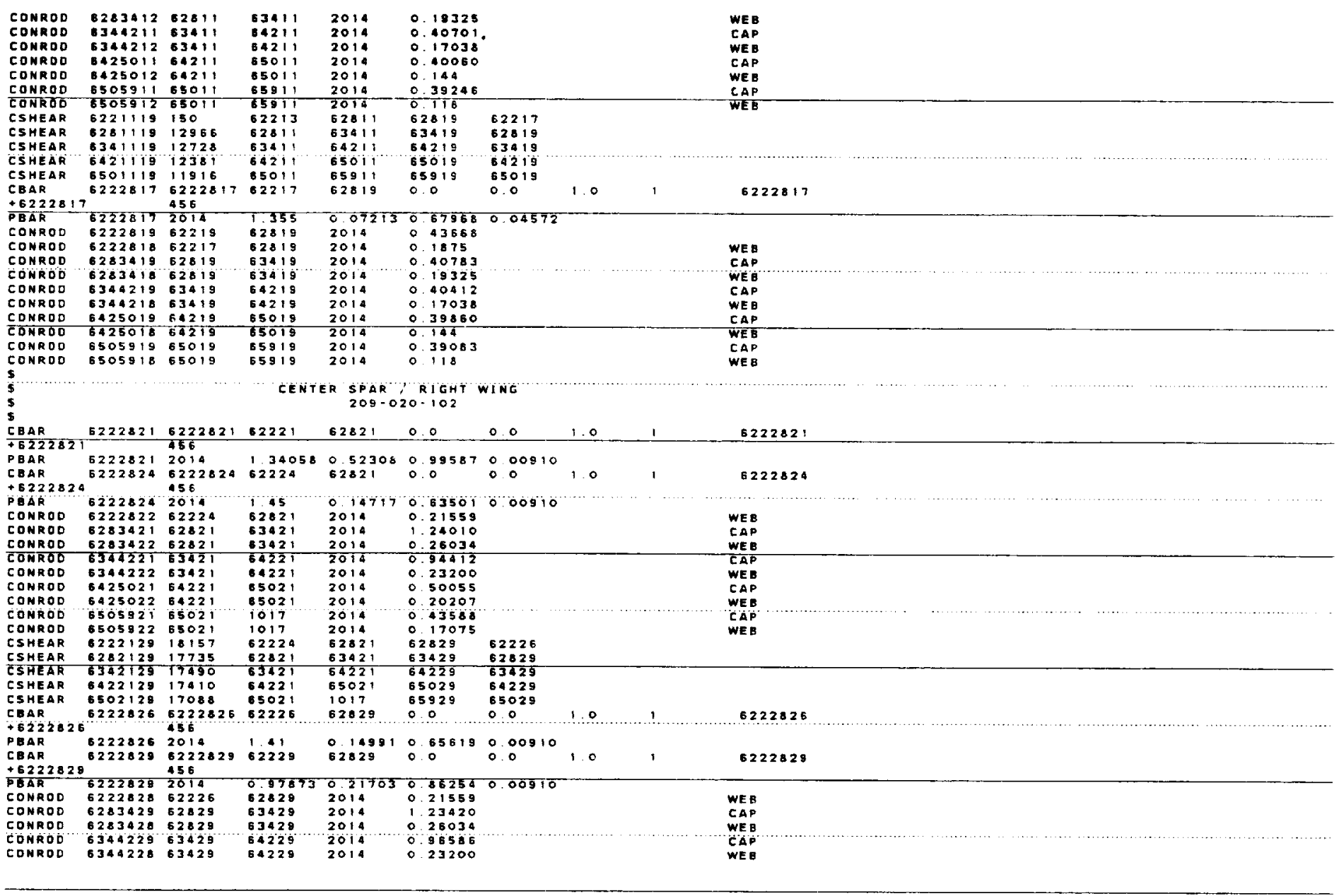

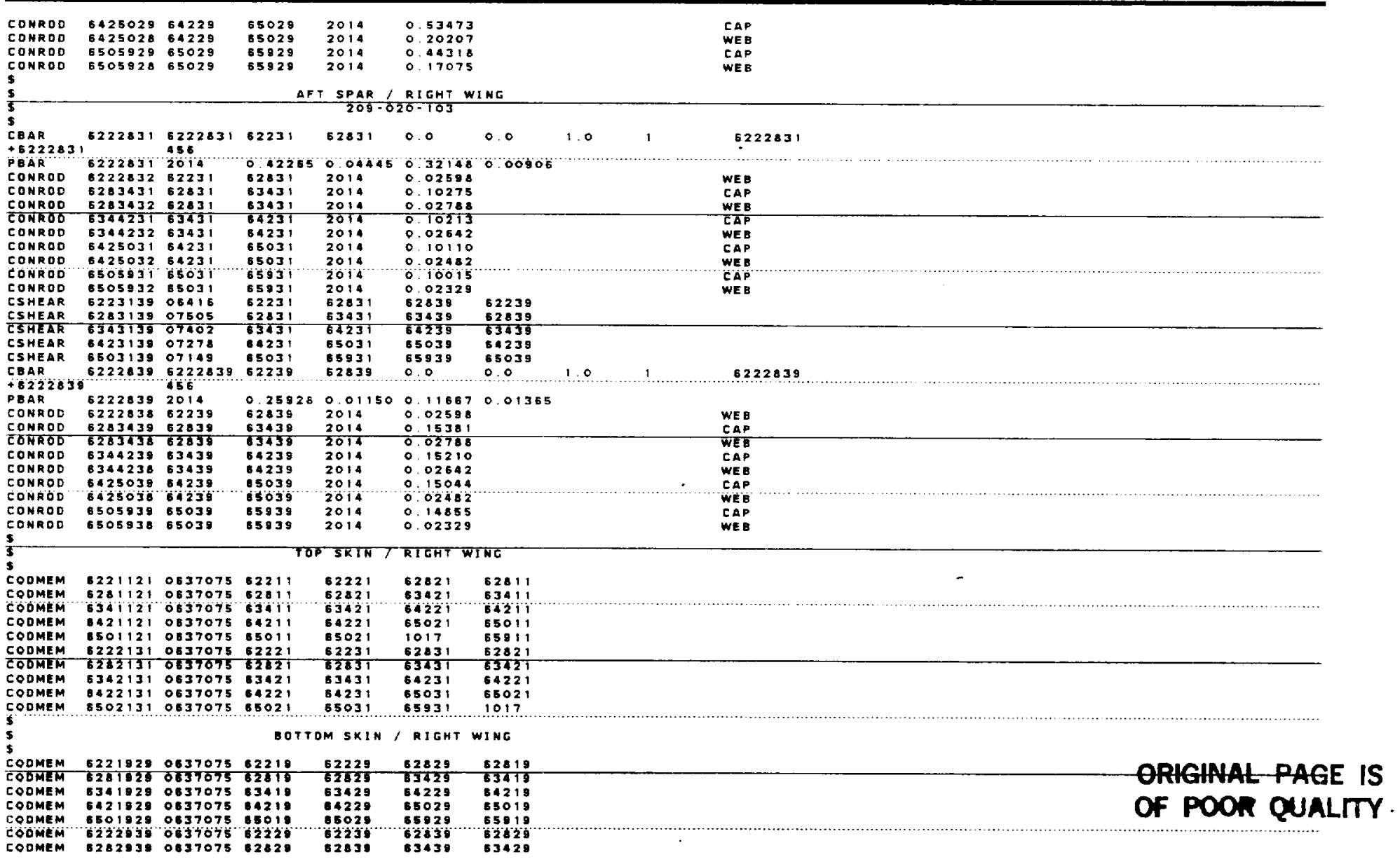




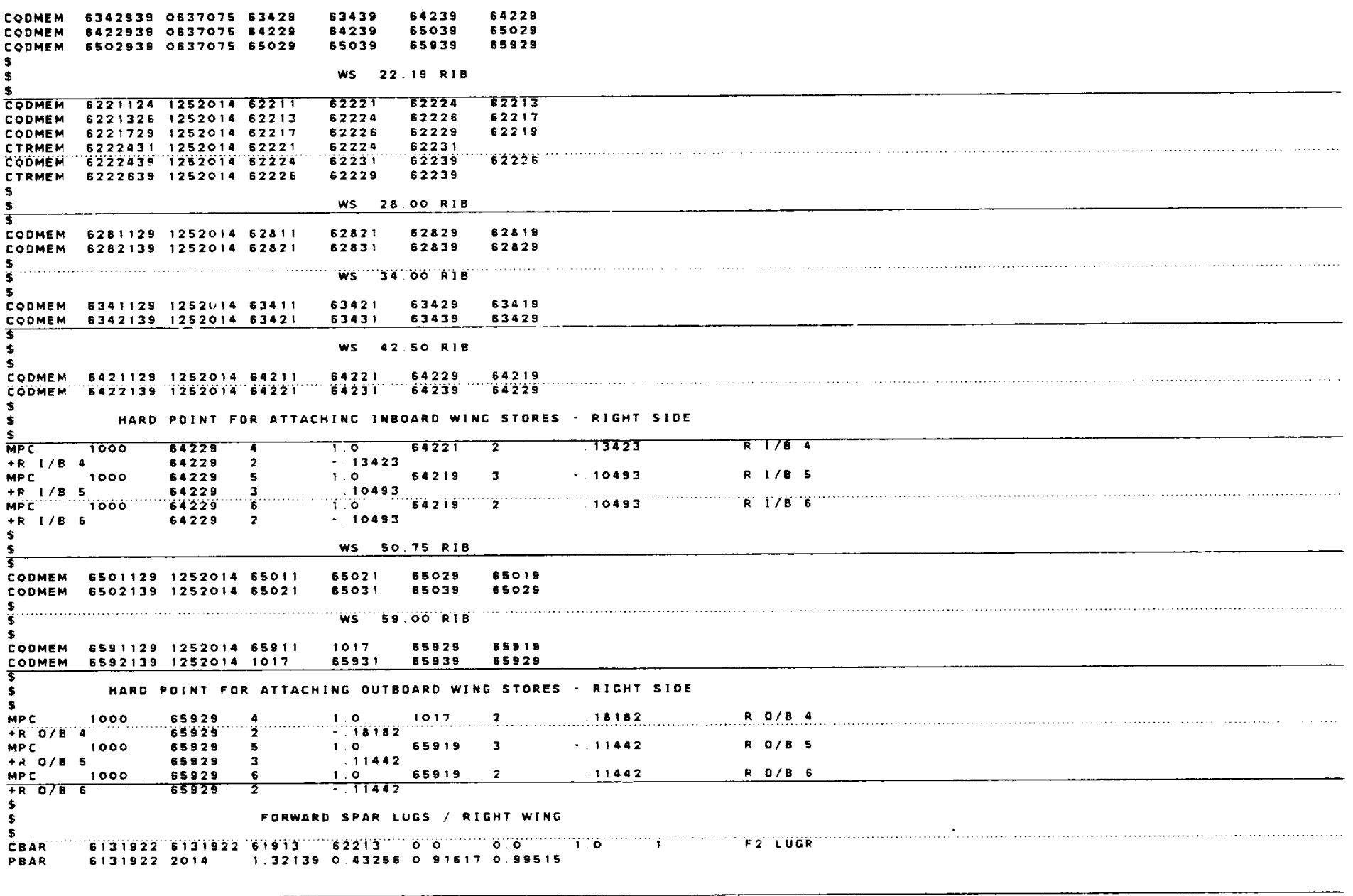

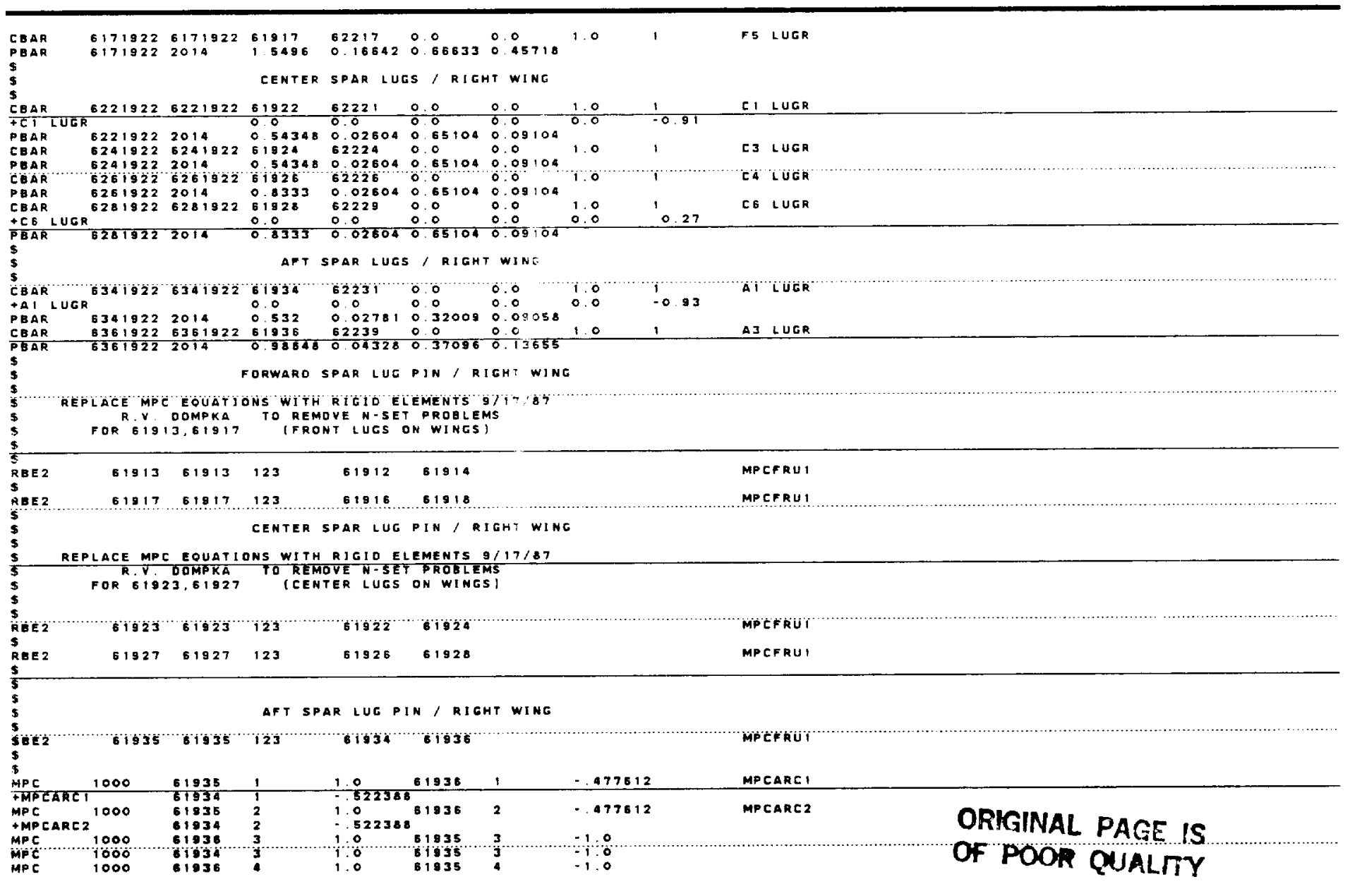




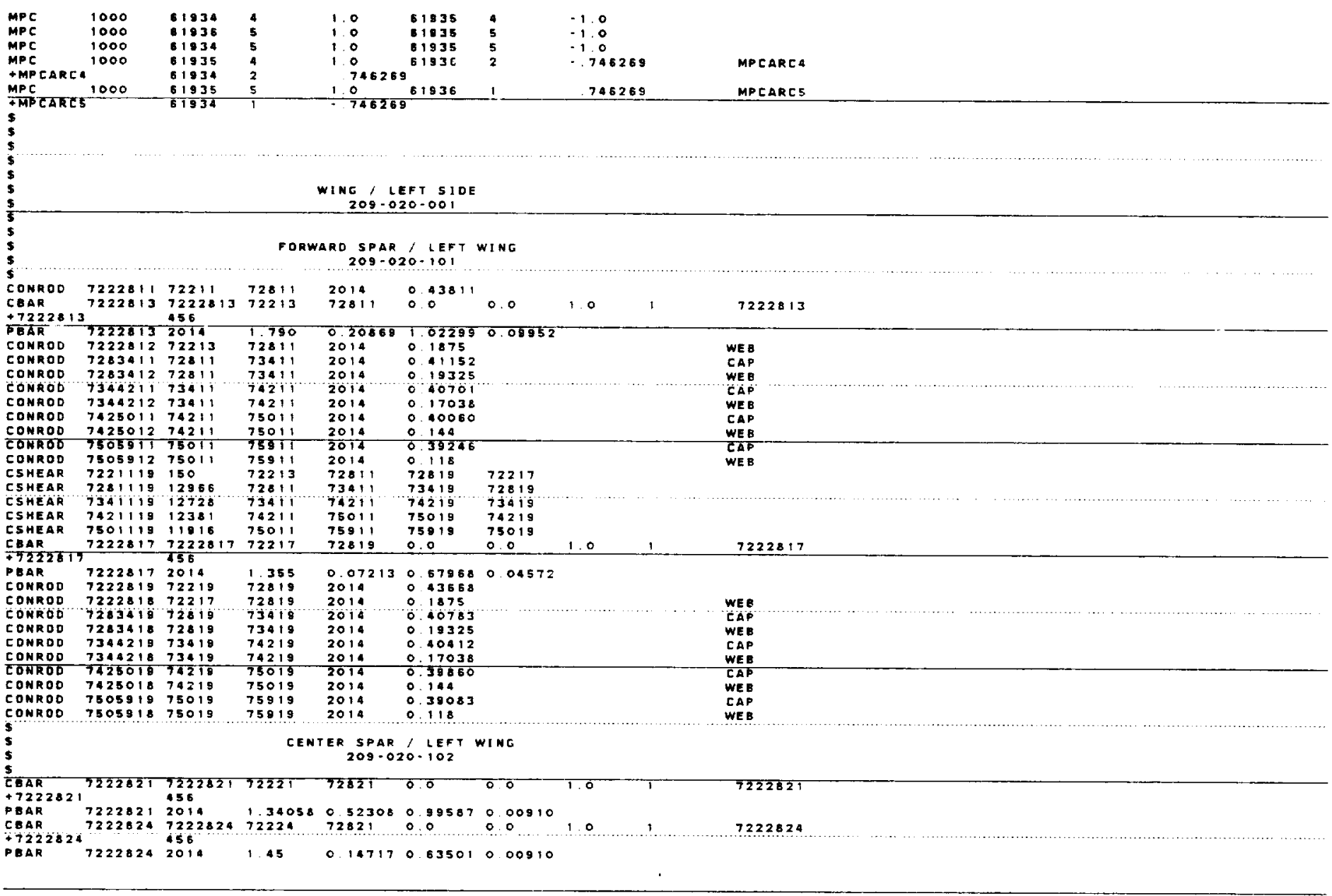

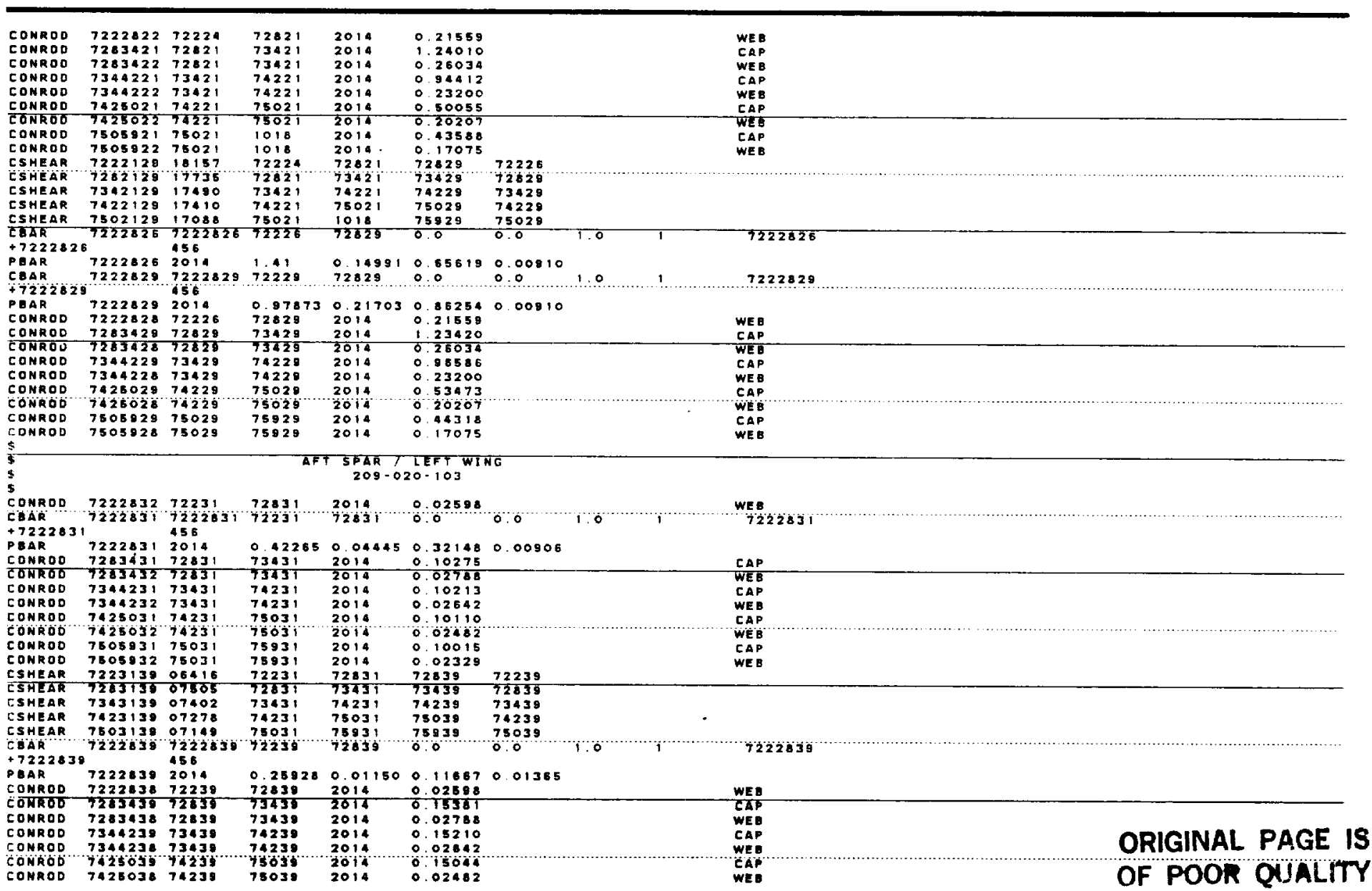




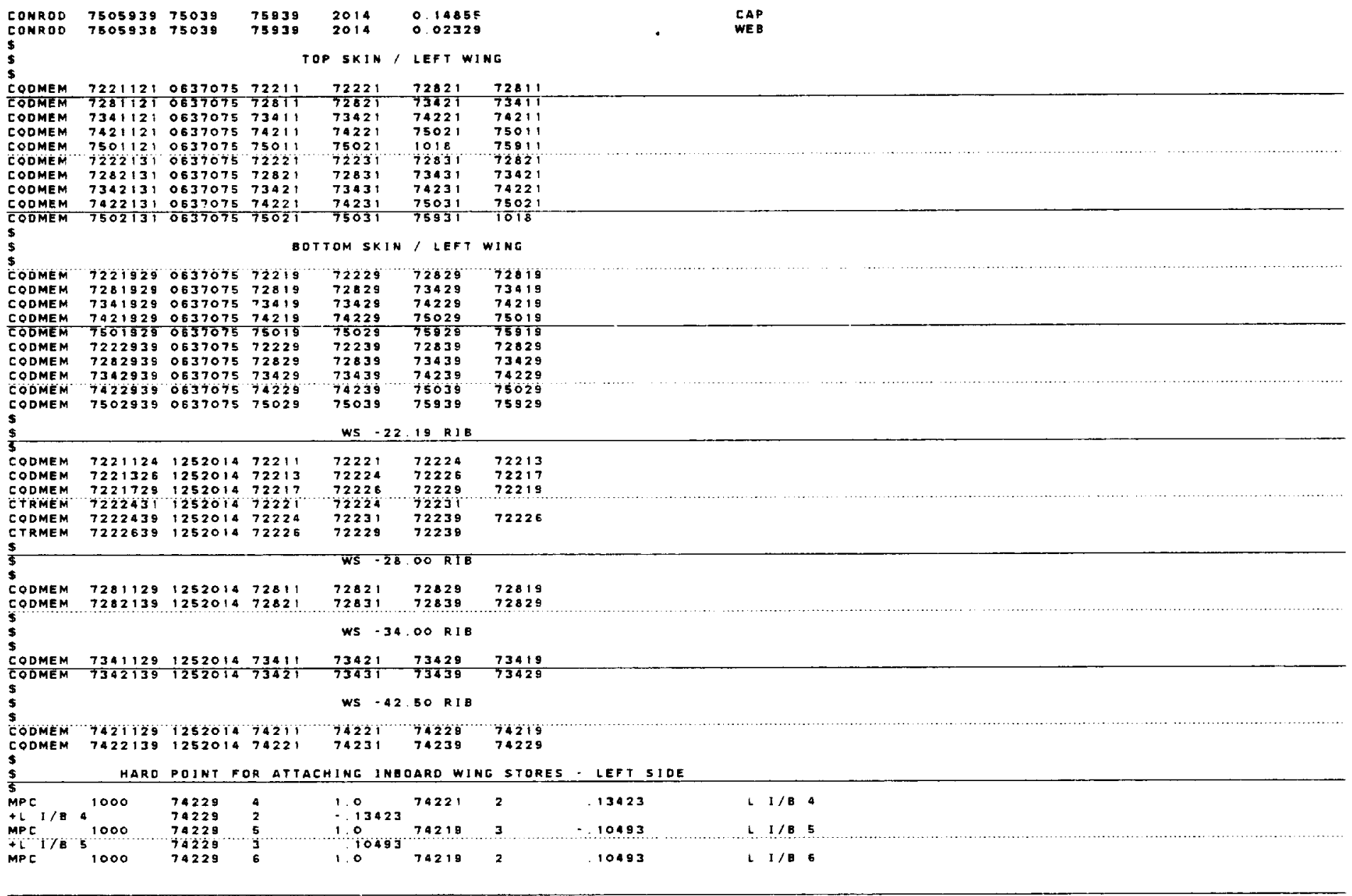

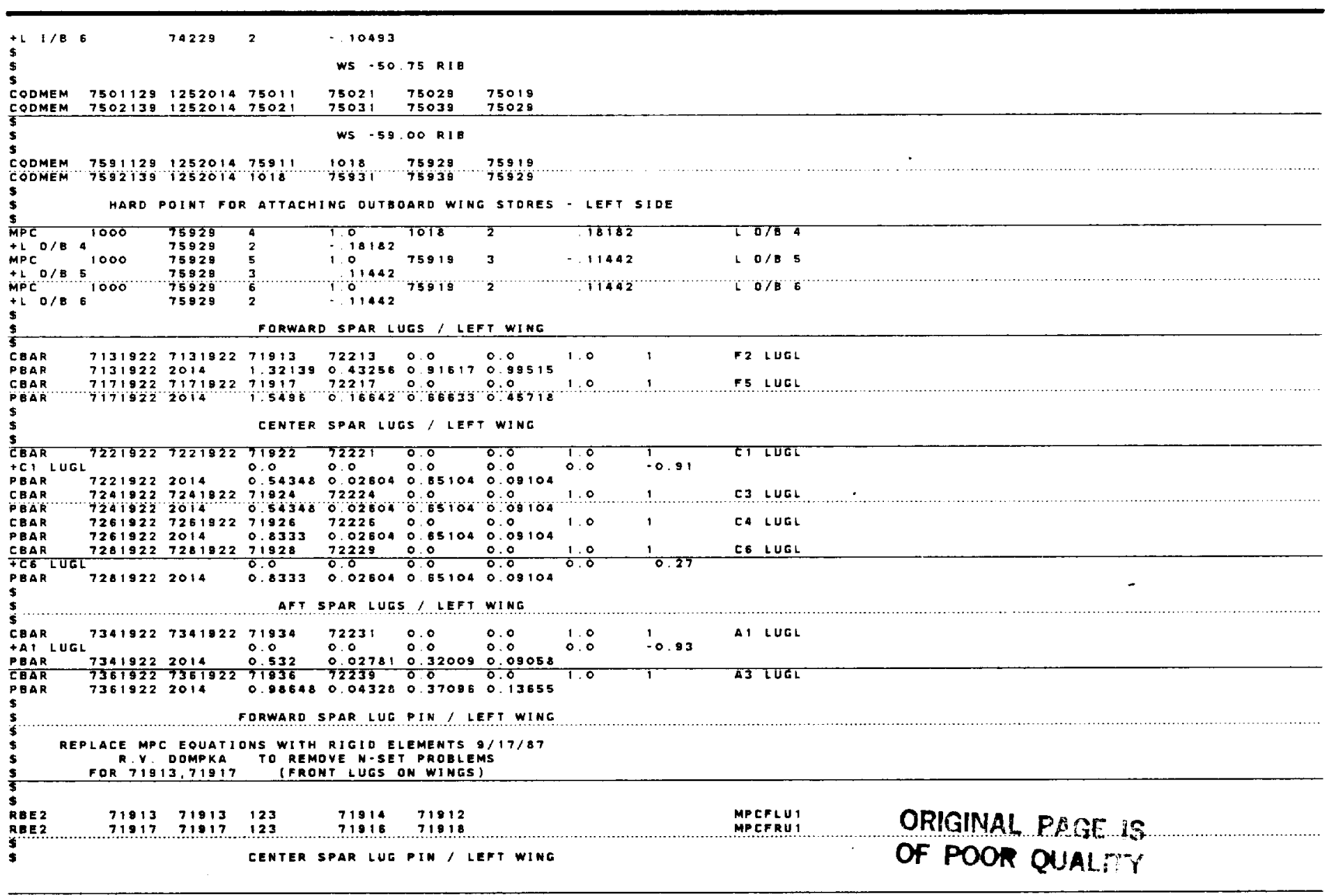




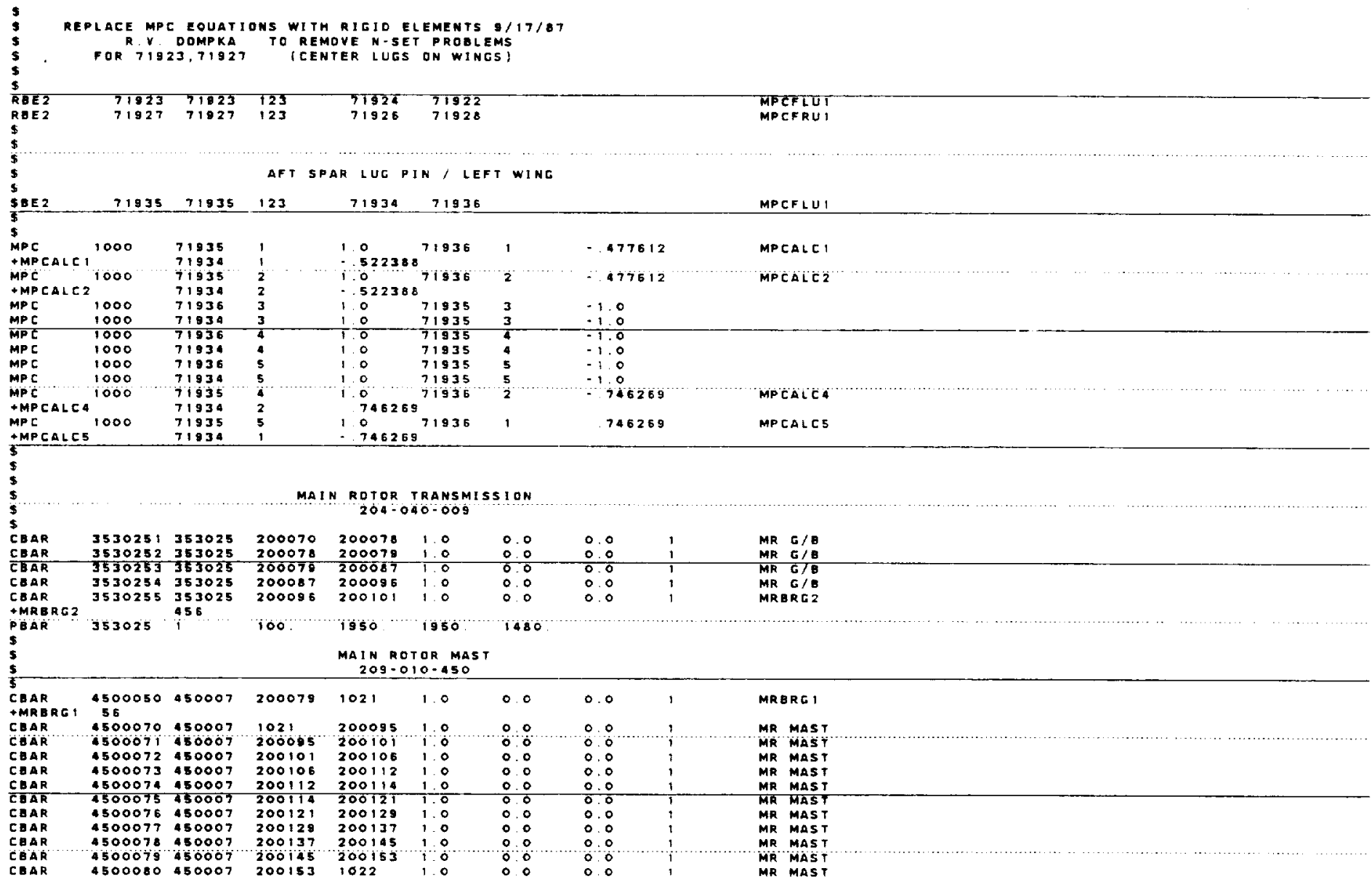

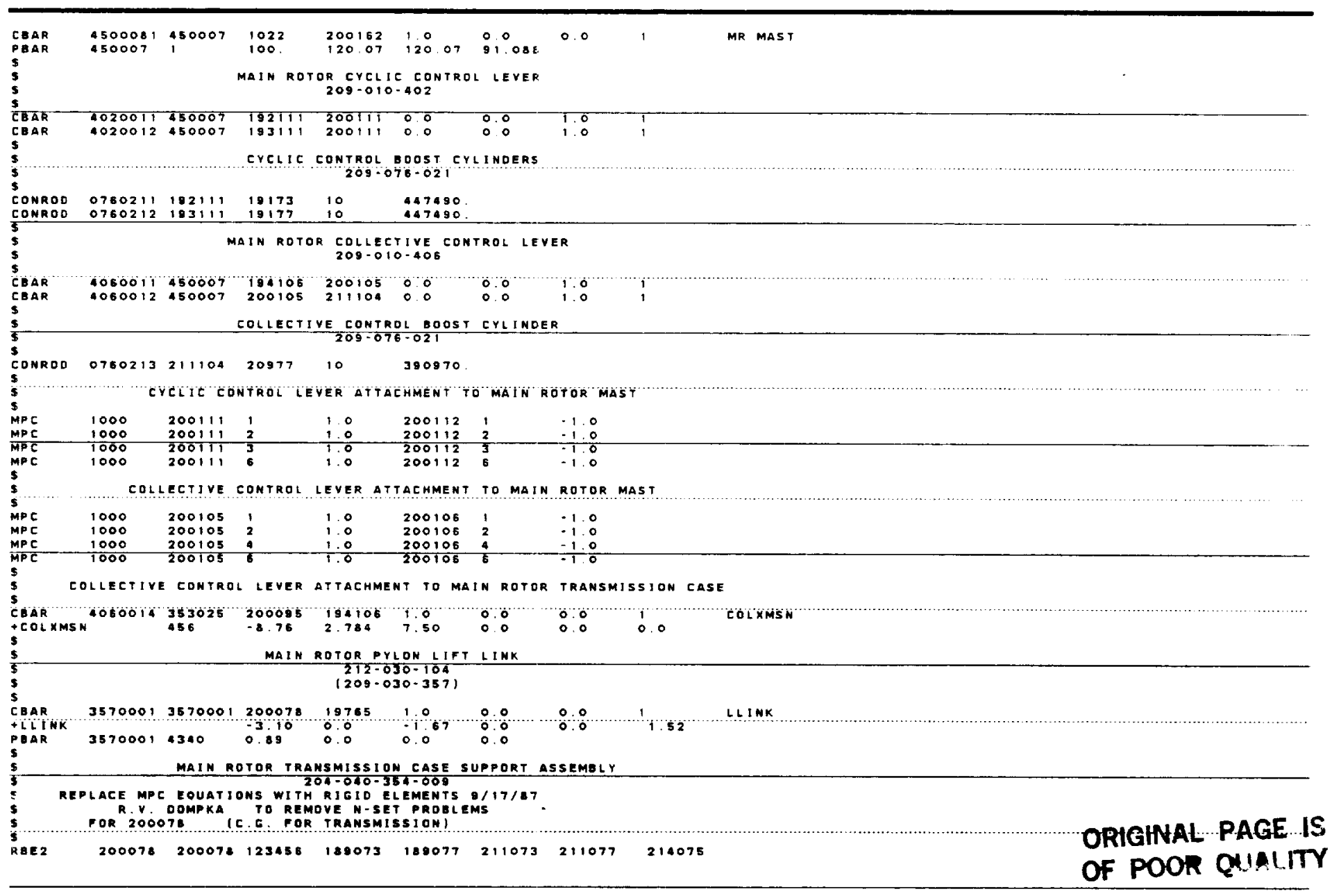




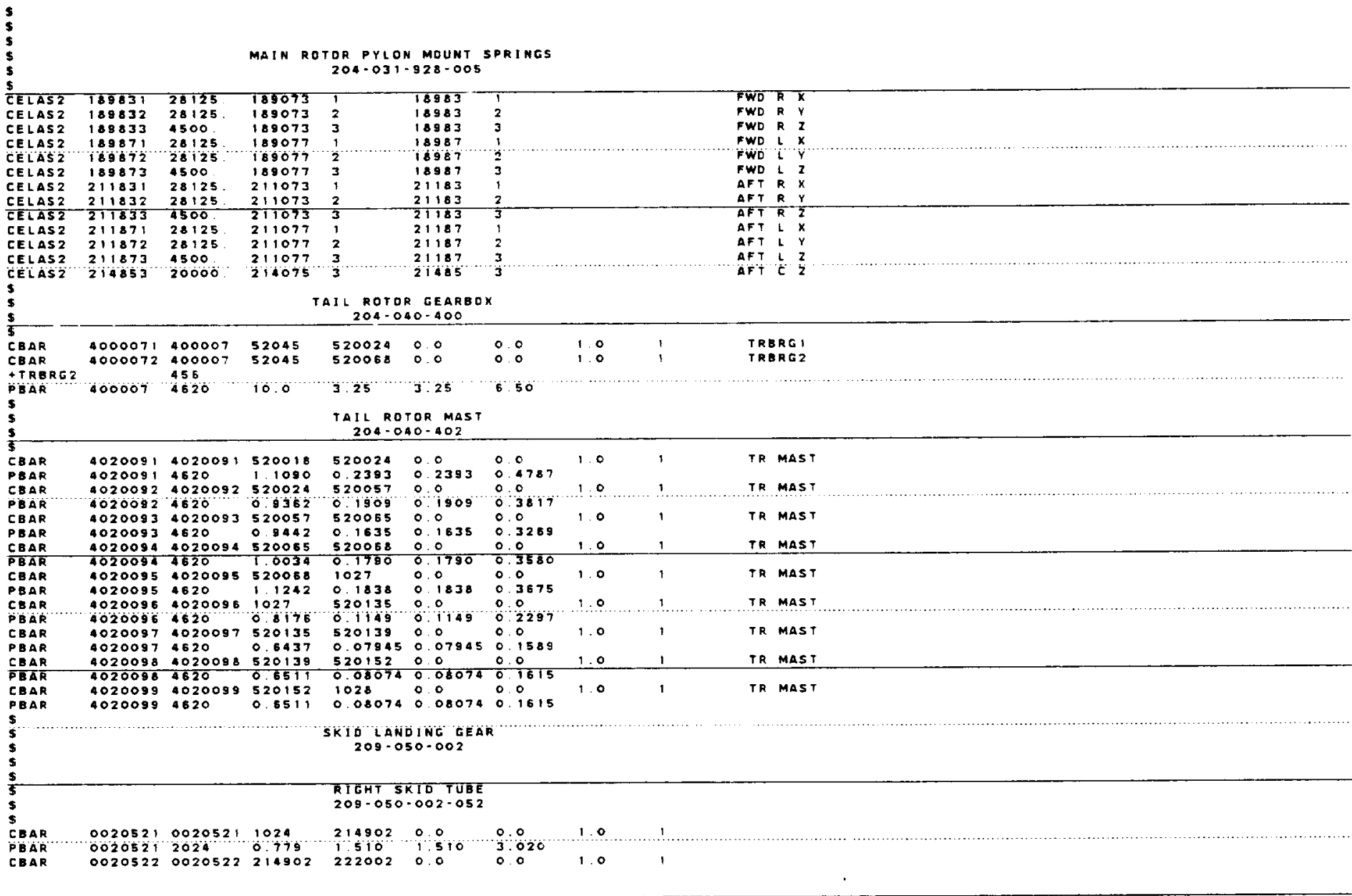

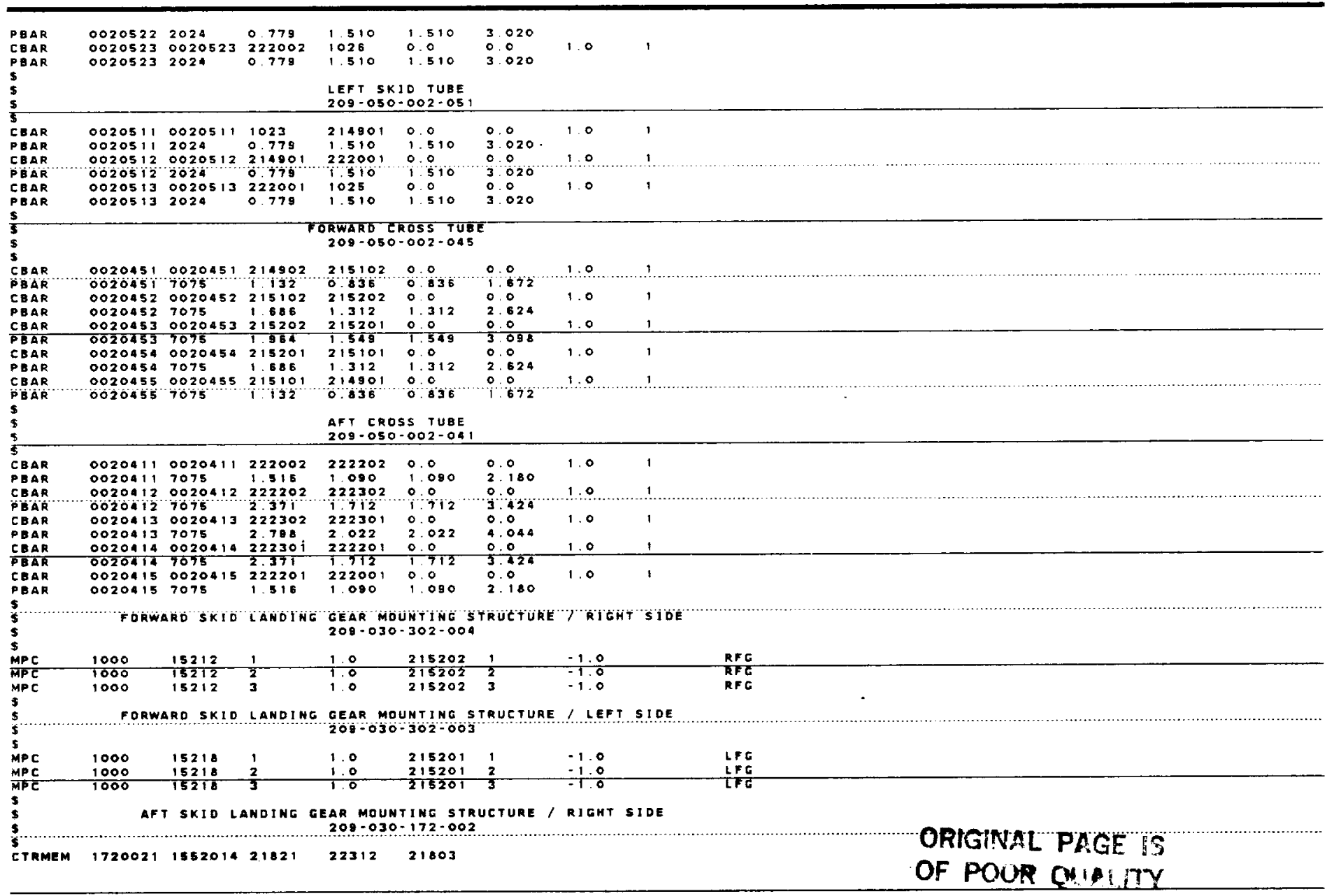




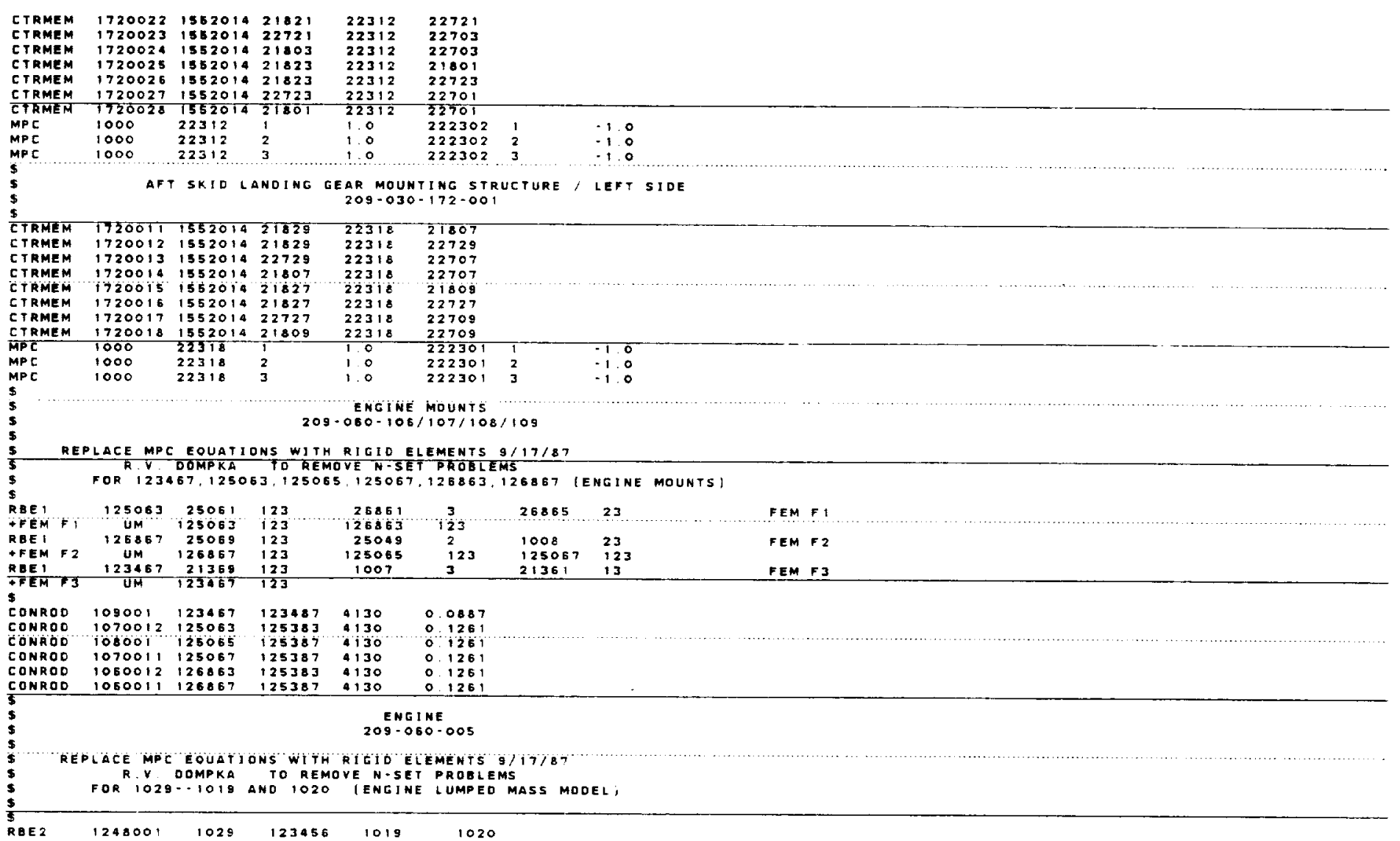

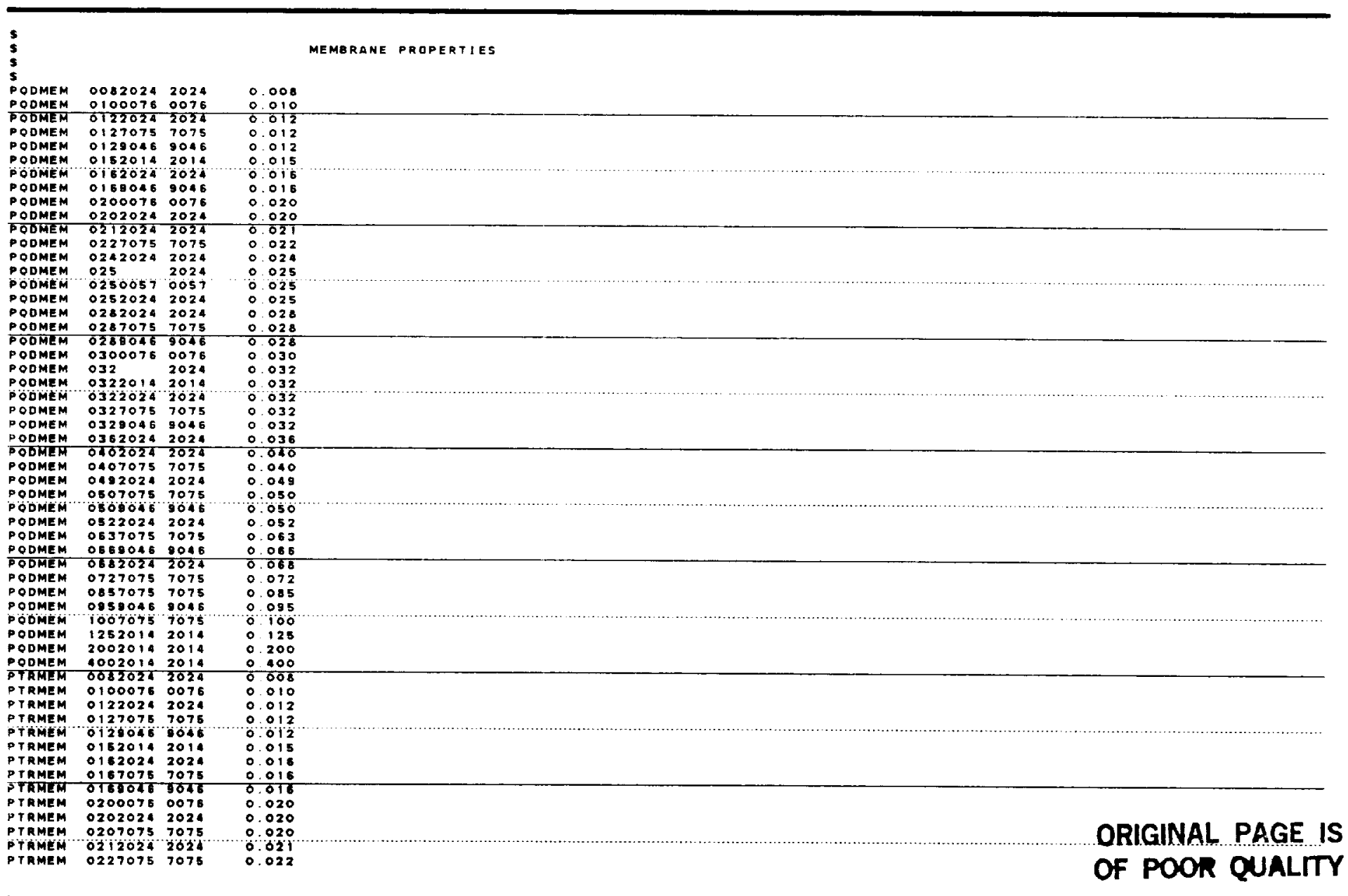




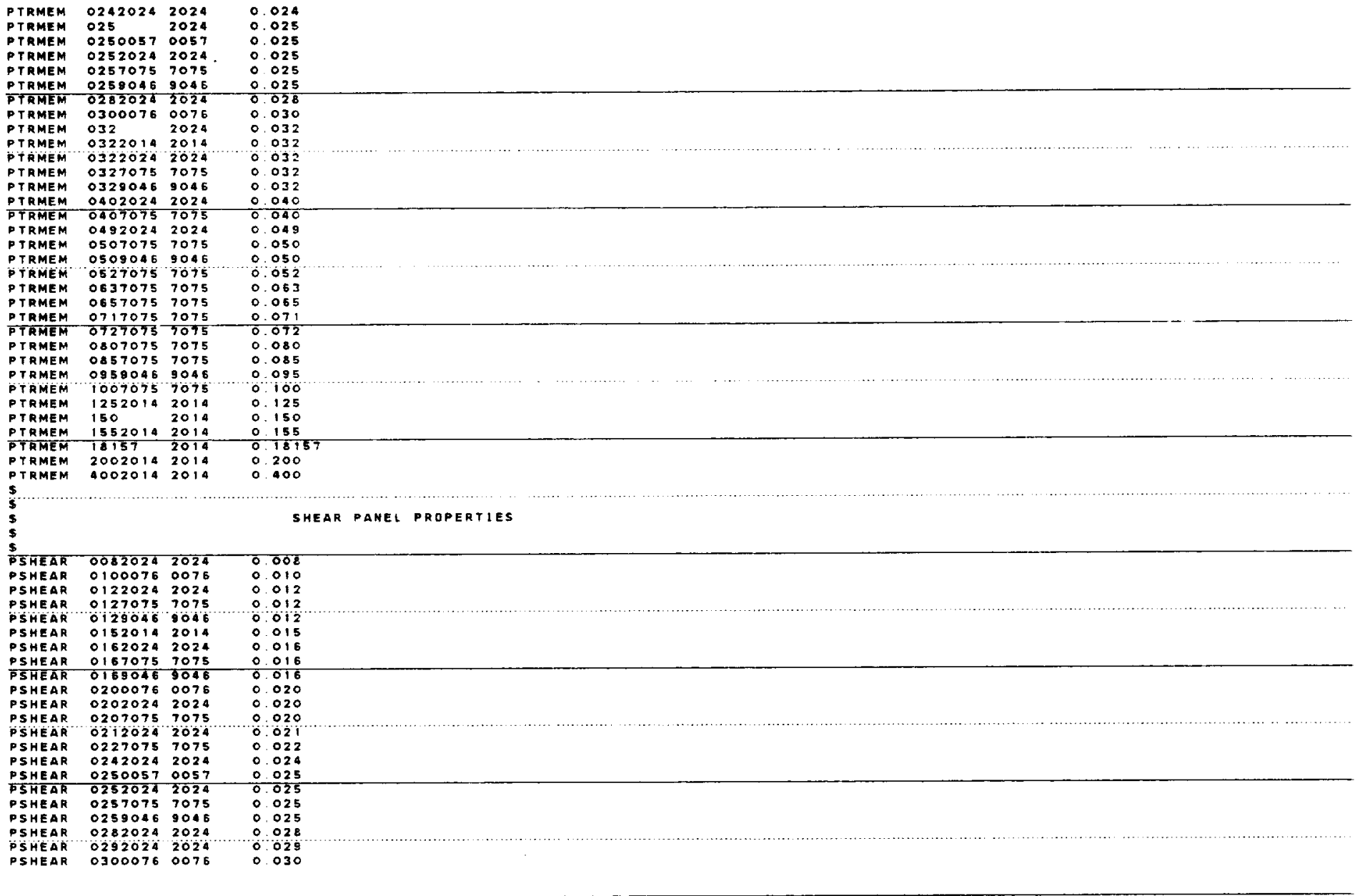

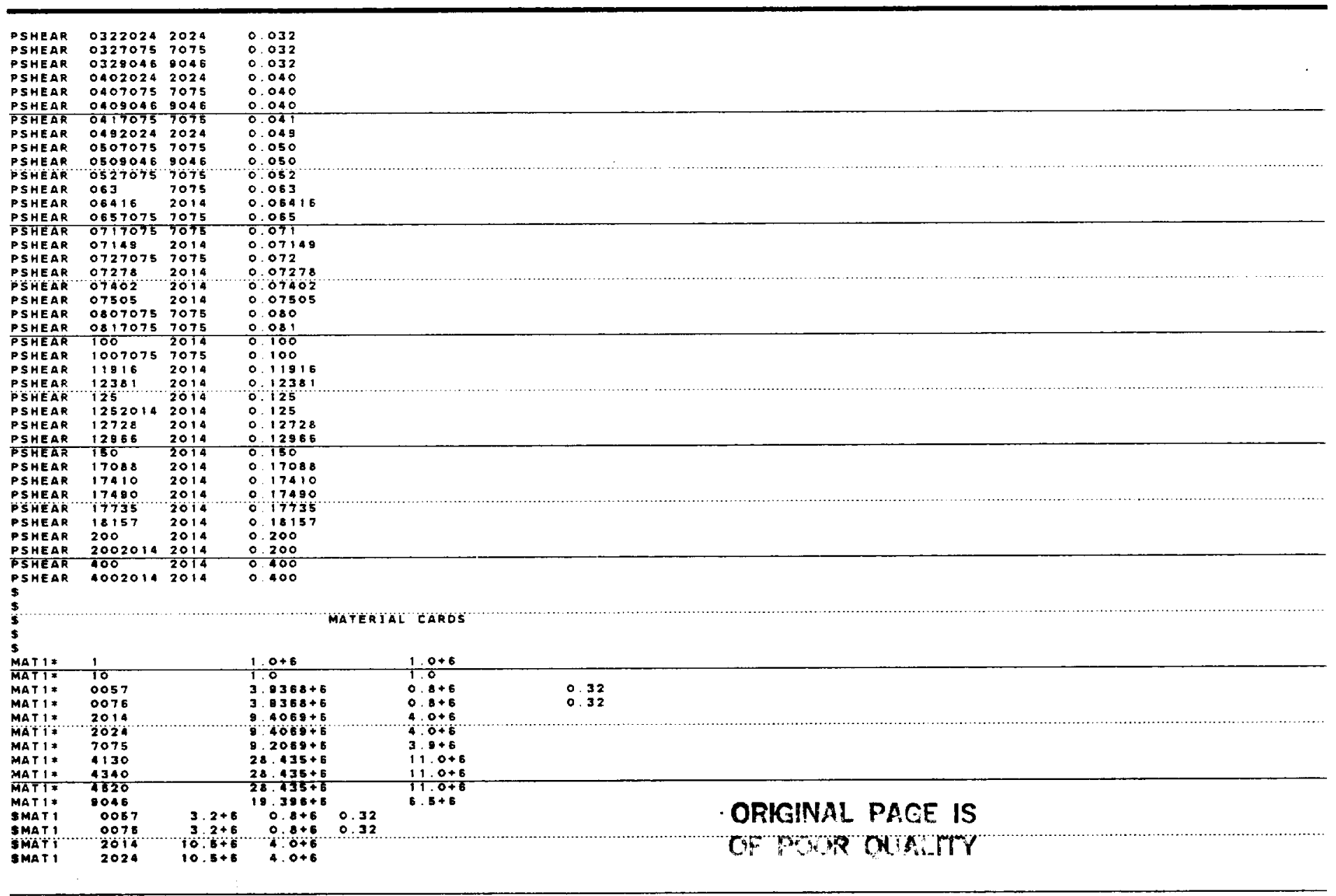




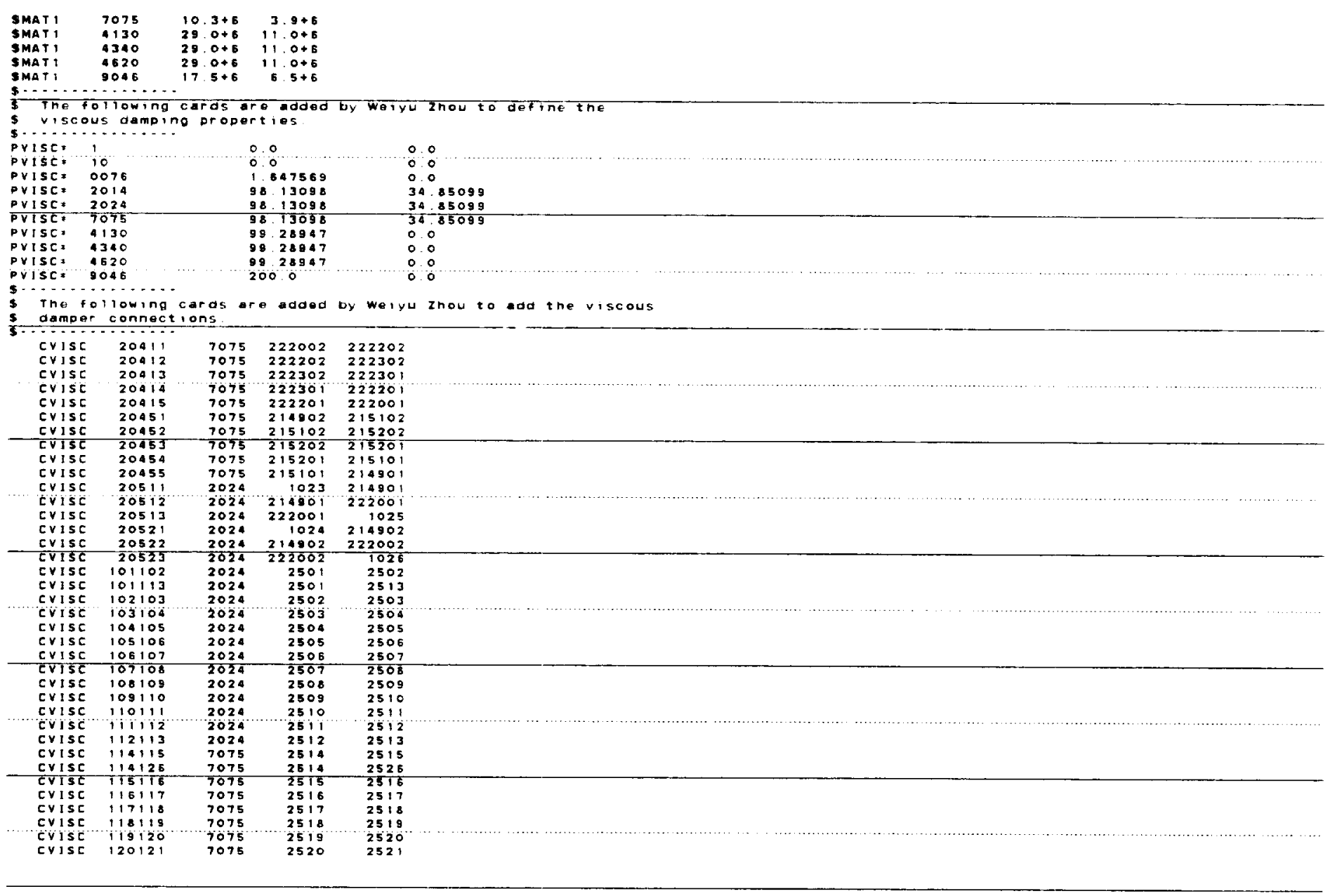

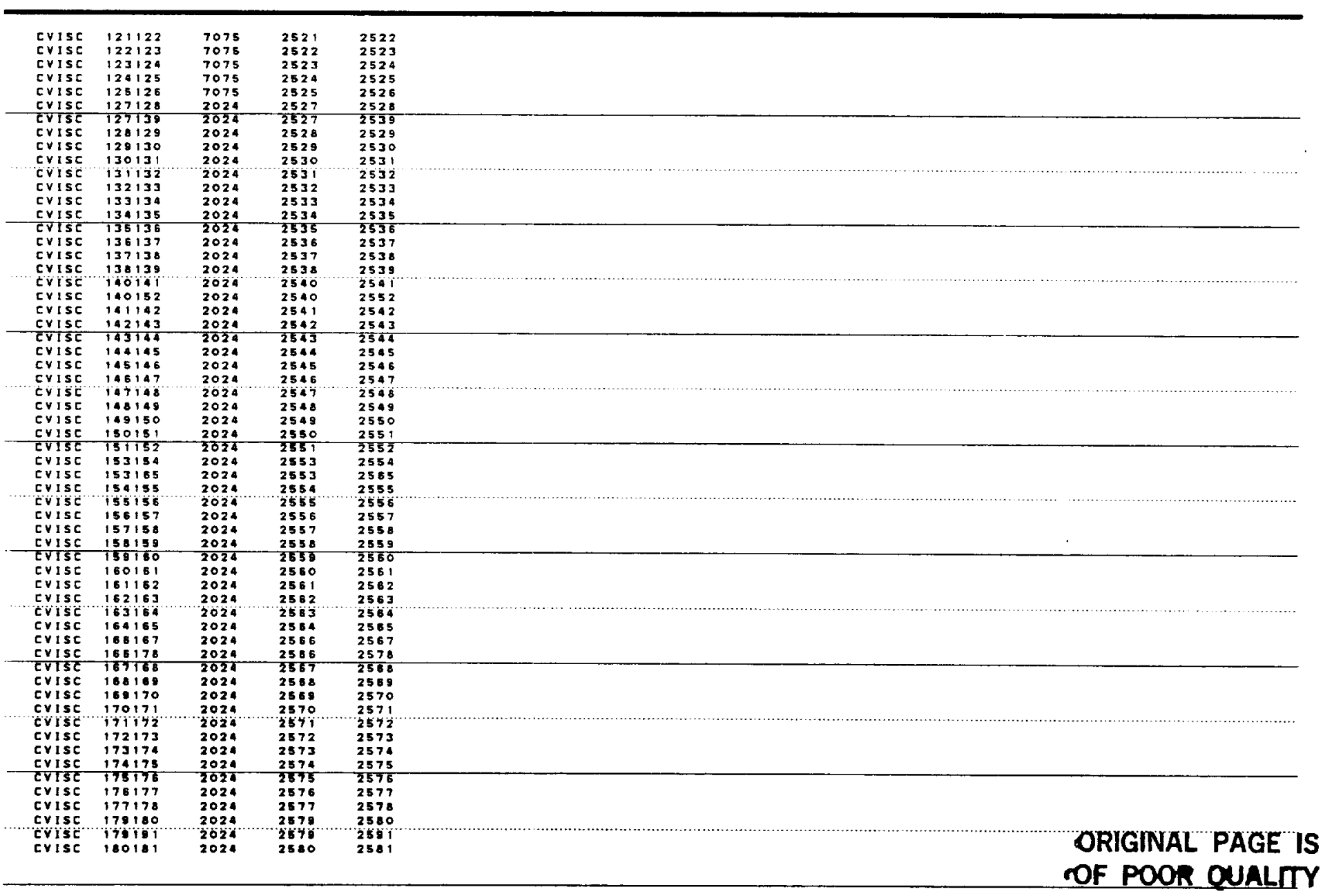




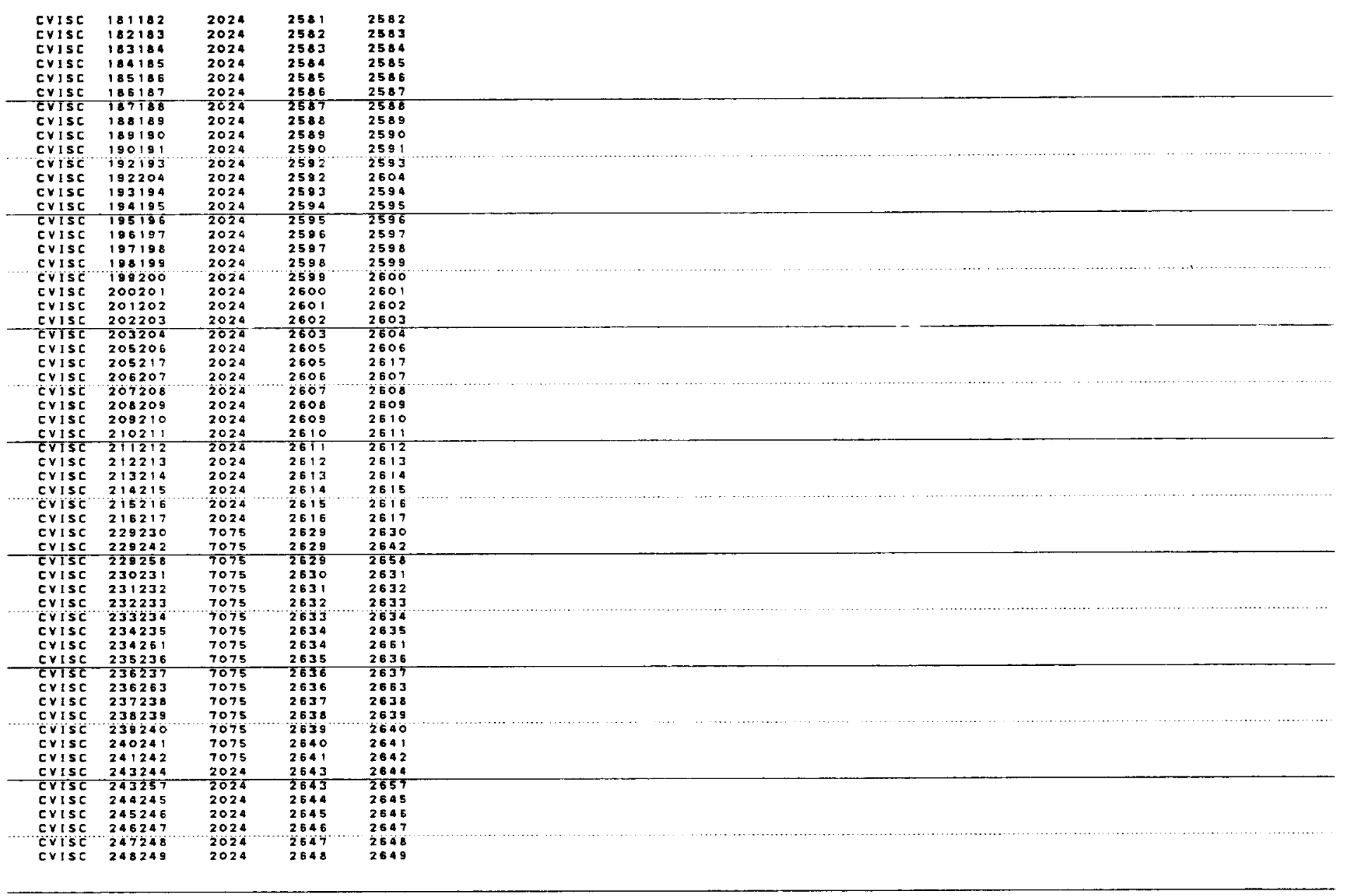

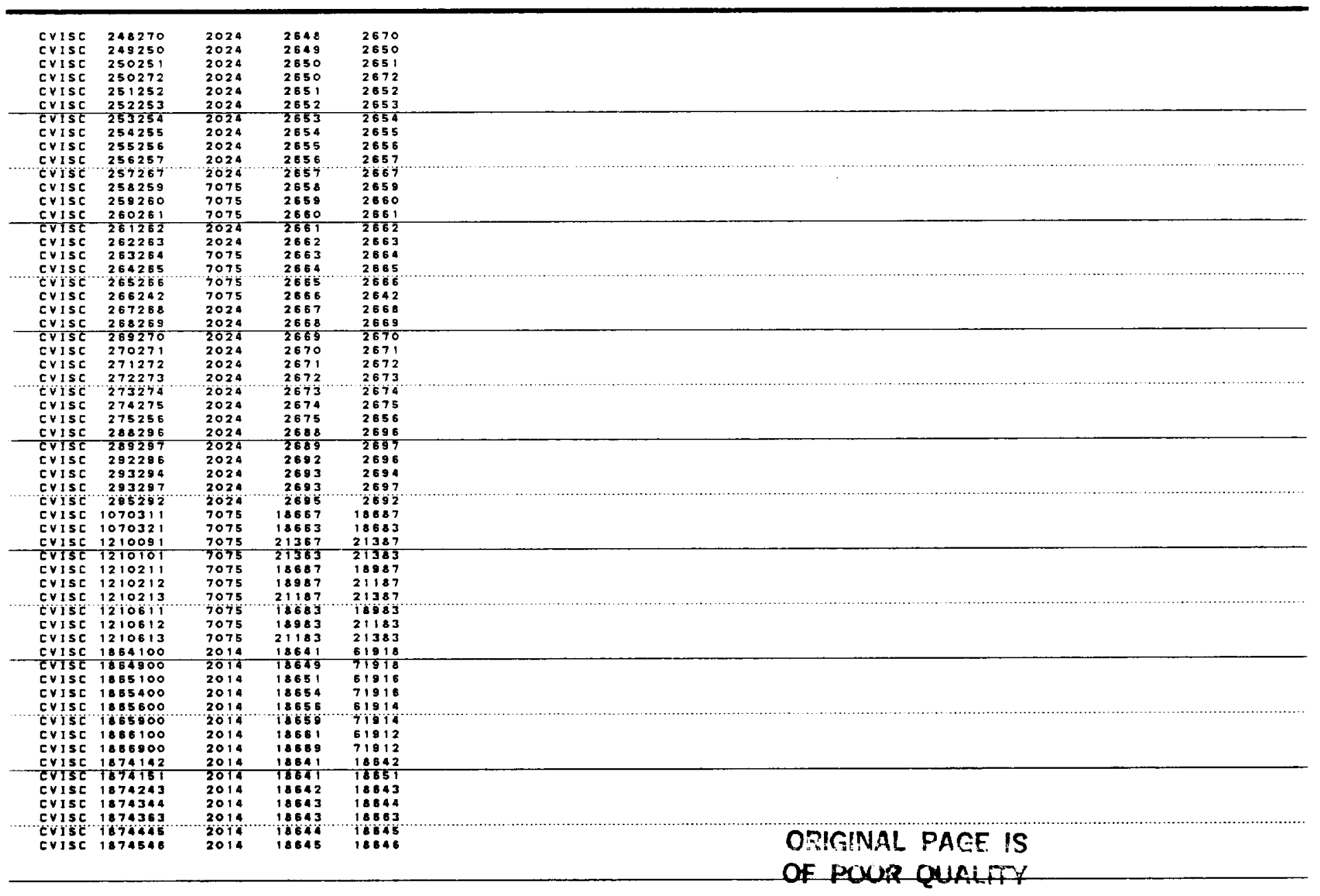




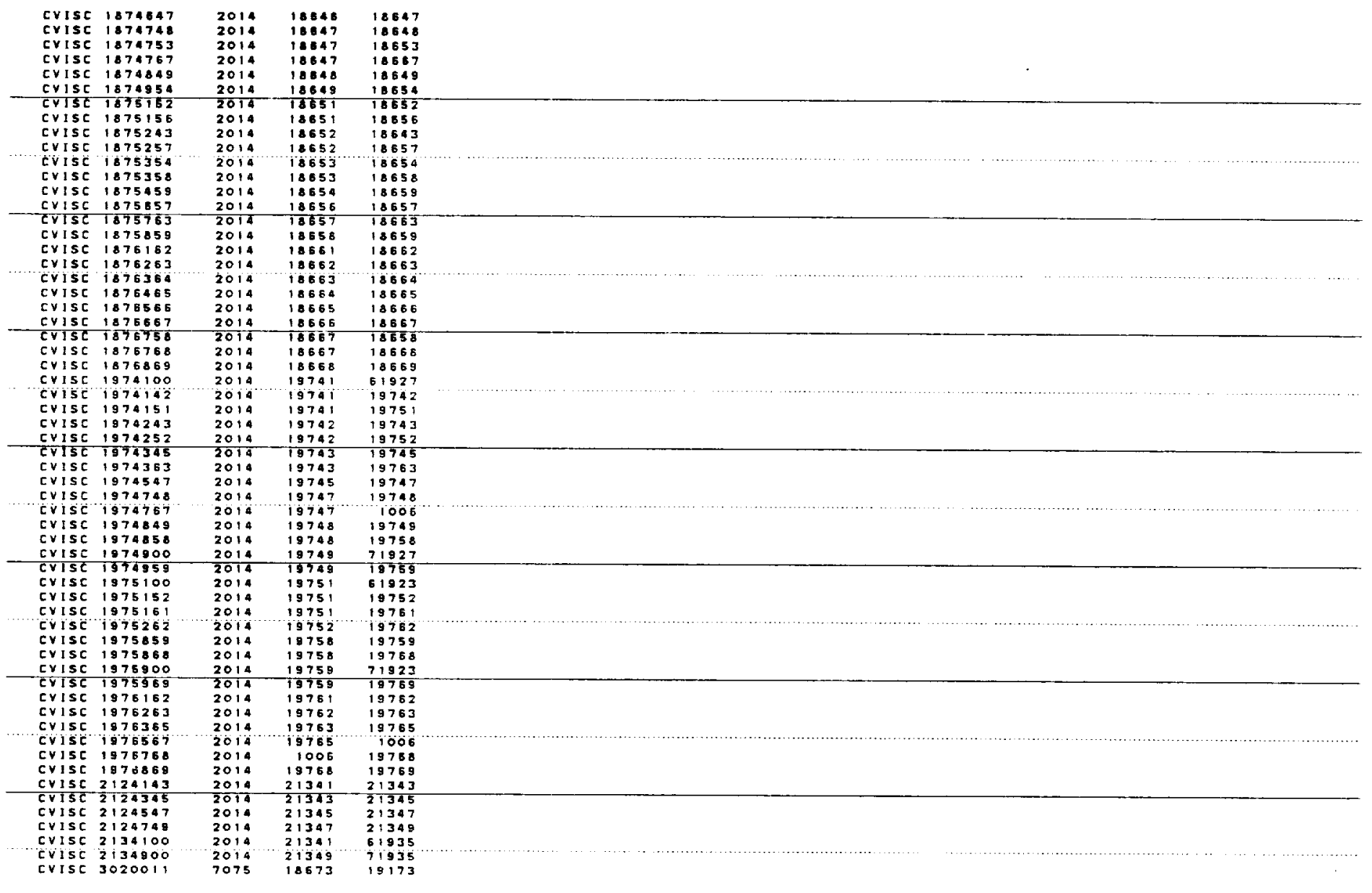

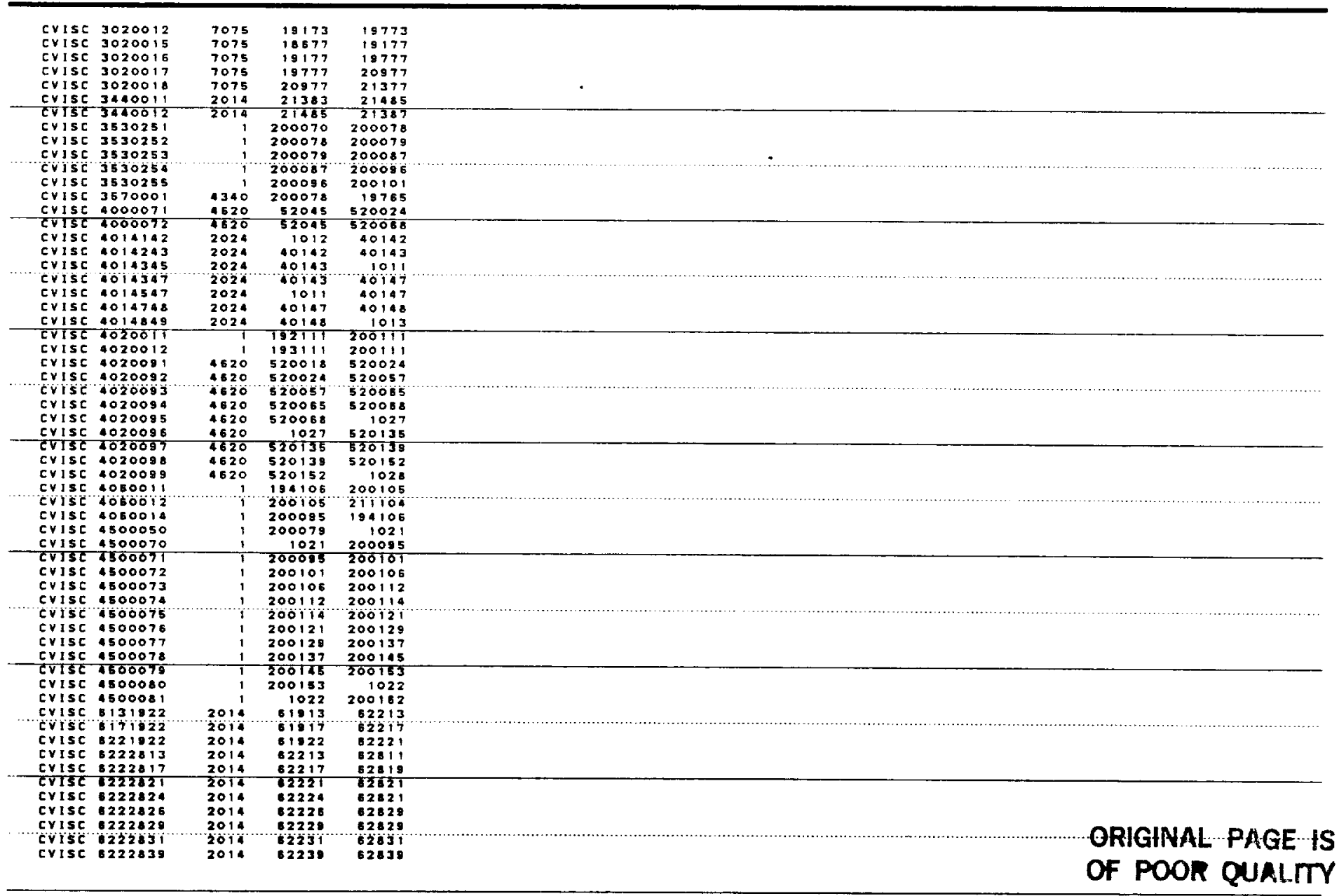




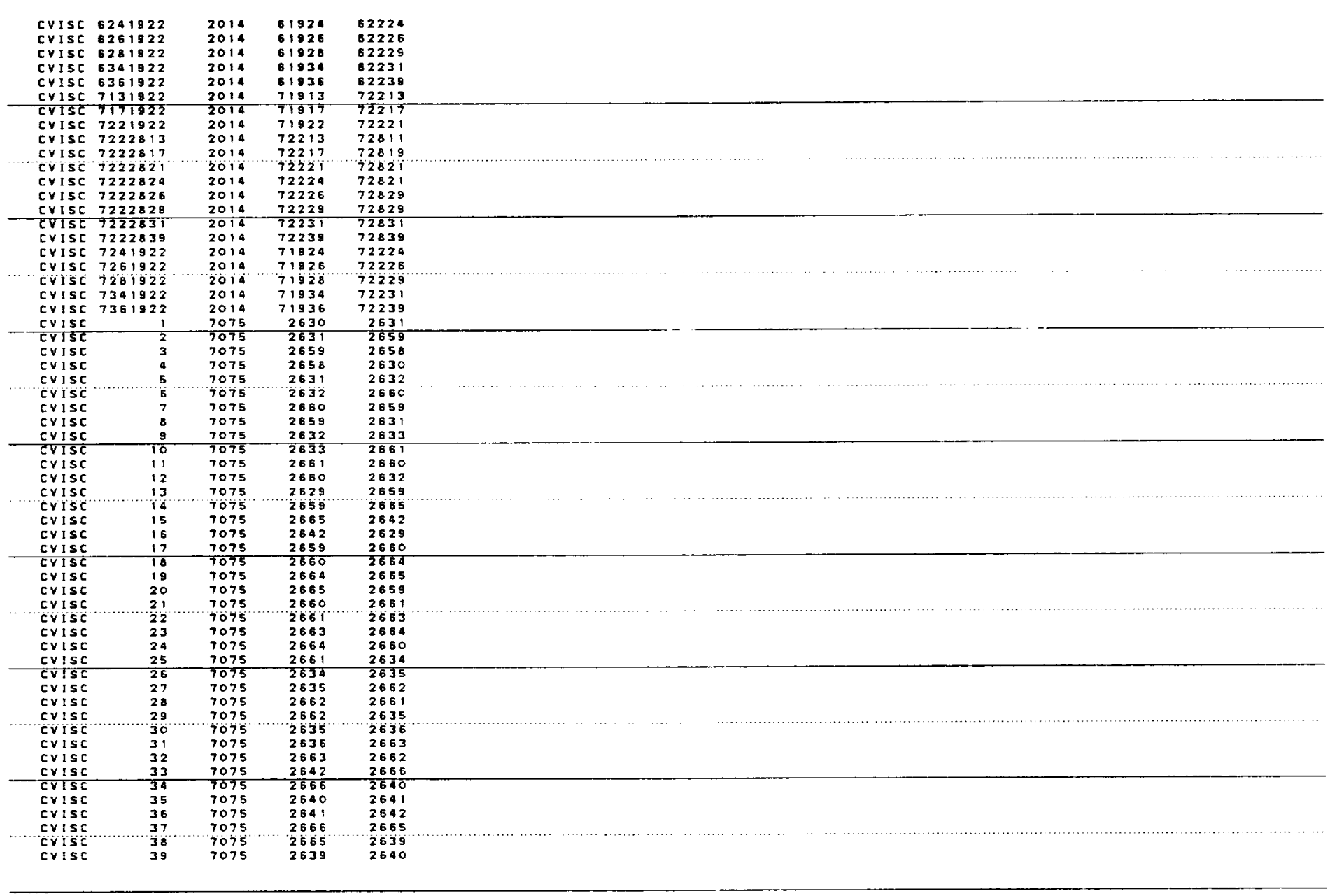

\begin{tabular}{|c|c|c|c|c|c|}
\hline 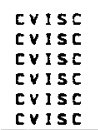 & $\begin{array}{l}40 \\
41 \\
42 \\
43 \\
44 \\
45\end{array}$ & $\begin{array}{l}7075 \\
7075 \\
7075 \\
7075 \\
7075 \\
7075\end{array}$ & $\begin{array}{l}2640 \\
25655 \\
25654 \\
25638 \\
2539 \\
2564\end{array}$ & $\begin{array}{l}2666 \\
2665 \\
2658 \\
2638 \\
2639 \\
2665 \\
2665 \\
2663\end{array}$ & \\
\hline 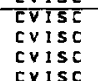 & $\begin{array}{l}46 \\
46 \\
48 \\
49\end{array}$ & $\begin{array}{l}7095 \\
7075 \\
7075 \\
7075 \\
07075\end{array}$ & $\begin{array}{l}2663 \\
2637 \\
2638 \\
2836\end{array}$ & $\begin{array}{l}2637 \\
2653 \\
2685 \\
2664\end{array}$ & \\
\hline $\begin{array}{l}\text { crisc } \\
\text { cystse } \\
\text { cylse }\end{array}$ & $\begin{array}{l}50 \\
51 \\
52\end{array}$ & $\begin{array}{l}7075 \\
7075 \\
7075\end{array}$ & $\begin{array}{l}2646 \\
26657 \\
2655\end{array}$ & $\begin{array}{l}2667 \\
2657 \\
2643\end{array}$ & \\
\hline 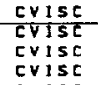 & $\begin{array}{l}53 \\
534 \\
55 \\
56\end{array}$ & $\begin{array}{l}7075 \\
7075 \\
7075 \\
7075\end{array}$ & $\begin{array}{l}2644 \\
2645 \\
26685 \\
2887\end{array}$ & $\begin{array}{l}2645 \\
2668 \\
2567 \\
2544\end{array}$ & \\
\hline 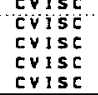 & $\begin{array}{l}57 \\
58 \\
58 \\
60 \\
61\end{array}$ & $\begin{array}{l}-175 \\
7075 \\
7075 \\
7075 \\
7075\end{array}$ & $\begin{array}{l}286 \\
2766 \\
2689 \\
2685 \\
2646\end{array}$ & 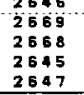 & \\
\hline 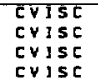 & $\begin{array}{l}62 \\
63 \\
64 \\
65\end{array}$ & $\begin{array}{l}7095 \\
7075 \\
7075 \\
7075\end{array}$ & $\begin{array}{l}2667 \\
2670 \\
2659 \\
2857\end{array}$ & $\begin{array}{l}2670 \\
2659 \\
2846 \\
2670\end{array}$ & \\
\hline $\begin{array}{l}\text { crise } \\
\text { crys } \\
\text { cystse }\end{array}$ & $\begin{array}{l}66 \\
66 \\
68 \\
68\end{array}$ & $\begin{array}{l}7005 \\
7075 \\
7075 \\
7075\end{array}$ & $\begin{array}{l}2670 \\
2675 \\
2565\end{array}$ & $\begin{array}{l}2672 \\
2556 \\
2557\end{array}$ & \\
\hline 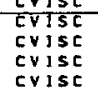 & $\begin{array}{l}0.09 \\
710 \\
72 \\
73\end{array}$ & $\begin{array}{l}7075 \\
7075 \\
7075 \\
7075 \\
7075\end{array}$ & $\begin{array}{l}2860 \\
28648 \\
2648 \\
2671 \\
2867\end{array}$ & $\begin{array}{l}2648 \\
2649 \\
2671 \\
2670 \\
2549\end{array}$ & \\
\hline $\begin{array}{l}\text { crisc } \\
\text { crist } \\
\text { crise } \\
\text { cystse }\end{array}$ & $\begin{array}{l}74 \\
75 \\
75 \\
75\end{array}$ & $\begin{array}{l}7005 \\
7075 \\
7075\end{array}$ & $\begin{array}{l}2649 \\
2650 \\
2657\end{array}$ & $\begin{array}{r}2650 \\
2672 \\
2571\end{array}$ & \\
\hline 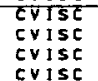 & $\begin{array}{l}78 \\
78 \\
80 \\
81\end{array}$ & $\begin{array}{l}7075 \\
7075 \\
7075 \\
7075\end{array}$ & $\begin{array}{l}2675 \\
28554 \\
2555 \\
2857\end{array}$ & $\begin{array}{l}2654 \\
2655 \\
2856 \\
2874\end{array}$ & \\
\hline 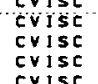 & $\begin{array}{l}8 \\
81 . \\
83 \\
84 \\
85\end{array}$ & $\begin{array}{l}\text { 7.755 } \\
77075 \\
7075 \\
7075 \\
7075\end{array}$ & $\begin{array}{l}-28 \\
2875 \\
2853 \\
2853 \\
2854 \\
3874\end{array}$ & $\begin{array}{l}2674 \\
2653 \\
2654 \\
2675 \\
2673\end{array}$ & \\
\hline 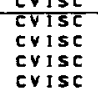 & $\begin{array}{l}\frac{85}{86} \\
87 \\
88 \\
89\end{array}$ & $\begin{array}{l}-7075 \\
77075 \\
77075 \\
7075 \\
7075\end{array}$ & $\begin{array}{l}\frac{2878}{2573} \\
2652 \\
2853 \\
2853\end{array}$ & $\begin{array}{l}2673 \\
2852 \\
2853 \\
2874 \\
2672\end{array}$ & \\
\hline $\begin{array}{l}\text { crise } \\
\text { crise } \\
\text { crise }\end{array}$ & $\begin{array}{l}80 \\
90 \\
92 \\
92\end{array}$ & $\begin{array}{l}7015 \\
7075 \\
7075 \\
7075 \\
7075\end{array}$ & $\begin{array}{l}287 \\
2851 \\
2551 \\
2852 \\
2501\end{array}$ & $\begin{array}{l}2651 \\
2655 \\
2652\end{array}$ & \\
\hline 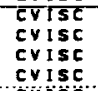 & $\begin{array}{l}9.94 \\
995 \\
98 \\
98\end{array}$ & $\begin{array}{l}7075 \\
77075 \\
7078 \\
7075\end{array}$ & $\begin{array}{l}2502 \\
2551 \\
2515 \\
2514 \\
.2502\end{array}$ & $\begin{array}{l}2515 \\
2515 \\
2501 \\
2503 \\
2503\end{array}$ & \\
\hline $\begin{array}{l}\text { curse } \\
\text { cyise }\end{array}$ & 8 & $\begin{array}{l}4645 \\
7075\end{array}$ & $\begin{array}{l}2503 \\
2511\end{array}$ & $\begin{array}{r}2516 \\
2515\end{array}$ & 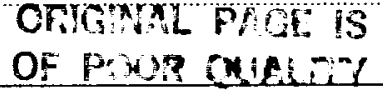 \\
\hline
\end{tabular}




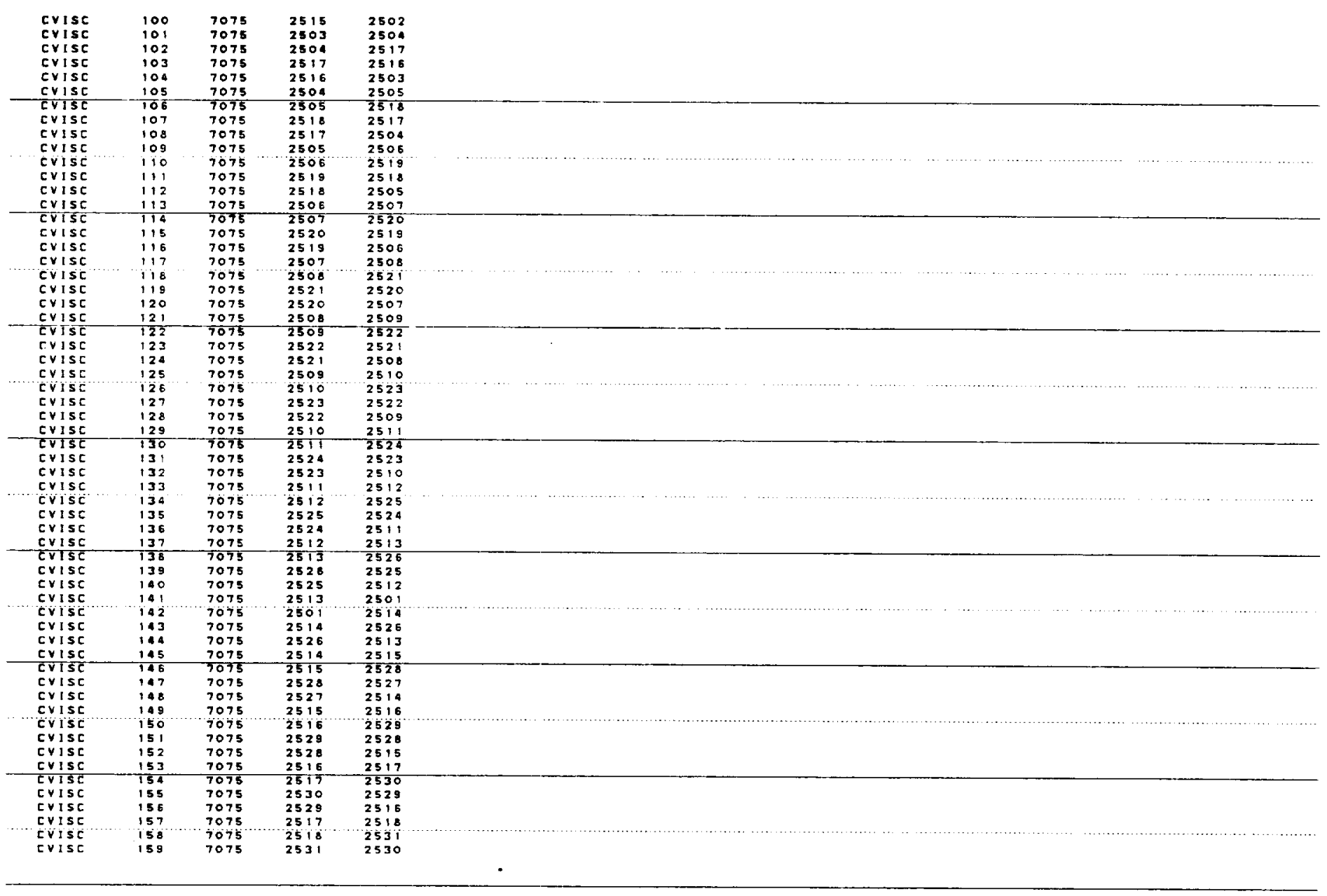

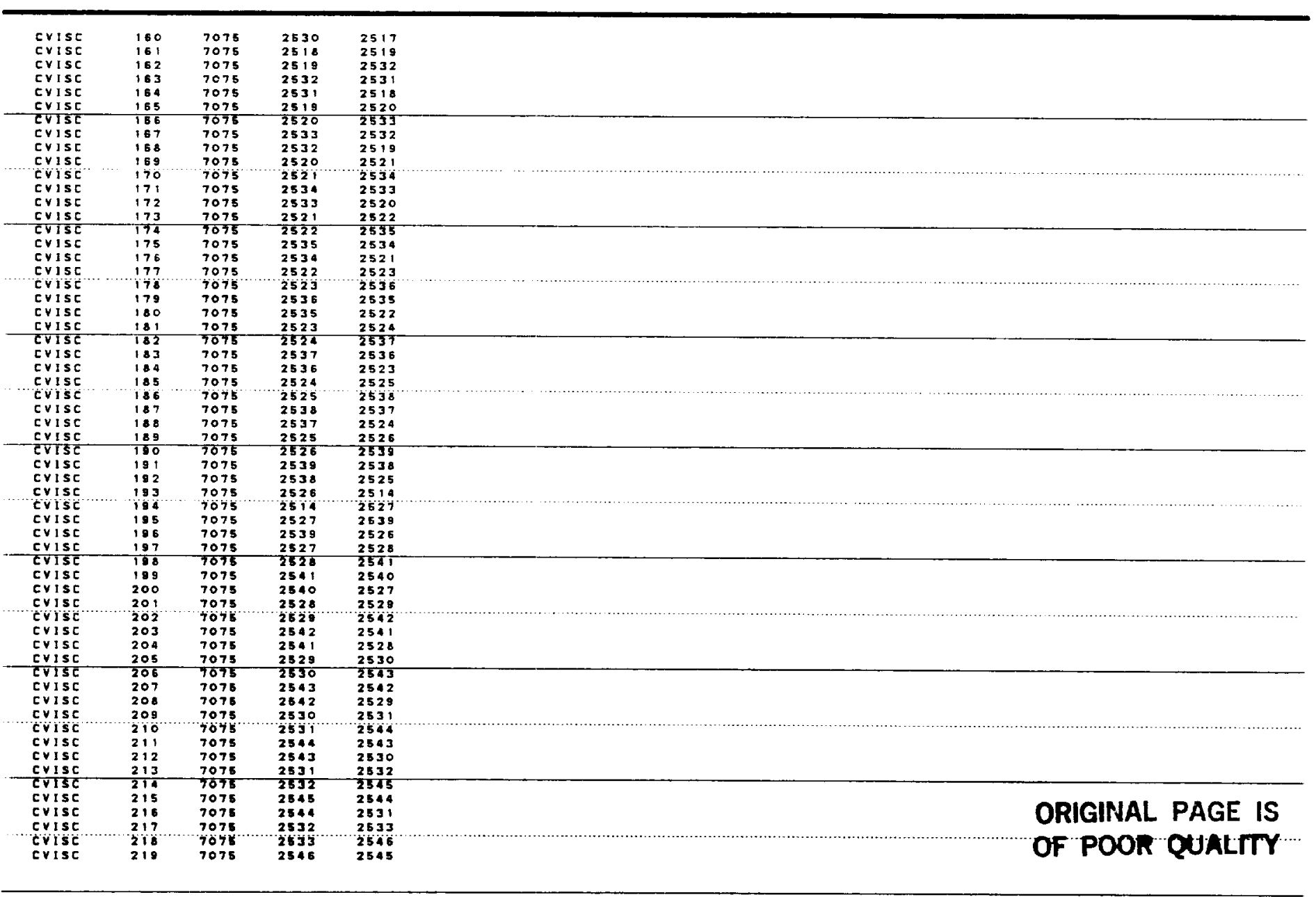




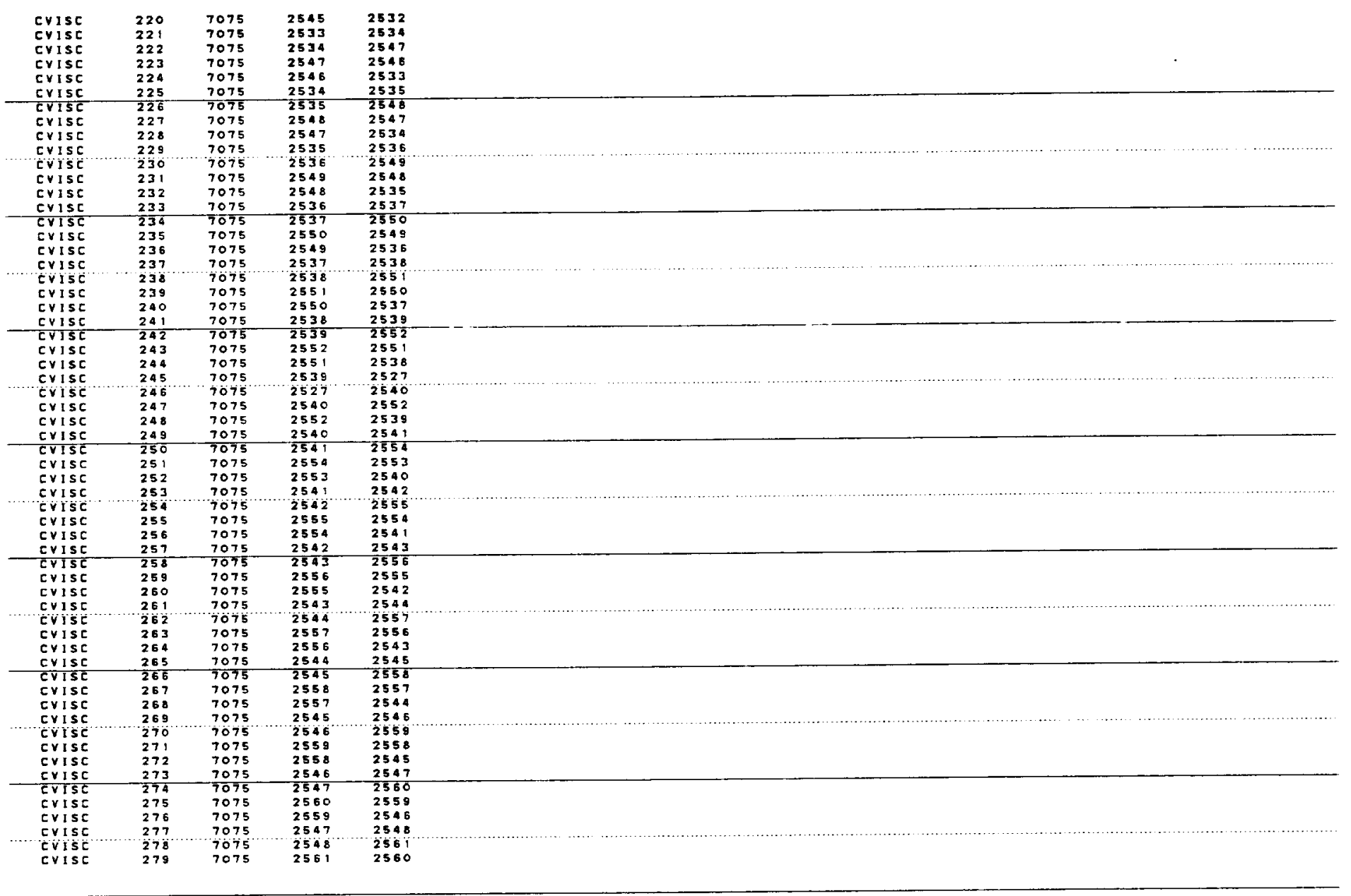

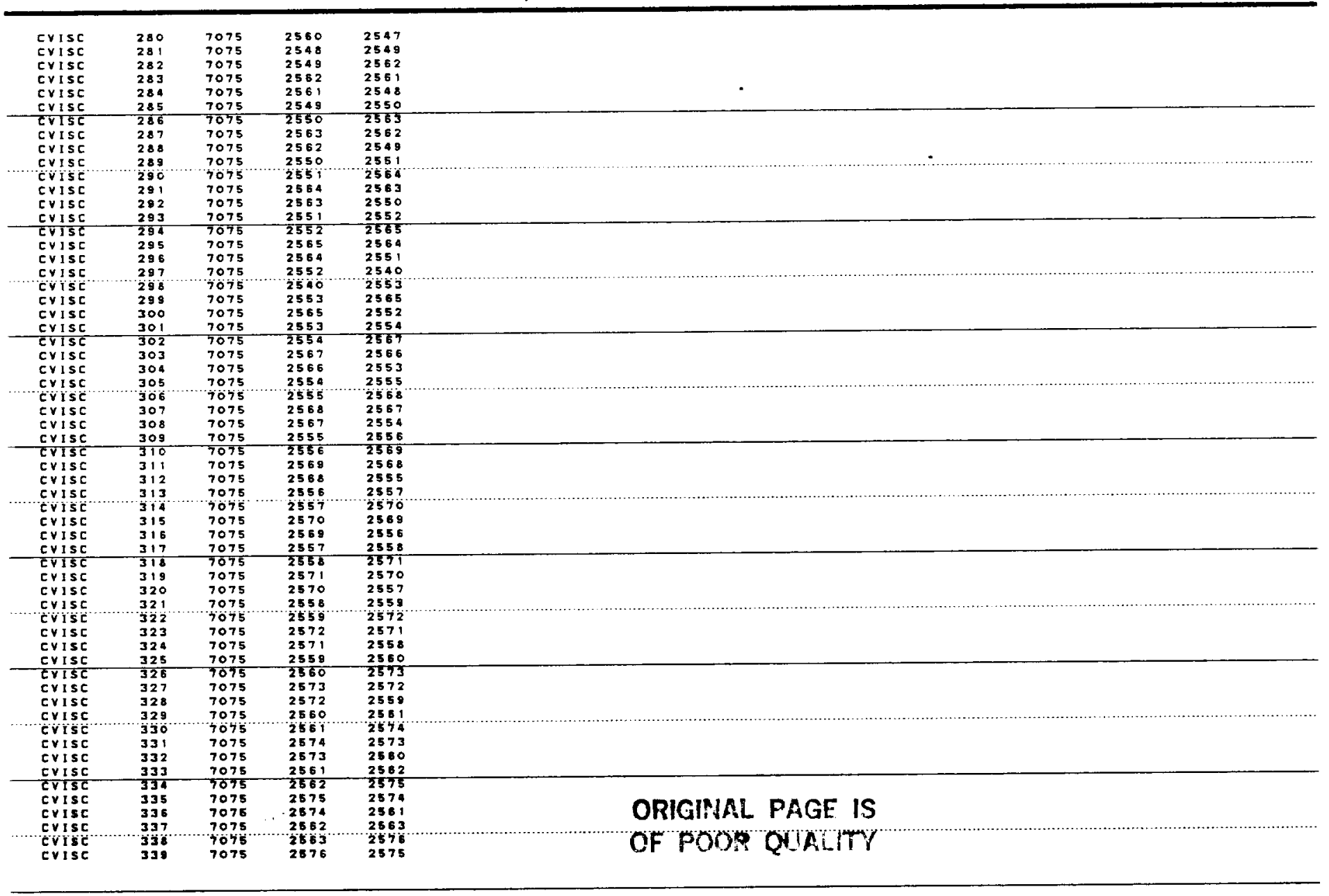




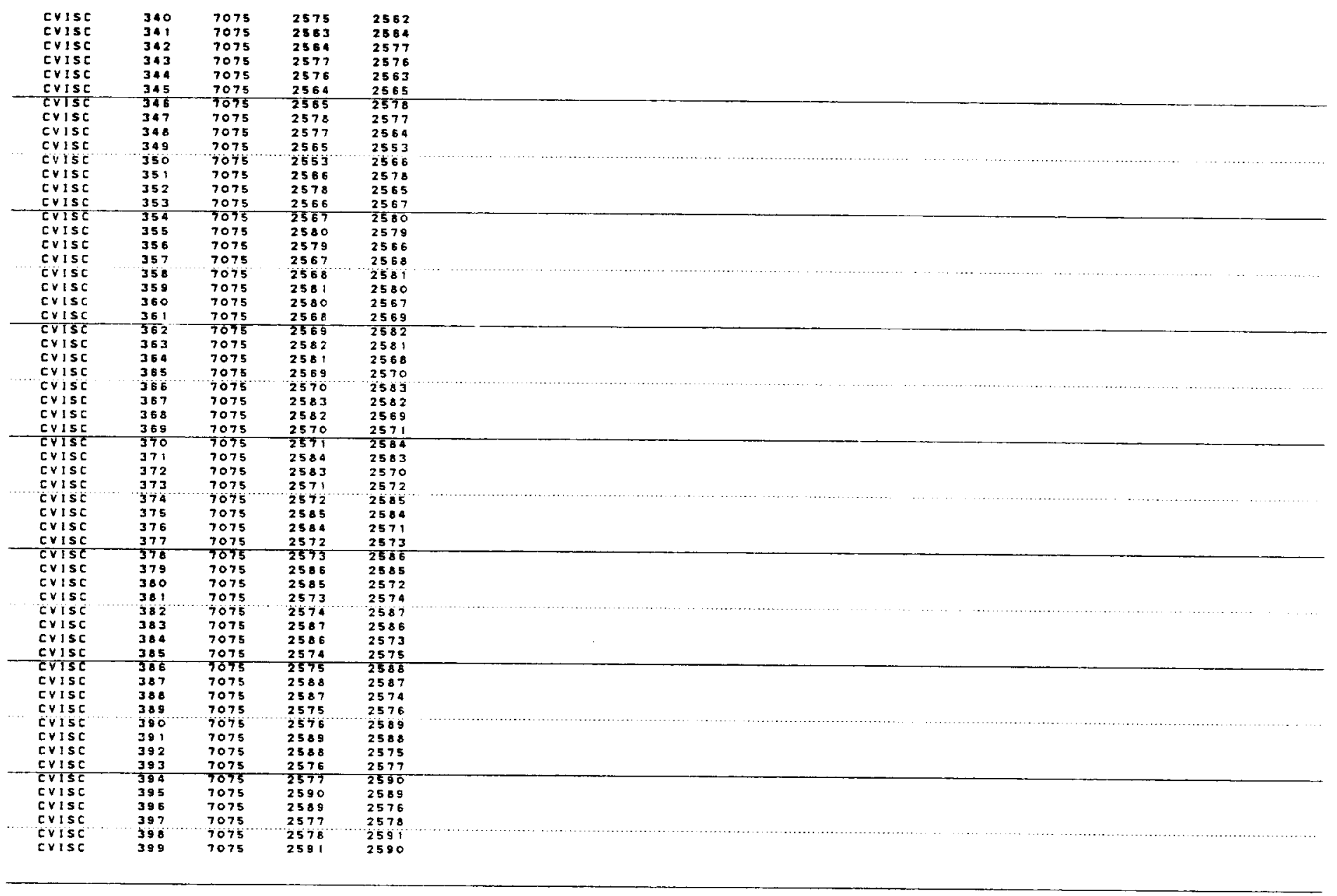

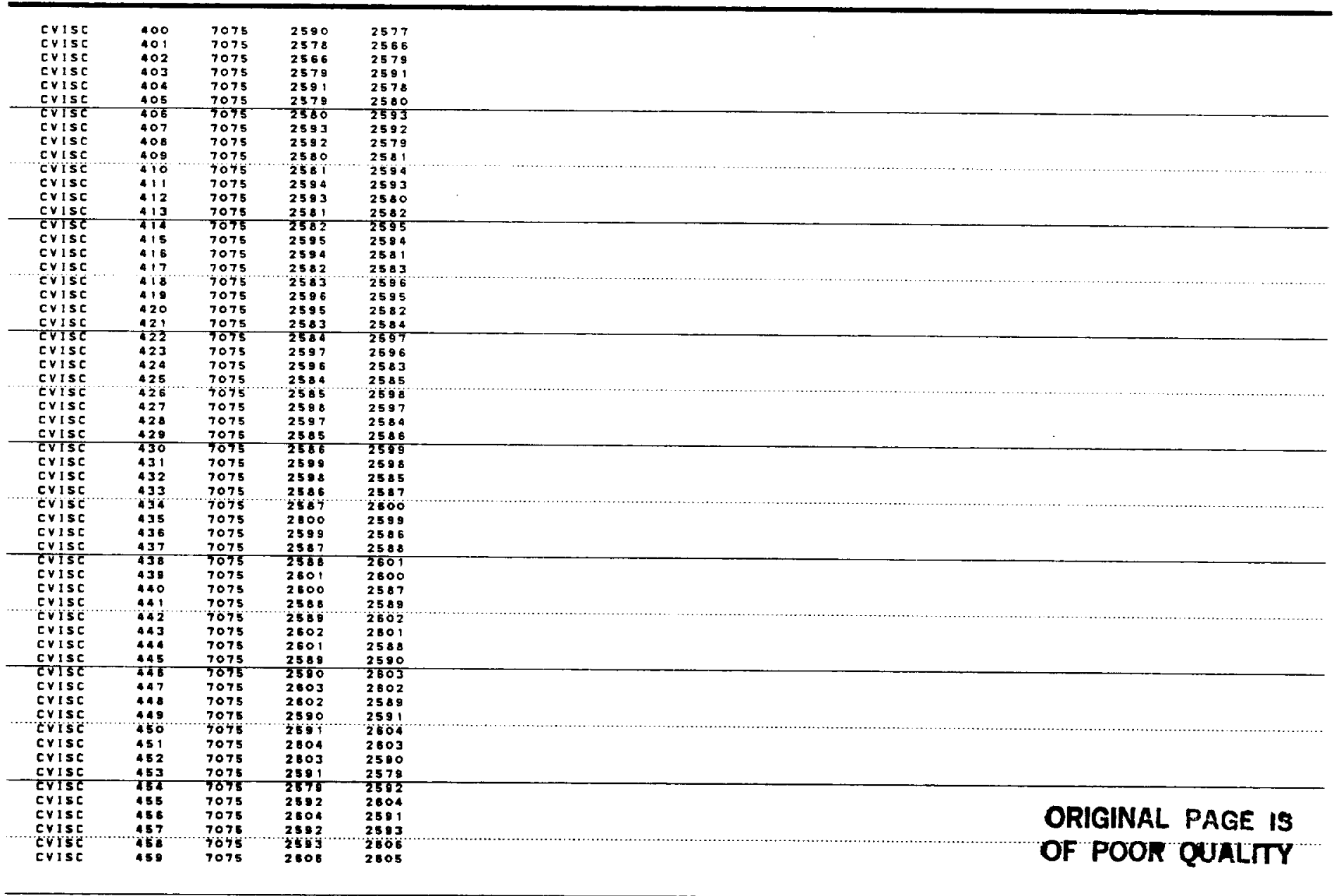




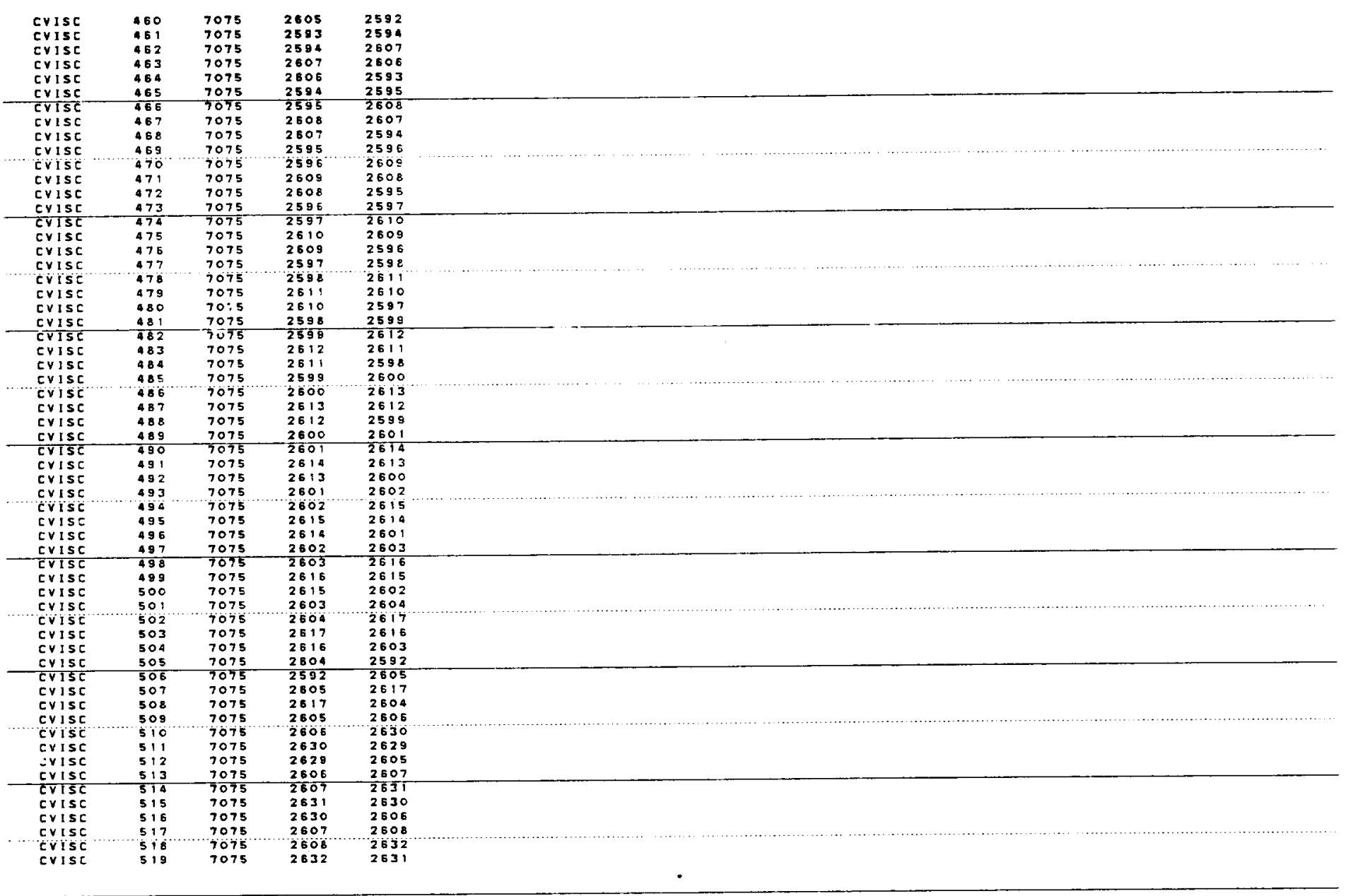

\begin{tabular}{|c|c|c|c|c|c|}
\hline 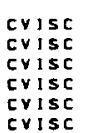 & $\begin{array}{l}\mathbf{5 2 0} \\
5221 \\
522 \\
523 \\
524 \\
524 \\
525\end{array}$ & $\begin{array}{l}7075 \\
7075 \\
7075 \\
7075 \\
7075 \\
7075 \\
075\end{array}$ & $\begin{array}{l}2831 \\
2808 \\
2808 \\
2853 \\
2633 \\
26532 \\
2609\end{array}$ & $\begin{array}{l}2607 \\
2609 \\
2653 \\
2632 \\
2608 \\
2600 \\
2610\end{array}$ & \\
\hline 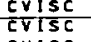 & $-\frac{525}{526}$ & $\frac{1075}{7075}$ & $\frac{2509}{2610}$ & $\frac{2610}{2634}$ & \\
\hline $\begin{array}{l}\text { crisc } \\
\text { crisc } \\
\text { crisc }\end{array}$ & $\begin{array}{l}527 \\
528 \\
529\end{array}$ & $\begin{array}{l}7095 \\
7075 \\
7075\end{array}$ & $\begin{array}{l}2634 \\
2633 \\
2650\end{array}$ & $\begin{array}{l}2633 \\
2609\end{array}$ & \\
\hline $\begin{array}{l}\text { crisc } \\
\text { crise } \\
\text { crise }\end{array}$ & $\begin{array}{l}529 \\
530 \\
531\end{array}$ & $\begin{array}{l}7785 \\
7075 \\
7075\end{array}$ & $\begin{array}{l}2560 \\
2661 \\
2635\end{array}$ & $\begin{array}{l}2511 \\
2635 \\
2534\end{array}$ & \\
\hline $\begin{array}{l}\text { crisc } \\
\text { crisc }\end{array}$ & $\begin{array}{l}532 \\
533\end{array}$ & $\begin{array}{l}7075 \\
7075\end{array}$ & $\begin{array}{l}2634 \\
2611\end{array}$ & $\begin{array}{l}2510 \\
2512\end{array}$ & \\
\hline $\begin{array}{l}c F 1 s c \\
c+15 t \\
c+15 t\end{array}$ & $\begin{array}{c}534 \\
535\end{array}$ & $\begin{array}{l}77075 \\
7075\end{array}$ & $\begin{array}{l}25612 \\
2636\end{array}$ & $\begin{array}{l}2536 \\
2635\end{array}$ & \\
\hline $\begin{array}{l}\text { crisc } \\
c y 1 s c\end{array}$ & $\begin{array}{l}536 \\
537\end{array}$ & $\begin{array}{l}7075 \\
7075\end{array}$ & $\begin{array}{l}2635 \\
2612\end{array}$ & $\begin{array}{l}28: 1 \\
26: 3\end{array}$ & \\
\hline $\begin{array}{ll}\text { Crist } \\
\text { crise }\end{array}$ & $\begin{aligned} 538 \\
539\end{aligned}$ & $\begin{array}{l}7075 \\
7075\end{array}$ & $\begin{array}{l}2613 \\
2637\end{array}$ & $\begin{array}{l}2637 \\
2636\end{array}$ & \\
\hline 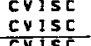 & $\begin{aligned} 540 \\
-547 \\
-593\end{aligned}$ & $\begin{array}{l}7075 \\
7075 \\
7075\end{array}$ & $\begin{array}{l}2536 \\
2513 \\
2813\end{array}$ & $\begin{aligned} 2612 \\
2614 \\
521\end{aligned}$ & \\
\hline 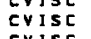 & 543 & 7075 & 2638 & 2837 & \\
\hline 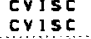 & $\begin{array}{r}544 \\
545\end{array}$ & $\begin{array}{l}7075 \\
7075\end{array}$ & $\begin{array}{l}2637 \\
2614\end{array}$ & $\begin{array}{l}2613 \\
2615\end{array}$ & \\
\hline $\begin{array}{l}\text { prist } \\
\text { cyisc } \\
\text { cyise }\end{array}$ & $\begin{array}{r}546 \\
547\end{array}$ & $\begin{array}{l}7075 \\
7075\end{array}$ & $\begin{array}{l}2665 \\
2639\end{array}$ & $\begin{array}{l}2639 \\
2638\end{array}$ & \\
\hline 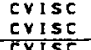 & $\begin{array}{l}548 \\
549 \\
550\end{array}$ & $\begin{array}{l}70075 \\
7075 \\
7078\end{array}$ & $\begin{array}{l}2638 \\
2515 \\
2815\end{array}$ & $\begin{aligned} 2814 \\
2616 \\
5810\end{aligned}$ & \\
\hline $\begin{array}{l}\text { arise } \\
\text { crise }\end{array}$ & $\begin{array}{l}550 \\
551\end{array}$ & $\begin{array}{l}7075 \\
7075\end{array}$ & $\begin{array}{l}26616 \\
2640\end{array}$ & $\begin{array}{l}2640 \\
2639\end{array}$ & \\
\hline $\begin{array}{l}\text { bise } \\
\text { cyise }\end{array}$ & $\begin{array}{r}552 \\
553 \\
55\end{array}$ & $\begin{array}{l}7075 \\
7075\end{array}$ & $\begin{array}{r}2838 \\
2815\end{array}$ & $\begin{array}{l}2615 \\
2617 \\
.261\end{array}$ & \\
\hline 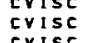 & $\begin{array}{c}554 \\
555 \\
558\end{array}$ & 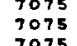 & $\begin{array}{l}2877 \\
2841 \\
2490\end{array}$ & $\begin{array}{l}2641 \\
2640\end{array}$ & \\
\hline 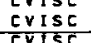 & $\begin{array}{r}550 \\
557\end{array}$ & 7075 & 2617 & $\begin{array}{l}2805 \\
\frac{2805}{2520}\end{array}$ & \\
\hline 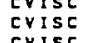 & $\begin{array}{c}558 \\
559 \\
569\end{array}$ & $\begin{array}{l}7075 \\
70075\end{array}$ & $\begin{array}{l}265 \\
2529 \\
289\end{array}$ & $\begin{aligned} 2628 \\
2841 \\
2617\end{aligned}$ & \\
\hline 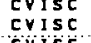 & $\begin{aligned} 560 \\
561 \\
261\end{aligned}$ & $\begin{array}{l}7075 \\
7075 \\
3075\end{array}$ & $\begin{aligned} 284 \\
2829 \\
0.83\end{aligned}$ & $\begin{array}{l}2630 \\
.2830\end{array}$ & \\
\hline $\begin{array}{l}\text { Evise } \\
\text { eyrse } \\
\text { ens }\end{array}$ & $\begin{array}{l}562 \\
563\end{array}$ & $\begin{array}{l}7075 \\
7075 \\
7075\end{array}$ & $\begin{array}{l}2630 \\
254 \\
253\end{array}$ & $\begin{array}{l}2644 \\
2843 \\
3843\end{array}$ & \\
\hline 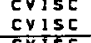 & $\begin{array}{l}564 \\
585 \\
\end{array}$ & $\begin{array}{l}7075 \\
7075\end{array}$ & $\begin{array}{l}2843 \\
2528 \\
25850\end{array}$ & $\begin{array}{r}2629 \\
2857 \\
-\end{array}$ & \\
\hline $\begin{array}{l}\text { Crist } \\
\text { evise }\end{array}$ & $\begin{array}{l}566 \\
567\end{array}$ & $\begin{array}{l}7075 \\
7075\end{array}$ & $\begin{array}{l}2857 \\
2878\end{array}$ & $\begin{array}{l}2578 \\
2677\end{array}$ & \\
\hline 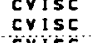 & $\begin{array}{l}568 \\
569\end{array}-12$ & $\begin{array}{l}7075 \\
.7075\end{array}$ & $\begin{array}{l}2877 \\
26530\end{array}$ & $\begin{array}{r}2529 \\
2631\end{array}$ & \\
\hline $\begin{array}{l}\text { hat } \\
\text { cyisc }\end{array}$ & $\begin{array}{l}570 \\
571 \\
571\end{array}$ & $\begin{array}{l}7075 \\
7075 \\
007\end{array}$ & $\begin{array}{l}2631 \\
2845\end{array}$ & $\begin{array}{l}2645 \\
2644\end{array}$ & \\
\hline $\begin{array}{ll}\text { Crisc } \\
\text { cerse }\end{array}$ & 573 & & & 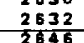 & \\
\hline 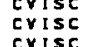 & $\begin{array}{c}574 \\
575 \\
578\end{array}$ & 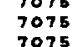 & 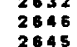 & $\begin{array}{l}2646 \\
2545 \\
2831\end{array}$ & \\
\hline $\begin{array}{l}\text { crisc } \\
\text { crist } \\
\text { cists }\end{array}$ & $\begin{array}{c}577 \\
548 \\
548\end{array}$ & $\begin{array}{l}7075 \\
7045 \\
7045\end{array}$ & $\begin{aligned} 2632 \\
2833 \\
283\end{aligned}$ & $\begin{aligned} 2633 \\
\ldots 2633 \\
.527\end{aligned}$ & ORFGANAL PAGF IS \\
\hline iris & 578 & 7075 & 2847 & 2846 & OF POOR CNA \\
\hline
\end{tabular}




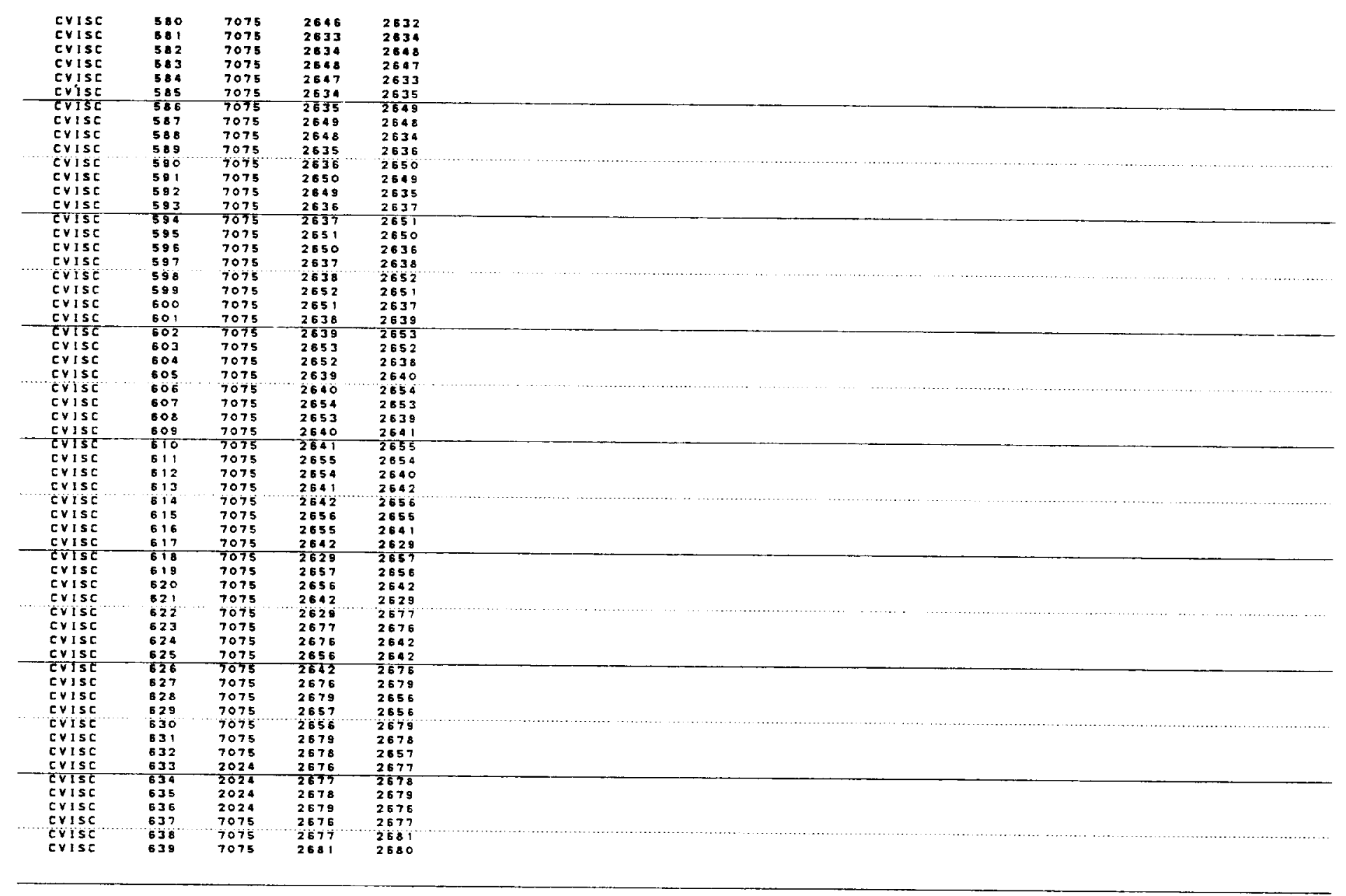

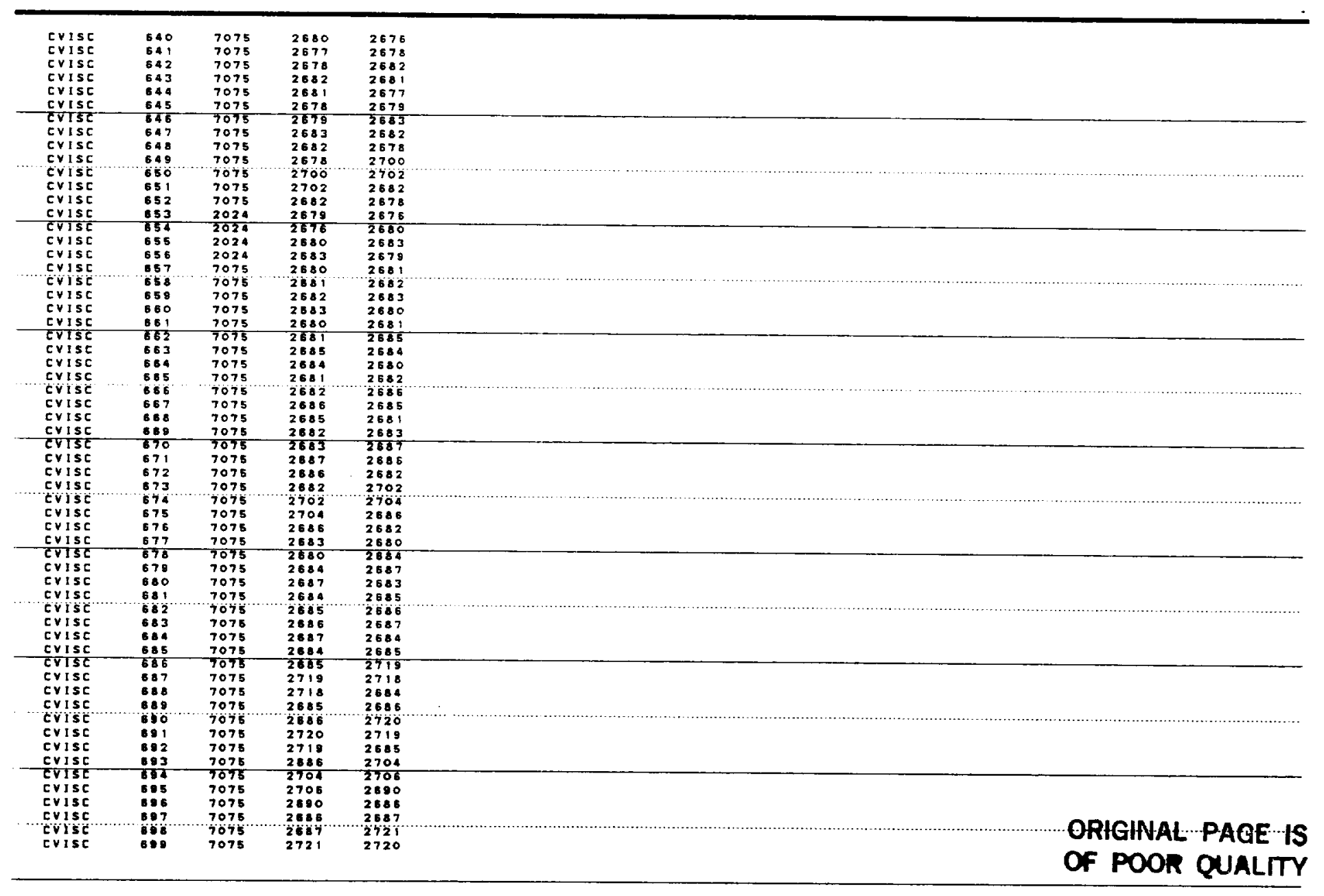




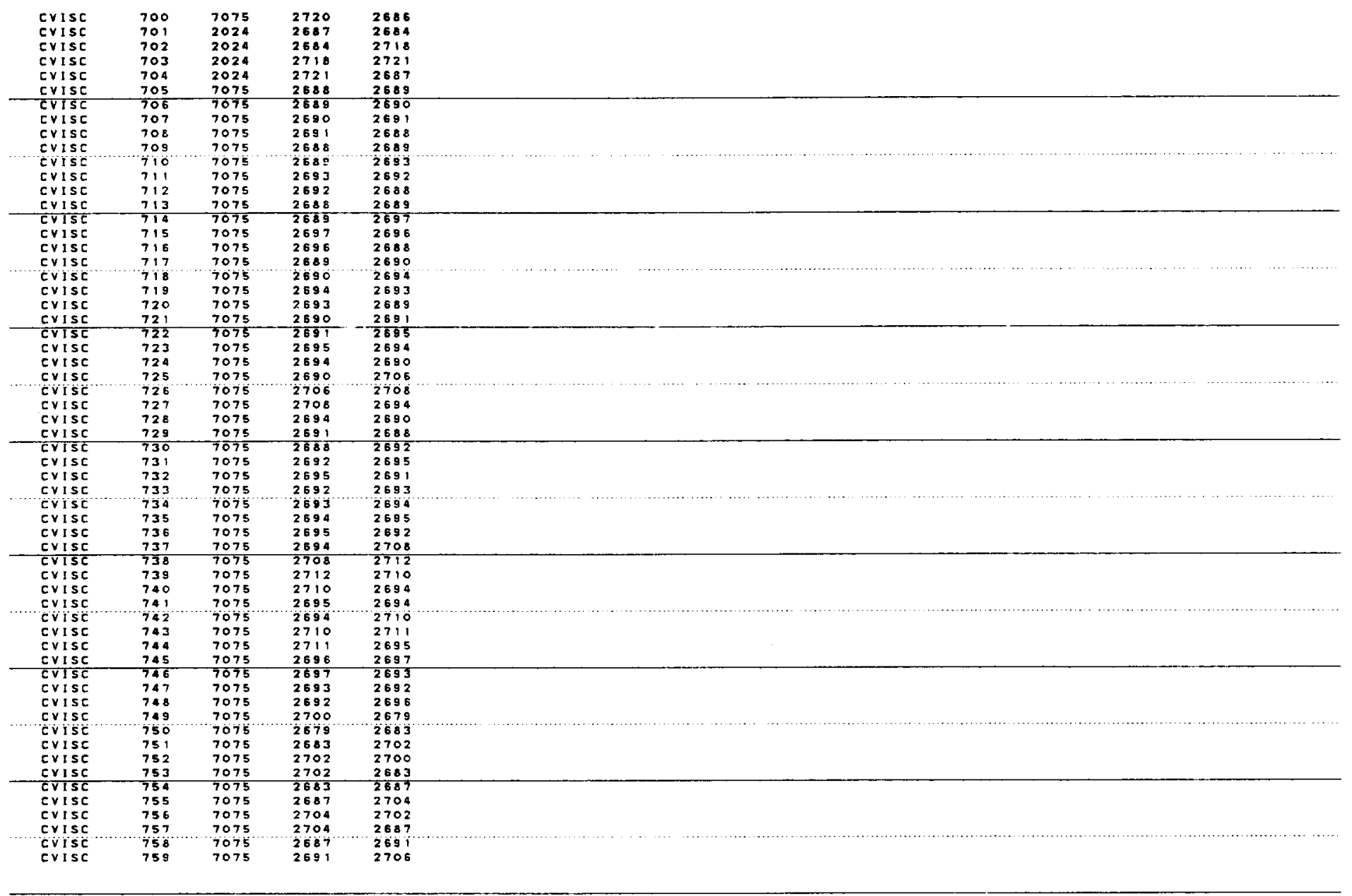

\begin{tabular}{|c|c|c|c|c|c|}
\hline 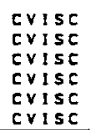 & $\begin{array}{l}760 \\
761 \\
752 \\
763 \\
764 \\
765\end{array}$ & $\begin{array}{l}7075 \\
7075 \\
7075 \\
7075 \\
7075 \\
7075\end{array}$ & $\begin{array}{l}2706 \\
2708 \\
2591 \\
2685 \\
2708 \\
2708 \\
\end{array}$ & $\begin{array}{l}2704 \\
2691 \\
2895 \\
2708 \\
2706 \\
2895\end{array}$ & \\
\hline crise & 766 & 7075 & 2695 & & \\
\hline crisc & 767 & 7075 & 2711 & 2712 & \\
\hline $\begin{array}{l}\text { cvise } \\
\text { crisc }\end{array}$ & $\begin{array}{l}768 \\
769\end{array}$ & $\begin{array}{l}7075 \\
7075\end{array}$ & $\begin{array}{l}2712 \\
2710\end{array}$ & $\begin{array}{l}2708 \\
2712\end{array}$ & \\
\hline $\begin{array}{l}\text { Cuse } \\
\text { cuiser }\end{array}$ & 770 & 7075 & & 2716 & \\
\hline $\begin{array}{l}\text { crisc } \\
\text { crise }\end{array}$ & $\begin{array}{l}771 \\
772\end{array}$ & $\begin{array}{l}7075 \\
7075\end{array}$ & $\begin{array}{l}2716 \\
2714\end{array}$ & $\begin{array}{l}2714 \\
2710\end{array}$ & \\
\hline$\frac{\text { crisc }}{\text { crisc }}$ & $\frac{773}{774}$ & $\begin{array}{l}7075 \\
9075\end{array}$ & $\frac{2712}{2711}$ & $\begin{array}{l}2711 \\
2715\end{array}$ & \\
\hline crisc & 775 & 7075 & 2715 & 2716 & \\
\hline $\begin{array}{l}\text { crise } \\
\text { crise }\end{array}$ & $\begin{array}{l}776 \\
777\end{array}$ & $\begin{array}{l}7075 \\
7075\end{array}$ & $\begin{array}{l}2716 \\
2718\end{array}$ & $\begin{array}{l}2712 \\
2719\end{array}$ & \\
\hline $\begin{array}{l}\text { evise } \\
\text { cuise }\end{array}$ & $\begin{array}{l}777 \\
778 \\
779\end{array}$ & $\begin{array}{l}7075 \\
7075 \\
7075\end{array}$ & $\begin{array}{l}2718 \\
2719 \\
2889\end{array}$ & $\begin{array}{r}27839 \\
2888\end{array}$ & \\
\hline $\begin{array}{ll}\text { c is ise } \\
\text { cyisc }\end{array}$ & 780 & $\begin{array}{l}7075 \\
2024\end{array}$ & $\begin{array}{l}2688 \\
2718\end{array}$ & 2718 & \\
\hline 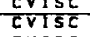 & $\frac{781}{982}$ & $\frac{2024}{2024}$ & $\frac{2718}{2719}$ & $\frac{2719}{2720}$ & \\
\hline $\begin{array}{l}\text { crisc } \\
\text { crise }\end{array}$ & $\begin{array}{l}783 \\
784\end{array}$ & $\begin{array}{l}2024 \\
2024\end{array}$ & $\begin{array}{l}2720 \\
2721\end{array}$ & $\begin{array}{l}2721 \\
2718\end{array}$ & \\
\hline crise & 785 & 7075 & 2719 & 2720 & \\
\hline $\begin{array}{l}\text { EVISE } \\
\text { cVise }\end{array}$ & $\begin{array}{l}786 \\
787\end{array}$ & $\begin{array}{l}7075 \\
7075\end{array}$ & $\begin{array}{l}2720 \\
2590\end{array}$ & $\begin{array}{l}2690 \\
2589\end{array}$ & \\
\hline $\begin{array}{l}\text { eVise } \\
\text { cVise }\end{array}$ & $\begin{array}{l}787 \\
788\end{array}$ & $\begin{array}{l}7075 \\
7075\end{array}$ & $\begin{array}{l}2690 \\
2689\end{array}$ & $\begin{array}{l}2589 \\
2719\end{array}$ & \\
\hline cvise & 789 & $\begin{array}{l}7075 \\
\end{array}$ & $\begin{array}{r}2721 \\
518\end{array}$ & 2718 & \\
\hline $\begin{array}{l}\text { Evise } \\
\text { cyise }\end{array}$ & $\begin{array}{l}780 \\
791\end{array}$ & $\begin{array}{l}7075 \\
7075\end{array}$ & $\begin{array}{l}2718 \\
2588\end{array}$ & $\begin{array}{l}2688 \\
2891\end{array}$ & \\
\hline cyise & 79 & 7075 & 2891 & 2721 & \\
\hline $\begin{array}{l}\text { crisc } \\
\text { citist }\end{array}$ & $\begin{array}{r}783 \\
794\end{array}$ & $\begin{array}{l}7075 \\
7075\end{array}$ & 2720 & $\begin{aligned} 2721 \\
2691\end{aligned}$ & \\
\hline crise & 795 & 7075 & 2591 & 2690 & \\
\hline $\begin{array}{l}\text { crisc } \\
\text { crise }\end{array}$ & $\begin{array}{l}796 \\
797\end{array}$ & $\begin{array}{l}7075 \\
7075\end{array}$ & $\begin{array}{l}2590 \\
2711\end{array}$ & $\begin{array}{l}2720 \\
2710\end{array}$ & \\
\hline crise & 798 & 7075 & 2910 & 2714 & \\
\hline $\begin{array}{l}\text { cyrisc } \\
\text { cyIsc }\end{array}$ & $\begin{array}{l}799 \\
800\end{array}$ & $\begin{array}{l}7075 \\
7075\end{array}$ & $\begin{array}{l}2714 \\
27115\end{array}$ & $\begin{array}{l}2715 \\
2711\end{array}$ & \\
\hline crise & 10091 & 2014 & $\begin{array}{r}24843 \\
\end{array}$ & 13500 & \\
\hline cyisc & $\begin{array}{l}10002 \\
10093\end{array}$ & 2014 & $\begin{array}{l}14847 \\
14883\end{array}$ & $\begin{array}{l}13500 \\
13500\end{array}$ & \\
\hline cyisc & 10094 & 2014 & 14887 & 13500 & \\
\hline $\begin{array}{l}\text { Crisc } \\
\text { CVISE }\end{array}$ & $\frac{20081}{20082}$ & $\frac{2014}{2014}$ & $\begin{array}{l}7033 \\
037\end{array}$ & $\begin{array}{r}8300 \\
3300\end{array}$ & \\
\hline $\begin{array}{l}\text { crisc } \\
\text { cyisc }\end{array}$ & $\begin{array}{l}20083 \\
20084\end{array}$ & 2014 & 9333 & 8300 & \\
\hline $\begin{array}{l}\text { crisc } \\
\text { crise }\end{array}$ & $\begin{array}{r}20084 \\
108001\end{array}$ & $\begin{array}{l}2014 \\
4130\end{array}$ & $\begin{array}{r}1003 \\
125065\end{array}$ & $\begin{array}{r}8300 \\
125387\end{array}$ & \\
\hline $\begin{array}{l}\text { cisisc } \\
\text { cyisc }\end{array}$ & $\begin{array}{l}100001 \\
333141\end{array}$ & 4130 & $\begin{array}{r}123467 \\
3331\end{array}$ & $\begin{array}{r}123487 \\
3341\end{array}$ & \\
\hline $\begin{array}{l}\text { crisc } \\
\text { crisc }\end{array}$ & $\begin{array}{l}333141 \\
333831\end{array}$ & $\begin{array}{l}2024 \\
2024\end{array}$ & $\begin{array}{l}3331 \\
3331\end{array}$ & $\begin{array}{l}3341 \\
3339\end{array}$ & Ofloming \\
\hline $\begin{array}{l}\text { crisc } \\
\text { crist }\end{array}$ & $\frac{333949}{334149}$ & $\frac{2024}{2024}$ & $\frac{3339}{3311}$ & $\frac{3349}{3349}$ & WFGMAL PAGE I \\
\hline $\begin{array}{l}\text { las } \\
\text { crise } \\
\text { crise }\end{array}$ & $\begin{array}{l}512327 \\
612739\end{array}$ & $\begin{array}{l}2024 \\
2024\end{array}$ & $\begin{array}{l}8123 \\
8127\end{array}$ & $\begin{array}{l}6127 \\
6139 \\
8139\end{array}$ & OF FOOR OUALT \\
\hline crise & $\begin{array}{l}813123 \\
.\end{array}$ & $\begin{array}{l}2024 \\
306\end{array}$ & 8123 & & \\
\hline $\begin{array}{l}\text { cyisé } \\
\text { crisc }\end{array}$ & $\begin{array}{l}6131419 \\
613337\end{array}$ & $\begin{array}{l}2024 \\
2024\end{array}$ & $\begin{array}{l}8131 \\
8133\end{array}$ & $\begin{array}{l}8141 \\
8139\end{array}$ & \\
\hline
\end{tabular}




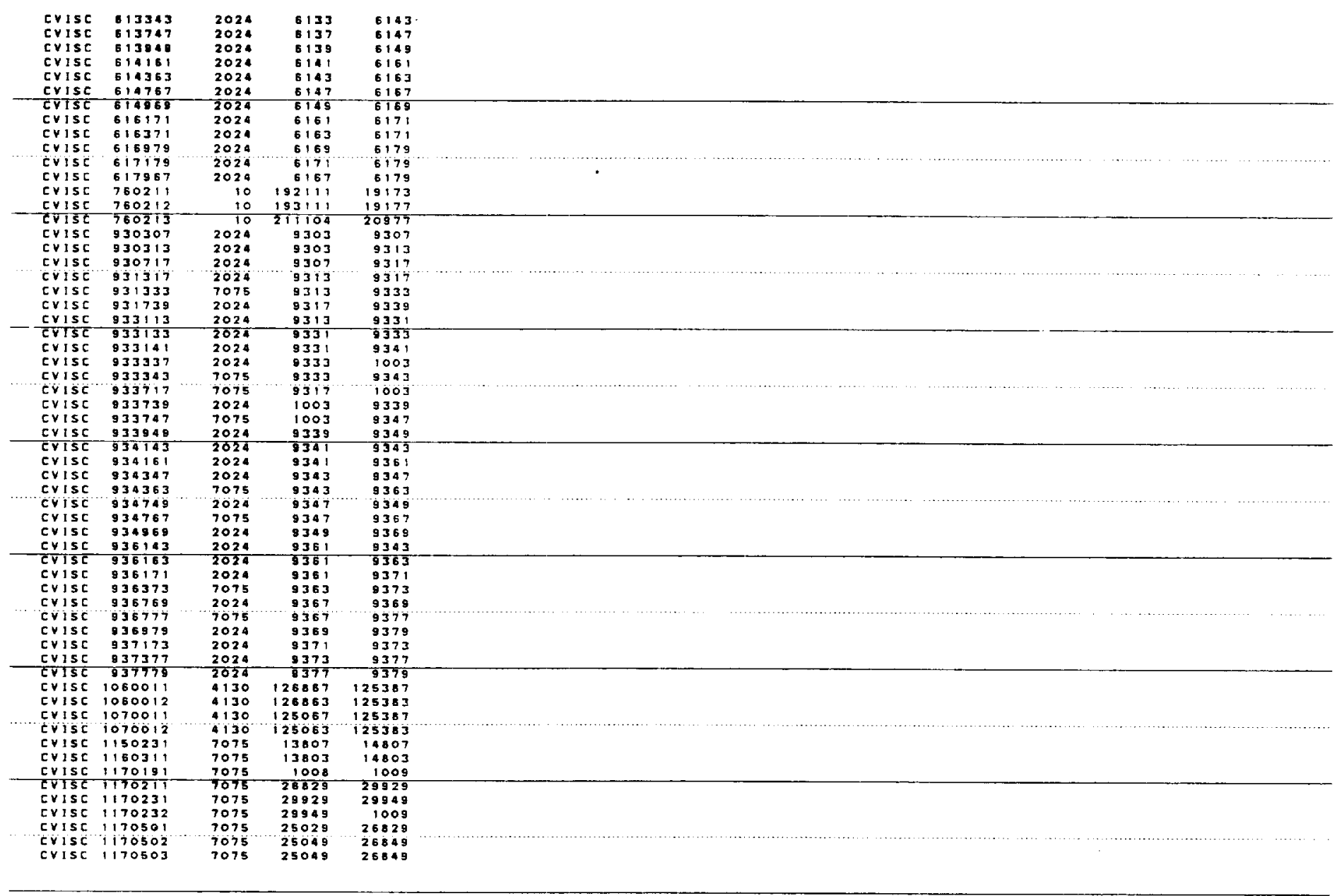

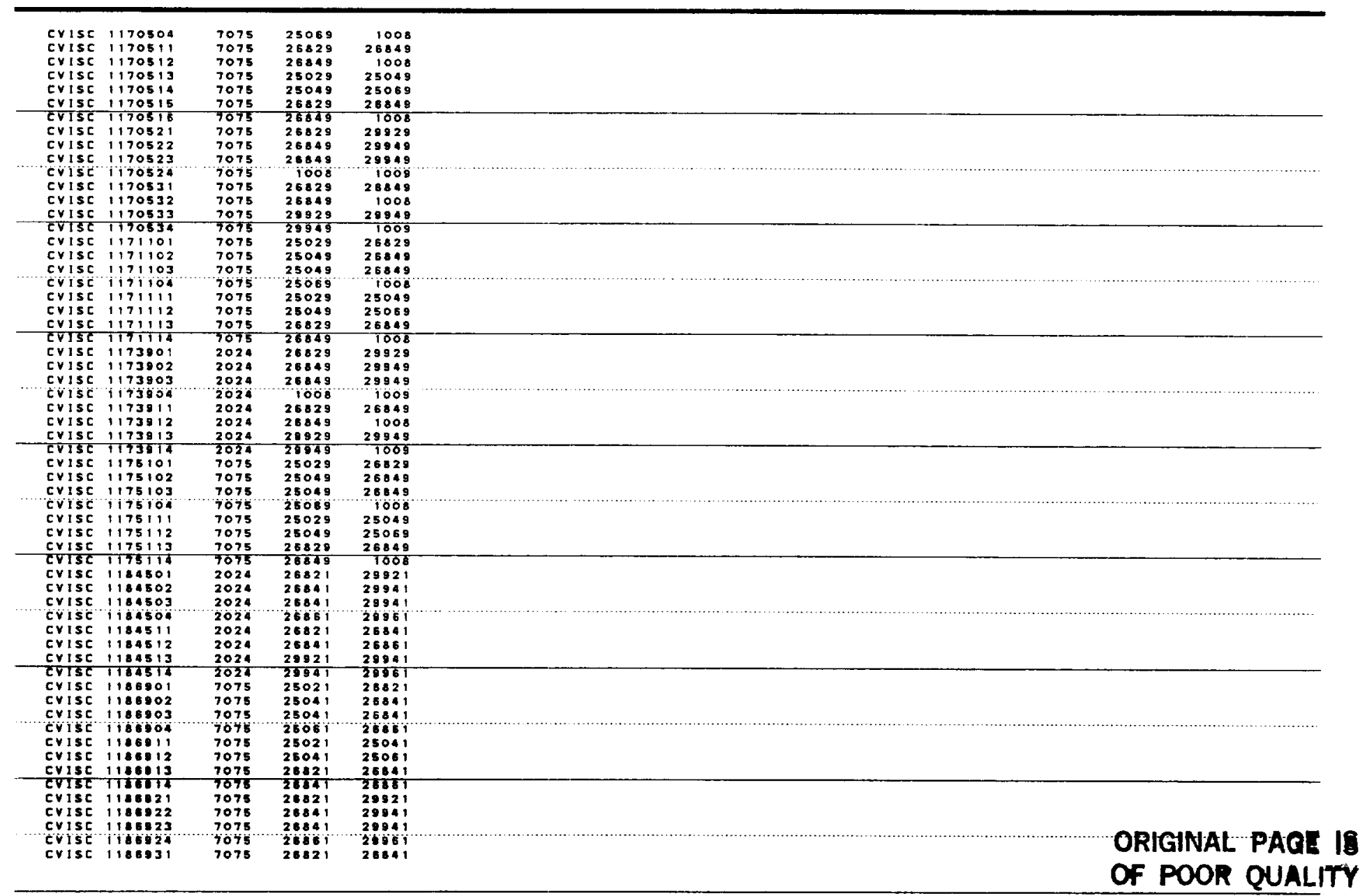




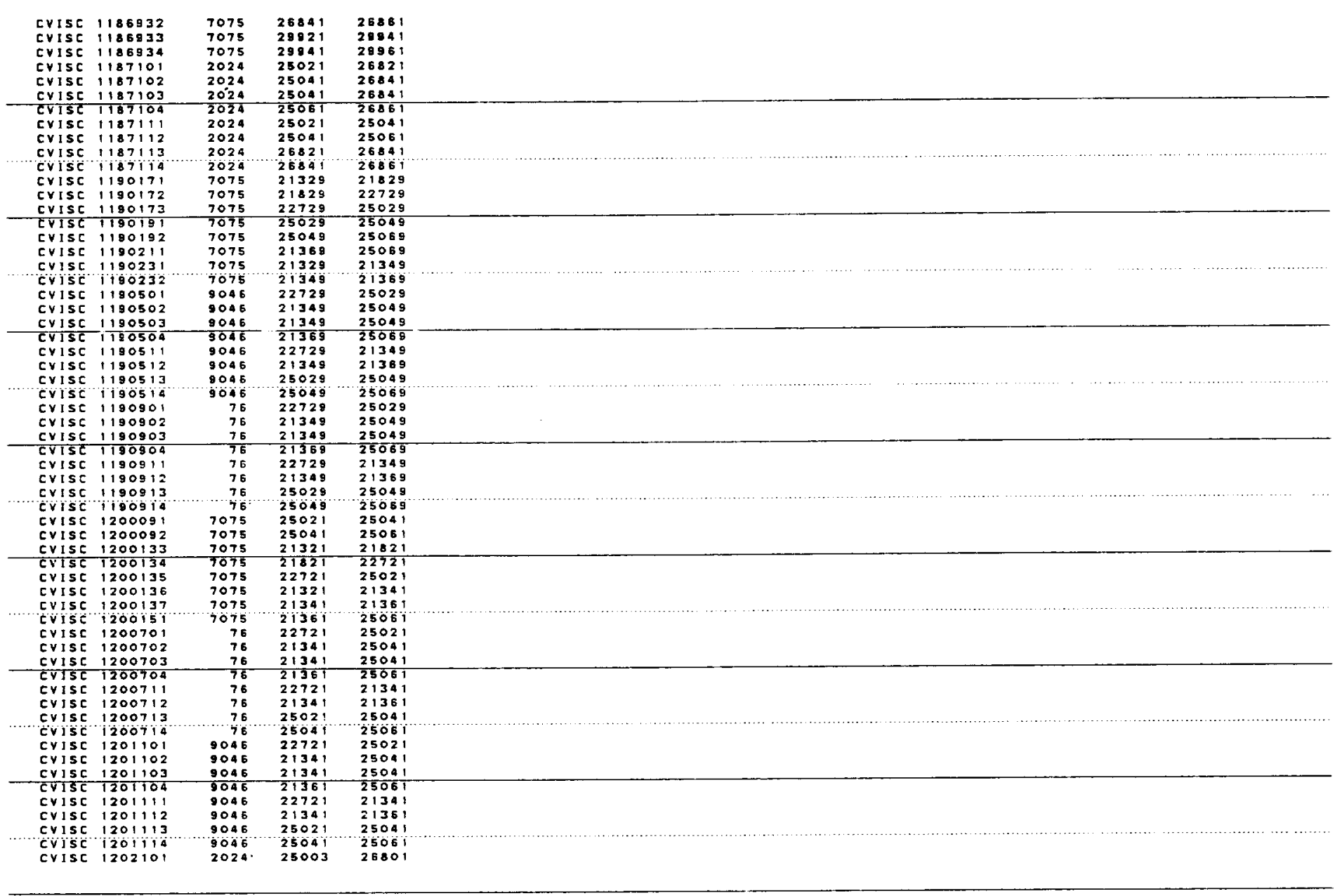

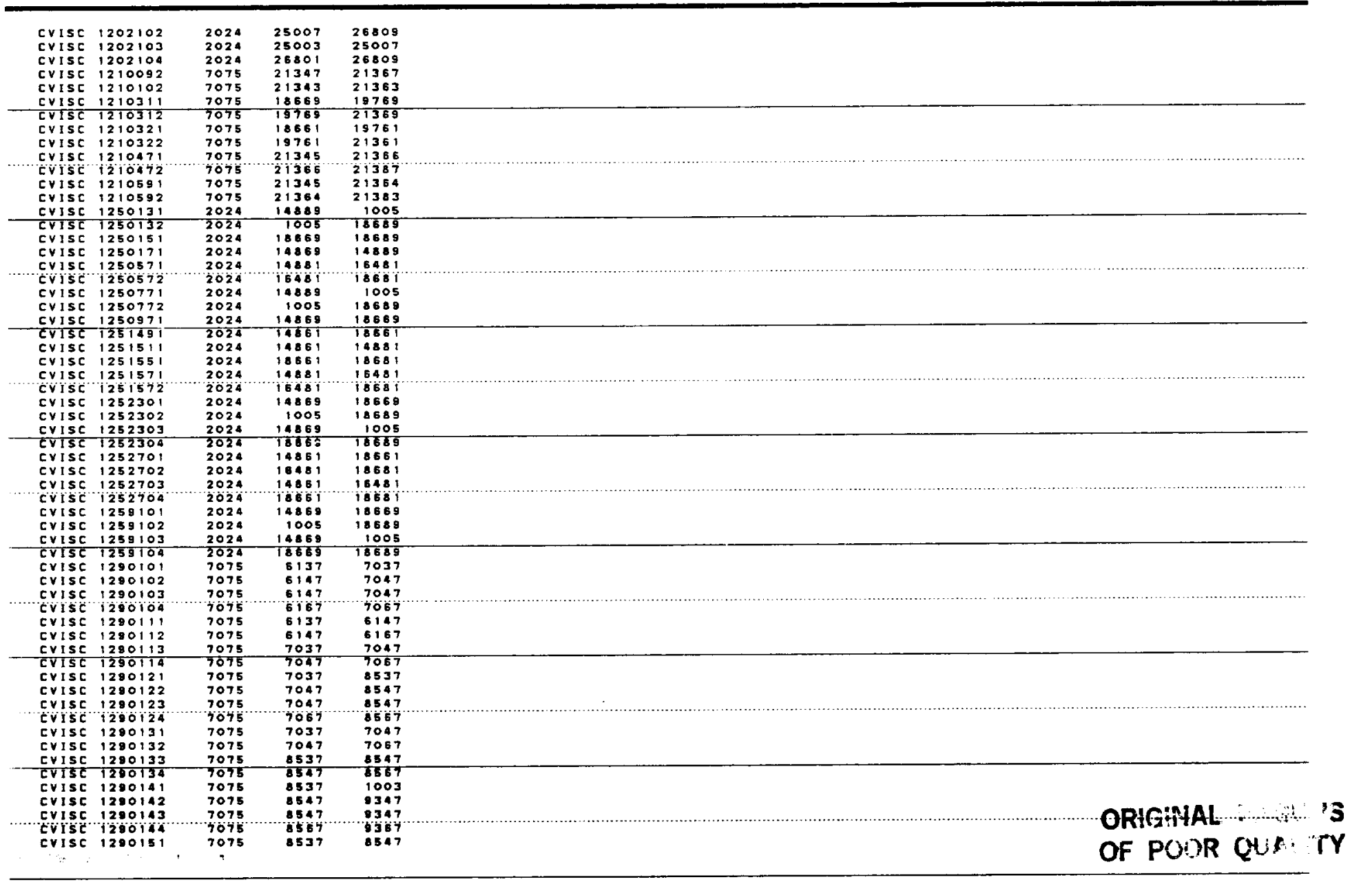




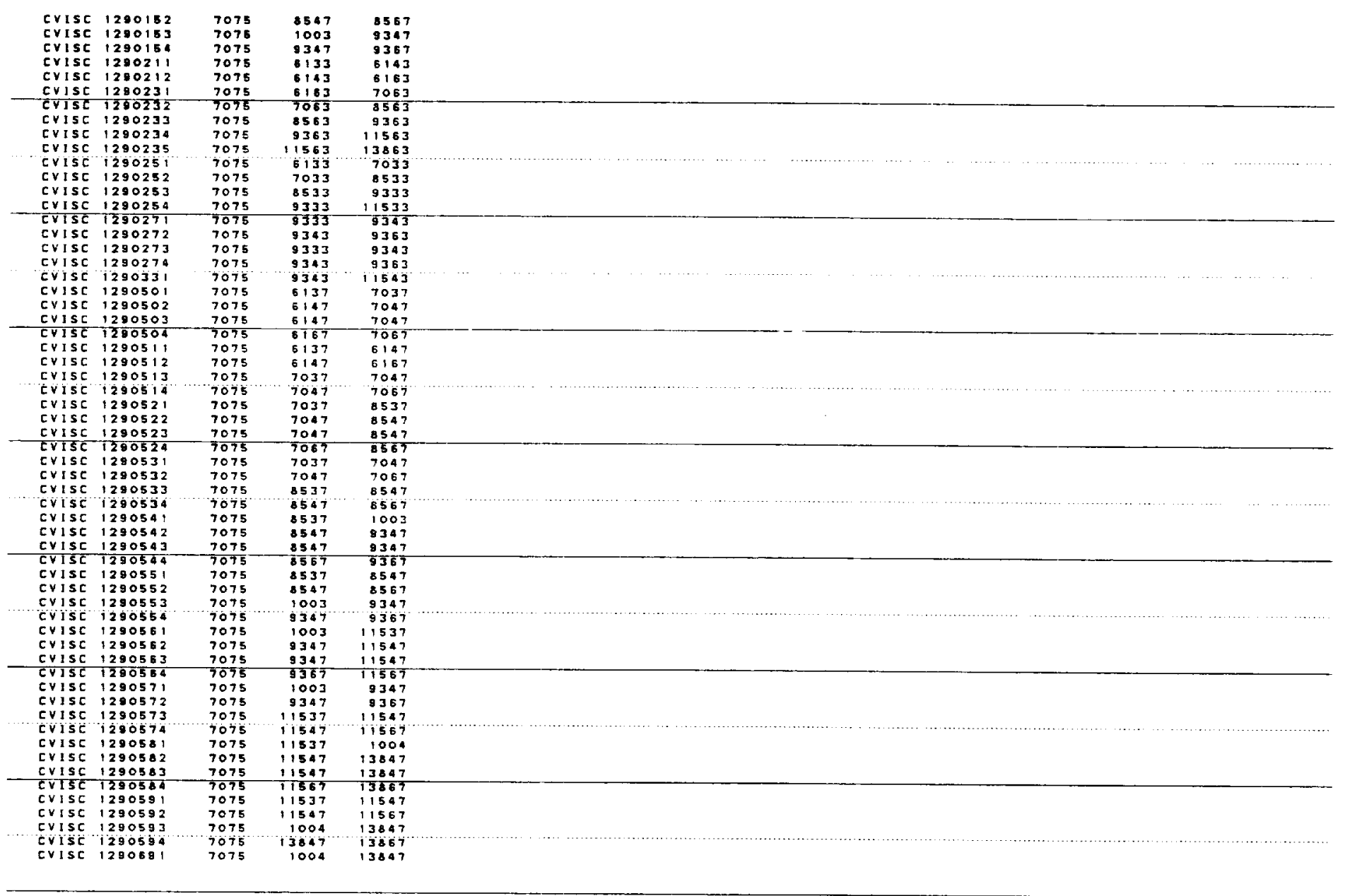

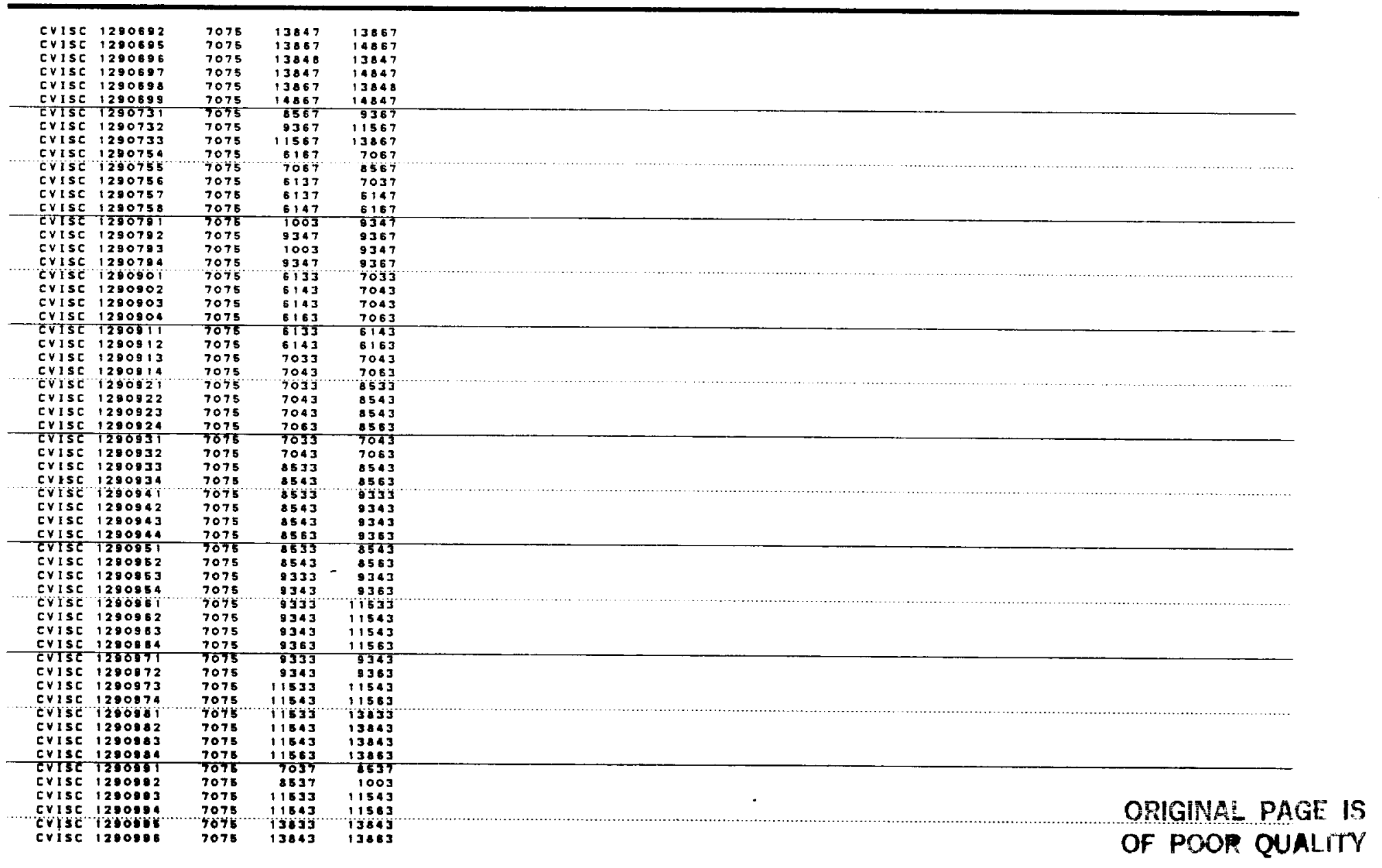




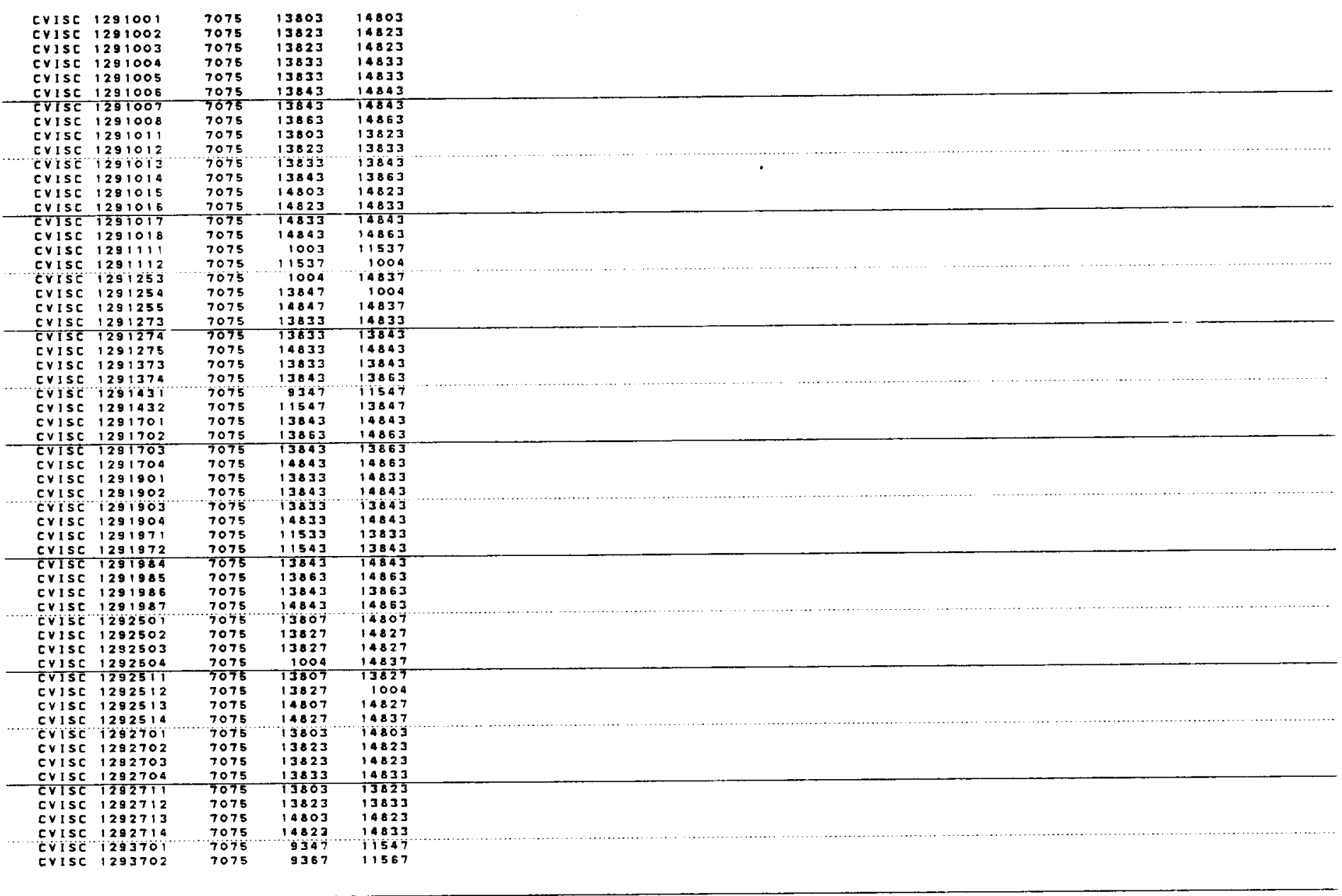

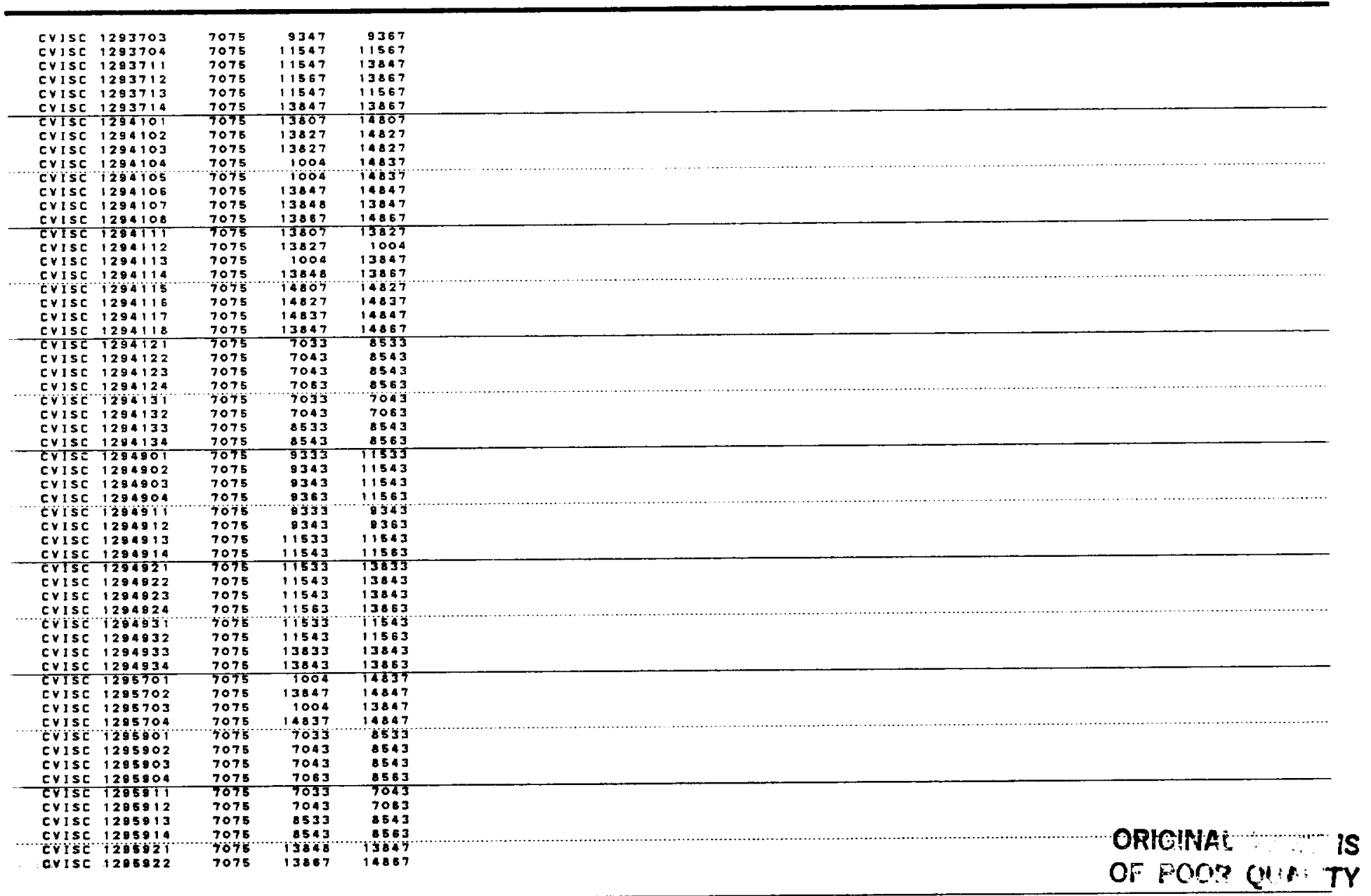




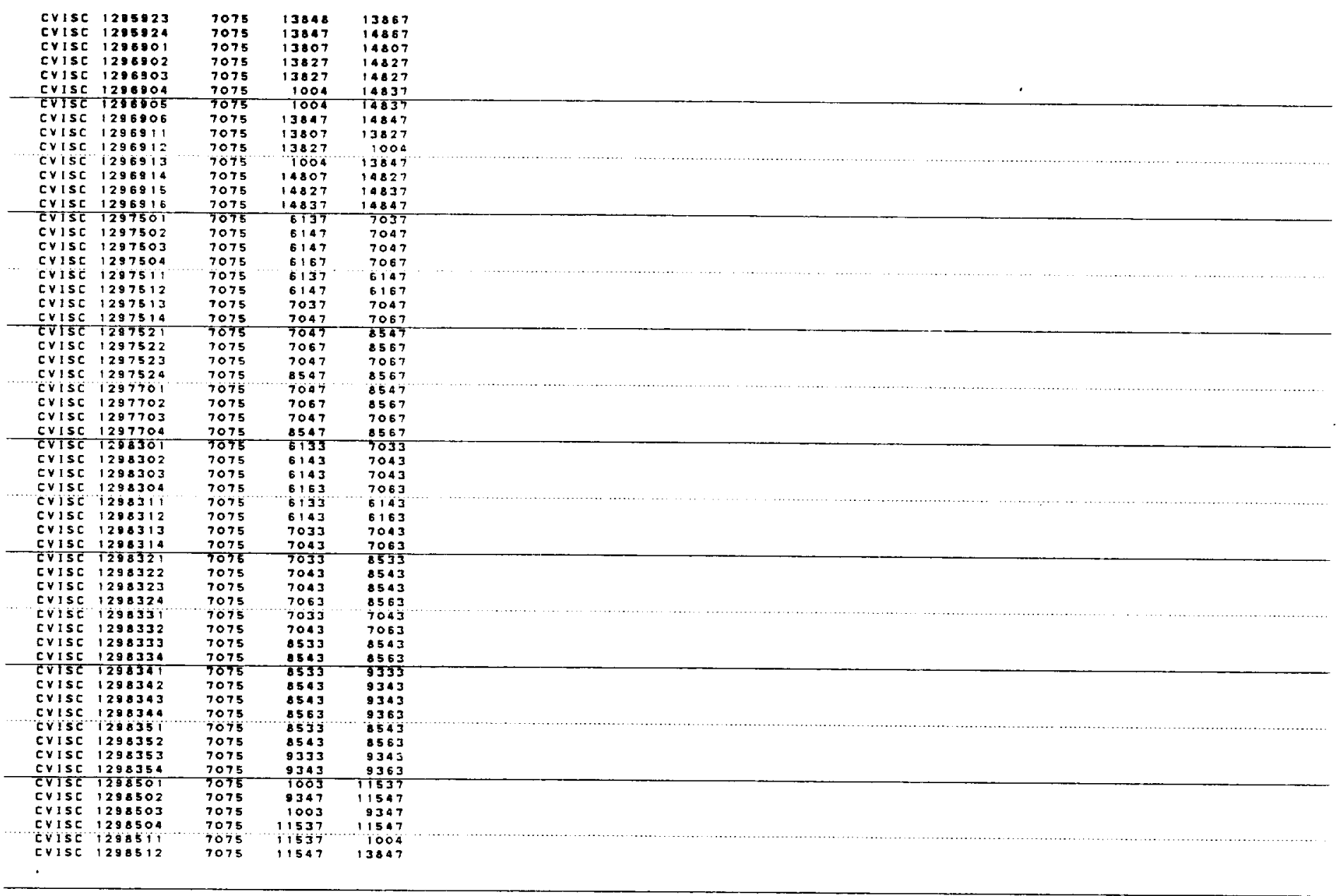

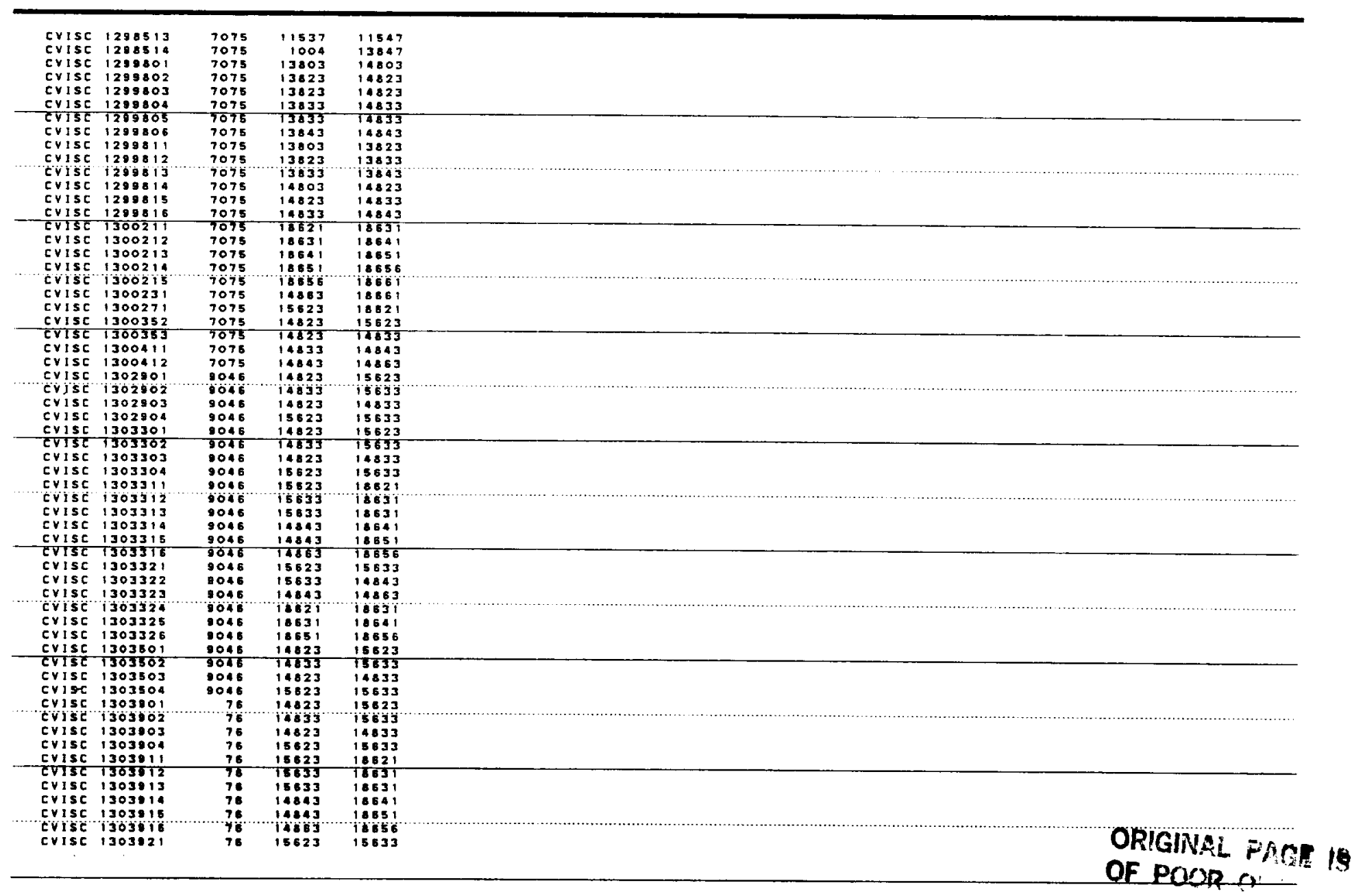




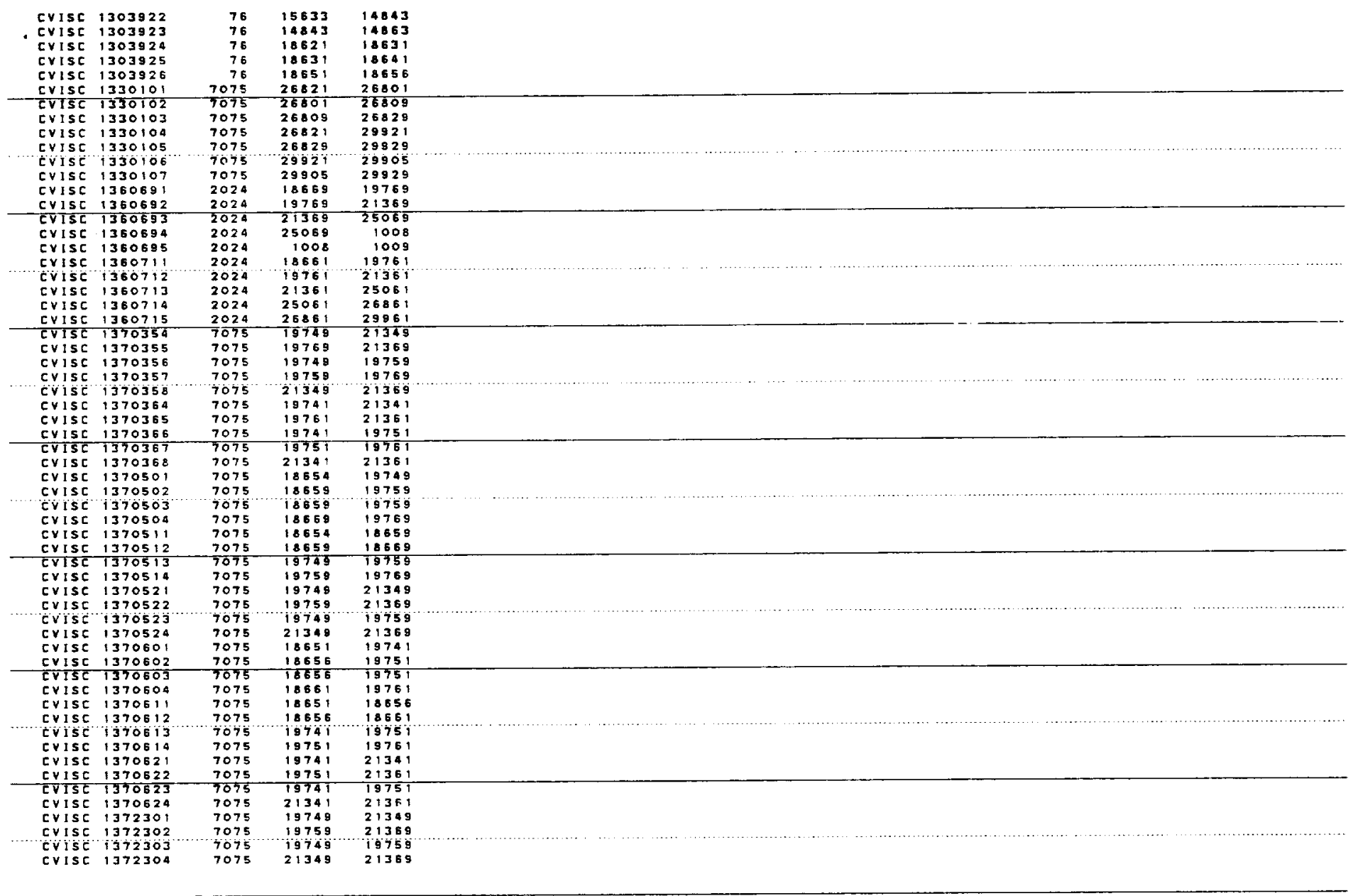

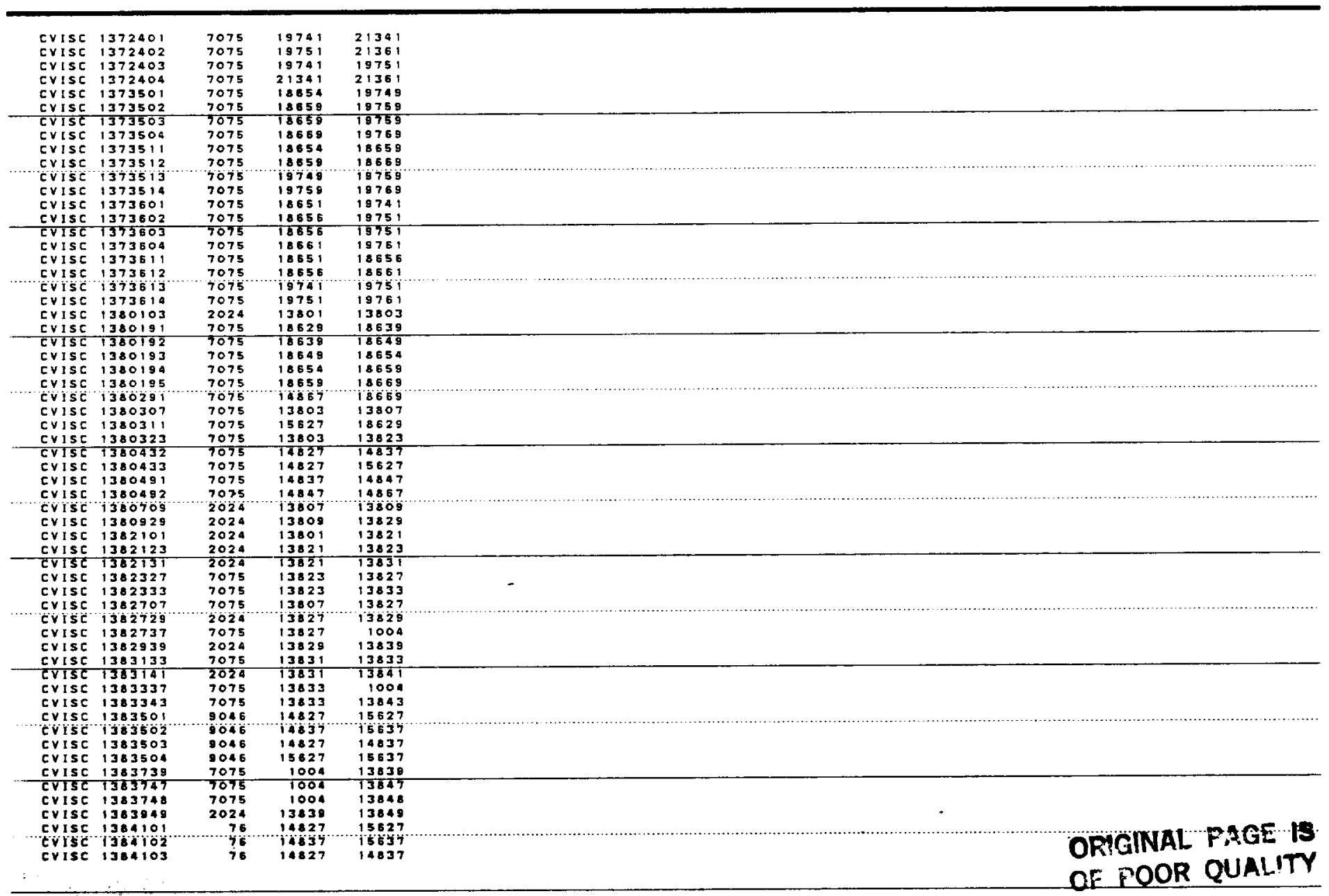




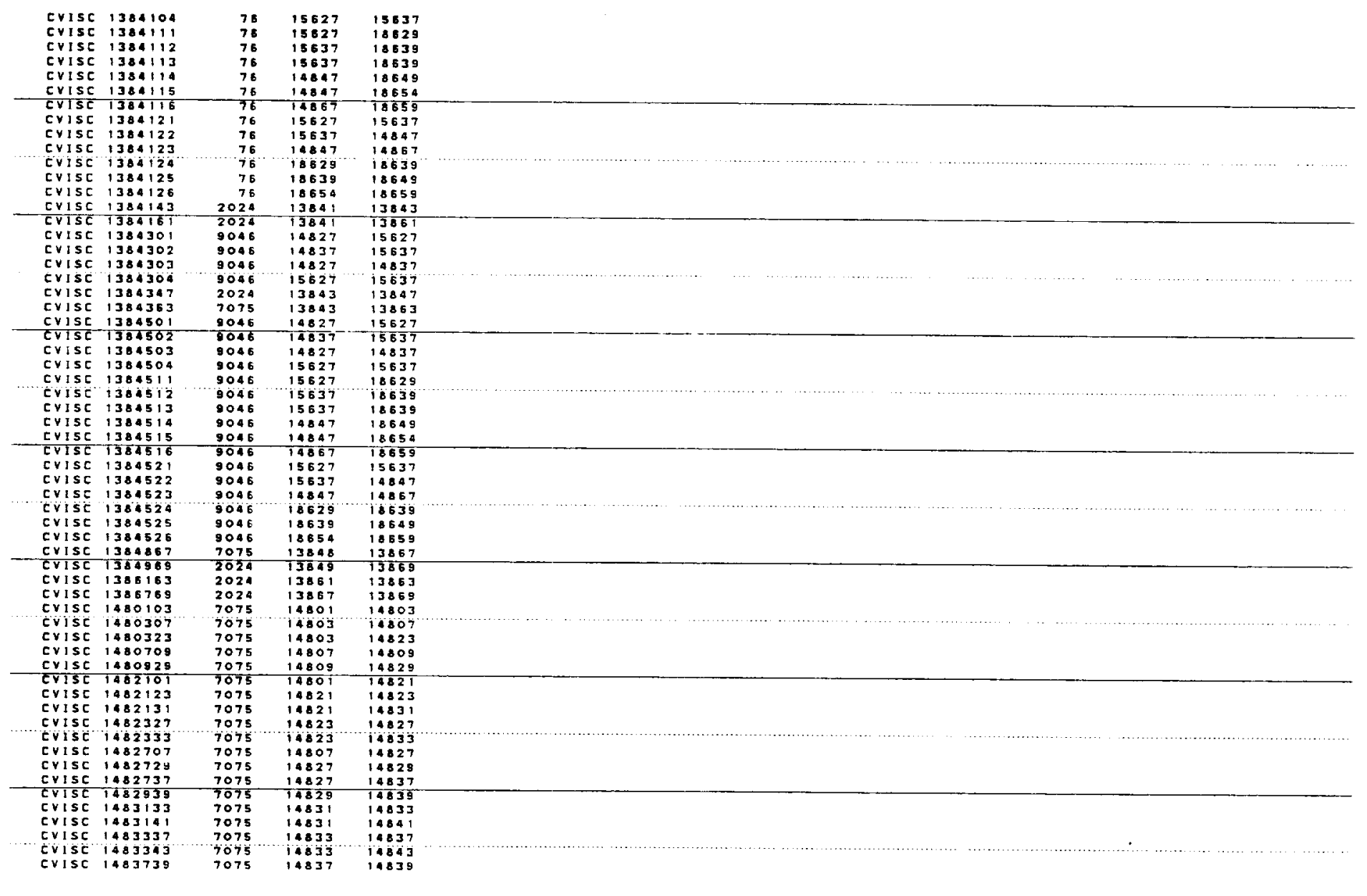

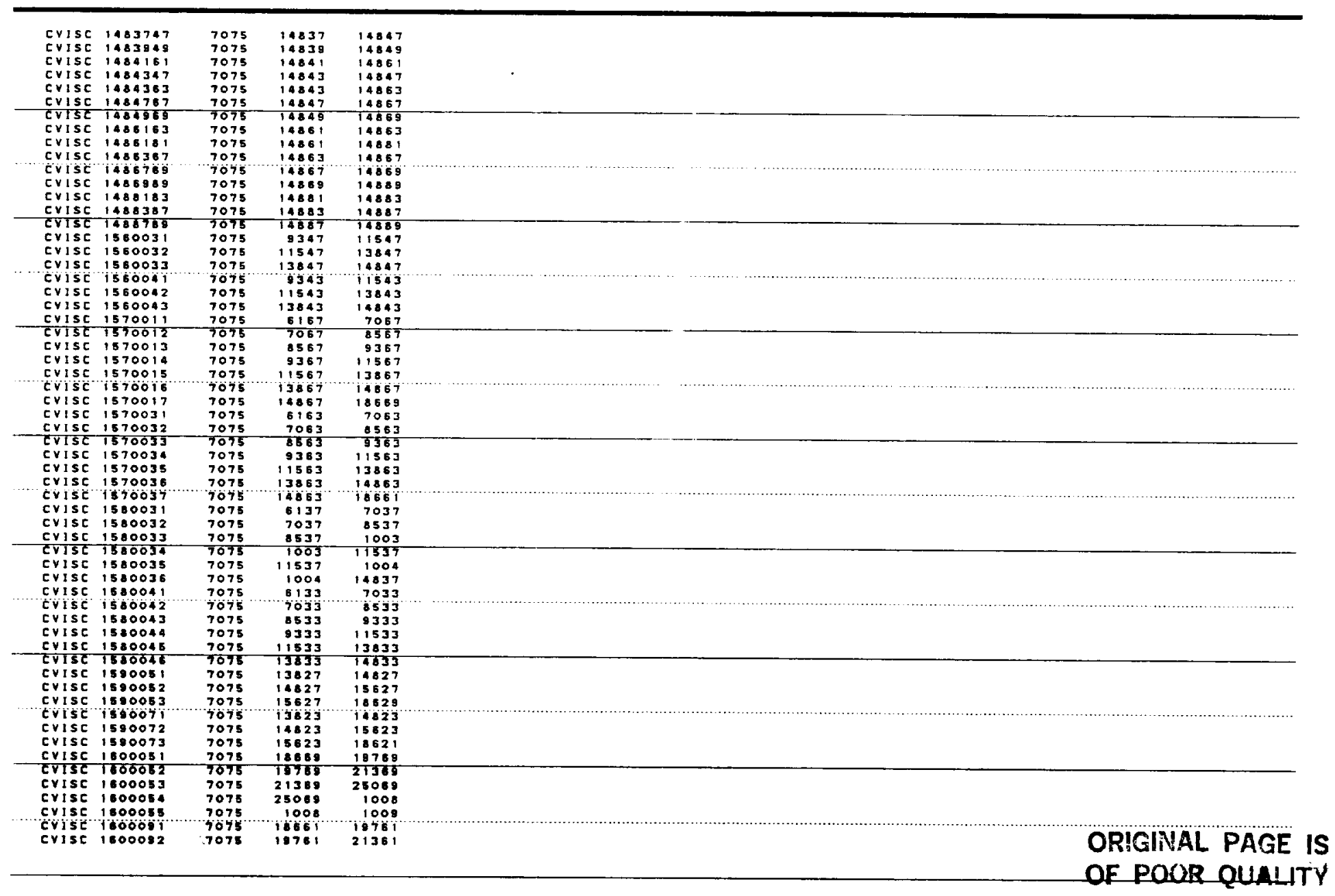




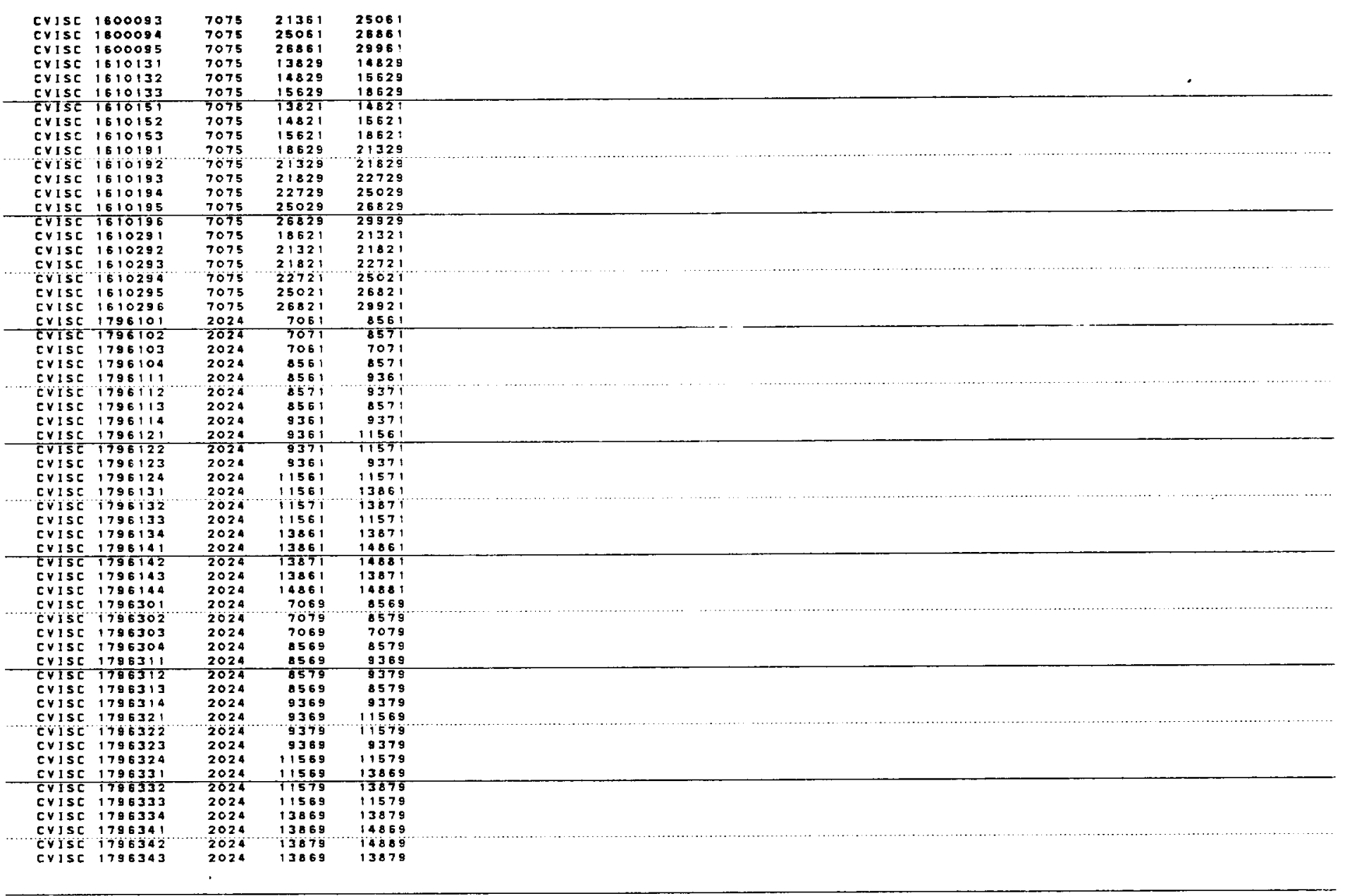

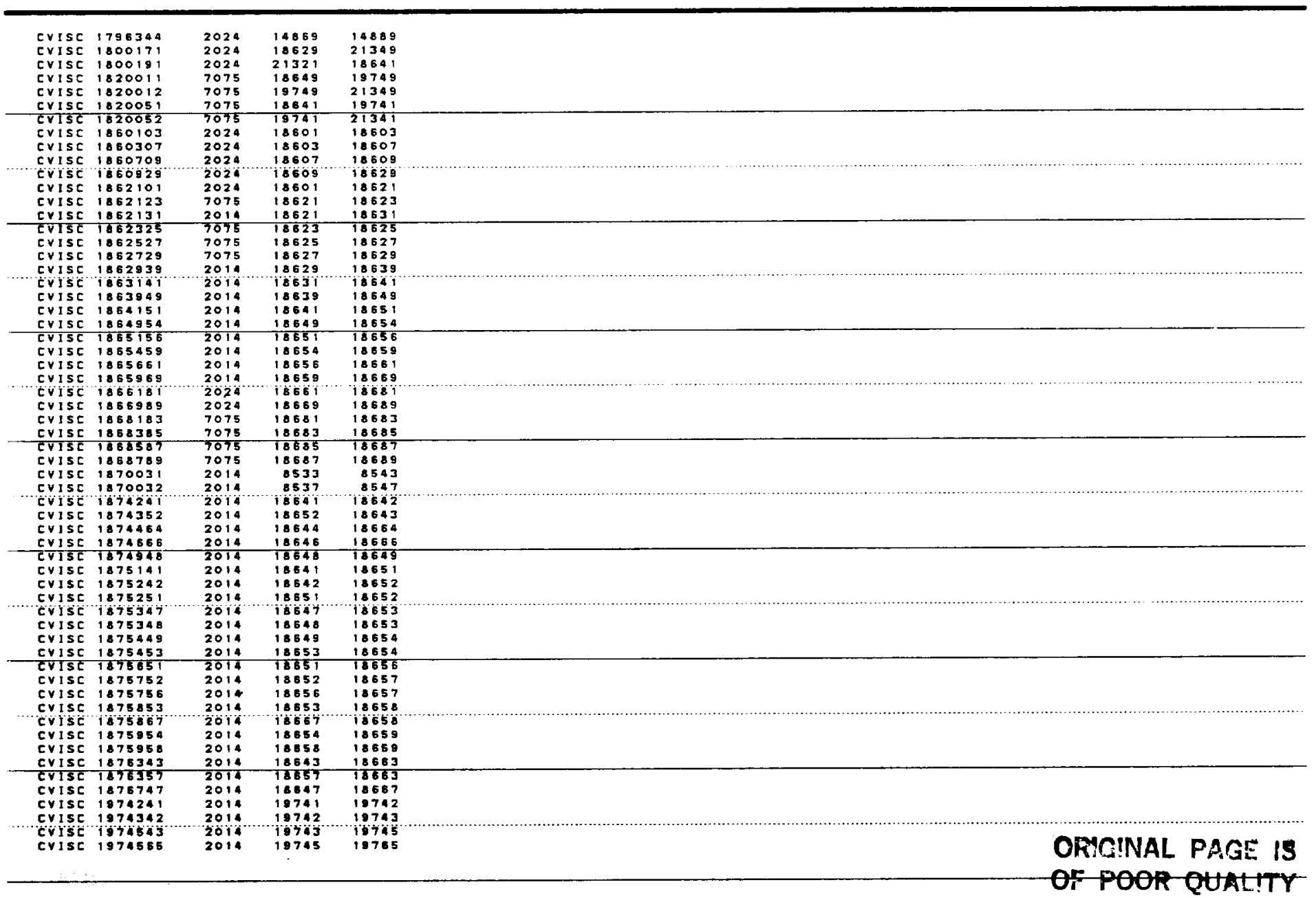




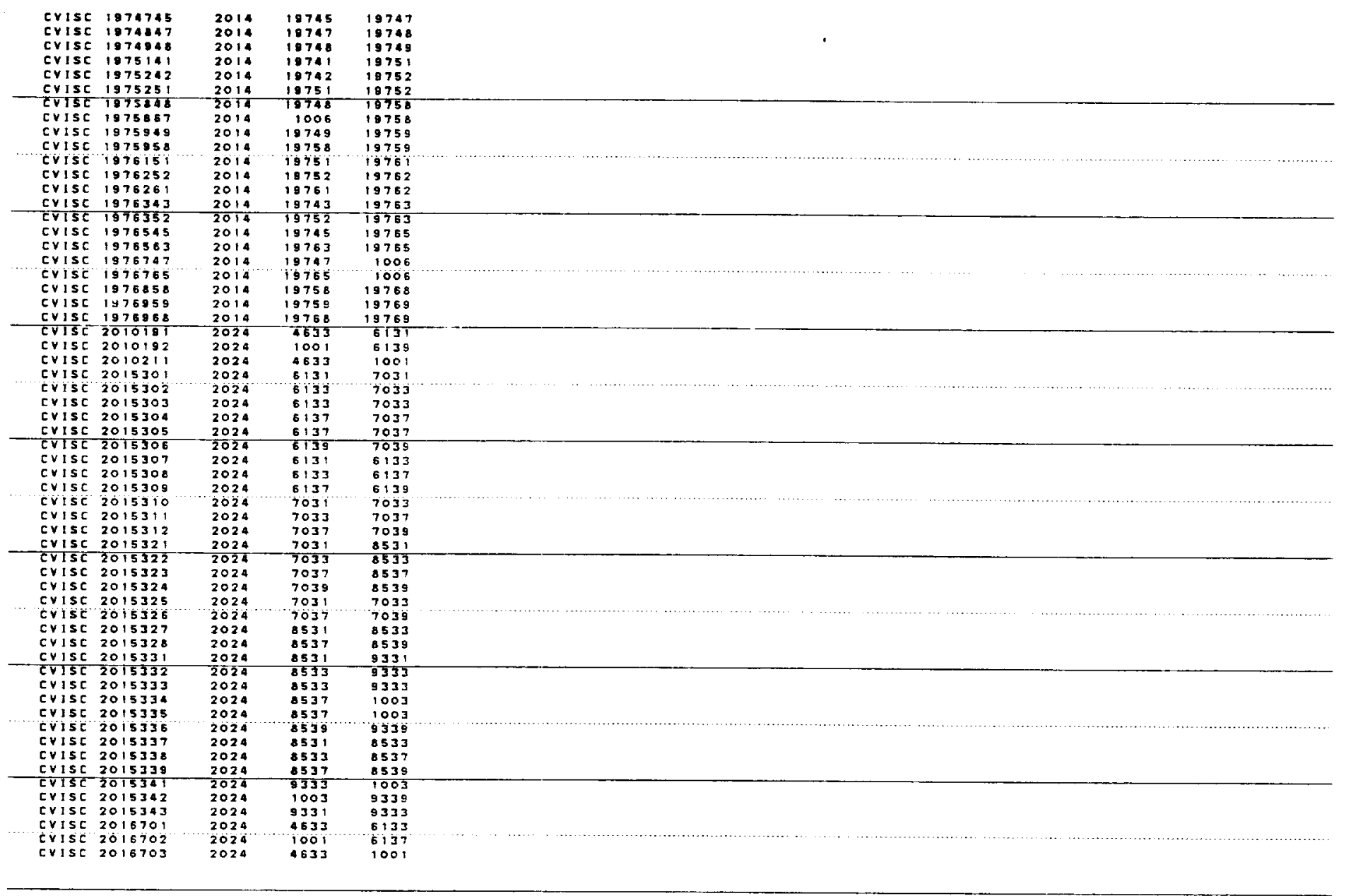

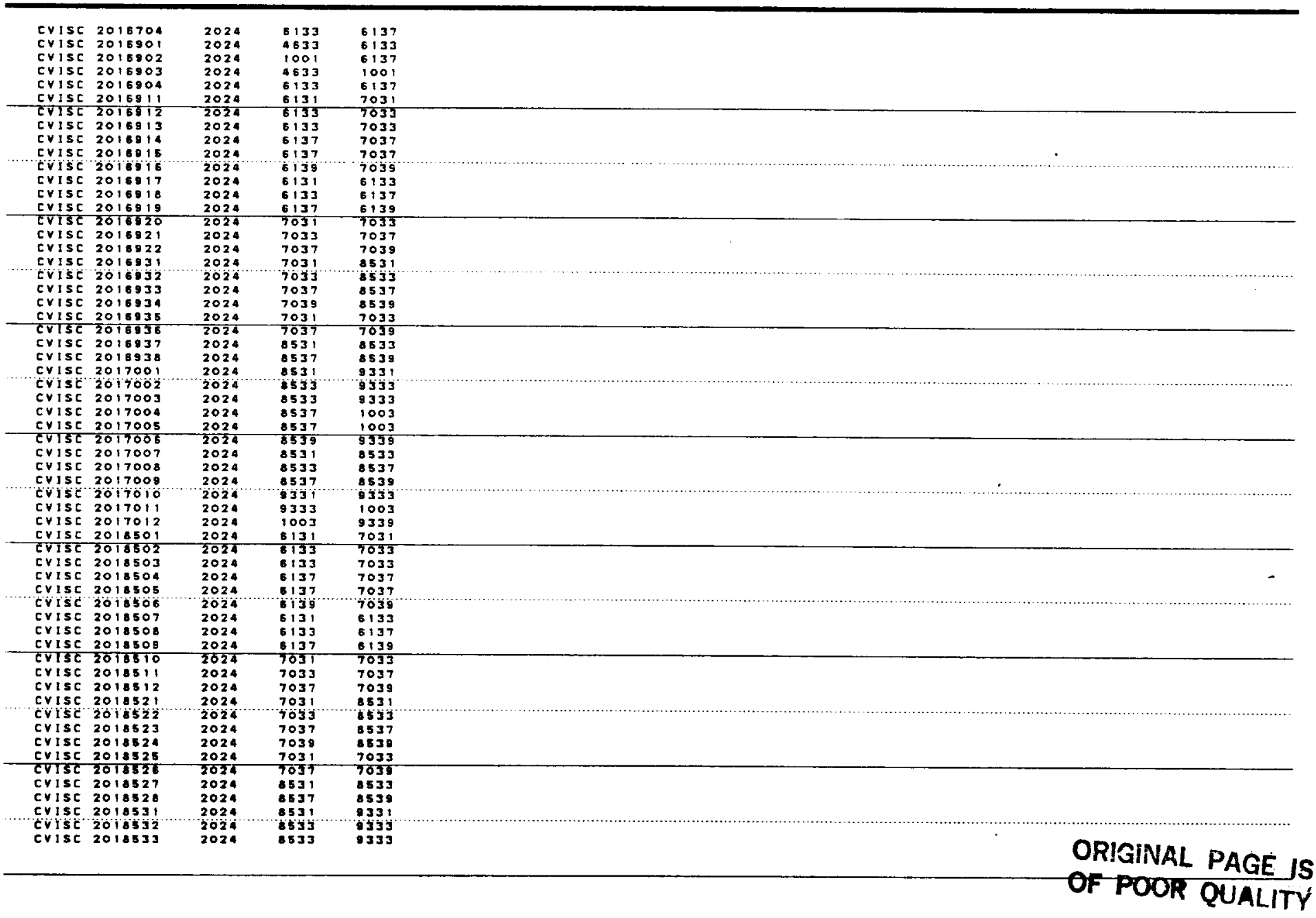




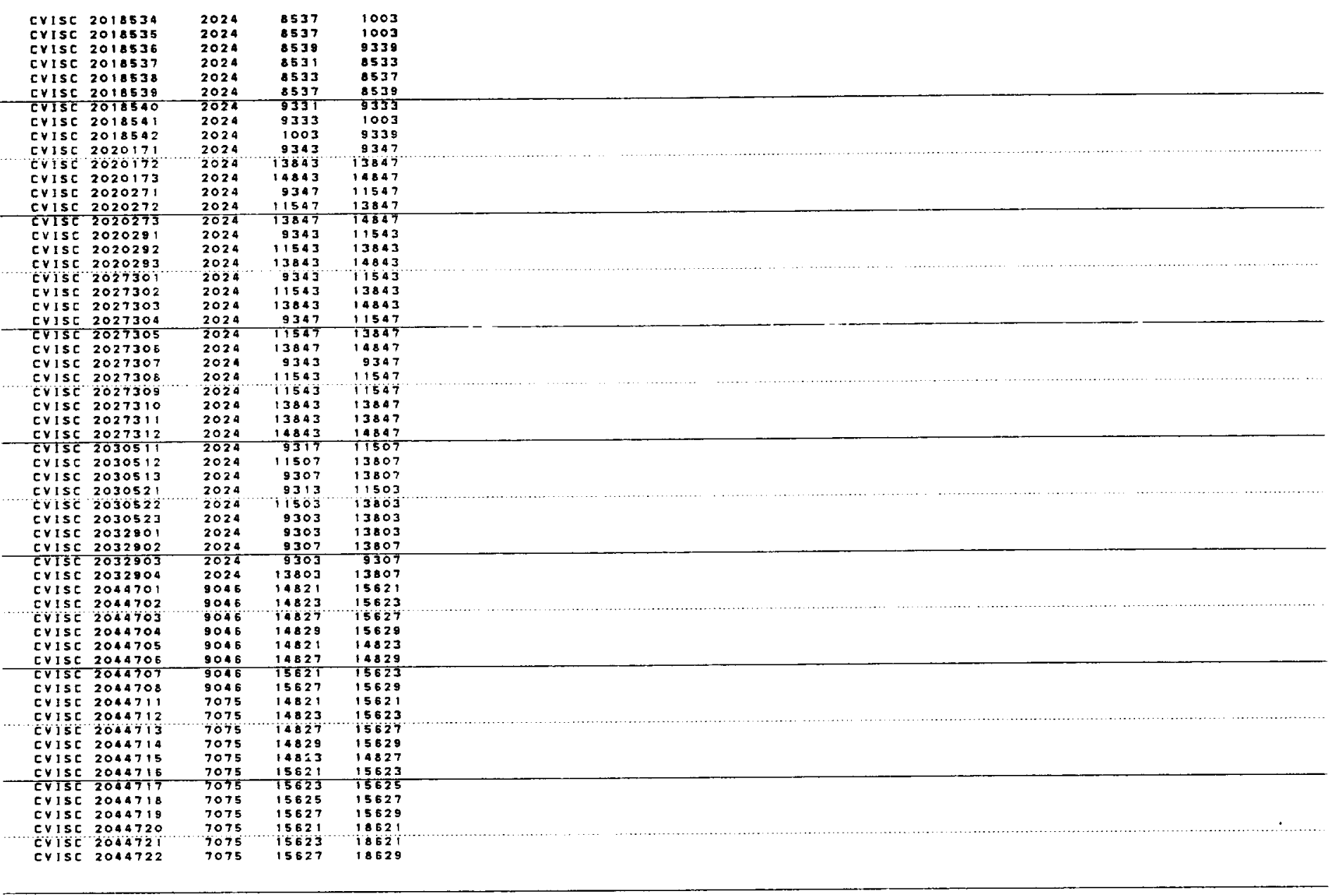

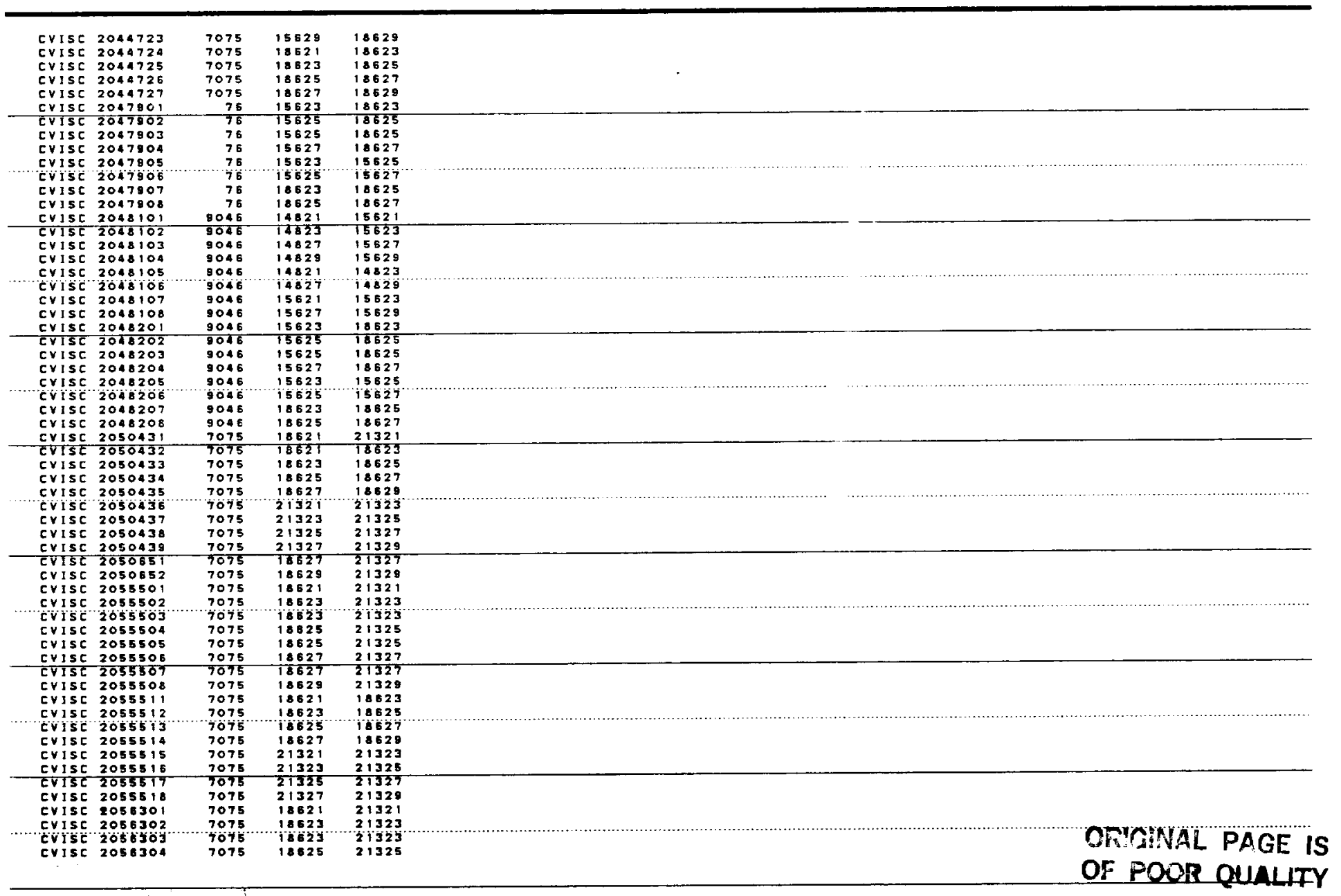




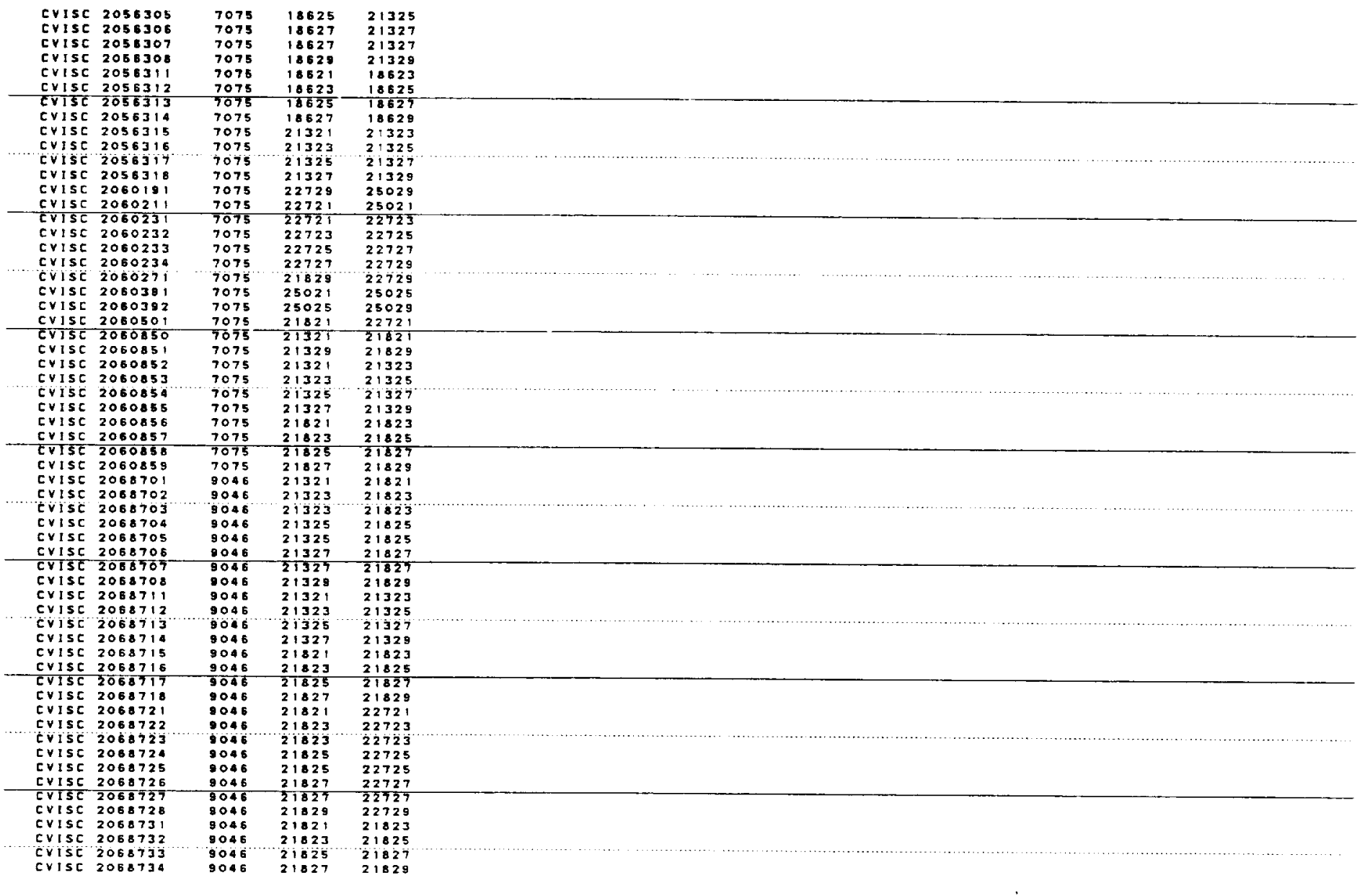

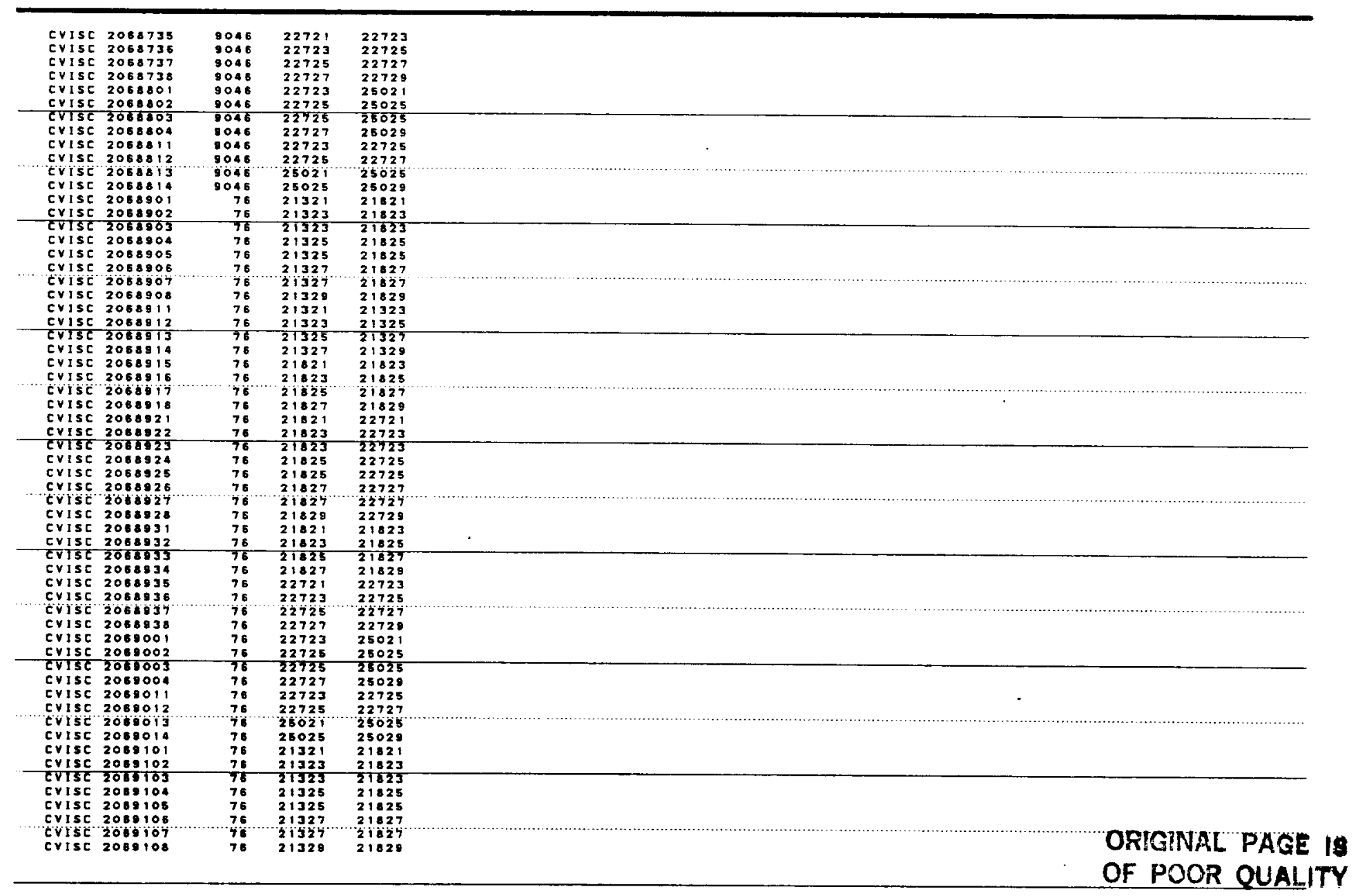




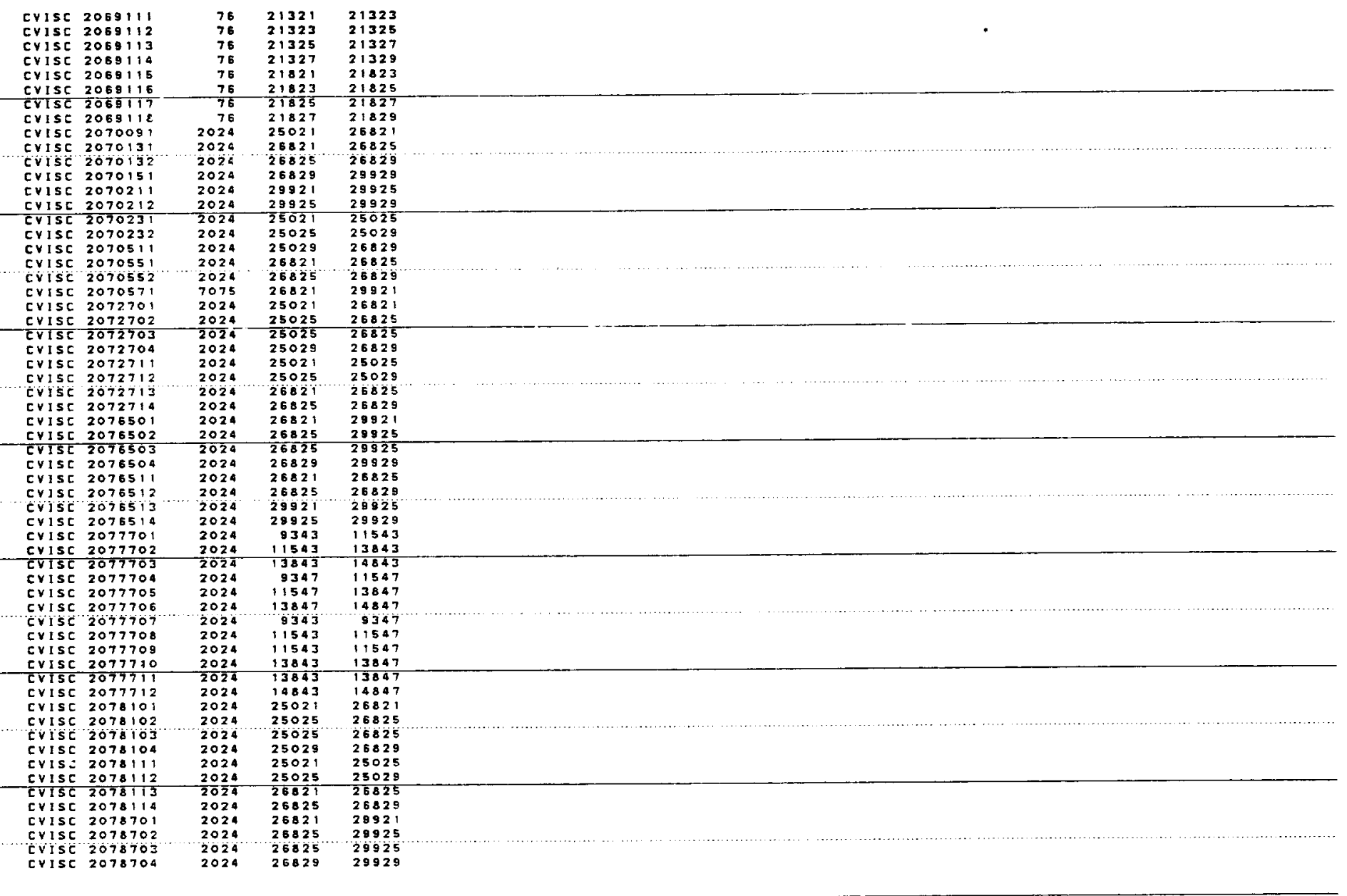

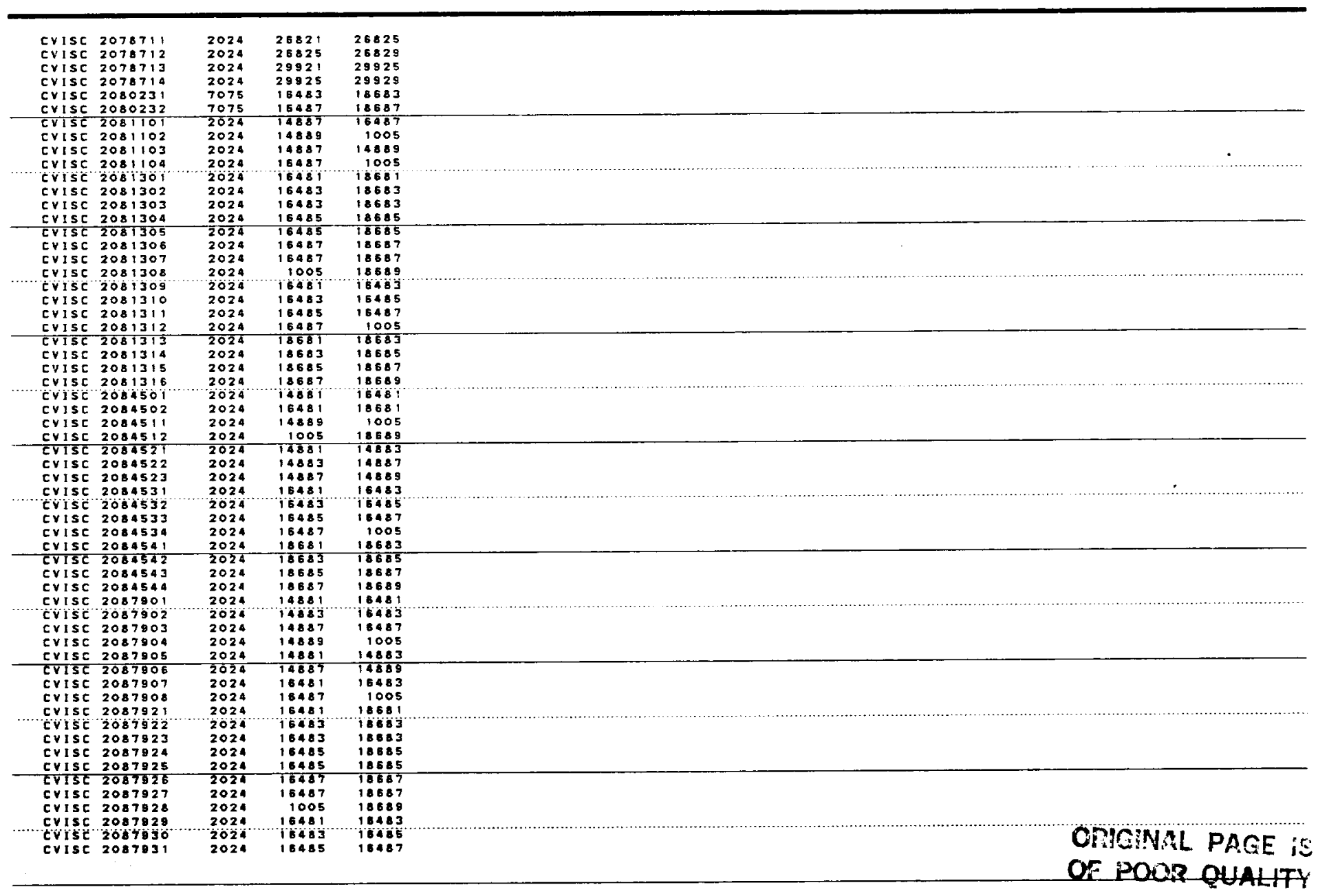


cVisC 208793

CVISC 208783

CVISC 200793

CVISE 2090051

CमIST 2090052

CVISC 2090053

eVise 209005 A

CVISC 2090055

Crise 209005

CVISC 209005 .

CrISC 2090411

crisc 2090431

CVISC 2090432

CVISC 2091011

CVISC 2091012

CYISC 209103

CVISC 2091270

CVISE 200127

CYISC 2091272

CY1SE 2091273

CYISC 2091274

CVISC 2091276

CVISC 2091277

CVISC 2091281

cVISC 2091283

CVISC 2091284

CVISC 2091285

cVisc 209128

CVISC 2092501

CVISC 2092503

crisc 2092504

CrISC 2092511

CVISC 2092513

CVISC 2092514

EVISC 2002522

CVISC 2092523

CVISC 209252

CVISC 2092532

CVISC 2092533

CVISE 2092534

CVist 2092541

CVISE 2092543

cVisc 2092551

CVISC 2092552

CVISE 2092553

CVISC 2092701

$16487 \quad 1005$

$202418683 \quad 1883$

$2024 \quad 1865518587$

$7075 \quad 25061 \quad 26861$

$\begin{array}{lll}7075 & 1007 & 26865 \\ 7075 & 1007 & 26865\end{array}$

$707525069 \quad 1008$

$\begin{array}{lll}7075 & 25081 & 1007\end{array}$

$\begin{array}{lll}7075 & 1007 & 25069 \\ 7075 & 2886, & 26855\end{array}$

$707525855 \quad 1008$

$\begin{array}{rrr}2024 & 25061 & 1007\end{array}$

$\begin{array}{lll}2024 & 26861 & 26855 \\ 2024 & 25855 & 1008\end{array}$

$\begin{array}{rrr}2024 & 25855 & 1008 \\ 2024 & 1007 & 25855\end{array}$

202425051008

$\begin{array}{lll}2024 & 25051 & 26861 \\ 2024 & 1007 & 26865\end{array}$

$\begin{array}{lll}7075 & 21361 & 25061 \\ 7075 & 21367 & 25069\end{array}$

70752136921363

$\begin{array}{lll}7075 & 21363 & 21364\end{array}$

$\begin{array}{lll}7075 & 21364 & 21366 \\ 7075 & 21365 & 21367\end{array}$

$\begin{array}{lll}7075 & 25061 & 1007\end{array}$

$7075 \quad 2685925069$

$7075 \quad 26859008$

$\begin{array}{rrr}7075 & 26865 & 26865 \\ 7075 & 28085 & 1008\end{array}$

$7075 \ldots 29969 \ldots 2985$

$\begin{array}{ll}9045 & 21363 \\ 95084\end{array}$

$9045 \quad 21364 \quad 1007$

$\begin{array}{rrr}9045 & 21365 & 1007 \\ 9046 & 21367 & 25069\end{array}$

$\begin{array}{lll}9045 & 21363 & 21364 \\ 9026 & 21366 & 21367\end{array}$

$\begin{array}{lll}9046 & 25061 \quad 1007 \\ 9066 & 1007 & 25069\end{array}$

$9066 \quad 100725069$

$\begin{array}{rrr}9046 & 25061 & 26861 \\ 9046 & 1007 & 28855\end{array}$

$\begin{array}{lrr}9046 & 1007 & 26855 \\ 9046 & 25069 & 1008\end{array}$

$9046 \quad 25051 \quad 1007$

$\begin{array}{lll}9046 & 1007 & 25069\end{array}$

904625855 100

$\begin{array}{lll}9046 & 25851 & 29961 \\ 9045 & 25855 & 29965\end{array}$

$\begin{array}{lll}9045 & 26855 & 29265 \\ 0046 & 26855 & 29965\end{array}$

$\begin{array}{rrr}9045 & 26851 & 1008 \\ 94655 & \end{array}$

$\begin{array}{lll}9045 & 26865 & 1008\end{array}$

$9046 \quad 29961 \quad 29955$

90462506125861

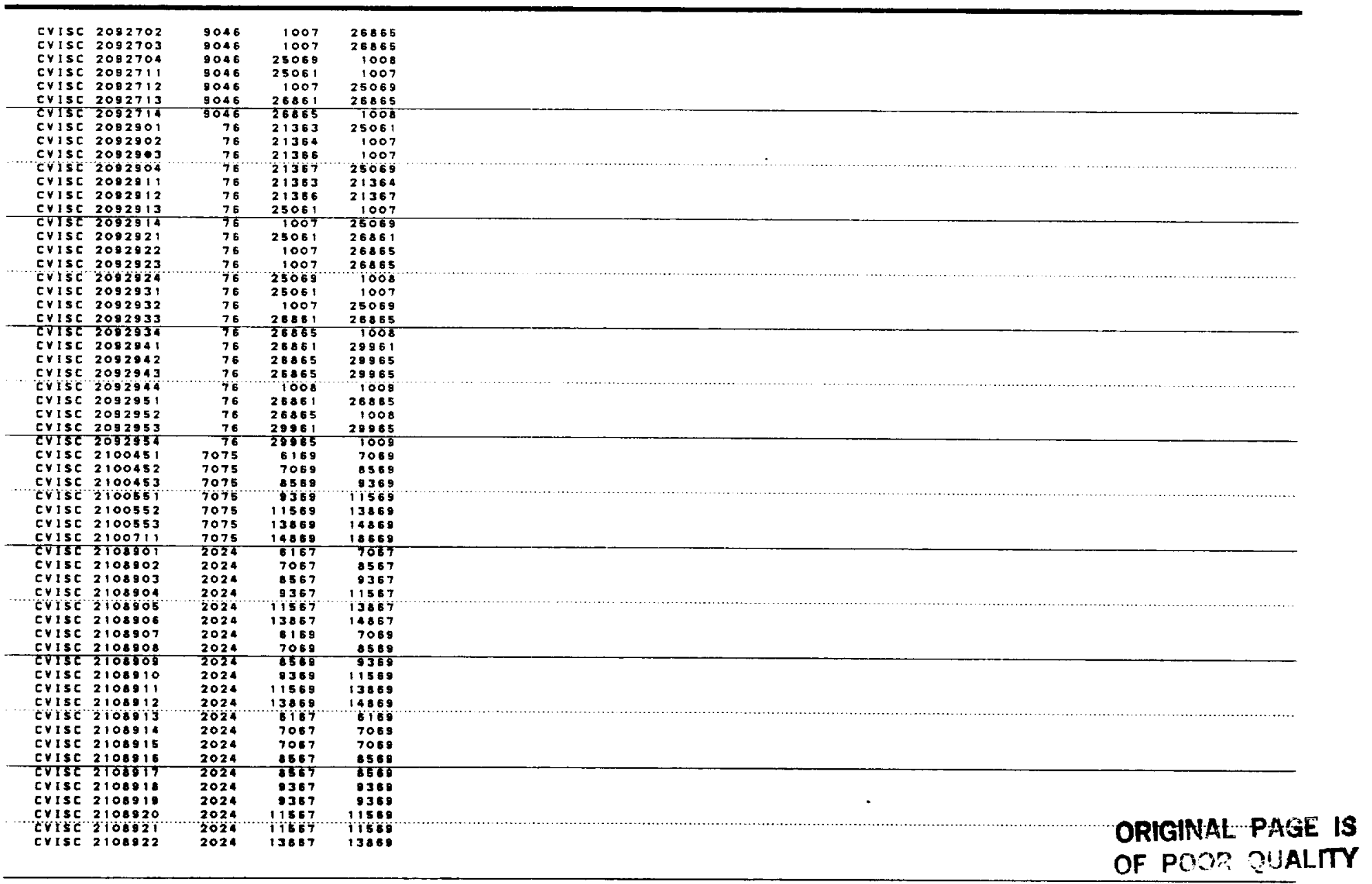




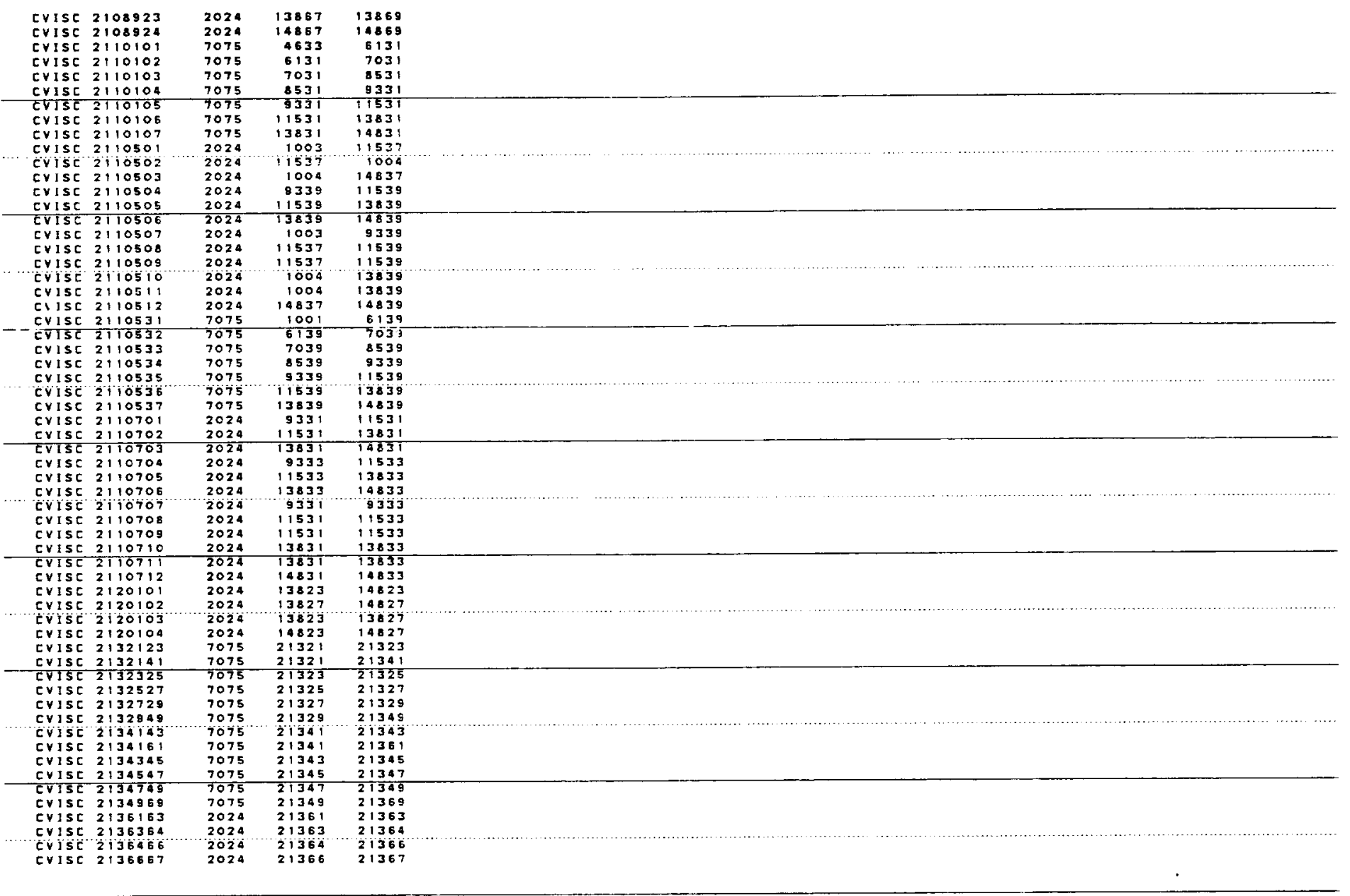

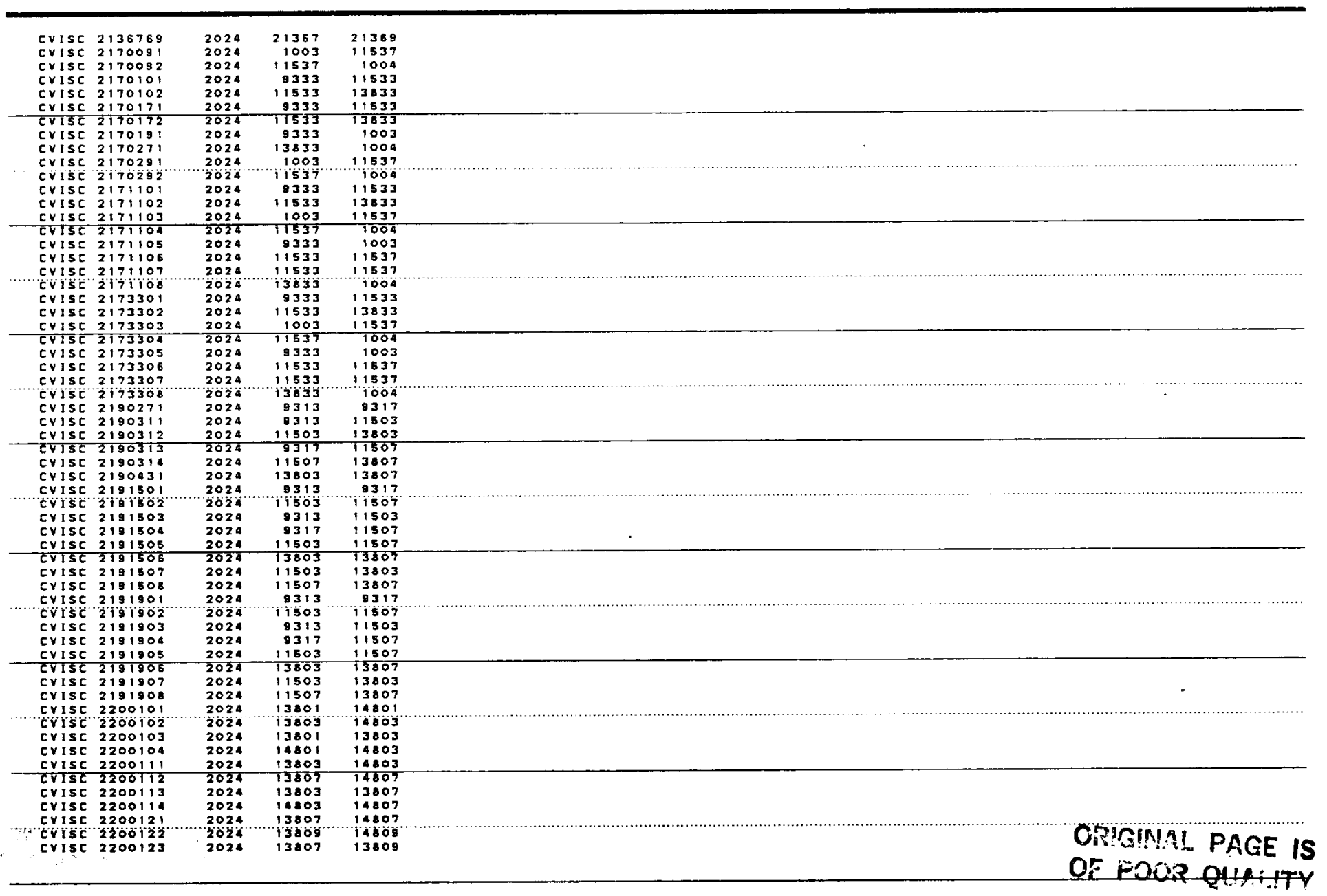




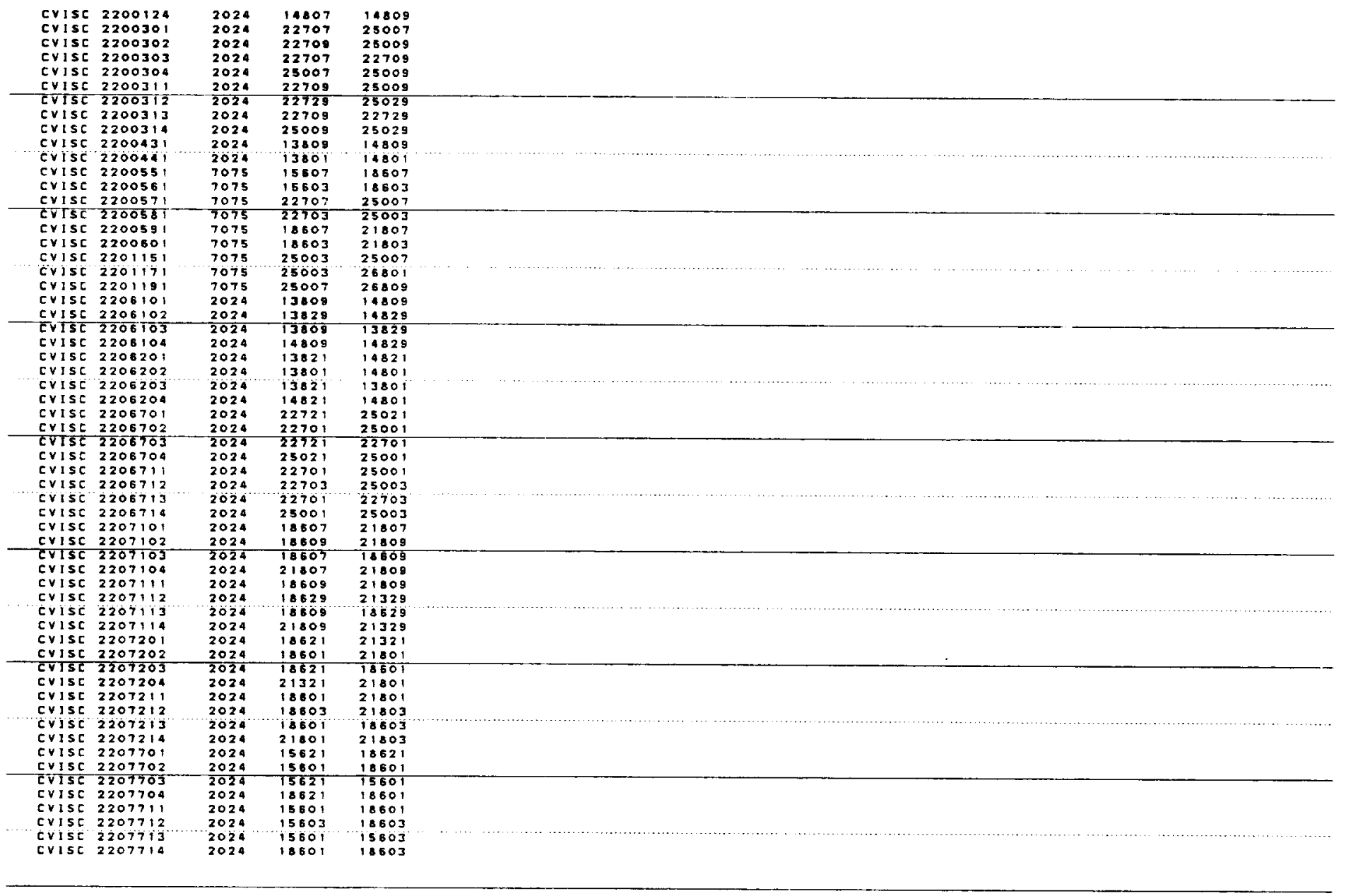

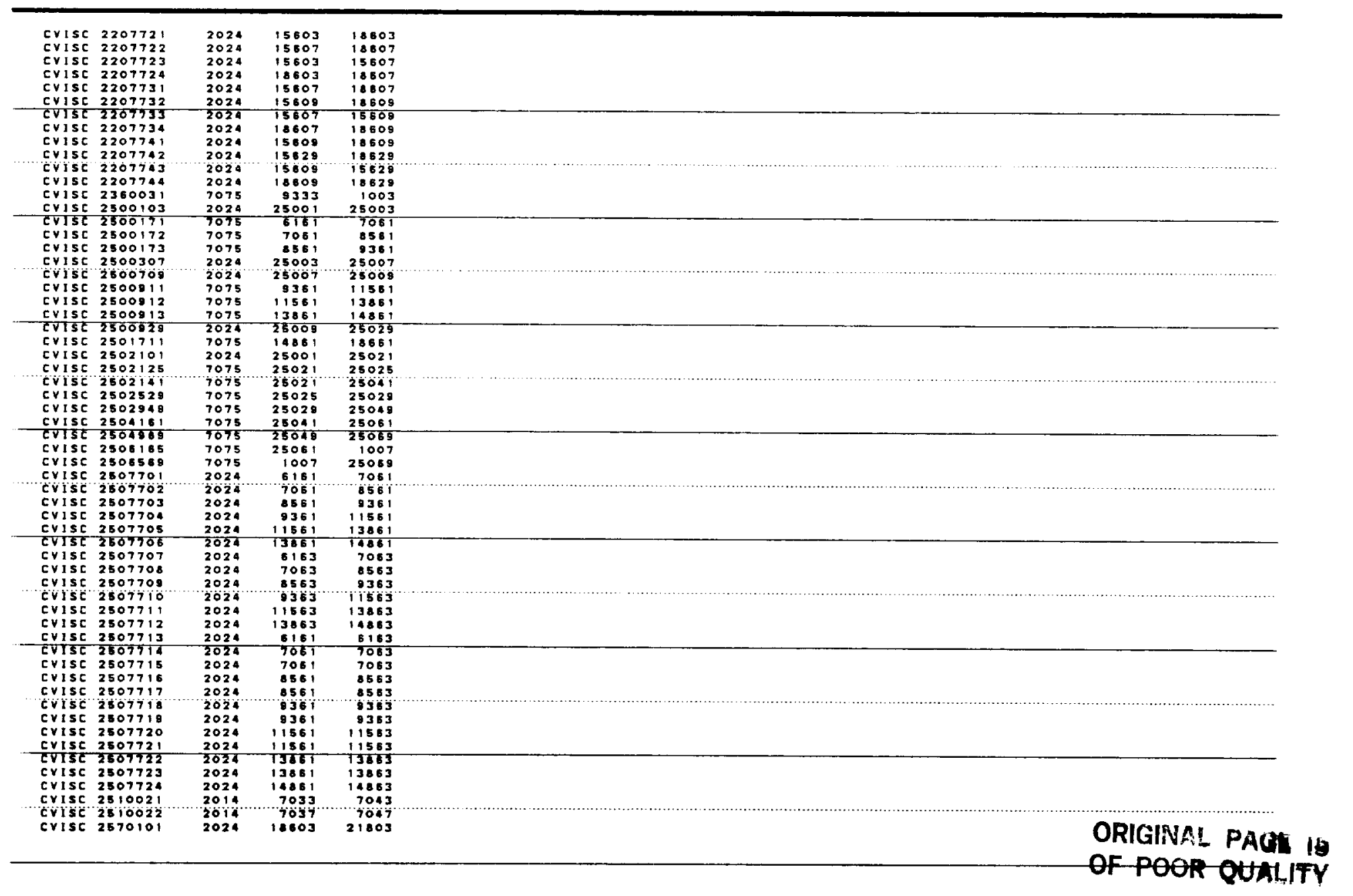




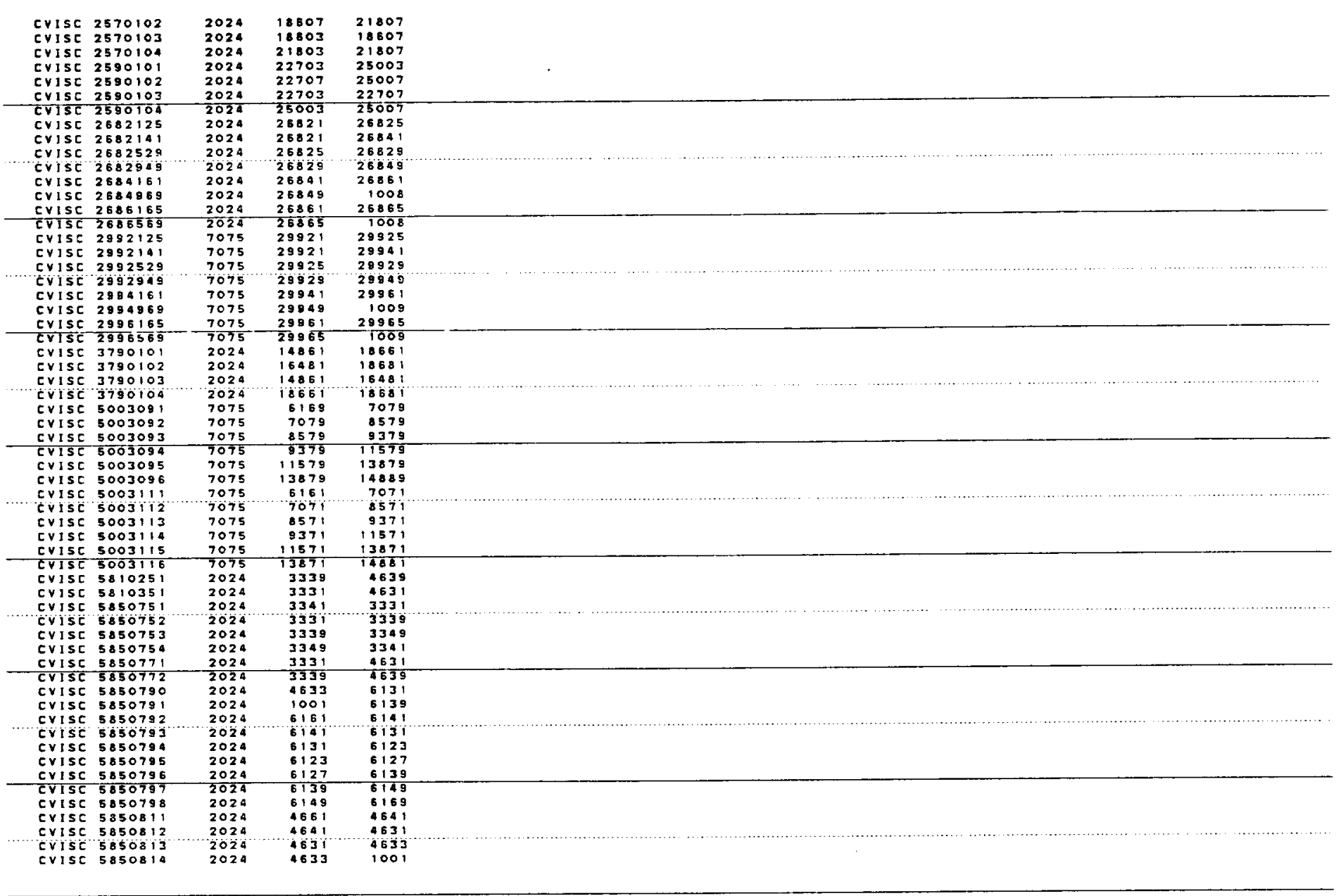

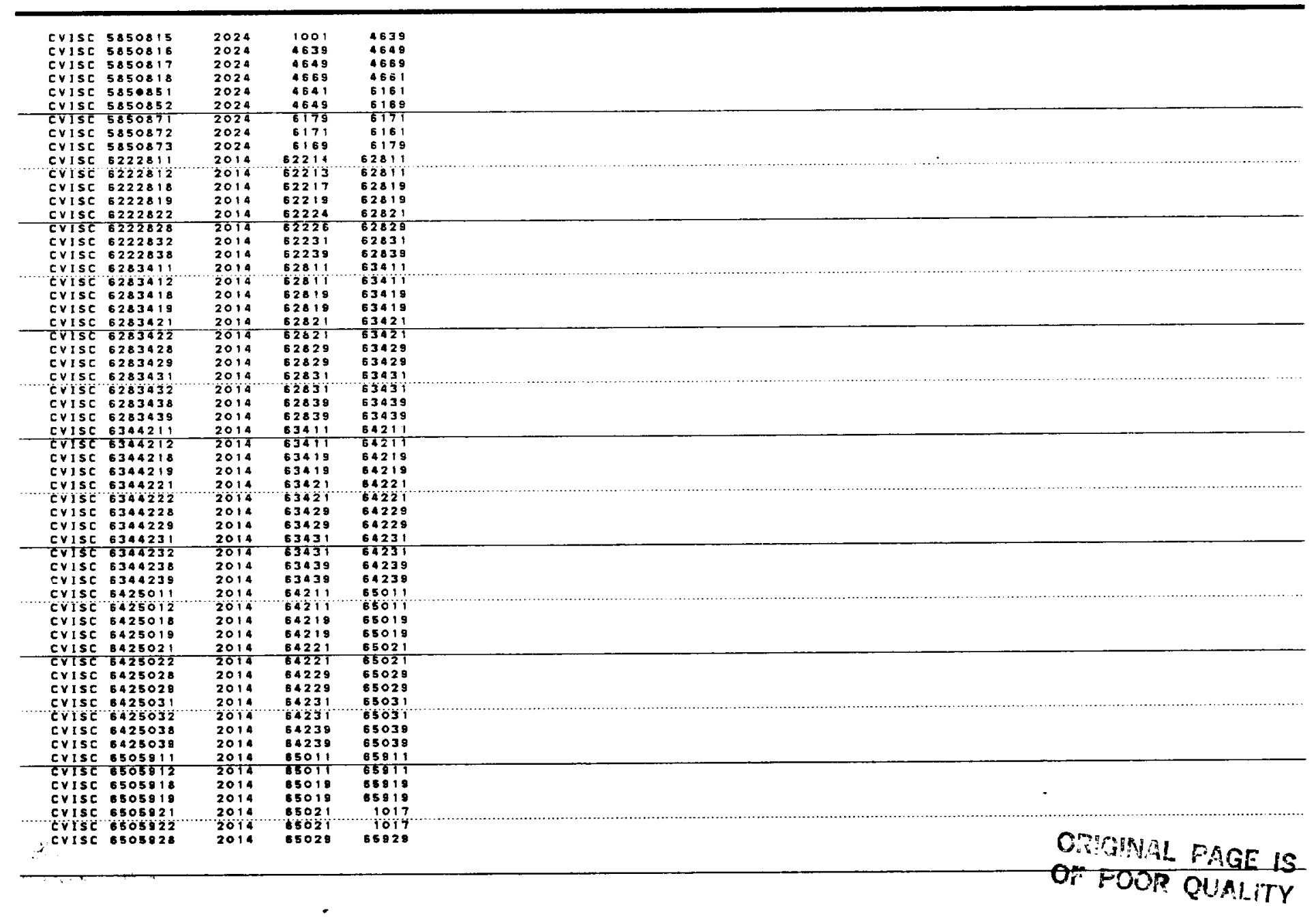




\begin{tabular}{|c|c|c|c|}
\hline 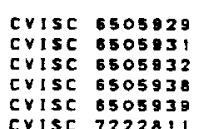 & $\begin{array}{l}2014 \\
2014 \\
2014 \\
2014 \\
2014 \\
2014 \\
2014\end{array}$ & $\begin{array}{l}55029 \\
85031 \\
65031 \\
65039 \\
65039 \\
65039\end{array}$ & $\begin{array}{l}65928 \\
55931 \\
65931 \\
65939 \\
65939 \\
72839\end{array}$ \\
\hline 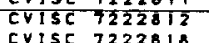 & 2014 & $\begin{array}{l}\frac{12211}{92213} \\
32217\end{array}$ & $\begin{array}{l}\frac{7811}{72811} \\
72816\end{array}$ \\
\hline $\begin{array}{l}\text { CVISC } 7222818 \\
\text { CVISC } 722819 \\
\text { CVISC } 7222822\end{array}$ & $\begin{array}{l}2014 \\
2014 \\
2014\end{array}$ & $\begin{array}{l}72219 \\
72224\end{array}$ & 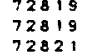 \\
\hline $\begin{array}{l}\text { CVISC } 7222828 \\
\text { CVISC } 7222832\end{array}$ & 2019 & $\begin{array}{l}72256 \\
72231\end{array}$ & $\begin{array}{l}72829 \\
72831\end{array}$ \\
\hline $\begin{array}{l}\text { CVISC } 7222838 \\
\text { Crise } 7283411\end{array}$ & $\begin{array}{l}2014 \\
2004\end{array}$ & $\begin{array}{l}7239 \\
72811\end{array}$ & $\begin{array}{l}72839 \\
7341\end{array}$ \\
\hline $\begin{array}{l}\text { Frisc } 7283412 \\
\text { CVISE } 728418\end{array}$ & $\begin{array}{l}2014 \\
2014\end{array}$ & $\begin{array}{l}72811 \\
72819\end{array}$ & 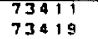 \\
\hline $\begin{array}{l}\text { CVVISC } 728319 \\
\text { CVISL } 7283421\end{array}$ & $\begin{array}{l}2014 \\
2014\end{array}$ & $\begin{array}{l}72819 \\
72821\end{array}$ & $\begin{array}{l}73419 \\
73421\end{array}$ \\
\hline $\begin{array}{l}C V I S C 728322 \\
\text { CVIST } 7283428\end{array}$ & $\begin{array}{l}2014 \\
2014\end{array}$ & $\begin{array}{l}72821 \\
72829\end{array}$ & $\begin{array}{l}73421 \\
73429\end{array}$ \\
\hline $\begin{array}{l}\text { CVISE } 7283429 \\
\text { CVISE } 7283431 \\
\end{array}$ & $\begin{array}{l}2014 \\
2014 \\
\end{array}$ & $\begin{array}{l}72829 \\
72831 \\
789\end{array}$ & $\begin{array}{l}73429 \\
73431 \\
7341\end{array}$ \\
\hline 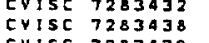 & $\begin{array}{l}2014 \\
2014\end{array}$ & $\begin{array}{l}72831 \\
72839\end{array}$ & $\begin{array}{l}73431 \\
73439\end{array}$ \\
\hline 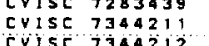 & $\begin{aligned} 2014 \\
2014 \\
2014\end{aligned}$ & $\begin{array}{l}72839 \\
73411 \\
73011\end{array}$ & $\begin{array}{l}73439 \\
74211 \\
7020\end{array}$ \\
\hline $\begin{array}{l}\text { CVISE } 734428 \\
\text { c }\end{array}$ & $\begin{array}{l}2014 \\
2014\end{array}$ & $\begin{array}{l}73411 \\
73419\end{array}$ & $\begin{array}{l}74211 \\
74218\end{array}$ \\
\hline 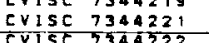 & $\begin{aligned} 2014 \\
2014\end{aligned}$ & $\begin{array}{l}73419 \\
73421 \\
3231\end{array}$ & $\begin{array}{l}74219 \\
74221 \\
-5231\end{array}$ \\
\hline $\begin{array}{l}\text { CVISC } 734428 \\
\text { Crist }\end{array}$ & 2014 & $\begin{array}{l}73429 \\
73429\end{array}$ & $\begin{array}{l}74221 \\
74229 \\
7.220\end{array}$ \\
\hline 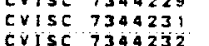 & $\begin{array}{l}2014 \\
2014 \\
2014\end{array}$ & 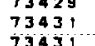 & $\begin{array}{l}7428 \\
74231 \\
34231\end{array}$ \\
\hline 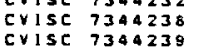 & $\begin{array}{l}2014 \\
2014 \\
2014\end{array}$ & $\begin{array}{l}73439 \\
73439\end{array}$ & 年 74239 \\
\hline $\begin{array}{l}\text { CVISC } 7425011 \\
\text { CVISC } 945512\end{array}$ & $\frac{2014}{2014}$ & $\begin{array}{l}74211 \\
74211\end{array}$ & $\begin{array}{l}75019 \\
75011 \\
75011\end{array}$ \\
\hline $\begin{array}{l}\text { Cvisc } 7425018 \\
\text { cvisc } 7425019\end{array}$ & $\begin{array}{l}2014 \\
2004\end{array}$ & $\begin{array}{l}74219 \\
74219\end{array}$ & $\begin{array}{l}75018 \\
75019\end{array}$ \\
\hline 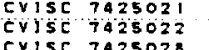 & $\begin{array}{l}2014 \\
2014\end{array}$ & $\begin{array}{l}74221 \\
74221 \\
7420\end{array}$ & $\begin{array}{l}75021 \\
75021 \\
75070\end{array}$ \\
\hline $\begin{array}{l}\text { CVISC } 7425028 \\
\text { CYISC } 7425029 \\
\text { CYISE } 7425031\end{array}$ & $\begin{array}{l}2014 \\
2014 \\
2014\end{array}$ & $\begin{array}{l}74229 \\
74229\end{array}$ & $\begin{array}{l}75028 \\
75028 \\
75031\end{array}$ \\
\hline 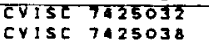 & $\begin{array}{l}2014 \\
2004\end{array}$ & $\begin{array}{l}74231 \\
74231 \\
74239\end{array}$ & $\begin{array}{l}75031 \\
75039\end{array}$ \\
\hline $\begin{array}{l}\text { crist } 7425039 \\
\text { cyisc } 7505911\end{array}$ & $\begin{array}{l}2016 \\
2014\end{array}$ & $\begin{array}{l}74239 \\
75011\end{array}$ & $\begin{array}{l}75039 \\
75911\end{array}$ \\
\hline $\begin{array}{l}\text { CYISC } 7505912 \\
\text { EYISC } 7505918\end{array}$ & $\begin{array}{l}2016 \\
2019\end{array}$ & $\begin{array}{l}75011 \\
75018\end{array}$ & $\begin{array}{l}75911 \\
75919\end{array}$ \\
\hline $\begin{array}{l}\text { CVISC } 7505819 \\
\text { CYISC } 7505927\end{array}$ & $\begin{array}{l}2014 \\
2018\end{array}$ & $\begin{array}{l}75019 \\
75021\end{array}$ & $\begin{aligned} 75819 \\
1018 \\
\end{aligned}$ \\
\hline 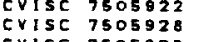 & $\begin{array}{l}2014 \\
2014\end{array}$ & $\begin{array}{l}75021 \\
75029\end{array}$ & $\begin{array}{l}1018 \\
75929\end{array}$ \\
\hline 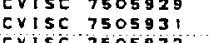 & & $\begin{array}{l}75029 \\
75031\end{array}$ & $\begin{array}{l}75929 \\
75931\end{array}$ \\
\hline $\begin{array}{l}7505938 \\
75058\end{array}$ & & 75039 & $\begin{array}{l}931 \\
939\end{array}$ \\
\hline
\end{tabular}

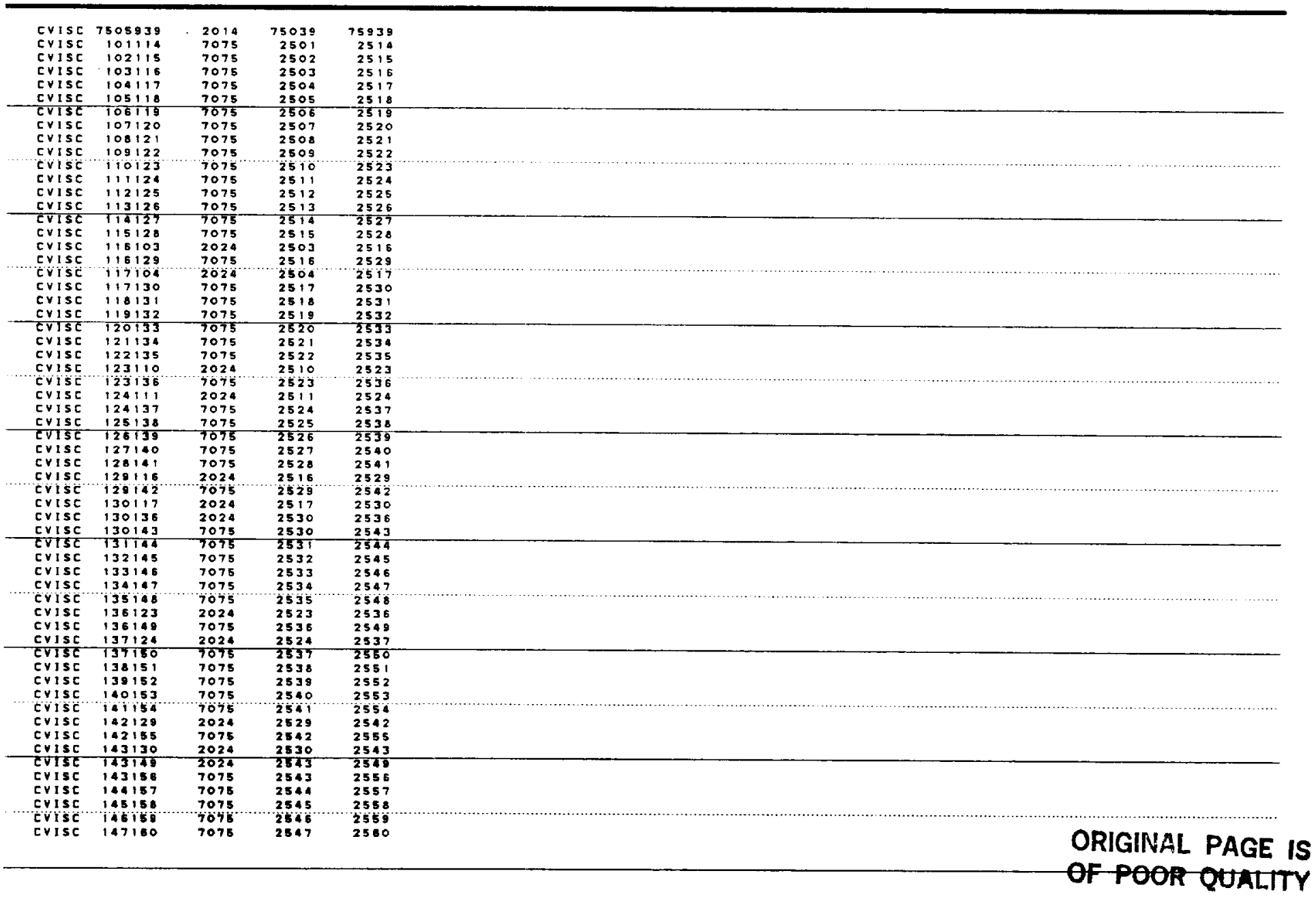




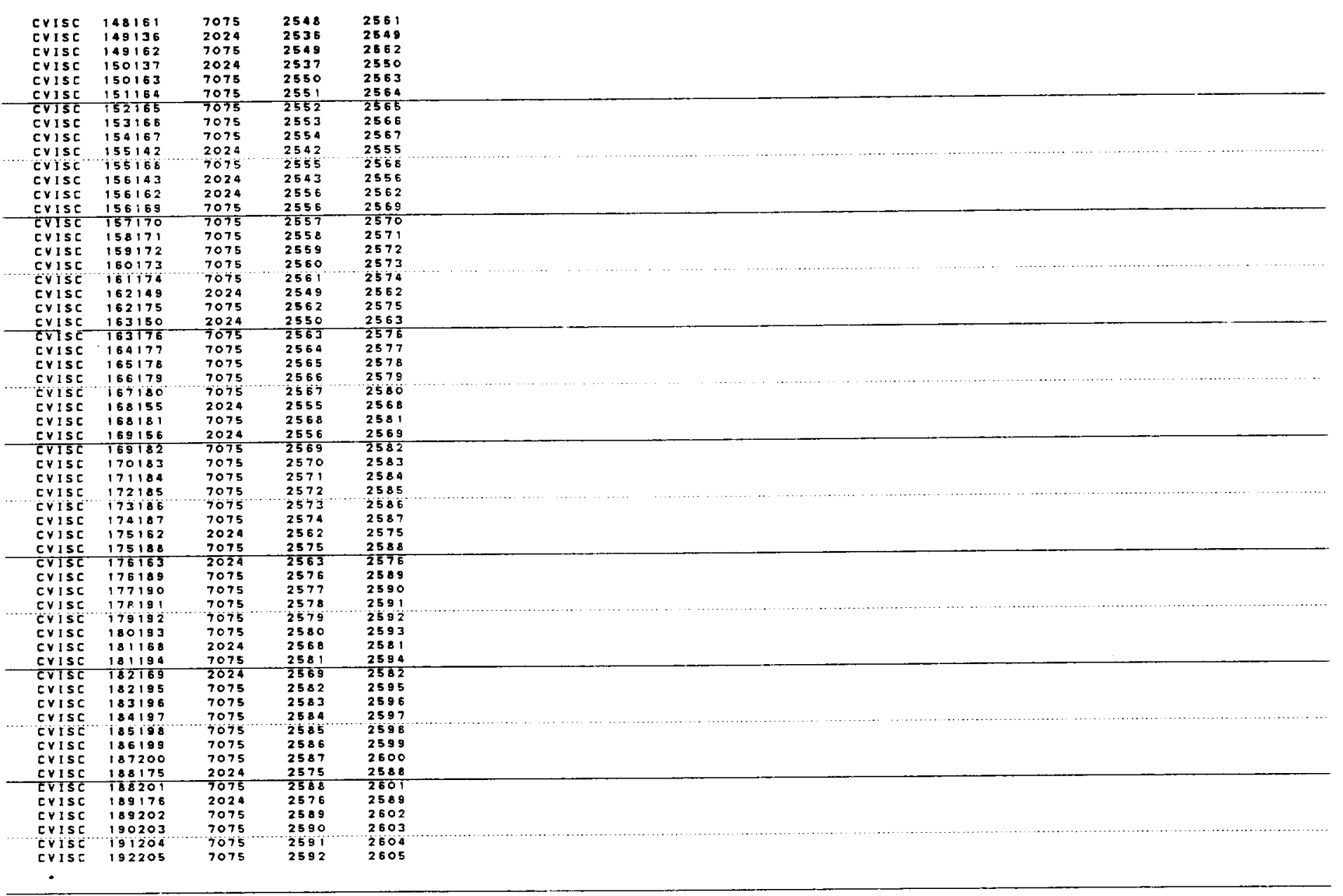

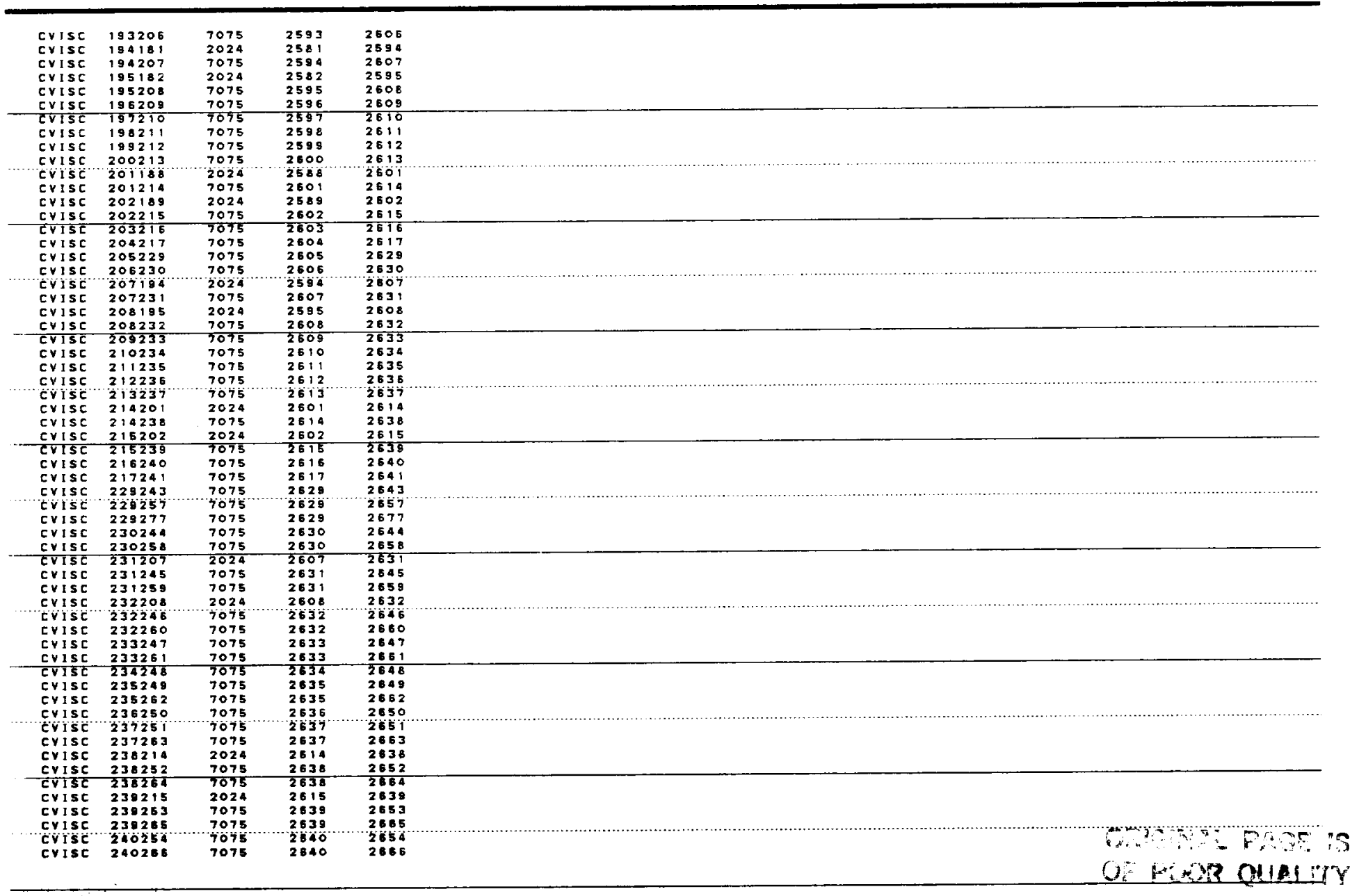




\begin{tabular}{|c|c|c|c|c|}
\hline 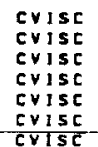 & $\begin{array}{r}241255 \\
242256 \\
242276 \\
244267 \\
245231 \\
245268 \\
246232\end{array}$ & $\begin{array}{l}7075 \\
7075 \\
7075 \\
7075 \\
2027 \\
7075 \\
7075 \\
2024\end{array}$ & $\begin{array}{l}2641 \\
2842 \\
2842 \\
2644 \\
2631 \\
2645 \\
2532\end{array}$ & $\begin{array}{l}2655 \\
2856 \\
2676 \\
2857 \\
2645 \\
2658 \\
2645\end{array}$ \\
\hline $\begin{array}{l}c \gamma 15 c- \\
c y 1 s c \\
c y 1 s c \\
c y 1 s c\end{array}$ & $\begin{array}{l}246232 \\
246289 \\
247270\end{array}$ & $\begin{array}{l}2024 \\
7075 \\
7075\end{array}$ & $\begin{array}{l}2632 \\
2645 \\
2647\end{array}$ & $\begin{array}{l}2645 \\
2669 \\
2570\end{array}$ \\
\hline $\begin{array}{l}\text { crist } \\
\text { cylst } \\
\text { cyisc }\end{array}$ & $\begin{array}{l}2433000 . \\
249271 \\
250300\end{array}$ & $\begin{array}{l}7075 \\
7075 \\
7075\end{array}$ & $\begin{array}{l}2588 \\
2643 \\
2650\end{array}$ & $\begin{array}{r}2700 \\
2571 \\
2700\end{array}$ \\
\hline 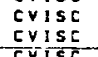 & $\begin{array}{r}251272 \\
252238 \\
\end{array}$ & $\begin{array}{r}7075 \\
-2024 \\
-2025\end{array}$ & $\begin{array}{r}2651 \\
2638 \\
-267 \\
\end{array}$ & $\begin{array}{r}2707 \\
2672 \\
2652 \\
\end{array}$ \\
\hline 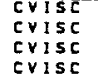 & $\begin{array}{l}252273 \\
25323 \\
25327 \\
25327\end{array}$ & $\begin{array}{l}7075 \\
2024 \\
7075 \\
7075\end{array}$ & $\begin{array}{l}25552 \\
2639 \\
2653 \\
2654\end{array}$ & $\begin{array}{l}2673 \\
2653 \\
2674 \\
2675\end{array}$ \\
\hline 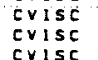 & $\begin{array}{r}256279 \\
256300 \\
25727\end{array}$ & $\begin{array}{l}7075 \\
70075 \\
7075\end{array}$ & $\begin{array}{l}2658 \\
2658 \\
2657\end{array}$ & $\begin{array}{l}2579 \\
2700 \\
2700\end{array}$ \\
\hline $\begin{array}{l}\text { crisc } \\
\text { evisc } \\
\text { EvIsc }\end{array}$ & $\begin{array}{r}257278 \\
257300 \\
259265\end{array}$ & $\begin{array}{l}7075 \\
7075 \\
70755\end{array}$ & $\begin{array}{r}2657 \\
2657 \\
\end{array}$ & $\begin{array}{r}2578 \\
-2700 \\
-2565\end{array}$ \\
\hline $\begin{array}{l}\text { cyise } \\
\text { cylse }\end{array}$ & $\begin{array}{l}250263 \\
25064 \\
276277\end{array}$ & $\begin{array}{l}7075 \\
7075\end{array}$ & $\begin{array}{l}2659 \\
2650 \\
2676\end{array}$ & $\begin{array}{l}2665 \\
2664\end{array}$ \\
\hline $\begin{array}{l}\text { crisc } \\
\text { cyist }\end{array}$ & $\begin{array}{l}276279 \\
275280\end{array}$ & $\begin{array}{l}2024 \\
7075 \\
7075\end{array}$ & $\begin{array}{l}2676 \\
2676 \\
2676\end{array}$ & $\begin{array}{l}2577 \\
2679 \\
2680\end{array}$ \\
\hline 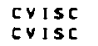 & $\begin{array}{l}277278 \\
277281\end{array}$ & $\begin{array}{l}2020 \\
7075\end{array}$ & $\begin{array}{l}2677 \\
2677\end{array}$ & $\begin{array}{l}2680 \\
2678 \\
2681\end{array}$ \\
\hline $\begin{array}{l}\frac{c}{v_{1}} \\
\text { cylstst }\end{array}$ & $\frac{27827}{278282}$ & $\frac{70975}{9075}$ & $\frac{2678}{2678}$ & $\begin{array}{r}2579 \\
2682\end{array}$ \\
\hline 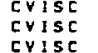 & $\begin{array}{l}278300 \\
279833 \\
2793000\end{array}$ & $\begin{array}{l}2024 \\
7075 \\
2024\end{array}$ & $\begin{array}{l}2678 \\
2879 \\
2579\end{array}$ & $\begin{array}{r}2700 \\
2683 \\
3700\end{array}$ \\
\hline $\begin{array}{l}\text { ivist } \\
\text { cy1sc }\end{array}$ & $\begin{array}{l}2808 \\
280283 \\
2803\end{array}$ & $\begin{array}{l}7075 \\
7075\end{array}$ & $\begin{array}{l}2680 \\
2580\end{array}$ & $\begin{array}{l}2700 \\
2681 \\
2683\end{array}$ \\
\hline 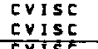 & $\begin{array}{r}2802884 \\
281282 \\
\end{array}$ & $\begin{array}{l}7075 \\
7075 \\
\end{array}$ & $\begin{array}{r}2680 \\
2881 \\
\end{array}$ & $\begin{array}{r}2684 \\
2682 \\
\end{array}$ \\
\hline cyisc & $\begin{array}{l}281185 \\
282283\end{array}$ & $\begin{array}{l}7075 \\
7075\end{array}$ & $\begin{array}{l}2681 \\
2582\end{array}$ & $\begin{array}{l}2685 \\
2683\end{array}$ \\
\hline 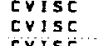 & $\begin{array}{l}2828286 \\
282302\end{array}$ & $\begin{array}{l}7075 \\
7075\end{array}$ & $\begin{array}{l}2682 \\
3682 \\
.2682\end{array}$ & $\begin{array}{l}2686 \\
2702\end{array}$ \\
\hline $\begin{array}{l}x=15 t \\
c y 1 s c \\
c y 1 s c\end{array}$ & $\begin{array}{l}283302 \\
284285\end{array}$ & $\begin{array}{l}7075 \\
7075 \\
7075\end{array}$ & $\begin{array}{l}2683 \\
2683 \\
2684\end{array}$ & $\begin{array}{l}2687 \\
2702 \\
2685\end{array}$ \\
\hline$\frac{c r s c}{c V i s c}$ & $\frac{28487}{284318}$ & $\frac{7075}{7075}$ & $\frac{2884}{2884}$ & $\frac{2887}{2418}$ \\
\hline $\begin{array}{l}\text { criss } \\
\text { cyisc } \\
\text { cyise }\end{array}$ & $\begin{array}{l}285886 \\
285319 \\
285897\end{array}$ & $\begin{array}{l}7075 \\
7075 \\
7075\end{array}$ & $\begin{array}{l}2685 \\
25655 \\
2585\end{array}$ & $\begin{array}{l}2686 \\
2719\end{array}$ \\
\hline $\begin{array}{l}\text { crisc } \\
\text { cyisc }\end{array}$ & $\begin{array}{l}286304 \\
286320\end{array}$ & $\begin{array}{l}7675 \\
7075 \\
7075\end{array}$ & $\begin{array}{l}2586 \\
2686 \\
2686\end{array}$ & $\begin{array}{l}2687 \\
2704 \\
2720\end{array}$ \\
\hline $\begin{array}{l}\text { cyisc } \\
\text { cyisc }\end{array}$ & $\begin{array}{l}287304 \\
287321\end{array}$ & $\begin{array}{l}7075 \\
7075\end{array}$ & $\begin{array}{l}26867 \\
2687\end{array}$ & $\begin{array}{l}2704 \\
2721 \\
2721\end{array}$ \\
\hline $\begin{array}{l}\text { erist } \\
\text { cyist }\end{array}$ & 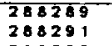 & 7075 & $\begin{array}{l}25888 \\
2688\end{array}$ & $\begin{array}{l}2689 \\
2699\end{array}$ \\
\hline & $\begin{array}{l}288292 \\
289290\end{array}$ & $\begin{array}{l}7075 \\
7075 \\
7075\end{array}$ & $\begin{array}{l}2888 \\
26899\end{array}$ & $\begin{array}{r}2592 \\
2650\end{array}$ \\
\hline & $\begin{array}{r}289293 \\
290291\end{array}$ & $\begin{array}{l}7075 \\
7075\end{array}$ & 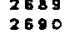 & $\begin{array}{l}2693 \\
2699\end{array}$ \\
\hline
\end{tabular}

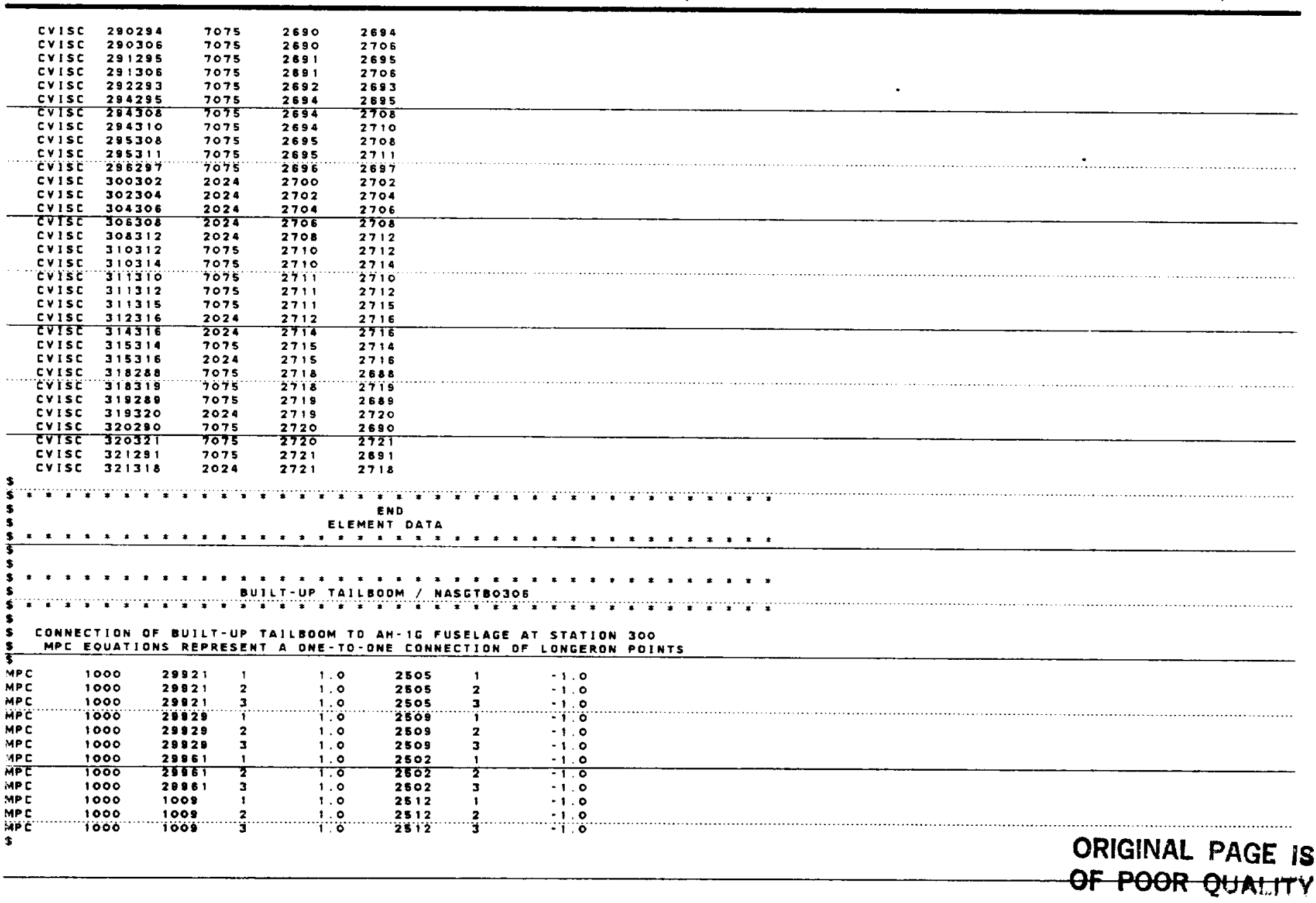


\$ CONHECTION OF TAIL ROTOR GEARBOX AND MAST TO BUILT-UP FIN AT STA 521
TIIR MAST MOOEL DIRECTLY FROM PREYIOUS AH- IE FEM FOR ELASTIE LINE

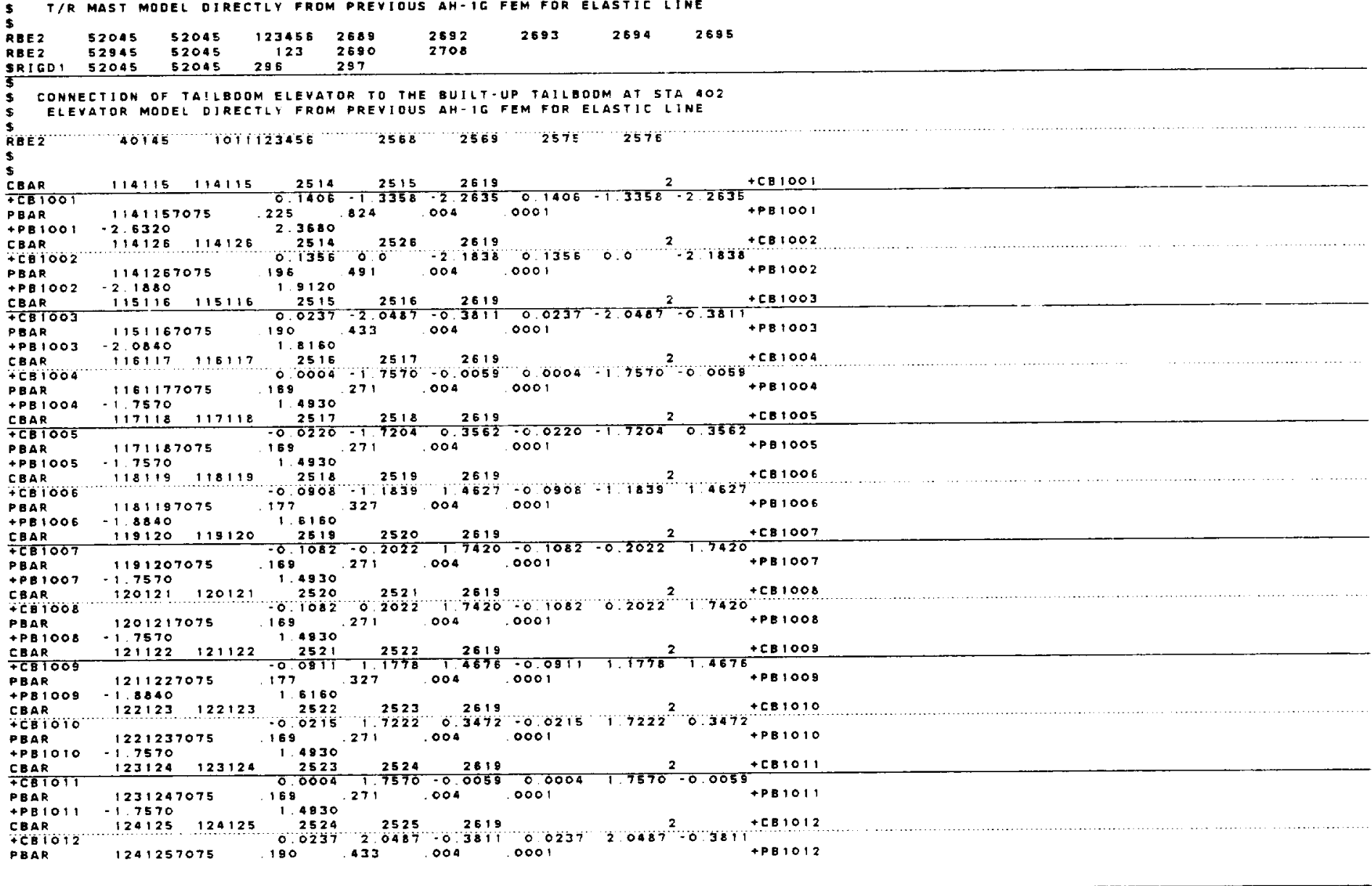

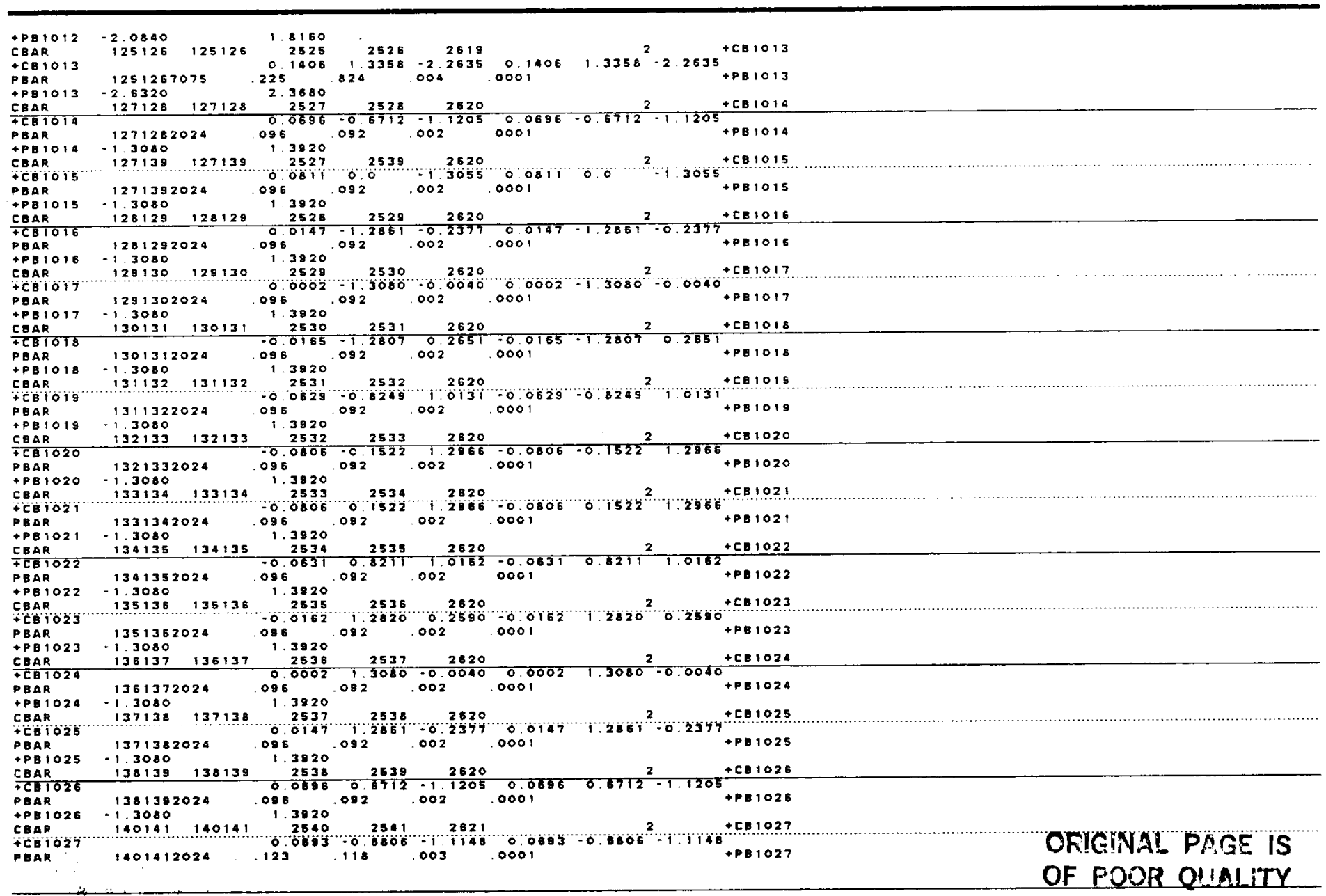




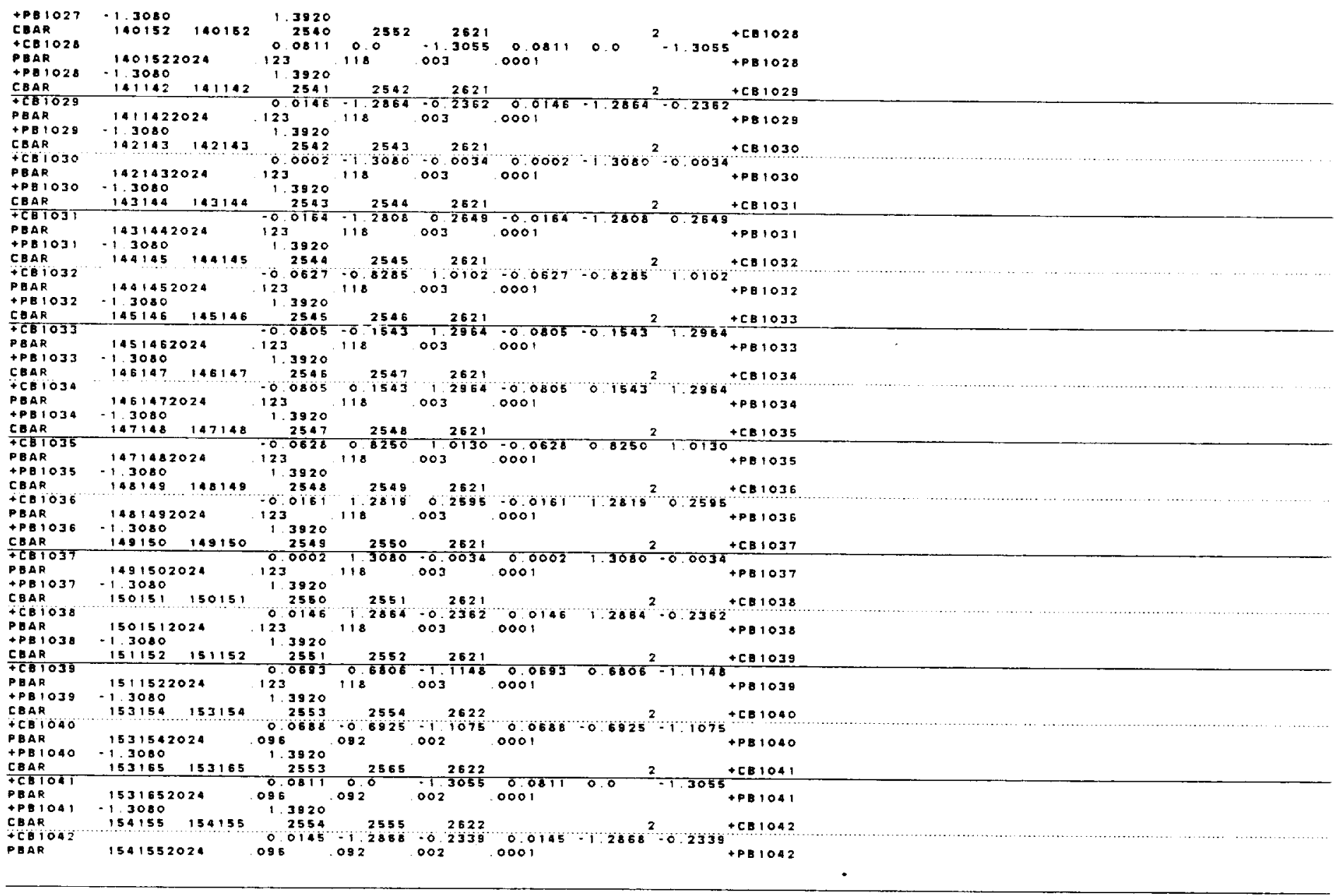

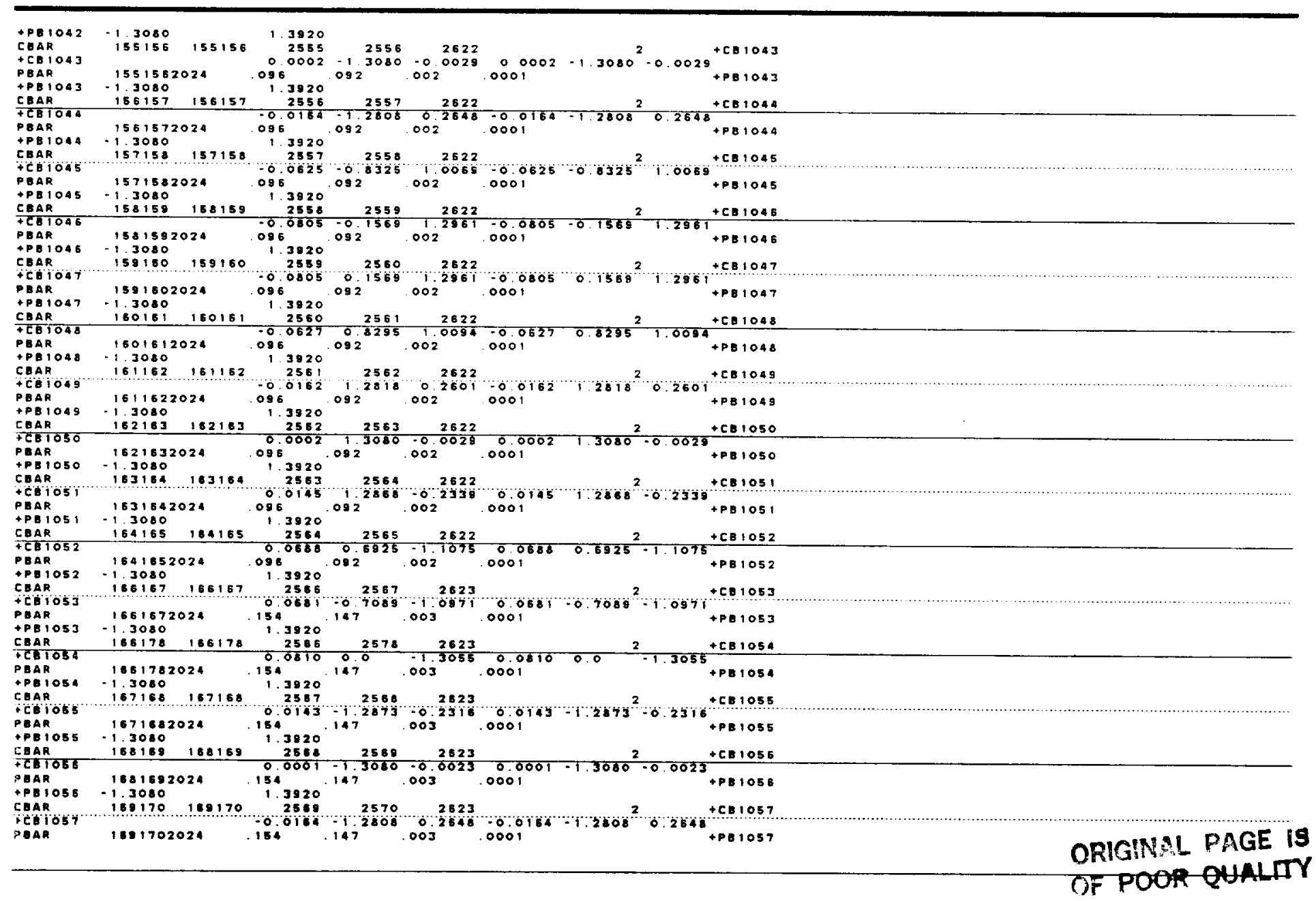




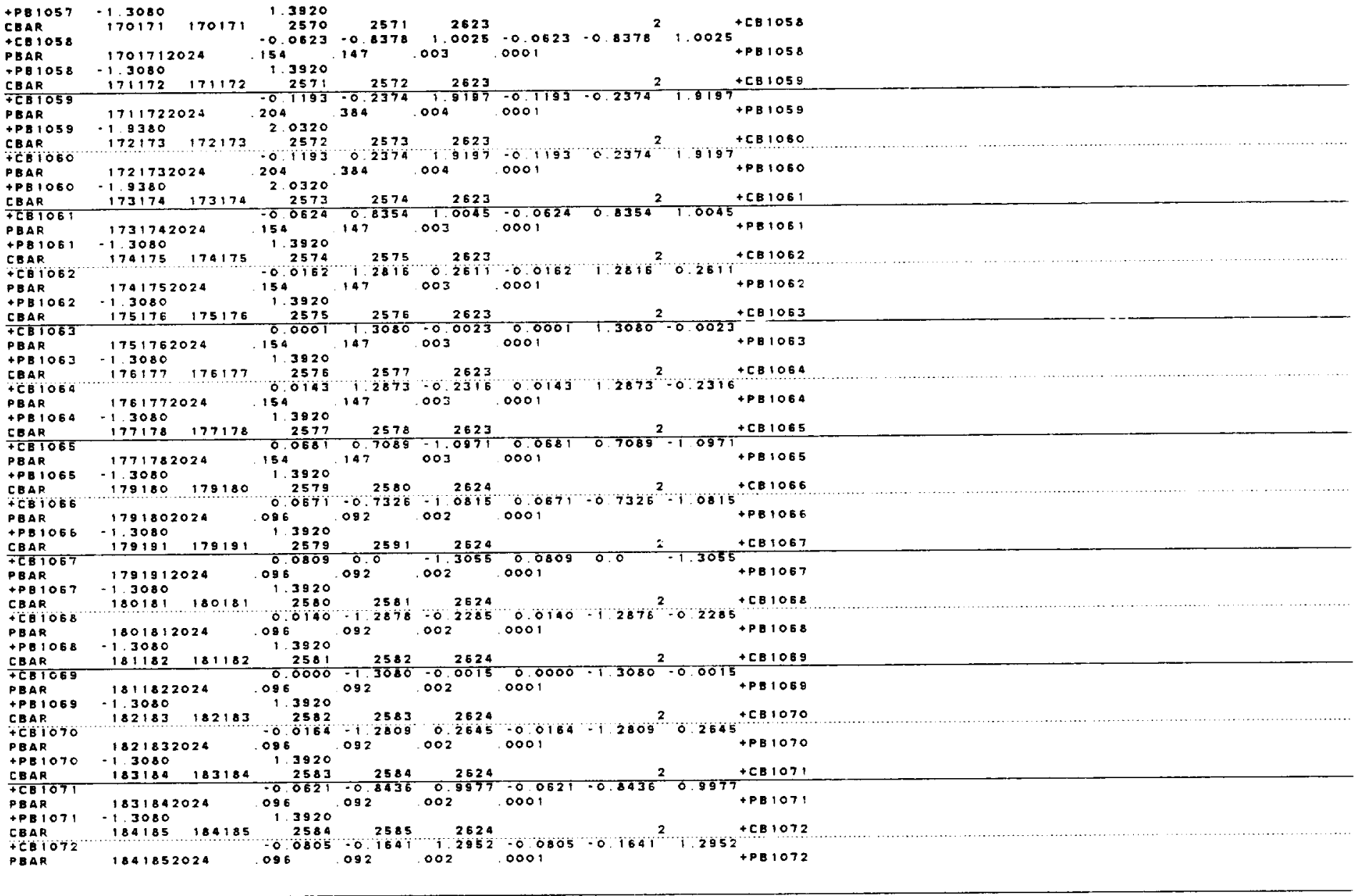

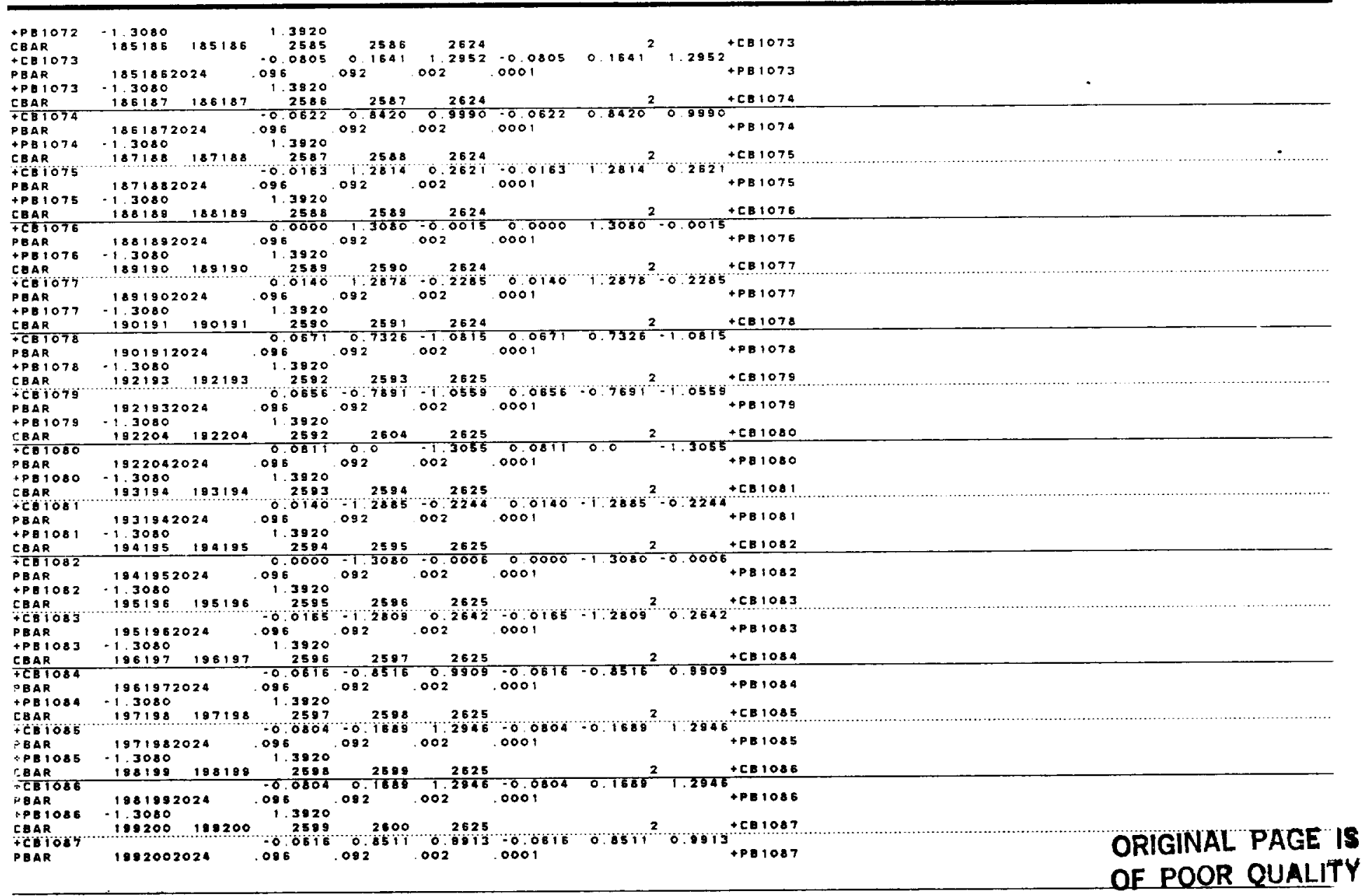




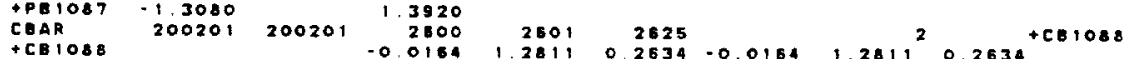

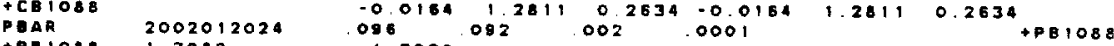

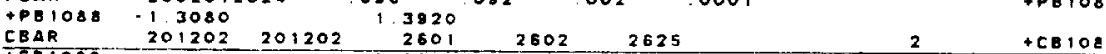

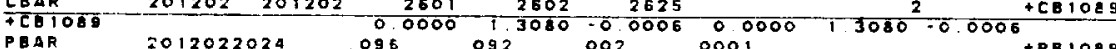

$P B A R$

TPB 1 OB
COAR

COAR
+CBIOSO

PEAR

CBAR

CBAR

PBAR
PBIIO

CEAR

PBAR

+PBiog

CEAR

PBAR

PB1093
CAAR

- CEloga

PQAR
$+P B$.

\begin{tabular}{l} 
CPB OY \\
CBAR \\
\hline CETOS
\end{tabular}

+CETO

PEAR
PBIOO

CBAR

PBAR

TPB IOQ
CEAR

CEAR

PBAR

CBA109
CBAR

CBAR
+ CEIOO

PEAR
P PAT

PQAR
COAB

$P B A R$

+PB 1099
COAR

TEB iloo

PEAR
+ PB 1100

CBAR

+CBT1

PEAR
$+P B 10$

PPBI
CBAR
+CB I

$2012022024 \quad 095$

$202203 \quad 202203 \quad 2602 \quad 2603 \quad 2625+C B 1090$

$2022032024 \quad 0.044001288500224000140128850 .2244+P B 1090$

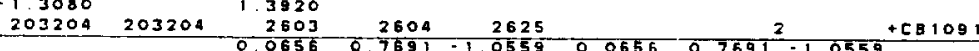

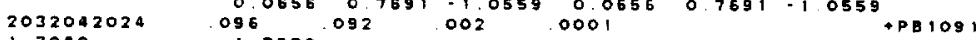

$205206205206 \quad 2605 \quad 2606 \quad 2626 \quad+581092$

$2052052024 \quad 13907371^{3137} .004^{7226} 0001070-1.3137-1.7226+081092$

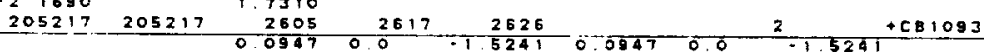

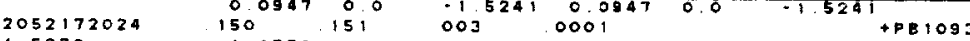

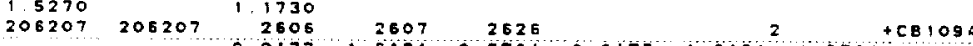

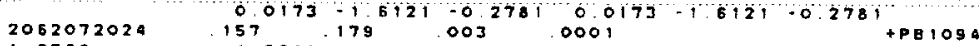

\begin{tabular}{llrrrrr}
207208 & 207208 & 2607 & 2608 & 2625 & 2 & 2 \\
\hline
\end{tabular}

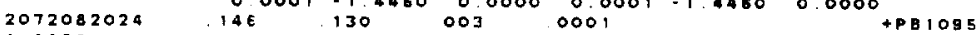

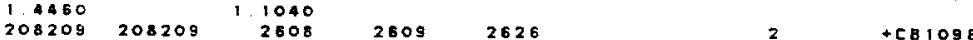

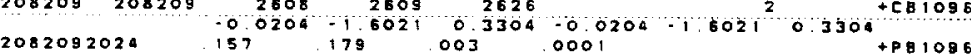

$\begin{array}{rrrrrrr}1.6360 & & 1.2640 & & & & \\ 209210 & 209210 & 2608 & 2510 & 2525 & 2 & +C 61097\end{array}$

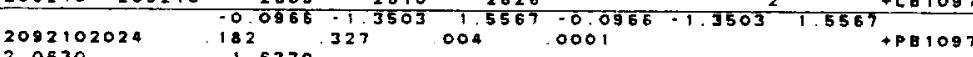

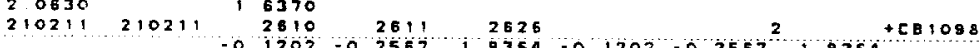

$2102112024 \quad 1^{-0} 1202-0.25670033640001202-0.25671 .9364+P B 1098$

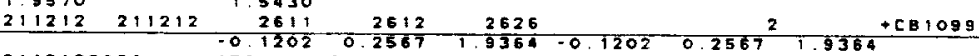

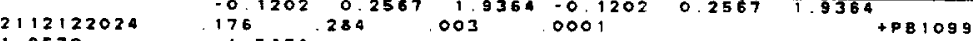

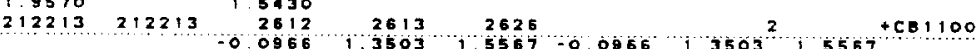

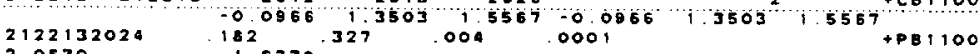

$\begin{array}{rrrrrrr}213314 & 213214 & 1.5370 & 2513 & 2614 & 2626 & 2\end{array}+C B 1101$

$2132142024 \quad 157 \quad 17900.00300010040013304+P B 110$

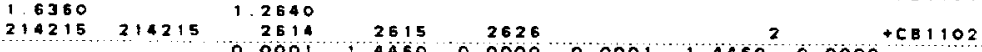

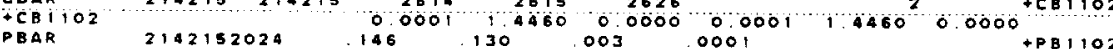

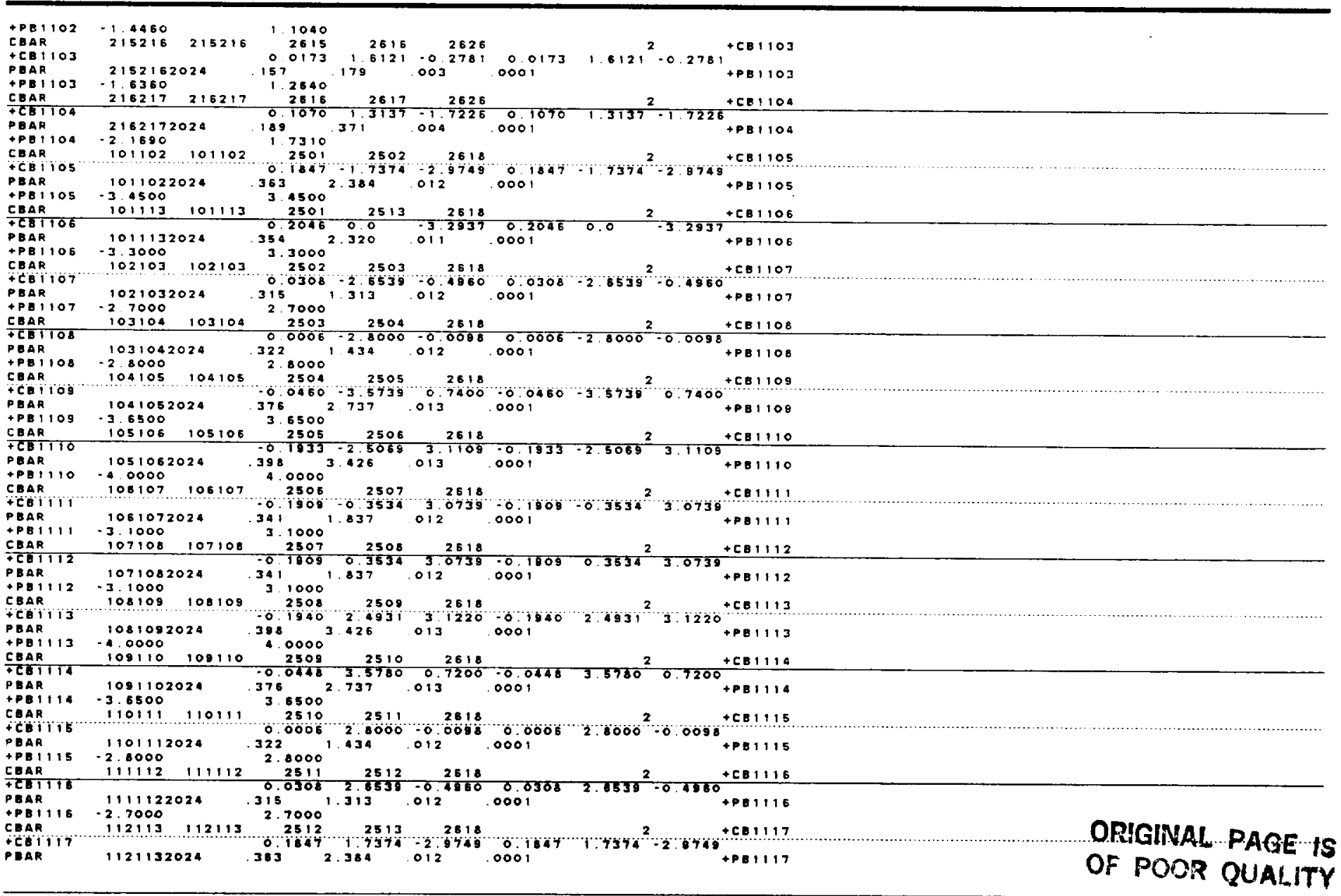




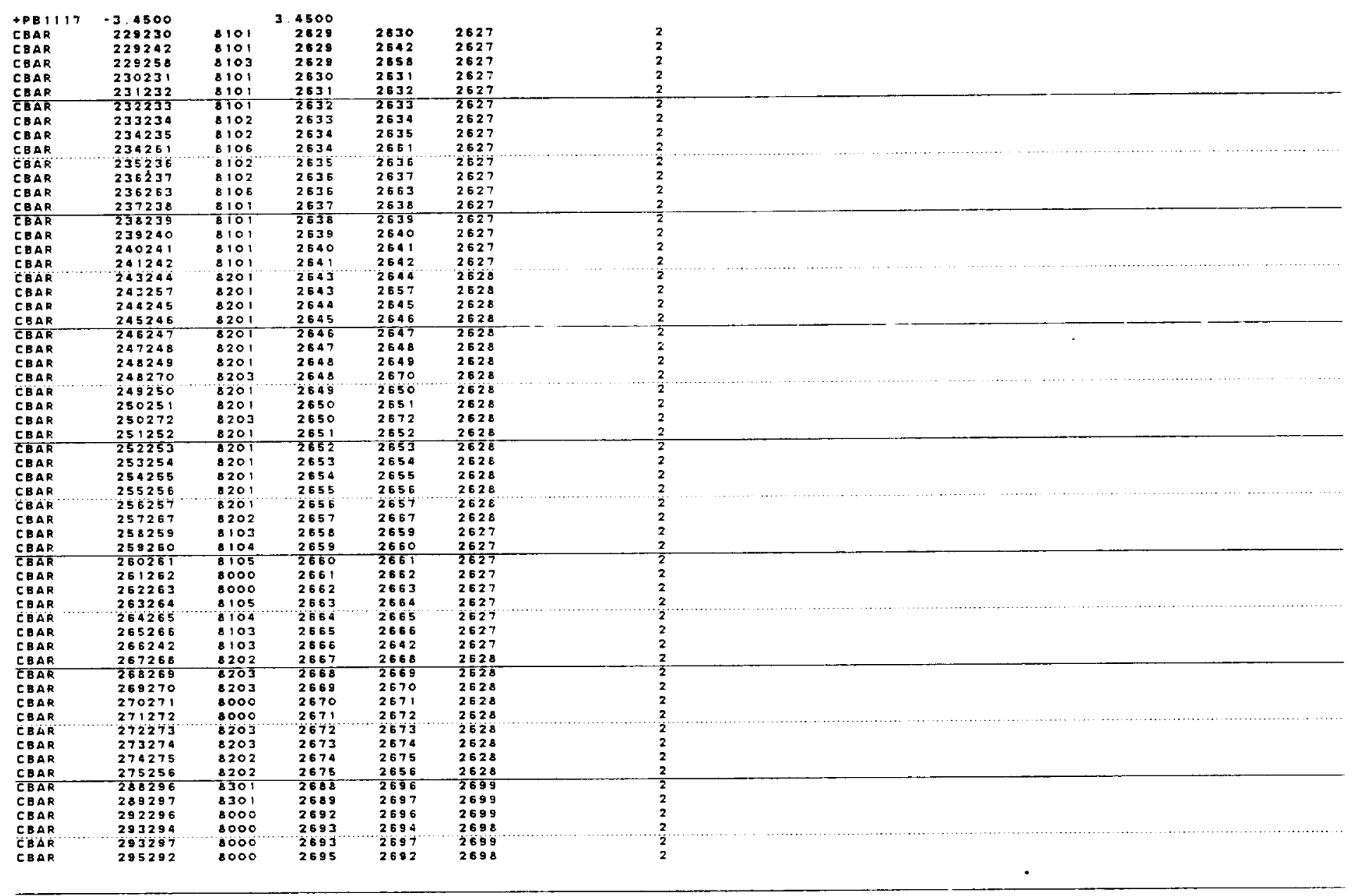

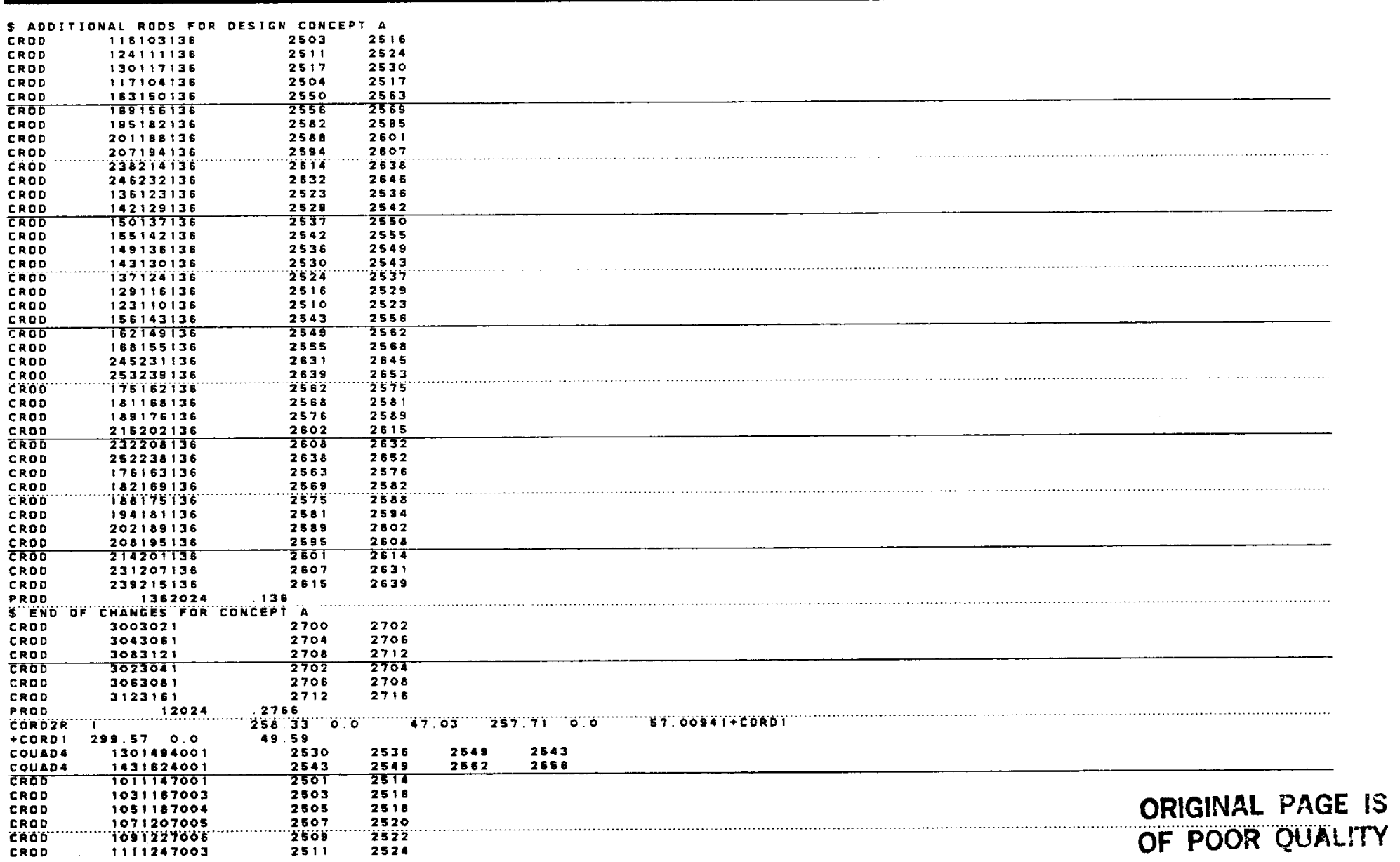




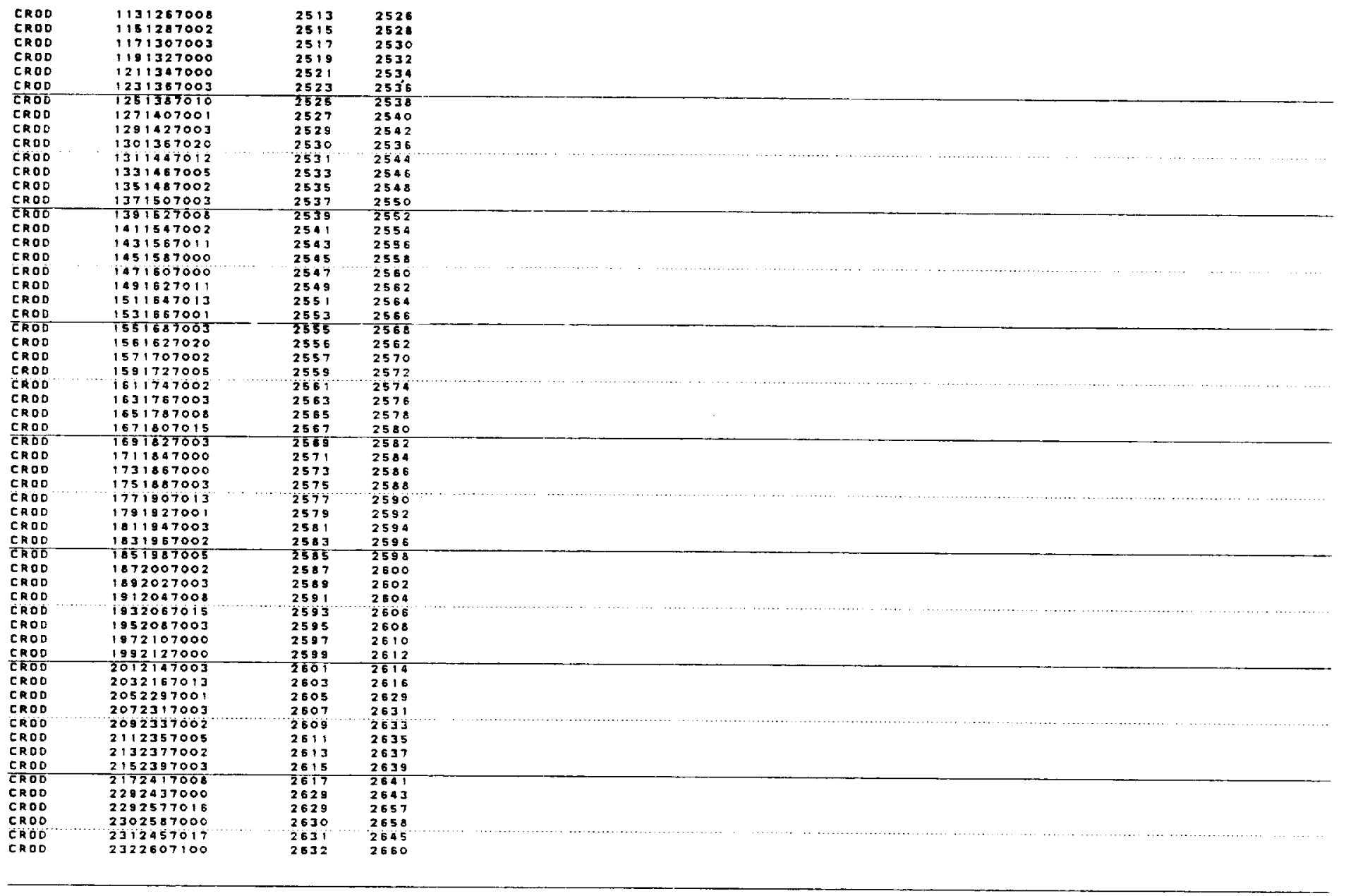

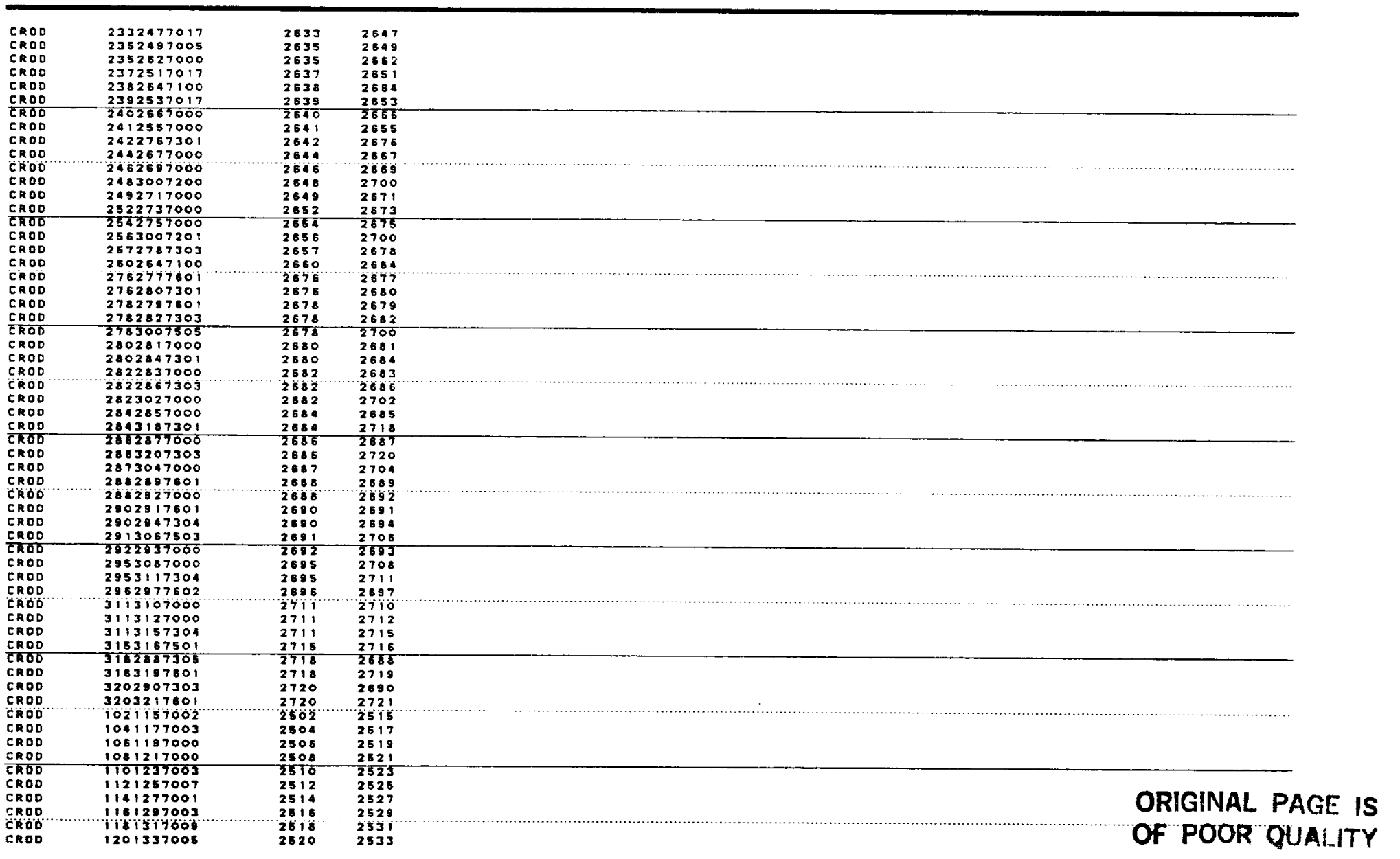




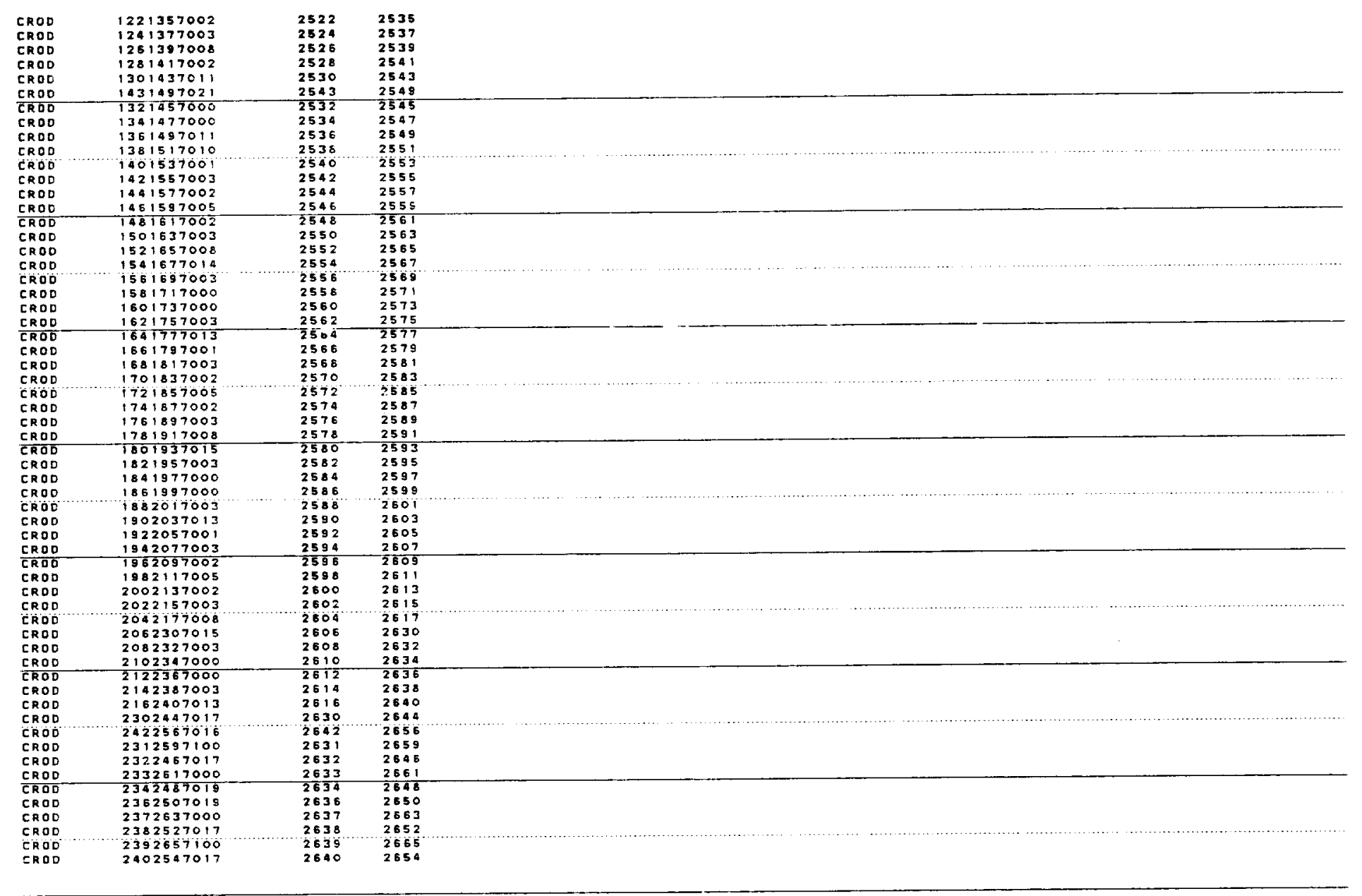

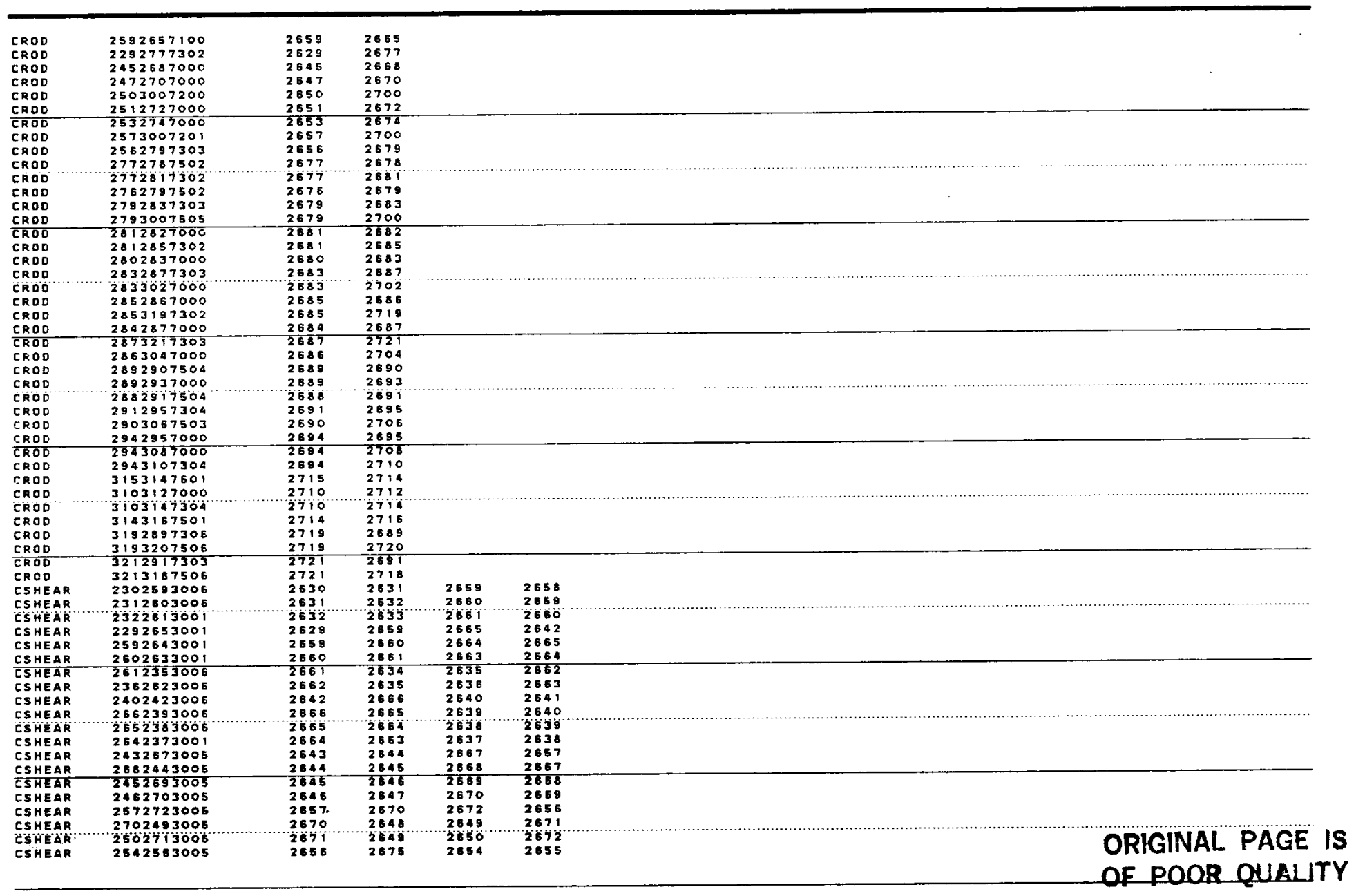




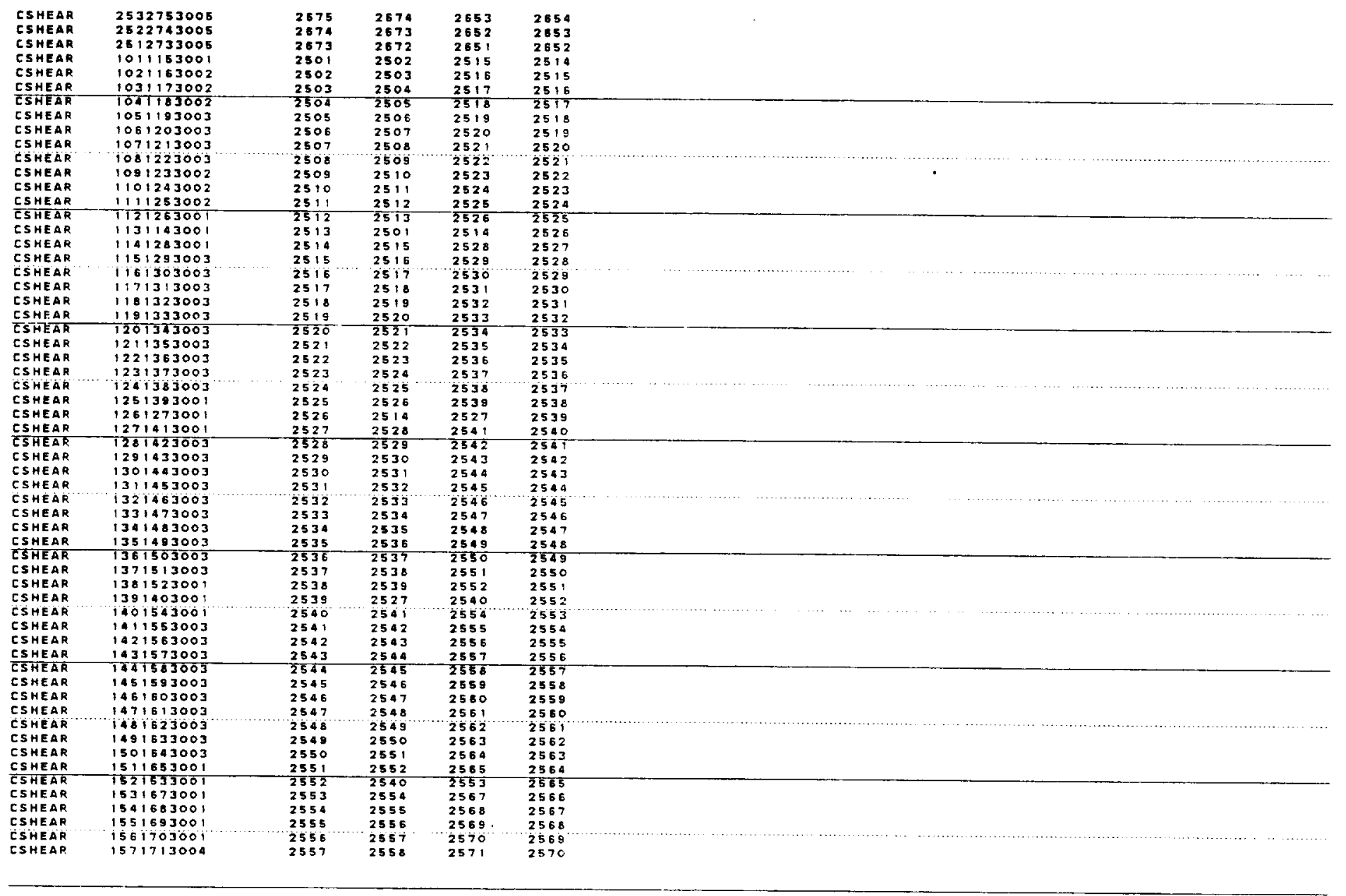

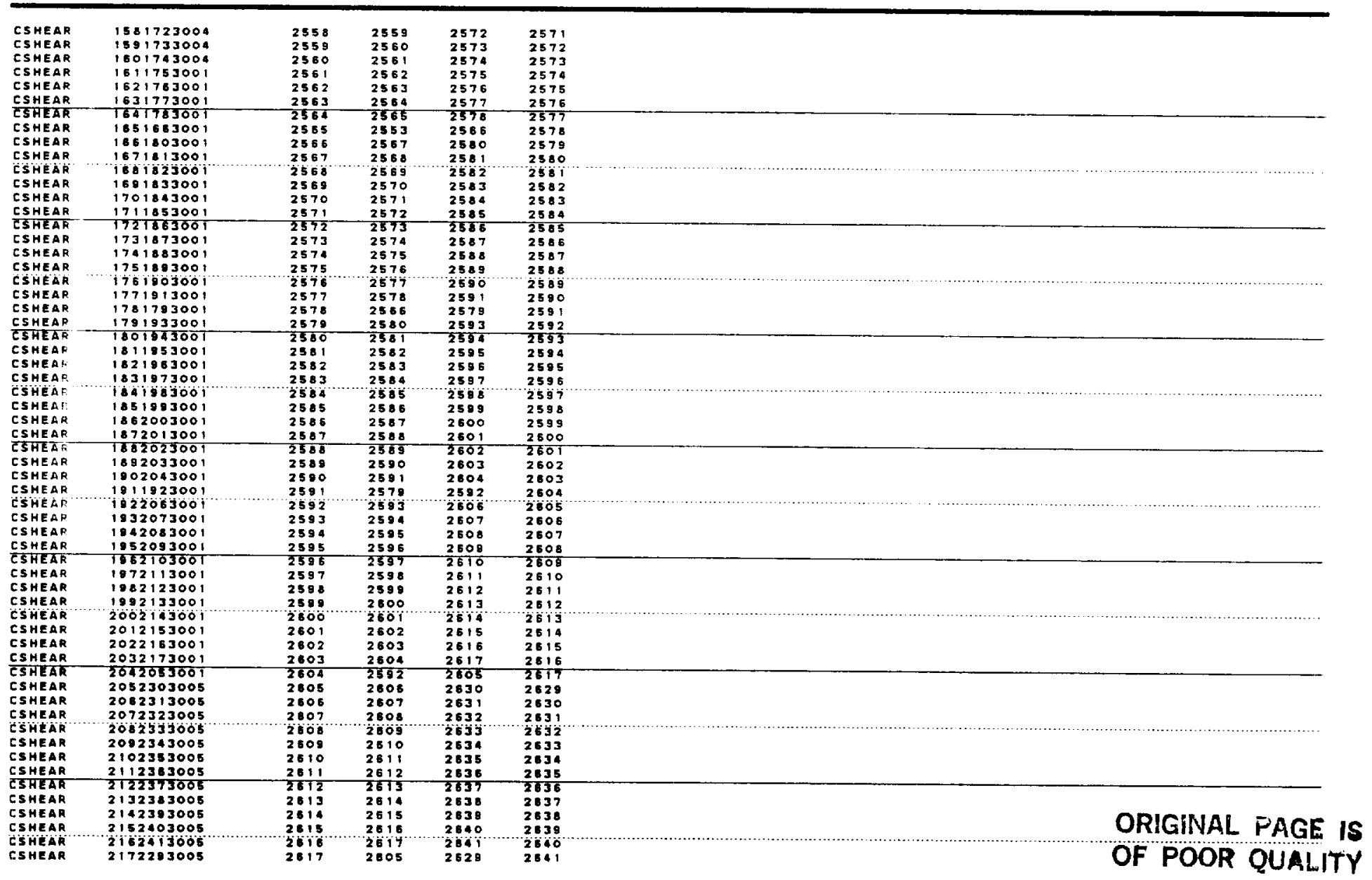




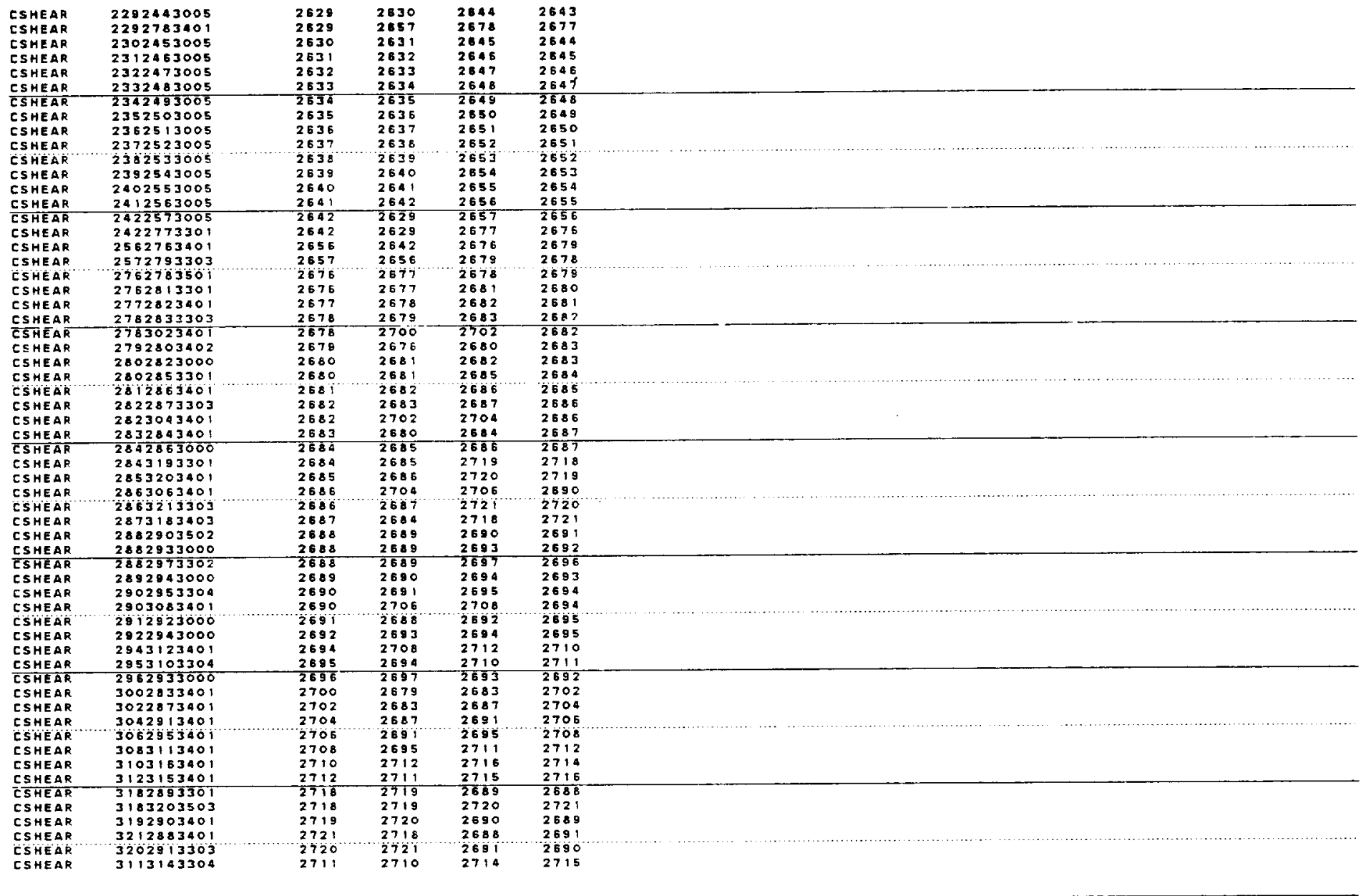

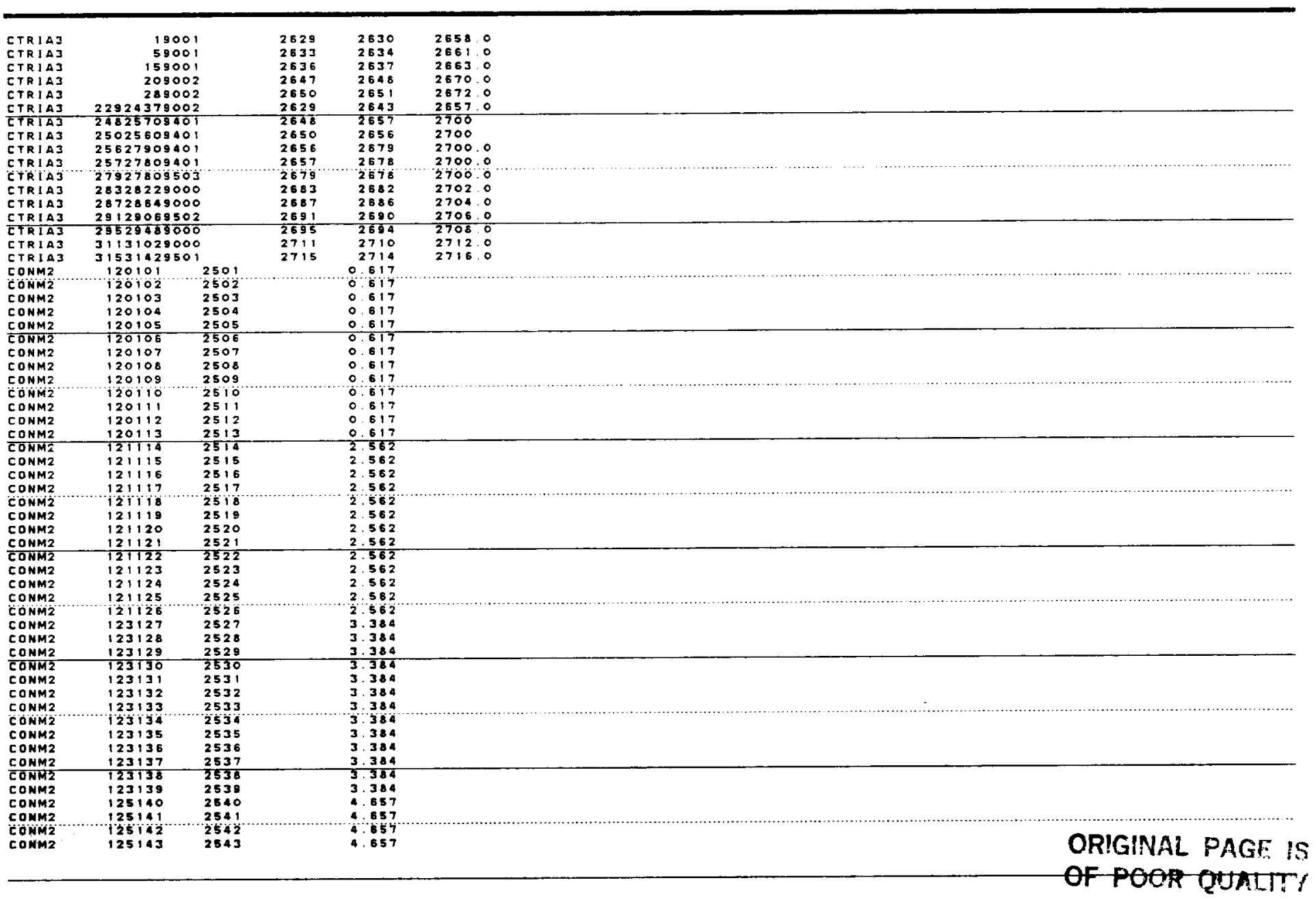




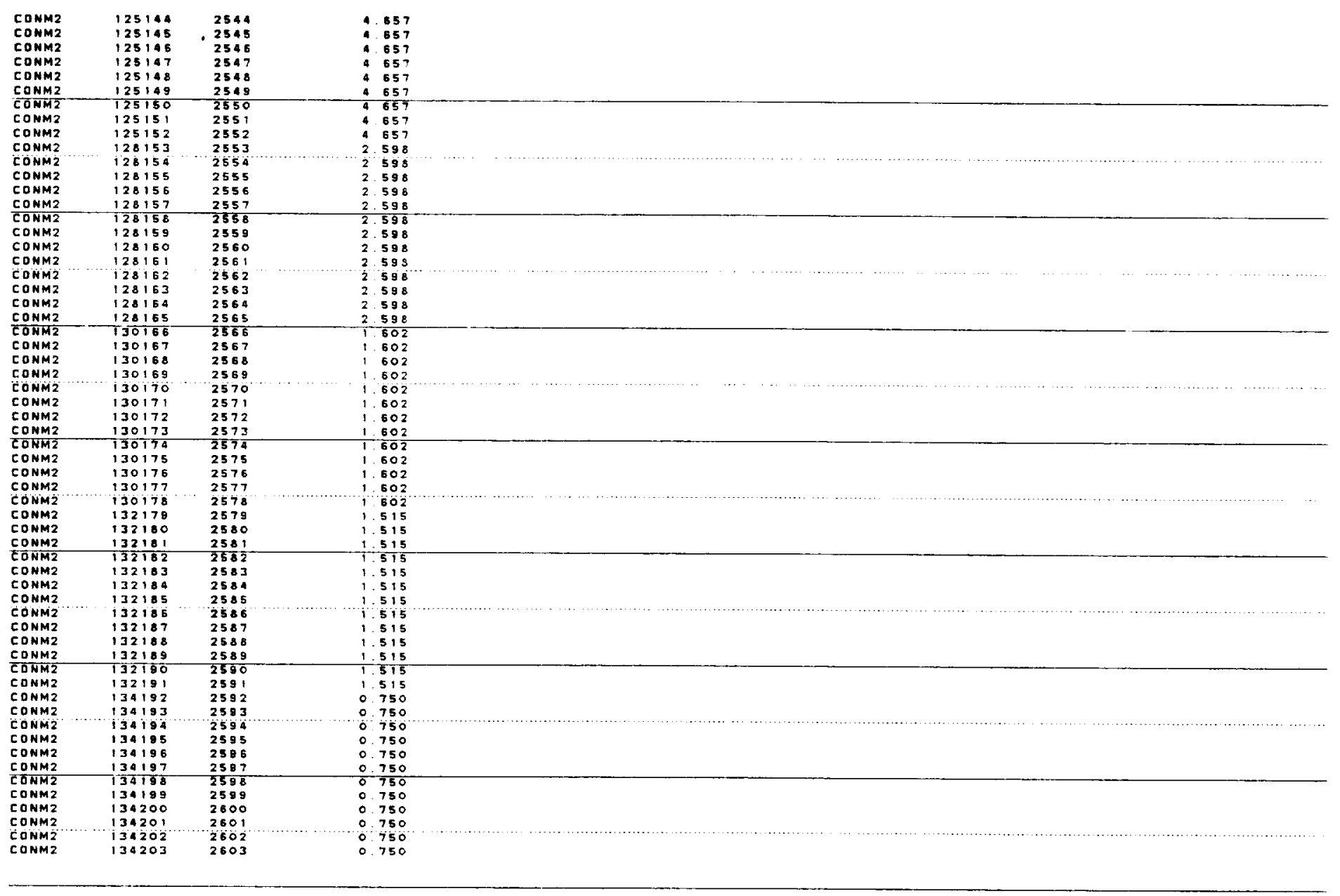

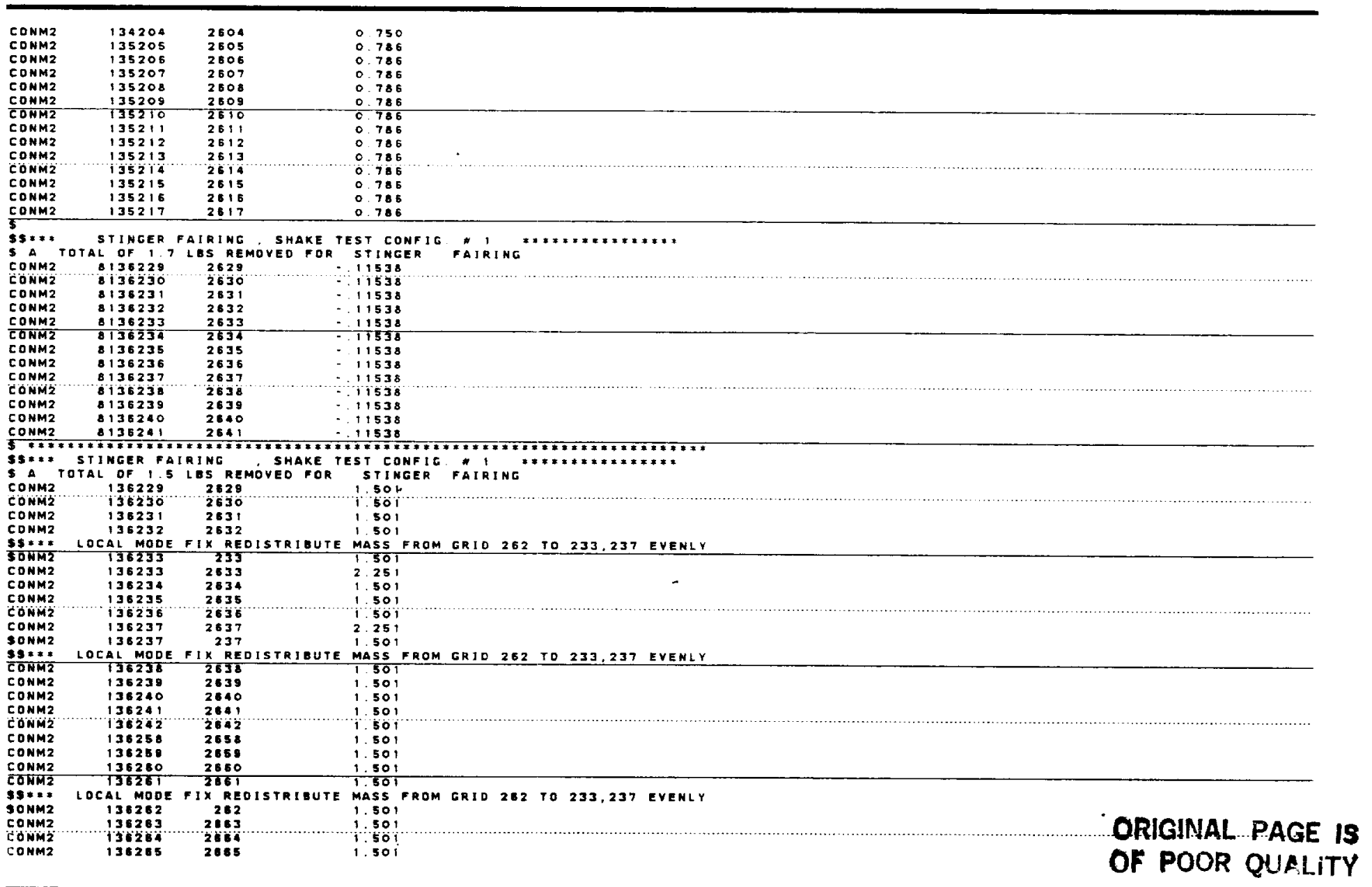




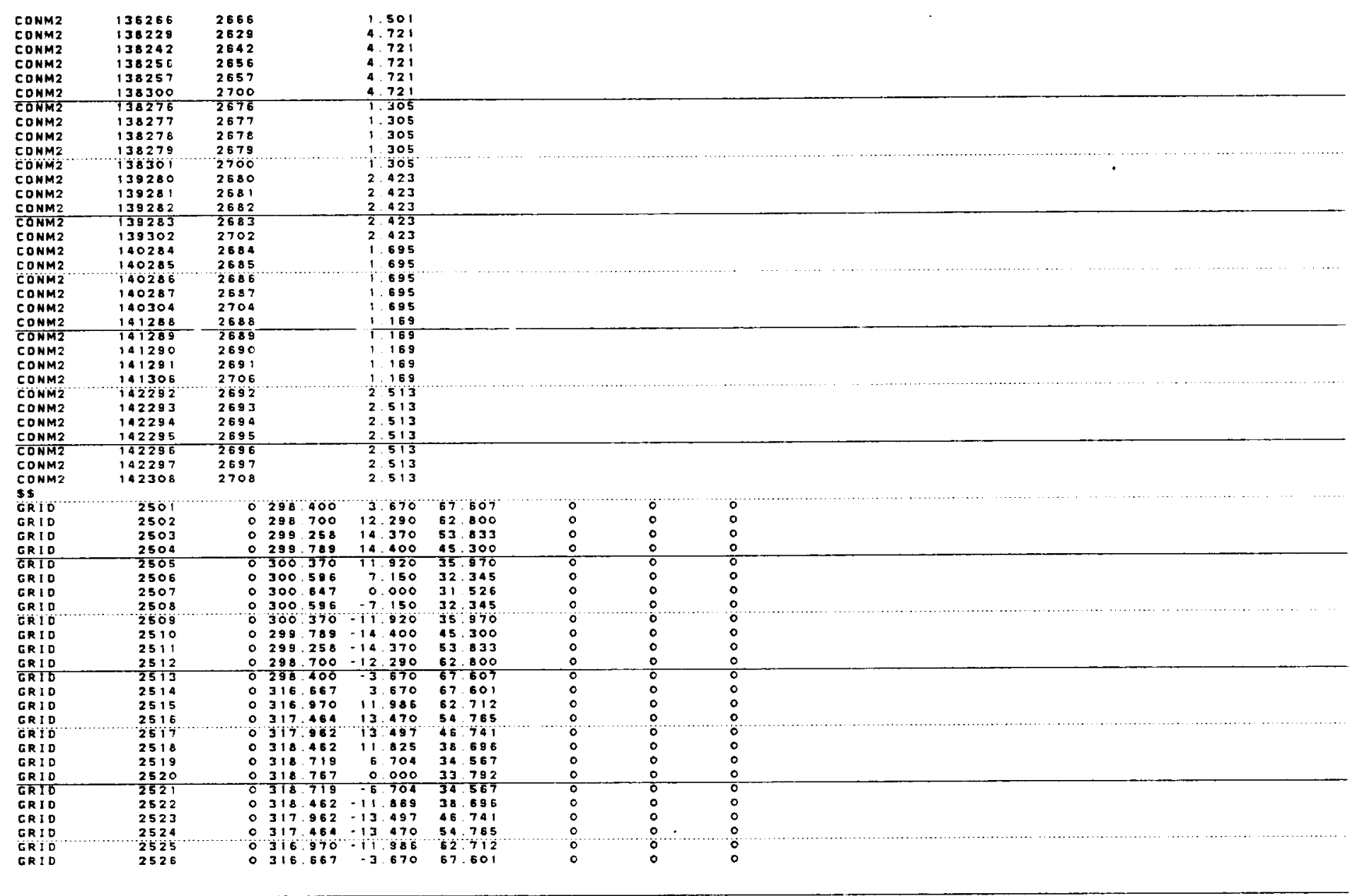

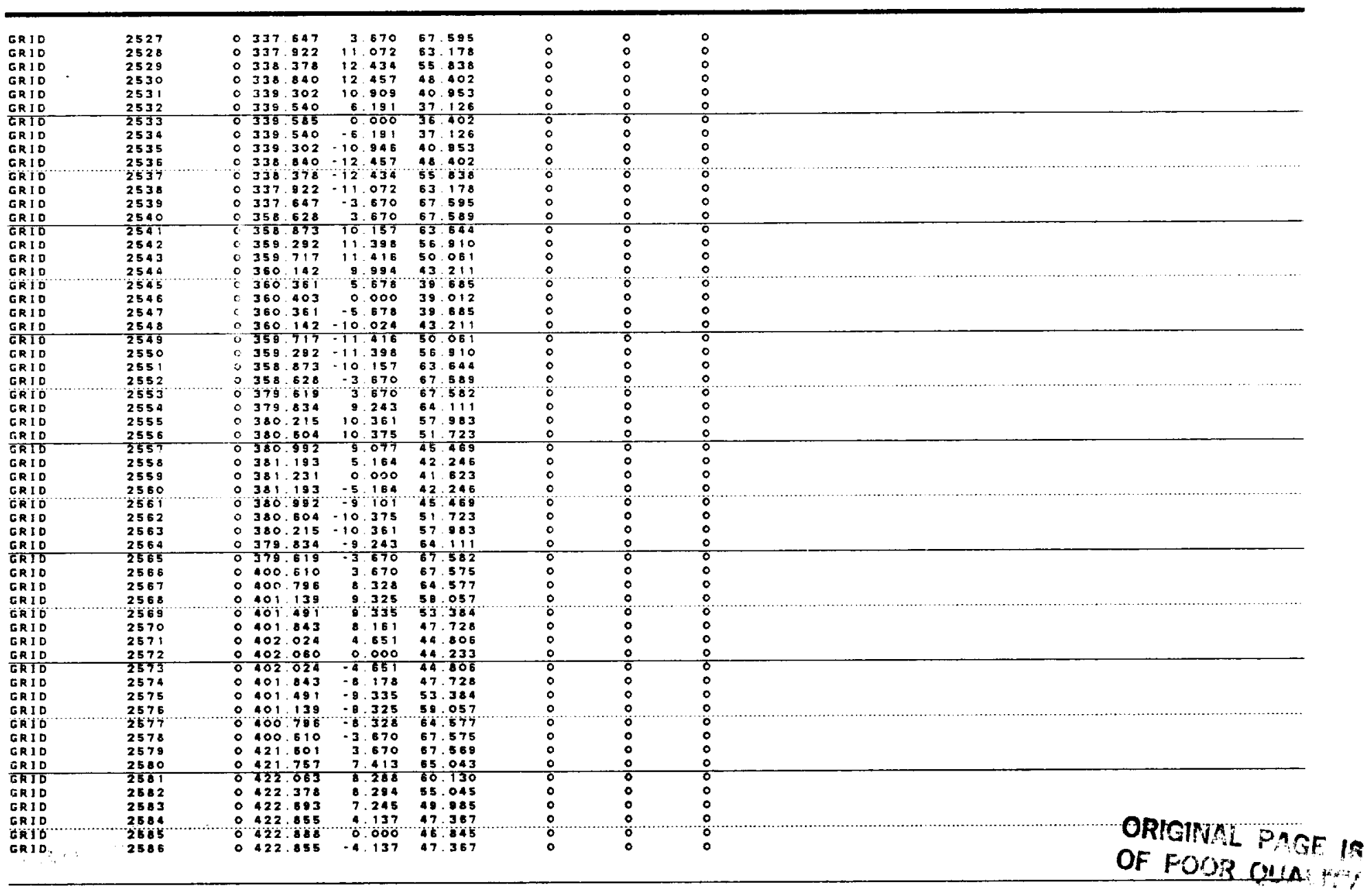




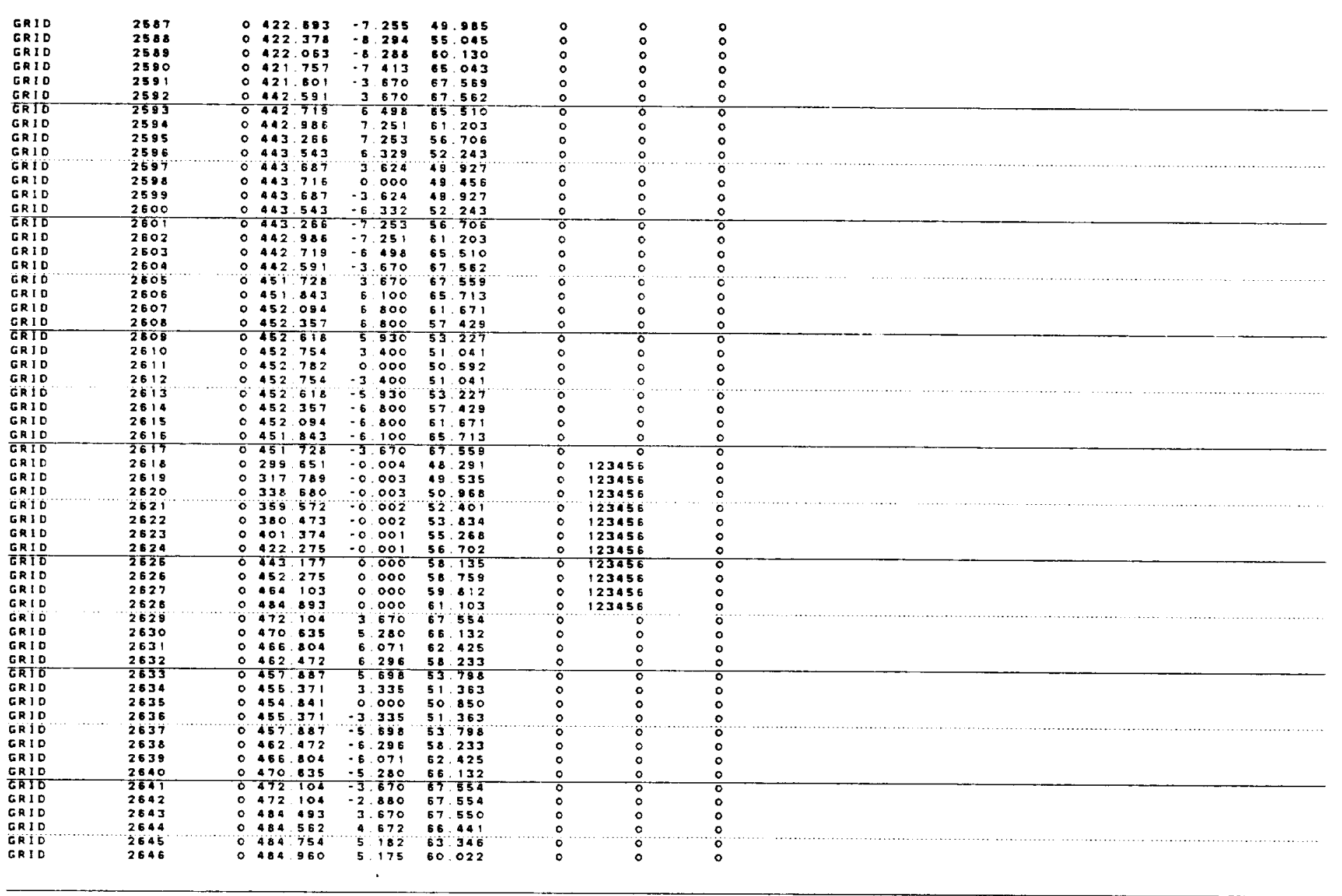

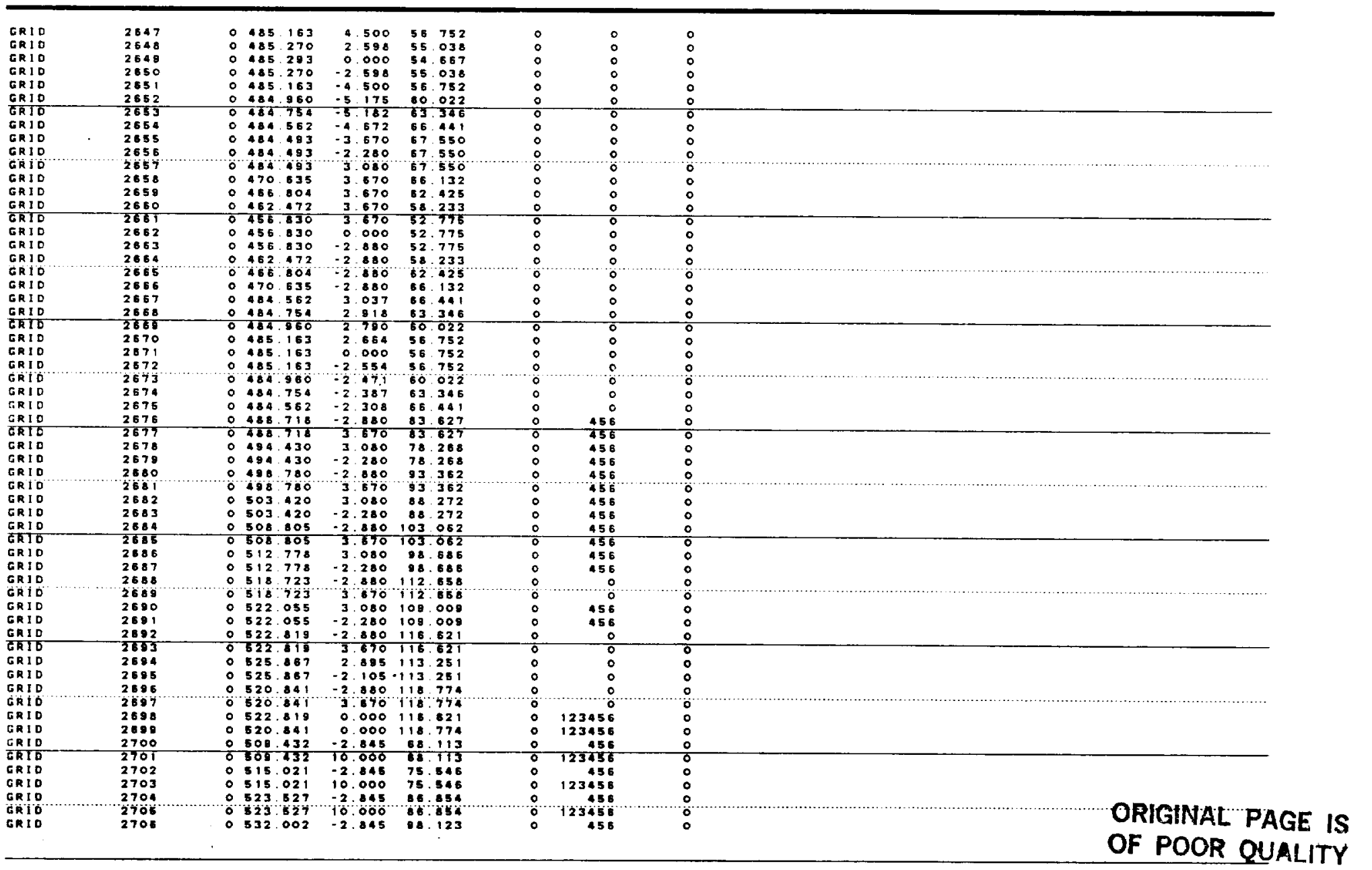




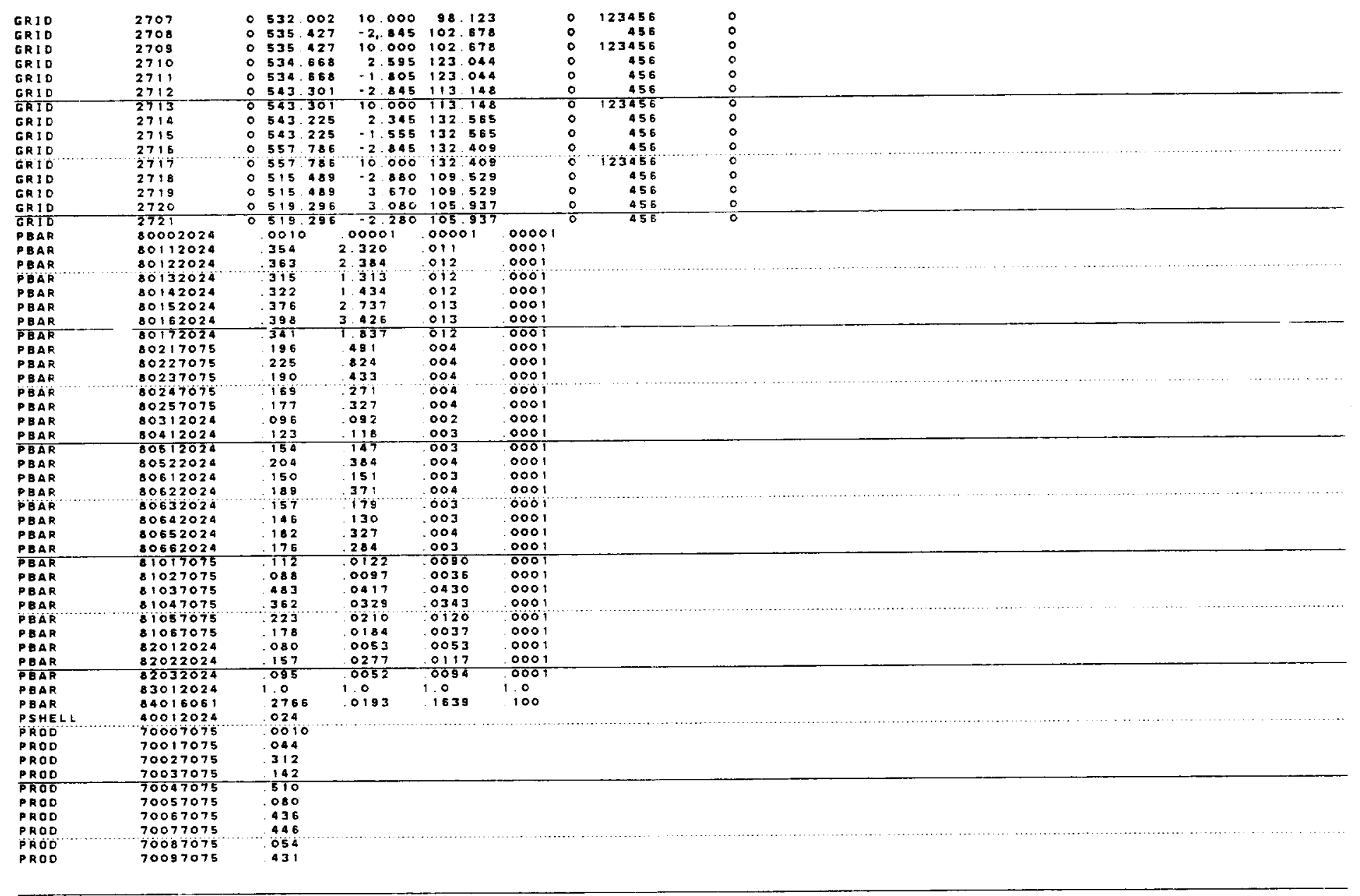

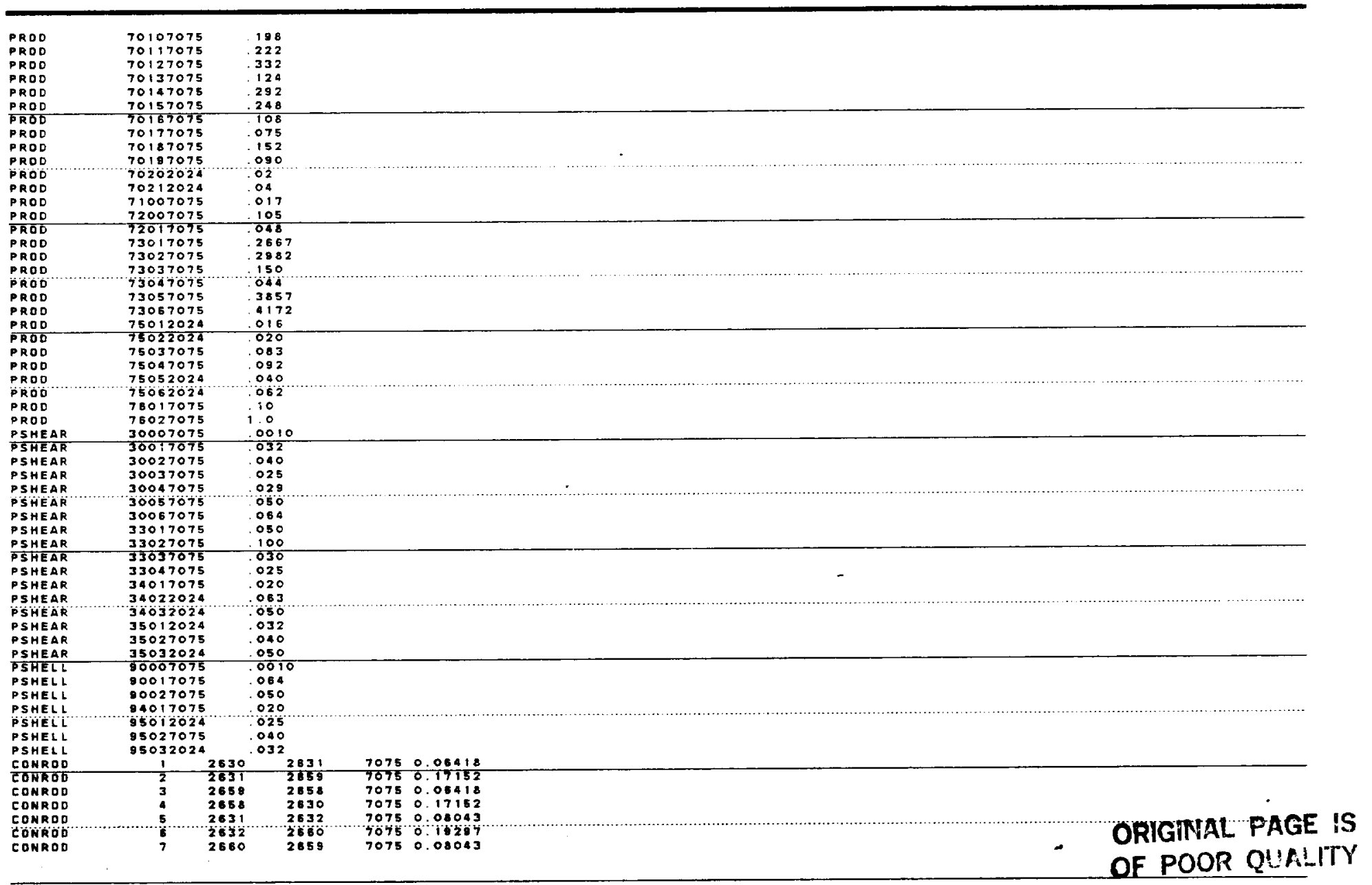




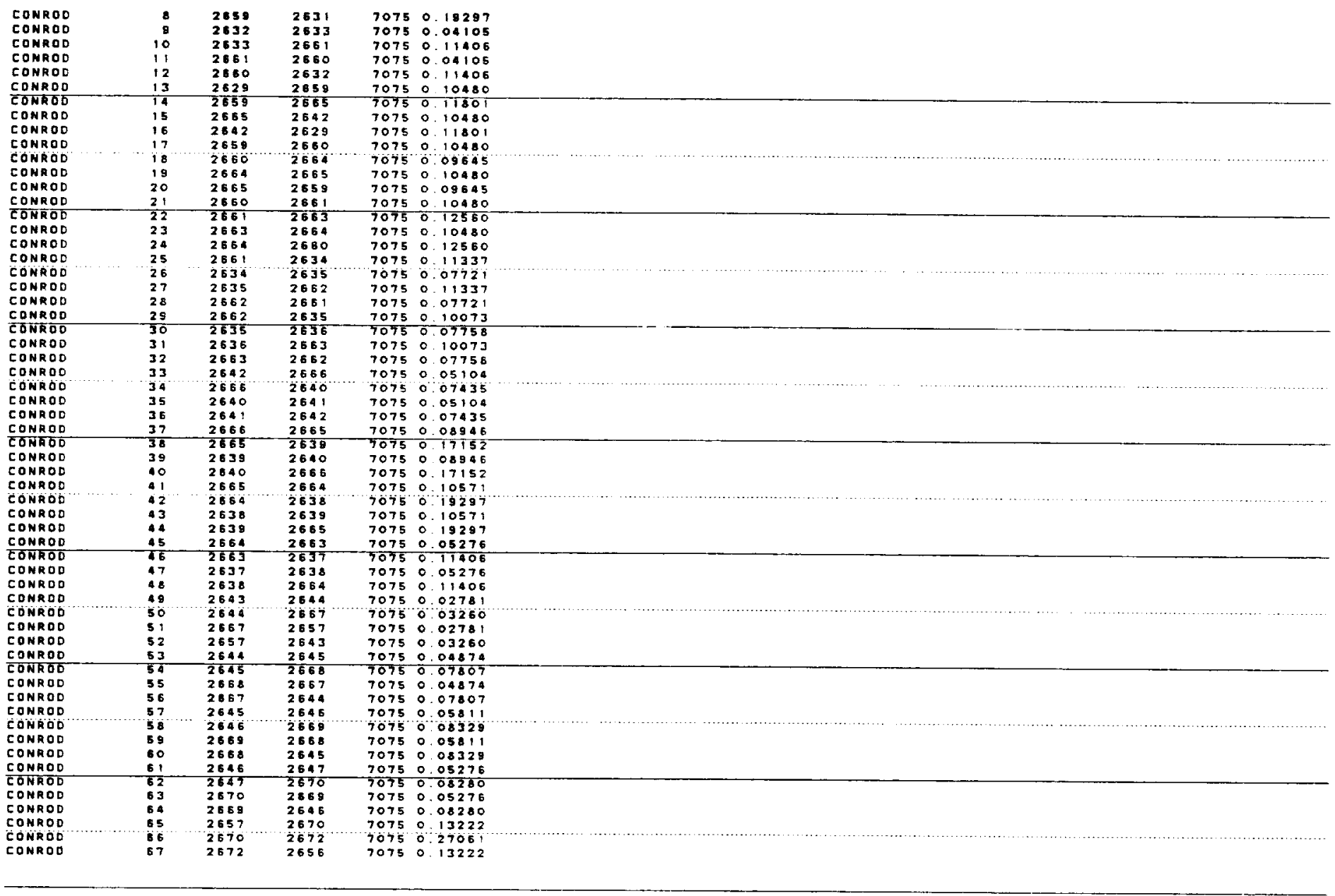

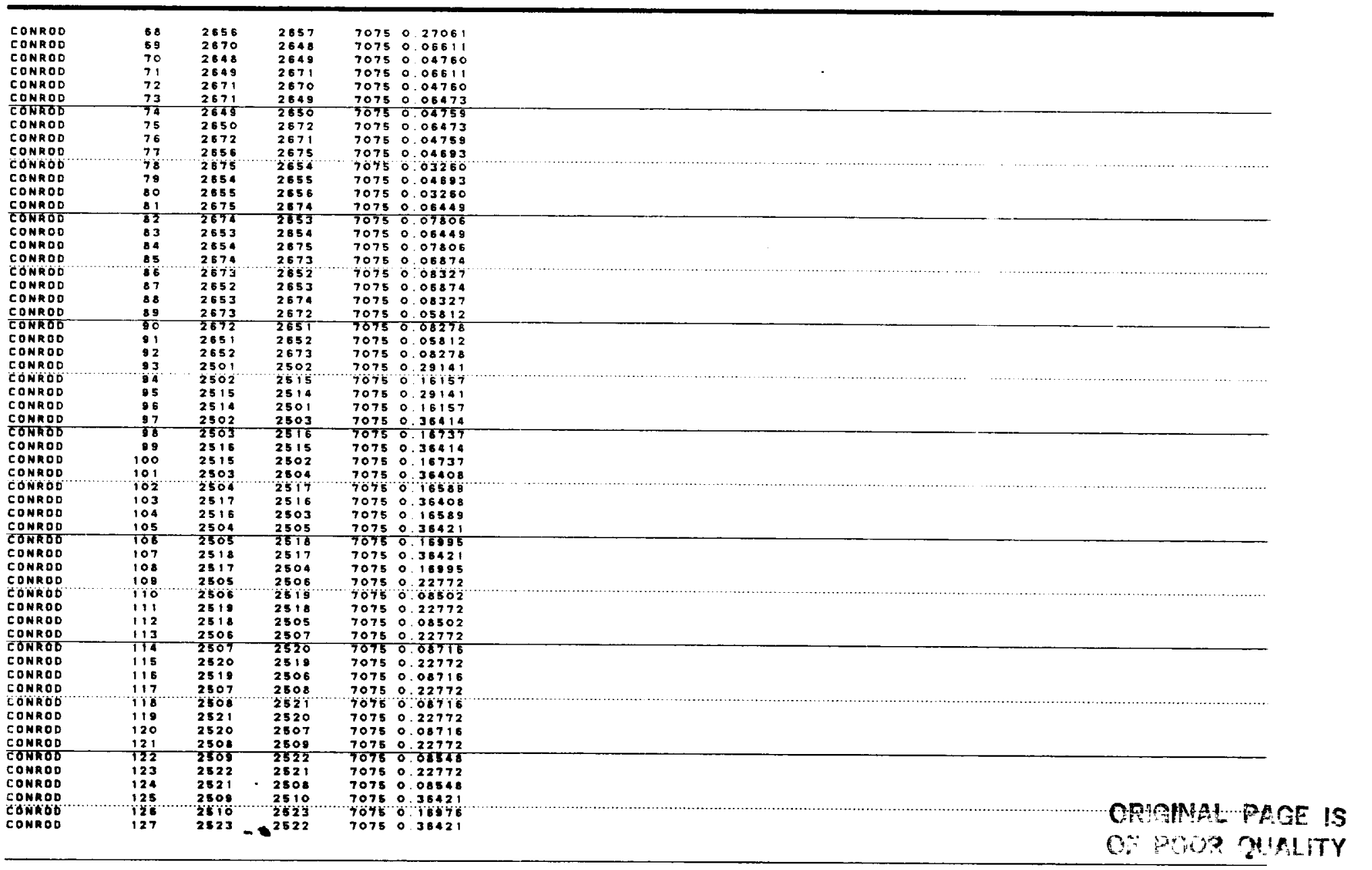




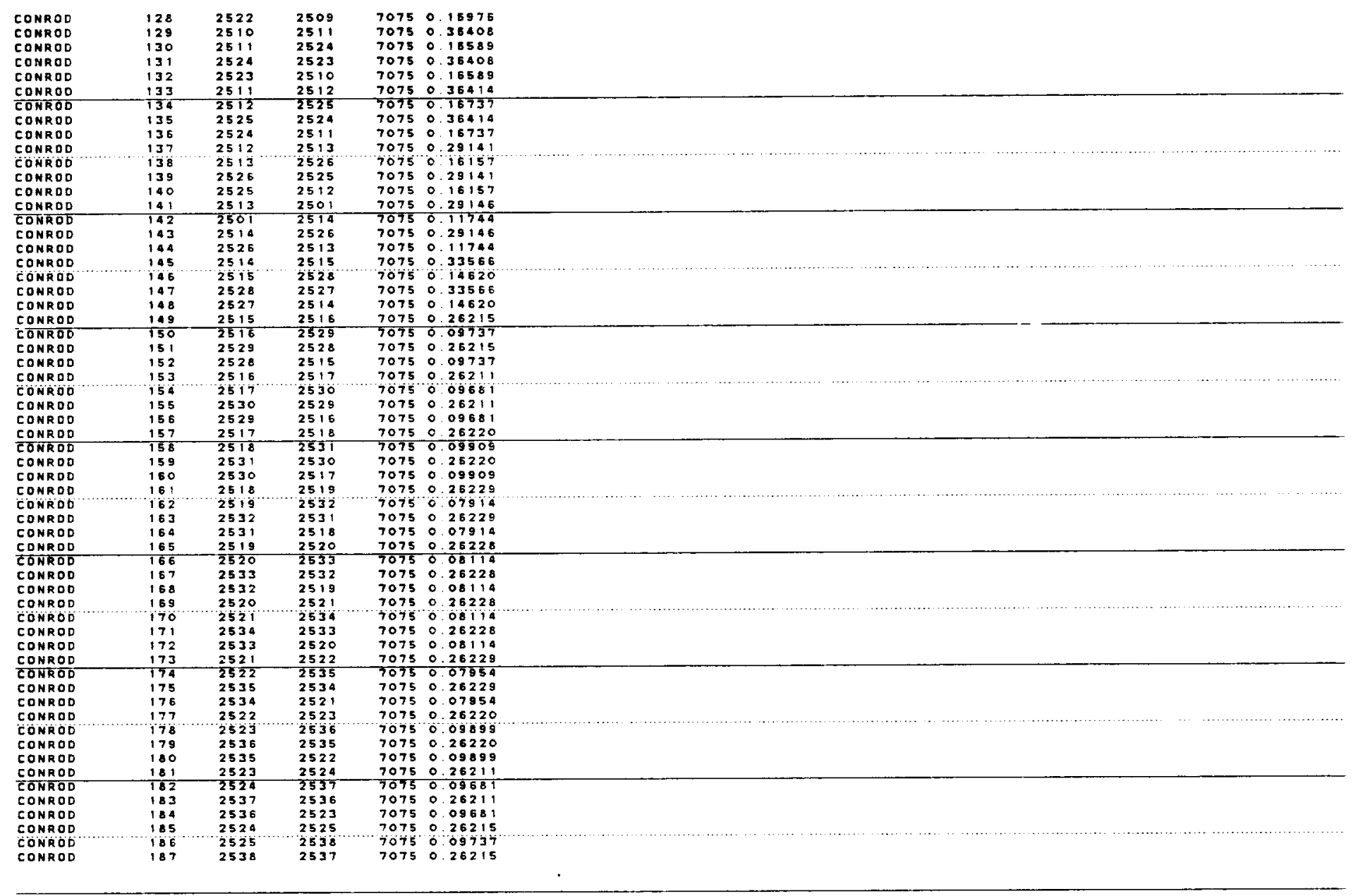

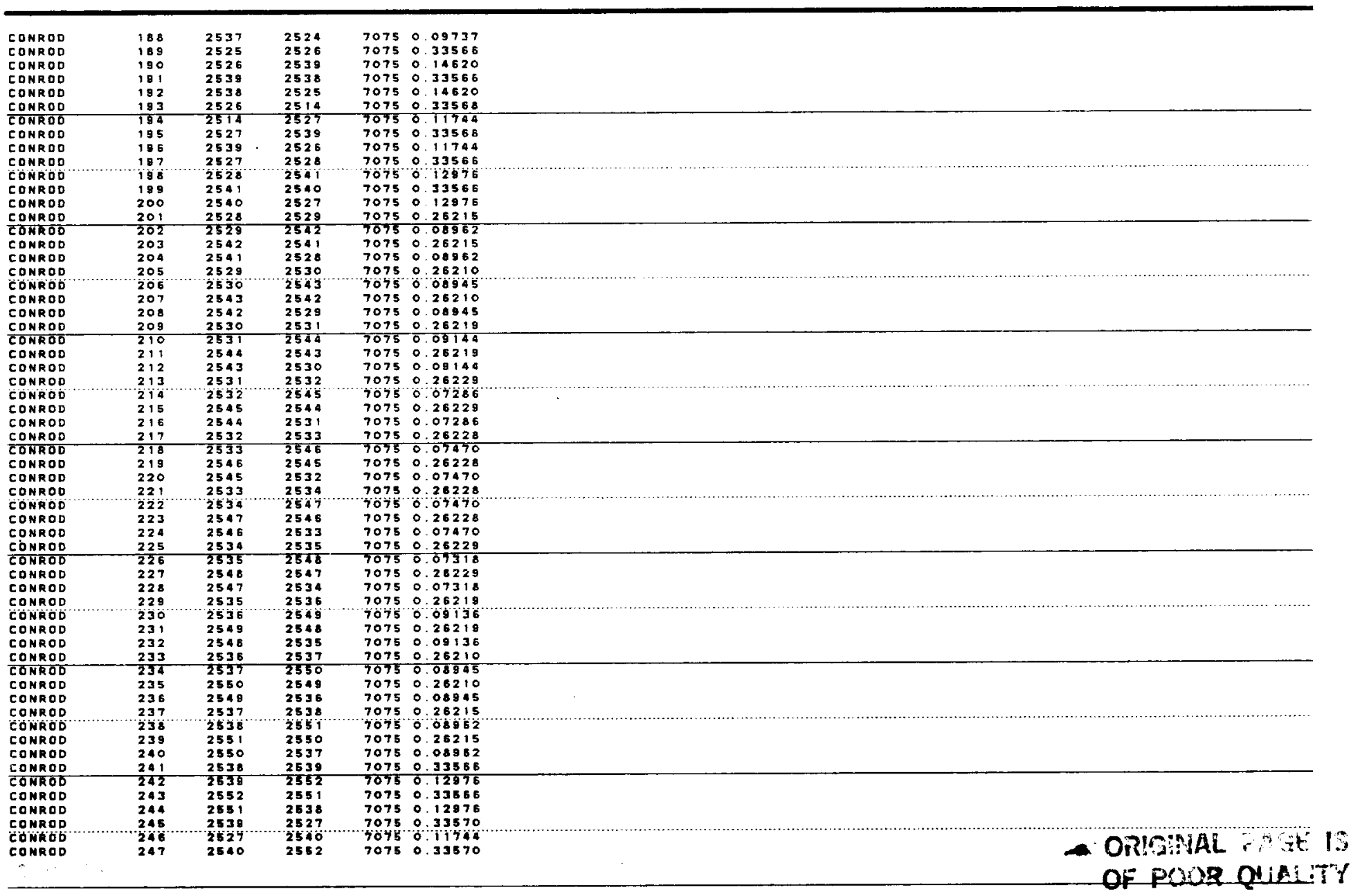




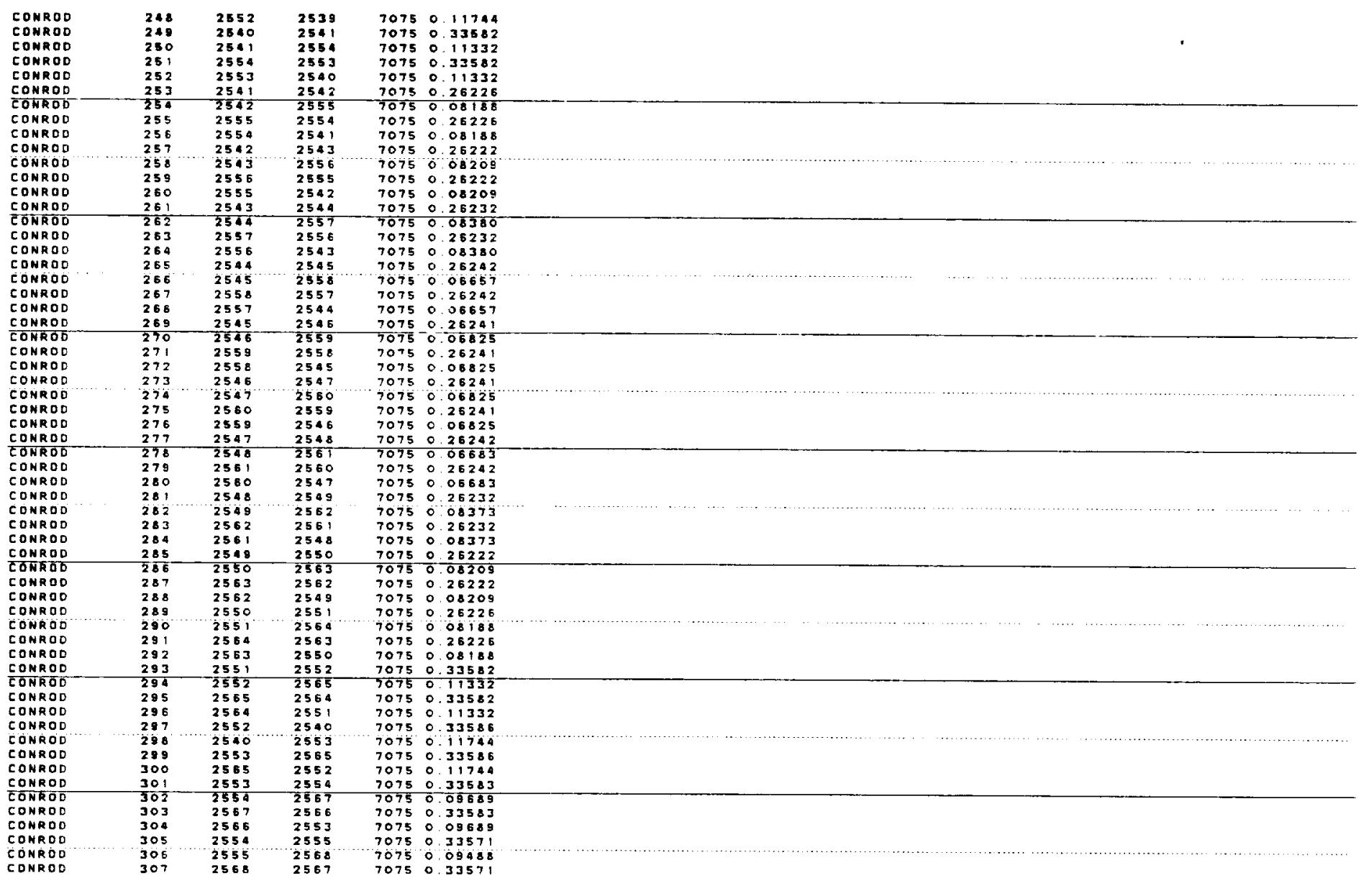

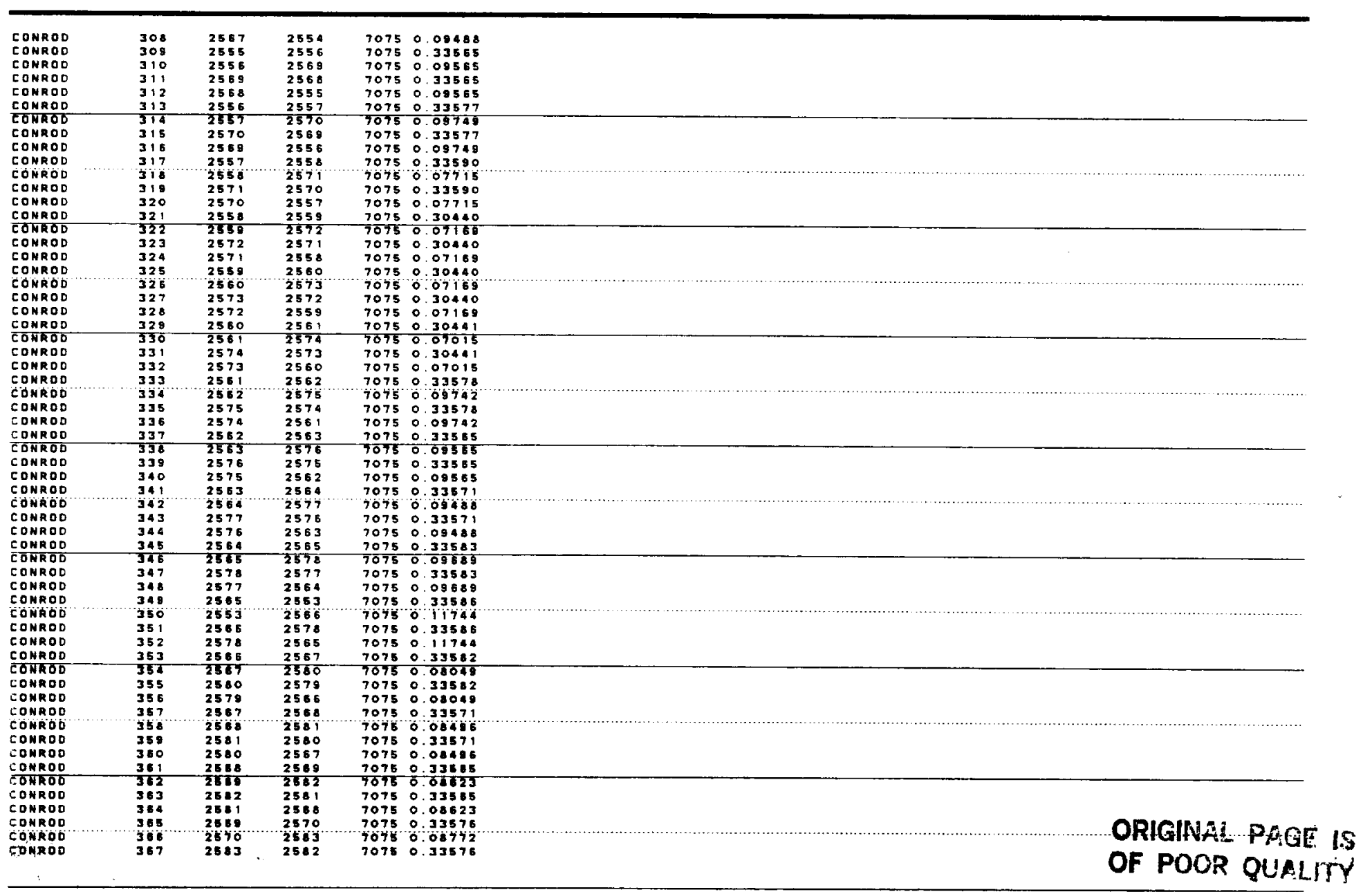




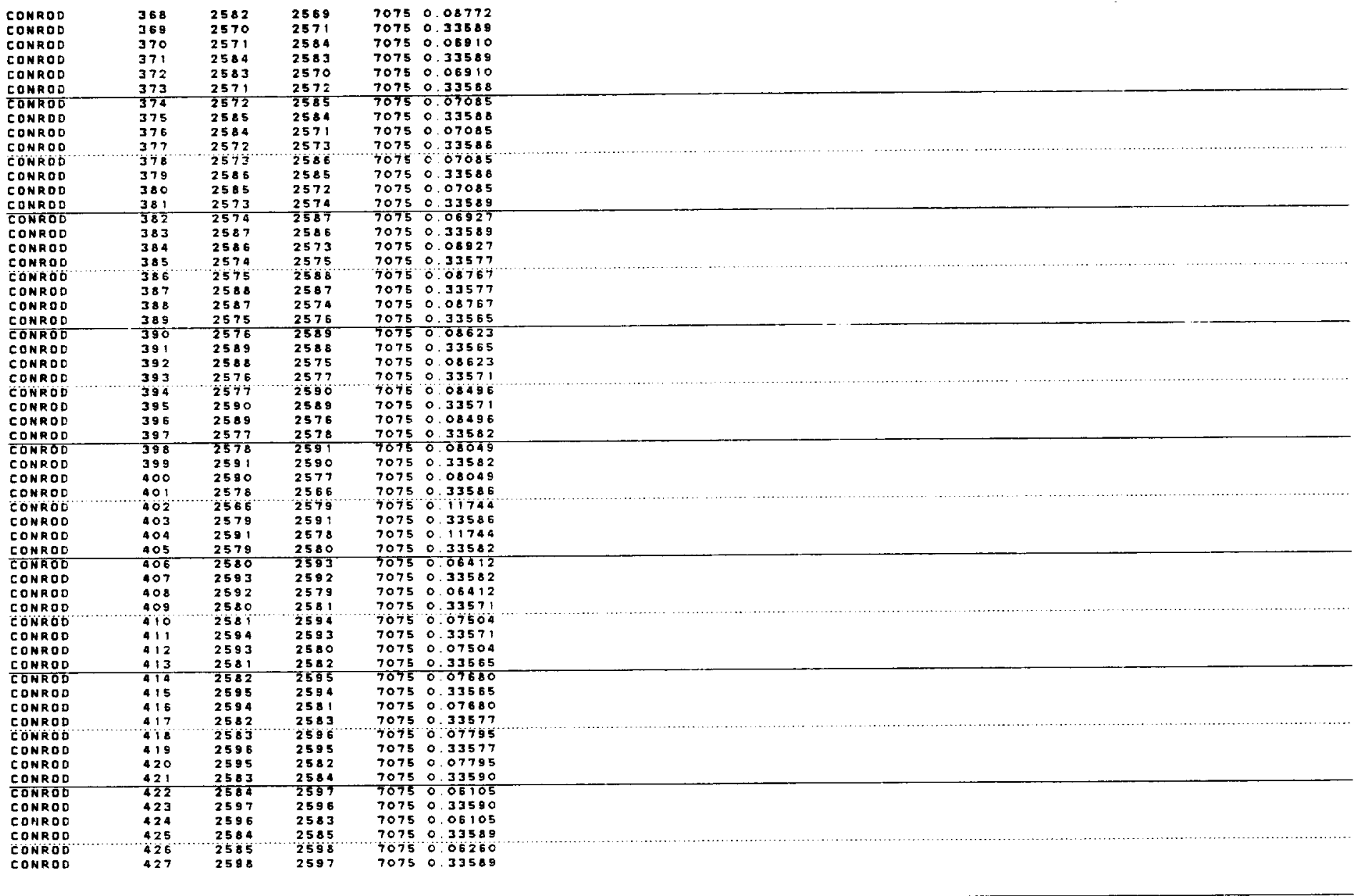

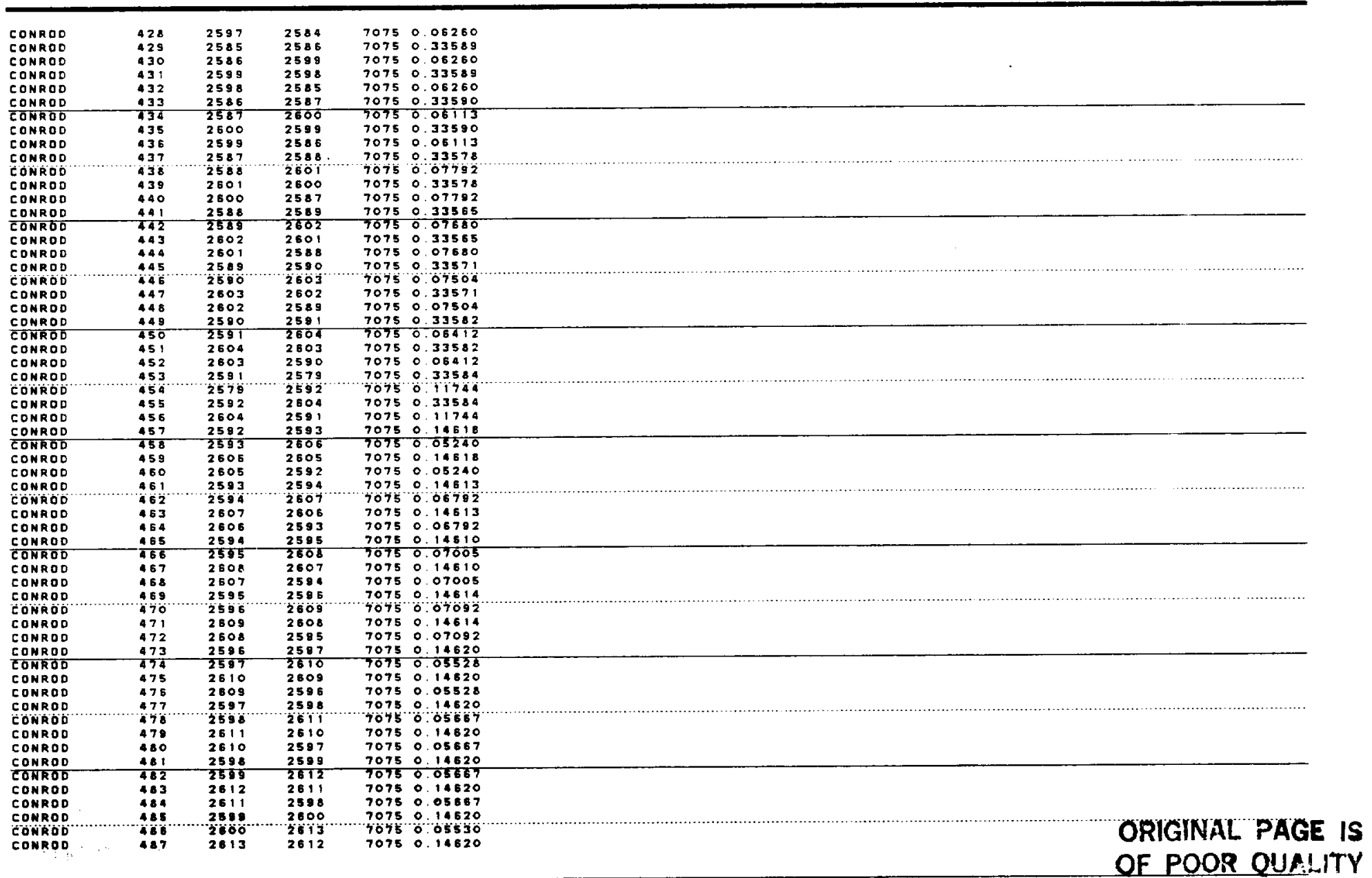




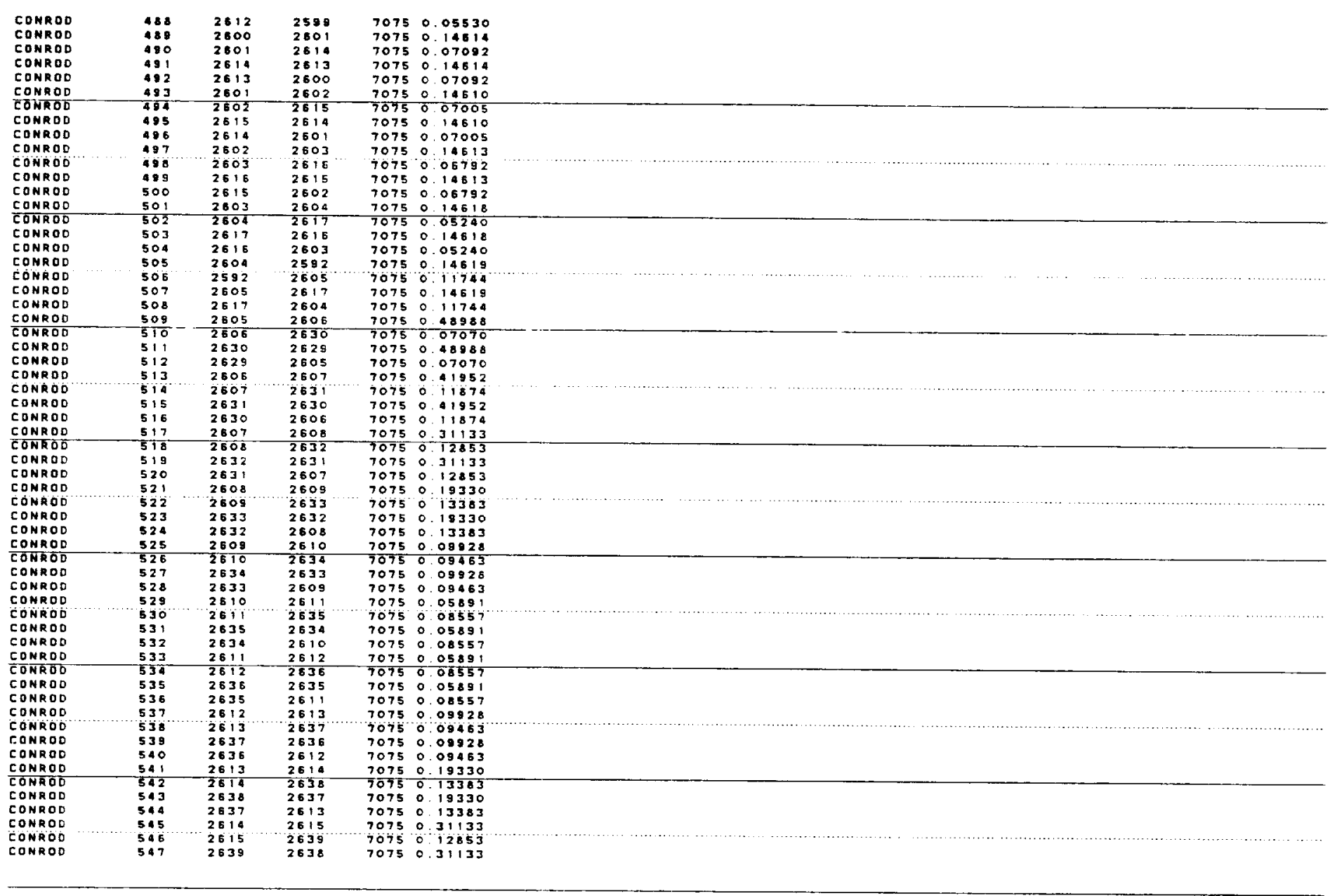

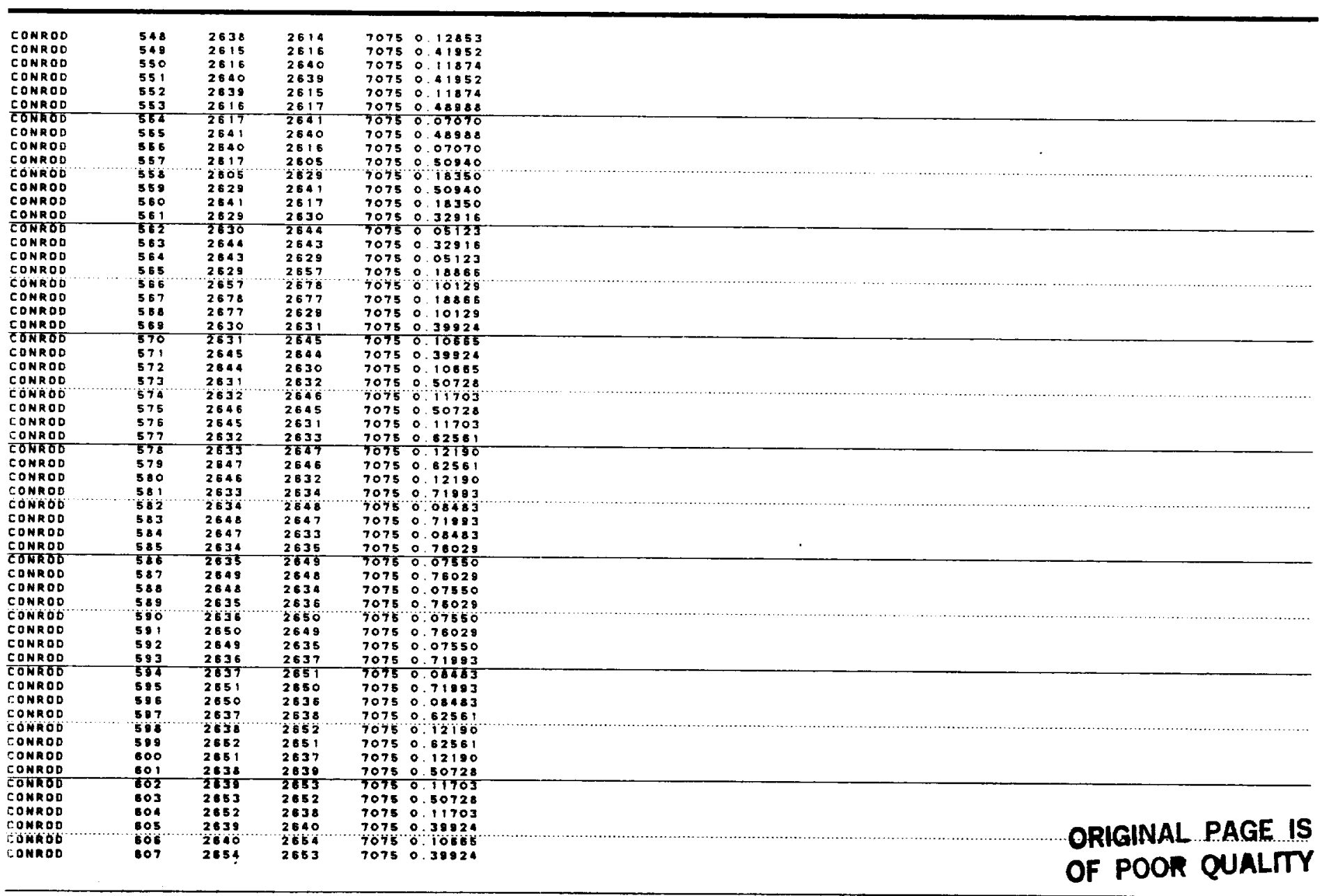




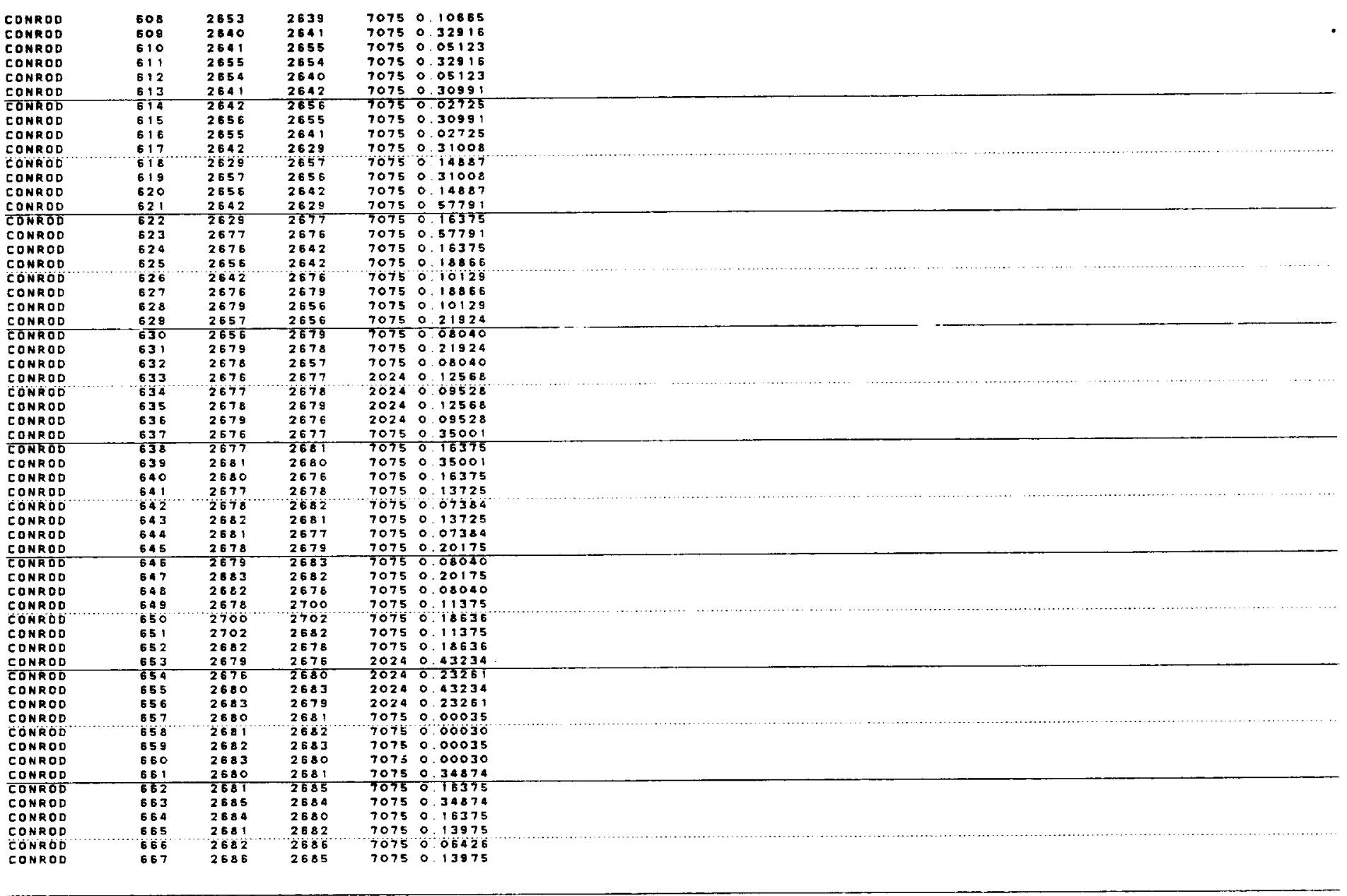

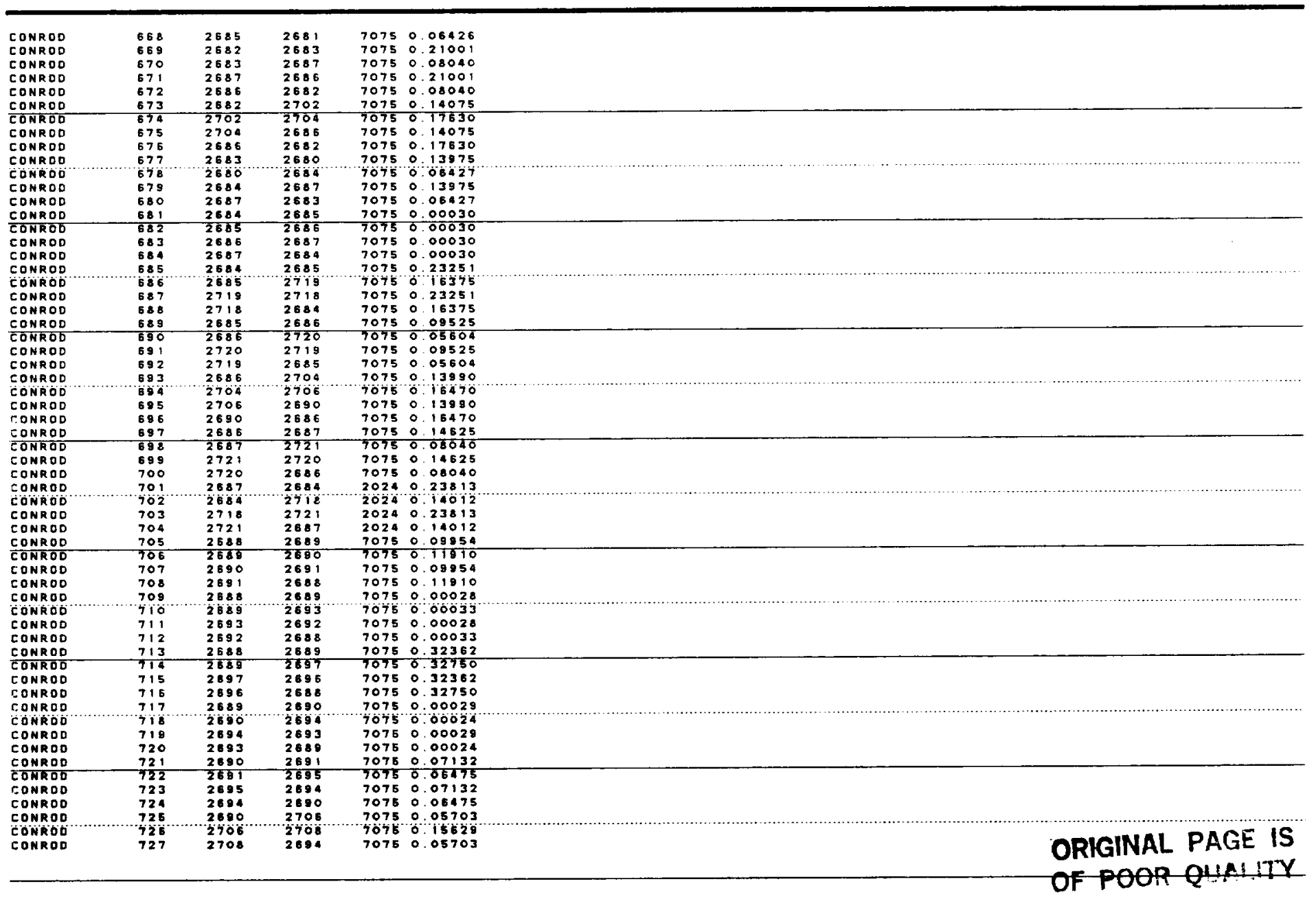




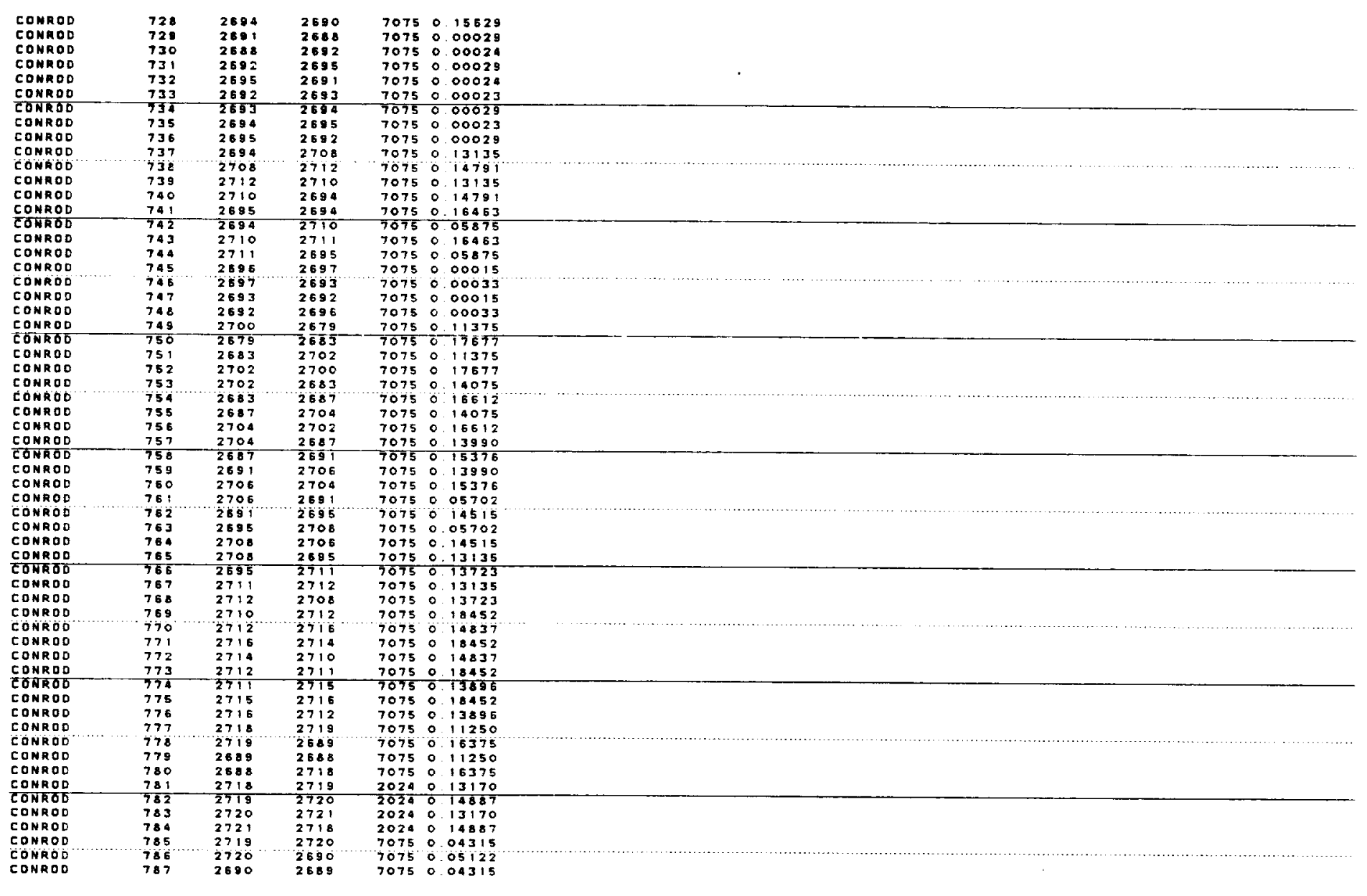

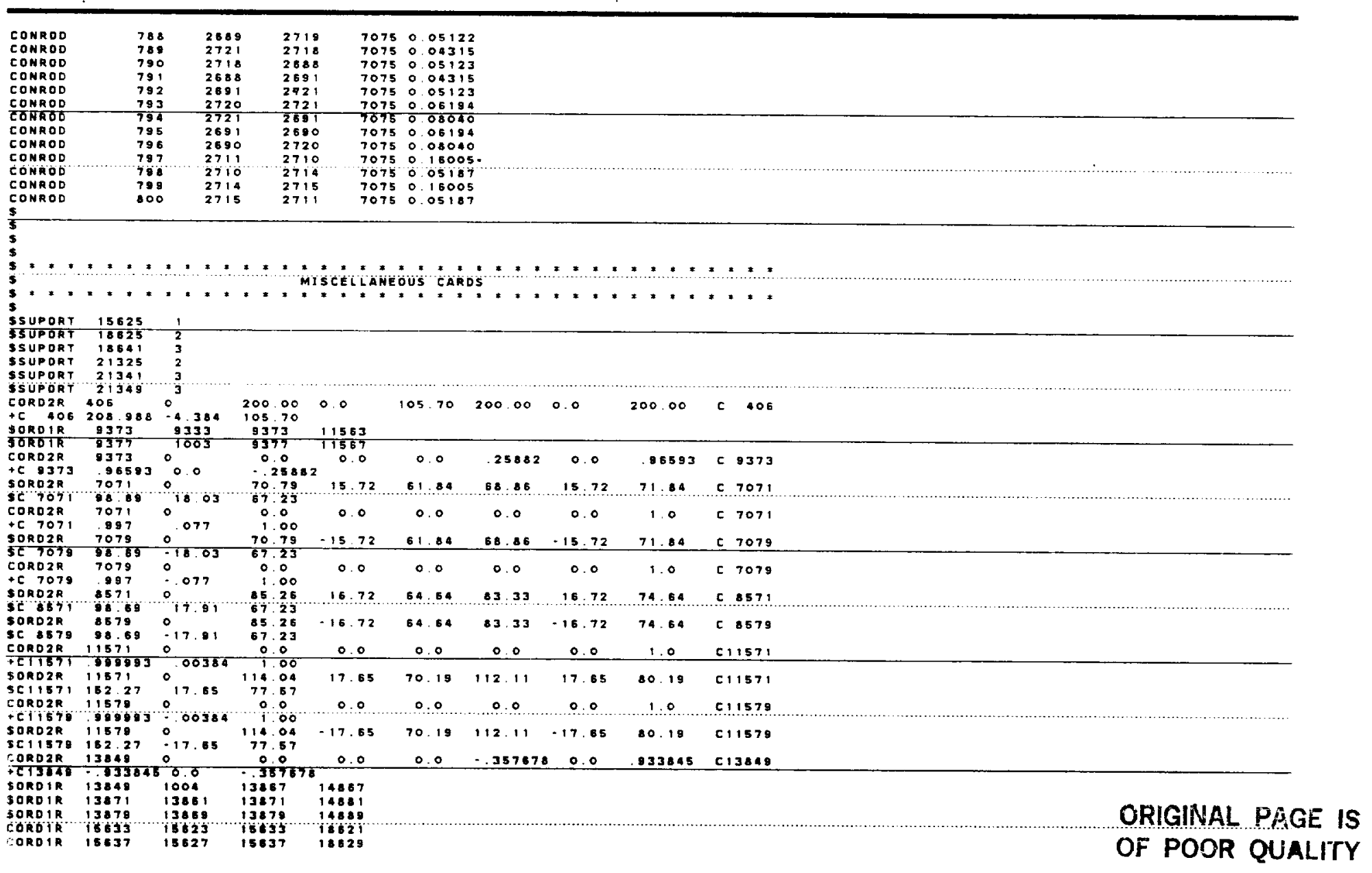


CORDIR $29945 \quad 29925 \quad 29965 \quad 2588$

she tollowing elac caras are added by weiyu znou for the complox s. eigenvalue analysis.

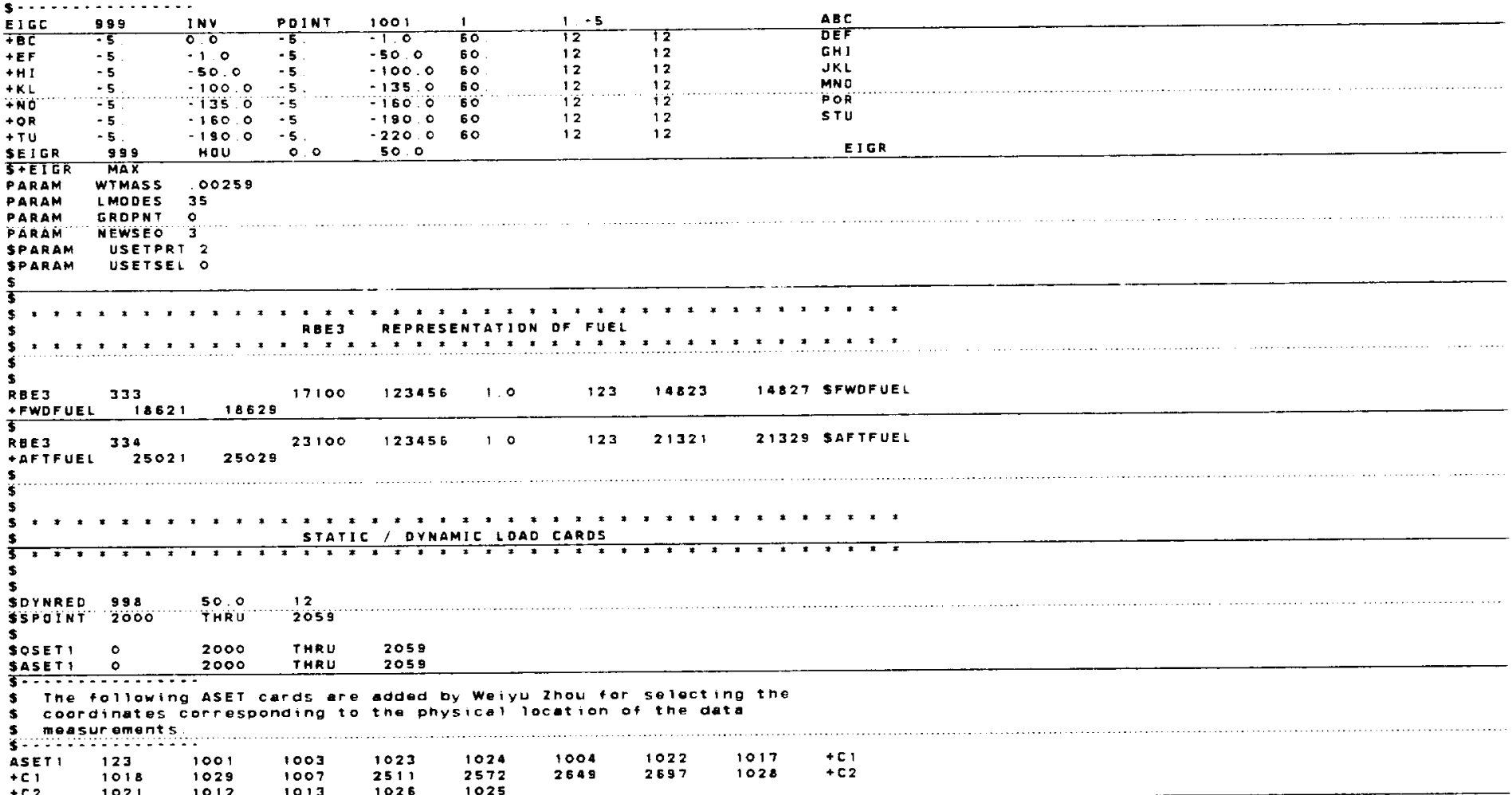

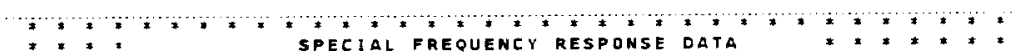

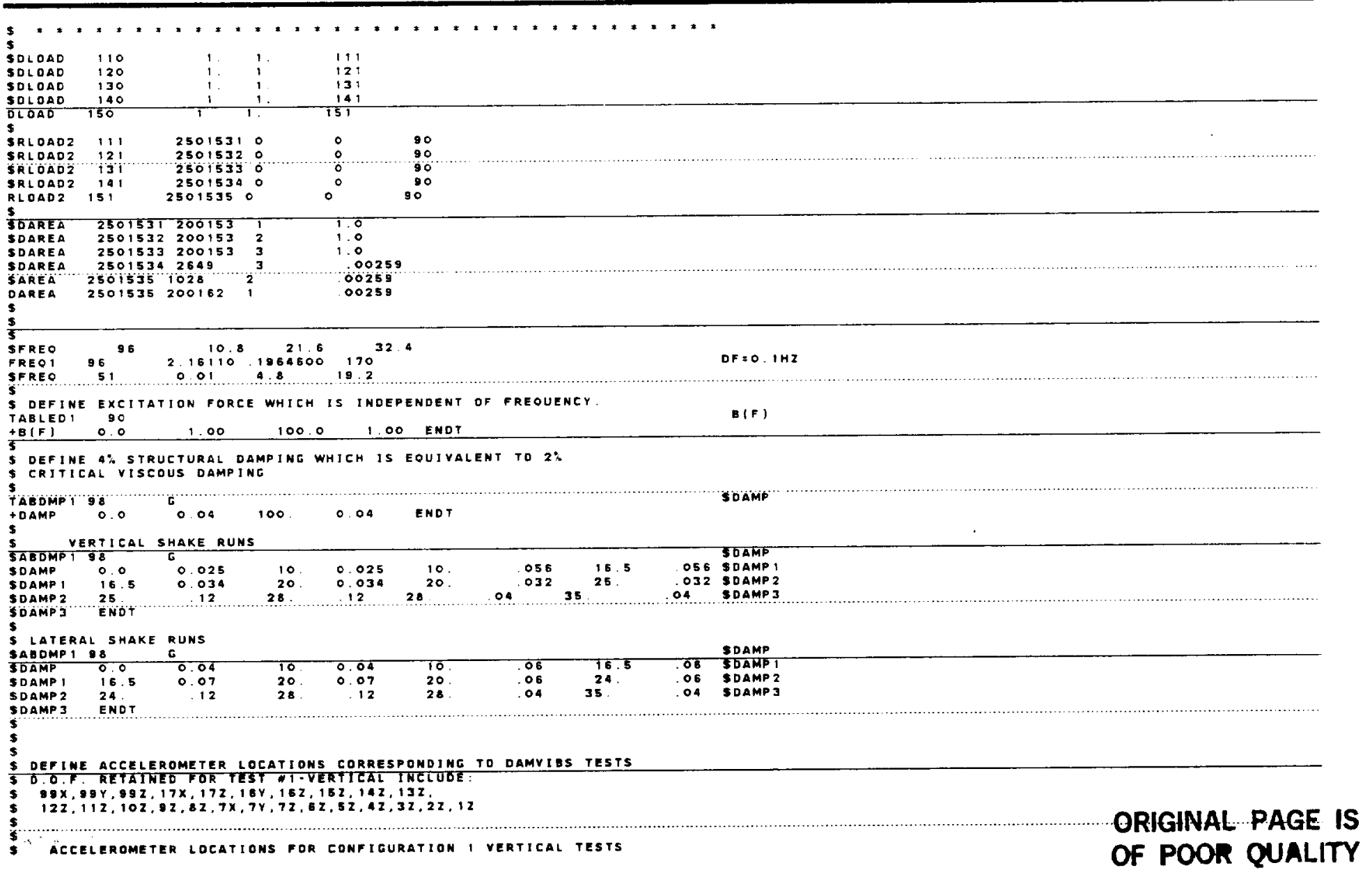


5 ACCELEROMETER LOCATIONS WITH ZERO APPENDED ARE FOR LATERAL OMLY
5 ACCELERMETER LOCATIONS W/DOUBLE IERO ARE FOR LOMGITUOINAL ONLY

3

GRID, $1,48.00,99.07,46,00,0,456$

$\frac{G R 10,2,95,00,-10,0,45,00,0,456}{\text { GRTB, } 3,110,20,-40,0,11,00,0.456}$

GRID.4.,110.20:40.00,11.00\%0\%456

GRID,5:,13670,-10.0.46.00\%,45E

GR $10.5,19690,-9.00,8460.0455$

GR ID, $7,20000,300,154,97,0,455$

GRID $9,20400,5900,63,95,0,455$

RID $80,23200,42$

Cर1D, $90,232,00,-38,00,7,35,0,456$

$\$$

GR1D, $800,398,00,33,30,57,0,0,056$

GRID, 900, 398,00,-32,80,57,0,0.456

CRID, 10, $232,30,0.00,95,60,0,456$

GRID 12 250 $00,00,9500,0,456$

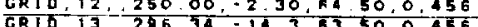

GRI0, $14,402 \quad 40-10042000450$

GR10 is 485300005470,456

ERID 15 521 5075012070456

RID.17, $52067,1960,119,7.45$

GR10, $180,200,00,-800,86,30,0.456$

CR10,190, 20400,5900,63,95,0,456

CRID. $200,20400,-5900,63,95,0,456$

$s$

REE 2, 301, 1001, 123,

REE2, $302,1003,123$,

REE2, $303,1004,123,5$

ROE2,305, $1006,2,6$

$\frac{\text { REE }, 305,1006,2,6}{\text { REE } 2,306,1007,123,12}$

REE 2, 307, 2511, 123,13

RBE2, 308.2572, 123,14

REEZ 310,1017, 123 190

REE 2,311,1018,123,9,200

REE $2,312,1019,123,10$

$\frac{R B E 2,313,1020,123,1}{R B E Z, 3 i 4,1023,133}$

REE2,315, 1023,123,3

AEE2,316,1024,123,

REE2, 317, $2897,123,16$
REEZ, 318, $1028,123,17$

SEE2, 319, $200015,123,99$

REE2, $320,1026,123,80$

REE 2,321, $1025,123,90$

REEZ, 323, 1012,123,800

REE2,324, $1021,123,180$

SBE2,325, 1017, 123,190

SEE2,336,1018,123,200

(2)

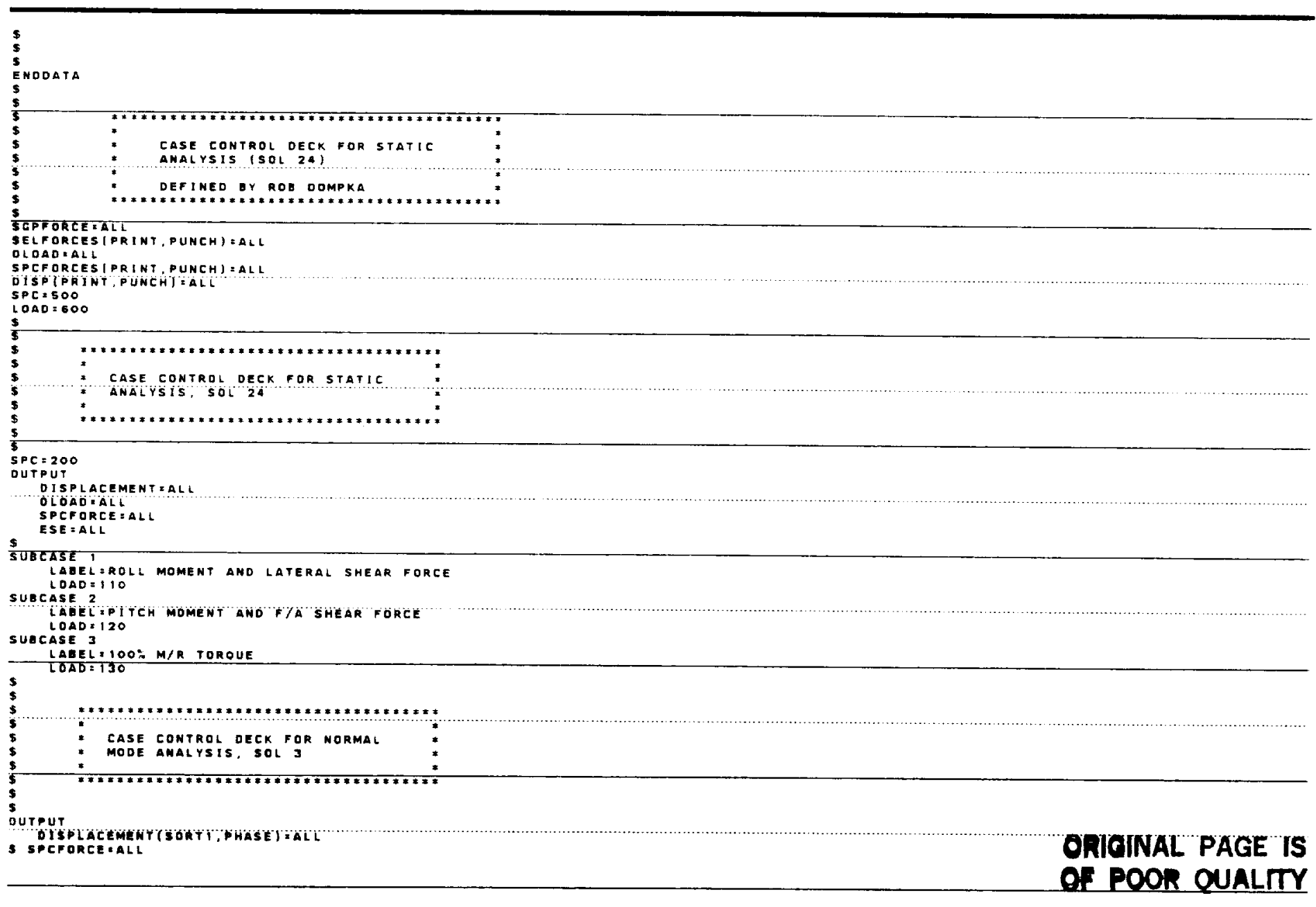


\$ ESE $\approx A L L$

DUTPUT (PLOT)

PLOTTER NAST

DET I INCLUDE

SEY I INCLUDE ALL
MAXIMUM DEFORMATION 30.0

\$ ***3-D VIEW

PTITLE = ISOMETRIC VIEW

FINÓ SCALE, ORIG IN TSETI

PLOT MODAL DEFORMATION O.RANGE 0,1,35, O, SET I, PEN 3, ORIGIN 1, SHAPE

$\$$

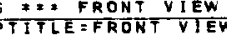

VIEW $0.0,0.0,0.0$

FIND SCALE ORIGIN 2 SET,

PLOT MODAL DEFORMATIDN O RANEE 0.1,35 OSET I,PEN 3, ORIGIN 2, SHAPE

$s$

S **TSIDE VIEW

PTITLE=SIDE V:EW

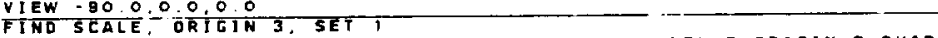

PLOT MODAL DEFORMATIÓNO, RANGE $0.1,35$ O, SET 1, PEN 3, ORIGIN 3,SHAPE

$5: *$ TOP VIEW

PTITLE = TOP VIEW

VIEW $0.0,900.0 .0$

FIND SCALE, ORICIN A, SET 1

5
$\$$
$\$$
$\$$
5
5

CASE CONTROL DECK FDR MODAL

FREQUENCY RESPOMSE, SOL 30

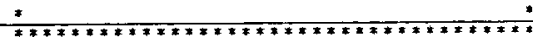

FREO: 95

SDAMP = 98

SPCEFORCE $=A L$

$\$$ ESEIALL

$\$$ FOLLOWINE SETS CORRESPOND TO THE ACCELEROMETER LDCATIONS

USED IN GVT FOR LONGI TOINAL (SET T) TLTERAL TSET 2 )
AND YERTICAL (SET 3 ) LOAD CONDITIONS /RESPONSE MEASUREMENT

SET T, 1,2,3,4,5,6,7,8,80,80,0,90,900,10,1i

$12,13,19,15,16,17,18,19,180,190,200$

SET, $1,1052,1054,1057,6701,6703,6711,6713,170014,183014,287001$

$256013,256018,92912,92962,340020,87004,132010,171000$

$256027,256028,4364,4456,82900,8050,8006$

SET 22:1052,1054,1057,6701,6703,6711,6713,97004,132010,143002,92962.

$170014,171009,183014,256023,256026,256035,256036,191,188,814$

SET J=1052,57016703,5711,5713,97004,132010,132017,143002,92900.8050

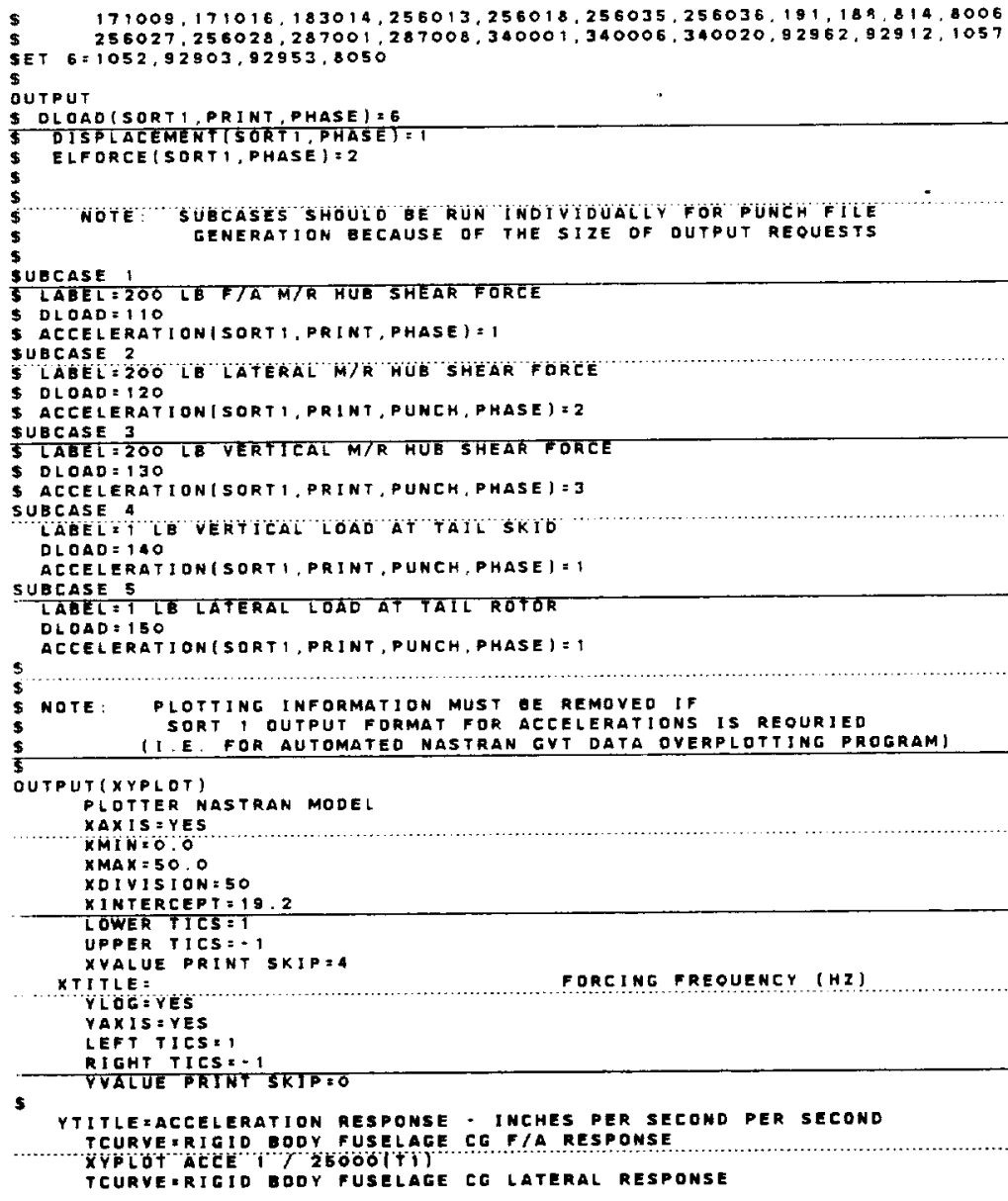



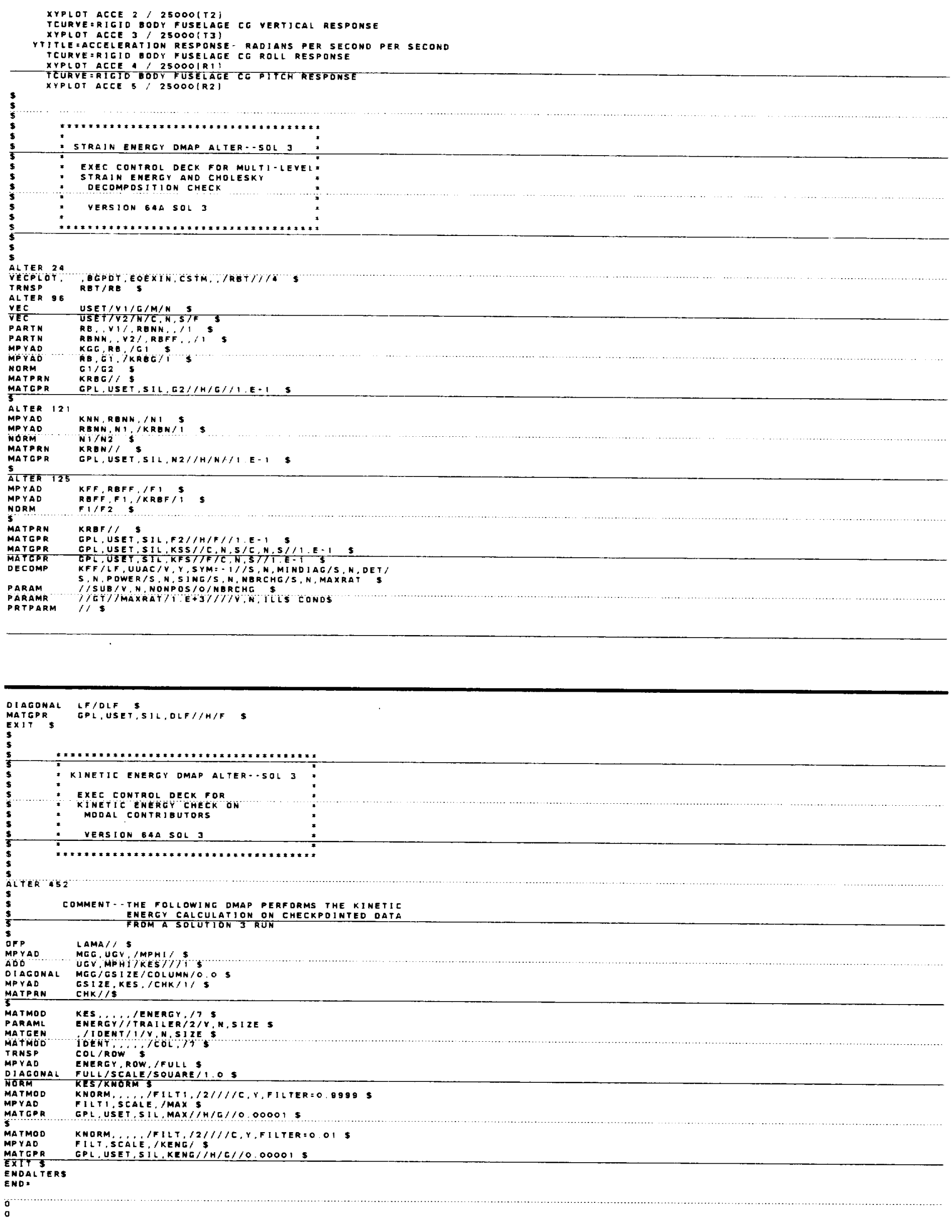


\section{APPENDIX C \\ MSC/NASTRAN OUTPUT FOR FINAL AH-1G SYSTEM IDENTIFICATION RUN}

The following pages include the listings of the output files from the final MSCNASTRAN run used to compute the structural eigenvalues and eigenvectors for the identified AH-1G structural model. 


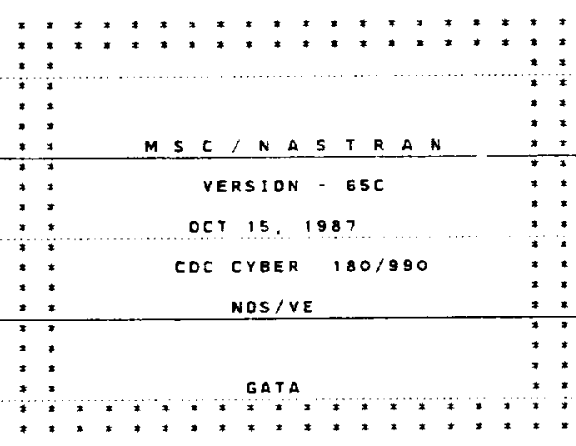

OETOBER 26, 1990 MSC/NASTRAN 10/15/87 PAGE

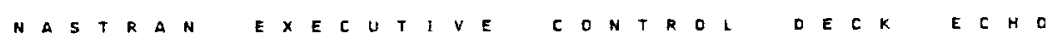

IO RVDOMPKA, GROUP 65

SOAE B 31 TILERTSTREF

TIAG $6,14,31$

IIME 240

SOL 25

MATPRT KAA

ALTER 3A?

MATPRT MAA//

ALTER 396

MATPRT BAA//

ALTER GEA
MATPRT UAV//,

SENO 
AH-IC THREE-DIMENSIONAL BUILTUP DYMAMICS MOOEL W/ CONTROLS MODELED

DIFE LCULT COMPONENTS GVT CDNF I GURATION "I IFULL UP I

THIS VERSIDN CONTAINS BUILTUP TAILBOQM MODEL

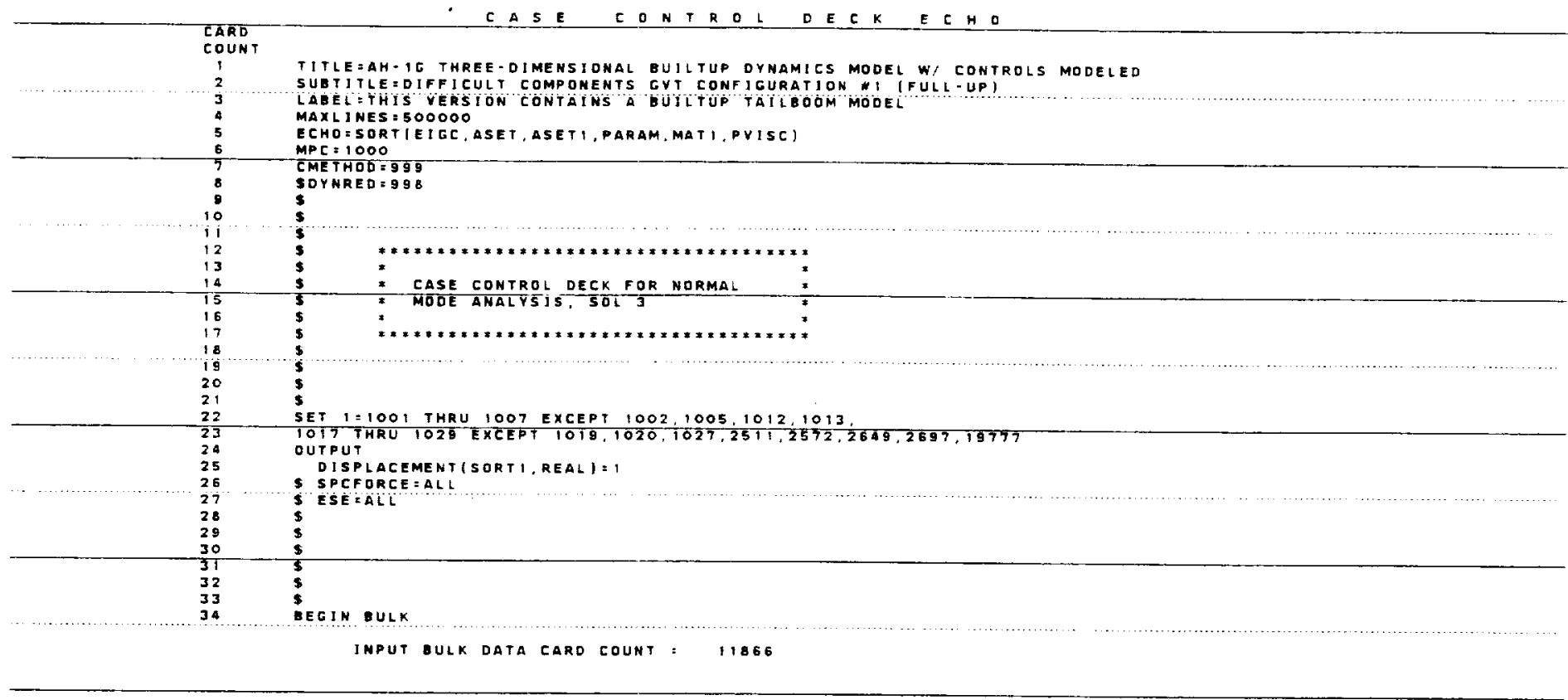

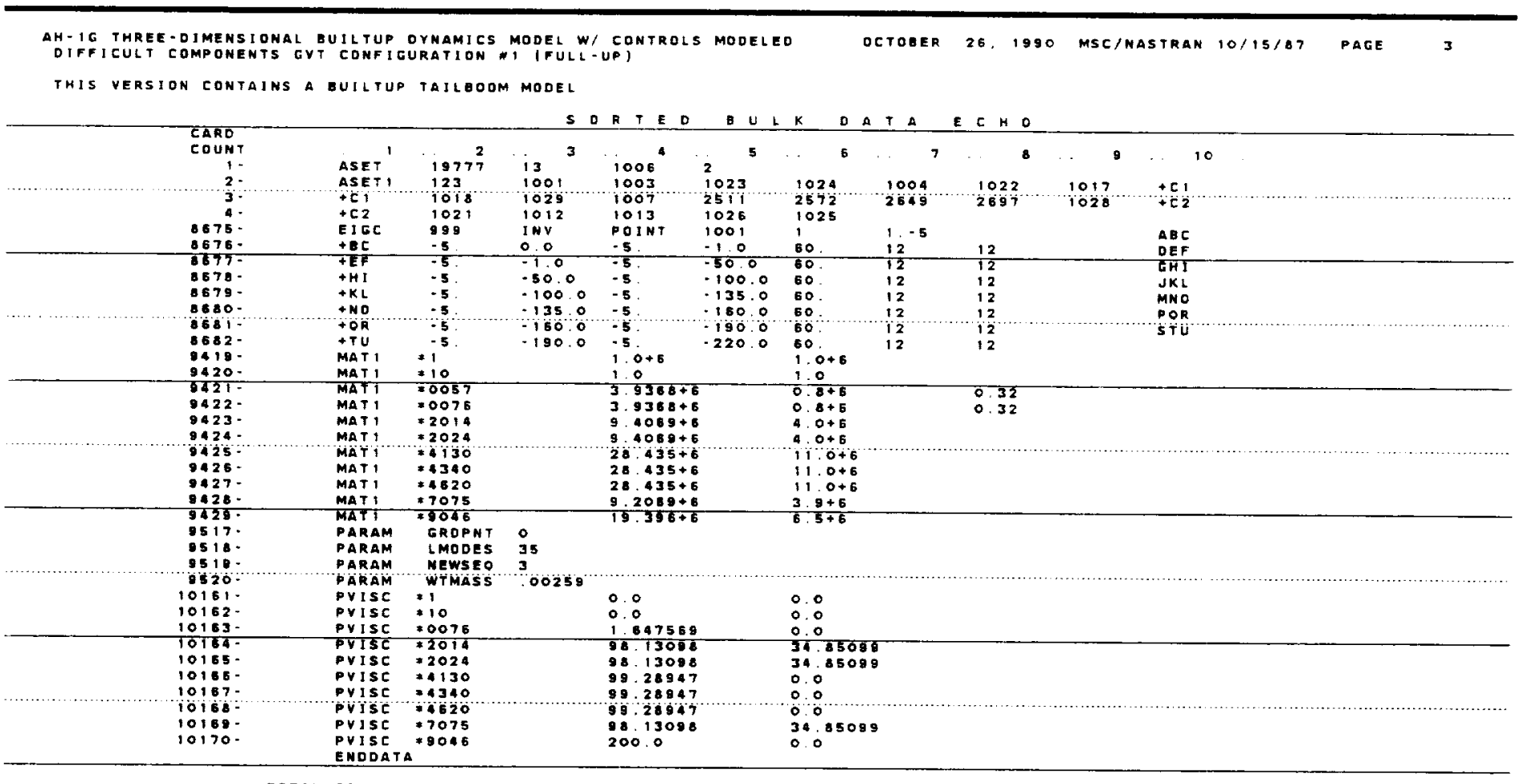

TOTAL COUNT = 10236

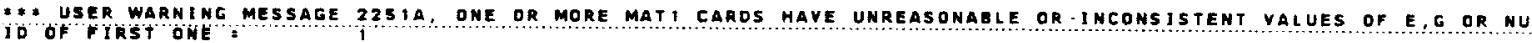

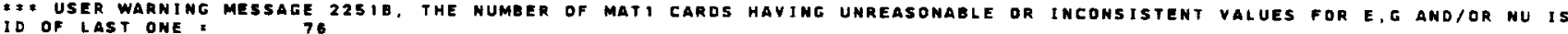


AH- IE THREE-OIMENSIONAL BUILTUP DYMAMICS MODEL W/ CONTROLS MOOELED

THIS VERSION CONTAINS A BUILTUP TAILBOOM MODE

NAS TRRA N SO UR C E PROERAM COMPILATIDN

WO

*** user warning message 54

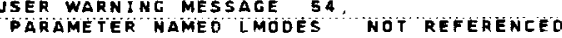

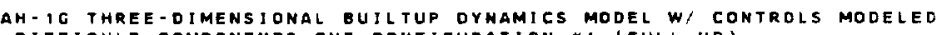

OCTOBER 26, 1990 MSC/NASTRAN 10/15/8T PAGE

DIFFICULT COMPONENTS GVT CONFIGURATION I IFULL-UP)

THIS VERSION CONTAINS A BUILTUP TAILBOOM MOOEL

SEQUENCE PROCESSOR OUTPUT

THERE ARE T35 POINTS DIVIDED INTO T GROUPIS

CONNECTIDN DATA

ELEMENT TYPE NUMEER ASSEMBLY TIME(SEC)

\begin{tabular}{lrr} 
ROD & & \\
SHEAR & 272 & 1.77 \\
TRMEE & 540 & 14.04 \\
CONROD & 243 & 3.16 \\
ELASZ & 2800 & 18.20 \\
ODMEM & 13 & .02 \\
\hline OUAD4 & 150 & 4.16 \\
BAR & 2 & .05 \\
TRIA3 & 358 & 4.05 \\
& 17 & 22
\end{tabular}

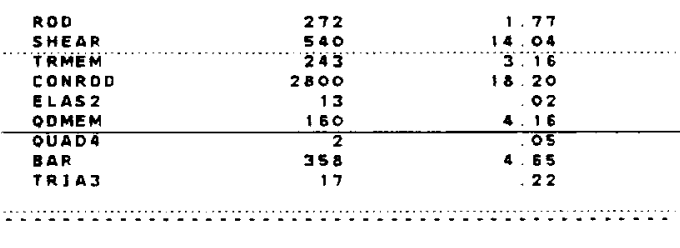

TOTAL MATRIX ASSEMBLY TIME FOR 4405 ELEMENTS IS 45.27 SECONOS

RIGID ELEMENT PROCESSINE COMPLETED

ORIGINAL PERFDRMANCE DATA

0

935

5.56

C-AVERAGE

C-RMS T-MAXIMUM P-GROUPS

P-AVERAGE

DECOMP TIME ISECS

CE DATA

resequenced performance data

$96.64-108.03$

190

13

$98 . \overline{2}$

10.320

SUPERTEROUP')

AV CONNETTIVIT

E-AVERAGE

C-RMS

c-maxtmúm p.ckótos

P-AVERAGE DECOMP TIMEISECSI

735

8. 66

18.1

20.30

38

$\circ$

0.00

16.0 DOF/GA1O

*** user waring message 20 o. an obsolete capability for element processing is being used.

THIS CAPABILITY MAY BE DELETED IN THE NEKT SYSTEM 
AH-IC THREE-DIMENSIONAL BUILTUP DYNAMICS MODEL W/ CONTROLS MODELED

THIS VERSION CONTAINS A BUILTUP TAILBDOM MOOEL

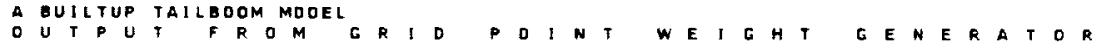

\section{REFERENEE POINT =}

- 5. $910710 E+03-8.573151 E-14-2.679110 E-15$ 1. $097363 E-11 \quad 5.225896 E+05 \quad 3.980523 E+02 *$

- $-8.573151 E-14$ S. $10710 E+03$ O 000000E+00 $-5.225896 E+05-1.097363 E-11 \quad 1.364420 E+06$,

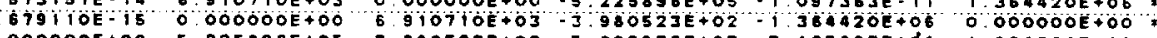

$000000 E+00-5.225896 E+05-3.980523 E+02 \quad 5.009936 E+07-7.165833 E+84-1.064261 E+08$,

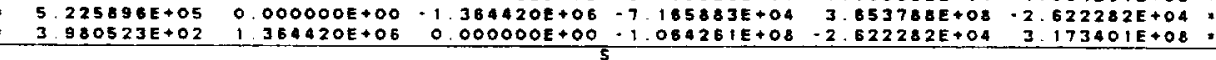

- 1,000000E+00 $0.000000 E+00 \quad 0.000000 E+00$.

* O.000000E+00 1.000000E+00 0.000000E+00:

MASS AXIS SYSTEM (S)

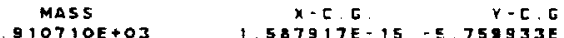

$\begin{array}{rl}Z-C & G \\ 75933 E-02 \quad 7.562025 E+0\end{array}$

$6.910710 E+05-1.974356 E+07-1.587917 E-15-7.562025 E+0$

* $1.058098 E+07 \quad 1.502485 E+05 \quad 3.248277 E+05 *$

* $502485 E+05 \quad 5.647519 E+07 \quad 553233 E+04$

- $243277 E+06$ S $632363 E+04$ A $11019490+07$

( $\triangle 23080 E+07$

5.647593E+07

- 8.596323E-02-2.883585E-03 9.962941E-01*

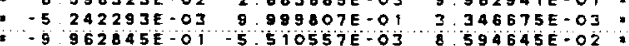

AH- 10 THREE-DIMENSIONAL BUILTUP OYNAMICS MOOELW/ CONTROLS MODELEO

DFF

DETDGER 26, 1990 MSC/NASTRAN 10/15/87 PAGE

THIS VERSION CONTAINS A GUILTUP TAILBOOM MODEL

* * USER INFORMATION MESSAGE 4158 - STATISTICS FOR SYMMETRIE OECOMPOSITION OF OATA OLOCK KOD 
intermediate MATRIX ... MAR

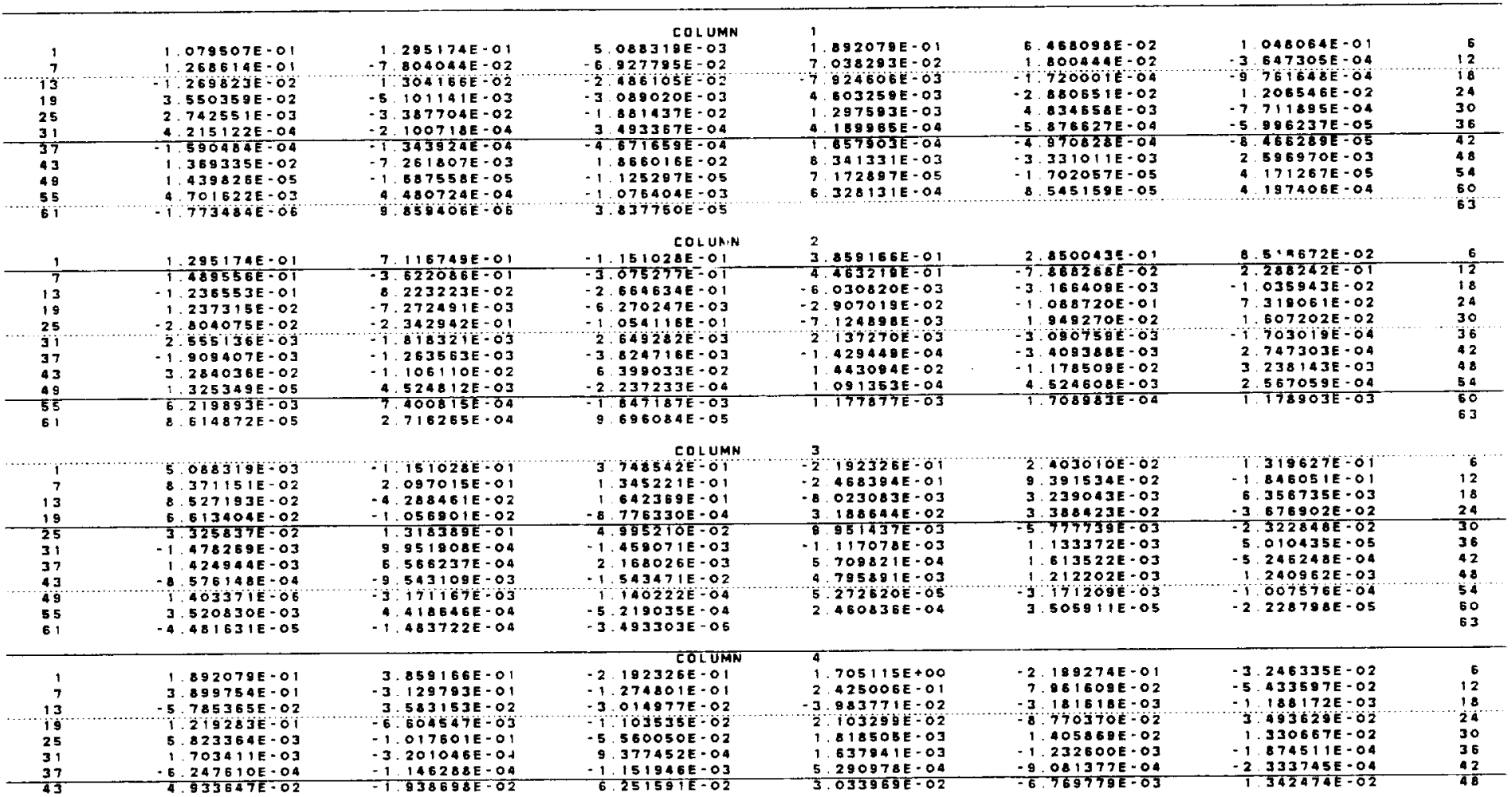

AH- TE THREE-DIMENSIONAL BUILTUP OYNAMICS MODEL W/ CONTRDLS MODELED

OCTOEER 26, 1990 MSE/NASTRAN 10/15/87 PAGE

THIS VERSION CONTAINS A BUILTUP TAILBOOM MODEL

INTERMEQIATE MATRIX ... MAa

\begin{tabular}{|c|c|c|c|c|c|c|c|c|c|c|c|c|}
\hline $\begin{array}{l}49 \\
55 \\
61\end{array}$ & 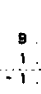 & $\begin{array}{l}586793 E-05 \\
965232 E-02 \\
101124 E-04\end{array}$ & $\begin{array}{r}-2 \cdot 7 \\
1: 3 \\
-1: 4\end{array}$ & $\begin{array}{l}788620 E-03 \\
383189 E-03 \\
451355 E-O A\end{array}$ & $\begin{array}{r}1 \\
-4.3 \\
i\end{array}$ & $\begin{array}{l}\text { COLUMN } \\
352868 E-04 \\
310357 E=03 \\
646676 E=04\end{array}$ & 2. & $\begin{array}{l}837343 E \cdot 04 \\
463698 E \cdot 03\end{array}$ & $\begin{array}{r}-2.789503 E-03 \\
2.8372308-04\end{array}$ & 4 & $\begin{array}{l}313241 E-05 \\
114213 E-03\end{array}$ & $\begin{array}{l}54 \\
60 \\
63\end{array}$ \\
\hline $\begin{array}{r}1 \\
7 \\
13 \\
19 \\
25 \\
31 \\
37 \\
43 \\
49 \\
55 \\
151\end{array}$ & $\begin{array}{r}6 \\
4 \\
-2 \\
6 \\
1 \\
2 \\
1 \\
2 \\
-4 \\
5 \\
1\end{array}$ & $\begin{array}{l}468098 E-O 2 \\
166411 E-O 2 \\
653463 E-O 2 \\
841136 E-02 \\
180798 E-O 2 \\
746302 E-O 2 \\
486248 E-O 4 \\
488431 E-O 2 \\
037469 E-O 5 \\
716583 E-03 \\
395633 E-O 4\end{array}$ & $\begin{array}{r}2.8 \\
1.8 \\
3 . \\
-3 . \\
-9.7 \\
-5.6 \\
-3.2 \\
-3.1 \\
3.7 \\
1.6 \\
3 .\end{array}$ & $\begin{array}{l}850043 E-01 \\
96371 E-04 \\
853278 E-02 \\
82477 E-02 \\
788851 E-02 \\
091809 E-04 \\
249708 E-04 \\
129894 E-02 \\
715839 E-03 \\
604520 E-03 \\
478709 E-04\end{array}$ & $\begin{array}{l}2 . \\
-9 . \\
-1 . \\
-5 . \\
-5 . \\
6 . \\
-1 . \\
4 . \\
-2 . \\
-1 . \\
9 .\end{array}$ & 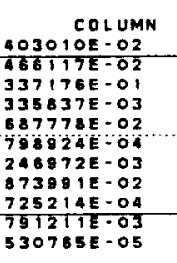 & $\begin{array}{r}5 \\
-2 . \\
-1 . \\
-3 \\
-8 . \\
4.2 \\
3 \\
7 . \\
1 . \\
1 . \\
1 .\end{array}$ & 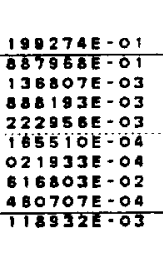 & $\begin{array}{r}1.313855 E+00 \\
-2.264071 E-02 \\
-2.125557 E-03 \\
-6.008707 E-02 \\
9.828508 E-02 \\
-2.305032 E-03 \\
-2.141183 E-03 \\
-1.602558 E-02 \\
3.715551 E-03 \\
1.86846 E-04\end{array}$ & $\begin{array}{r}1 \\
-8 \\
-5 \\
3 \\
-2 \\
-5 \\
-5 \\
8 \\
-3 \\
2 \\
1\end{array}$ & $\begin{array}{l}511446 E-01 \\
624073 E-02 \\
226126 E-03 \\
841716 E-02 \\
534327 E-02 \\
398982 E-04 \\
125953 E-05 \\
316405 E-05 \\
544987 E-04 \\
373507 E-03\end{array}$ & $\begin{array}{l}6 \\
12 \\
18 \\
24 \\
30 \\
36 \\
42 \\
48 \\
54 \\
60 \\
63\end{array}$ \\
\hline $\begin{array}{l}71 \\
7 \\
13 \\
19 \\
25 \\
31 \\
37 \\
43 \\
49 \\
55 \\
61\end{array}$ & $\begin{array}{r}1 \\
1 \\
-3 \\
2 \\
-4 \\
1 \\
-1 \\
1 \\
4 \\
5 \\
-3\end{array}$ & $\begin{array}{l}O 48064 E-O 1 \\
362726=01 \\
183450 E-02 \\
707719 E=02 \\
974994 E=03 \\
273449 E-03 \\
071681 E=03 \\
808271 E-02 \\
365573 E=05 \\
366013 E=03 \\
609000-O 5\end{array}$ & $\begin{array}{r}8.5 \\
-1.3 \\
2.8 \\
1.5 \\
-7.5 \\
-5.1 \\
-2.5 \\
3.7 \\
-7.5 \\
-1.2 \\
-1.0\end{array}$ & $\begin{array}{l}516672 E-O 2 \\
392082 E-01 \\
613238 E-O 2 \\
615598 E-02 \\
513281 E-02 \\
124160 E-04 \\
316115 E-04 \\
712522 E-03 \\
514568 E-O 5 \\
468737 E-O 4 \\
007628 E-O 4\end{array}$ & 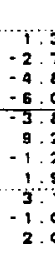 & 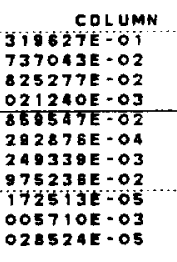 & $\begin{array}{r}6 \\
-3 . \\
1.2 \\
-1 . \\
4 \\
2 \\
9 \\
-2 . \\
8 \\
3 \\
6 .\end{array}$ & $\begin{array}{l}246335 E-02 \\
919407 E-01 \\
740239 E-02 \\
118017 E-03 \\
708042 E-04 \\
539098 E-04 \\
11064 A E-04 \\
364712 E-03 \\
967280 E-O S \\
297192 E-O 4\end{array}$ & 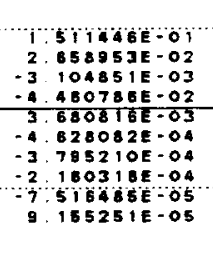 & $\begin{array}{r}1 \\
-9 \\
-1 \\
2 \\
1 \\
-3 \\
-8 \\
4 \\
1 \\
3 \\
3\end{array}$ & $\begin{array}{l}802126 E+O O \\
493938 E-O 3 \\
879803 E-03 \\
333850 E-02 \\
103127 E-02 \\
765196 E-05 \\
145731 E-05 \\
020725 E-03 \\
520228 E-O 5 \\
238162 E-O A\end{array}$ & $\begin{array}{l}\cdots 1 \\
12 \\
18 \\
24 \\
30 \\
36 \\
42 \\
48 \\
54 \\
60 \\
63\end{array}$ \\
\hline $\begin{array}{r}1 \\
7 \\
13 \\
19 \\
25 \\
31 \\
37 \\
43 \\
49 \\
55 \\
61\end{array}$ & $\begin{array}{r}1 \\
1 \\
\therefore 3 \\
4 \\
3 \\
4 \\
1 \\
8 \\
-8\end{array}$ & $\begin{array}{l}268514 E-O 1 \\
043134 E+00 \\
696278 E-02 \\
231194 E-O 1 \\
122344 E-02 \\
003528 E-O 3 \\
332683 E-04 \\
321718 E-O 1 \\
809806 E-O 4 \\
170762 E-02 \\
465193 E-O 4\end{array}$ & $\begin{array}{r}1.5 \\
-2.2 \\
4.2 \\
-1 . \\
-1 . \\
-1 . \\
-8.2 \\
-1.5 \\
-7.5 \\
-1.2\end{array}$ & $\begin{array}{l}489556 E-O 1 \\
29751 E-01 \\
105105 E-O 2 \\
437416 E-O 3 \\
028044-01 \\
5957311-03 \\
490415 E-03 \\
233329 E-03 \\
940923 E-02 \\
090878 E-O 6 \\
744501 E-O 3\end{array}$ & $\begin{array}{r}8 . \\
-5 \\
-9 \\
-1 \\
-8 \\
2 . \\
-2 . \\
1 \\
1 \\
-1 \\
2 .\end{array}$ & 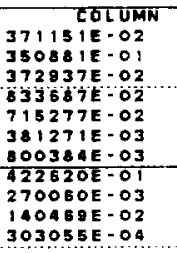 & $\begin{array}{r}7 \\
3 . \\
4 . \\
-4 . \\
4 . \\
-1 . \\
3 . \\
2 . \\
7 . \\
6 . \\
6 .\end{array}$ & $\begin{array}{l}898754 E-01 \\
145523 E-01 \\
386767 E-02 \\
610612 E-O 2 \\
250895-02 \\
396189 E-03 \\
584205 E-03 \\
685252 E-02 \\
551216 E-04 \\
596336 E-03\end{array}$ & $\begin{array}{r}166411 E-02 \\
2.479675 E-01 \\
-1.323198 E-02 \\
-1.286536 E-61 \\
1.8846840-02 \\
-2.071839 E-03 \\
-1.649327 E-03 \\
1.545215 E-02 \\
-1.941159 E-02 \\
6.04293 E-04\end{array}$ & $\begin{array}{r}1 \\
-1 \\
-3 \\
5 \\
9 \\
3 \\
-9 \\
5 \\
-4 \\
7\end{array}$ & 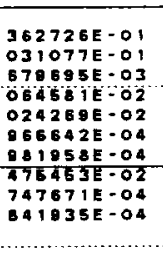 & $\begin{array}{r}6 \\
12 \\
18 \\
24 \\
30 \\
38 \\
42 \\
48 \\
54 \\
60 \\
63\end{array}$ \\
\hline $\begin{array}{r}1 \\
7 \\
13\end{array}$ & $\begin{array}{l}-7 \\
-2 \\
-2\end{array}$ & $\begin{array}{l}804044 E-02 \\
29751 E-01 \\
06789 E-01\end{array}$ & $\begin{array}{r}-3 . \\
-1 \\
-1\end{array}$ & $\begin{array}{l}522088 E-01 \\
931726 E+00 \\
130855=01\end{array}$ & 2 & $\begin{array}{r}\text { COLUMM } \\
097015 E-01 \\
221881 E-01 \\
130388 E-01\end{array}$ & $\begin{array}{l}8 \\
-3 \\
-4 \\
1 .\end{array}$ & $\begin{array}{l}129793 E-01 \\
083494 E-01 \\
.861798 E-02\end{array}$ & $\begin{array}{r}1.004371 E-01 \\
9.755871 E-02 \\
5.373653 E-03\end{array}$ & $\begin{array}{r}-1 \\
-3 \\
1\end{array}$ & $\begin{array}{l}392082 E-01 \\
105808 E-01 \\
615425 E-02\end{array}$ & $\begin{array}{r}6 \\
12 \\
18\end{array}$ \\
\hline
\end{tabular}


AH- 1 G THREE-DJMENSIONAL BUILTUP OYNAMICS MODEL W/ CONTROLS MODELED

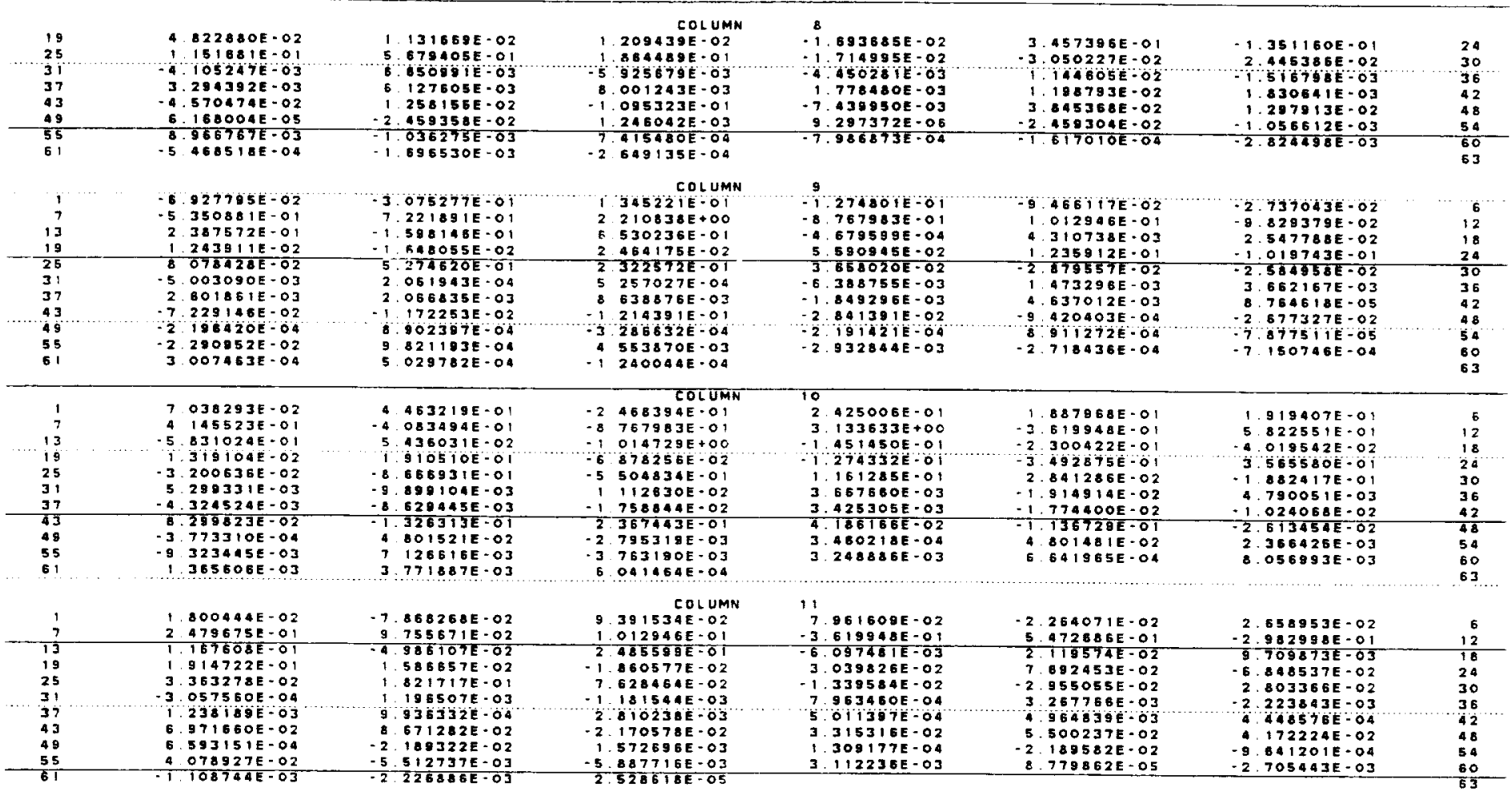

\footnotetext{
AH- IG THREE-DIMENSIONAL BUILTUP OYNAMICS MODEI W/ CONTROLS MODELED
}

DCTOBER

26. 1990 MSC/MASTRAN $10 / 15 / 07$

THIS VERSION CONTAINS A BUILTUP TALLBOOM MODEL

INTERMEDIATE MATRIX ... MAA

\begin{tabular}{|c|c|c|c|c|c|c|c|}
\hline $\begin{array}{l}1 \\
7 \\
13 \\
19 \\
25 \\
31 \\
39 \\
43 \\
49 \\
55 \\
61\end{array}$ & 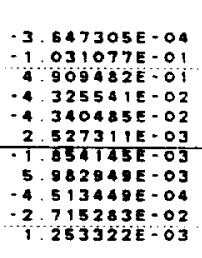 & 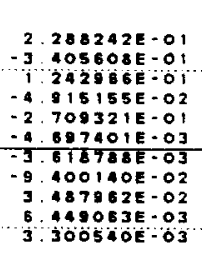 & 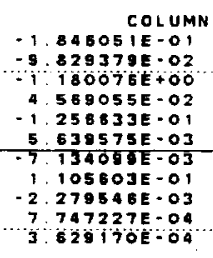 & 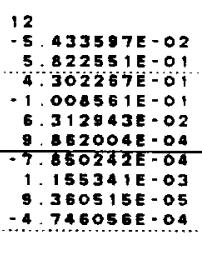 & $\begin{array}{r}8924073 E-02 \\
2.982998-01 \\
-4.340419 E-02 \\
-2.450577 E-01 \\
2.373971 E-02 \\
-8.586130 E-03 \\
1.437908-02 \\
-7.747821 E-02 \\
3.487942 E-02 \\
1.496173 E-04\end{array}$ & 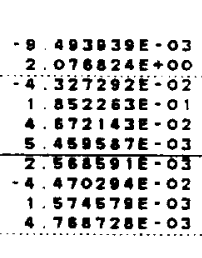 & $\begin{array}{l}5 \\
12 \\
18 \\
24 \\
30 \\
38 \\
42 \\
48 \\
54 \\
60 \\
63\end{array}$ \\
\hline $\begin{array}{l}1 \\
7 \\
13 \\
18 \\
25 \\
31 \\
37 \\
43 \\
48 \\
55 \\
51\end{array}$ & 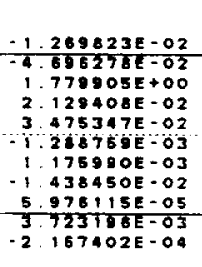 & 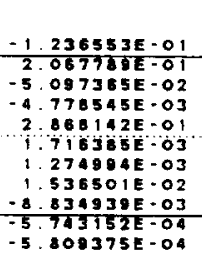 & 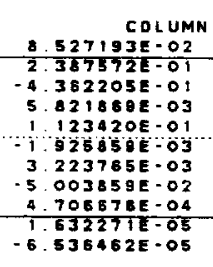 & 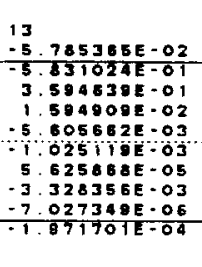 & 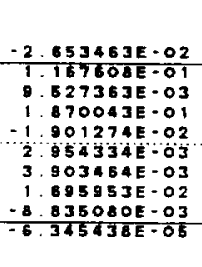 & 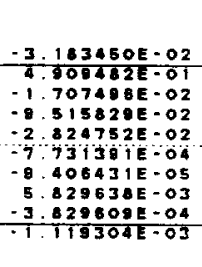 & $\begin{array}{l}5 \\
12 \\
18 \\
28 \\
30 \\
36 \\
42 \\
48 \\
54 \\
60 \\
63\end{array}$ \\
\hline $\begin{array}{r}1 \\
13 \\
13 \\
19 \\
25 \\
31 \\
37 \\
43 \\
49 \\
55 \\
61\end{array}$ & 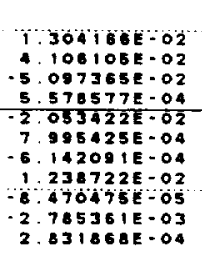 & 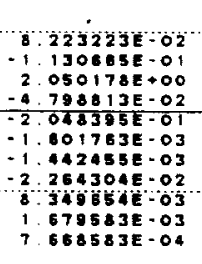 & 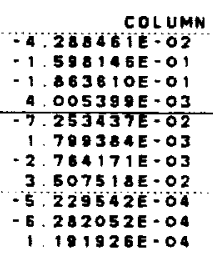 & 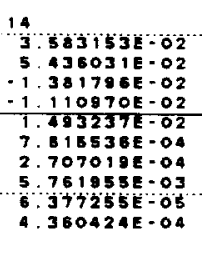 & 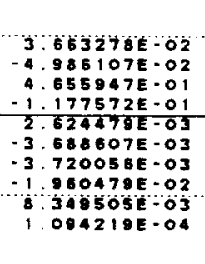 & 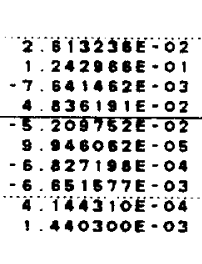 & $\begin{array}{l}6 \\
12 \\
18 \\
24 \\
310 \\
36 \\
42 \\
48 \\
56 \\
60 \\
63\end{array}$ \\
\hline $\begin{array}{l}1 \\
7 \\
13 \\
13 \\
25 \\
31 \\
37 \\
43\end{array}$ & 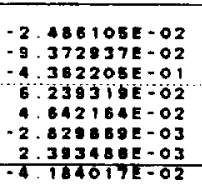 & 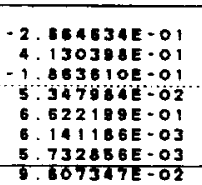 & 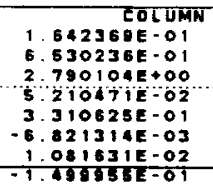 & 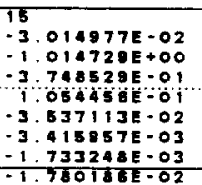 & 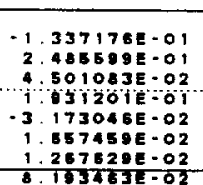 & 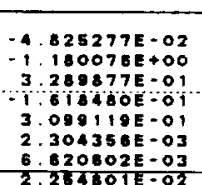 & $\begin{array}{l}5 \\
12 \\
18 \\
24 \\
30 \\
36 \\
42 \\
48 \\
\end{array}$ \\
\hline
\end{tabular}




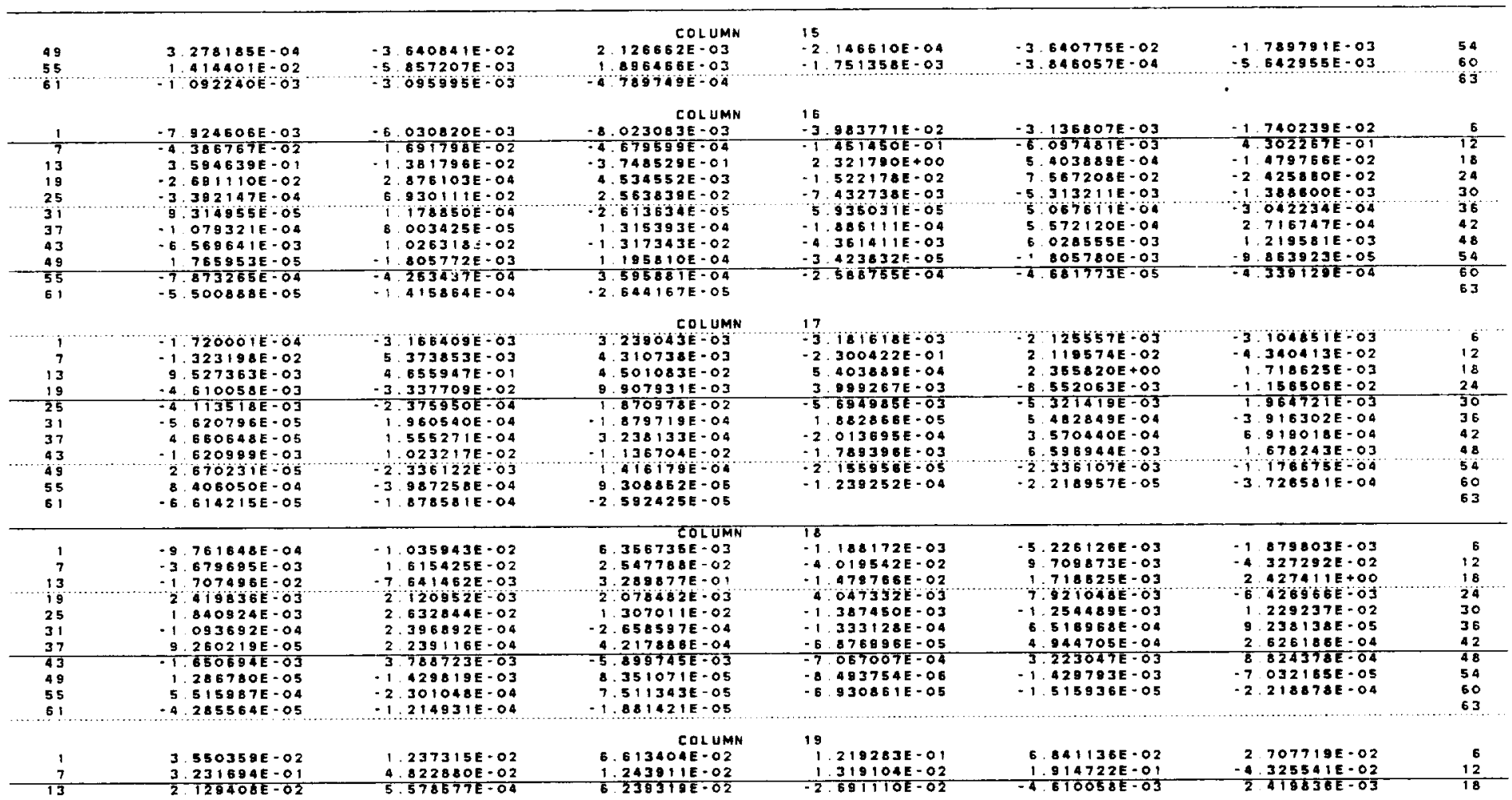

AH - IG THREE-DIMENSIONAL BUILTUP DYNAMICS MOOEL W/ CONTROLS MODELEO
OIFFI CULT COMPONENTS GVT CONFI GURATION I IFULL-UP)

DCtOGER 26,1090 MSE/NASTRAN 10/15/87 PAG

THIS VERSION CONTAINS A BUILTUP TAILBODM MODEL

INTERMEDIATE MATRIX ... MAA

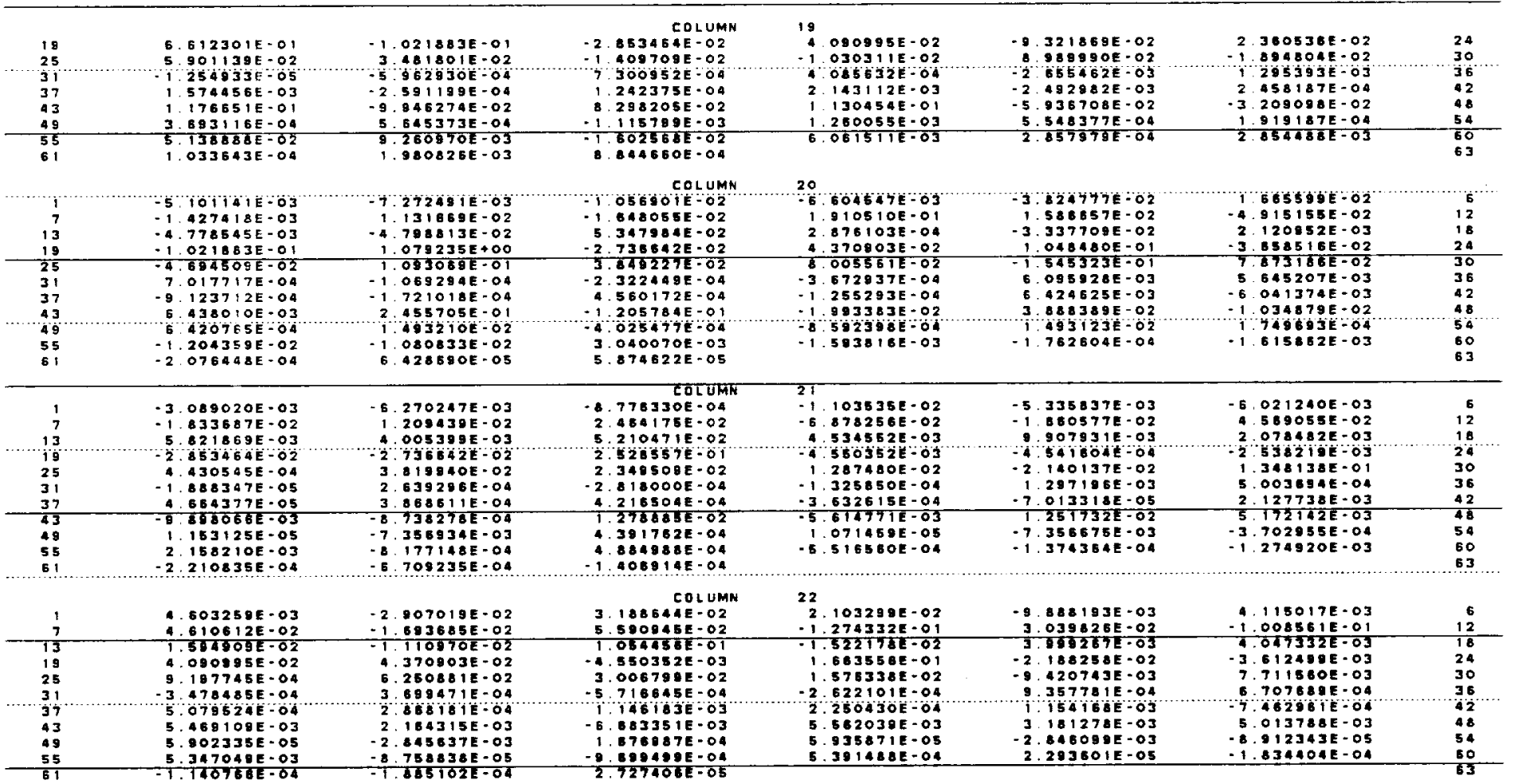




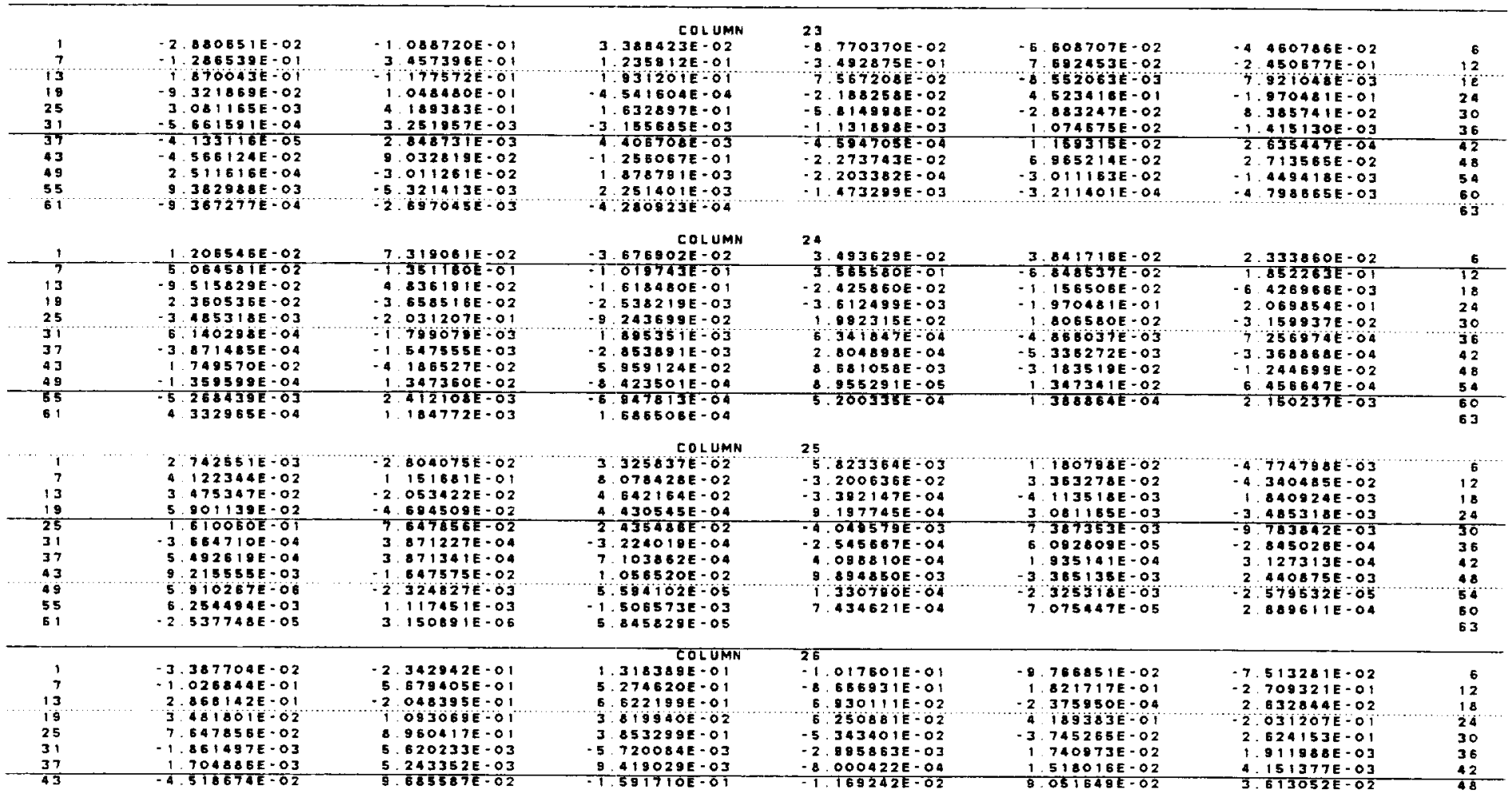

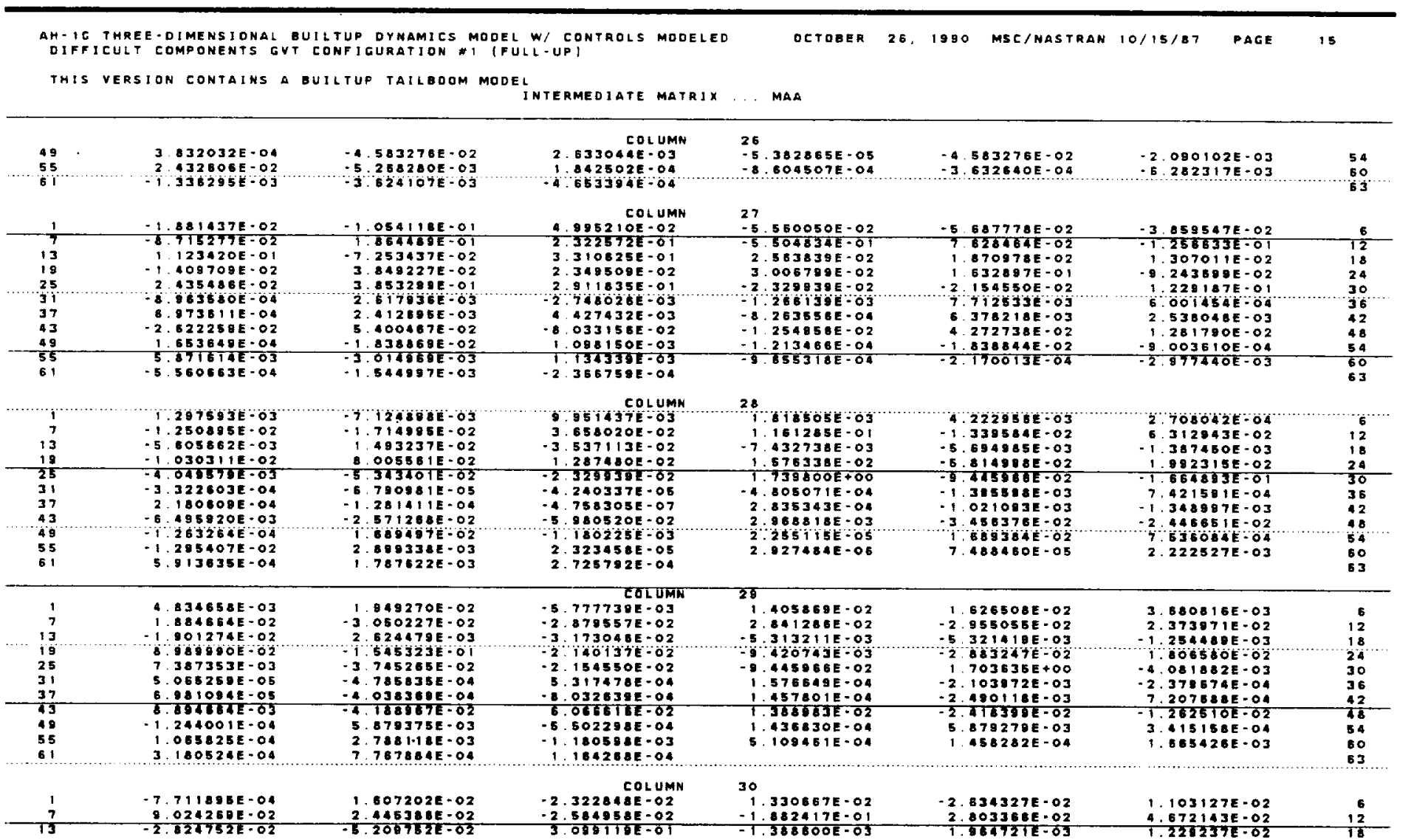


THIS VERSION CONTAINS A BUILTUP TAJLBOOM MODEL

INTERMEDIATE MATRIX ... MAA

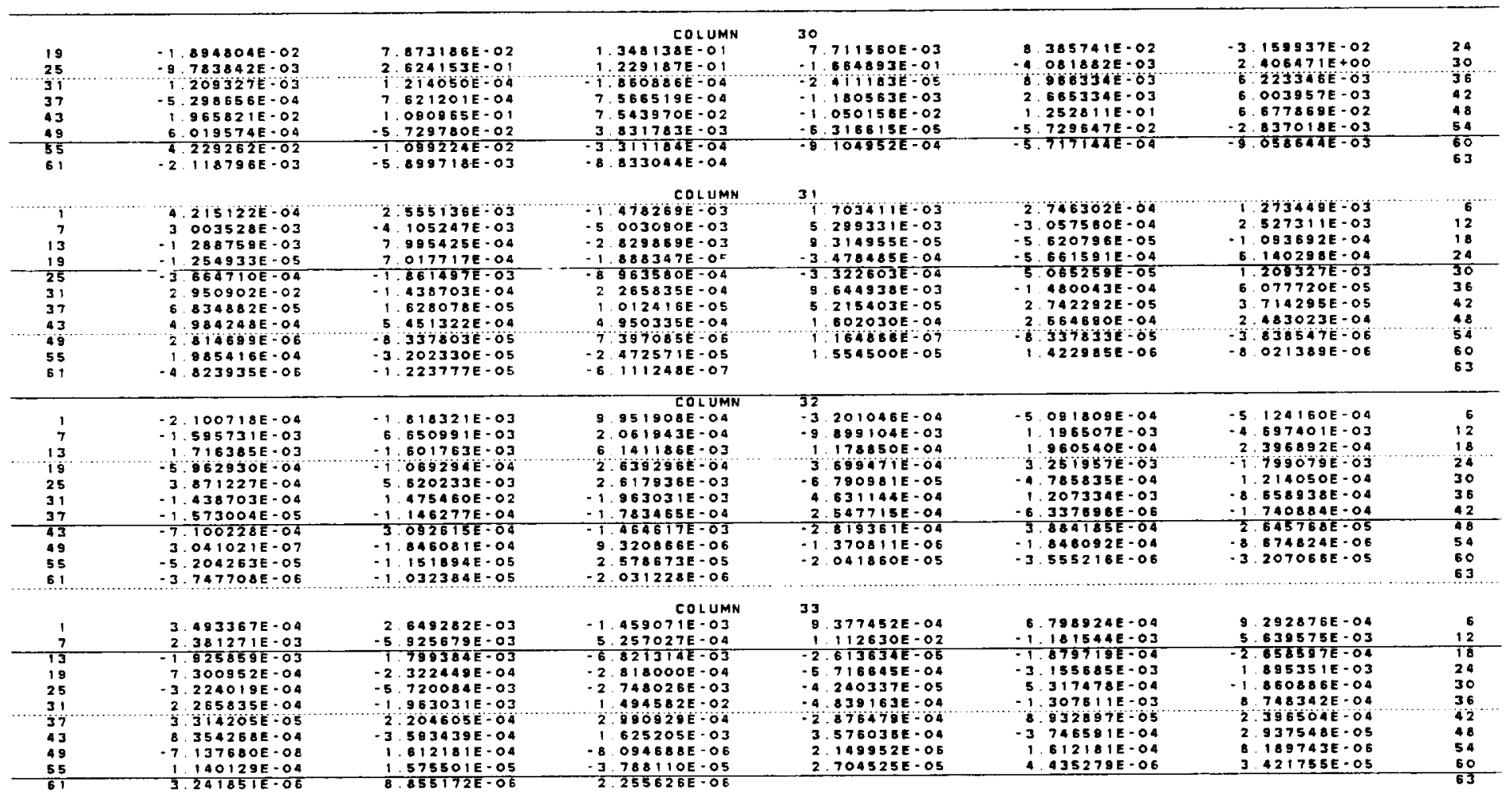

AH- 1 G THREE-Dimensional BUILTUP DYNAMICS MODEL W/ CONTROLS MODELEO

DCTOBER 26, 1990 MSE/NASTRAN 10/15/87 PAGE 17

THIS VERSION CONTAINS A BUILTUP TAILBOOM MOOEL

NTERMEDIATE MATRIX ... MAA

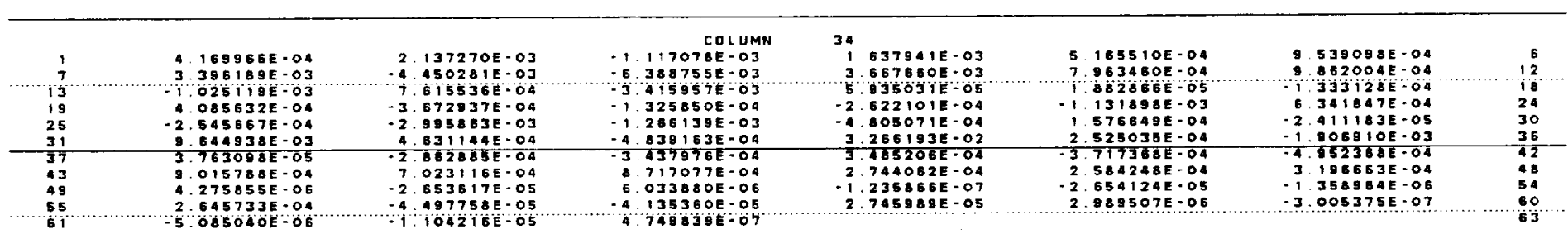

\begin{tabular}{|c|c|c|c|c|c|c|c|c|c|}
\hline 1 & & & & $\begin{array}{r}\text { CDLUMN } \\
133372 E-03\end{array}$ & $\begin{array}{l}35 \\
-1.232800 E-03\end{array}$ & $-2.305032 E .03$ & & $628082 E-04$ & 6 \\
\hline$\frac{1}{7}$ & $-5.041539 E-03$ & $-1.04605 E-02$ & $\frac{1}{1}$ & $473296 E-03$ & $=1.9145$ IAE-OL & $3.2697664-03$ & .9 & $585130 E-03$ & 12 \\
\hline 13 & $2.954334 E-03$ & $-3.888607 E-03$ & 1 & $657459 E-02$ & $5.0576111 E-04$ & 3. $482849 E-04$ & 5 & $516868 E-04$ & 18 \\
\hline 19 & $-2.655462 \mathrm{E}-03$ & $\begin{array}{l}\text { 6. } 095828 E-03 \\
740973 E-02\end{array}$ & 1 & $\begin{array}{l}297196 E-03 \\
712533 E-03\end{array}$ & $\begin{array}{r}9.357781 E-O 4 \\
-1.38559 E-O 3\end{array}$ & $\begin{array}{r}1.074675 E-02 \\
-2.103972 E-03\end{array}$ & $\begin{array}{r}-4 \\
8\end{array}$ & $\begin{array}{l}868037 E-03 \\
966334 E-03\end{array}$ & $\begin{array}{l}24 \\
30\end{array}$ \\
\hline $\begin{array}{l}25 \\
31\end{array}$ & $\begin{array}{r}5.092809 E-05 \\
-1.480043 E-04\end{array}$ & $\begin{array}{r}140973 E=02 \\
1.207334 E=03\end{array}$ & 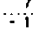 & $\begin{array}{l}712533 E-03 \\
307611 E-03\end{array}$ & 2 525035E-04 & $970422 E=02$ & -5 & IE IOQBEE-O3 & 36 \\
\hline 37 & $-4.697873 E-05$ & $-1.642946 E-05$ & -3 & $O B 2938 E-05$ & $2.9648338-04$ & $4.075020 E-04$ & -2 & $250358 E \cdot 04$ & 42 \\
\hline 43 & $-1.529635 E-03$ & 4. $360773 E-03$ & .5 & $198747 E-03$ & $-8.332804 E-O A$ & 3. $101769 E-03$ & 1 & $089107 E \cdot 03$ & 48 \\
\hline 49 & $1.347188 E-05$ & $-1.278383 \mathrm{E}-03$ & 7 & O5E 141E-05 & $-9.540797 E-06$ & $-1,278362 \mathrm{E}-03$ & -6 & $302026 E-05$ & 54 \\
\hline 55 & $4.926320 E-04$ & $-2.420102 E-04$ & 7 & $110028 E-05$ & $803850 E \cdot 05$ & $.1 .392996 E-65$ & & $091186 E-04$ & 50 \\
\hline 6 & $-4.118361 E-05$ & $-1,140636 E-04$ & $-t$ & $689201 E-05$ & & & & & 53 \\
\hline
\end{tabular}

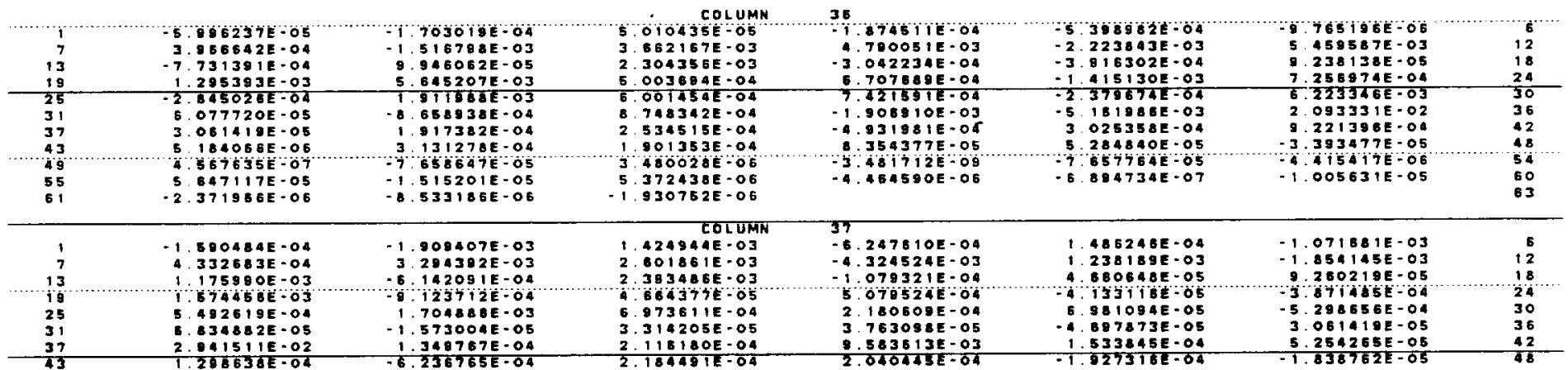




\begin{tabular}{|c|c|c|c|c|c|c|c|}
\hline 49 & 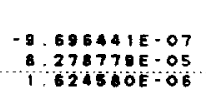 & $\begin{array}{r}5.00082 E-06 \\
3.5757175-05 \\
4.456909 E-06\end{array}$ & 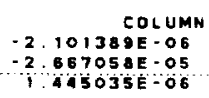 & 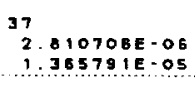 & $\begin{array}{rl}-5 & 006988 E-06 \\
2 & 0073800-06\end{array}$ & 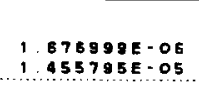 & $\begin{array}{l}54 \\
60 \\
63\end{array}$ \\
\hline $\begin{array}{l}17 \\
13 \\
19 \\
25 \\
31 \\
37 \\
43 \\
40\end{array}$ & 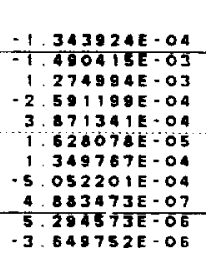 & 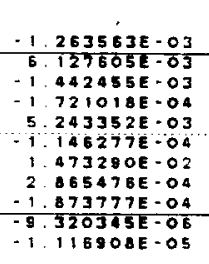 & 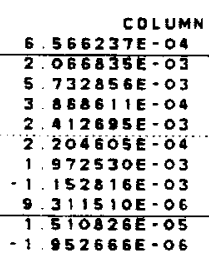 & 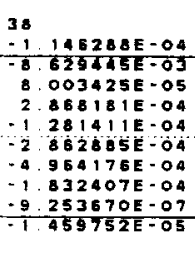 & 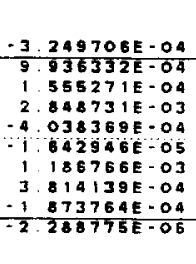 & 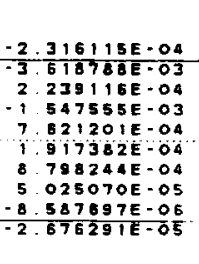 & $\begin{array}{l}6 \\
12 \\
18 \\
24 \\
30 \\
36 \\
42 \\
48 \\
54 \\
60 \\
63\end{array}$ \\
\hline $\begin{array}{r}1 \\
13 \\
19 \\
19 \\
25 \\
31 \\
37 \\
43 \\
49 \\
55 \\
6\end{array}$ & 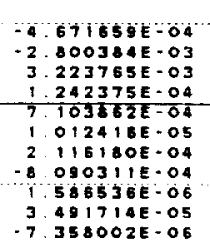 & 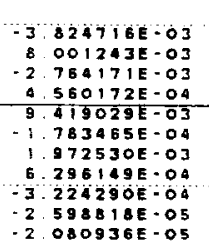 & 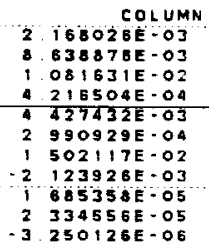 & 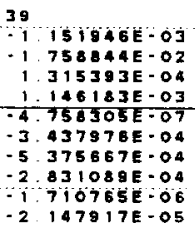 & 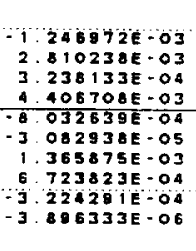 & 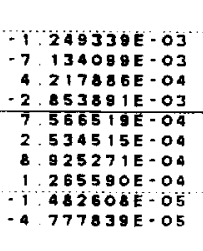 & $\begin{array}{l}16 \\
12 \\
18 \\
24 \\
30 \\
36 \\
42 \\
48 \\
54 \\
60 \\
63\end{array}$ \\
\hline $\begin{array}{r}1 \\
7 \\
13 \\
19 \\
25 \\
31 \\
37 \\
47 \\
49 \\
55 \\
51\end{array}$ & 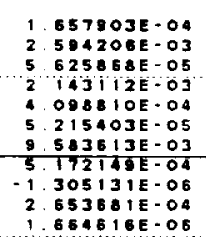 & 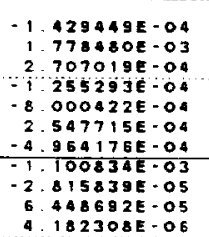 & 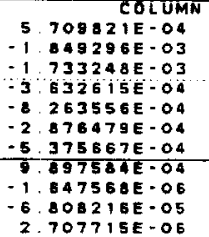 & 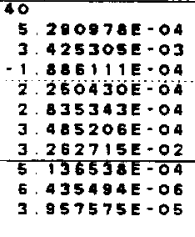 & 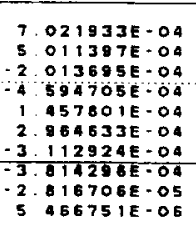 & 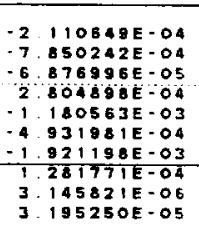 & $\begin{array}{r}5 \\
12 \\
18 \\
24 \\
30 \\
36 \\
42 \\
48 \\
54\end{array}$ \\
\hline$\frac{1}{13}$ & 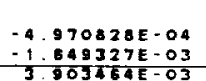 & 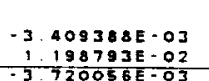 & 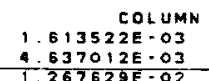 & 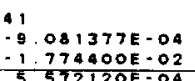 & $\begin{array}{r}-2.14183 E-03 \\
4.964838 E-03 \\
\end{array}$ & 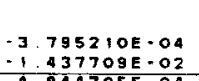 & $\frac{12}{18}$ \\
\hline
\end{tabular}

AH-IE THREE-DIMENSIONAL BUILYUP OYMAMICS MOOEL W/ CONTROLS MODELEO DIFFICULT COMPONENTS GVT CONFIGURATION 1 (FULL-UP)

OCTOBER $944705 E-04$

THIS VERSION CONTAINS A BUILTUP TAILBDOM MODEL

inteRmediate matrix ... MAa

\begin{tabular}{|c|c|c|c|c|c|c|c|c|c|c|c|c|}
\hline $\begin{array}{l}19 \\
25 \\
31 \\
37 \\
43 \\
49 \\
55 \\
61\end{array}$ & $\begin{array}{r}-2 \\
\therefore \\
2 \\
1 \\
-1 \\
1 \\
5 \\
-3\end{array}$ & 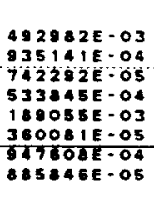 & $\begin{array}{r}6 \\
1 \\
-6 \\
1 \\
4 \\
-1 \\
-2 \\
-1\end{array}$ & $\begin{array}{l}424625 E-03 \\
518016 E-02 \\
337666 E-06 \\
186766 E-03 \\
050964 E-03 \\
100855 E-03 \\
178145 E-04 \\
054117 E-04\end{array}$ & $\begin{array}{r}-7 \\
5 \\
8 \\
1 \\
-5 \\
7 \\
7 \\
-1 \\
-1\end{array}$ & $\begin{array}{l}\text { COLUMN } \\
013318 E-05 \\
378218 E-03 \\
932978-05 \\
3658750-03 \\
140470 E-03 \\
498988-05 \\
283362 E-05 \\
356291 E-05\end{array}$ & $\begin{array}{l}41 \\
1.154168 E-03 \\
-1.021083 E-03 \\
-3.717368-04 \\
-3.112924 E-04 \\
-5.923310 E-04 \\
-7.440783 E-06 \\
-2.67480 B E-05\end{array}$ & $\begin{array}{r}1 \\
-2 \\
2 \\
1 \\
2 \\
-1 \\
-9\end{array}$ & $\begin{array}{l}159315 E-02 \\
490118 E-03 \\
0750201-04 \\
955713 E-02 \\
827963 E-03 \\
180642 E-03 \\
297769 E-06\end{array}$ & $\begin{array}{r}-5 \\
2 \\
3 \\
5 \\
1 \\
-5 \\
-1\end{array}$ & 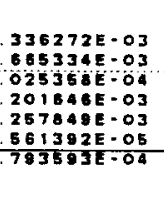 & $\begin{array}{l}28 \\
30 \\
38 \\
42 \\
48 \\
58 \\
60 \\
63\end{array}$ \\
\hline $\begin{array}{l}1 \\
7 \\
13 \\
19 \\
25 \\
31 \\
37 \\
43 \\
49 \\
55 \\
61\end{array}$ & $\begin{array}{r}-8 \\
-9 \\
-9 \\
2 \\
3 \\
3 \\
5 \\
-1 \\
6 \\
4 \\
-3\end{array}$ & $\begin{array}{l}466286 E-O 5 \\
981958 E-04 \\
406431 E-05 \\
458187 E-04 \\
127313 E-04 \\
714295 E-05 \\
254285 E-05 \\
653588 E-05 \\
173668 E-O 5 \\
974846 E-O 5 \\
932061 E-O 5\end{array}$ & $\begin{array}{r}2 \\
1 \\
-6 \\
-6 \\
-6 \\
-1 \\
-1 \\
8 \\
2 . \\
-2 . \\
-1 . \\
-1 .\end{array}$ & 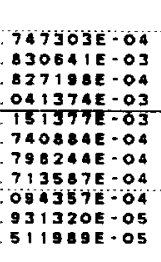 & $\begin{array}{r}-5 \\
8 \\
6 \\
2 \\
2 \\
2 \\
2 \\
8 \\
2 . \\
1 . \\
1 . \\
-3 .\end{array}$ & $\begin{array}{l}\text { COLUMN } \\
246248 E-O 4 \\
764618 E-05 \\
820802 E-03 \\
127738 E-03 \\
538048 E-03 \\
398504 E-04 \\
8252716-04 \\
560703 E-05 \\
.026459 E-O 5 \\
271930 E-O 5 \\
.332386 E-O 6\end{array}$ & 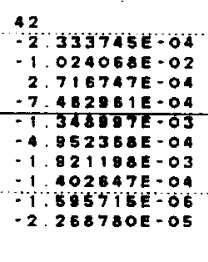 & $\begin{array}{r}8 \\
4 \\
6 \\
2 \\
7 \\
-2 \\
5 \\
5 \\
5 \\
-2 \\
-2\end{array}$ & 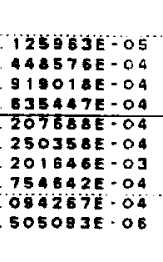 & $\begin{array}{r}-8 \\
2 \\
2 \\
-3 \\
6 \\
9 \\
2 \\
2 \\
-4 \\
-1 \\
-3\end{array}$ & $\begin{array}{l}145731 E-O S \\
588591 E-03 \\
625188 E-04 \\
388868 E-04 \\
003856-03 \\
2213962-04 \\
098702 E-02 \\
264375 E-05 \\
1157632 E-05 \\
116771 E-05\end{array}$ & $\begin{array}{l}12 \\
12 \\
28 \\
30 \\
36 \\
42 \\
48 \\
54 \\
60 \\
62\end{array}$ \\
\hline $\begin{array}{r}1 \\
7 \\
13 \\
19 \\
25 \\
31 \\
37 \\
43 \\
49 \\
55 \\
51\end{array}$ & \begin{tabular}{r}
$\vdots$ \\
\hdashline$:$ \\
$\vdots$ \\
1 \\
1 \\
3 \\
3
\end{tabular} & 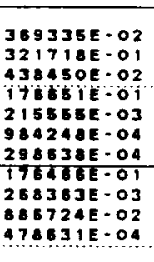 & $\begin{array}{r}3 \\
-4 \\
1 \\
6 \\
-4 \\
-7 \\
-5 \\
5 \\
1 \\
-2 \\
2 \\
2\end{array}$ & $\begin{array}{l}284038 E-02 \\
570474 E-02 \\
238722 E-02 \\
3180702-03 \\
518674 E-02 \\
100228 E-04 \\
052201 E-04 \\
351388 E-02 \\
994898-02 \\
363320 E-03 \\
323878 E-03\end{array}$ & $\begin{array}{r}-8 \\
-8 \\
-2 \\
-6 \\
-2 \\
8 \\
-8 \\
-8 \\
-6 \\
-1 \\
11\end{array}$ & $\begin{array}{l}. \text { COLUMA } \\
.576148 E-04 \\
.229146 E-02 \\
184017 E-02 \\
896066 E-03 \\
622259 E-02 \\
354268 E-06 \\
090311 E-04 \\
154094 E-02 \\
941080 E-04 \\
574452 E-02 \\
248048 E-03\end{array}$ & 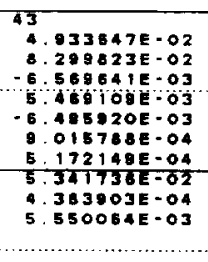 & $\begin{array}{r}2 \\
6 \\
-1 \\
-4 \\
0 \\
-1 \\
-1 \\
2 \\
1 \\
-2\end{array}$ & 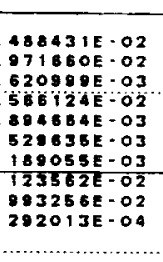 & $\begin{array}{r}1 \\
5 \\
-1 \\
1 \\
1 \\
1 \\
5 \\
-1 \\
3 \\
5 \\
-8\end{array}$ & 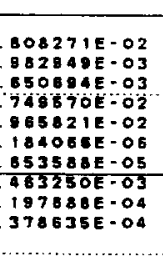 & $\begin{array}{l}12 \\
18 \\
28 \\
30 \\
38 \\
42 \\
48 \\
50 \\
60 \\
63\end{array}$ \\
\hline $\begin{array}{r}1 \\
7 \\
13 \\
19 \\
25 \\
31 \\
37 \\
43 \\
19 \\
55 \\
11\end{array}$ & $\begin{array}{r}-7 \\
-8 \\
1 \\
-8 \\
-1 \\
5 \\
8 \\
5 \\
1 . \\
1 . \\
-9 .\end{array}$ & $\begin{array}{l}261807 E-03 \\
233329 E-03 \\
536501 E-02 \\
946274 E-02 \\
947575-02 \\
451322 E-04 \\
236785 E-O 4 \\
351385-02 \\
258491 E-03 \\
078573 E-03 \\
72255 B E-O 4\end{array}$ & $\begin{array}{r}-1 \\
1 \\
-\overline{2} \\
2 . \\
9 \\
3 \\
2 . \\
3 \\
1 \\
-2 . \\
-1 .\end{array}$ & 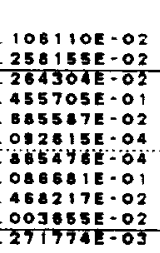 & $\begin{array}{r}-9 \\
-1 \\
8 \\
-8 \\
5 \\
-3 \\
6 . \\
-6 \\
3 \\
1 . \\
1 .\end{array}$ & $\begin{array}{l}\text { COL UMN } \\
543109 E-O 3 \\
172253 E-02 \\
607347 E-02 \\
738278 E-04 \\
400467 E-02 \\
593430 E-04 \\
2081196-O 4 \\
657180 E-02 \\
647123 E-04 \\
088592 E-03 \\
602990 E-05\end{array}$ & $\begin{array}{l}44 \\
-1.938698 E-02 \\
-1.326313 E-01 \\
1.026318 E-62 \\
2.154315 E-03 \\
-2.571268 E-02 \\
7.023118 E-04 \\
-1.100834 E-O 3 \\
-2.504892 E=02 \\
-1.267430 E-03 \\
-9.991758 E-O 4 \\
\end{array}$ & $\begin{array}{r}-3 \\
8 \\
1 \\
8 \\
-4 \\
4 \\
1 . \\
9 \\
-3 \\
-3\end{array}$ & $\begin{array}{l}129994 E-02 \\
571282 E-02 \\
023217 E-02 \\
032819 E-02 \\
188967-02 \\
380773 E-03 \\
050966 E-03 \\
081147 E-02 \\
467987 E-02 \\
031064 E-04\end{array}$ & $\begin{array}{r}3 \\
-9 \\
3 \\
3 \\
-4 \\
1 \\
3 \\
3 \\
1 \\
1 \\
-5 \\
-4 \\
-4\end{array}$ & 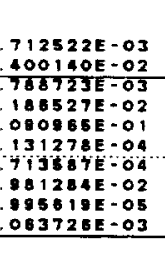 & $\begin{array}{l}1 \\
12 \\
18 \\
24 \\
30 \\
38 \\
42 \\
48 \\
50 \\
60 \\
63\end{array}$ \\
\hline
\end{tabular}


NTERMEDIate Matrix ... MAa

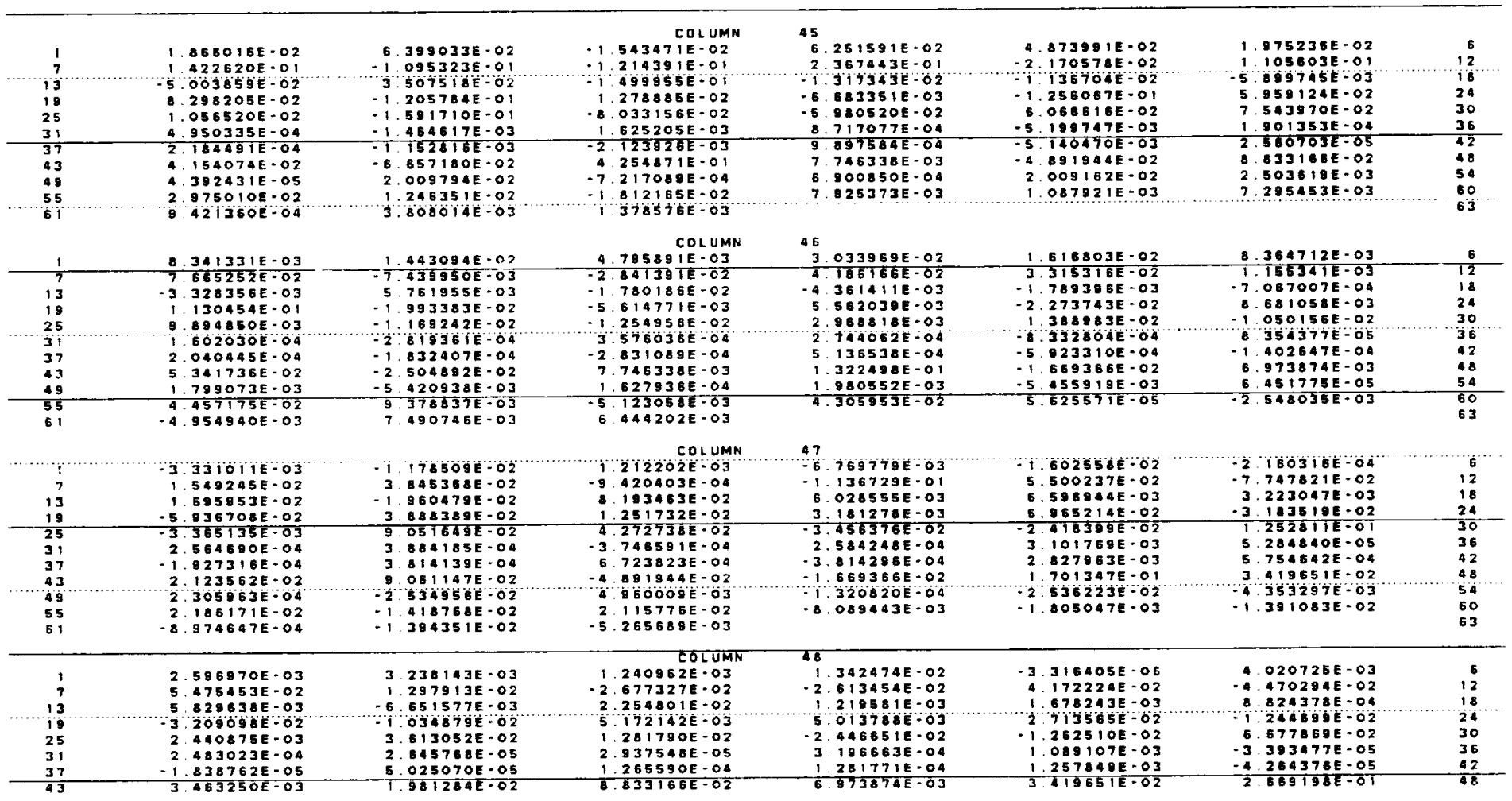

AH- IC THRE -dimensidnal BUILTUP DYNamics MOdel W/ CONTROLS MOdeleo

OCTOBER

2E, 1990 MSC/NASTRAN 10/15/87 PAGE

THIS YERSION CONTAINS B BUILUP TAILBOOM MODEL

INTERMEDIATE MATRIX ... MAA

\begin{tabular}{|c|c|c|c|c|c|c|c|c|c|}
\hline $\begin{array}{l}49 \\
55 \\
69\end{array}$ & $\begin{array}{c}4 \\
-1 \\
-4\end{array}$ & $\begin{array}{l}693498 E-05 \\
257653 E-03 \\
136076 E-03\end{array}$ & $\begin{array}{r}1.777640 E-02 \\
3.43748 E-03 \\
\text { i. }\end{array}$ & $\begin{array}{r}6 \\
1 \\
4 \\
4\end{array}$ & $\begin{array}{l}\text { COLUMN } \\
386748 E-03 \\
7360820-02 \\
458814 E-03\end{array}$ & $\begin{array}{r}48 \\
-6.085389 E-05 \\
4.163789 E-02\end{array}$ & $\begin{array}{r}1.777414 E-02 \\
3.36205 E-03\end{array}$ & $\begin{array}{r}365179 E-03 \\
935787 E-03\end{array}$ & $\begin{array}{l}54 \\
50 \\
63\end{array}$ \\
\hline $\begin{array}{l}1 \\
7 \\
13 \\
19 \\
25 \\
31 \\
37 \\
43 \\
49 \\
55 \\
61\end{array}$ & $\begin{array}{r}1 \\
4 \\
5 \\
3 \\
5 \\
2 \\
-9 \\
1 \\
1 \\
1 \\
-7\end{array}$ & $\begin{array}{l}439826 E-05 \\
809806 E-04 \\
976115 E-05 \\
693115 E-04 \\
910267 E-06 \\
8146996-06 \\
696411 E-07 \\
268363 E-03 \\
222084 E-02 \\
16219 E-03 \\
481315 E-05\end{array}$ & $\begin{array}{r}1.325349 E-05 \\
6.188004 E-05 \\
-8.470475 E-05 \\
6.420785 E-04 \\
3.832032 E-04 \\
3.041021 E=07 \\
4.883473 E-07 \\
1.259491 E-03 \\
-1.139472 E-04 \\
-8.148074 E-04 \\
-1.051231 E-04\end{array}$ & $\begin{array}{r}1 \\
-2 \\
3 \\
1 \\
\vdots \\
-7 \\
1 \\
4 \\
7 \\
-5 \\
8\end{array}$ & $\begin{array}{l}\text { COLUMN } \\
403371 E-06 \\
196420 E-04 \\
278185 E-04 \\
153125 E-05 \\
853649 E-04 \\
137680 E-08 \\
58 B 538 E-05 \\
392431 E-05 \\
645235 E-05 \\
203168 E-05 \\
321271 E-07\end{array}$ & $\begin{array}{r}49 \\
9.586793 E-05 \\
-3.773310 E-04 \\
1.78593 E-05 \\
5.902335-05 \\
-1.283254 E-04 \\
9.275855-06 \\
-1.305131 E-06 \\
1.789073 E-03 \\
-3.395810 E-04 \\
8.47982 E-05\end{array}$ & 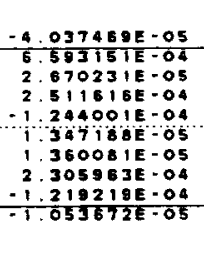 & 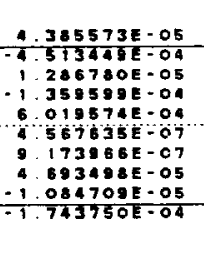 & $\begin{array}{l}6 \\
12 \\
18 \\
24 \\
30 \\
36 \\
42 \\
48 \\
54 \\
60 \\
63\end{array}$ \\
\hline $\begin{array}{l}1 \\
7 \\
13 \\
19 \\
25 \\
31 \\
37 \\
43 \\
49 \\
55 \\
61\end{array}$ & $\begin{array}{r}-1 . \\
-1 \\
-8 \\
5 \\
-2 . \\
-8 \\
-5 \\
1 \\
-1 . \\
-1 . \\
3\end{array}$ & $\begin{array}{l}687558 E-05 \\
840923 E-02 \\
834939 E-03 \\
645373 E-04 \\
324827 E-03 \\
337803 E-05 \\
000892 E-06 \\
994898 E-02 \\
139472 E-04 \\
904976 E-02 \\
510730 E-03\end{array}$ & 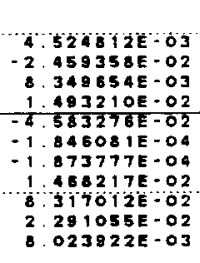 & $\begin{array}{r}-3 \\
8 \\
-3 \\
-7 \\
-7 \\
1 \\
1 \\
-3 \\
2 \\
2 \\
-1 \\
-1 \\
1\end{array}$ & 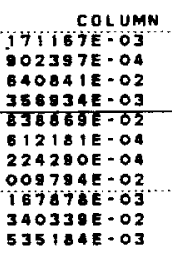 & $\begin{array}{r}50 \\
-2.780620 E-03 \\
4.001521 E-02 \\
-1.805772 E-03 \\
-2.845637 E-03 \\
1.88997 E-02 \\
-2.653817 E-05 \\
-2.815839 E-05 \\
-5.420938 E-03 \\
-6.738940 E=05 \\
3.603781 E-03\end{array}$ & $\begin{array}{r}3.715639 E-O J \\
-2.189322 E-O 2 \\
-2.336122 E-03 \\
-3.011261 E-02 \\
5.879375 E-03 \\
-1.27833 E-03 \\
-1.180555-03 \\
-2.534956 E-02 \\
\text {. } 058293 E-O 2 \\
\text { 1. B28331E-OJ }\end{array}$ & 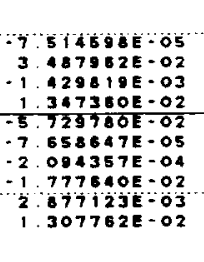 & $\begin{array}{l}16 \\
12 \\
18 \\
24 \\
30 \\
38 \\
42 \\
40 \\
54 \\
60 \\
63\end{array}$ \\
\hline $\begin{array}{r}1 \\
7 \\
13 \\
19 \\
25 \\
35 \\
37 \\
43 \\
19 \\
55 \\
61\end{array}$ & $\begin{array}{r}-1 \\
1 \\
4 \\
-1 . \\
5 \\
7 \\
-2 . \\
-6 \\
7 \\
7 \\
2 \\
2\end{array}$ & $\begin{array}{l}125297 E-05 \\
270060 E-03 \\
706575 E-04 \\
1155780 E-03 \\
594102 E-05 \\
397085 E-06 \\
101380 E-06 \\
941080 E-04 \\
646235 E-06 \\
012673 E-04 \\
754407 E-04\end{array}$ & 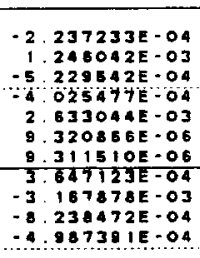 & $\begin{array}{r}1 \\
-3 \\
2 \\
4 \\
1 \\
-8 \\
1 \\
-7 \\
1 \\
1 \\
\therefore\end{array}$ & 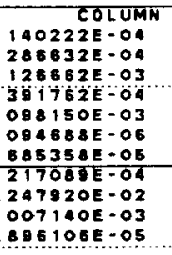 & 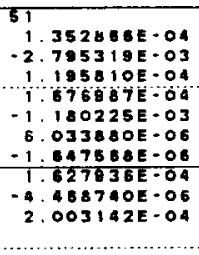 & 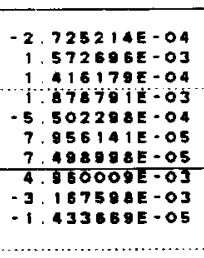 & 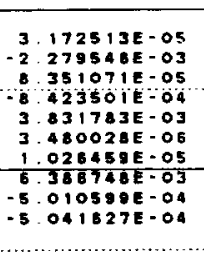 & $\begin{array}{l}6 \\
12 \\
18 \\
24 \\
30 \\
36 \\
42 \\
48 \\
54 \\
60 \\
63\end{array}$ \\
\hline $\begin{array}{r}1 \\
\frac{7}{13}\end{array}$ & $\begin{array}{l}7 \\
6 \\
-7\end{array}$ & $\begin{array}{l}172897 E-05 \\
551216 E-04 \\
027349 E-O E\end{array}$ & $\begin{array}{r}1.081353 E-04 \\
9.287372 E-00 \\
6.377255 E-05\end{array}$ & $\begin{array}{r}5 \\
-2 \\
-2 \\
\end{array}$ & $\begin{array}{l}\text { COLUMN } \\
.272620 E-05 \\
191421 E-04 \\
14 B 610 E-04\end{array}$ & $\begin{array}{l}52 \\
2.837343 E-04 \\
3.480218 E-04 \\
-3.423832 E-05\end{array}$ & $\begin{array}{r}1.400707 E-04 \\
1.308177 E-04 \\
-2.158958 E-05\end{array}$ & $\begin{array}{r}3.967290 E-05 \\
9.360515 E-05 \\
8.493754 E-06\end{array}$ & $\begin{array}{r}5 \\
12 \\
18\end{array}$ \\
\hline
\end{tabular}




\begin{tabular}{|c|c|c|c|c|c|c|c|c|c|c|c|c|c|}
\hline $\begin{array}{l}19 \\
25 \\
311 \\
37 \\
13 \\
19 \\
55 \\
61\end{array}$ & $\begin{array}{r}1 \\
1 \\
1 \\
2 \\
4 \\
-3 \\
1 \\
5\end{array}$ & $\begin{array}{l}260055 E-03 \\
330790 E-04 \\
16486 E=07 \\
110708 E-06 \\
3139030-04 \\
395810 E-04 \\
299080 E-03 \\
428227 E-05\end{array}$ & $\begin{array}{r}-8 \\
-5 \\
-1 \\
-9 \\
-9 \\
-6 \\
-6 \\
5 \\
9 \\
9\end{array}$ & $\begin{array}{l}592398 E-O 4 \\
382855-O 5 \\
370811 E-O S \\
253570 E-O 7 \\
267430 E-03 \\
738940 E-O 5 \\
154785 E-04 \\
529143 E-O 5\end{array}$ & $\begin{array}{r}1 \\
-1 \\
2 \\
-1 \\
5 \\
-4 \\
-5 \\
2\end{array}$ & $\begin{array}{l}\text { COLUMN } \\
071459 E-05 \\
213685-04 \\
14952 E-08 \\
710765 E-06 \\
900850 E-08 \\
168740 E-06 \\
153604 E-04 \\
164672 E-05\end{array}$ & $\begin{array}{r}52 \\
5 \\
2 \\
-1 \\
6 \\
1 \\
1 \\
2\end{array}$ & $\begin{array}{l}935871 E-05 \\
255115 E-05 \\
235866-07 \\
435494 E-O B \\
280552 E-03 \\
219541 E-02 \\
002044 E-04\end{array}$ & $\begin{array}{r}-2 \\
1 \\
-9 \\
-7 \\
-1 \\
-7 \\
3\end{array}$ & $\begin{array}{l}203382 E-O 4 \\
435830 E-O 4 \\
540797 E-O 5 \\
140783 E-O 6 \\
320820 E-04 \\
502654 E-O 5 \\
575714 E-05\end{array}$ & $\begin{array}{r}8 \\
-6 \\
-3 \\
-3 \\
-6 \\
-3 \\
2\end{array}$ & $\begin{array}{l}955291 E-O 5 \\
316515 E-O 5 \\
481712 E-O 9 \\
585715 E-06 \\
055389 E-05 \\
751309 E-07 \\
561100 E-04\end{array}$ & $\begin{array}{l}24 \\
30 \\
36 \\
42 \\
48 \\
54 \\
50 \\
53\end{array}$ \\
\hline $\begin{array}{l}1 \\
7 \\
12 \\
19 \\
25 \\
31 \\
37 \\
43 \\
49 \\
55 \\
61\end{array}$ & $\begin{array}{r}-1 \\
-1 \\
-8 \\
5 \\
-2 \\
-8 \\
-5 \\
1 \\
-1 \\
-1 \\
3\end{array}$ & $\begin{array}{l}702057 E-05 \\
941159 E-02 \\
835080 E-03 \\
548377 E-04 \\
325318 E=03 \\
337833 E-05 \\
008998 E-O 5 \\
99325 E E-02 \\
219219 E-04 \\
907125 E-02 \\
510592 E-03\end{array}$ & $\begin{array}{r}4 \\
-2 \\
8 \\
1 \\
-4 \\
-1 \\
-1 \\
1 \\
7 \\
2 \\
8\end{array}$ & $\begin{array}{l}524808 E-O 3 \\
459304 E-02 \\
349505 E-03 \\
493123 E-02 \\
583276 E-02 \\
846092 E-04 \\
873754 E-04 \\
467987 E-02 \\
058293 E-02 \\
291038 E-02 \\
023912 E-03\end{array}$ & $\begin{array}{r}-3 \\
8 \\
-3 \\
-7 \\
-1 \\
1 \\
-3 \\
2 \\
-3 \\
-1 \\
1\end{array}$ & $\begin{array}{l}\text { COLUMN } \\
171206 E-O 3 \\
911272 E-04 \\
840775 E-02 \\
358675 E-03 \\
838544 E-02 \\
612181 E-04 \\
224291 E-04 \\
009162 E-02 \\
167596 E-03 \\
339917 E-02 \\
535117 E-03\end{array}$ & $\begin{array}{r}53 \\
-2 \\
4 \\
-1 \\
-2 \\
1 \\
-2 \\
-2 \\
-5 \\
-7 \\
3\end{array}$ & $\begin{array}{l}789503 E-03 \\
801481 E-02 \\
805700 E-03 \\
846099-03 \\
665384 E-02 \\
654124 E-05 \\
816706 E-05 \\
455919 E-03 \\
502654 E-05 \\
601313 E-03\end{array}$ & $\begin{array}{r}3 \\
-2 \\
-2 \\
-3 \\
5 \\
-1 \\
-1 \\
-2 \\
8 \\
8 \\
1\end{array}$ & $\begin{array}{l}715551 E-03 \\
189582 E-02 \\
336107 E-03 \\
011163 E-02 \\
879279 E-03 \\
278362-03 \\
180642 E-03 \\
536223 E-02 \\
296298 E-02 \\
627823 E-03\end{array}$ & $\begin{array}{r}-7 \\
3 \\
-1 \\
1 \\
-5 \\
-7 \\
-2 \\
2 \\
21 \\
2 \\
1\end{array}$ & $\begin{array}{l}516485 E-O 5 \\
487942 E-02 \\
429783 E-03 \\
347341 E-02 \\
72964 E-O 2 \\
657764 E-05 \\
094267 E-04 \\
777414 E-02 \\
876895 E-O 3 \\
307482 E-O 2\end{array}$ & $\begin{array}{r}6 \\
12 \\
18 \\
24 \\
-30 \\
36 \\
42 \\
48 \\
54 \\
60 \\
63\end{array}$ \\
\hline $\begin{array}{r}1 \\
7 \\
13 \\
19 \\
25 \\
31 \\
37 \\
43 \\
18 \\
55 \\
61\end{array}$ & $\begin{array}{r}4 \\
-4 \\
-3 \\
1 \\
-2 \\
-3 \\
1 \\
1 \\
-1 \\
-1 \\
1 \\
1\end{array}$ & 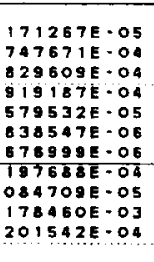 & $\begin{array}{r}2 \\
-1 \\
4 \\
1 \\
-2 \\
-8 \\
-8 \\
-5 \\
2 \\
8 \\
4\end{array}$ & $\begin{array}{l}567059 E-04 \\
056612 E-03 \\
144110 E-04 \\
789693 E-04 \\
090102 E-03 \\
574824 E-O E \\
587597 E-O E \\
985619 E-05 \\
877123 E-03 \\
544944 E-04 \\
404987 E-04\end{array}$ & $\begin{array}{r}-1 \\
-7 \\
-1 \\
-3 \\
-9 \\
8 \\
-1 \\
2 \\
-5 \\
-5 \\
1\end{array}$ & $\begin{array}{l}\text { COLUMN } \\
007576 E-04 \\
877511 E-05 \\
789791 E-03 \\
702955 E-04 \\
003610 E-04 \\
189743 E-06 \\
482808 E-05 \\
503618 E-03 \\
010598 E-04 \\
433230 E-04 \\
368962 E-04\end{array}$ & $\begin{array}{r}54 \\
4 . \\
2 . \\
-6 \\
-8 \\
7 \\
-1 \\
3 \\
3 \\
6 \\
-3 . \\
6 .\end{array}$ & $\begin{array}{l}313241 E-O S \\
366425 E-03 \\
853923 E-05 \\
912343 E-05 \\
536084-04 \\
358964 E-O 6 \\
145821 E-O 6 \\
451778 E-O 5 \\
751309 E-07 \\
601528 E-04\end{array}$ & $\begin{array}{r}2 \\
-9 \\
-1 \\
-1 \\
3 \\
-6 \\
-5 \\
-5 . \\
-4 . \\
2 . \\
1 .\end{array}$ & $\begin{array}{l}544987 E-04 \\
641201 E-04 \\
176575 E-04 \\
4494118 E-03 \\
415158 E-04 \\
302026 E-05 \\
561392 E-05 \\
353299 E-03 \\
876895 E-03 \\
305302 E-04\end{array}$ & $\begin{array}{r}1 \\
1 \\
-7 \\
-2 \\
-2 \\
-4 \\
-1 \\
4 \\
1 \\
8\end{array}$ & $\begin{array}{l}520228 E-O 5 \\
57579 E-O 3 \\
032155 E-O 5 \\
15664 E-O 4 \\
837018 E-O 3 \\
415417 E-O 5 \\
157632 E-05 \\
365179 E-03 \\
241901 E-02 \\
718512 E-O 4\end{array}$ & $\begin{array}{r}6 \\
12 \\
18 \\
24 \\
30 \\
36 \\
42 \\
48 \\
54 \\
60 \\
63\end{array}$ \\
\hline $\begin{array}{r}1 \\
7 \\
13 \\
19 \\
25 \\
31 \\
37 \\
43 \\
49 \\
53 \\
11\end{array}$ & $\begin{array}{c}4 . \\
-\frac{6}{3} \\
5 \\
5 \\
\vdots \\
3 . \\
3 . \\
1 . \\
5\end{array}$ & $\begin{array}{l}701622 E-03 \\
170762 E-02 \\
723196 E-03 \\
13888 E-02 \\
254494 E-03 \\
885416 E-04 \\
278779 E-O 5 \\
848724 E-02 \\
418219 E-03 \\
055186 E-01 \\
750925 E-03\end{array}$ & $\begin{array}{r}6 \\
8 \\
-2 \\
-1 \\
2 \\
2 \\
-5 \\
5 \\
1 \\
-1 \\
-2 \\
-1 \\
-1\end{array}$ & $\begin{array}{l}219893 E-03 \\
968767 E-03 \\
785361 E-03 \\
204595-02 \\
432608 E-02 \\
204263 E-05 \\
294573 E-06 \\
078573 E-03 \\
904975 E-02 \\
72911 B E-02 \\
866604 E-02\end{array}$ & $\begin{array}{r}3 \\
-2 \\
1 \\
2 \\
5 \\
1 \\
3 \\
2 \\
7 \\
-1 \\
-9\end{array}$ & $\begin{array}{l}\text { COLUMN } \\
520830 E-O 3 \\
290982 E-02 \\
414401 E-02 \\
158210 E-03 \\
871614 E-03 \\
140129 E-04 \\
991714 E-05 \\
975010 E-02 \\
012673 E-04 \\
489678 E-02 \\
702409 E-03\end{array}$ & $\begin{array}{r}55 \\
1 . \\
-9 \\
-7 \\
5 \\
-1 . \\
2 . \\
2 . \\
4 . \\
1 \\
-1 . \\
\end{array}$ & 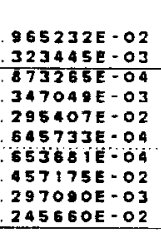 & $\begin{array}{r}5 \\
4 \\
-8 \\
8 \\
1 \\
4 \\
5 \\
2 \\
-1 \\
-2 \\
\end{array}$ & 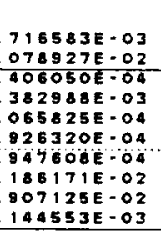 & $\begin{array}{r}5 \\
-2 \\
5 \\
-5 \\
5 \\
5 \\
4 \\
-1 \\
-1 \\
-1 \\
\end{array}$ & 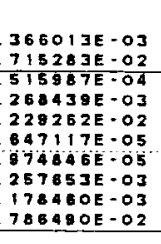 & $\begin{array}{l}6 \\
12 \\
18 \\
24 \\
30 \\
36 \\
42 \\
48 \\
54 \\
60 \\
63\end{array}$ \\
\hline
\end{tabular}

AH - IG THREE-DJMENSIONAL BUILTUP OYNAMICS MOOEL W/ CONTROLS MODELEO

OCTOBER 26, 1990 MSC/MASTRAN $10 / 15 / 87$ PAGE

THIS VERSION CONTAINS A GUILTUP TAILBOOM MODEL

INTERMEDIATE MATRIX ... MAA

\begin{tabular}{|c|c|c|c|c|c|c|c|}
\hline $\begin{array}{l}1 \\
7 \\
13 \\
19 \\
25 \\
31 \\
37 \\
43 \\
49 \\
55 \\
61\end{array}$ & 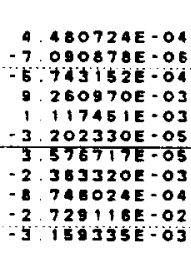 & 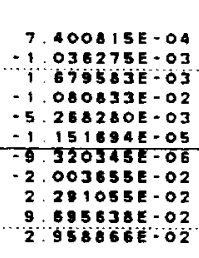 & 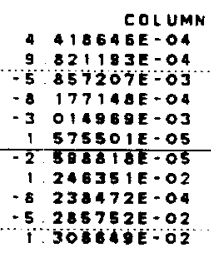 & 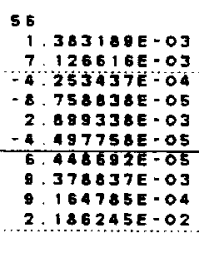 & 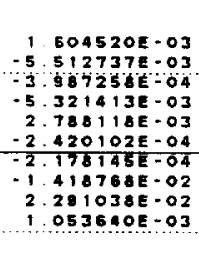 & 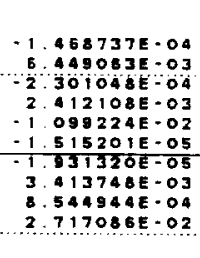 & $\begin{array}{r}6 \\
12 \\
18 \\
24 \\
30 \\
36 \\
42 \\
48 \\
54 \\
60 \\
63\end{array}$ \\
\hline $\begin{array}{l}1 \\
7 \\
13 \\
13 \\
25 \\
31 \\
37 \\
43 \\
48 \\
38 \\
61\end{array}$ & 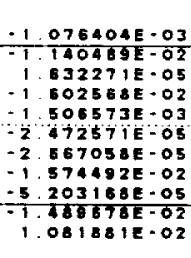 & 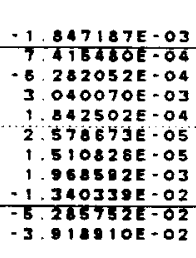 & 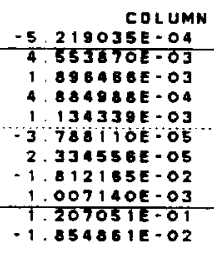 & 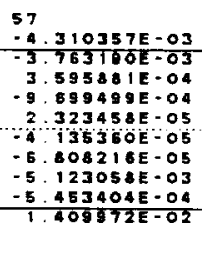 & 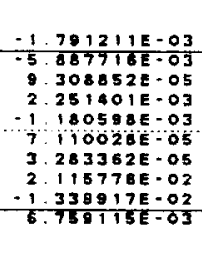 & 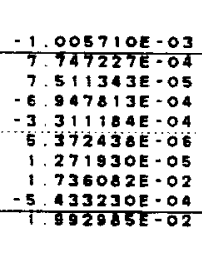 & $\begin{array}{l}6 \\
12 \\
18 \\
24 \\
30 \\
36 \\
42 \\
48 \\
54 \\
60 \\
63\end{array}$ \\
\hline $\begin{array}{r}1 \\
7 \\
13 \\
13 \\
25 \\
31 \\
37 \\
43 \\
49 \\
55 \\
81\end{array}$ & 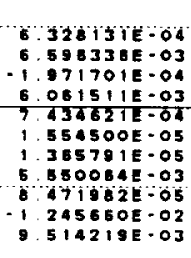 & 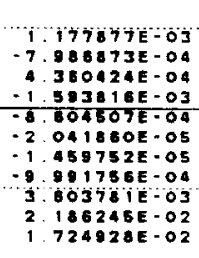 & 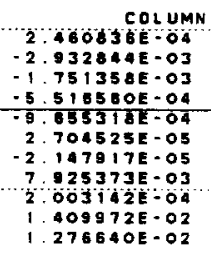 & 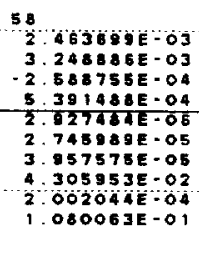 & 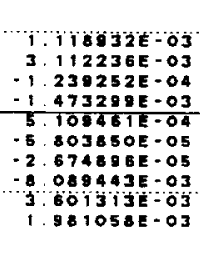 & 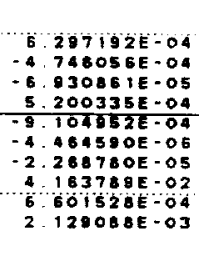 & $\begin{array}{l}6 \\
12 \\
18 \\
24 \\
30 \\
36 \\
42 \\
48 \\
54 \\
60 \\
63\end{array}$ \\
\hline $\begin{array}{r}1 \\
7 \\
13 \\
19 \\
25 \\
31 \\
37 \\
13\end{array}$ & 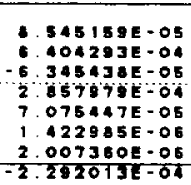 & 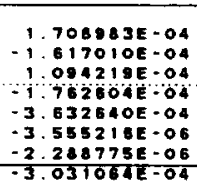 & 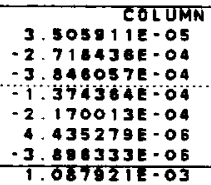 & $\begin{array}{r}58 \\
2.837230 E-04 \\
5.841985 E-04 \\
-481773 E-05 \\
2.2936010-06 \\
7.488460-05 \\
2.989507 E-06 \\
5.4867515-06 \\
5.625571 E-\delta 5\end{array}$ & 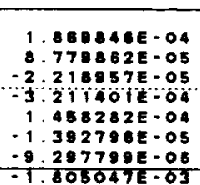 & 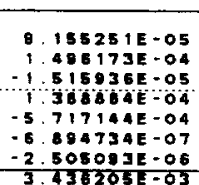 & $\begin{array}{l}16 \\
12 \\
18 \\
24 \\
30 \\
36 \\
42 \\
48\end{array}$ \\
\hline
\end{tabular}


AH-IG THREE-DIMENSIONAL BUILTUP DYHAMICS MODEL W/ CONTROLS MDDELEO

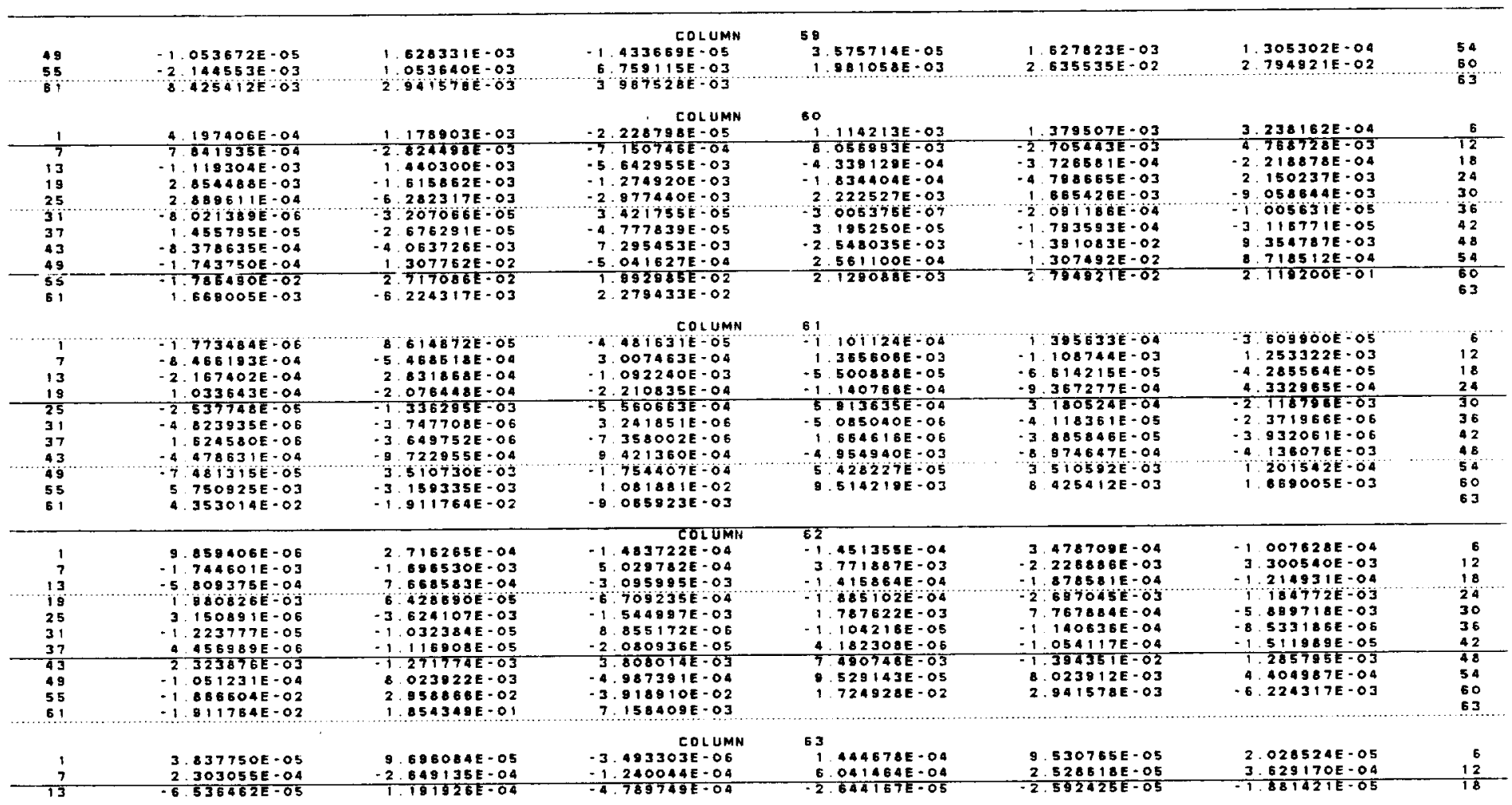

AH - 1 G THREE-DIMFNSIONAL UULTUP DYHAMICS MODEL W' CONTROLS MODELED

OCTOBER 26, 1990 MSE/NASTRAN 10/15/B7 PAGE 25

THIS VERSION CONTAINS A BUILTUP TAILBOOM MOOEL

intermediate matrix ... MaA

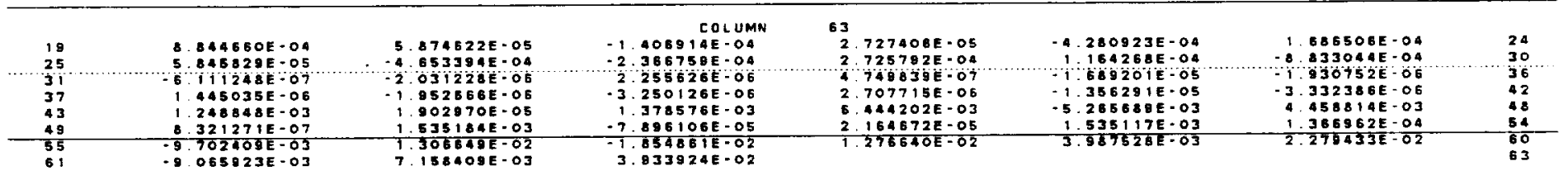




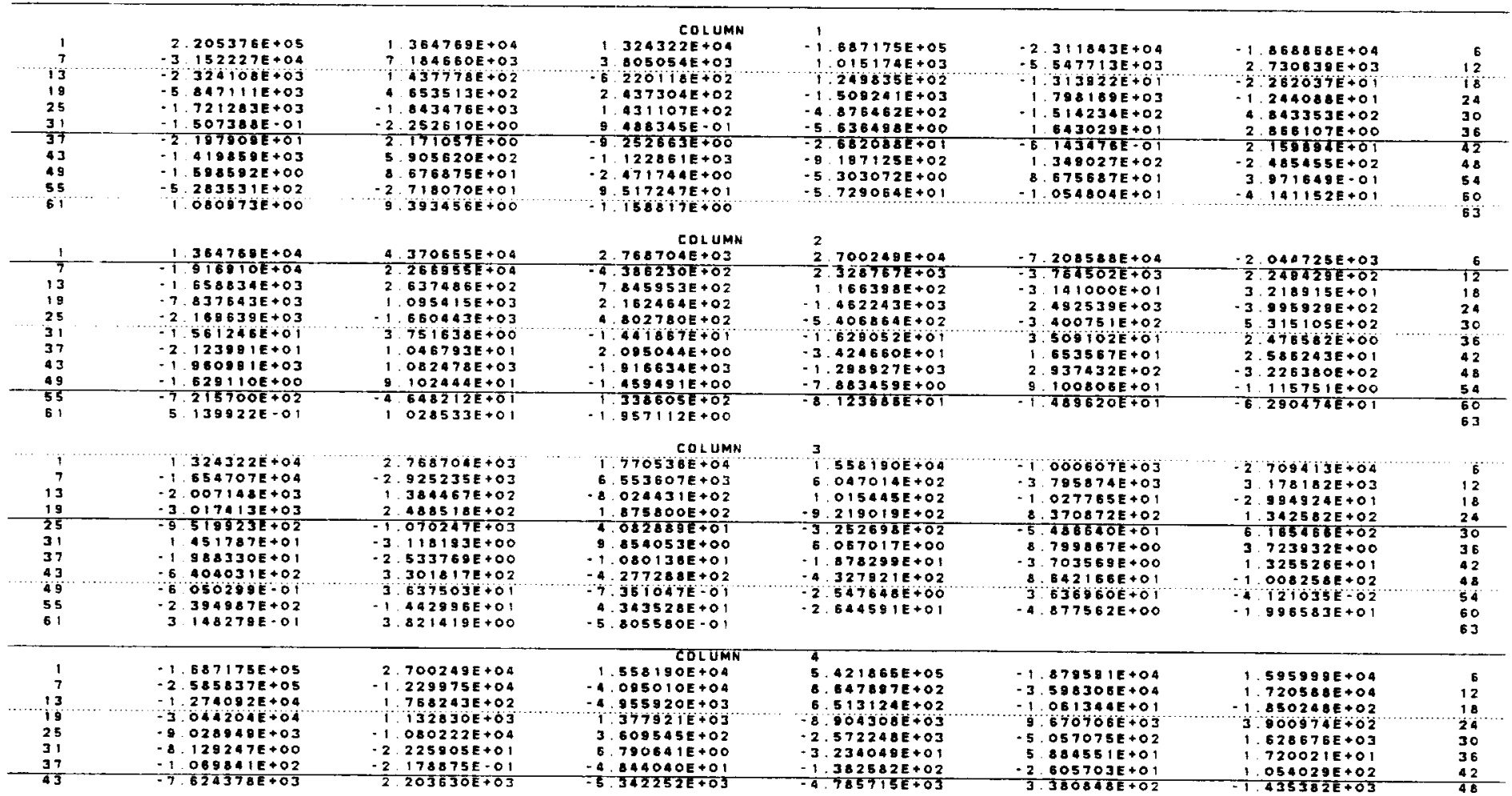

\begin{tabular}{|c|c|c|c|c|c|c|c|c|c|c|}
\hline $\begin{array}{l}\text { AH - } \\
\text { OIF } \\
\text { THI }\end{array}$ & $\begin{array}{l}\text { THREE - DIMENSIONAL } \\
\text { ICULT COMPONENTS GV } \\
\text { VERSION CONTAINS A }\end{array}$ & $\begin{array}{l}\text { BUILTUP OYNAMICS } \\
\text { T CONFIGURATION } \\
\text { BUILTUP TAILBOOM }\end{array}$ & $\begin{array}{l}\text { MODEL W/ CONTRDLS } \\
\text { IFULL-UP) } \\
\text { MDOEL } \\
\text { INTERMEDIATE }\end{array}$ & $\begin{array}{l}\text { MOOELEO } \\
\text { MATRIX }\end{array}$ & & $\begin{array}{l}\text { OCTOAER } \\
\text { KAG }\end{array}$ & $25, \quad 1990$ & MSC / NASTRAN & $10 / 15 / 87$ & 27 \\
\hline $\begin{array}{l}49 \\
55 \\
61\end{array}$ & $\begin{array}{r}-1.057958 E+01 \\
-2.850897 E+03 \\
9062160 E+00\end{array}$ & $\begin{array}{r}5.510211 E+02 \\
-1.132902 E+02 \\
5.9108716+0\end{array}$ & $\begin{array}{r}\text { col } \\
-1.99044 E+ \\
5.02879 E+ \\
-5.056232 E+\end{array}$ & $\begin{array}{l}2 u \text { um } \\
+01 \\
+02 \\
+02 \\
+00\end{array}$ & $\begin{array}{r}4 \\
-2 \\
-2 \\
-2\end{array}$ & $\begin{array}{r}633434 E+01 \\
904723 E+02\end{array}$ & & $\begin{array}{l}509575 E+02 \\
526183 E+01\end{array}$ & $\begin{array}{r}731706 E+00 \\
-2 \quad 020752 E+02\end{array}$ & $\begin{array}{l}54 \\
50 \\
63\end{array}$ \\
\hline $\begin{array}{l}1 \\
-7 \\
13 \\
19 \\
25 \\
31 \\
37 \\
43 \\
49 \\
35 \\
61\end{array}$ & 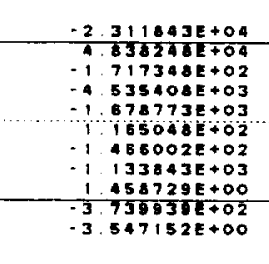 & 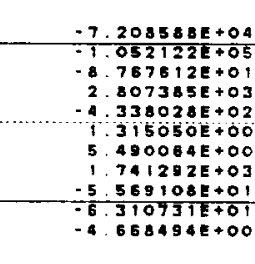 & 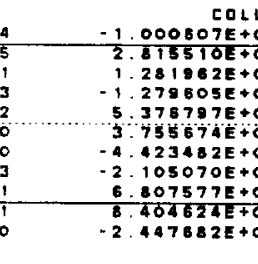 & 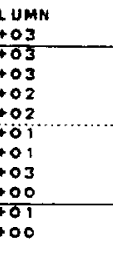 & $\begin{array}{r}5 \\
-1 \\
3 \\
2 \\
3 \\
-1 \\
5 \\
-9 \\
-9 \\
-5 \\
-5 \\
-5\end{array}$ & $\begin{array}{l}879591 E+04 \\
056257 E+09 \\
870038 E+01 \\
861437 E+02 \\
689379 E+02 \\
7151185 E+01 \\
73324 E+01 \\
561308 E+02 \\
747508 E+00 \\
350046 E+01\end{array}$ & $\begin{array}{r}1.8 \\
2.1 \\
-1.5 \\
7.7 \\
-8.0 \\
9.1 \\
8.1 \\
5.8 \\
-5.5 \\
-9.6\end{array}$ & 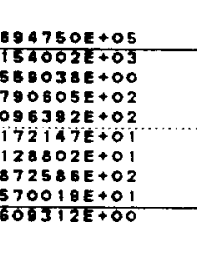 & 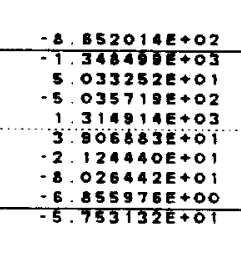 & $\begin{array}{l}6 \\
12 \\
18 \\
24 \\
30 \\
36 \\
42 \\
48 \\
54 \\
60 \\
63\end{array}$ \\
\hline $\begin{array}{l}1 \\
7 \\
13 \\
19 \\
25 \\
35 \\
37 \\
43 \\
43 \\
45 \\
55 \\
61\end{array}$ & 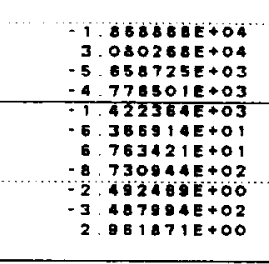 & 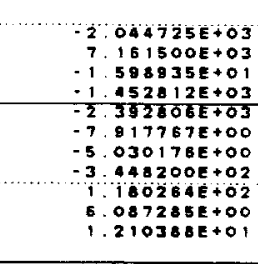 & 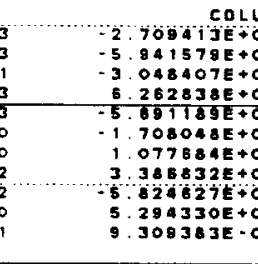 & 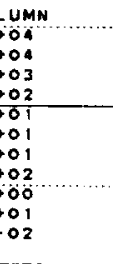 & $\begin{array}{r}6 \\
1 \\
-2 \\
2 \\
-2 \\
-7 \\
-3 \\
2 \\
2 \\
-5 \\
-1 \\
-3\end{array}$ & 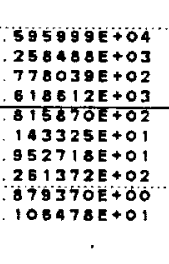 & $\begin{array}{r}-8.8 \\
-1.1 \\
9.5 \\
1.9 \\
-2.5 \\
-3.8 \\
-6.7 \\
-1.5 \\
-1.5 \\
-5.9\end{array}$ & 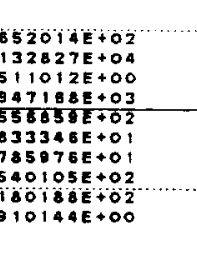 & 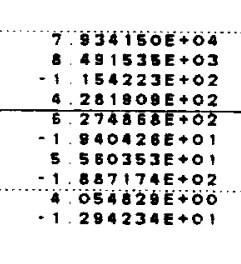 & $\begin{array}{l}6 \\
12 \\
18 \\
24 \\
30 \\
36 \\
42 \\
48 \\
54 \\
60 \\
63\end{array}$ \\
\hline $\begin{array}{l}1 \\
13 \\
13 \\
25 \\
31 \\
37 \\
43 \\
49 \\
55 \\
61\end{array}$ & 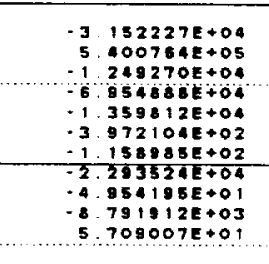 & 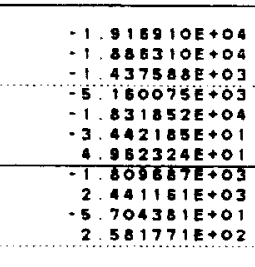 & 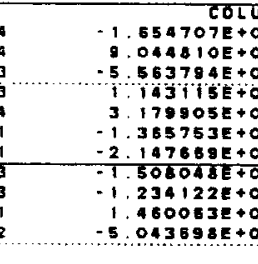 & $\begin{array}{l}0 \mathrm{MN} \\
04 \\
03 \\
03 \\
03 \\
03 \\
01 \\
02 \\
02 \\
01 \\
04 \\
02 \\
02 \\
03 \\
00\end{array}$ & 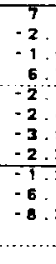 & 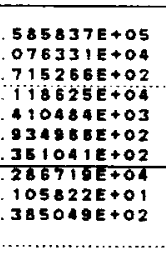 & $\begin{array}{rl}4 & 8 \\
-8 & 4 \\
1 & 2 \\
2 & 4 \\
1 & 1 \\
-1 & 0 \\
-1 & 2 \\
-2 & 5 \\
2 & 4 \\
-1 & 5\end{array}$ & 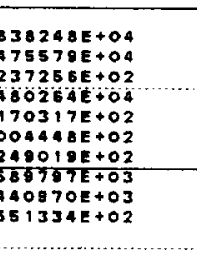 & 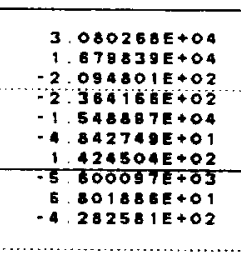 & $\begin{array}{r}5 \\
12 \\
18 \\
28 \\
30 \\
36 \\
42 \\
48 \\
54 \\
60 \\
53\end{array}$ \\
\hline$\frac{1}{7}$ & $\begin{array}{r}7.184680 E+03 \\
-1.886310 E+0.04 \\
-1.011136 E+003\end{array}$ & $\begin{array}{l}2.266955 E+04 \\
1.519179 E+05 \\
1.400197 E+03\end{array}$ & $\begin{array}{r}\operatorname{colU} \\
-2.925235 E+0 \\
-5.458380 E+0 \\
-1.3503 \delta E+0\end{array}$ & $\begin{array}{l}\text { UMN } \\
03 \\
0.03 \\
04\end{array}$ & $\begin{array}{l}8 \\
-1 \\
-2 \\
-3\end{array}$ & $\begin{array}{l}229975 E+04 \\
071751 E+03 \\
272836+02\end{array}$ & $\begin{array}{r}-1.0 \\
1.8 \\
2.80\end{array}$ & $\begin{array}{l}052122 E+05 \\
07091 E+04 \\
087348 E+01\end{array}$ & $\begin{array}{r}7.181800 E+03 \\
1.822094 E+0.4 \\
-6.271919 E+02\end{array}$ & $\begin{array}{r}5 \\
12 \\
18\end{array}$ \\
\hline \multicolumn{11}{|c|}{$\begin{array}{l}\text { CRIGINAL PAGE IS } \\
\text { OF POOR QUALITY }\end{array}$} \\
\hline
\end{tabular}


AH. 1G THREE-DIMENSIONAL BUILTUP DYNAMICS MOOEL W/ CONTROLS MODELEO

THIS VERSION CONTAINS A BUILTUP TAILBODM MODEL

NTERMEDIATE MATRIX ... KAA

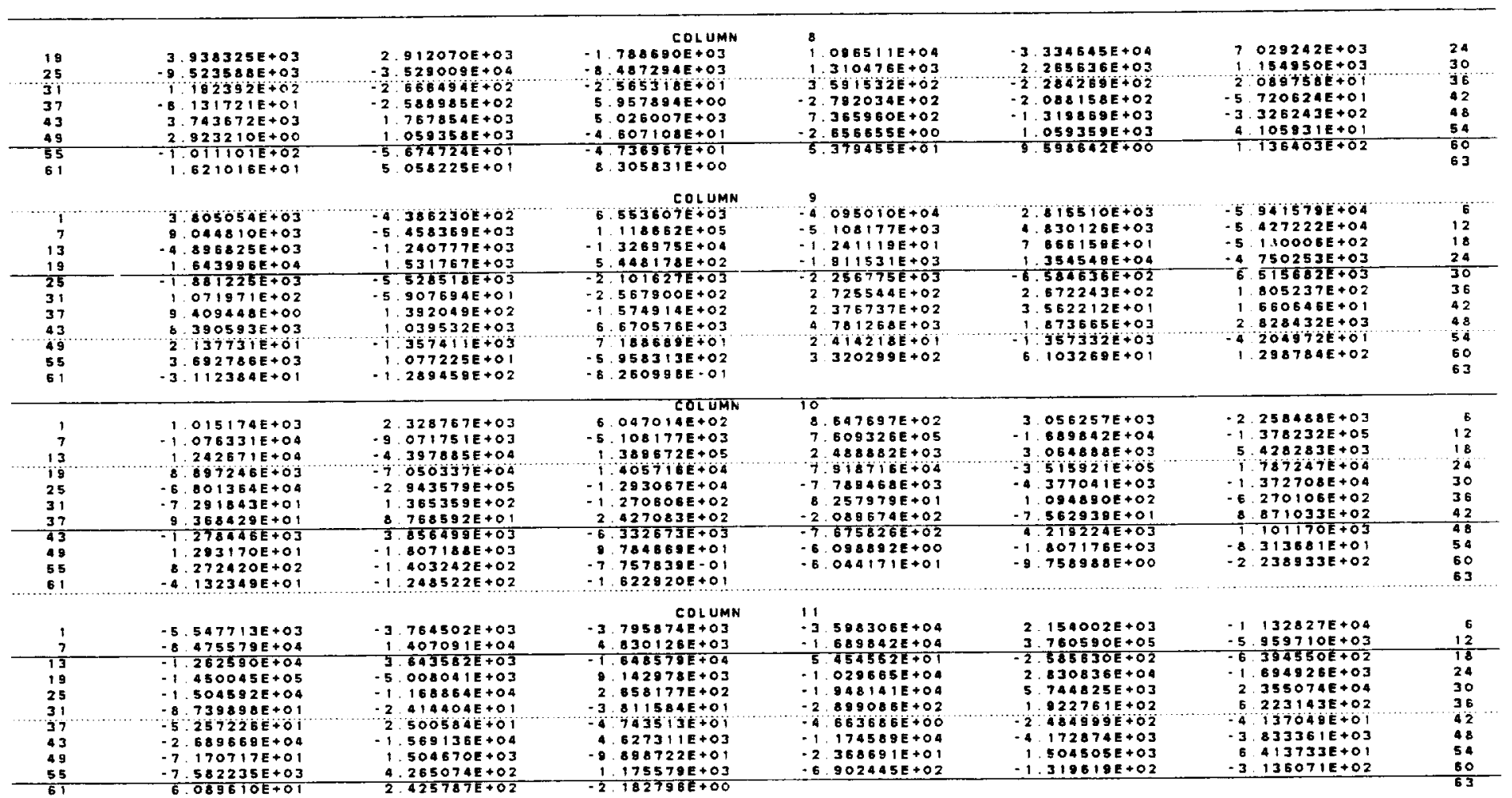

AH - I G THREE-DIMENSIONAL BUILUP DYMAMICS MODEL W/ CONTROLS MOOELEO

OCTODER 26, 1990 MSC/NASTRAN 10/15/87 PACE 29

THIS VERSION CONTAINS A BUILTUP TAILBOOM MODE

INTERMEDIATE MATRIX ... KaA

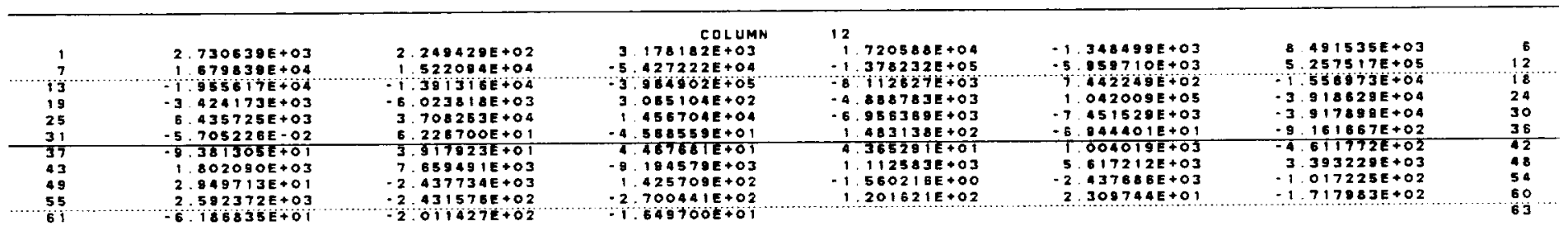

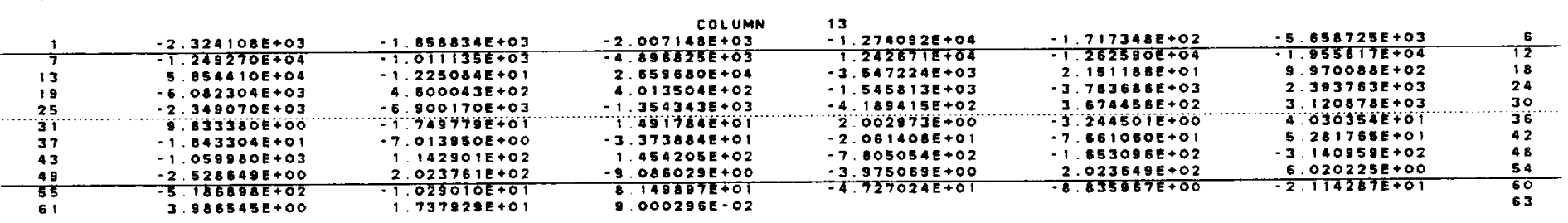

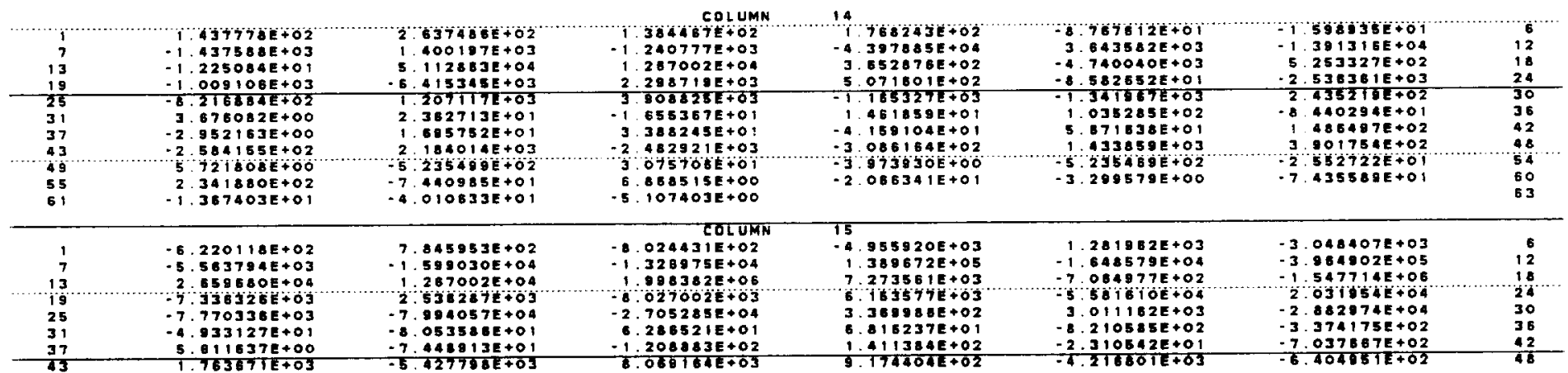


THIS VERSIOM COMTAIMS A BUILTUP TALLBOOM MODEL

INTERMEDiate MATRIX: . KaA

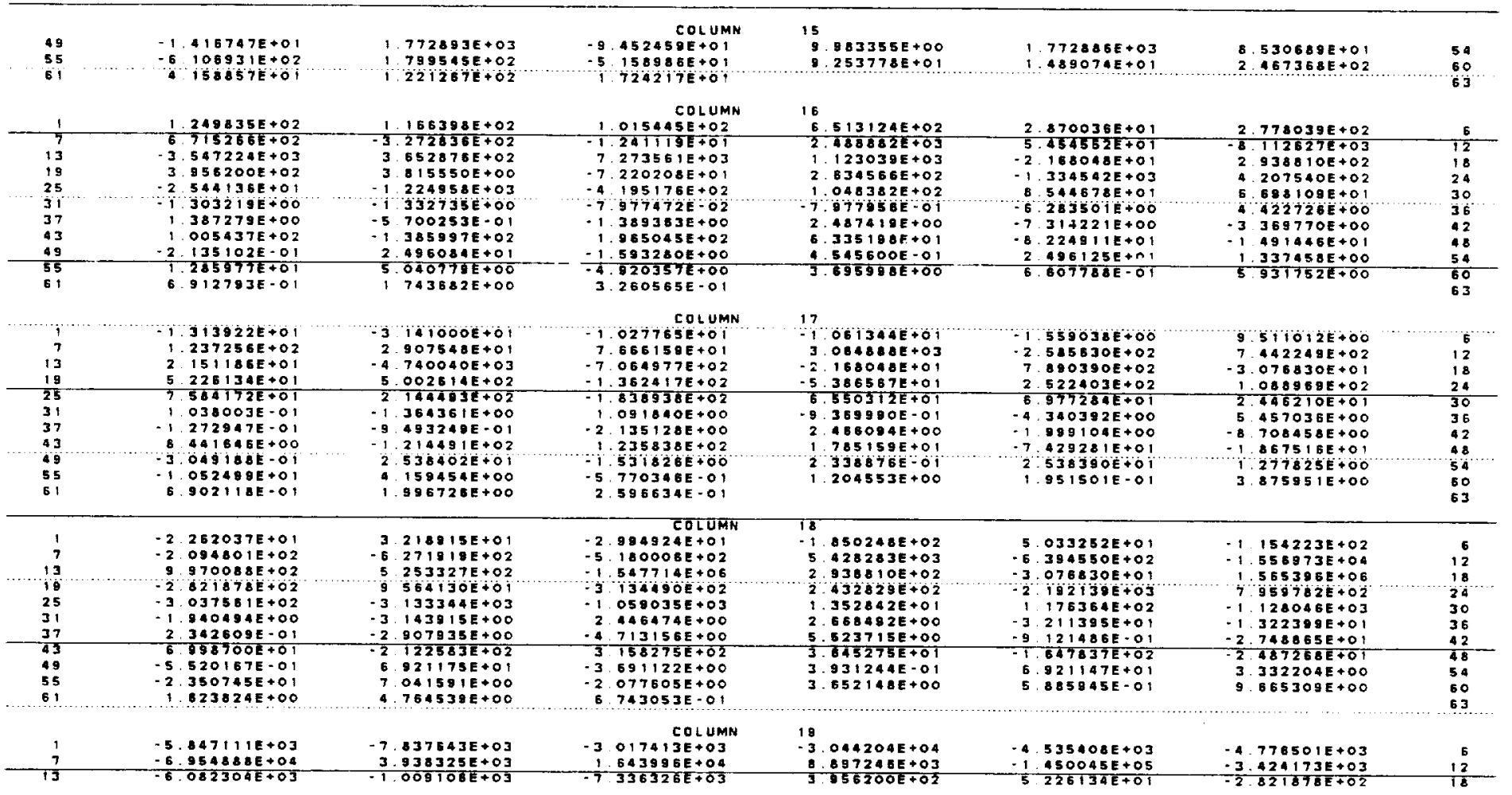

AH-1G THRE - DIMENSIONAL BUILTUP DYNAMICS MOOEL W/ CONTROLS MODELED

OCTOBER 28, 1990 MSC/NASTAAN 10/15/87 PAGE

3

THIS VERSION CONTAINS A BUILTUP TAILBOOM MODEL

INTERMEDIATE MATRIX ... KAA

\begin{tabular}{|c|c|c|c|c|c|c|c|c|c|}
\hline $\begin{array}{l}19 \\
25 \\
311 \\
37 \\
43 \\
49 \\
55 \\
61\end{array}$ & 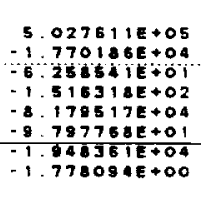 & $\begin{array}{r}-3 \\
-2 \\
-1 \\
1 \\
5 \\
3 \\
-4 \\
2\end{array}$ & $\begin{array}{l}986633 E+03 \\
885128 E+04 \\
243704 E+00 \\
521793 E+01 \\
714742 E+03 \\
059037 E+02 \\
30143 E+02 \\
0369540+02\end{array}$ & $\begin{array}{r}\text { COLUMN } \\
-1.834870 E+04 \\
-2.022428 E+03 \\
-3.41770601 \\
-2.000709 E+01 \\
1.014051 E+04 \\
1.262261 E+02 \\
3.323469 E+03 \\
-4.840110 E+01\end{array}$ & $\begin{array}{l}19 \\
-1.832403 E+04 \\
-6.34797 E+04 \\
-8.080331+01 \\
-2.583808+02 \\
-1.465529 E+04 \\
-1.687435 E+02 \\
-1.969813 E+03\end{array}$ & $\begin{array}{r}2 \\
-3 \\
5 \\
1 \\
8 \\
3 \\
-3\end{array}$ & 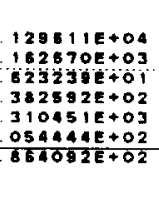 & $\begin{array}{r}-2.129740 E+03 \\
7.87089 E+03 \\
-.177629 E+02 \\
3.671549 E+01 \\
5.356158 E+03 \\
-1.304433 E+01 \\
-1.723601 E+03\end{array}$ & $\begin{array}{l}24 \\
30 \\
36 \\
42 \\
18 \\
54 \\
60 \\
63\end{array}$ \\
\hline $\begin{array}{l}1 \\
7 \\
13 \\
19 \\
25 \\
31 \\
37 \\
43 \\
49 \\
55 \\
61\end{array}$ & $\begin{array}{r}653513 E+02 \\
-5.160075 E+03 \\
400043 E+02 \\
-3.96833 E+03 \\
1.206847 E+04 \\
-835788 E+01 \\
9.2575818+01 \\
927980+03 \\
-636733 E+01 \\
1.439501 E+03 \\
-1.000446 E+01\end{array}$ & $\begin{array}{r}1 \\
2 \\
-6 \\
1 \\
-1 \\
4 \\
4 \\
-5 \\
-3 \\
1 \\
-8\end{array}$ & $\begin{array}{l}095415 E+03 \\
912070 E+03 \\
415345 E+03 \\
855742 E+05 \\
548863 E+04 \\
154764 E+01 \\
579718 E+01 \\
295793 E+04 \\
150866 E+03 \\
25327 E+03 \\
540480 E+01\end{array}$ & 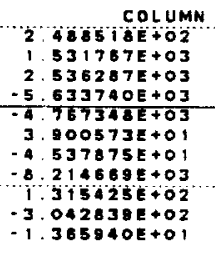 & 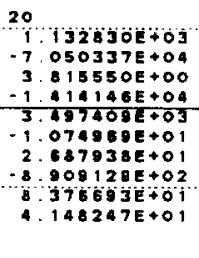 & $\begin{array}{r}2 \\
-5 \\
5 \\
-1 \\
-3 \\
-7 \\
-7 \\
2 \\
2 \\
-3 \\
-6\end{array}$ & $\begin{array}{l}0073856+03 \\
008041 E+03 \\
002614 E+02 \\
308800+04 \\
164670 E+04 \\
458845 E+02 \\
729661 E+02 \\
686001 E+03 \\
150639 E+03 \\
482355 E-01\end{array}$ & $\begin{array}{r}-152812 E+03 \\
-6.023818 E+03 \\
9.55130 E+01 \\
5.142213 E+03 \\
.4275218+04 \\
-8.525552 E+02 \\
8.851350 E+02 \\
2575293 E+03 \\
-9.843056 E+01 \\
-5.809879 E+01\end{array}$ & $\begin{array}{l}16 \\
12 \\
18 \\
24 \\
30 \\
36 \\
42 \\
48 \\
56 \\
60 \\
63\end{array}$ \\
\hline $\begin{array}{r}1 \\
7 \\
13 \\
19 \\
25 \\
31 \\
37 \\
43 \\
49 \\
55 \\
61\end{array}$ & 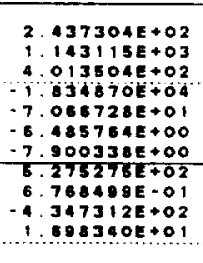 & $\begin{array}{r}2 \\
-1 \\
2 \\
-5 \\
-4 \\
-2 \\
-2 \\
1 \\
1 \\
-1 \\
5\end{array}$ & $\begin{array}{l}162464 E+02 \\
788890 E+03 \\
298719 E+03 \\
633740 E+03 \\
1758418+03 \\
195164 E+00 \\
695258 E+01 \\
724543 E+02 \\
046240 E+03 \\
918008 E+01 \\
278065 E+01\end{array}$ & $\begin{array}{l}\text { COLUMN } \\
1.875800 E+02 \\
5.48178 E+02 \\
-8.027002 E+03 \\
2.007684 E+03 \\
-2.463160 E+03 \\
1.235118 E+00 \\
1.038476 E+01 \\
-1.428088 E+04 \\
-1670182 E+01 \\
3.313434 E+01 \\
932941 E+00\end{array}$ & 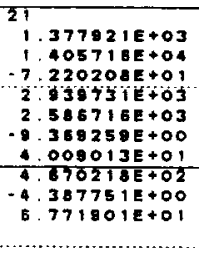 & $\begin{array}{r}-1 \\
9 \\
-1 \\
-2 \\
-3 \\
-3 \\
1 \\
-1 \\
1 \\
1\end{array}$ & $\begin{array}{l}279805 E+02 \\
142978 E+03 \\
382417 E+02 \\
35243 E+03 \\
5398712+03 \\
586537+01 \\
065204 E+02 \\
88624 E+03 \\
048234 E+03 \\
231184 E+01\end{array}$ & 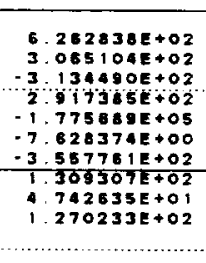 & $\begin{array}{l}5 \\
12 \\
18 \\
24 \\
30 \\
36 \\
42 \\
48 \\
54 \\
60 \\
63\end{array}$ \\
\hline $\begin{array}{r}1 \\
7 \\
13 \\
19 \\
25 \\
31 \\
37 \\
43 \\
49 \\
55 \\
61\end{array}$ & 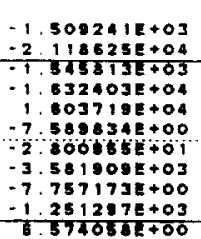 & $\begin{array}{r}-1 \\
1 \\
5 \\
-1 \\
1 \\
1 \\
1 \\
1 . \\
-5 \\
2 . \\
2 . \\
3 .\end{array}$ & 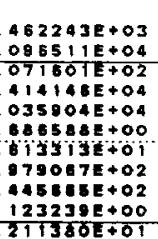 & $\begin{array}{r}\text { COLUMN } \\
-9.219019 E+02 \\
-1.911531 E+03 \\
6.163597 E+03 \\
2.039731 E+03 \\
-7.659513 E+02 \\
5.445513 E+00 \\
-3.167112 E+01 \\
-4.197542 E+02 \\
-1.195592 E+01 \\
2.084328 E+02 \\
\end{array}$ & 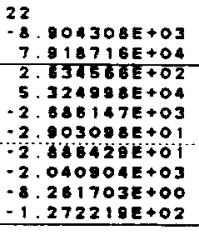 & $\begin{array}{r}3 \\
-1 \\
-5 \\
-8 \\
8 \\
-8 \\
-1 \\
-1 \\
-1 \\
2 \\
-2 \\
-2\end{array}$ & 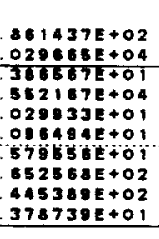 & 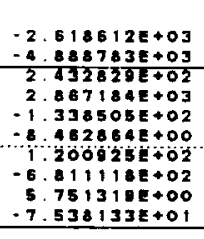 & $\begin{array}{r}6 \\
12 \\
118 \\
24 \\
30 \\
38 \\
42 \\
48 \\
54 \\
60\end{array}$ \\
\hline
\end{tabular}


AH- 1 G THREE-DIMENSIONAL QUILTUP OYNAMICS MOOEL W/ CONTROLS MODELEO

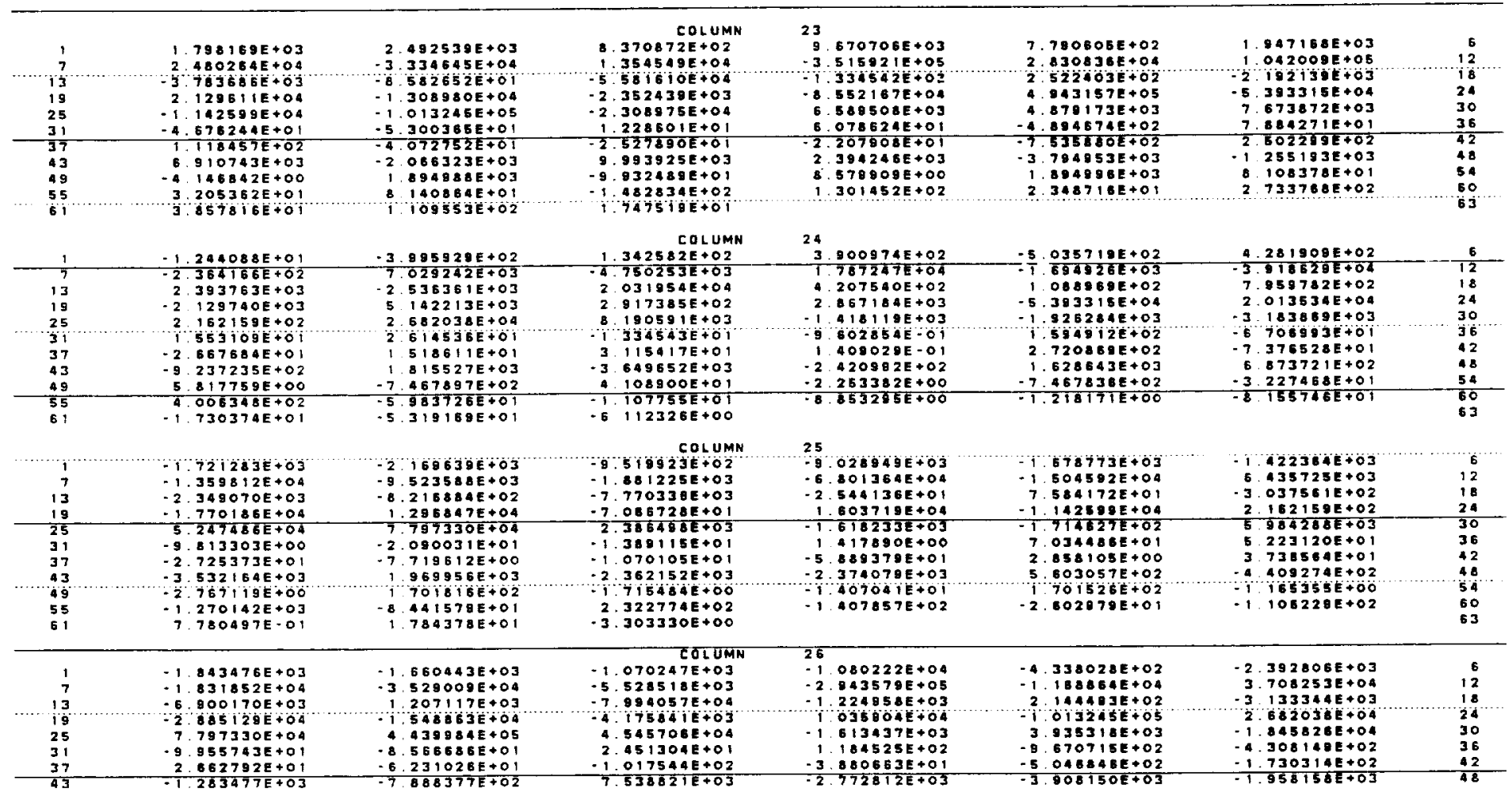

AH- IG THREE-DIMENSIONAL DUILTUP DYNAMICS MODEL W/ CONTROLS MOdELED

\begin{tabular}{|c|c|c|c|c|c|c|c|c|c|c|c|c|}
\hline $\begin{array}{l}49 \\
55 \\
61\end{array}$ & $\begin{array}{l}-1 \\
-2 \\
-2 \\
5\end{array}$ & $\begin{array}{l}705335 E+01 \\
930902 E+03 \\
201683 E+01\end{array}$ & $\begin{array}{r}2 \\
-8 \\
i\end{array}$ & $\begin{array}{l}725794 E+03 \\
332161 E+00 \\
855057 E+02\end{array}$ & $\begin{array}{r}\text { COLUMN } \\
-1.289861 E+02 \\
3.34857 E+02 \\
580685 E+01\end{array}$ & $\begin{array}{l}26 \\
-1.5 \\
-1.2\end{array}$ & $\begin{array}{l}683774 E+01 \\
387323 E+02\end{array}$ & $\begin{array}{r}2 \\
-2\end{array}$ & $\begin{array}{r}725735 E+03 \\
772014 E+01\end{array}$ & & $\begin{array}{l}054887 E+02 \\
108815 E+02\end{array}$ & $\begin{array}{l}54 \\
60 \\
63\end{array}$ \\
\hline 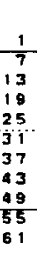 & $\begin{array}{r}1 \\
-3 \\
-1 \\
-2 \\
2 \\
2 \\
-2 \\
1 \\
1 \\
-6 \\
-4 \\
-4 \\
2\end{array}$ & $\begin{array}{l}431107 E+O 2 \\
176905 E+O 1 \\
354343 E+03 \\
022428 E+03 \\
386496 E+03 \\
883792 E+01 \\
430971 E+01 \\
028479 E+03 \\
270117 E+00 \\
73710 E+02 \\
032867 E+O 1\end{array}$ & $\begin{array}{r}4 \\
-8 \\
3 \\
-4 \\
4 \\
4 \\
-3 \\
-2 \\
-2 \\
9 \\
6 \\
6\end{array}$ & 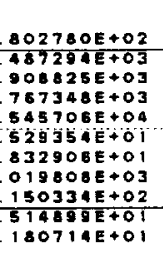 & 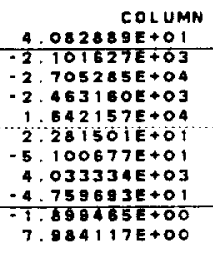 & $\begin{array}{l}27 \\
3 . \\
-1.2 \\
-4 . \\
7 . \\
3 . \\
3 . \\
3 . \\
2.7 \\
2 . \\
2 .\end{array}$ & $\begin{array}{l}500545 E+02 \\
293687 E+04 \\
195178 E+02 \\
859513 E+02 \\
886445 E+02 \\
448128 E+01 \\
563985 E+01 \\
7337716+02 \\
884848 E+00 \\
753153 E+01\end{array}$ & $\begin{array}{r}5 \\
2 \\
-1 \\
-2 \\
1 \\
-3 \\
-1 \\
-1 \\
9 \\
9\end{array}$ & $\begin{array}{l}376797 E+02 \\
858177 E+02 \\
23838 E+02 \\
308975 E+04 \\
8589850+03 \\
736718 E+02 \\
372306 E+02 \\
936180 E+03 \\
150272 E+02 \\
210516 E+00\end{array}$ & $\begin{array}{r}-5 \\
1 \\
-1 \\
0 \\
-8 \\
-1 \\
-1 \\
-4 \\
4 \\
1\end{array}$ & $\begin{array}{l}681189 E+01 \\
4567011+04 \\
059035 E+03 \\
180591 E+03 \\
803327 E+03 \\
400210 E+02 \\
704526 E+02 \\
848858+02 \\
118378 E+01 \\
0947191+02\end{array}$ & $\begin{array}{l}6 \\
12 \\
18 \\
24 \\
30 \\
36 \\
42 \\
48 \\
54 \\
60 \\
63\end{array}$ \\
\hline $\begin{array}{r}7 \\
7 \\
13 \\
19 \\
25 \\
31 \\
37 \\
43 \\
49 \\
55 \\
61\end{array}$ & $\begin{array}{l}-4 \\
-2 \\
-4 \\
-6 \\
-1 \\
1 \\
-8 \\
-6 \\
1 \\
1 \\
-3\end{array}$ & $\begin{array}{l}\text { OT6462E+OZ } \\
110484 E+03 \\
18915 E+02 \\
347787 E+04 \\
618235 E+03 \\
175153 E+01 \\
293295+00 \\
238985+03 \\
365533 E+01 \\
340030 E+01 \\
816804 E+O 1\end{array}$ & $\begin{array}{r}-5 \\
1 \\
-1 \\
3 \\
-1 \\
-7 \\
1 \\
1 \\
-1 \\
-1 \\
-2 \\
-1\end{array}$ & $\begin{array}{l}406864 E+02 \\
310476 E+03 \\
165327 E+03 \\
497409 E+03 \\
61337 E+03 \\
281409 E=02 \\
544767 E+00 \\
129115 E+03 \\
883762 E+03 \\
369433 E+01 \\
080493 E+02\end{array}$ & $\begin{array}{r}\text { COLUMN } \\
-3.252693 E+O 2 \\
-2.256775 E+03 \\
3.369986 E+02 \\
2.586716 E+03 \\
3.36645 E+02 \\
2.693610 E+00 \\
-3.956181 E+00 \\
2.616080 E+04 \\
1.121706 E+O 2 \\
5.36883 E+O 1 \\
1.755076 E+O 1\end{array}$ & $\begin{array}{l}28 \\
-2 . \\
-7 . \\
1.9 \\
-2 . \\
1.5 \\
3.1 \\
-1 . \\
-3 . \\
-5 . \\
-1 .\end{array}$ & 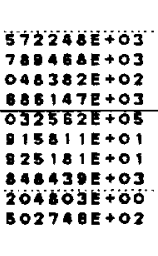 & $\begin{array}{r}-1 \\
-1 \\
6 \\
6 \\
-2 \\
2 \\
3 \\
4 \\
-1 \\
-3\end{array}$ & 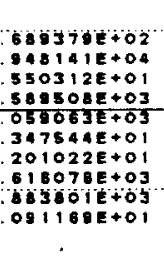 & $\begin{array}{l}-7 \\
-6 \\
1 \\
-1 \\
-1 \\
-7 \\
6 \\
2 \\
27 \\
-3\end{array}$ & $\begin{array}{l}815670 E+O 2 \\
956369 E+03 \\
352842 E+01 \\
418119 E+03 \\
996498+04 \\
312729 E+01 \\
12455 E+01 \\
287329 E+03 \\
942168 E+01 \\
047028 E+02\end{array}$ & $\begin{array}{l}6 \\
12 \\
18 \\
24 \\
30 \\
36 \\
42 \\
48 \\
184 \\
60 \\
63\end{array}$ \\
\hline $\begin{array}{r}1 \\
7 \\
13 \\
19 \\
25 \\
31 \\
37 \\
43 \\
49 \\
55 \\
61\end{array}$ & $\begin{array}{r}-1 \\
1 \\
3 \\
-3 \\
-1 \\
1 \\
-2 \\
-2 \\
-2 \\
8 \\
3 \\
-1 .\end{array}$ & $\begin{array}{l}519234 E+02 \\
170317 E+02 \\
674456 E+02 \\
162670 E+03 \\
714627 E+02 \\
769357 E+01 \\
257686 E+01 \\
085727 E+02 \\
047072 E+00 \\
026722 E+02 \\
781677 E+01\end{array}$ & $\begin{array}{r}-3 \\
2 \\
-1 \\
-3 \\
3 \\
2 \\
-2 \\
2 \\
2 \\
-8 \\
-1 \\
-5\end{array}$ & $\begin{array}{l}400751 E+02 \\
28538 E+03 \\
341867 E+03 \\
154570 E+04 \\
035318 E+03 \\
695827 E+00 \\
282878 E+00 \\
798575 E+03 \\
313845 E+02 \\
071592 E+02 \\
274976 E+01\end{array}$ & 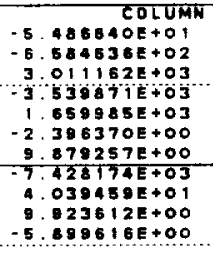 & $\begin{array}{l}29 \\
-5 . \\
-4 . \\
8 \\
0 . \\
-2 . \\
1 . \\
-1 . \\
-4 . \\
-5 . \\
-1 .\end{array}$ & $\begin{array}{l}057075 E+02 \\
377041 E+03 \\
544678 E+01 \\
026933 E+01 \\
059083 E+03 \\
286722 E+01 \\
330640 E+01 \\
169618 E+02 \\
803403 E+00 \\
125880 E+01\end{array}$ & $\begin{array}{r}-8 \\
5 \\
6 \\
4 \\
4 . \\
2 . \\
1 \\
1 . \\
1 \\
-8 \\
-1 .\end{array}$ & 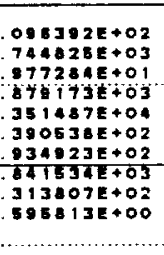 & $\begin{array}{r}2 \\
-7 \\
1 \\
-1 \\
1 \\
2 \\
-7 \\
8 \\
-3 \\
-8\end{array}$ & $\begin{array}{l}556859 E+02 \\
451529 E+03 \\
176364 E+02 \\
9262840+03 \\
53444 E+04 \\
19710 E+00 \\
104287 E+01 \\
026081 E+02 \\
001348 E+01 \\
573117 E+01\end{array}$ & $\begin{array}{r}6 \\
12 \\
18 \\
24 \\
30 \\
38 \\
42 \\
48 \\
54 \\
60 \\
63\end{array}$ \\
\hline $\begin{array}{r}1 \\
\frac{7}{13}\end{array}$ & $\begin{array}{r}4 \\
-3\end{array}$ & $\begin{array}{l}843353 E+02 \\
548887 E+04 \\
120878 E+03\end{array}$ & $\begin{array}{l}5 \\
\frac{1}{2}\end{array}$ & $\begin{array}{l}.315105 E+02 \\
154950 E+03 \\
.435219 E+02\end{array}$ & $\begin{array}{r}\text { COLUMN } \\
6.185486 E+02 \\
6.51562 E+03 \\
.2 .882974 E+04\end{array}$ & $\begin{array}{r}30 \\
1 . \\
-1 . \\
6 .\end{array}$ & $\begin{array}{l}828678 E+03 \\
372708 E+04 \\
658109 E+O 1\end{array}$ & $\frac{1}{2}$. & $\begin{array}{r}314914 E+03 \\
385074 E+04 \\
426210 E+01\end{array}$ & $\begin{array}{r}6 \\
-3 \\
-1 \\
\end{array}$ & $\begin{array}{l}274868 E+02 \\
6178096+04 \\
128046 E+03\end{array}$ & $\begin{array}{r}6 \\
12 \\
18\end{array}$ \\
\hline
\end{tabular}


AH-IG THREE-DIMENSIONAL GUILTUP DYNAMICS MOOEL W/ EONTROLS MODELED

\begin{tabular}{|c|c|c|c|c|c|c|c|c|c|c|c|c|c|}
\hline $\begin{array}{r}19 \\
55 \\
61\end{array}$ & $\begin{array}{r}2 . \\
-1 . \\
-1 .\end{array}$ & $\begin{array}{l}838641 E-02 \\
288311 E+01 \\
053953 E-01\end{array}$ & $\begin{array}{l}-2 \\
-2 \\
\because 1\end{array}$ & $\begin{array}{l}000651 E+00 \\
801349 E+00 \\
2247951 E=01\end{array}$ & $\begin{array}{r} \\
2 \\
2 \\
-7\end{array}$ & $\begin{array}{r}\text { COLUMN } \\
117832 E=01 \\
853028 E+00 \\
941783 E=02\end{array}$ & $\begin{array}{l}37 \\
-2 \\
-1\end{array}$ & $\begin{array}{l}085944 E-01 \\
782854 E+00\end{array}$ & $\begin{array}{l}-2 \\
-3\end{array}$ & $\begin{array}{l}0909 B I E+00 \\
208435 E-01\end{array}$ & $\begin{array}{l}-2 \\
-1 \\
-1\end{array}$ & $\begin{array}{l}187324 E-O 1 \\
865099 E+O O\end{array}$ & $\begin{array}{r}54 \\
60 \\
63\end{array}$ \\
\hline $\begin{array}{r}1 \\
7 \\
13 \\
19 \\
25 \\
311 \\
37 \\
43 \\
49 \\
55 \\
61\end{array}$ & $\begin{array}{r}2 \\
4 \\
-7 \\
1 \\
-7 \\
2 \\
-1 \\
1 \\
3 \\
3 \\
-2\end{array}$ & 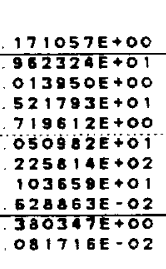 & $\begin{array}{r}1 \\
-2 . \\
1 \\
4 \\
-8 \\
5 . \\
2 . \\
1 \\
-4 . \\
-2 . \\
-8 .\end{array}$ & 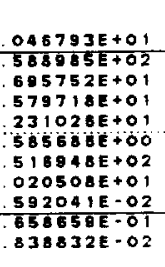 & $\begin{array}{r}-2 . \\
1 \\
-7 \\
-1 \\
-2 \\
-2 \\
-1 \\
3 \\
3 \\
2 . \\
-5 \\
7 .\end{array}$ & 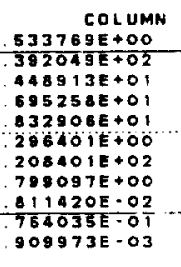 & $\begin{array}{r}38 \\
-2 \\
8 \\
-5 \\
1 \\
1 \\
9 \\
-2 \\
5 \\
6 \\
3\end{array}$ & $\begin{array}{l}178875 E-01 \\
.78659 E+01 \\
700253 E-01 \\
613313 E+01 \\
544757 E+00 \\
488088 E+00 \\
150093 E+01 \\
039058 E+00 \\
316350 E-03 \\
917375 E-01\end{array}$ & $\begin{array}{r}5 \\
2 \\
-2 \\
-4 \\
-2 . \\
3 \\
-7 \\
-7 \\
-4 \\
7\end{array}$ & $\begin{array}{r}490054 E+00 \\
500584 E+01 \\
493249 E-01 \\
072752 E+01 \\
292678 E+00 \\
172017 E+00 \\
282504 E+01 \\
020108 E+00 \\
584705 E-02 \\
.226535 E-C 2\end{array}$ & $\begin{array}{r}-5 \\
3 \\
-2 \\
1 \\
-2 \\
2 \\
7 \\
3 \\
5 \\
-2\end{array}$ & $\begin{array}{l}030176 E+00 \\
919923 E+01 \\
907935 E+00 \\
518611 E+01 \\
077021 E+01 \\
031927 E+00 \\
389431 E+01 \\
164259 E+00 \\
279238 E-03 \\
243998 E-01\end{array}$ & $\begin{array}{l}5 \\
12 \\
18 \\
24 \\
30 \\
36 \\
42 \\
18 \\
54 \\
60 \\
63\end{array}$ \\
\hline $\begin{array}{l}7 \\
7 \\
13 \\
19 \\
25 \\
31 \\
37 \\
43 \\
49 \\
55 \\
61\end{array}$ & $\begin{array}{l}-9 \\
-2 \\
-3 \\
-2 \\
-1 \\
-1 \\
1 \\
1 \\
2 \\
-3 \\
-2 \\
7 \\
7\end{array}$ & $\begin{array}{l}252663 E+00 \\
147669 E+01 \\
373884 E+01 \\
000709 E+01 \\
090105 E+01 \\
003301 E+01 \\
057694 E+02 \\
201277 E+00 \\
171522 E-02 \\
184743 E+00 \\
167343 E-02\end{array}$ & $\begin{array}{r}2 \\
5 \\
3 \\
-4 \\
-1 \\
3 \\
-1 \\
-1 \\
-1 \\
2 \\
3 \\
2\end{array}$ & $\begin{array}{l}095044 E+O C \\
957894 E+00 \\
38245 E+01 \\
537875 E+01 \\
017544 E+01 \\
712042 E+00 \\
208401 E+02 \\
090759 E+01 \\
940175 E+00 \\
155806 E-01 \\
242498 E-O 1\end{array}$ & $\begin{array}{r}-1 . \\
-1 \\
-1 \\
1 \\
-5 \\
-1 \\
2 . \\
2 . \\
2 . \\
-1 \\
1 \\
2 .\end{array}$ & $\begin{array}{l}\text { COLUMN } \\
080138 E+01 \\
.574914 E+02 \\
20883 E+02 \\
.036475 E+01 \\
100677 E+01 \\
266815 E+01 \\
763224 E+02 \\
081009 E+01 \\
592115 E=01 \\
158871 E=01 \\
280873 E-02\end{array}$ & $\begin{array}{r}39 \\
-4 \\
2 \\
-1 \\
-3 \\
-3 \\
1 \\
5 \\
-5 \\
9 \\
-3\end{array}$ & $\begin{array}{l}164040 E+01 \\
427083 E+02 \\
389353 E+00 \\
157142 E+01 \\
956181 E+00 \\
180830 E+00 \\
180088 E+01 \\
584975 E-01 \\
653502 E-03 \\
307265 E-02\end{array}$ & $\begin{array}{r}-4 \\
-1 \\
-2 \\
-2 \\
-2 \\
-4 \\
-4 \\
5 \\
-5 \\
-2 \\
-9 \\
-9\end{array}$ & $\begin{array}{l}423382 E+01 \\
743513 E+01 \\
135128 E+00 \\
527890 E+01 \\
87257 E+00 \\
86148 E-01 \\
14258 E+01 \\
080318 E+00 \\
940137 E+00 \\
321423 E-O 3\end{array}$ & $\begin{array}{r}1 \\
4 \\
-4 \\
3 \\
1 \\
2 \\
-4 \\
2 \\
1 \\
2 \\
2\end{array}$ & $\begin{array}{l}077884 E+01 \\
487881 E+01 \\
713158 E+00 \\
115417 E+01 \\
198985+01 \\
647772 E+00 \\
892278 E+01 \\
632405 E+00 \\
271050 E-O 1 \\
738968 E-O 1\end{array}$ & $\begin{array}{r}6 \\
12 \\
18 \\
24 \\
30 \\
36 \\
42 \\
48 \\
56 \\
60 \\
63\end{array}$ \\
\hline $\begin{array}{r}1 \\
7 \\
13 \\
19 \\
25 \\
31 \\
37 \\
43 \\
49 \\
55 \\
61\end{array}$ & $\begin{array}{r}-2 \\
-2 \\
-2 \\
-2 \\
-5 \\
-1 \\
-5 \\
-5 \\
8 \\
-3 \\
-3\end{array}$ & 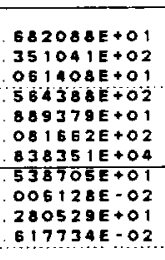 & $\begin{array}{r}-3 \\
-2 \\
-4 \\
2 \\
-3 \\
-9 \\
-2 \\
-2 \\
7 \\
7 \\
-4 \\
3\end{array}$ & $\begin{array}{l}424660 E+01 \\
792034 E+02 \\
159104 E+01 \\
687336 E+01 \\
880663 E+01 \\
076902 E+00 \\
150093 E+01 \\
257411 E+02 \\
914438 E+00 \\
683954 E+00 \\
755021 E+01\end{array}$ & $\begin{array}{r}-1 \\
2 \\
1 \\
4 \\
3 \\
5 \\
5 \\
-8 \\
-8 \\
6 \\
-9\end{array}$ & $\begin{array}{l}\text { COLUMN } \\
878299 E+01 \\
376737 E+02 \\
11384 E+02 \\
009013 E+01 \\
558985+01 \\
305650 E+01 \\
180088 E+01 \\
958701 E+01 \\
53292 E \cdot 02 \\
34938 E+00 \\
432348 E+02\end{array}$ & $\begin{array}{r}40 \\
-1 \\
-2 \\
2 \\
-2 \\
-1 \\
-3 \\
5 \\
-5 \\
-5 \\
-3\end{array}$ & 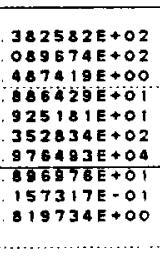 & $\begin{array}{r}-9 \\
-9 \\
-4 \\
2 \\
-2 \\
-1 \\
9 \\
4 \\
4 \\
7 \\
7 \\
-6\end{array}$ & 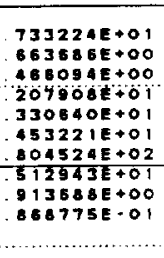 & $\begin{array}{r}2 \\
4 \\
5 \\
1 \\
7 \\
9 \\
-5 \\
-1 \\
-4 \\
-3 \\
-3\end{array}$ & $\begin{array}{l}952718 E+O 1 \\
355291 E+01 \\
523715 E+0 O \\
409029 E=01 \\
458207 E+01 \\
947843 E+01 \\
284458 E+02 \\
599058 E+01 \\
364223 E-02 \\
149846 E+00\end{array}$ & $\begin{array}{r}6 \\
12 \\
18 \\
24 \\
30 \\
36 \\
42 \\
48 \\
54 \\
60 \\
63\end{array}$ \\
\hline $\begin{array}{r}1 \\
7 \\
13\end{array}$ & & $\begin{array}{r}143476 E-01 \\
249019 E+02 \\
861060 E+01\end{array}$ & $\begin{array}{r}1 \\
-2 \\
5\end{array}$ & $\begin{array}{r}653587 E+O 1 \\
08 B 158 E+02 \\
671538 E+01\end{array}$ & $\begin{array}{r}-3 \\
-3 \\
-2\end{array}$ & $\begin{array}{r}\text { COLUMN } \\
.0356 E+00 \\
56212 E+01 \\
310542 E+01\end{array}$ & $\begin{array}{l}41 \\
-2 \\
-7 \\
-7\end{array}$ & $\begin{array}{r}605703 E+01 \\
562939 E+01 \\
314251 E+00\end{array}$ & $\begin{array}{r}8 \\
-2 \\
-1\end{array}$ & $\begin{array}{r}128802 E+01 \\
48499 E+02 \\
999104 E+00\end{array}$ & $\begin{array}{r}-6 \\
1 \\
-9\end{array}$ & $\begin{array}{r}785976 E+01 \\
004019 E+03 \\
121486 E \div 0 !\end{array}$ & $\begin{array}{r}5 \\
12 \\
18\end{array}$ \\
\hline
\end{tabular}

AH- IG THREE-DIMENSIONAL OUILTUP DYMAMICS MODEL CONTROLS MODELED

OCTOEER 2 2. $4849998+02$

\begin{tabular}{|c|c|c|c|c|c|c|c|}
\hline $\begin{array}{l}19 \\
25 \\
31 \\
37 \\
43 \\
49 \\
55 \\
61\end{array}$ & 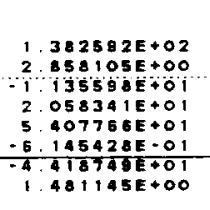 & 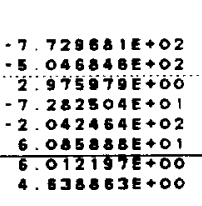 & 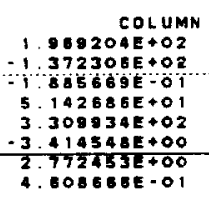 & 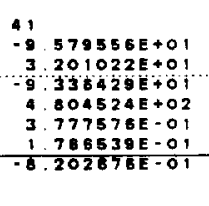 & 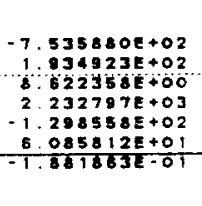 & 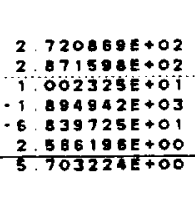 & $\begin{array}{l}24 \\
30 \\
36 \\
42 \\
48 \\
54 \\
68 \\
63\end{array}$ \\
\hline $\begin{array}{l}1 \\
7 \\
13 \\
19 \\
255 \\
31 \\
37 \\
43 \\
49 \\
85 \\
61\end{array}$ & 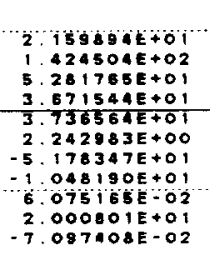 & 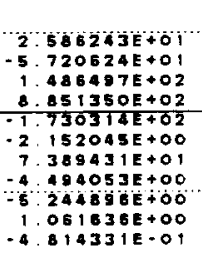 & 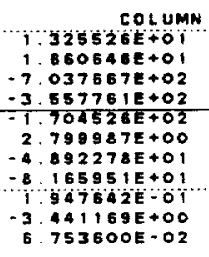 & 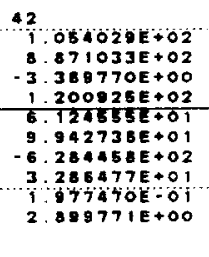 & 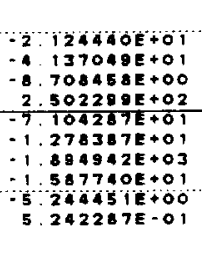 & 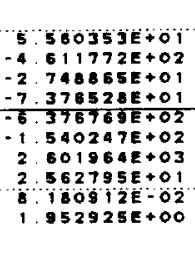 & $\begin{array}{r}6 \\
12 \\
18 \\
24 \\
-30 \\
36 \\
42 \\
48 \\
48 \\
50 \\
63 \\
63\end{array}$ \\
\hline $\begin{array}{r}1 \\
7 \\
13 \\
19 \\
25 \\
31 \\
37 \\
37 \\
43 \\
45 \\
55 \\
61 \\
1.1\end{array}$ & 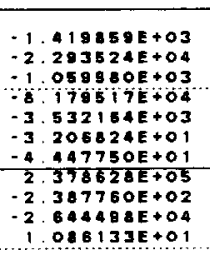 & 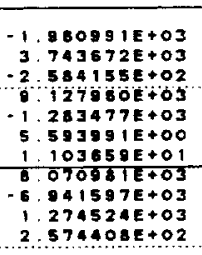 & 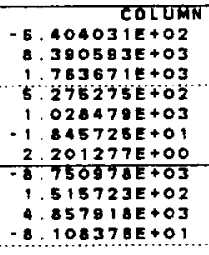 & 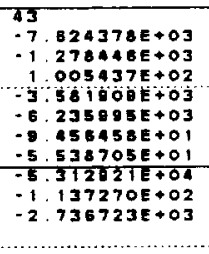 & 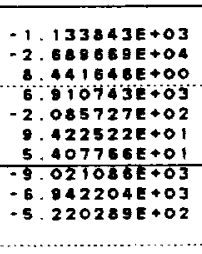 & 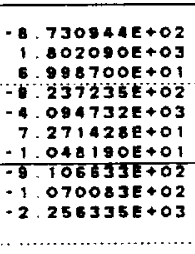 & $\begin{array}{l}15 \\
12 \\
18 \\
28 \\
30 \\
30 \\
36 \\
42 \\
48 \\
54 \\
50 \\
83 \\
63\end{array}$ \\
\hline $\begin{array}{l}13 \\
18 \\
25 \\
31 \\
37 \\
43 \\
43 \\
55 \\
19\end{array}$ & 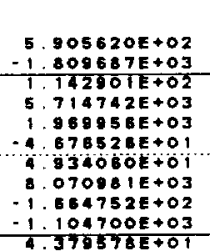 & 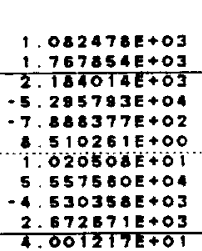 & 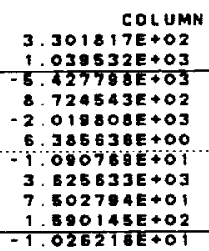 & 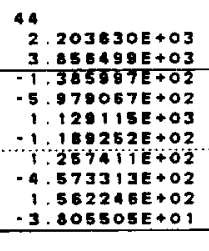 & 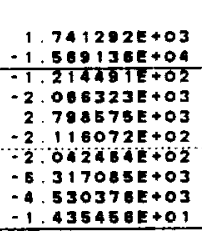 & 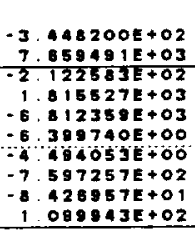 & $\begin{array}{l}5 \\
12 \\
18 \\
28 \\
30 \\
36 \\
42 \\
48 \\
54 \\
80 \\
63\end{array}$ \\
\hline
\end{tabular}




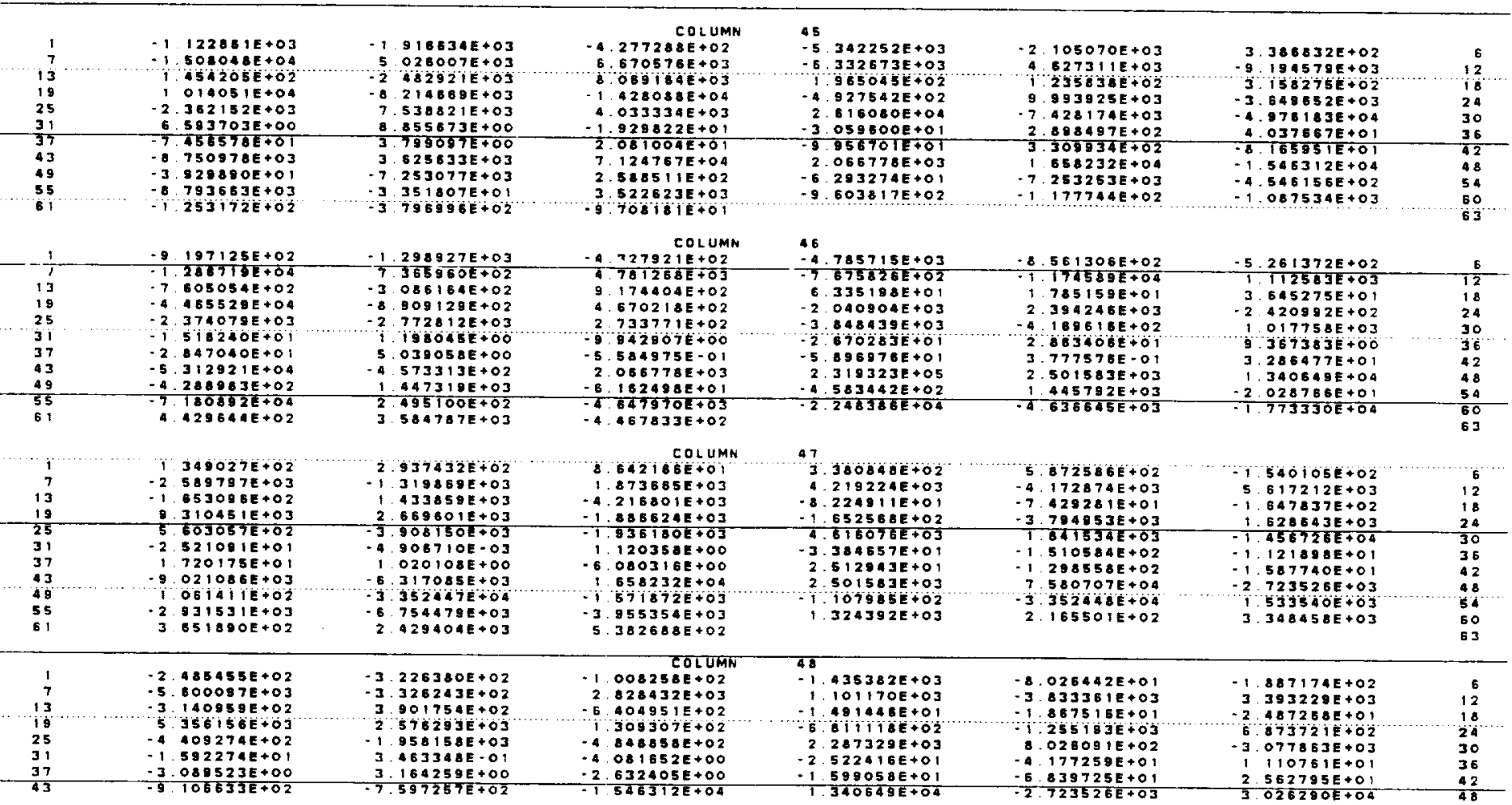

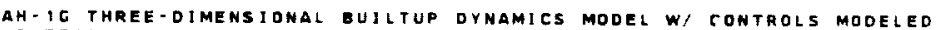

OCTOBER 26, 1990 MSC/NASTRAN 10/15/B7 PAGE

THIS VERSION CONTAINS A BUILTUP TAILBOOM MOOEL

INTERMEDIATE MATRIX ... KAA

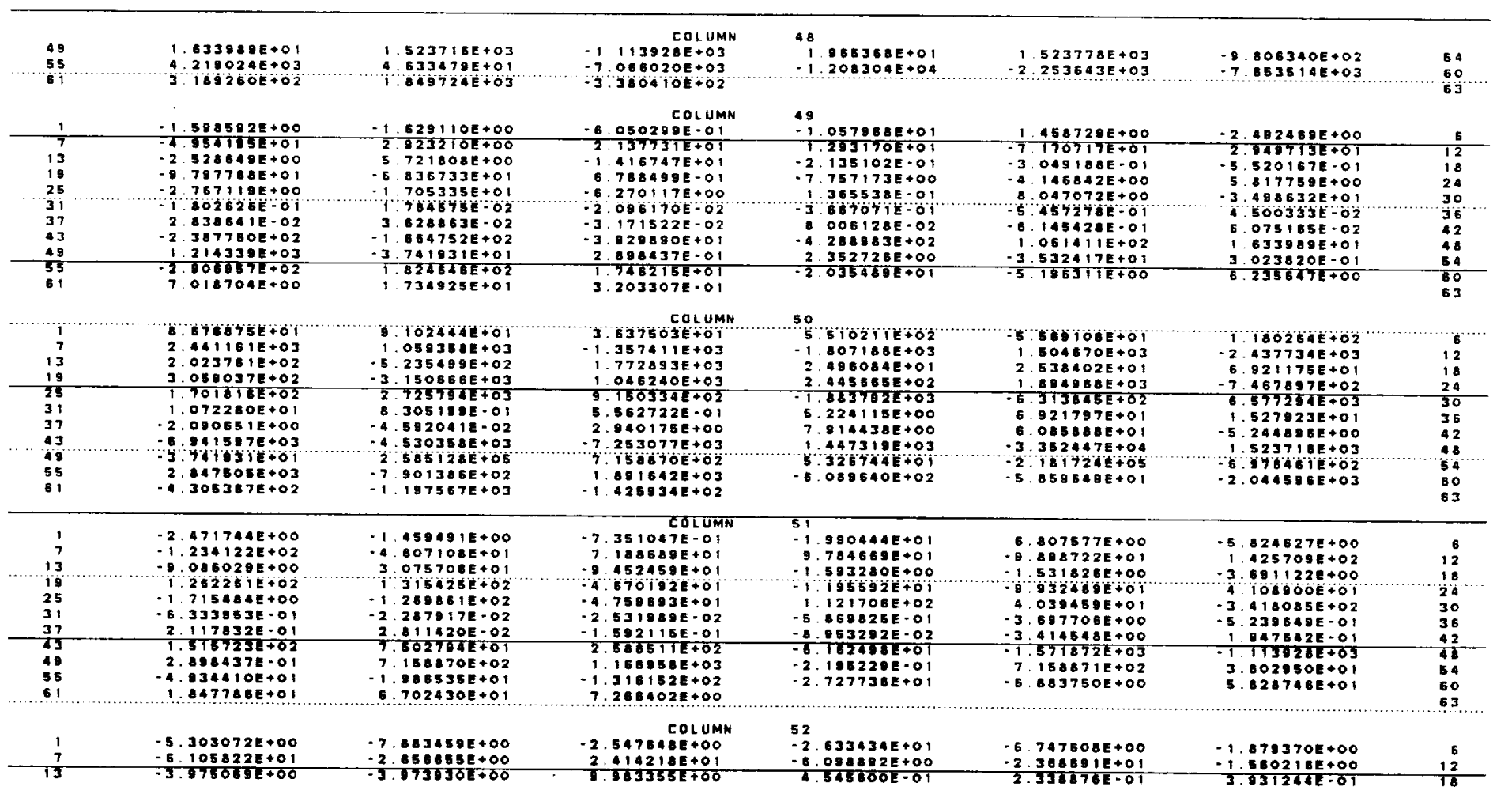


AH - IG THREE-DIMENSIONAL OUILTUP DYNAMICS MOOEL W/ CONTROLS MODELED

\begin{tabular}{|c|c|c|c|c|c|c|c|}
\hline $\begin{array}{l}19 \\
25 \\
31 \\
37 \\
43 \\
49 \\
55 \\
61\end{array}$ & 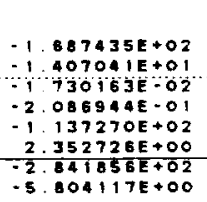 & 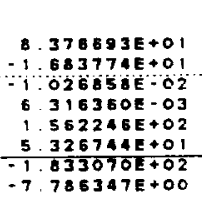 & $\begin{array}{r}\text { COLUMA } \\
-4.387751 E+00 \\
2.684846 E+00 \\
-5.811436 E-02 \\
-9.653502 E-03 \\
-6.293274 E+01 \\
-2.195229 E-01 \\
7.842347 E+01 \\
-1.422009 E+00\end{array}$ & $\begin{array}{l}52 \\
-2.261703 E+\infty \\
-5.204803 E+00 \\
6.939716 E=02 \\
-5.157317 E-01 \\
-4.5834428+02 \\
1.213873 E+03 \\
-3.729785 E+01\end{array}$ & 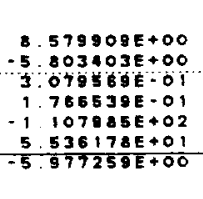 & 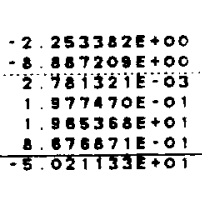 & $\begin{array}{l}24 \\
30 \\
36 \\
42 \\
48 \\
54 \\
60 \\
63\end{array}$ \\
\hline $\begin{array}{l}1 \\
7 \\
13 \\
19 \\
25 \\
31 \\
37 \\
43 \\
49 \\
55 \\
61\end{array}$ & 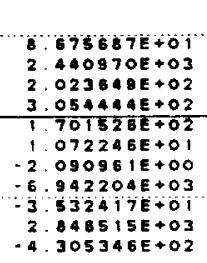 & 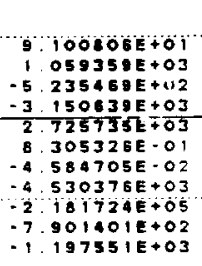 & 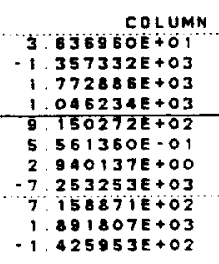 & 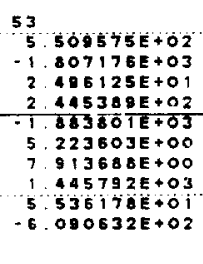 & 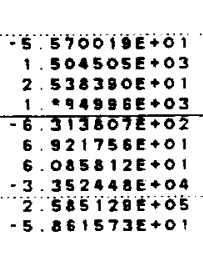 & 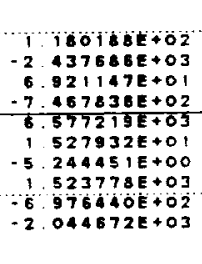 & $\begin{array}{l}6 \\
12 \\
18 \\
24 \\
30 \\
36 \\
42 \\
48 \\
54 \\
60 \\
63\end{array}$ \\
\hline $\begin{array}{l}1 \\
7 \\
13 \\
13 \\
25 \\
31 \\
37 \\
43 \\
49 \\
55 \\
51\end{array}$ & 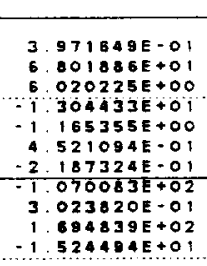 & 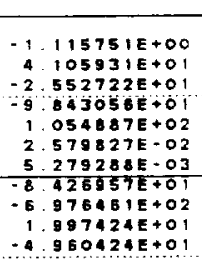 & 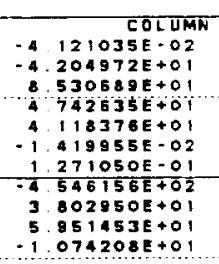 & $\begin{array}{r}54 \\
7.341706 E+00 \\
-813681 E+0 \\
1.337458 E+00 \\
5751139 E+00 \\
7.942198 E+01 \\
3.040732 E-01 \\
-4.364223 E-02 \\
-2.028766 E+01 \\
8.678871 E-01 \\
-8.800877 E+01\end{array}$ & 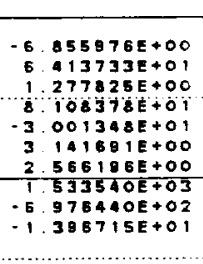 & 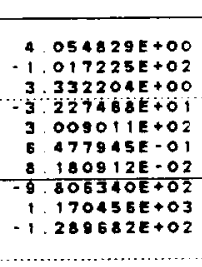 & $\begin{array}{l}6 \\
12 \\
18 \\
24 \\
30 \\
36 \\
42 \\
48 \\
54 \\
60 \\
63\end{array}$ \\
\hline $\begin{array}{r}1 \\
7 \\
13 \\
19 \\
25 \\
31 \\
37 \\
43 \\
49 \\
55\end{array}$ & 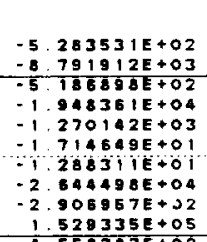 & 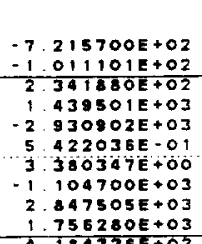 & 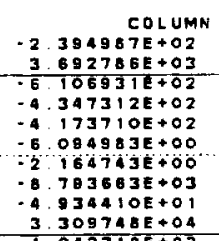 & 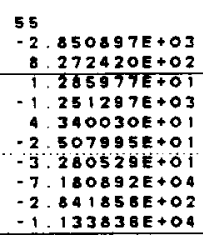 & 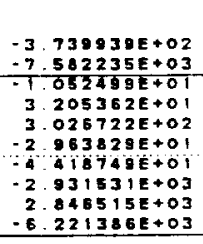 & 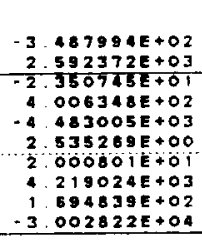 & $\begin{array}{l}6 \\
12 \\
18 \\
28 \\
30 \\
36 \\
62 \\
48 \\
54 \\
60 \\
\end{array}$ \\
\hline
\end{tabular}

AH- IG THREE-DIMENSIONAL BUILTUP DYNAMICS MODEL W/ CONTROLS MOQELEO

OCTOBER 26 , 1990 MSE/MASTRAN 10/15/87 PAGE GI

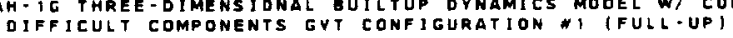

THIS VERSION CONTAINS A BUILTUP TAILBOOM MODEL

intermediate MATRIX ... KaA

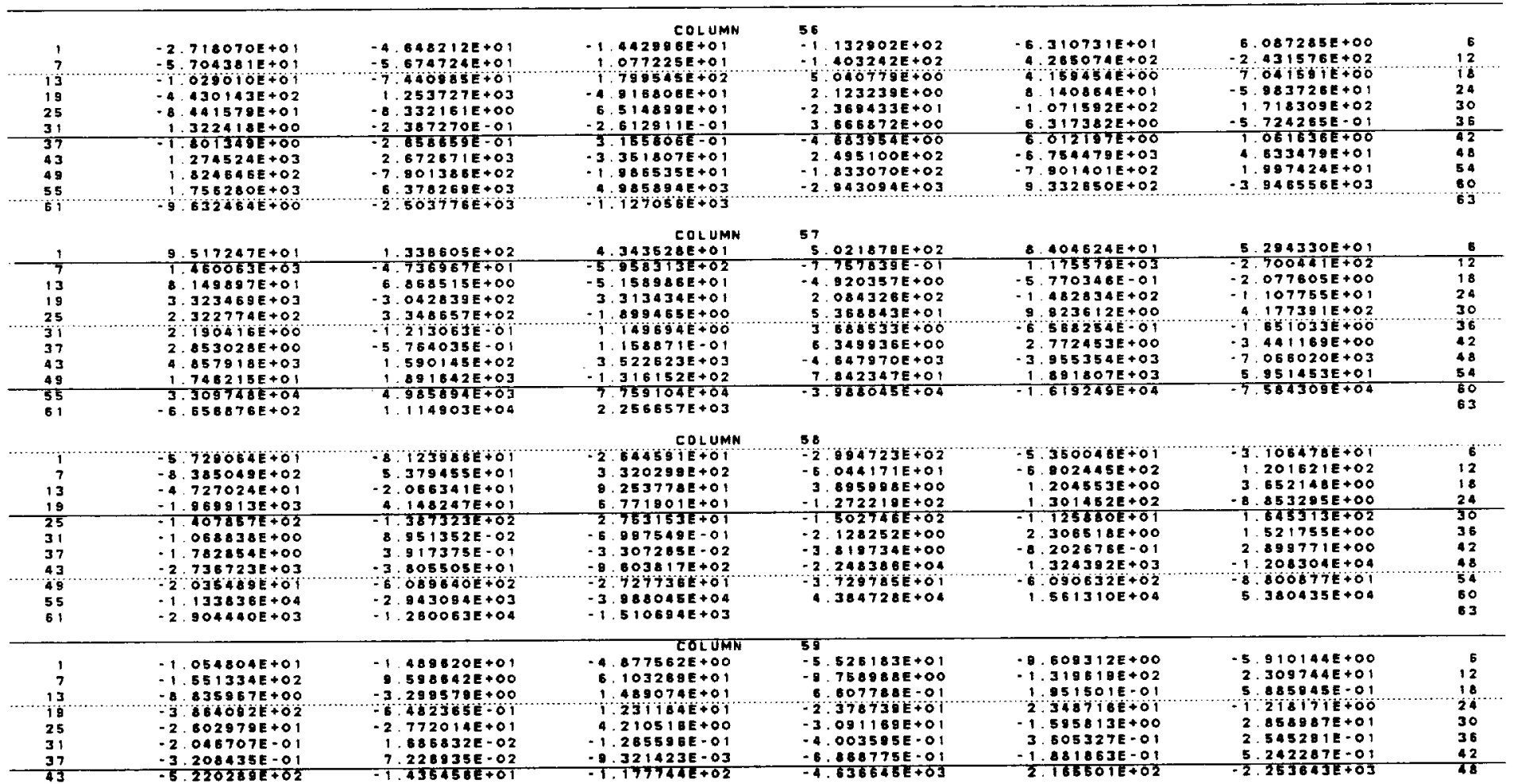


AH - IC TMREE-DIMENSIONAL BUILTUP DYMAMICS MODELW/ CONTROLS MOOELED DIPFICULT COMPONENTS GVT CONFIGURATION 1 IFULL-UPI

THIS VERSION CONTAINS A BUILTUP TAILBOOM MOOEL

INTERMEDIATE MATRIX ... KRA

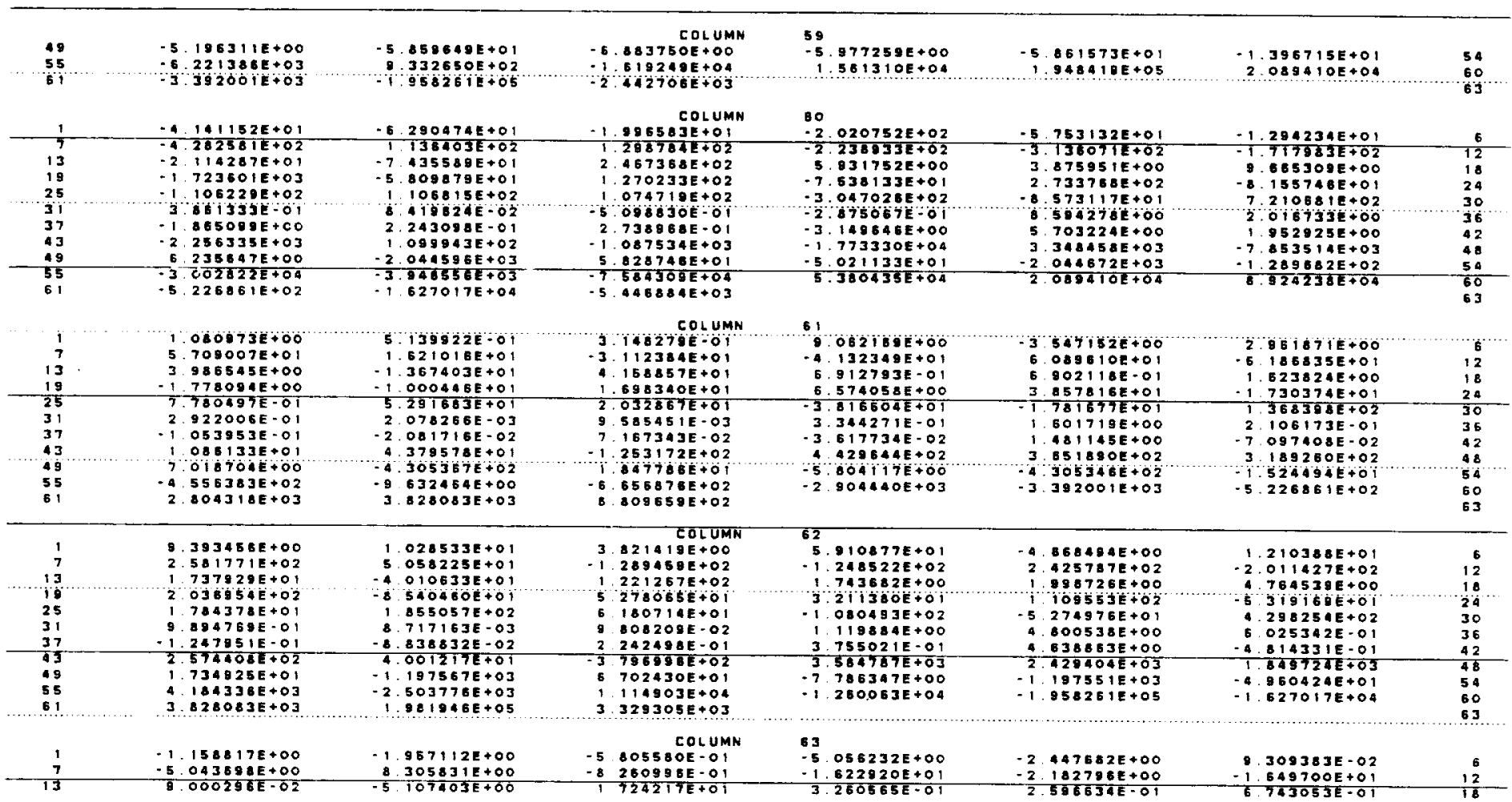

AH-IG THREE-DIMENSIONAL BUILTUP OYNAMICS MODEL W/ CONTROLS MOdELED

OCTOBER 2E, 1990 MSC/MASTRAM 10/15/87 PAGE 43

THIS VERSION CONTAINS A BUILTUP TAILOOOM MODEL

INTERMEOIATE MATRIX ... KAA

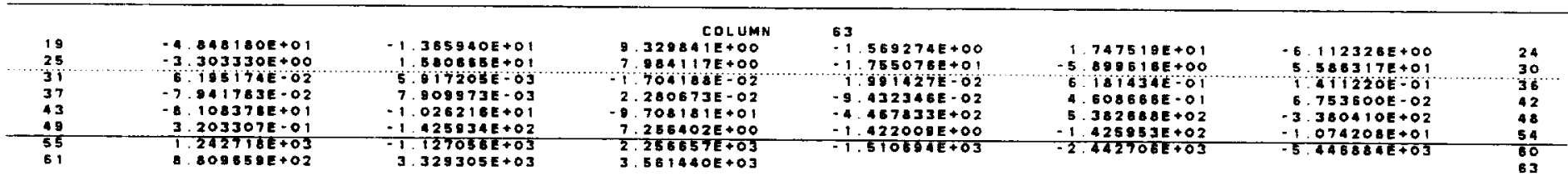


INTERMEDIATE MATRIX ... BaA

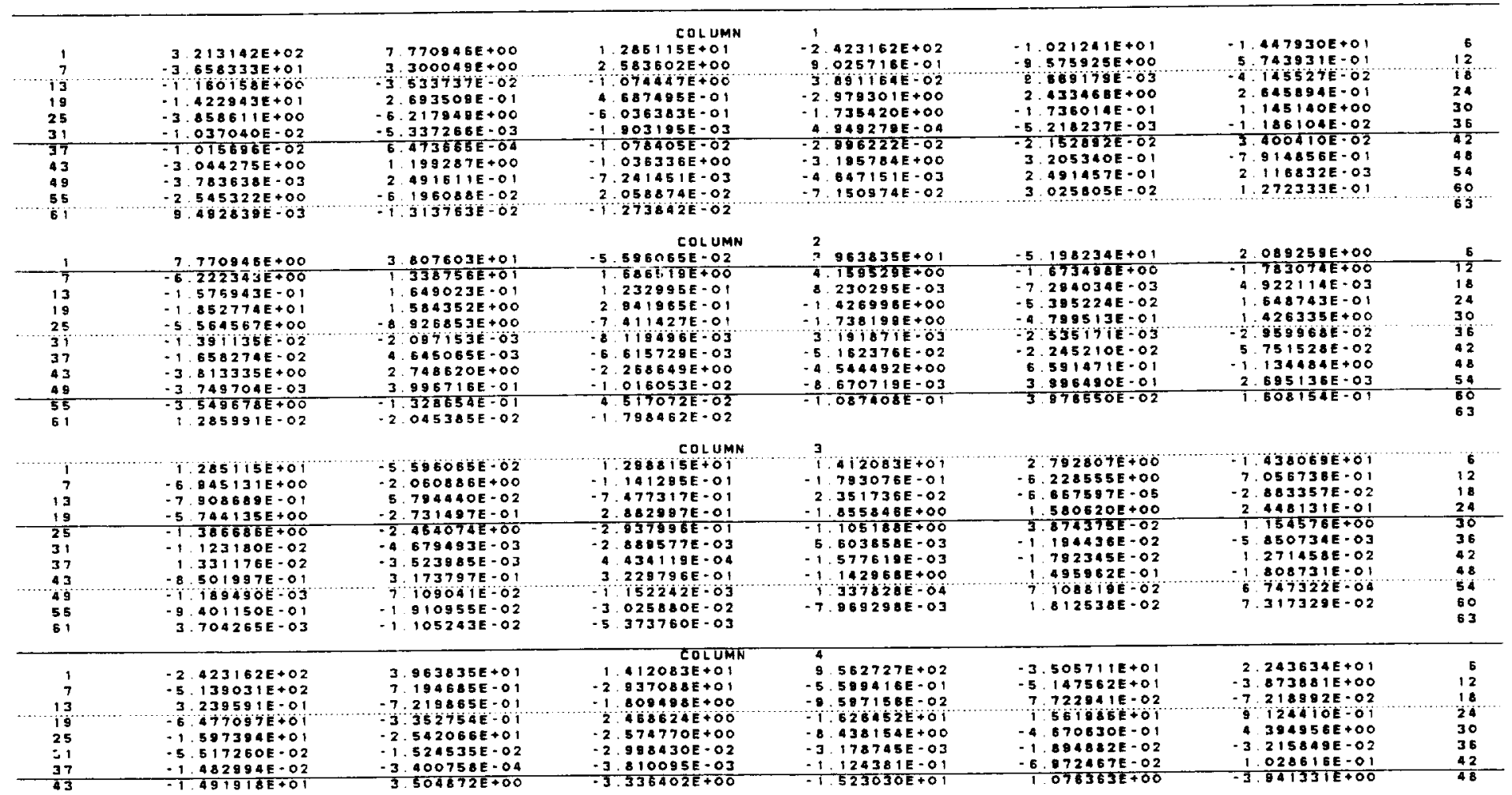

AH-IG THREE-DIMENSIONAL BUILTUP DYNAMICS MODEL W/ CONTROLS MODELEO

OCTOBER 26, 1990 MSC/NASTRAN 10/15/87 PAGE

45 DIFFICULY COMPONENTS GVT CONFIGURATION * 1 IFULL-UP

THIS VERSION CONTAINS A BUILTUP TAILBOOM MODEL

INTERMEOIATE MATRIX ... BAa

\begin{tabular}{|c|c|c|c|c|c|c|c|}
\hline $\begin{array}{l}49 \\
55 \\
51\end{array}$ & $\begin{array}{r}-1.820228 E-02 \\
-1.270241 E+01 \\
4.741528 E-02\end{array}$ & $\begin{array}{r}1.0050148+00 \\
-1.888048 E-01 \\
-8.175835 E-02\end{array}$ & $\begin{array}{c}\text { COLUMM } \\
-3.282023 E-02 \\
-7.979568 E-02 \\
-6.6161616 E-02\end{array}$ & $\begin{array}{r}4 \\
-1.317845 E \cdot 02 \\
-2.801419 E \cdot 01\end{array}$ & $\begin{array}{r}1.005954 E+00 \\
1.819300 E-01\end{array}$ & $\begin{array}{r}780299 E-03 \\
710003 E-01\end{array}$ & $\begin{array}{l}54 \\
60 \\
63\end{array}$ \\
\hline $\begin{array}{l}1 \\
77 \\
13 \\
19 \\
25 \\
31 \\
37 \\
43 \\
49 \\
55 \\
61\end{array}$ & $\begin{array}{r}-1.021241 E+01 \\
3.067575 E+01 \\
8.336909 E-01 \\
1.276721 E+00 \\
-1.250392 E-01 \\
5.001088 E-03 \\
6.0190611 E-02 \\
1.727234 E+00 \\
2.076321 E-03 \\
-971580 E-01 \\
\text { B. } 153483 E-03\end{array}$ & 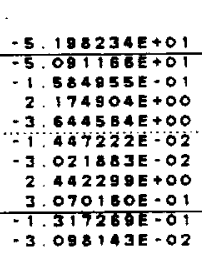 & 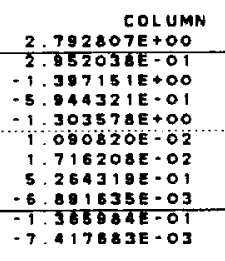 & 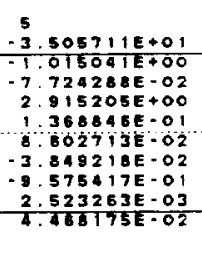 & 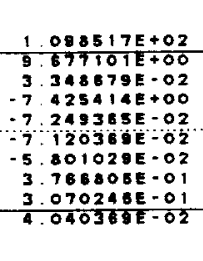 & 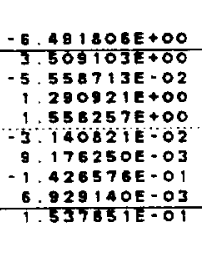 & $\begin{array}{l}8 \\
12 \\
18 \\
24 \\
30 \\
36 \\
42 \\
48 \\
54 \\
60 \\
63\end{array}$ \\
\hline $\begin{array}{l}71 \\
7 \\
13 \\
19 \\
25 \\
31 \\
37 \\
43 \\
48 \\
55 \\
61\end{array}$ & 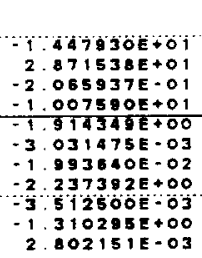 & 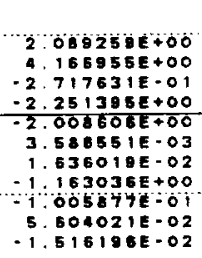 & 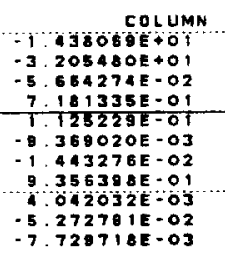 & 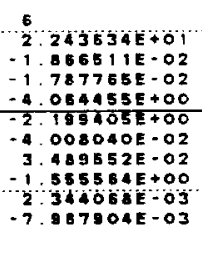 & 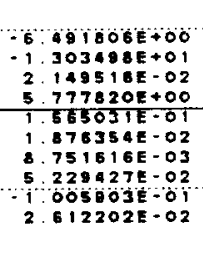 & 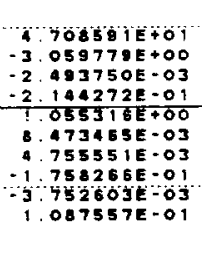 & $\begin{array}{l}16 \\
12 \\
18 \\
24 \\
30 \\
36 \\
42 \\
48 \\
54 \\
60 \\
63\end{array}$ \\
\hline $\begin{array}{r}1 \\
7 \\
13 \\
118 \\
25 \\
31 \\
37 \\
43 \\
49 \\
55 \\
61\end{array}$ & 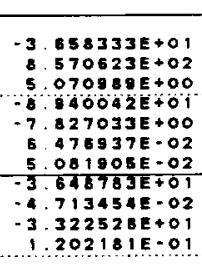 & 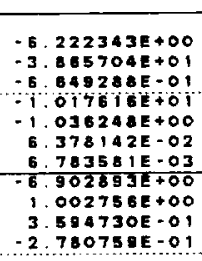 & 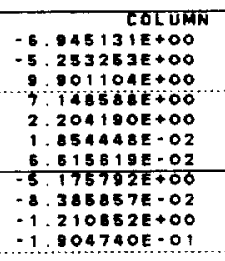 & 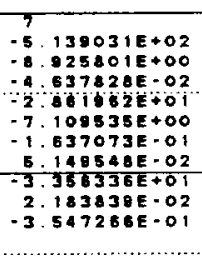 & 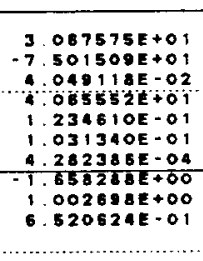 & 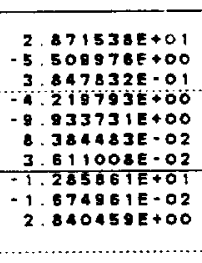 & $\begin{array}{l}6 \\
12 \\
18 \\
24 \\
30 \\
38 \\
42 \\
48 \\
54 \\
50 \\
63\end{array}$ \\
\hline $\begin{array}{r}1 \\
7 \\
-13\end{array}$ & 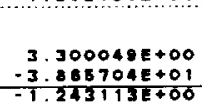 & $\begin{array}{r}.338758 E+01 \\
8.311592 E+01 \\
.31292 E-0.8\end{array}$ & $\begin{array}{r}\text { COLUMN } \\
-2.080886+00 \\
-3.915147 E+00 \\
-192179 E+00\end{array}$ & $\begin{array}{r}8.194885 E-01 \\
-2.1941711+00 \\
-2.083141 E-02\end{array}$ & $\begin{array}{r}-5.021188 E+01 \\
1.242198 E+01 \\
1.833250 E=02\end{array}$ & $\begin{array}{r}1.165985 E+00 \\
8.223697 E+00 \\
-2.182222 E-01\end{array}$ & $\frac{5}{12}$ \\
\hline
\end{tabular}

ORICINAL B... Or prope ?hing 


\begin{tabular}{|c|c|c|c|c|c|c|c|}
\hline $\begin{array}{l}19 \\
25 \\
31 \\
37 \\
43 \\
49 \\
55 \\
51\end{array}$ & $\begin{array}{r}6.731810 E+00 \\
-1.1898175+00 \\
-3.0187778 .02 \\
4.268558 E-02 \\
7.698642 E+00 \\
1.476360 E-03 \\
1.031241 E E+00 \\
-2.539111 E-03\end{array}$ & 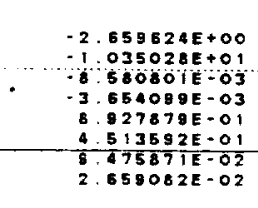 & $\begin{array}{r}\text { COL UMN } \\
-105293 E+00 \\
-3.029437 E+00 \\
3.798759 E=03 \\
-1.415621 E-02 \\
3.284398 E+00 \\
-5.987475 E=03 \\
-2.698395 E-01 \\
3.2619318-03\end{array}$ & $\begin{array}{l}8 \\
5.637648 E+00 \\
3.323114 E-01 \\
1.325206 E=02 \\
3.337208 E-02 \\
2.995840+00 \\
1.508532 E-02 \\
1.804994 E-01\end{array}$ & 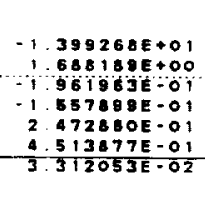 & 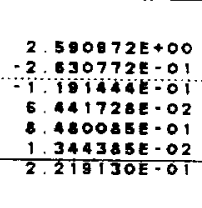 & $\begin{array}{l}24 \\
30 \\
36 \\
42 \\
48 \\
54 \\
60 \\
63\end{array}$ \\
\hline $\begin{array}{l}1 \\
7 \\
13 \\
19 \\
25 \\
31 \\
37 \\
43 \\
49 \\
55 \\
6\end{array}$ & $\begin{array}{r}2.533502 E+00 \\
-5.253253 E+00 \\
4.173739 E+00 \\
6.596208 E-01 \\
-4.40897 E-01 \\
-3.759279 E-02 \\
-4.220170 E-02 \\
7.239938 E+00 \\
6281672 E-03 \\
1.167670 E+01 \\
-4039803 E-02\end{array}$ & 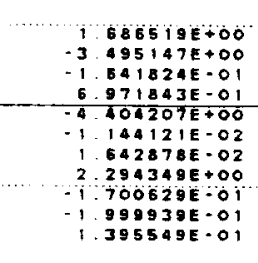 & 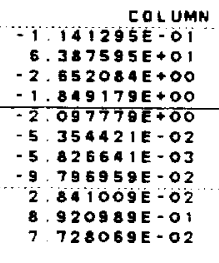 & $\begin{array}{r}9 \\
-2.937088 E+01 \\
-3.692458 E+00 \\
-1.847075 E-01 \\
8.871453 E-02 \\
-3.6711968+00 \\
2.457048 E-02 \\
-7.231030 E-02 \\
9.584881 E+00 \\
-2.377817 E=02 \\
-2.491373 E-02\end{array}$ & 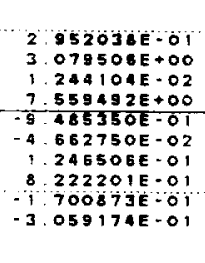 & 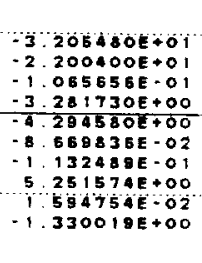 & $\begin{array}{l}6 \\
12 \\
18 \\
24 \\
-30 \\
36 \\
42 \\
48 \\
54 \\
60 \\
63\end{array}$ \\
\hline $\begin{array}{l}1 \\
7 \\
13 \\
119 \\
25 \\
31 \\
37 \\
43 \\
49 \\
55 \\
61\end{array}$ & 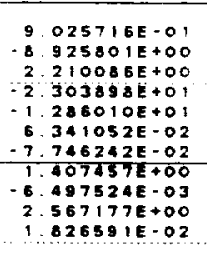 & 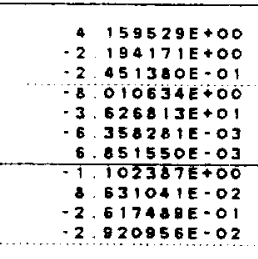 & 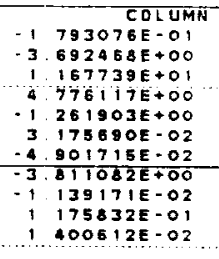 & 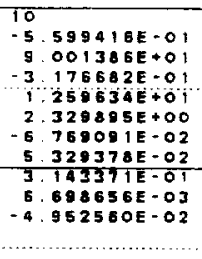 & 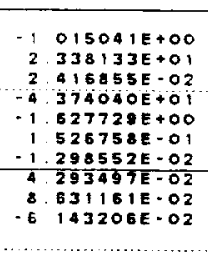 & $\begin{array}{r}-186511 E-02 \\
-1.227739 E+01 \\
4525911 E-01 \\
2.736474 E+00 \\
1.18819 E+00 \\
7314106 E-02 \\
1.360409 E-01 \\
4.505465 E-01 \\
1.465159 E-02 \\
-3.674597-01\end{array}$ & $\begin{array}{l}6 \\
12 \\
18 \\
24 \\
30 \\
36 \\
42 \\
48 \\
54 \\
60 \\
63\end{array}$ \\
\hline $\begin{array}{l}1 \\
7 \\
13 \\
19 \\
25 \\
31 \\
37 \\
43 \\
49 \\
55 \\
61\end{array}$ & 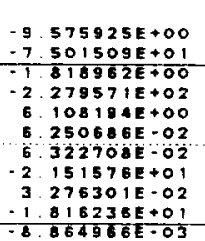 & 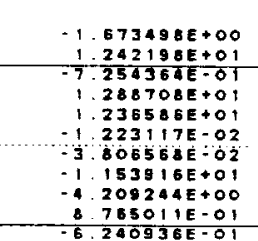 & 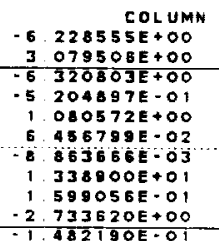 & $\begin{array}{r}1 \\
-5.147562 E+01 \\
2.338133 E+01 \\
22.440844 E-01 \\
3.189940 E+01 \\
1.363734 E+01 \\
2.164748 E-01 \\
6.915087 E-02 \\
1.480716 E+01 \\
1.610148 E-02 \\
9.102783 E-01 \\
\end{array}$ & 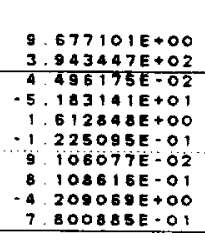 & 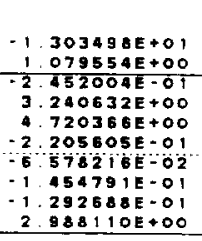 & $\begin{array}{r}6 \\
12 \\
18 \\
24 \\
30 \\
36 \\
42 \\
48 \\
54 \\
50\end{array}$ \\
\hline
\end{tabular}

\footnotetext{
AH- IC THREE-DIMENS IONAL GUILTUP DYNAMITS MOOEL W/ CONTROLS MOOELED

DETOBER 26. 1990 MSC/NASTRAN 10/15/87 PAGE 47

THIS VERSION CONTAINS A BUILTUP TAILBOOM MODEL
}

NTERMEDIATE MATRIX ... BAA

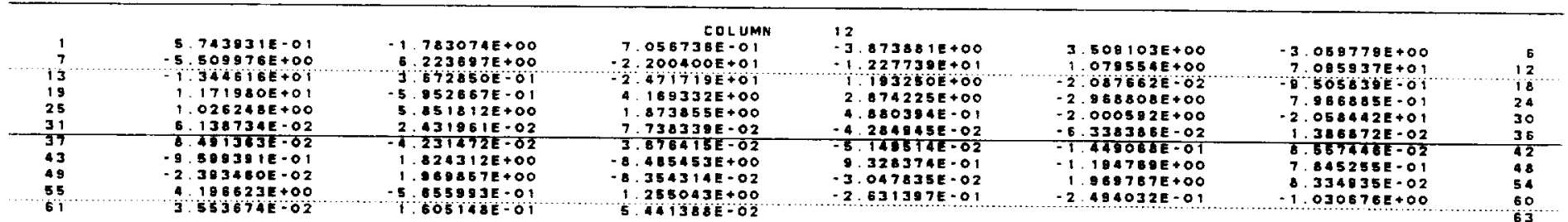

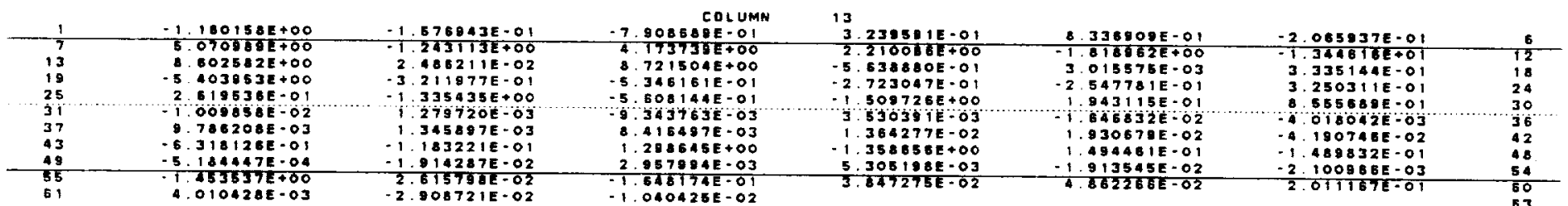

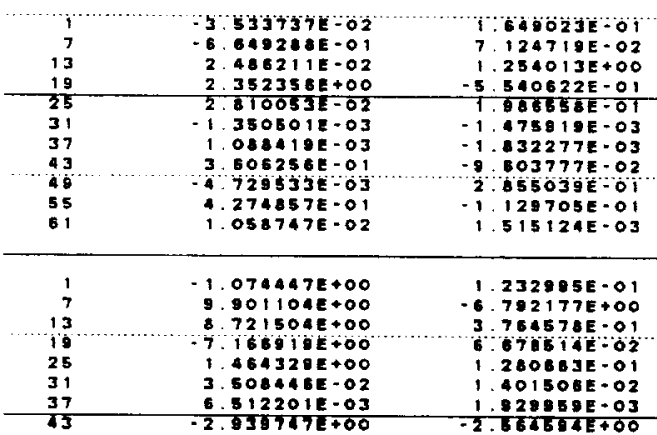

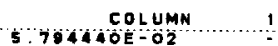

3.641824E- 76

1. $148809 E-02$

$3.238213 E-03$

3.238213E-OJ

$3.124001 E-01$

1. $150867 \mathrm{TH}-02$

$7.358149 E-02$
$4.354105 E-03$

2 TBBGEE 61 .

$2.451380 E-01 \quad-7.254384 E-01$

$5.163460 E-03 \quad-9.855605 E-02$

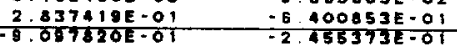

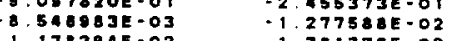

1. $178294 E-02$

$-1.27758 \Delta E-02$
$-1,381336 E-02$

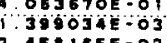

$-5.078458 E-02$
2.0548610 .01

2. $58185 E-02 \quad-9.964820 E-02$

\begin{tabular}{|c|c|c|}
\hline & TESTE-OI & 6 \\
\hline & $672850 E-01$ & 12 \\
\hline & $524124 E-02$ & 18 \\
\hline & $526318 \mathrm{E}-02$ & 24 \\
\hline & $\begin{array}{l}\text { 630348E-Ot } \\
432311 E-03\end{array}$ & $\begin{array}{l}30 \\
36\end{array}$ \\
\hline & $887742 E-03$ & 42 \\
\hline$\ldots$ & $548936 E-01$ & 48 \\
\hline & $\begin{array}{l}327410 E-02 \\
012630 E-01\end{array}$ & 54 \\
\hline
\end{tabular}

C. COLUMN

$15.009498 E+00 \quad-1.397151 E+00$

$7.477317 E-01$
$-2.652004 E+00$

$1673306+0$

3
$320003 E * 00$

$7.805847 E-01 \quad-3.784001=-02$

3. $164372 E+01$
$3.236826 E+00$

3. $571599-01$

$4.036174 E-03$
1.732785 .02
$1.03060-01$

$321313 E-01$
9.22435

$214919 E-02$

$0.637193-02$
$2.60717 E-02$

\begin{tabular}{|c|c|}
\hline $\begin{array}{r}-5.854274 E-02 \\
-2.471719 E+01 \\
1.226782 E+00 \\
7.300860000 \\
-8.734532 E+00 \\
1.254114 E-00 \\
-3.018885-02 \\
\end{array}$ & $\begin{array}{r}6 \\
12 \\
18 \\
28 \\
30 \\
30 \\
36 \\
22 \\
\end{array}$ \\
\hline
\end{tabular}


AH- IE THREE-DIMENSIONAL BUILTUP DYNAMICS MODEL W/ CONTROLS MODELED

THIS VERSION CONTAINS A BUILTUP TAILBOOM MODEL

NTERMEDIATE MATRIX ... GAR

\begin{tabular}{|c|c|c|c|c|c|c|c|}
\hline $\begin{array}{l}49 \\
55 \\
61\end{array}$ & $\begin{array}{r}-1.375102 E-03 \\
-2.075598 E+00 \\
-1.94162 E-02\end{array}$ & $\begin{array}{r}-3.847250 E-01 \\
4.43716 E-01 \\
4.63897 E=02\end{array}$ & $\begin{array}{r}\text { COLUMN } \\
1.019834 E-02 \\
-11253293 E-02 \\
\hdashline 8.581677 E-03\end{array}$ & $\begin{array}{l}15 \\
-1.072567 E-03 \\
6449825-02 \\
2.02\end{array}$ & $\begin{array}{r}-3.847297 E-01 \\
4.523450 E-02 \\
4\end{array}$ & $\begin{array}{r}-2.015878 E-02 \\
3.208353 E-01 \\
Y\end{array}$ & $\begin{array}{l}54 \\
60 \\
63\end{array}$ \\
\hline $\begin{array}{r}1 \\
7 \\
13 \\
19 \\
25 \\
31 \\
37 \\
43 \\
49 \\
55 \\
61\end{array}$ & 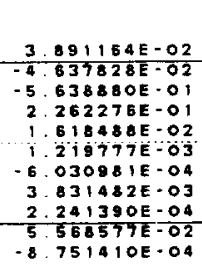 & 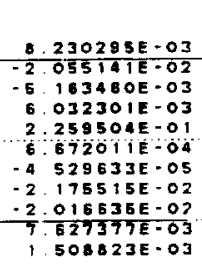 & 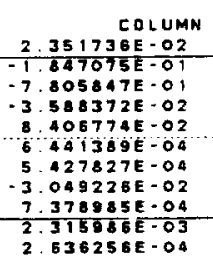 & 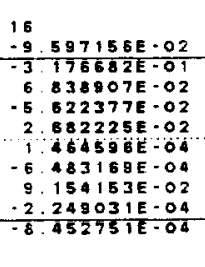 & 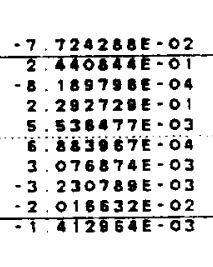 & 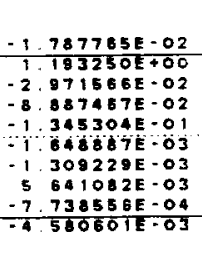 & $\begin{array}{l}6 \\
12 \\
18 \\
24 \\
30 \\
36 \\
42 \\
48 \\
54 \\
60 \\
63\end{array}$ \\
\hline $\begin{array}{r}1 \\
7 \\
13 \\
19 \\
25 \\
31 \\
37 \\
43 \\
49 \\
55 \\
61\end{array}$ & 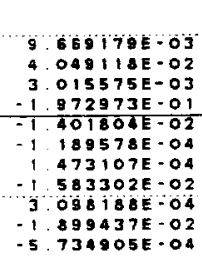 & 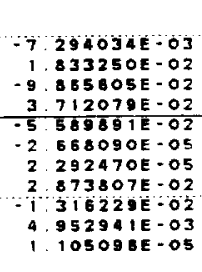 & 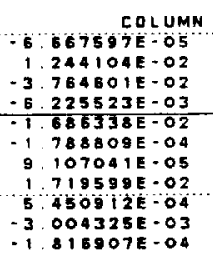 & 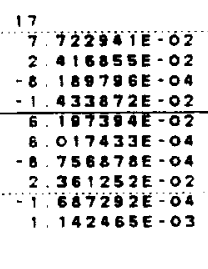 & 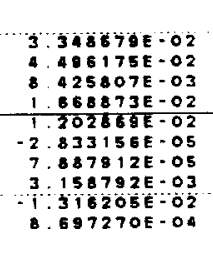 & 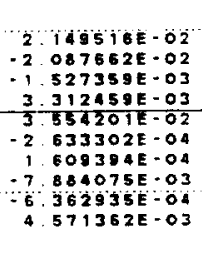 & $\begin{array}{l}6 \\
12 \\
18 \\
24 \\
30 \\
36 \\
42 \\
18 \\
184 \\
60 \\
63\end{array}$ \\
\hline $\begin{array}{r}1 \\
7 \\
13 \\
13 \\
25 \\
31 \\
37 \\
43 \\
49\end{array}$ & 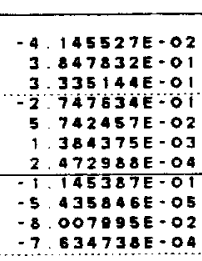 & 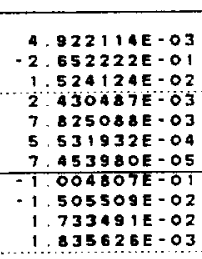 & 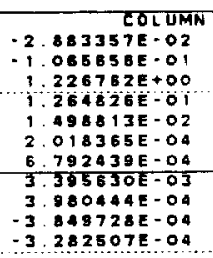 & 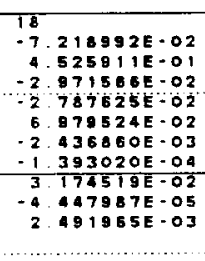 & 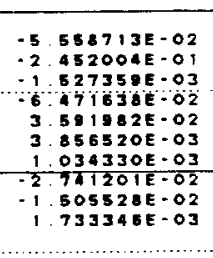 & 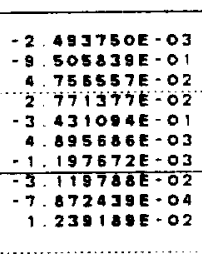 & $\begin{array}{r}5 \\
12 \\
18 \\
24 \\
30 \\
38 \\
62 \\
48 \\
54 \\
60 \\
63\end{array}$ \\
\hline$\frac{t}{13}$ & $\begin{array}{l}-1.422943 E+0, \\
-8.940062 E+0 \\
-5.403953 E+00\end{array}$ & $\begin{array}{r}-1.852774 E+01 \\
6.731810 E+00 \\
2.35235 E+00\end{array}$ & $\begin{array}{r}\text { COLLMM } \\
-5.744135 E+00 \\
6.595208 E-01 \\
-7.186919 E+00\end{array}$ & $\begin{array}{l}19 \\
-6477097 E+01 \\
-2.303898 E+01 \\
2.262296 E-01\end{array}$ & $\begin{array}{r}1.276721 E+00 \\
-2.27851 E++02 \\
-1.992973 E=01\end{array}$ & $\begin{array}{r}1.007590 E+01 \\
1.171980 E+01 \\
-274634 E-01\end{array}$ & $\begin{array}{r}6 \\
-12 \\
18\end{array}$ \\
\hline
\end{tabular}

AH-IC THREE-DIMENSIONAL BUILTUP OYNAMICS MODEL W/ CONTROLS MOOELED
OIFFICULT COMPONEMTS GVT CONFIGURATION I (FULL-UP)

OCTOBER 26, 1990 MSE/NASTRAN 10/15/87 PAGE 49

THIS VERSION CONTAINS A BUILTUP TAILBOOM MODEL

intermediate matrix ... BaA

\begin{tabular}{|c|c|c|c|c|c|c|c|c|}
\hline $\begin{array}{l}18 \\
25 \\
31 \\
37 \\
43 \\
49 \\
55 \\
61\end{array}$ & 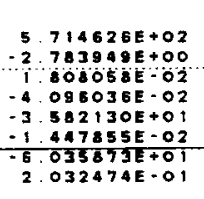 & $\begin{array}{r}7 \\
4 \\
-3 \\
3 \\
3 \\
5 \\
-1 \\
-1\end{array}$ & $\begin{array}{l}22241 B E+00 \\
830006 E+00 \\
180269 E-02 \\
704575 E-03 \\
434892 E+00 \\
514000 E-01 \\
230344 E-02 \\
513140 E+00\end{array}$ & $\begin{array}{l}\text { COLUMN } \\
-1.030129 E+01 \\
6.967461 E-01 \\
-4212110.02 \\
-3.519723 E-02 \\
4.925230 E+00 \\
5.893489 E-02 \\
-4.64960 E+00 \\
-3.380885 E-01\end{array}$ & $\begin{array}{l}19 \\
-3.733998 E+00 \\
-1.80076 E+01 \\
7.10235 E-O 2 \\
4.462506 E-02 \\
-5.2770876+01 \\
1.759858-01 \\
3.1981333+00\end{array}$ & 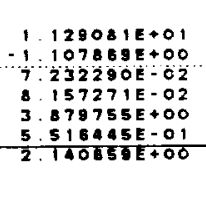 & 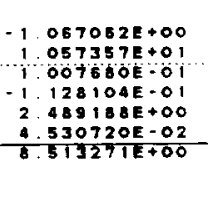 & $\begin{array}{l}24 \\
30 \\
36 \\
42 \\
48 \\
54 \\
60 \\
63\end{array}$ \\
\hline $\begin{array}{l}1 \\
7 \\
13 \\
19 \\
25 \\
31 \\
37 \\
43 \\
49 \\
55 \\
6\end{array}$ & $\begin{array}{r}2.693509 E-O 1 \\
-1.017618 E+01 \\
-3.211977 E-01 \\
7.222116 E+00 \\
-2.744893 E+00 \\
-1.062291 E-O 3 \\
1.640248 E-O 3 \\
-1.212770 E+01 \\
-1.046230 E-O 4 \\
2.612601 E+0 O \\
-2.653557 E-O 2\end{array}$ & $\begin{array}{r}1 \\
-2 \\
-5 \\
5 \\
-1 \\
-1 \\
-1 \\
-8 \\
-2 \\
1 \\
1 \\
6\end{array}$ & $\begin{array}{l}584352 E+0 O \\
65924 E+00 \\
540522 E-01 \\
780072 E+01 \\
280998 E+01 \\
249322 E-02 \\
371168 E-02 \\
784545 E+00 \\
248272 E+00 \\
537338 E+00 \\
505752 E-02\end{array}$ & 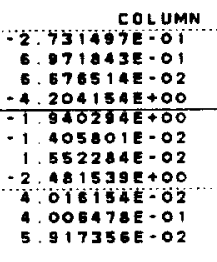 & 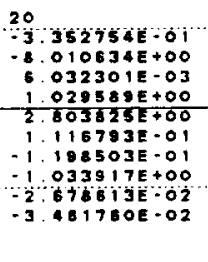 & 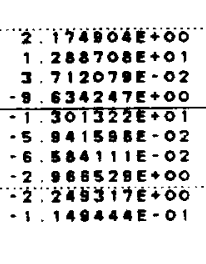 & 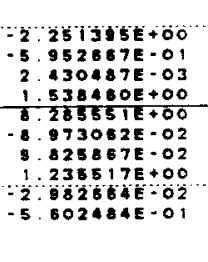 & $\begin{array}{l}15 \\
12 \\
18 \\
24 \\
30 \\
36 \\
42 \\
48 \\
54 \\
50 \\
63\end{array}$ \\
\hline $\begin{array}{l}1 \\
7 \\
13 \\
13 \\
25 \\
31 \\
37 \\
43 \\
49 \\
55 \\
61\end{array}$ & $\begin{array}{r}4.687495 E-01 \\
7.148588 E+00 \\
-5.348181 E-01 \\
-1.030129 E+01 \\
-8973210 E-01 \\
1.863924 E-02 \\
4.187038 E-03 \\
3.875854 E+00 \\
4.204408 E-03 \\
-5.245728 E-01 \\
-1.840175 E-02\end{array}$ & $\begin{array}{r}2 \\
-1 \\
-1 \\
-1 \\
-4 \\
1 \\
-1 \\
8 \\
9 \\
-5 \\
3 \\
-3\end{array}$ & $\begin{array}{l}941985 E-01 \\
105193 E+00 \\
146809 E-02 \\
264154 E+00 \\
739549 E+00 \\
1175575-02 \\
294661 E-03 \\
962975 E-01 \\
939998 E-01 \\
576434 E-02 \\
899718 E-02\end{array}$ & $\begin{array}{r}\text { COLUMN } \\
2.882897 E-0, \\
-1.84979 E+00 \\
3.238828 E+00 \\
3.0318100+01 \\
1.083801 E+00 \\
-7.543745 E-03 \\
-1.227821 E-02 \\
2.570874 E+00 \\
2.146387 E-02 \\
-1.555380-02 \\
-1.454740 E-02\end{array}$ & $\begin{array}{r}21 \\
2.488624 E+00 \\
4.776117 E+00 \\
-3.588372 E-02 \\
-5.856656=01 \\
-3.608775+00 \\
-1.257118 E-03 \\
-6.529347 E-03 \\
3.122942 E+00 \\
7.785382 E-03 \\
1.233042 E-01\end{array}$ & 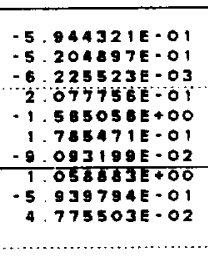 & 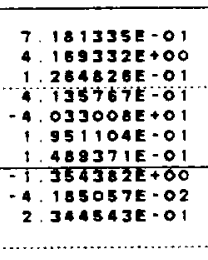 & $\begin{array}{r}6 \\
12 \\
18 \\
24 \\
30 \\
36 \\
42 \\
48 \\
54 \\
60 \\
63\end{array}$ \\
\hline $\begin{array}{l}1 \\
7 \\
13 \\
19 \\
25 \\
31 \\
37 \\
43 \\
49 \\
55 \\
5\end{array}$ & 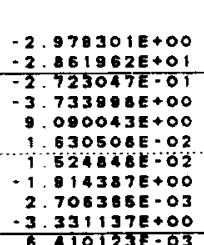 & $\begin{array}{r}-1 \\
5 \\
-3 \\
1 \\
2 \\
-7 \\
-7 \\
-6 \\
-2 \\
4 \\
\end{array}$ & 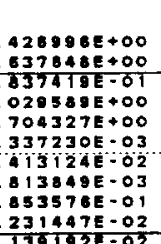 & 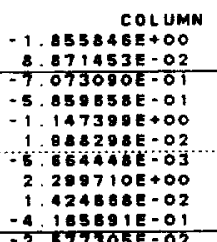 & 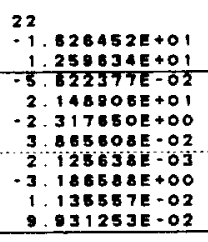 & 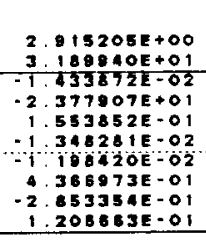 & 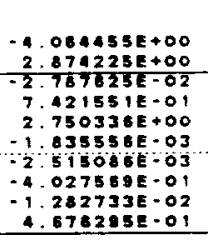 & $\begin{array}{r}6 \\
12 \\
18 \\
24 \\
30 \\
36 \\
12 \\
18 \\
54 \\
80\end{array}$ \\
\hline
\end{tabular}


INTERMEDIATE MATRIX ... BAA

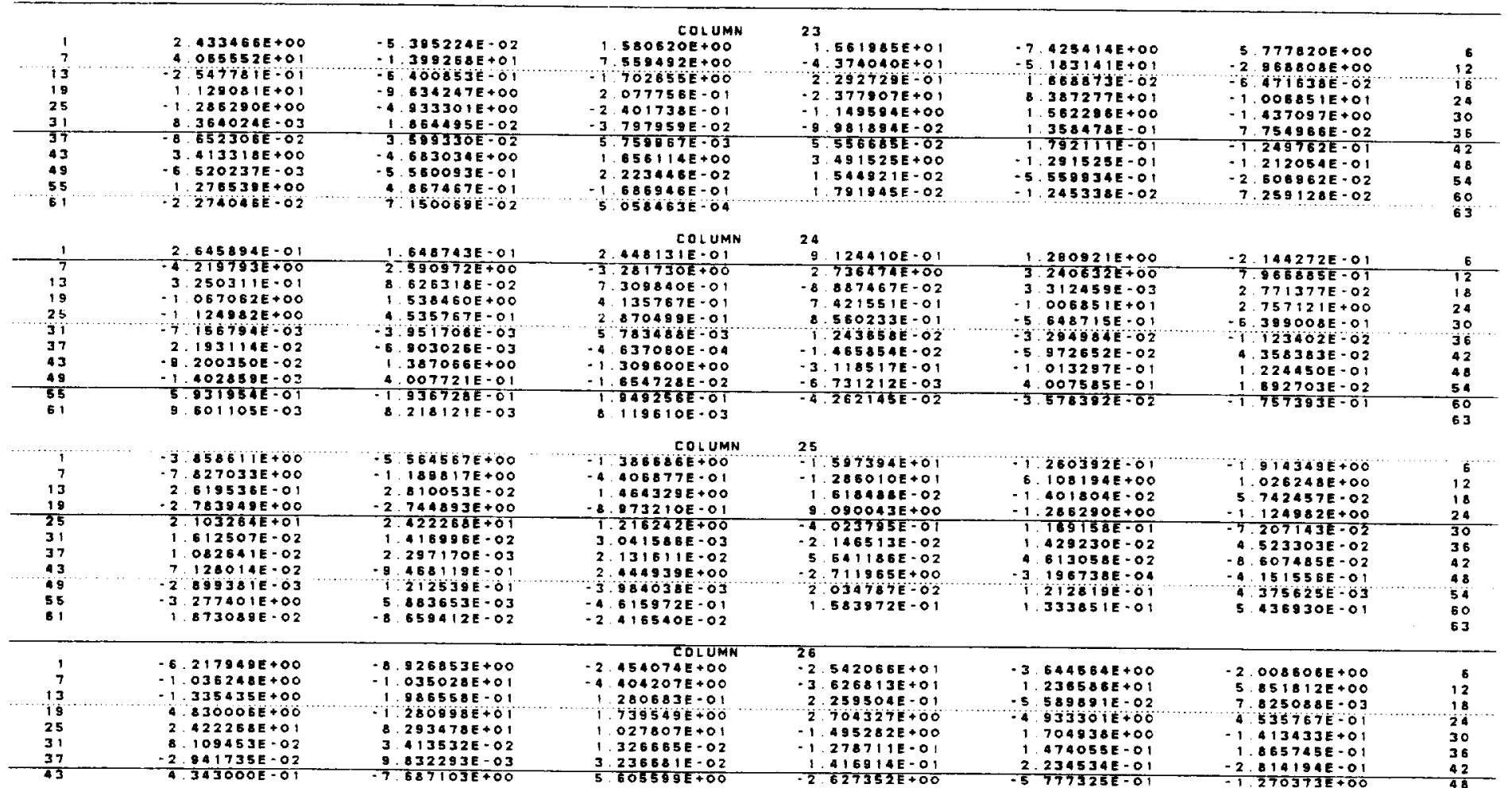

AH- IC THREE-DIMENSIONAL BUILTUP DYNAMICS MODEL W/ CONTAOLS MODELED
DIFFICULT COMPONENTS GYT COMFIGURATION

OCTOBER 26, 1990 MSC/NASTRAN 10/15/87 PAGE

5

THIS VERSION CONTAINS A BUILTUP TAILBOOM MOOEL

INTERMEDIATE MATRIX ... BAA

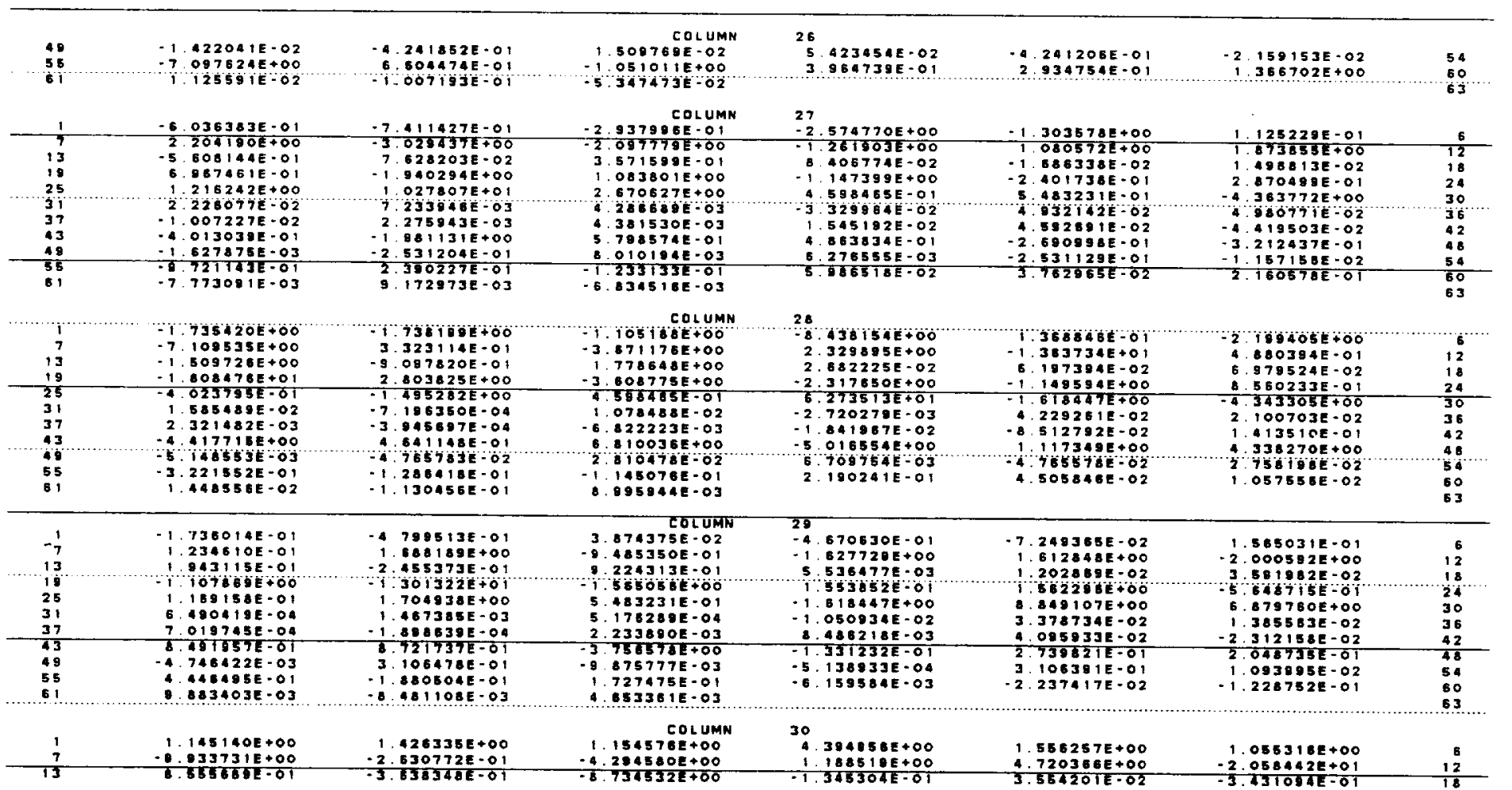




\begin{tabular}{|c|c|c|c|c|c|c|c|}
\hline $\begin{array}{l}19 \\
25 \\
31 \\
37 \\
43 \\
49 \\
55 \\
51\end{array}$ & 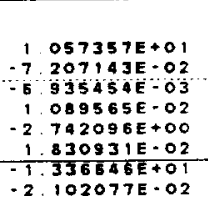 & 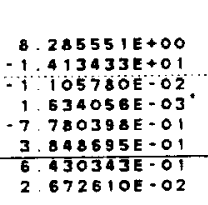 & 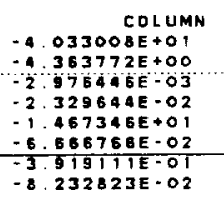 & $\begin{array}{l}30 \\
2.750338 E+00 \\
-4.343305 E+00 \\
6+73550 E-02 \\
1.228201 E-02 \\
5.590972 E+00 \\
6.538790 E-03 \\
5.241715 E-01\end{array}$ & $\begin{array}{r}-1.437097 E+00 \\
6.879760 E+00 \\
1889426 E-01 \\
8.560096 E-03 \\
-4.0645007 E+00 \\
3.849068 E-01 \\
.053843 E-01\end{array}$ & 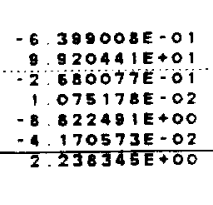 & $\begin{array}{l}24 \\
30 \\
36 \\
42 \\
48 \\
54 \\
60 \\
63\end{array}$ \\
\hline $\begin{array}{r}1 \\
7 \\
13 \\
19 \\
19 \\
31 \\
37 \\
43 \\
49 \\
55 \\
61\end{array}$ & 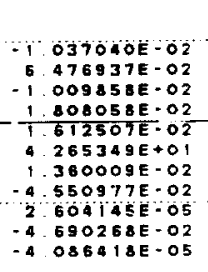 & 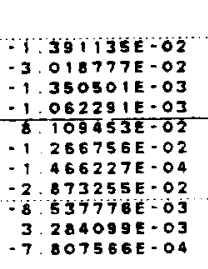 & 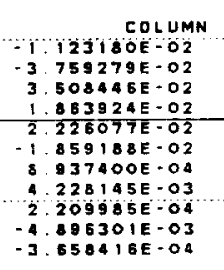 & 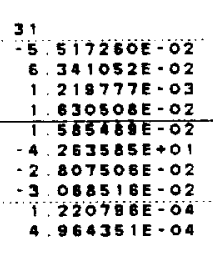 & 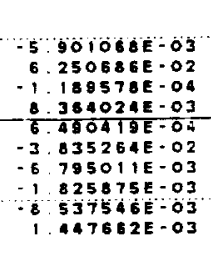 & 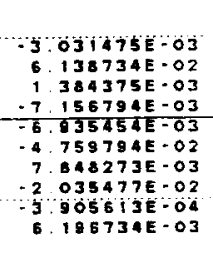 & $\begin{array}{l}6 \\
12 \\
18 \\
28 \\
30 \\
36 \\
42 \\
48 \\
54 \\
50 \\
63\end{array}$ \\
\hline $\begin{array}{r}1 \\
7 \\
13 \\
19 \\
25 \\
31 \\
37 \\
43 \\
49 \\
55 \\
61\end{array}$ & 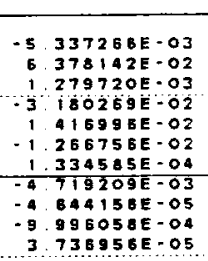 & 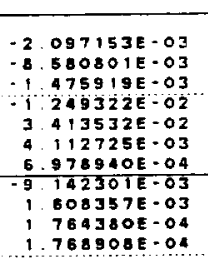 & 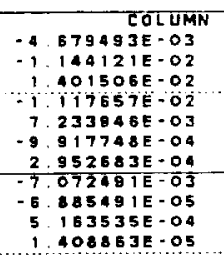 & 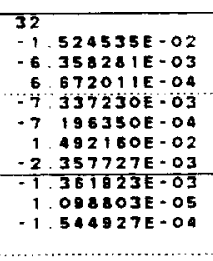 & 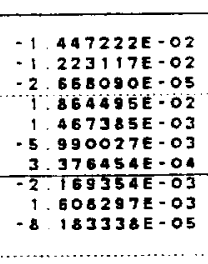 & 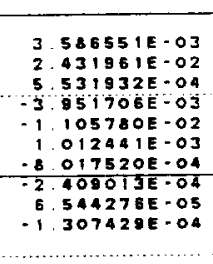 & $\begin{array}{l}6 \\
12 \\
18 \\
24 \\
30 \\
36 \\
42 \\
48 \\
54 \\
60 \\
63\end{array}$ \\
\hline $\begin{array}{r}1 \\
7 \\
13 \\
19 \\
25 \\
31 \\
37 \\
43 \\
49 \\
55\end{array}$ & 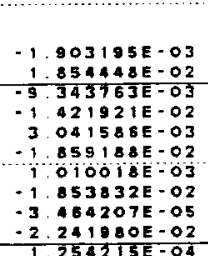 & 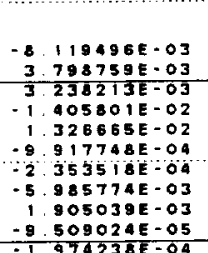 & 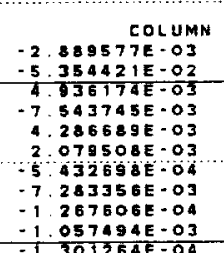 & 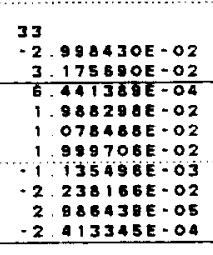 & 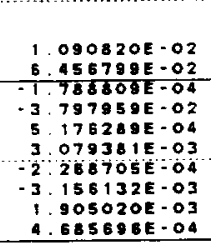 & 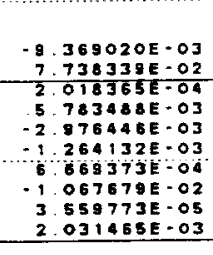 & $\begin{array}{r}6 \\
12 \\
18 \\
24 \\
30 \\
36 \\
42 \\
48 \\
54 \\
60 \\
63\end{array}$ \\
\hline
\end{tabular}

AM-IG THREE-DIMENSIONAL BUILTUP DYNAMICS MOOEL W/ CONTROLS MODELED

\begin{tabular}{|c|c|c|c|c|c|c|c|}
\hline $\begin{array}{r}1 \\
7 \\
13 \\
19 \\
25 \\
31 \\
37 \\
43 \\
49 \\
55 \\
61\end{array}$ & 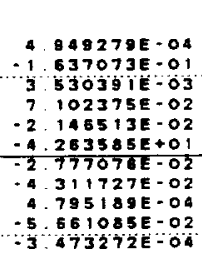 & 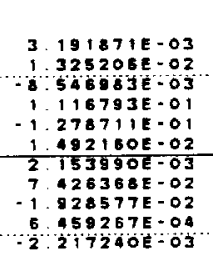 & 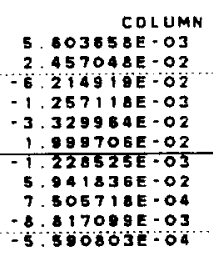 & 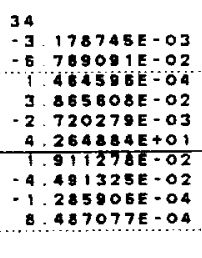 & 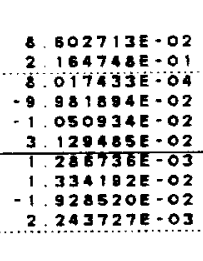 & 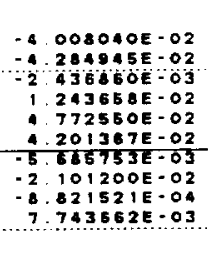 & $\begin{array}{r}6 \\
12 \\
18 \\
24 \\
30 \\
38 \\
62 \\
48 \\
54 \\
60 \\
63\end{array}$ \\
\hline $\begin{array}{l}1 \\
77 \\
13 \\
19 \\
25 \\
31 \\
37 \\
43 \\
49 \\
55 \\
81\end{array}$ & 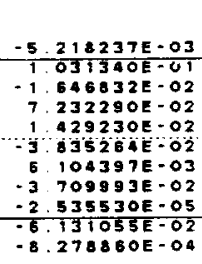 & 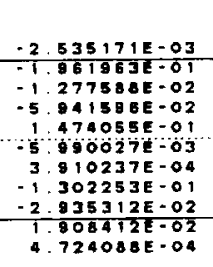 & 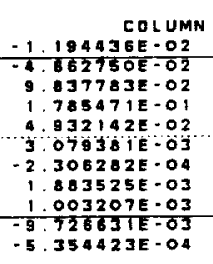 & 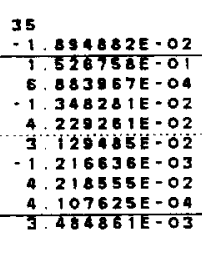 & 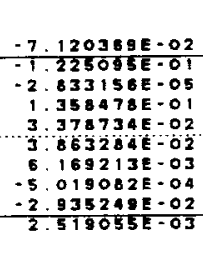 & 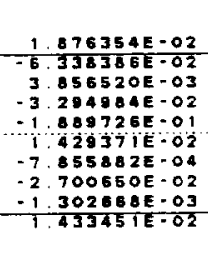 & $\begin{array}{l}6 \\
12 \\
18 \\
24 \\
30 \\
36 \\
42 \\
48 \\
54 \\
60 \\
63\end{array}$ \\
\hline $\begin{array}{r}1 \\
7 \\
13 \\
19 \\
25 \\
31 \\
37 \\
43 \\
48 \\
55 \\
61\end{array}$ & 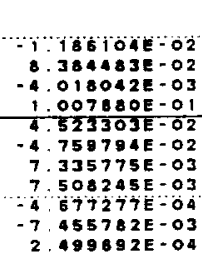 & 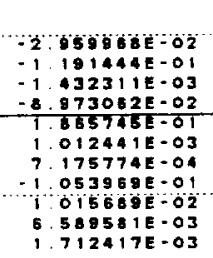 & 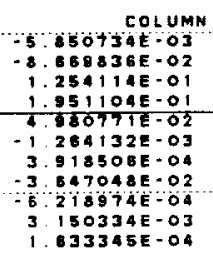 & 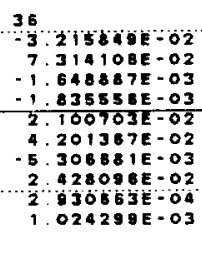 & 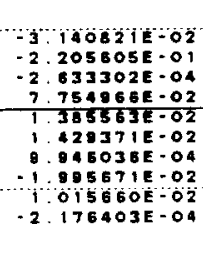 & 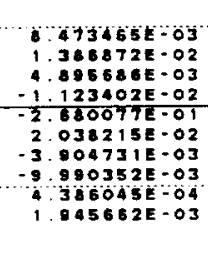 & $\begin{array}{l}6 \\
12 \\
18 \\
18 \\
24 \\
30 \\
35 \\
42 \\
48 \\
56 \\
60 \\
63\end{array}$ \\
\hline $\begin{array}{r}7 \\
7 \\
13 \\
16 \\
25 \\
31 \\
37 \\
43\end{array}$ & 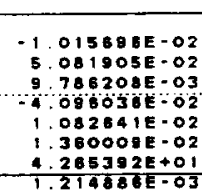 & 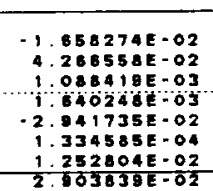 & 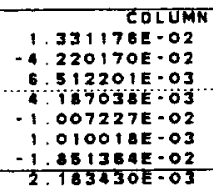 & 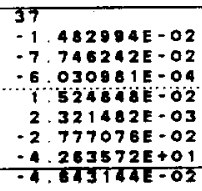 & 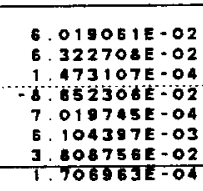 & 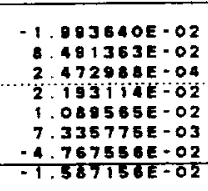 & $\begin{array}{r}6 \\
12 \\
18 \\
214 \\
30 \\
36 \\
12 \\
18\end{array}$ \\
\hline
\end{tabular}


INTERMEDIATE MATRIX .. BAA

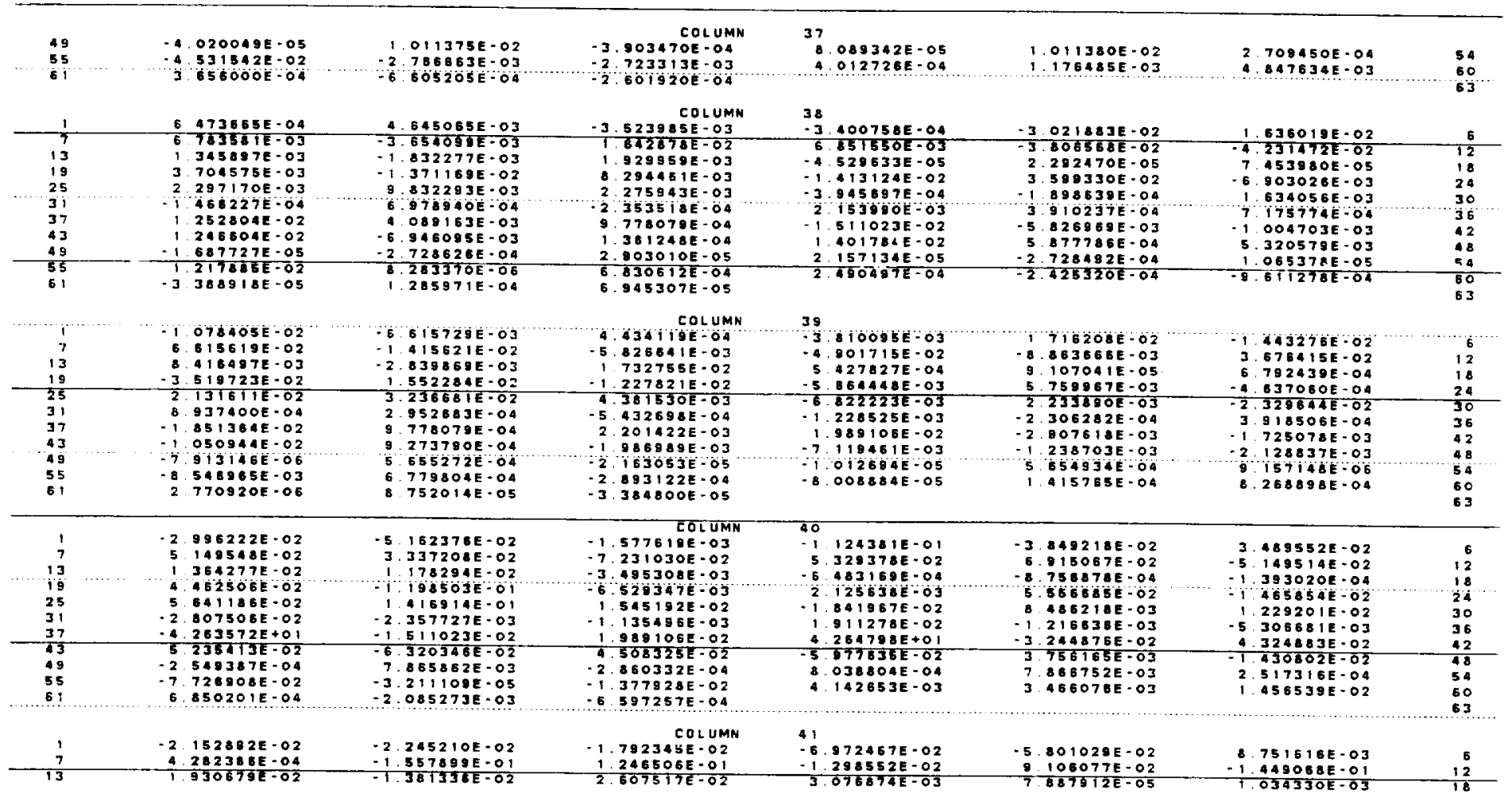

AH - IG THREE- DIMENSIONAL OUILTUP OYMAMICS MODEL W/ CONTROLS MODELED

OCTOBER 26, 1980 MSC/NASTRAN $10 / 15 / 87$ D

PACE

5

THIS VERSION CONTAINS A BUILUP TAILOOOM MODE

INTERMEDIATE MATRIX ... BAA

\begin{tabular}{|c|c|c|c|c|c|c|c|c|c|c|}
\hline $\begin{array}{l}19 \\
25 \\
31 \\
37 \\
43 \\
49 \\
55 \\
51\end{array}$ & $\begin{array}{r}8 \\
-4 \\
-6 \\
3 \\
3 \\
2 \\
-6 \\
-6\end{array}$ & $\begin{array}{l}157271 E-02 \\
813058 E-02 \\
7630111-03 \\
808756 E-02 \\
355029 E-03 \\
149475 E-04 \\
302369 E-02 \\
119720 E-04\end{array}$ & $\begin{array}{r}-6 \\
2 \\
3 \\
-5 \\
-1 \\
-4 \\
1 \\
-1\end{array}$ & $\begin{array}{l}584111 E-02 \\
234534 E-01 \\
376456 E-O 4 \\
826960-03 \\
135536 E-01 \\
1412511-02 \\
786056 E-02 \\
874508 E-O 3\end{array}$ & $\begin{array}{r}-8 \\
4 \\
-2 \\
-2 \\
1 \\
1 \\
-2 \\
-8 \\
-8\end{array}$ & $\begin{array}{l}\text { COLUMN } \\
003198 E-02 \\
502681 E-02 \\
268705 E-04 \\
007818 E-03 \\
188646 E-01 \\
782639 E-03 \\
736376 E-032 \\
701604 E-04\end{array}$ & $\begin{array}{l}41 \\
-1.198420 E-02 \\
-8.312792 E-02 \\
1.286736 E-O 3 \\
-3.244875 E-02 \\
8.313277 E-04 \\
7.518402 E-04 \\
5.104626 E-03\end{array}$ & 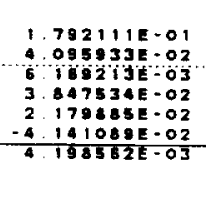 & $\begin{array}{r}-5.972652 E-02 \\
8.580098 E-03 \\
9.96636 E-04 \\
-1.407286-02 \\
1.456525 E-03 \\
1.584475 E-03 \\
1.876278 E-02\end{array}$ & $\begin{array}{l}24 \\
30 \\
36 \\
42 \\
48 \\
54 \\
60 \\
63\end{array}$ \\
\hline $\begin{array}{r}1 \\
13 \\
13 \\
18 \\
25 \\
31 \\
37 \\
43 \\
49 \\
55 \\
61\end{array}$ & $\begin{array}{r}3 \\
3 \\
-4 \\
-1 \\
-8 \\
7 \\
-4 \\
-5 \\
1 \\
2 \\
2 \\
-3\end{array}$ & $\begin{array}{l}400410 E-O 2 \\
611008 E-02 \\
190746 E-02 \\
124104 E-01 \\
607465 E-02 \\
648273 E-03 \\
767565-02 \\
6015875-02 \\
5515235 E-04 \\
071070 E-02 \\
140002 E-04\end{array}$ & $\begin{array}{r}5: \\
5 \\
1 \\
9 \\
-2 \\
-8 \\
-1 \\
8 \\
5 \\
-4 . \\
1 .\end{array}$ & $\begin{array}{l}751528 E-O 2 \\
441728 E-02 \\
807742 E-03 \\
825867 E-02 \\
814194 E-01 \\
017520 E-04 \\
004703 E-03 \\
308709 E-02 \\
222984 E-03 \\
977181 E-03 \\
524819 E-03\end{array}$ & $\begin{array}{r}1 \\
-3 \\
-3 \\
1 \\
-4 \\
8 \\
-1 \\
-1 \\
-3 \\
1 \\
3 \\
3\end{array}$ & 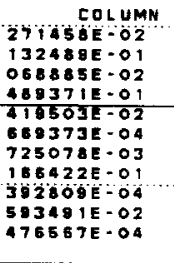 & $\begin{array}{r}42 \\
1.02061 E E-O 1 \\
1.380409 E-01 \\
-1.30929 E-03 \\
-2.515086 E-03 \\
1.113510 E-01 \\
-5.185753 E-03 \\
4.324883 E-02 \\
3.844707 E-02 \\
-7.74892 E=04 \\
-3.260450 E-03\end{array}$ & 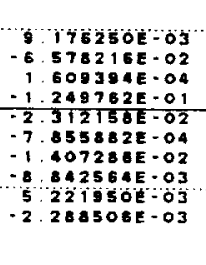 & 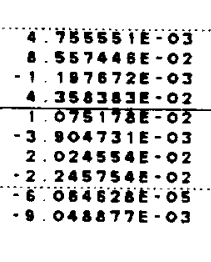 & $\begin{array}{r}16 \\
12 \\
18 \\
24 \\
30 \\
36 \\
42 \\
48 \\
54 \\
80 \\
63\end{array}$ \\
\hline $\begin{array}{r}1 \\
7 \\
13 \\
13 \\
25 \\
31 \\
37 \\
43 \\
49 \\
55 \\
61\end{array}$ & $\begin{array}{r}-3 \\
-3 \\
-6 \\
-3 \\
7 \\
-4 \\
1 \\
2 \\
3 \\
-7 \\
2\end{array}$ & 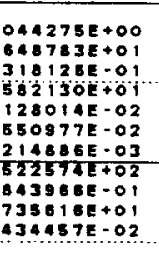 & $\begin{array}{r}-3 . \\
7 \\
3 . \\
1 \\
-4 \\
1 \\
1 \\
-5 \\
-3 \\
-2 .\end{array}$ & 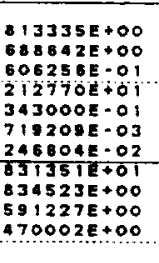 & $\begin{array}{r}-8 \\
7 \\
-2 \\
-3 \\
-4 \\
-1 \\
-1 \\
2 \\
1 \\
-5 \\
-4 \\
-4\end{array}$ & 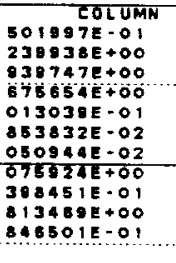 & 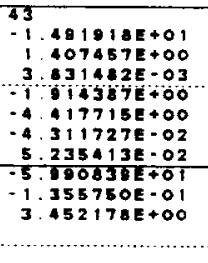 & 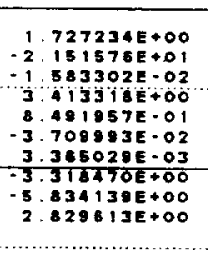 & $\begin{array}{r}-237392 E+00 \\
-9.59931 E-01 \\
-1.145387 E-01 \\
-2.200350 E-02 \\
-2.722098 E+00 \\
7.508245 E-03 \\
-5.601587 E-02 \\
-6.608044 E+00 \\
-1.246198 E-01 \\
1.031418 E+01\end{array}$ & $\begin{array}{r}5 \\
12 \\
18 \\
24 \\
30 \\
36 \\
42 \\
48 \\
54 \\
60 \\
63\end{array}$ \\
\hline $\begin{array}{l}1 \\
7 \\
13 \\
19 \\
25 \\
31 \\
37 \\
43 \\
49 \\
55 \\
61\end{array}$ & $\begin{array}{r}1 \\
-6 \\
-1 \\
3 \\
-8 \\
-2 \\
2 \\
1 \\
1 \\
-4 \\
-1\end{array}$ & 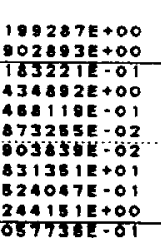 & 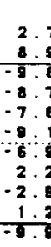 & $\begin{array}{l}748620 E+00 \\
927870 E-01 \\
603797=02 \\
784645 E+00 \\
687103 E+00 \\
142301 E-03 \\
968093 E=03 \\
200220 E+01 \\
990838 E+00 \\
273838 E+00 \\
960424 E-02\end{array}$ & $\begin{array}{r}3 \\
2 \\
-2 \\
4 \\
-1 \\
-5 \\
6 \\
8 \\
7 \\
4 \\
3\end{array}$ & 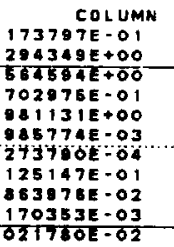 & $\begin{array}{r}44 \\
3.504872 E+00 \\
-1.102387 E+00 \\
-2.175515 E-02 \\
-8.813849 E-03 \\
4.841148 E-01 \\
7.428388 E-02 \\
-8.320346 E-02 \\
-3.104280 E+00 \\
-1.058773 E-01 \\
7.322175 E-02 \\
\end{array}$ & 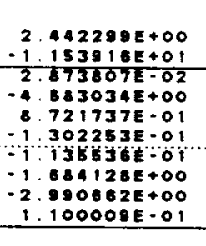 & 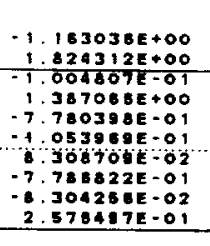 & $\begin{array}{r}5 \\
12 \\
18 \\
24 \\
30 \\
36 \\
42 \\
88 \\
34 \\
80 \\
63\end{array}$ \\
\hline
\end{tabular}


AH- IC THREE-DIMENSIONAL BUILTUP DYMAMICS MODEL W/ CONTROLS MODELED

THIS VERSION CONTAINS A BUILTUP TAILBOOM MODEL

INTERMEDIATE MATRIX ... BAa

\begin{tabular}{|c|c|c|c|c|c|c|c|c|c|c|}
\hline $\begin{array}{r}1 \\
7 \\
13 \\
19 \\
25 \\
31 \\
35 \\
43 \\
49 \\
55 \\
611\end{array}$ & $\begin{array}{r}-1.036336 E+00 \\
-5.175792 E+00 \\
1.29845 E+00 \\
4.925230+00 \\
2.444939 E+00 \\
4.228145 E-03 \\
2183430 E-03 \\
2.075924 E+00 \\
3563307 E-02 \\
1918079 E+01 \\
1591683 E-01\end{array}$ & $\begin{array}{r}-2 \\
3 \\
-3 \\
-2 \\
5 \\
-7 \\
-7 \\
1 \\
8 \\
-2 \\
-8 \\
-8 \\
-8\end{array}$ & 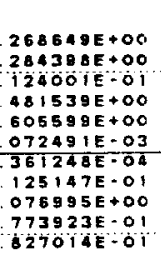 & $\begin{array}{r}3 \\
-9 \\
1 \\
2 \\
5 \\
-7 \\
-1 \\
2 \\
4 \\
-1 \\
-2 \\
-2\end{array}$ & 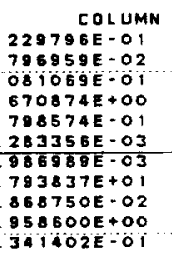 & $\begin{array}{r}45 \\
-3 \\
-3 \\
-3 \\
2 \\
6 \\
5 \\
5 \\
4 \\
-4 . \\
8 . \\
-5 .\end{array}$ & $\begin{array}{l}336402 E+00 \\
81082 E+00 \\
049226 E-02 \\
299710 E+00 \\
810036 E+00 \\
941836 E-02 \\
508325 E-02 \\
820158 E+00 \\
386510 E-02 \\
30431 E-02\end{array}$ & $\begin{array}{r}5.264319 E-01 \\
1338900+01 \\
171958 E-02 \\
1.656114 E+00 \\
-356578+00 \\
1.883525 E-03 \\
178666 E-01 \\
5.773593 E+00 \\
-2.076802 E+00 \\
7.493636 E-01\end{array}$ & 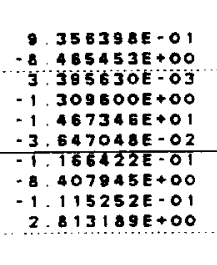 & $\begin{array}{r}5 \\
12 \\
18 \\
24 \\
30 \\
36 \\
42 \\
48 \\
54 \\
60 \\
63\end{array}$ \\
\hline $\begin{array}{l}1 \\
7 \\
13 \\
19 \\
25 \\
31 \\
37 \\
43 \\
49 \\
55 \\
61\end{array}$ & $\begin{array}{r}-3 \\
-395784 E+0 O \\
-356336 E+01 \\
-1.358656 E+00 \\
-5.277087 E+01 \\
-271985 E+00 \\
=3068516 E-02 \\
-4.643144 E-02 \\
-5.990839 E+01 \\
1.163184 E-01 \\
-2.761694 E+02 \\
9.637412 E-01\end{array}$ & $\begin{array}{r}-4 \\
2 \\
-4 \\
-1 \\
-2 \\
-1 \\
1 \\
1 \\
-3 \\
1 \\
-3 \\
-3 \\
-3\end{array}$ & $\begin{array}{l}544492 E+00 \\
993849 E+00 \\
063670 E=01 \\
033917 E+00 \\
627352 E+00 \\
0361923 E-03 \\
401734 E-02 \\
104280 E+00 \\
278647 E+00 \\
297093 E-01 \\
125438 E+00\end{array}$ & $\begin{array}{r}-1 \\
8 \\
7 \\
3 \\
4 \\
=2 \\
-7 \\
-4 \\
-1 \\
=4 \\
-1\end{array}$ & 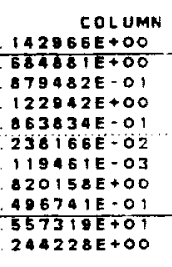 & $\begin{array}{r}46 \\
-1 \\
-1 \\
3 \\
9 \\
-3 \\
-5 \\
-4 \\
-5 \\
4 \\
1 \\
-1 \\
-1\end{array}$ & $\begin{array}{l}523030 E+01 \\
143371 E-01 \\
154153 E-02 \\
186588+00 \\
016554 E+00 \\
191325 E-02 \\
977635 E-02 \\
786309 E+02 \\
736608 E-01 \\
786739 E+01\end{array}$ & $\begin{array}{r}-9.575417 E-01 \\
1.480716 E+01 \\
2.31252 E-02 \\
3.491525 E+00 \\
-1331232 E-01 \\
421855=02 \\
8313279 E-04 \\
309345 E+00 \\
1279035 E+00 \\
3738694 E+00\end{array}$ & 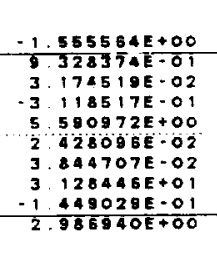 & $\begin{array}{r}6 \\
12 \\
18 \\
24 \\
30 \\
36 \\
42 \\
48 \\
54 \\
60 \\
63\end{array}$ \\
\hline $\begin{array}{r}7 \\
7 \\
13 \\
19 \\
25 \\
31 \\
37 \\
43 \\
49 \\
55 \\
61\end{array}$ & 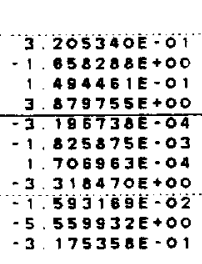 & $\begin{array}{r}5 \\
2 \\
-5 \\
-2 \\
-5 \\
-2 \\
5 \\
-1 \\
-1 \\
-5 \\
-5 \\
1\end{array}$ & $\begin{array}{l}591471 E-O 1 \\
472860 E-O 1 \\
978458 E-02 \\
886529 E+00 \\
777325 E-01 \\
189354 E-03 \\
877786 E-04 \\
684128 E+00 \\
903303 E+00 \\
004300 E+00 \\
080527 E+00\end{array}$ & $\begin{array}{r}1 \\
8 \\
-5 \\
1 \\
-2 \\
-3 \\
-1 \\
5 \\
-1 \\
-2 \\
-2\end{array}$ & 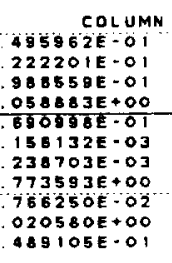 & $\begin{array}{r}47 \\
1 \\
4 \\
-3 \\
4 \\
1 \\
1 \\
3 \\
3 \\
5 \\
-7\end{array}$ & $\begin{array}{l}076363 E+00 \\
293497 E-02 \\
230789-03 \\
386973 E-01 \\
117349 E+00 \\
334192 E-02 \\
756165 E-03 \\
093435 E+00 \\
269681 E-02 \\
796201 E-02\end{array}$ & 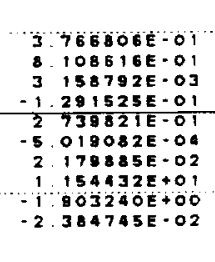 & 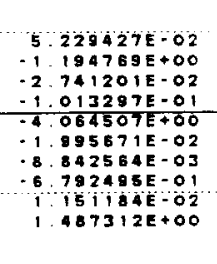 & $\begin{array}{l}6 \\
12 \\
18 \\
24 \\
30 \\
36 \\
42 \\
48 \\
54 \\
60 \\
63\end{array}$ \\
\hline $\begin{array}{r}1 \\
7 \\
13 \\
19 \\
25 \\
31 \\
37 \\
43\end{array}$ & $\begin{array}{r}-7.914856 E-01 \\
-1.285861 E+01 \\
-1.489832-01 \\
2.489188 E+00 \\
-4.151556 E-01 \\
-2.035477 E-02 \\
-1.587156 E-02 \\
-1 \\
-6.608044 E+D 0\end{array}$ & $\begin{array}{r}-1 \\
3 \\
1 \\
-1 \\
-2 \\
5 \\
-7\end{array}$ & $\begin{array}{l}134494 E+00 \\
48005 E-01 \\
648936 E-01 \\
235517 E+00 \\
270373 E+00 \\
400013 E=04 \\
320579 E=03 \\
78622 E=01\end{array}$ & $\begin{array}{r}-1 \\
5 \\
-8 \\
-1 \\
-3 \\
-1 \\
-2 \\
-8\end{array}$ & $\begin{array}{l}\text { COLUMH } \\
808731 E-01 \\
251574 E+00 \\
021335 E-01 \\
3543626+00 \\
212437 E-01 \\
057679 E-02 \\
128375-03 \\
407945 E+00\end{array}$ & $\begin{array}{r}48 \\
-3 \\
4 \\
5 \\
-4 \\
4 \\
-2 \\
-1 \\
3\end{array}$ & $\begin{array}{l}941331 E+00 \\
50545 E-01 \\
641082 E-03 \\
02758 E-01 \\
338270 E+00 \\
101200 E-02 \\
430802 E-02 \\
12846 E E+O 1\end{array}$ & $\begin{array}{r}-1.426576 E-01 \\
-1.4547915-01 \\
-7.804075 E-03 \\
-1.212054 E-01 \\
2.048735=01 \\
-2.700650 E-02 \\
1.456525 E-03 \\
-6792495 E-01\end{array}$ & $\begin{array}{r}-1.758268 E-01 \\
7.845255 E-01 \\
-3.119788 E-02 \\
1.22450 E-01 \\
-8.82491210+00 \\
-8.990352 E-03 \\
-2.245754 E-02 \\
3.435907 E+01\end{array}$ & $\begin{array}{r}5 \\
12 \\
18 \\
24 \\
30 \\
36 \\
42 \\
48\end{array}$ \\
\hline
\end{tabular}

AH- IG THRE - OIMENS IONAL DUILTUP DYNAMICS MOOEL W/ CONTROLS MOOELEO

OCTOBER 26. 1990 MSC/NASTRAN 10/15/87 PRG 57 THIS VERSION CONTAINS A BUILTUP TAILBODM MODEL

INTERMEDIATE MATRIX ... BAA

\begin{tabular}{|c|c|c|c|c|c|c|c|c|c|c|}
\hline $\begin{array}{l}49 \\
55 \\
61\end{array}$ & $\begin{array}{r}-3.254721 E-02 \\
1.108498 E+00 \\
2.916895 E-O 1\end{array}$ & 5 & $\begin{array}{l}422396 E-01 \\
554719 E-01 \\
045415 E=02\end{array}$ & $\begin{array}{r}\text { COLUMN } \\
1.377501 E-01 \\
-8.454251 E+00 \\
-55.455076 E-02\end{array}$ & $\begin{array}{l}48 \\
-4 \\
-1\end{array}$ & $\begin{array}{r}564259 E-02 \\
405285 E+01\end{array}$ & & $\begin{array}{r}421094 E-01 \\
274550 E-01 \\
\therefore\end{array}$ & $\begin{array}{r}1.820016 E-01 \\
-1118724 E+01 \\
1.1187240\end{array}$ & $\begin{array}{l}54 \\
80 \\
63\end{array}$ \\
\hline $\begin{array}{l}1 \\
7 \\
13 \\
19 \\
25 \\
31 \\
37 \\
43 \\
49 \\
55 \\
61\end{array}$ & $\begin{array}{r}-3.783638 E-03 \\
-4.713454 E-02 \\
-5.1844475-04 \\
-1.44785 E-02 \\
-2.099381 E-03 \\
2.604145 E-05 \\
-4.020049 E-05 \\
3.843965 E-01 \\
2.072014 E-02 \\
-5.237891 E-01 \\
-8.150596 E-03\end{array}$ & $\begin{array}{r}-3 \\
-4 \\
-4 \\
-1 \\
-1 \\
4 \\
-1 \\
1 \\
-7 \\
2 \\
-2 \\
-2\end{array}$ & 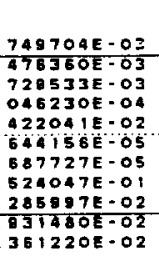 & $\begin{array}{r}\text { COLUMM } \\
-1.189490 E-03 \\
6.281672 E-03 \\
-1.375102 E-03 \\
1.204408 E-03 \\
-1.627875 E-03 \\
-3.464207 E-05 \\
-7.913146 E-05 \\
3.563307 E-02 \\
2.963408 E-03 \\
1.421094 E-01 \\
7.188007 E-05\end{array}$ & $\begin{array}{r}49 \\
-1 \\
-6 \\
2 \\
2 \\
-5 \\
-5 \\
-2 \\
-2 \\
-1 \\
8\end{array}$ & $\begin{array}{l}.920220 E-02 \\
197524 E-03 \\
241390 E-04 \\
708365 E-03 \\
148553-03 \\
7951186 E-04 \\
549387 E-04 \\
163196 E-01 \\
350830 E-02 \\
227445 E-02\end{array}$ & $\begin{array}{r}2 \\
3 \\
3 \\
-6 \\
-4 \\
-2 \\
2 \\
-1 \\
-7 \\
3\end{array}$ & $\begin{array}{l}876321 E-03 \\
.276301 E-02 \\
098188 E-04 \\
520237 E-03 \\
745425-03 \\
73530 E-05 \\
149475 E-04 \\
593169 E-02 \\
268449 E-02 \\
914307 E-02\end{array}$ & $\begin{array}{r}-3.512500 E-03 \\
-2.393460 E-02 \\
-5.43546 E-05 \\
-1.02859-03 \\
1.830931 E-02 \\
-4.67277 E-04 \\
1.553237 E-04 \\
-3.254721 E-02 \\
-3.183531 E-03 \\
1.438525-01\end{array}$ & $\begin{array}{l}6 \\
12 \\
18 \\
24 \\
30 \\
36 \\
42 \\
18 \\
54 \\
60 \\
63\end{array}$ \\
\hline $\begin{array}{l}11 \\
7 \\
13 \\
19 \\
25 \\
31 \\
37 \\
43 \\
49 \\
55 \\
61\end{array}$ & $\begin{array}{r}2.491611 E-01 \\
1.002756 E+00 \\
-1.919287 E-02 \\
5.514000 E-01 \\
1.212539 E-01 \\
-8.537776 E-03 \\
1.011375 E-02 \\
-5.834523 E+00 \\
-7.285997 E-02 \\
6.656662 E+00 \\
2.369822 E-01\end{array}$ & $\begin{array}{r}3 \\
4 \\
2 \\
-2 \\
-4 \\
1 \\
-2 \\
-2 \\
2 \\
-2 \\
-4\end{array}$ & $\begin{array}{l}996716 E-O 1 \\
513592 E-O 1 \\
855039 E-01 \\
249272 E+00 \\
241852 E-01 \\
608357 E-03 \\
728626 E-04 \\
900338 E+00 \\
668905 E+01 \\
087919 E+00 \\
408974 E-01\end{array}$ & 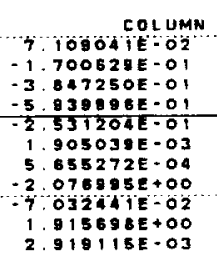 & $\begin{array}{r}50 \\
1 \\
8 \\
-2 \\
-2 \\
-4 \\
-1 \\
7 \\
1 \\
6 \\
-6\end{array}$ & $\begin{array}{l}006014 E+00 \\
631041 E \cdot 02 \\
018635 E-02 \\
853576 E-01 \\
765783 E-02 \\
928577 E-02 \\
885862 E-03 \\
278647 E+00 \\
368415 E-02 \\
492572 E-01\end{array}$ & $\begin{array}{l}3 \\
-4 \\
-1 \\
-5 \\
3 \\
-2 \\
-2 \\
-1 \\
-1 \\
-1 \\
-2 .\end{array}$ & $\begin{array}{l}070160 E-01 \\
209244 E+00 \\
315229 E-02 \\
560093 E-01 \\
106478 E-09 \\
935312 E-02 \\
141251 E-02 \\
903303 E+00 \\
783887 E+01 \\
152443 E-01\end{array}$ & 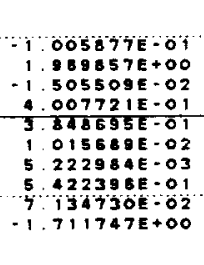 & $\begin{array}{l}\cdots 1 \\
12 \\
18 \\
24 \\
30 \\
36 \\
42 \\
48 \\
54 \\
60 \\
63\end{array}$ \\
\hline $\begin{array}{r}1 \\
7 \\
13 \\
13 \\
25 \\
31 \\
37 \\
43 \\
49 \\
55 \\
61\end{array}$ & $\begin{array}{r}-7.241451 E-03 \\
-8.385857 E-O 2 \\
2.957994 E-03 \\
5.683489 E-O 2 \\
-3.984038 E-03 \\
2.20985 E-04 \\
-3.903470 E-04 \\
1.398451 E-01 \\
2.063408 E-03 \\
-7.522257 E-02 \\
-1.140879 E-02\end{array}$ & $\begin{array}{r}-1 \\
-5 \\
-1 \\
2 \\
1 \\
-6 \\
2 \\
2 \\
-7 \\
3 \\
3 \\
1\end{array}$ & $\begin{array}{l}018053 E-O 2 \\
987475 E-O 3 \\
150857 E-O 2 \\
0191515-O 2 \\
509769 E-02 \\
08549110-05 \\
003010 E-05 \\
063975 E-02 \\
0324115-02 \\
224714 E-02 \\
781938 E-02\end{array}$ & 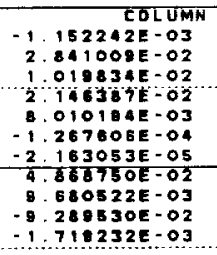 & $\begin{array}{r}51 \\
-3 \\
-1 \\
7 \\
7 \\
2 \\
7 \\
-2 \\
-1 \\
-2 \\
-3\end{array}$ & 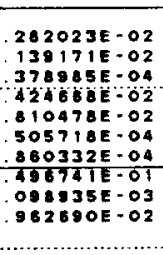 & $\begin{array}{r}-6 \\
1 \\
5 \\
2 \\
-8 \\
1 \\
1 \\
-1 \\
-7 \\
-7 \\
4\end{array}$ & 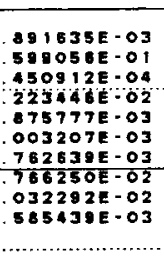 & $\begin{array}{r}-O 42032 E-03 \\
-3.35414 E-02 \\
3.980444 E-04 \\
-1.854726 E-02 \\
-6.868768 E-02 \\
-6.218974 E-04 \\
-3.392809 E-04 \\
-1.377501 E-01 \\
-6.404864 E-03 \\
1.398112 E-03\end{array}$ & $\begin{array}{r}6 \\
12 \\
18 \\
24 \\
30 \\
36 \\
42 \\
48 \\
54 \\
60 \\
63\end{array}$ \\
\hline $\begin{array}{r}1 \\
-13\end{array}$ & $\begin{array}{r}-4.647151 E-03 \\
2.183839 E-02 \\
5.305198 E-03\end{array}$ & $\begin{array}{r}-8 \\
1 \\
1\end{array}$ & $\begin{array}{l}670719 E-03 \\
508532 E-02 \\
35034 E-03\end{array}$ & $\begin{array}{r}\text { COLUMN } \\
1.337828 E-O 4 \\
-2.377817 E-02 \\
-1.072587 .03\end{array}$ & $\begin{array}{r}52 \\
-1 \\
6 \\
-2\end{array}$ & $\begin{array}{l}317945 E-02 \\
.89686 E-03 \\
.240031 E-04\end{array}$ & $\begin{array}{r}2 \\
7 \\
-1\end{array}$ & $\begin{array}{r}523263 E-03 \\
\text { B10146E-O2 } \\
\text { B87292E-OA }\end{array}$ & $\begin{array}{r}2.344088 E-03 \\
-3.047835 E-02 \\
-447087 E-05\end{array}$ & $\begin{array}{r}6 \\
12 \\
18\end{array}$ \\
\hline
\end{tabular}


AH-IC THREE-DIMENSIOMAL BUILTUP DYNAMILS MODEL W/ CONTROLS MODELED DIFFICULT COMPONENTS GVT CONFIGURATION I [FULL-UP]

INTERMEDIATE MATRIX , BAA

\begin{tabular}{|c|c|c|c|c|c|c|c|c|c|c|c|c|c|}
\hline $\begin{array}{l}19 \\
25 \\
31 \\
37 \\
13 \\
49 \\
55 \\
81\end{array}$ & $\begin{array}{r}1 \\
2 \\
1 \\
8 \\
-1 \\
-1 \\
-9 \\
1\end{array}$ & $\begin{array}{l}750858 E-01 \\
034787 E-02 \\
220796 E-04 \\
080342 E-05 \\
35570 E-01 \\
350830 E-02 \\
25873 E-01 \\
222121 E-O 2\end{array}$ & $\begin{array}{r}2 \\
5 \\
1 \\
2 \\
-1 \\
4 \\
4.4 \\
-3\end{array}$ & $\begin{array}{l}678613 E-02 \\
423454 E-02 \\
098803 E-05 \\
157134 E-05 \\
656773 E-01 \\
38841 B E-02 \\
001213 E-02 \\
059191 E-02\end{array}$ & $\begin{array}{r}7 \\
6 \\
2 \\
-1 \\
8 \\
-2 \\
-1 \\
-8 \\
-8\end{array}$ & $\begin{array}{l}\text { COLUMN } \\
785382-03 \\
276555 E-03 \\
986439 E-05 \\
012604 E-05 \\
385510 E-02 \\
098935-03 \\
391057 E-01 \\
947998 E-03\end{array}$ & $\begin{array}{r}52 \\
1 \\
5 \\
-1 \\
8 \\
1 \\
2 . \\
6\end{array}$ & $\begin{array}{l}135557 E-O 2 \\
709754-03 \\
208506 E-O 4 \\
038804 E-04 \\
736808 E-01 \\
252931 E-02 \\
608784 E-02\end{array}$ & $\begin{array}{r}1 \\
-5 \\
4 \\
7 \\
5 \\
4 \\
3\end{array}$ & 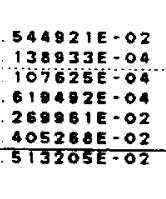 & $\begin{array}{r}-6 \\
6 \\
2 \\
-7 \\
-1 \\
1 \\
1 \\
1\end{array}$ & $\begin{array}{l}731212 E-03 \\
538799 E-03 \\
930663 E-04 \\
748952-04 \\
564259 E-02 \\
745858 E-03 \\
496530 E-01\end{array}$ & $\begin{array}{l}24 \\
30 \\
36 \\
42 \\
48 \\
54 \\
60 \\
63\end{array}$ \\
\hline $\begin{array}{r}1 \\
7 \\
13 \\
19 \\
25 \\
31 \\
37 \\
43 \\
49 \\
55 \\
61\end{array}$ & $\begin{array}{r}2 \\
1 \\
-1 \\
5 \\
1 \\
-8 \\
1 \\
-5 \\
-7 \\
6 \\
2 \\
2\end{array}$ & $\begin{array}{l}O Q 1357 E-01 \\
002698 E+00 \\
913545 E-02 \\
518445 E-01 \\
212819 E-01 \\
537548 E-03 \\
011380 E-02 \\
834139 E+00 \\
261449 E-02 \\
654954 E+00 \\
36987 B E-01\end{array}$ & $\begin{array}{r}3 \\
4 \\
2 \\
-2 \\
-4 \\
1 \\
-2 \\
-2 \\
-2 . \\
-2 \\
-4 \\
-4\end{array}$ & $\begin{array}{l}996490 E-O 1 \\
513877 E-01 \\
854084 E-01 \\
249317 E+00 \\
241206 E-01 \\
608287 E-03 \\
7284920-09 \\
990682 E+00 \\
783887 E+01 \\
087938 E+00 \\
407896 E-01\end{array}$ & $\begin{array}{r}7 \\
-1 \\
-3 \\
-5 \\
-2 \\
1 \\
5 \\
-2 \\
-7 \\
1 \\
2\end{array}$ & $\begin{array}{l}\text { COLUMN } \\
108 B 19 E-O 2 \\
700873 E-01 \\
867297 E-01 \\
939794-01 \\
531129 E-O 1 \\
905020 E-03 \\
659942-04 \\
076802 E+00 \\
032292 E=02 \\
915226 E+00 \\
903692 E-03\end{array}$ & $\begin{array}{r}53 \\
1 \\
8 \\
-2 \\
-2 \\
-4 \\
-1 \\
7 \\
1 \\
4 \\
-6\end{array}$ & $\begin{array}{l}005954 E+00 \\
631161 E-02 \\
016632 E-02 \\
853354 E-01 \\
765578 E-02 \\
928520 E-02 \\
886752 E-03 \\
279035 E+00 \\
05266 E-O 2 \\
490090 E-01\end{array}$ & $\begin{array}{r}3 \\
-4 \\
-1 \\
-5 \\
-5 \\
-2 \\
-2 \\
-4 \\
-1 \\
2 \\
-2\end{array}$ & $\begin{array}{l}070246 E-01 \\
209069 E+00 \\
316205 E-02 \\
559934 E-01 \\
106351 E-01 \\
035249 E-02 \\
141089 E-02 \\
903240 E+00 \\
698966+01 \\
551178 E-01\end{array}$ & $\begin{array}{r}1 \\
1 \\
-1 \\
4 \\
3 \\
1 \\
5 \\
5 \\
-1 \\
-1\end{array}$ & 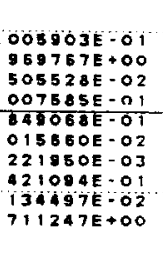 & $\begin{array}{r}6 \\
12 \\
18 \\
24 \\
-30 \\
36 \\
42 \\
48 \\
54 \\
60 \\
63\end{array}$ \\
\hline $\begin{array}{r}1 \\
7 \\
13 \\
19 \\
25 \\
31 \\
37 \\
43 \\
49 \\
55 \\
61\end{array}$ & $\begin{array}{r}2 \\
-1 \\
-2 \\
4 \\
4 \\
-3 \\
2 \\
-1 \\
-3 \\
4 \\
1\end{array}$ & $\begin{array}{l}116832 E-03 \\
674961 E-02 \\
100968 E-03 \\
530720 E-02 \\
375625 E-03 \\
105613 E-04 \\
709450 E-04 \\
246168 E-01 \\
183631 E-03 \\
131473 E-01 \\
321236 E-02\end{array}$ & $\begin{array}{r}2 \\
1 \\
1 \\
2 \\
-2 \\
6 \\
1 \\
1 \\
-8 \\
7 \\
-2 \\
-1 \\
-1\end{array}$ & $\begin{array}{l}695138 E-03 \\
34355-02 \\
327410 E-02 \\
962564 E-02 \\
158153 E-02 \\
544276 E-05 \\
055378 E-05 \\
304256 E-02 \\
13430 E-02 \\
319037 E-02 \\
782905 E-02\end{array}$ & $\begin{array}{r}6 \\
1 \\
-2 \\
-2 \\
-1 \\
3 \\
9 \\
9 \\
-1 \\
-6 \\
4 \\
2 \\
2\end{array}$ & 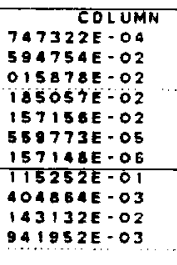 & $\begin{array}{r}54 \\
7 \\
-7 \\
-1 \\
2 \\
-8 \\
2 \\
-1 \\
1 \\
-7\end{array}$ & $\begin{array}{l}160290 E-03 \\
465159 E-02 \\
738555 E-04 \\
262733 E-02 \\
758198 E-02 \\
821521 E-04 \\
517316 E-04 \\
449028 E-01 \\
745858 E-03 \\
949550 E-02\end{array}$ & $\begin{array}{r}6 \\
.1 \\
-5 \\
-2 \\
1 \\
1 \\
-1 \\
-1 \\
1 \\
7 \\
.1\end{array}$ & $\begin{array}{l}929140 E-03 \\
292688 E-01 \\
362935 E-04 \\
606826=02 \\
093995-02 \\
302688 E-03 \\
584955-03 \\
151184 E=02 \\
134997 E-02 \\
097100 E-02\end{array}$ & 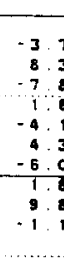 & $\begin{array}{l}752603 E-03 \\
334935-02 \\
872439 E-04 \\
892703 E-02 \\
170573 E-02 \\
386045 E-04 \\
064628 E-05 \\
820016 E-01 \\
895500 E-03 \\
158347 E-01\end{array}$ & $\begin{array}{l}6 \\
12 \\
18 \\
24 \\
30 \\
36 \\
42 \\
18 \\
54 \\
60 \\
63\end{array}$ \\
\hline $\begin{array}{r}1 \\
7 \\
13 \\
18 \\
25 \\
31 \\
37 \\
43 \\
48 \\
55 \\
61\end{array}$ & $\begin{array}{l}-2 \\
-3 \\
-1 \\
-6 \\
-3 \\
-4 \\
-4 \\
-7 \\
-5 \\
5 \\
-9 \\
-9\end{array}$ & $\begin{array}{l}545322 E+00 \\
322528 E+01 \\
133537+00 \\
035873 E+01 \\
277011 E+00 \\
890268 E-02 \\
5311426-02 \\
735618 E+01 \\
2378912-01 \\
119942 E+02 \\
745900 E-01\end{array}$ & $\begin{array}{r}-3 \\
1 \\
4 \\
2 \\
-7 \\
-9 \\
1 \\
-4 \\
6 \\
4 \\
8\end{array}$ & $\begin{array}{l}549678 E+00 \\
031241 E+00 \\
277857 E=01 \\
612601 E+00 \\
097624 E+00 \\
996058 E=04 \\
21785 E=02 \\
244151 E+00 \\
656562 E+00 \\
754483 E+00 \\
884848 E+00\end{array}$ & $\begin{array}{l}-9 \\
1 \\
-2 . \\
-5 \\
-8 \\
-2 . \\
-8 \\
-1 \\
-7 . \\
9 \\
2 \\
2\end{array}$ & $\begin{array}{l}\text { COLUMN } \\
101150 E-01 \\
167670 E+01 \\
075558 E+00 \\
245729 E-01 \\
721143 E-01 \\
241980 E-02 \\
546865-03 \\
918079 E+01 \\
5222570-02 \\
122854 E+01 \\
420084 E+00\end{array}$ & $\begin{array}{r}55 \\
-1 \\
2 \\
-5 \\
-3 \\
-3 \\
-5 \\
-5 \\
-2 \\
-2 \\
-2 \\
-2 \\
\end{array}$ & 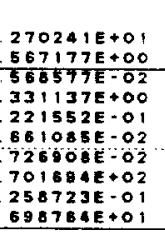 & $\begin{array}{r}-9 \\
-1 \\
-1 \\
1 \\
4 \\
-6 \\
-6 \\
-5 \\
6 \\
-1 \\
\end{array}$ & 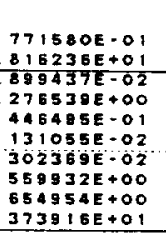 & $\begin{array}{r}-1.3 \\
4.1 \\
-8.0 \\
5.8 \\
-1.3 \\
-7.2 \\
2.0 \\
1.1 \\
4.1 \\
-7.3 \\
\end{array}$ & $\begin{array}{l}310295 E+00 \\
198523 E+00 \\
00765 E-02 \\
931954 E-01 \\
336548 E+01 \\
455782 E-03 \\
071070 E-02 \\
108498 E+00 \\
131473 E-01 \\
309400 E+01 \\
\end{array}$ & $\begin{array}{r}6 \\
12 \\
18 \\
24 \\
30 \\
36 \\
42 \\
48 \\
54 \\
60\end{array}$ \\
\hline
\end{tabular}

AH. IC THREE-DIMENSIONAL OUILTUP DYNAMICS MODEL W/ CONTROLS MODELED

OCTOBEA

1990

MSC/NASTRAN $10 / 15 / 07$

PAGE

50

THIS VERSION CONTAINS A BUILTUP TAILBOOM MOOEL

INTERMEOIATE MATRIX ... BaA

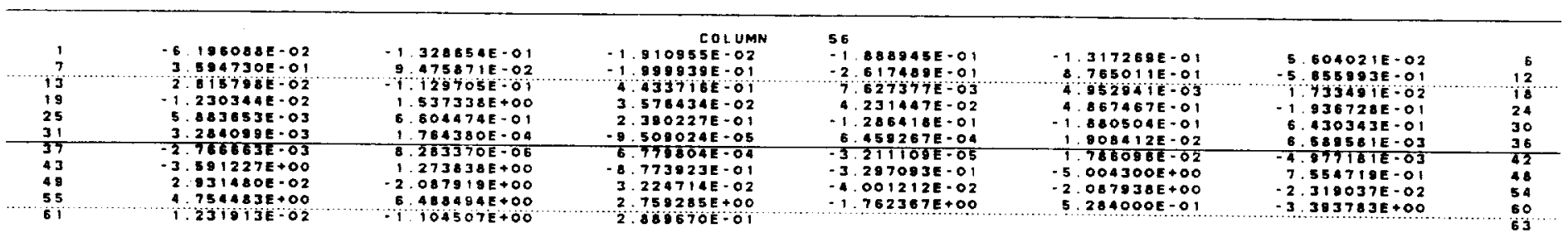

\begin{tabular}{|c|c|c|c|c|c|c|c|c|c|c|}
\hline & $2.058874 E \cdot 02$ & $4.517072 E .02$ & $\begin{array}{l}\text { COL UMN } \\
\text { SBSOE-O2 }\end{array}$ & 57 & & & & & & \\
\hline 7 & $-1.210652 E+00$ & $-2.688335 E-01$ & $\frac{-3.025880 E-02}{8.9205802-01}$ & -7 & $078568 E-02$ & & $365984 E-01$ & & $272791 E-02$ & E \\
\hline 13 & $-1.6481745-01$ & $7.358149 E-02$ & $-1.253203 E-02$ & 2 & Jis985E-03 & -3 & $004325 E=03$ & -3 & $\begin{array}{l}25 B 043 E+00 \\
B 49728 E-04\end{array}$ & $\begin{array}{l}12 \\
18\end{array}$ \\
\hline $\begin{array}{l}19 \\
25\end{array}$ & $\begin{array}{l}-4.649780 E+00 \\
-.615872 E-01\end{array}$ & $\begin{array}{r}4006478 E-01 \\
051011 E+00\end{array}$ & $\begin{array}{l}-1,5553 B 9 E=02 \\
-1.233133 E-01\end{array}$ & -9 & $165881 E-09$ & -1 & $886946 E-01$ & 1 & QA9256E-OI & 24 \\
\hline 31 & $-4.896301 \mathrm{~L}-03$ & 5 O63535E-OA & $\begin{array}{r}-1.233133 E-01 \\
\because 1.057494 E=03\end{array}$ & $\because 6$ & $\begin{array}{l}1450785-01 \\
617096003\end{array}$ & $\begin{array}{l}1 \\
-9 \\
-9\end{array}$ & $\begin{array}{l}727475 E-01 \\
725631=03\end{array}$ & $\therefore 3$ & $918111 E-01$ & 30 \\
\hline 37 & $-2.7233138-03$ & C. $830612 E-04$ & $-2.093122 E-04$ & -1 & $377928 E-02$ & -2 & $365376 E-02$ & 1 & $\begin{array}{l}150334 E-03 \\
593481 E-02\end{array}$ & $\begin{array}{l}36 \\
42\end{array}$ \\
\hline 43 & $-5.013489 E+00$ & $4-170353 E-03$ & $-1.958000 E+00$ & -4 & $557319 E+01$ & -2 & $020580 E+00$ & -9 & $454251 E+00$ & 48 \\
\hline 49 & $-1.121074 E-01$ & 1.915598E+00 & $-9.289530 E-02$ & -1 & $381057 E-01$ & & $013226 E+00$ & 4 & $143132 E-02$ & 54 \\
\hline 85 & $\begin{array}{r}\text { T.122854E+O1 } \\
-4 . \text { OASBEE-OI }\end{array}$ & $\begin{array}{l}2.753285 E+00 \\
2.611028 E+00\end{array}$ & $\begin{array}{l}6.883353 E+01 \\
7.539868 E-01\end{array}$ & -2 & Q25043E+01 & & $331431 E+00$ & $=5$ & OSE $639 E+01$ & 60 \\
\hline
\end{tabular}

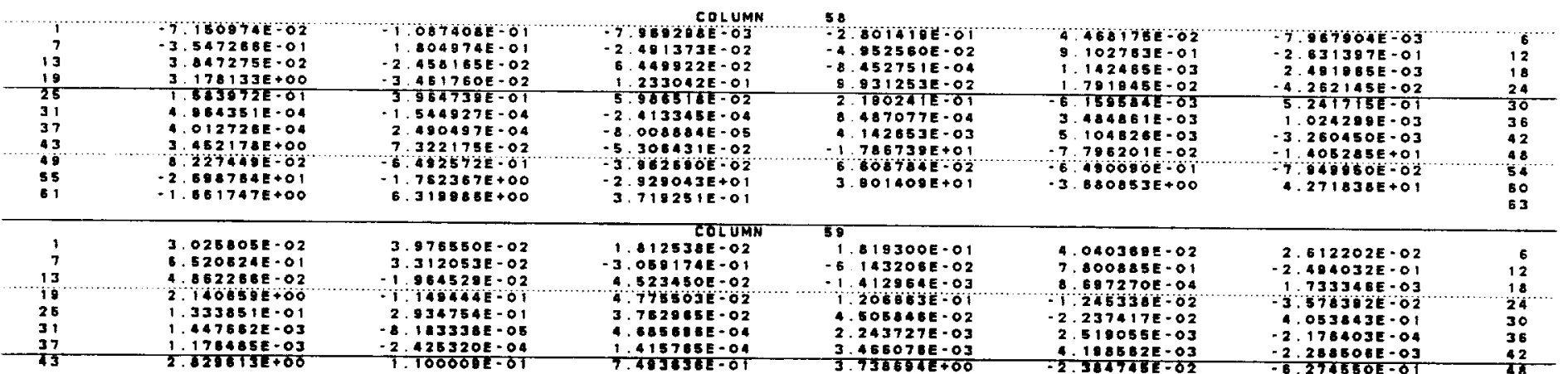


AH-IG THREE-DIMEHSIOMAL BUILTUP OYNAMICS MODEL W/ COMTROLS MODELED

THIS VERSION CONTAINS A BUILTUP TAILBDOM MODEL

INTERMEDIATE MATRIX ... BAA

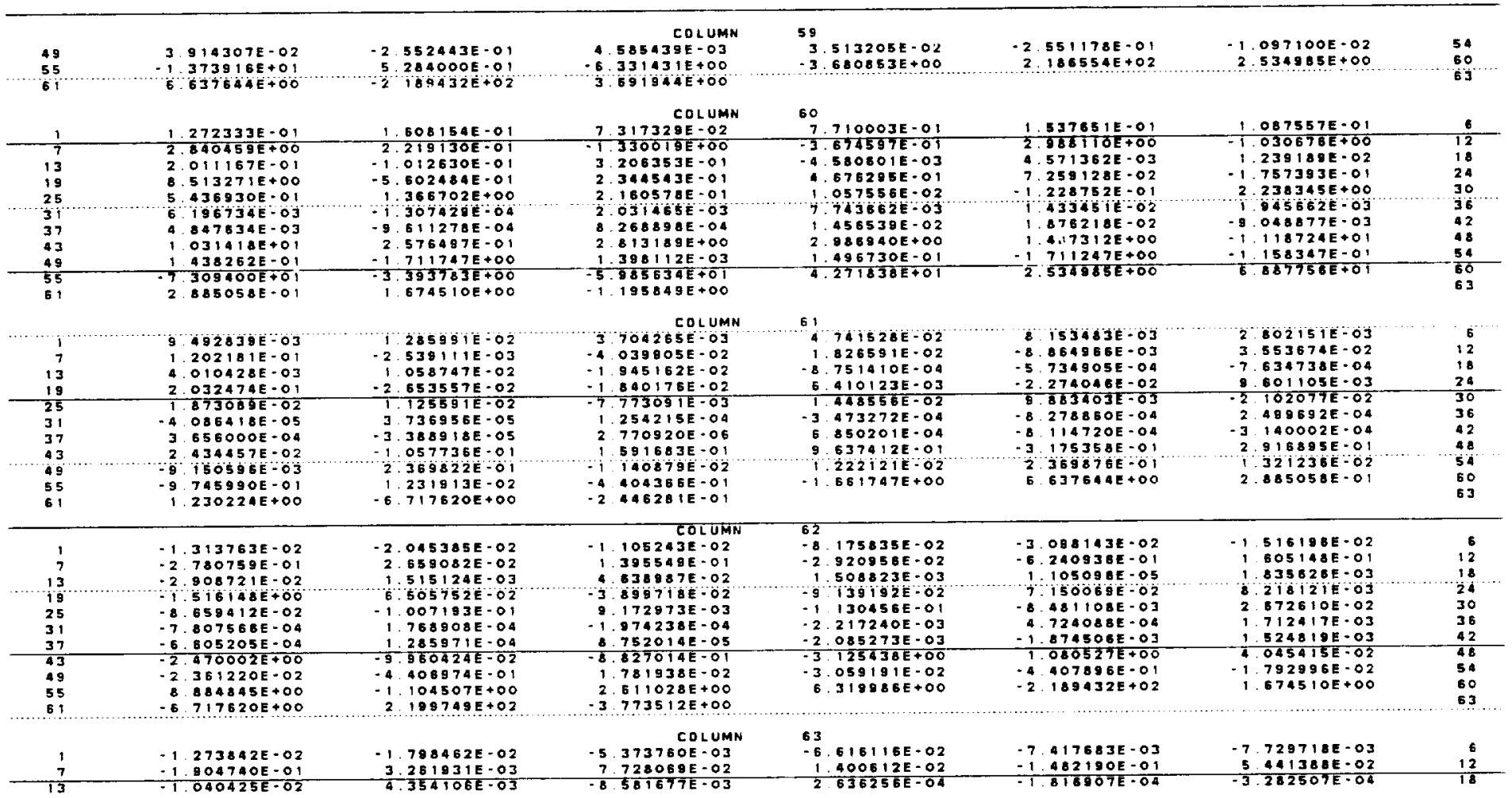

AH-IG THRE -DIMENSIONAL BUILTUP OYNAMICS MODEL W/ CONTROLS MODELED

DCTOBER

26, 1890 MSE/NASTRAN $10 / 15 / 87$ PAGE

DIFE ICULT COMPONENTS GVT COMFIGURATION I (FULL-UPI

THIS VERSIDN CONTAINS A BUILTUP TAILBOOM MODEL

INTERMEDIATE MATRIX ... BAA

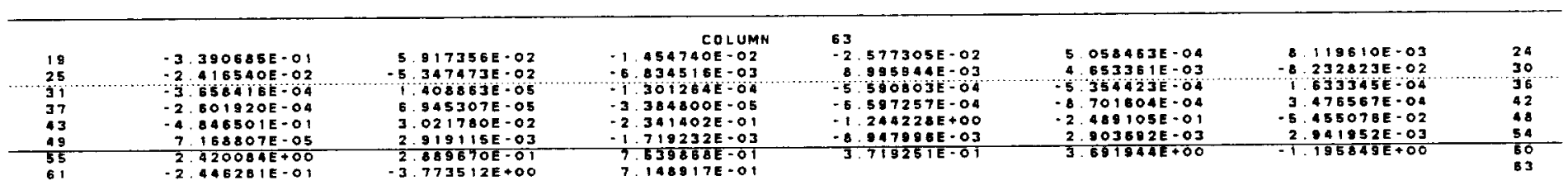


THIS VERSION CONTAINS A BULLTUP TALLOOOM MODEL

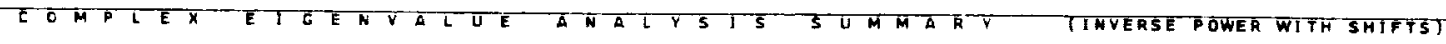

NUMEER OF EIGENVALUES EXTRACTED . . . . . 12

MUMEER OF STARTINE POINTS USED

NUMEER OF STARTING POINT OR SHIFT POINT MOVES O

TOTAL NUMBER OF TRIANGULAR DECOMPOS ITIOUONS

TOTAL NUMEER OF VECTOR JTERATIONS 257

REASON FOR TERMINATION

6

AH - I T THREE-DIMENSIONAL BUILTUP DYMAMICS MODEL W/ CONTROLS MODELEO

THIS VERSION CONTAINS A BUILTUP TAILBOOM MODEL

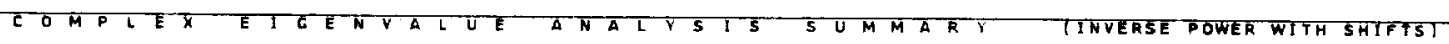

MUMEER OF EIGENVALUES EXTRACTED

10

NUMBER OF STARTTHE POTNTS USEO

1

MUMEER OF STARTINE POINT OR SHIFT POINT MOVES

TOTAL NUMEER OF TRIANGULAR DECOMPOSTTIONS

1

TOTAL NUMEER OF VECTOR ITERATIONS

8

REASON FOR TERMINATION

, 

OIFFICULT COMPONENTS GVT CONFTGURATION 1 (FULL-UP)

THIS VERSION CONTAINS A BUILTUP TAILBOOM MOOEL

COMPLEX ETGENVALUE ANALYSIS 5 U MMA A Y TINVERE POWER WITH SHTFTSI

NUMBER OF EIGENVALUES Extracteo . . . . . . . . 26

NUMBER OF STARTINC POTNTS USED?

NUMBER OF STARTINE POINT OR SHIFT POINT MOVES.

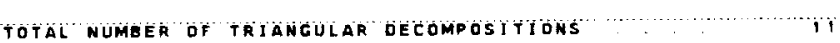

TOTAL NUMEER OF VECTOR ITERATIONS 104

REASON FOR TERMINATION

7

AH- IG THREE-DIMENSIONAL BUILTUP DYMAMIES MODEL W CONTROLS MODELED

DCTOOER 26, 1990 MSC/NASTRAN 10/15/87 PAGE

65

THIS VERSION COMTAINS A BUILTUP TALLBOOM MODEL

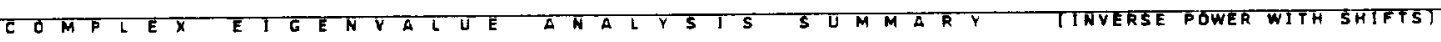

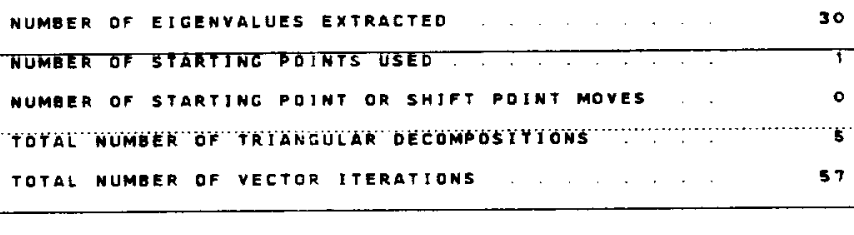

REASON FOR TERMINATION 
THIS VERSION CONTAINS A BUILTUP TAILOOOM MODEL

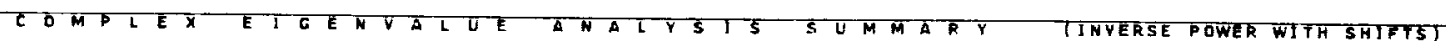

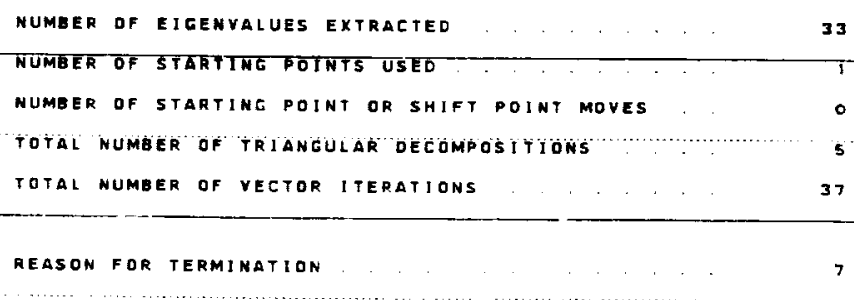

DIFF THREE-DIMENSIONAL OUILTUP DYNAMICS MODEL W/ CONTROLS MODELED

THIS VERSION CONTAINS A BUILTUP TAILBOOM MODEL

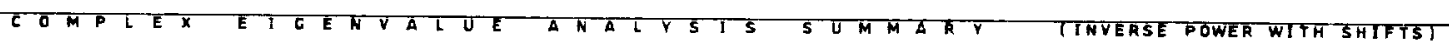

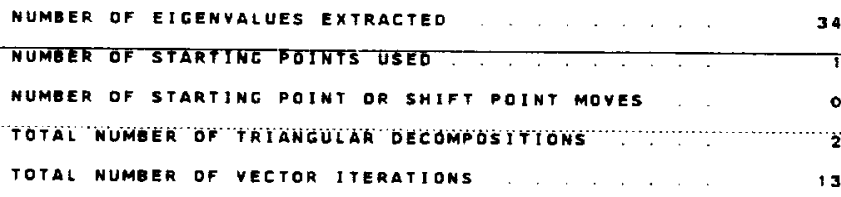

REASON FOR TERMINATION

7 
AH-IC THREE-DIMENSIOMAL BUILTUP OYMAMICS MODEL W/ CONTROLS MDDELED

THIS YERSION CONTAINS A BUILTUP TAILBOOM MODEL

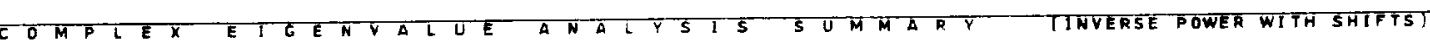

HUMBER OF EIGENYALUES EXTRACTED

35

NUMEER DF ETARTINC POINTS USEO

NUMBER DF STARTING POINT DR SHIFT POINT MOVES

TOTAL NUMBEE OF TAIANCULAR DECOMPOSITIONS

TOTAL NUMBER OF VECTDR ITERATIONS

AH - 10 THREE- DIMENSIOMAL BUILTUP OYNAMICS MODEL W/ CONTRDLS MODELED

OCTOBER 26, 1990 MEC/NASTRAN 10/15/87 PAGE

DIFF ICULT COMPONENTS GVT CONFIGURATION I (FULL-UP)

THIS VERSION CONTAINS A BUILTUP TAILBOOM MODEL

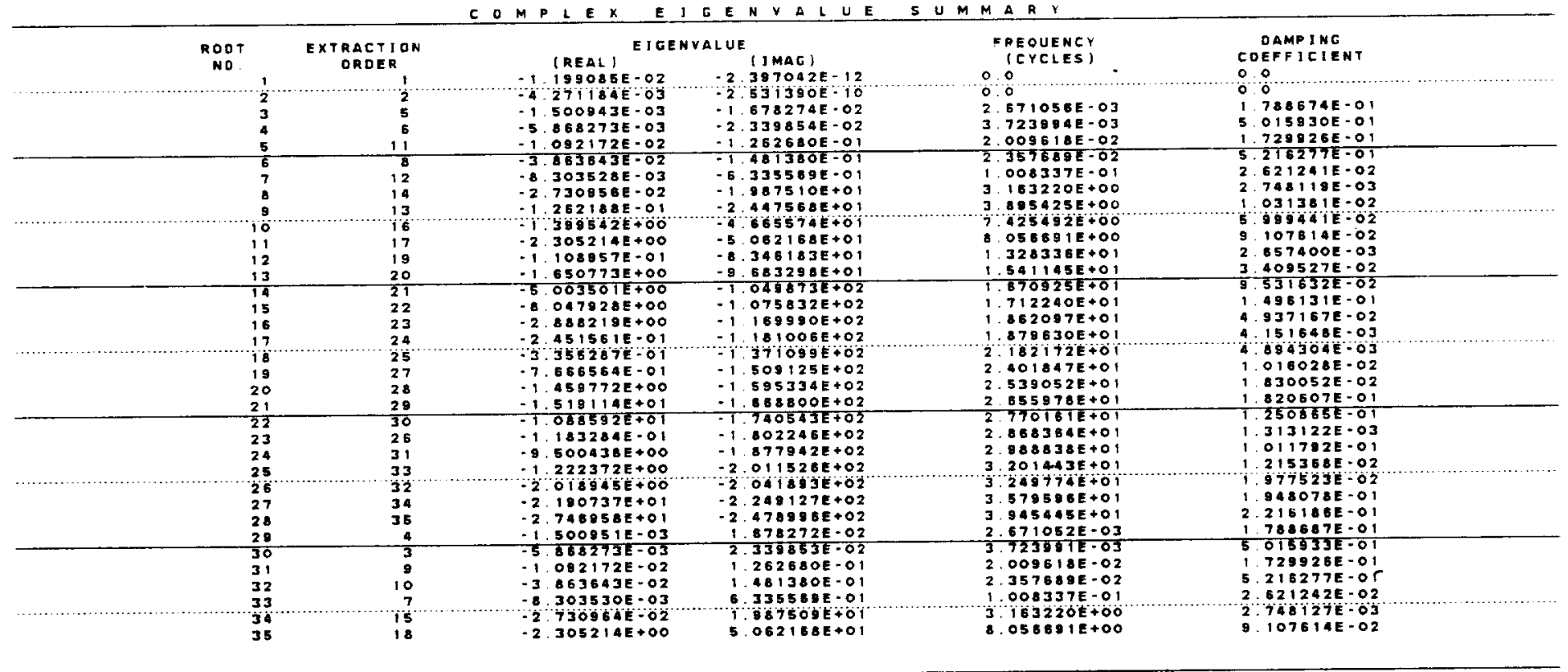


AH-IG THRE - DIMENSIONAL BUILTUP DYMAMICS MODEL W/ CONTROLS MDDELED

DIFFICULT COMPONENTS GVT CONFIGURATION I IFULL-UPI

DCtober 26,1890 MSC/nAStran 10/15/8? PAEE, 7

THIS VERSION CONTAINS A QUILTUP TAILBOOM MODEL

inTERMEDIATE MATRIX ... UaV

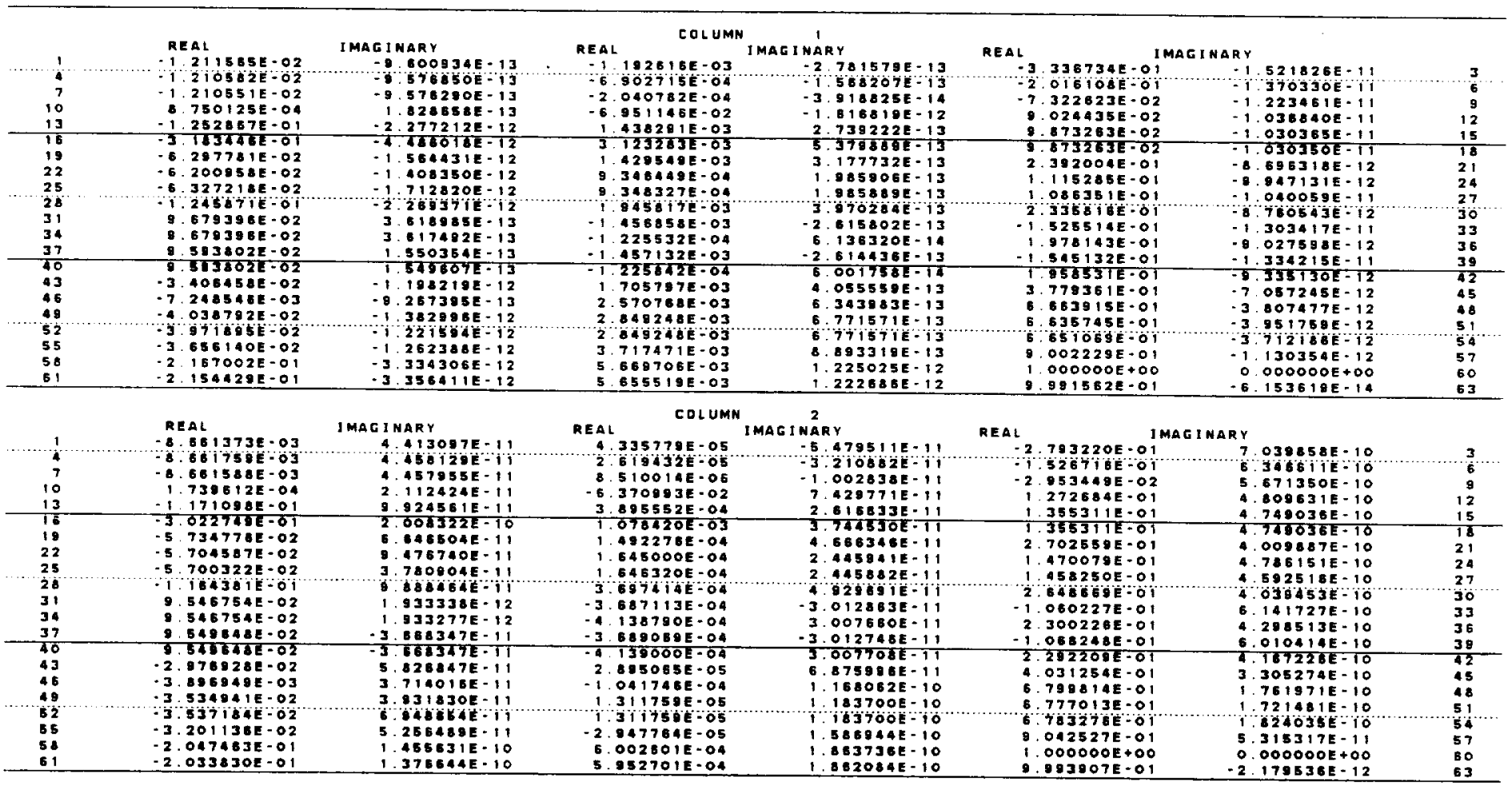




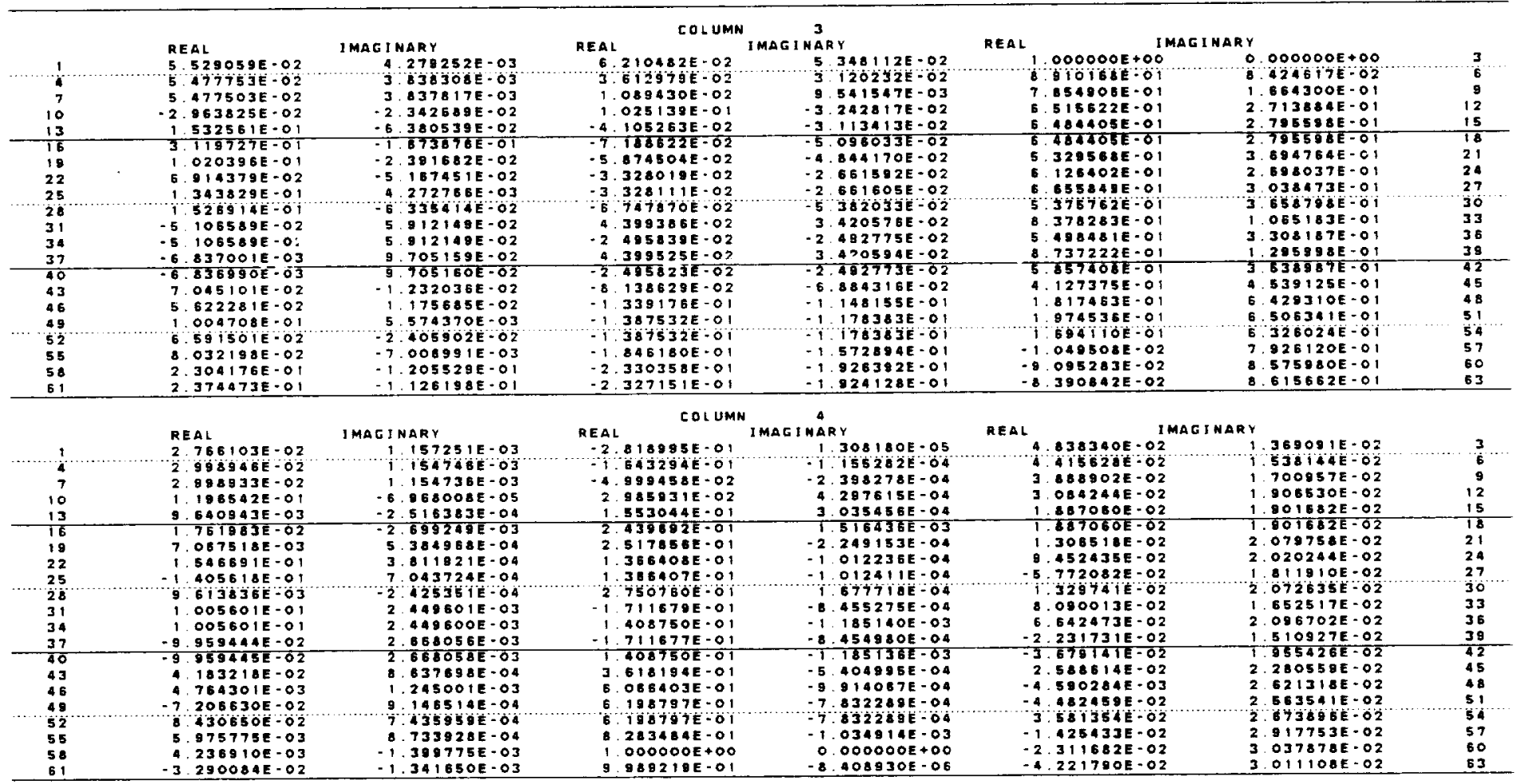

AH-IE THREE-DIMENSIONAL BUILTUP DYMAMICS MODEL W/ CONTROLS MODELED

\begin{tabular}{|c|c|c|c|c|c|c|c|}
\hline $\begin{array}{l}1 \\
1 \\
7 \\
10 \\
13 \\
16 \\
19 \\
22 \\
25 \\
28 \\
31 \\
34 \\
37 \\
40 \\
43 \\
46 \\
49 \\
52 \\
55 \\
58 \\
61\end{array}$ & 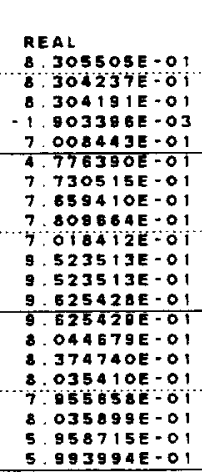 & 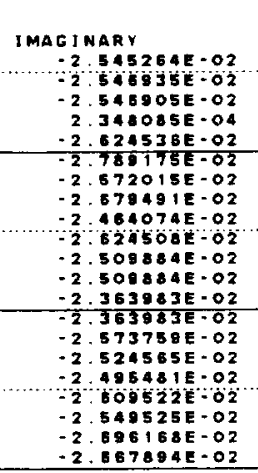 & 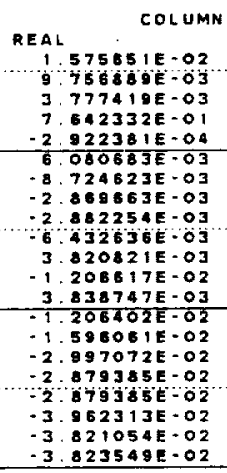 & 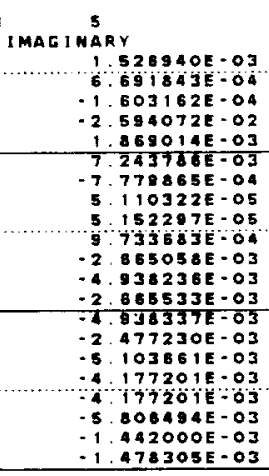 & 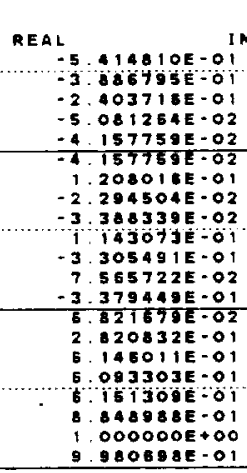 & 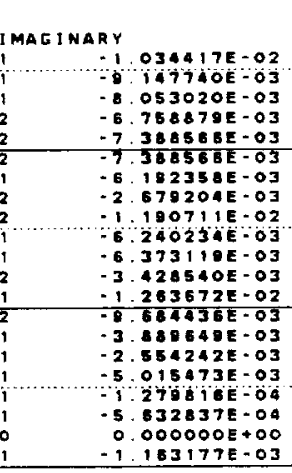 & $\begin{array}{r}3 \\
3 \\
9 \\
12 \\
15 \\
18 \\
18 \\
21 \\
24 \\
27 \\
30 \\
33 \\
36 \\
39 \\
42 \\
45 \\
48 \\
51 \\
54 \\
57 \\
60 \\
53 \\
\end{array}$ \\
\hline $\begin{array}{r}1 \\
4 \\
7 \\
10 \\
13 \\
16 \\
16 \\
22 \\
25 \\
28 \\
31 \\
34 \\
37 \\
40 \\
43 \\
48 \\
48 \\
52 \\
58 \\
58 \\
81\end{array}$ & 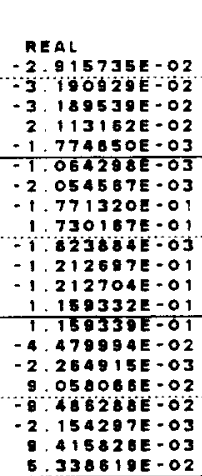 & 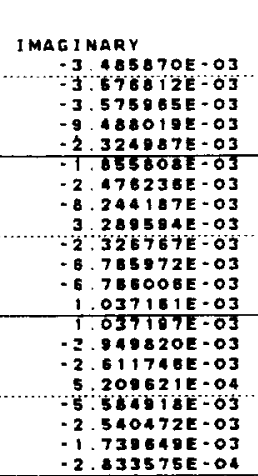 & 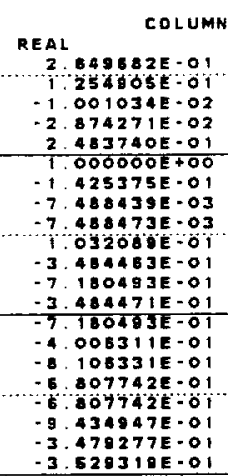 & 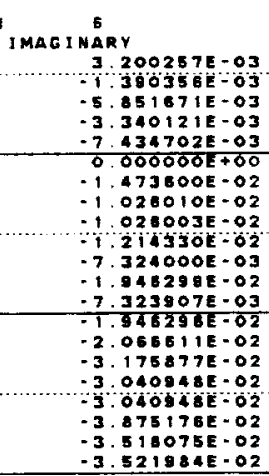 & 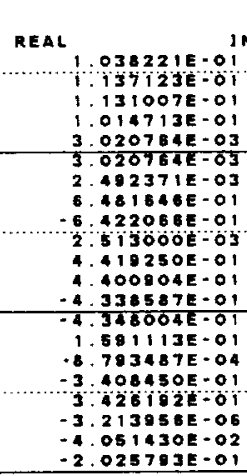 & 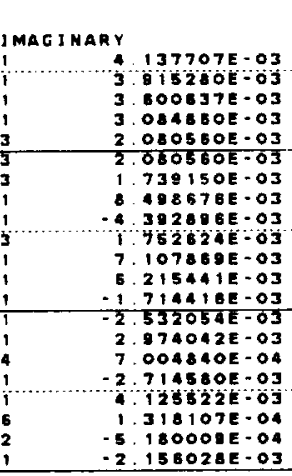 & $\begin{array}{l}3 \\
36 \\
8 \\
22 \\
15 \\
18 \\
21 \\
24 \\
27 \\
30 \\
33 \\
36 \\
30 \\
32 \\
45 \\
48 \\
51 \\
54 \\
57 \\
50 \\
53\end{array}$ \\
\hline
\end{tabular}




\begin{tabular}{|c|c|c|c|c|c|c|c|c|c|c|c|c|}
\hline & R & EAL & I MAG INARY & REAL & COLUMN & 1 MAG I NAPA & & REAL & & ARY & & \\
\hline 1 & $\therefore 2$ & $115519 E-02$ & $-1.293298 E-03$ & 8 & $150287 E-01$ & 2 & $663443 E-02$ & -4 & $.887138 E \cdot 02$ & 4. & $.548651 E-03$ & \\
\hline 4 & -2 & उ $17779 E-O 2$ & $\because 1016861 E-03$ & 8 & $126887 E-O$ & 2 & $03509 A E-O Z$ & $\because 5$ & MQ8B IIE 02 & 4 & OOtsडiE-O3 & $\frac{3}{6}$ \\
\hline 7 & -2 & $324441 E-02$ & $-1.417515 E-03$ & 7 & $130508 E-01$ & 1. & $42449 B E-02$ & -5 & $.582487 E-02$ & & $.808428 E \cdot 03$ & 9 \\
\hline 10 & 4 & $781860 E-01$ & 1. $516969 \mathrm{E}-02$ & -2 & $053382 E-02$ & -1 & $241463 E-03$ & -5 & $.0733 B 0 E \cdot 02$ & 4. & $231516 E-03$ & 12 \\
\hline 13 & .4 & $687927 E-04$ & $5.635769 E .06$ & 3. & $471460 E-01$ & 2. & $489328 E-02$ & 8. & $.591244 E-04$ & 8. & $723723 E-06$ & 15 \\
\hline $\begin{array}{l}15 \\
19\end{array}$ & 1 & & $1.769568 E-04$ & 4 & $7528735 \cdot 02$ & 5 & $7225201-02$ & 8. & $.591249 E-04$ & & $.723746 E-06$ & 18 \\
\hline $\begin{array}{l}19 \\
22\end{array}$ & -1 & $.058517 E-03$ & $-4.485183 E-05$ & 3 . & $657695 E-01$ & 7 & $.810210 E-03$ & -2 & $.218044 E \cdot 04$ & - 5 & $297303 E-05$ & 21 \\
\hline $\begin{array}{l}22 \\
25\end{array}$ & $-?$ & $.292073 E-01$ & $-7,924987 E-03$ & 4. & $.585521 E-01$ & 1. & $389745 E-02$ & -3 & $.379548 E-01$ & 2. & $763385 E-02$ & 24 \\
\hline 25 & $\cdot i$ & $\begin{array}{l}271132 E-01 \\
708168 E-04\end{array}$ & $7.8358505-03$ & 4 & $655502 E-01$ & 1. & $389738 E-02$ & 3. & $.304882 \mathrm{E}-01$ & 2 & $763106 \mathrm{E}-02$ & 27 \\
\hline 31 & .8 & $\begin{array}{l}708168 E-04 \\
984834 E-02\end{array}$ & $\begin{array}{r}7.7231416-07 \\
-5.514383 E-03\end{array}$ & 2 & $4409711-01$ & $i$. & $.834588 E 02$ & & $6 A 295 E=0 A$ & 8. & $85+3368-05$ & 30 \\
\hline 34 & -8 & $984368 E-02$ & $-5.514353 E-03$ & 7 & $\begin{array}{l}000000 E+00 \\
245778 E-01\end{array}$ & $\begin{array}{r}0 \\
-1\end{array}$ & $\begin{array}{l}000000 E+00 \\
673238 E-02\end{array}$ & $\begin{array}{l}-2 \\
-2 .\end{array}$ & $303173 E-01$ & 1. & $.887885 E-02$ & 33 \\
\hline 37 & & $5304280-02$ & $5.181483 E-03$ & 9. & $\begin{array}{l}245778 E-01 \\
999849 E-01\end{array}$ & $\begin{array}{l}-1 \\
-3\end{array}$ & $\begin{array}{l}673238 E-02 \\
979621 E-07\end{array}$ & $\begin{array}{r}-2 . \\
2 .\end{array}$ & $.299086 E \cdot 0 !$ & 1 & $866500 E-02$ & 35 \\
\hline 20 & & $.530147 E \cdot 02$ & $5.101440 \mathrm{E}-03$ & 7. & $245747 E-01$ & $=1$ & $\begin{array}{r}979621 E-07 \\
693246 E-02\end{array}$ & $\frac{2}{2}$ & $\begin{array}{l}360677 E \cdot 0: \\
.301212 E-6 !\end{array}$ & $\frac{-1}{-1}$ & $\frac{.04123 E-02}{.875054 E-02}$ & $\frac{39}{42}$ \\
\hline 43 & -3 & $257600 E-02$ & $-1.886508 E-03$ & 3. & $172856 E-09$ & -3 & $567727 E-03$ & -8 & .38514 BE-O2 & 6 & $533702 E-03$ & $\begin{array}{l}42 \\
45\end{array}$ \\
\hline 46 & -1 & $.483122 E-03$ & $-8.528076 E-05$ & 1. & $485401 E-01$ & -2 & $180226 E-02$ & -3. & $.463671 E-03$ & -4. & $.044601 E-04$ & 48 \\
\hline 49 & 8 & $683648 E-02$ & $4115684 E-03$ & 8. & $302114 E-02$ & -1. & $623960 E-02$ & 1. & $761502 E-01$ & -1. & $502880 E-02$ & 51 \\
\hline 52 & -6 & $930366 E-02$ & -423 B 49 IE & 8 & $302114 E-02$ & 11 & $623960 E-O 2$ & -1 & $830464 E-01$ & $i$ & $422276 E-02$ & 54 \\
\hline 55 & -1 & $.260166 \mathrm{E} \cdot 03$ & $.8 .391983 E-05$ & -9. & $273228 E-02$ & -2. & $004104 E \cdot 02$ & -5. & $.241606 E-03$ & -5 & $.742689 E-04$ & 57 \\
\hline 58 & 8 & $\begin{array}{l}100725 E \cdot 03 \\
037533 E \cdot 02\end{array}$ & $\begin{array}{l}5.521141 \mathrm{E}-04 \\
2.526885 \mathrm{E}-03\end{array}$ & $\begin{array}{l}-5 \\
-5\end{array}$ & $386625 E-01$ & -3 & $842734 E \cdot 03$ & 1 & $508788 E-02$ & -2 & $356338 E-03$ & EO \\
\hline & & $037533 E \cdot 02$ & $2.5268 \Delta 5 E-03$ & -5 . & $353929 E-01$ & -3 & $.055544 E-03$ & 1. & $.002928 E-01$ & -9 & $267196 E-03$ & 63 \\
\hline & & & & & COLUMN & -1 & & & & & & \\
\hline & $-\mathbf{R}$ & $532+78 E-01$ & IMAE INARY & REAL & & I MAEINAR & & REAL & & ARY & & \\
\hline 4 & $\cdots$ & & $\begin{array}{l}004152 E-04 \\
001622 E-04\end{array}$ & $\ldots n^{2}$ & $063131 E-03$ & 9 & $565451 E \cdot 05$ & $\therefore 2$ & $414964 E-01$ & & $387604 E \cdot 03$ & 3 \\
\hline 4 & -1 & $\begin{array}{l}53280 E=01 \\
53240 E=01\end{array}$ & $\begin{array}{l}1.001022 E-04 \\
7.63762 E-05\end{array}$ & है. & $9078611-04$ & 4 & $.535146 E-05$ & $\because \mathrm{i}$ & $627937 E-O i$ & s. & $684176 E-04$ & 6 \\
\hline 10 & & $.400937 E \cdot 03$ & & $\begin{array}{l}-7 \\
-1\end{array}$ & $389512 E-04$ & -6. & $605218 E \cdot 06$ & -8 & $759537 \mathrm{E}-02$ & 5. & $061846 E-04$ & $\mathbf{9}$ \\
\hline 13 & & $100220 E-01$ & $\begin{array}{r}1.054104 E-04 \\
4.281835 E-04\end{array}$ & $\begin{array}{l}-1 \\
-1\end{array}$ & $\begin{array}{l}84053 E-01 \\
535033 E-003\end{array}$ & 3 & $558792 E \cdot 04$ & 5 & $778042 E-03$ & -2 & $570415 E-04$ & 12 \\
\hline T6 & & $.00 \delta 600 E+00$ & $0.000000 E+00$ & $\frac{-1}{3}$ & $\frac{535933 E-03}{517024 E-03}$ & $-\frac{1}{2}$ & $008646 E-04$ & $=1$. & $878560 E-02$ & -2 & $927782 E-0.4$ & 15 \\
\hline 19 & $-i$ & O4073IE-01 & $1.841643 E-0 Q$ & -8 & $223231 E-04$ & $\begin{array}{c}2 . \\
-8\end{array}$ & $\begin{array}{l}078536 E-04 \\
131718 E-05\end{array}$ & $\begin{array}{r}-1 \\
7\end{array}$ & $8802665-02$ & -2 & 920204E-04 & 18 \\
\hline 22 & -1 & $838293 E-01$ & $2.514800 E-0 Q$ & -1 & $757392 E-03$ & $\begin{array}{l}-8 \\
-1\end{array}$ & $\begin{array}{l}B, 31718 E=05 \\
115034 E=04\end{array}$ & $?$ & $632538 E-02$ & -8 & $921999 E-04$ & 21 \\
\hline 25 . & -1 & $8185808-01$ & $2923056 E-00$ & -4 & $104780 E-04$ & $\begin{array}{l}-1 \\
-9\end{array}$ & $015034 E \cdot 04$ & 9 & $544524 E-03$ & -5 & $215141 E-O 4$ & 24 \\
\hline 28 & -2 & $233524 E-O I$ & J $278535 E=04$ & $i$ & $275952 E-03$ & -1 & प32381E-OA & -1 & $010446 E-03$ & -2 & $407575 E-04$ & 27 \\
\hline 31 & $-g$ & $088850 E-02$ & -4 40A4BJE-04 & -5 & $251486 E-04$ & 5 & $477894 E-05$ & $\begin{array}{l}7 \\
-1\end{array}$ & $310231 E-02$ & 8 & $674380 E-04$ & 30 \\
\hline 34 & -9 & $.87840 E-02$ & $-4,308483 E-04$ & -6 & $629441 E \cdot 03$ & 2 & $701809 E-05$ & -1. & $\begin{array}{l}275860 E-O 1 \\
180470 E-02\end{array}$ & 8 & A44986E-04 & 33 \\
\hline 37 & & $885027 E \cdot 02$ & $-4.288921 E-04$ & -6. & $834058 E-03$ & $i$ & $217828 E-04$ & $-i$ & $\begin{array}{r}180479 E-02 \\
323651 E-01\end{array}$ & -9 & $039076 E-04$ & 36 \\
\hline 40 & -9 & $885511 E \cdot 02$ & $-4.2526348-04$ & -6 & $256563 E-03$ & $i$ & $021118 E-05$ & 4. & $\begin{array}{r}.323651 E-01 \\
.349952 E-02\end{array}$ & $\frac{1}{-7}$ & $\frac{132602 E-03}{091322 E-04}$ & $-\frac{39}{42}$ \\
\hline 43 & -1 & $681191 E-01$ & 1.224924E-04 & $-\mathbf{g}$ & $316362 E-04$ & -1 & $256871 E-06$ & 1. & $654023 E-01$ & -1 & $152140 E-03$ & 45 \\
\hline 46 & & $461846 E \cdot 01$ & 2.965601E-04 & 1 & $431204 E-03$ & 2 . & $498484 E-04$ & 3. & S491AOE-OI & $-i$ & $599688 E \cdot O 4$ & 48 \\
\hline 49 & $\therefore 1$ & $758016 E-01$ & $-8 \quad 140873 E-05$ & 2 & $239098 E-03$ & 2 . & $074704 E-04$ & 3 & $829772 E-01$ & -7 & $362901 E-05$ & 51 \\
\hline 32 & & $763458 E-01$ & $9.620030 \mathrm{E}-05$ & 2 & $23992 \mathrm{E}-03$ & 2 & $074703 E=04$ & 3 & $873783 E-01$ & -2 & $941671 E=O A$ & 54 \\
\hline 55 & & $697388 E-01$ & $1.586353 E-04$ & 4 & $394588 E-03$ & 3. & $.310770 E-04$ & 6 . & $.065567 E-01$ & 2. & $747857 E \cdot 03$ & 57 \\
\hline 58 & -3 & $602760 E-01$ & $-2.915147 E-03$ & -5 & $635612 E-03$ & -3 & $064025 E-04$ & 7. & $147202 E-01$ & 4. & $539274 E-03$ & BO \\
\hline 61 & -3 & $052657 E-01$ & $-3.016591 \mathrm{E}-03$ & -5 & $483424 E-03$ & 2. & $883725 E-04$ & 7. & $.253358 \mathrm{E}-01$ & 4. & $847234 E-03$ & 63 \\
\hline
\end{tabular}

\footnotetext{
AH-1E THREE-OIMENSIONAL BUILTUP DYNAMICS MODEL W/ CONTROLS MDOELEO

OCTOBER

26, 1990 MSC/NASTRAN 10/15/87

PAGE 75

THIS VERSION CONTAINS A BUITUP TAILBOOM MOOEL

INTERMEDIATE MATRIX ... UAV
}

\begin{tabular}{|c|c|c|c|c|c|c|c|}
\hline $\begin{array}{r}1 \\
4 \\
7 \\
10 \\
13 \\
16 \\
19 \\
22 \\
25 \\
28 \\
31 \\
34 \\
37 \\
40 \\
43 \\
45 \\
49 \\
52 \\
55 \\
58 \\
51 \\
\end{array}$ & 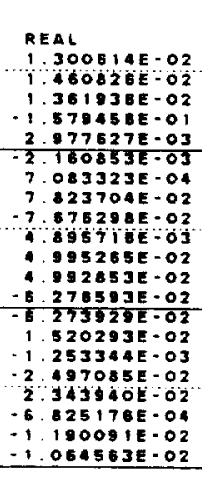 & 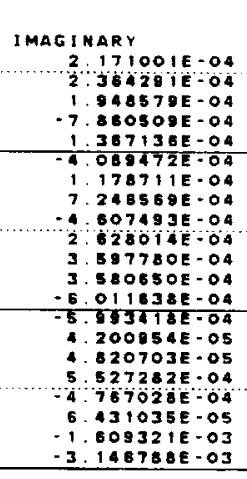 & 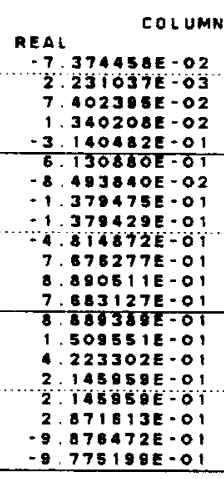 & 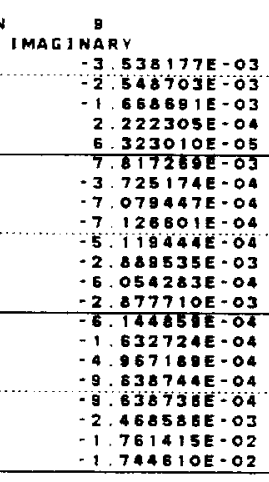 & 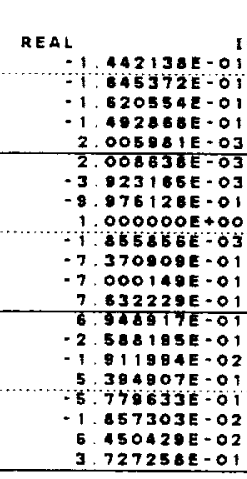 & 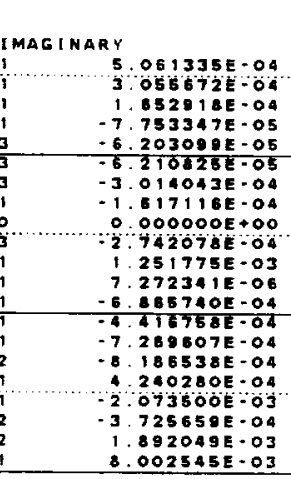 & $\begin{array}{r}3 \\
6 \\
9 \\
12 \\
15 \\
15 \\
21 \\
24 \\
27 \\
30 \\
33 \\
36 \\
39 \\
42 \\
45 \\
48 \\
51 \\
54 \\
57 \\
60 \\
63\end{array}$ \\
\hline $\begin{array}{r}1 \\
4 \\
7 \\
10 \\
13 \\
18 \\
19 \\
22 \\
25 \\
23 \\
31 \\
34 \\
37 \\
40 \\
43 \\
46 \\
40 \\
82 \\
55 \\
88 \\
61\end{array}$ & 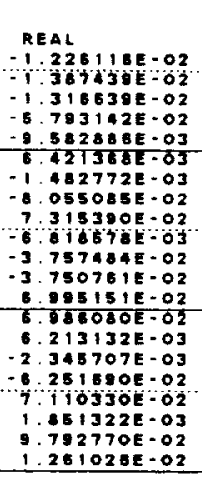 & 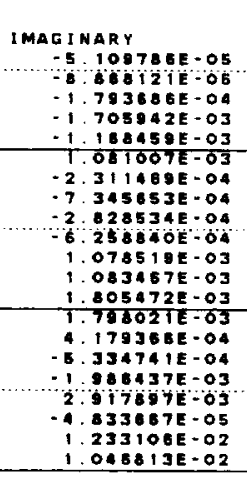 & 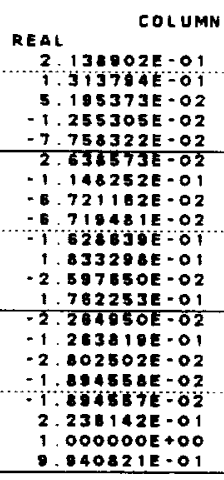 & 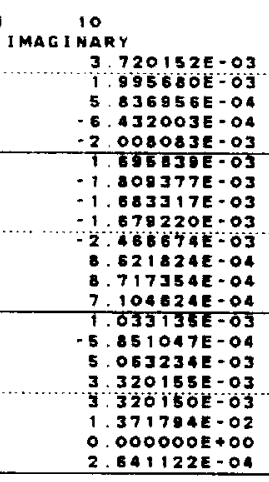 & 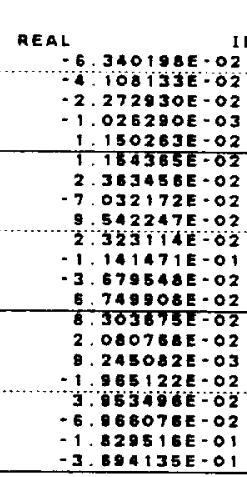 & 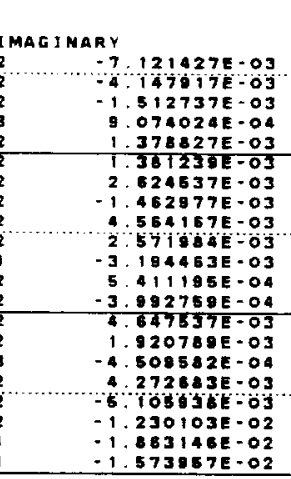 & $\begin{array}{r}3 \\
36 \\
9 \\
12 \\
15 \\
18 \\
21 \\
24 \\
27 \\
30 \\
33 \\
33 \\
39 \\
42 \\
45 \\
48 \\
51 \\
54 \\
57 \\
60 \\
63\end{array}$ \\
\hline
\end{tabular}


AH - IC THREE-DIMENSIONAL OUILTUP DYNAMICS MODEL W/ CONTROLS MODELED

\begin{tabular}{|c|c|c|c|c|c|c|c|c|}
\hline $\begin{array}{r}1 \\
4 \\
7 \\
10 \\
13 \\
16 \\
19 \\
22 \\
25 \\
28 \\
31 \\
31 \\
37 \\
40 \\
43 \\
46 \\
49 \\
52 \\
55 \\
58 \\
61 \\
\end{array}$ & 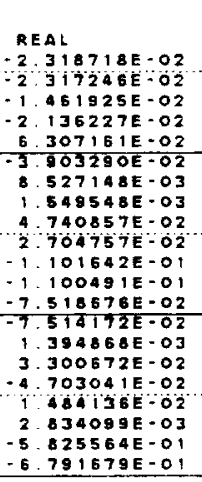 & 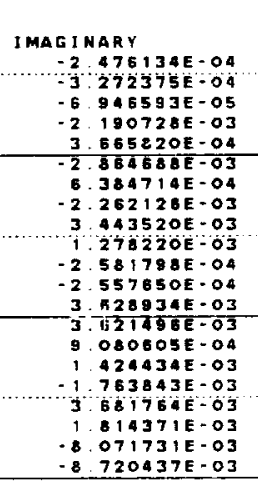 & 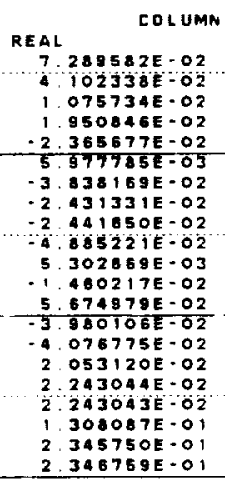 & 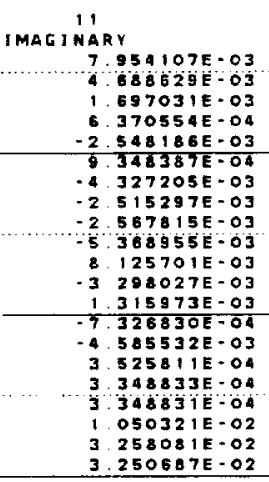 & 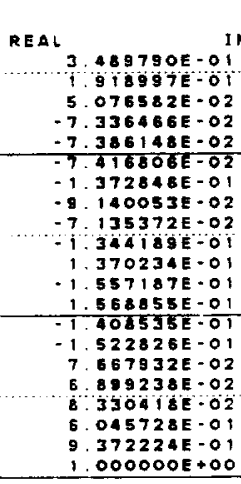 & 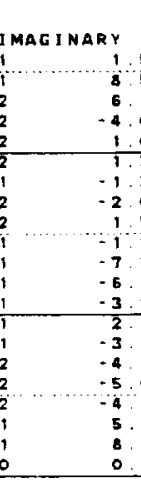 & 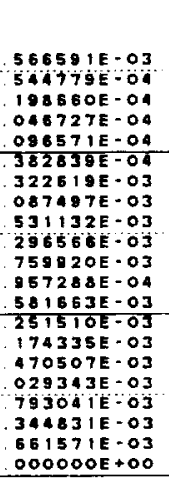 & $\begin{array}{r}3 \\
35 \\
9 \\
12 \\
15 \\
15 \\
18 \\
21 \\
24 \\
27 \\
30 \\
33 \\
36 \\
39 \\
-39 \\
45 \\
48 \\
51 \\
54 \\
57 \\
60 \\
63 \\
\end{array}$ \\
\hline $\begin{array}{l}1 \\
24 \\
7 \\
10 \\
13 \\
16 \\
16 \\
22 \\
25 \\
28 \\
31 \\
34 \\
37 \\
40 \\
43 \\
46 \\
49 \\
52 \\
55 \\
58 \\
61\end{array}$ & 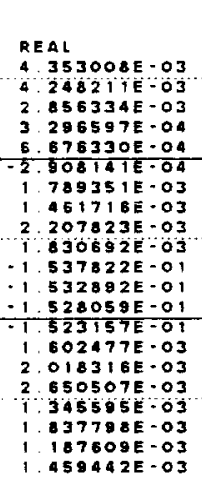 & 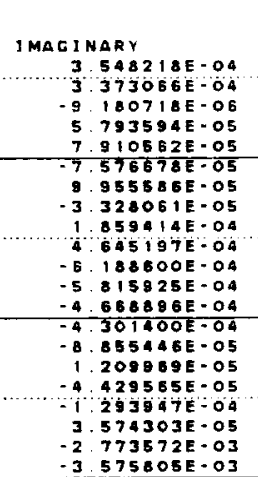 & 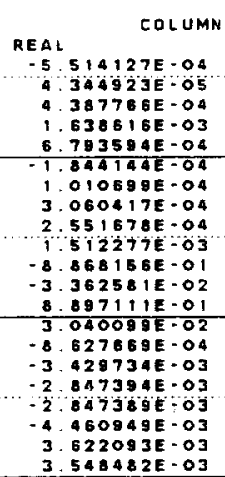 & 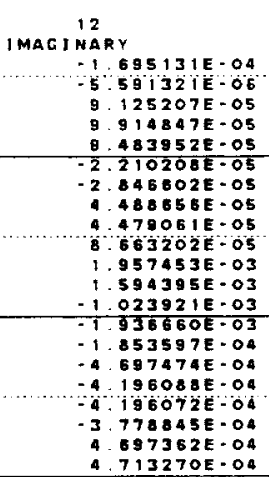 & 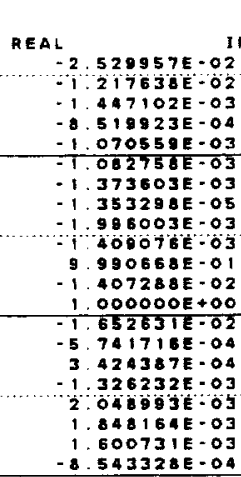 & $\begin{array}{r}\text { I NARY } \\
-2 \\
-3 \\
7 \\
3 \\
3 \\
3 \\
-3 \\
3 \\
3 \\
-5 \\
-5 \\
-1 \\
0 \\
-1 \\
-1 \\
-1 \\
-1 \\
-1 \\
-1\end{array}$ & 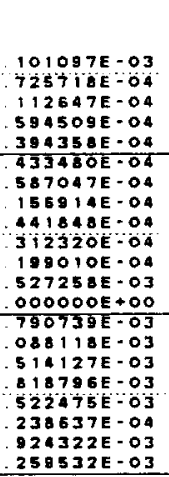 & $\begin{array}{r}3 \\
6 \\
9 \\
9 \\
12 \\
15 \\
18 \\
21 \\
24 \\
27 \\
30 \\
33 \\
36 \\
39 \\
42 \\
45 \\
48 \\
51 \\
54 \\
57 \\
60 \\
63\end{array}$ \\
\hline
\end{tabular}

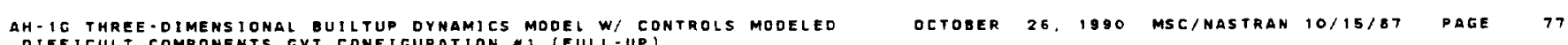
DHF ISERSION CONTAINS A BUILTUP TAILOOOM MODE NTERMEDIATE MATRIX ... UaY

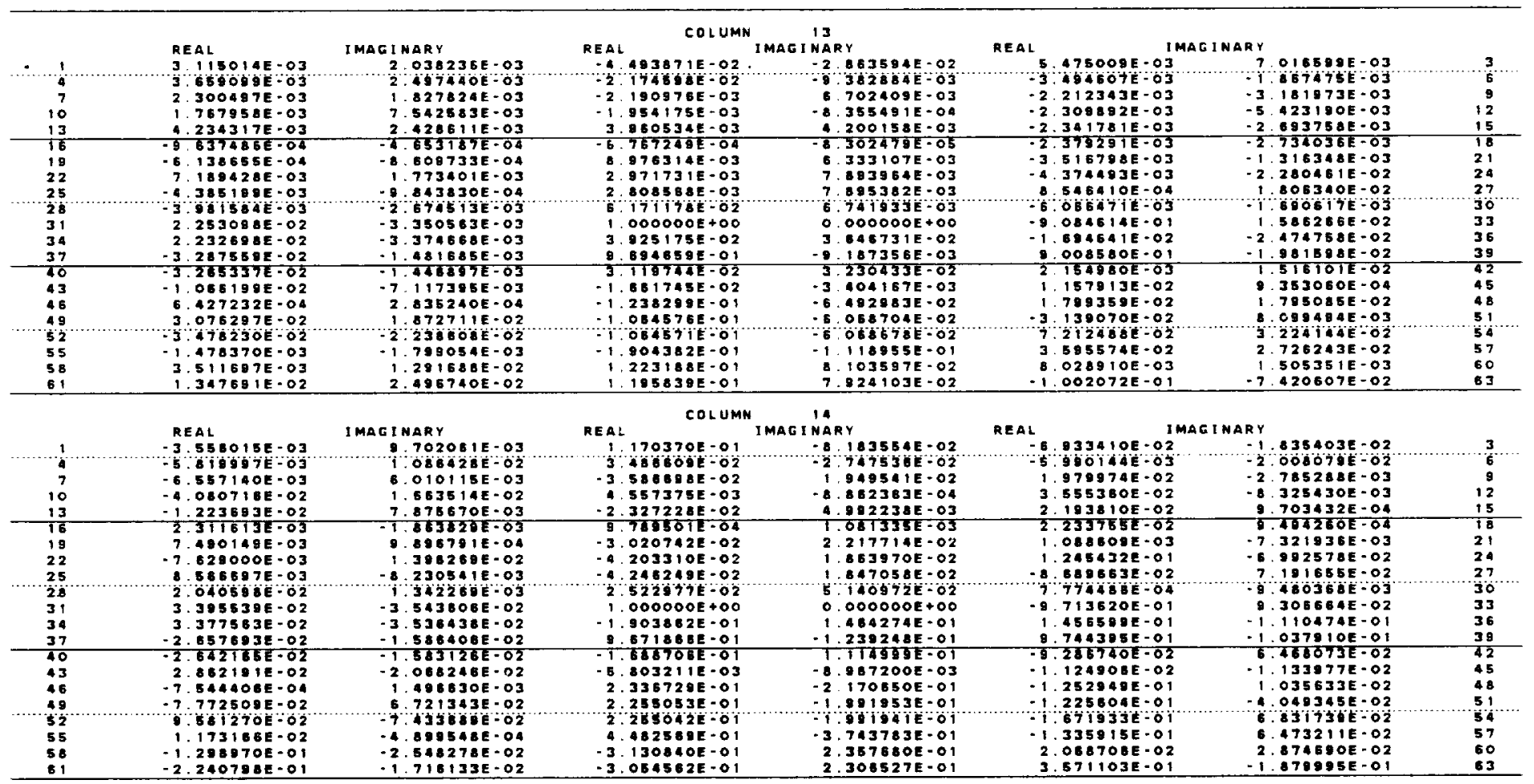




\begin{tabular}{|c|c|c|c|c|c|c|c|c|c|c|c|c|c|}
\hline & & & IMAG II & NARY & REAL & COLUMM & IMAGJ 15 & RY $Y$ & REAL & & I MAC I MARY & & \\
\hline 1 & -5 & $458273 E-02$ & -5 & $559814 E-03$ & & $000180 E-01$ & a & $295: 27 E-02$ & 3 & $000828 E-01$ & 1 क्षा & $330581 E+02$ & 3 \\
\hline 4 & -5 & $327127 E=02$ & $\because 6$ & $163789 E-03$ & 2 & USBTSGE-OZ & $\because$ & 2 ITASEE-O2 & $\mathrm{i}$ & $402878 E-01$ & 9 & OESZGBE OJ & $\mathbf{6}$ \\
\hline 7 & -1 & $.822859 E-02$ & -7 & $000361 E-04$ & -3. & $.520837 E-02$ & -1 & $291562 E-02$ & -8. & $.76116 E-O 2$ & 2. & $800045 E-03$ & 9 \\
\hline 10 & -3 & $.483170 \mathrm{E}-02$ & -1 & $.191328 E-02$ & -2 & $026510 E-02$ & 1 & $337058 E-03$ & -8 & $16918 E-02$ & 3 & Q72537E-03 & 12 \\
\hline-13 & $\frac{1}{3}$ & $.756014 E-02$ & -1 & $083398 E-03$ & -9. & $800395 E-02$ & -5 & $301765 E-03$ & -8. & $235298 \mathrm{BE}-02$ & -4 & $123008 E-04$ & 15 \\
\hline $\begin{array}{l}16 \\
19\end{array}$ & $\begin{array}{l}-2 \\
.3\end{array}$ & $\begin{array}{l}.659080 E-03 \\
382,11 E-02\end{array}$ & -1 & $.844035 E-04$ & $\overline{9}$ & $521696 E-03$ & -3 & $17697406-04$ & -9 & $410698 E-02$ & -1 & 517890E-OA & 18 \\
\hline 22 & 1 & $262034 E .02$ & -1 & $\begin{array}{r}\text { O8B429E-O3 } \\
451881 E-03\end{array}$ & $\begin{array}{l}-1 \\
-3\end{array}$ & $200032 E-03$ & -1 & $111280 E-02$ & 3 & $354159 E-02$ & -1 & $507078 E-03$ & 21 \\
\hline 25 & +3 & $758733 E-02$ & 1 & $077501 E-03$ & $\begin{array}{l}-3 \\
-3\end{array}$ & $\begin{array}{l}274840 E-02 \\
O 87650 E-02\end{array}$ & $\begin{array}{l}-1 \\
-1\end{array}$ & $\begin{array}{l}502449 E-02 \\
540327 E-02\end{array}$ & $\begin{array}{l}-7 \\
-7\end{array}$ & $\begin{array}{l}966019 E-02 \\
160874 E-02\end{array}$ & $\begin{array}{r}3 \\
-4\end{array}$ & $746847 E-O 2$ & 24 \\
\hline 28 & -1 & $112+37 E=01$ & -2 & OSOGOTE-OS & $\because 8$ & $714545 E=03$ & $\because 1$ & $\begin{array}{l}540327 E-02 \\
242007 E-04\end{array}$ & $\begin{array}{r}-7 \\
\cdots 2\end{array}$ & $\begin{array}{l}160874 E-02 \\
870713 E-O 2\end{array}$ & -4 & $189057 \mathrm{E}-02$ & 27 \\
\hline 31 & $i$ & $.055185 E-0$ & -5 & $174748 E-02$ & -2 & $848331 E-01$ & 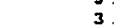 & उ73AT3E-O1 & .8 & $121744 E-02$ & $\begin{array}{l}-1 \\
-2\end{array}$ & $\begin{array}{l}362032 E-O 3 \\
30775 A E-01\end{array}$ & $\begin{array}{l}30 \\
\mathbf{3 3}\end{array}$ \\
\hline 34 & & $.052823 E-01$ & -5 & $157828 E-02$ & -7 & $105782 E-03$ & -5 & $288103 E-02$ & .4 & $006092 E-02$ & $\begin{array}{r}-2 \\
1\end{array}$ & $\begin{array}{l}30775 \Delta E-O 1 \\
\text { ST71 }\end{array}$ & 36 \\
\hline 37 & 3 & $.819400 E-02$ & -6 & $.001037 E-02$ & 3 & $167092 E-01$ & 2. & $.826882 E-01$ & 1 & $585088 \mathrm{~B}-01$ & 3 & $776299 E-01$ & 39 \\
\hline 40 & 3 & $.8150 \overline{61 E .02}$ & -6 & $580050 \overline{0}-02$ & 2 & $558199 E-02$ & $=8$ & $281395 E-O 2$ & -4 & 63983ठE-Ó & -7 & $215055 E=02$ & 42 \\
\hline 43 & a & $.468896 E-03$ & 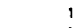 & $071320 E-02$ & 5 & $201170 E-02$ & 2. & $.006977 E-03$ & $i$ & $002651 E-01$ & 9 & $255030 E-03$ & 45 \\
\hline 46 & -5 & $.40055 B E-03$ & 8 & $908151 E-04$ & 2 & $024878 E-01$ & 9. & $979301 E-02$ & 4 & $358533 \mathrm{EE}-01$ & 1 & $514562 E-02$ & 48 \\
\hline 49 & -4 & $263464 E-02$ & $\therefore 3$ & $124985 E-02$ & 1 & $\triangle 84873 E-01$ & 9. & 5692995.02 & 5 & $559378 E-01$ & 1. & $544748 E-02$ & 51 \\
\hline 52 & 2 & $164965 E-02$ & 3 & 4010 AIEOQZ & $i$ & $864862 E-01$ & 9 & $569261 E-02$ & 4 & BSIAZSE-OI & -5 & $54894 J E=O 3$ & 54 \\
\hline 55 & -3 & $706245 E-02$ & -3 & $828551 E-03$ & 2 & $121825 E-01$ & $i$. & $704677 E-01$ & 1. & $448916 E-01$ & -2 & $450554 E-O 2$ & 57 \\
\hline $5 a$ & 6 & $458613 E \cdot 01$ & 2 & $982302 E \cdot 02$ & $-i$ & $481012 E-01$ & $-i$ & $2467 B 1 E-01$ & -2 & $443300 E-01$ & -1 & $592531 E-O 2$ & 60 \\
\hline & 1 & $000000 E+00$ & 0 & $000000 E+00$ & -1 & $494482 E-01$ & -1 & $22938 \Delta E-01$ & -3 & $560432 E-01$ & 1. & $124220 E-01$ & 63 \\
\hline & & & & & & COLUMN & 16 & & & & & & \\
\hline & & & IMACIN & NARY & REAL & & IMAGINAR & & REAL & & MAG INARY & & \\
\hline & 1 & $525051 E-02$ & -6 & $944949 E-04$ & -2 & $693039 E-01$ & 5 . & $O B 4816 E-O 2$ & 6 . & $.384829 E-O 2$ & -2 & $696142 E-02$ & 3 \\
\hline 4 & & $770021 \mathrm{E}-02$ & $\because$ & SOS1AEE OJ & -1 & $119833 E=0 t$ & 2 & $007249 E-02$ & 3 & $740730 E=O Z$ & $B$ & $O T G 3 B 5 E-O J$ & 6 \\
\hline 7 & & $.35: 205 E-02$ & -2 & $252999 E \cdot 03$ & 3 & $044206 E-02$ & -5 & $046892 E-03$ & 2 & $468201 E-02$ & 1. & $018913 E-03$ & 9 \\
\hline 10 & & $587378 E-01$ & -2 & $791906 E-02$ & 3 & $009202 E-03$ & -8 & $485918 E-04$ & $i$ & $829472 E-02$ & G. & 314150E-03 & 12 \\
\hline 13 & & $308981 E-03$ & -3 & $512187 E-03$ & 3 & $145806 E-01$ & -5 & $502770 E-02$ & -2 & $215418 E-02$ & B & $645303 E-03$ & 15 \\
\hline 16 & & BSOMSAE-O3 & 9 & $028551 E-04$ & -7 & 1230008.02 & 1 & $1857825-02$ & -2 & 2649 IAE-OZ & 8 & 866950-03 & 18 \\
\hline 10 & .6 & $387390 E-03$ & 2 & $029884 E-03$ & -5 & $902757 E-03$ & .1 & $391162 E-02$ & $i$ & $966076 \mathrm{E}-02$ & -1 & $668768 E-03$ & 21 \\
\hline 22 & & $.853789 E-02$ & -1 & $003959 E-02$ & 1 & $476706 E-01$ & -2 & $918406 E-02$ & 2. & BI264IE-OI & -6 & $433652 E-03$ & 24 \\
\hline 25 & & & 1 & $402458 E-02$ & 1 & $499327 E-01$ & -2 & $971495 E-02$ & -3 & $223352 E-0$ & 2 & $199093 E-02$ & $\begin{array}{l}24 \\
27\end{array}$ \\
\hline 28 & & OOEBIEE-OZ & 3 & QBETHJE $O 3$ & $-\mathbf{s}$ & OLTIB LE-OA & 7 & & 4 & $054806 E=02$ & $\overline{6}$ & IAISZTE-OS & 30 \\
\hline 31 & $i$ & $035956 E .01$ & .7 & $7395 / 4 E-O Z$ & 7 & $275767 E-O A$ & 1. & $721817 E-01$ & -6 & $261705 E-01$ & -5 & 1078B4E-02 & 33 \\
\hline 34 & & $.030862 E \cdot 01$ & -7 & $722951 E-02$ & -1 & $202160 E-01$ & $-i$ & $410315 E-02$ & 2 & & -2 & $853436 E-02$ & 36 \\
\hline 37 & .2 & $.39904 E \cdot 03$ & -4 & $.97363 E-02$ & 1 & $000000 E+00$ & 0. & $.000000 E+00$ & 5 & $.786188 \mathrm{E}-01$ & 1 & $278626 E-01$ & 39 \\
\hline 40 & -2 & $.7655365 \cdot 03$ & .4 & $775 B D E-02$ & -7 & $620426 E-02$ & -8 & 4622180.02 & -1 & $.788004 E-0 \mid$ & 5 & $887889 E-02$ & 42 \\
\hline 43 & 2 & $.989535 E \cdot 03$ & 9 & $103249 E-03$ & 2 & B9, 102E-O3 & -1. & $779087 E-02$ & 2 & $.073739 E-03$ & 1. & $410583 E-03$ & 45 \\
\hline 45 & -1 & $.876411 E \cdot 03$ & & $806796 \mathrm{E}-03$ & 8 & $416502 E-02$ & 6. & $.202162 E-02$ & 5. & $.36007 E-02$ & -3 & $432232 E-02$ & 48 \\
\hline 48 & -3 & $.077098 E-02$ & -3 & $043228 E-02$ & 5 & $472256 \mathrm{E}-02$ & 6 . & $109008 E-02$ & & $319264 \mathrm{E}-01$ & -4 & $0 T 1770 E-02$ & 51 \\
\hline 52 & $z$ & $032936 E-02$ & 4 & $086972 E-02$ & E & 4722 BEE-OZ & 6 & $100960 E-O 2$ & 8 & $1795896-03$ & $\because 4$ & 32+BSBE-O2 & 54 \\
\hline 55 & -7 & $185793 E-03$ & .3 & $666380 E-03$ & 1 & $328228 E-01$ & 1 & $578774 E-01$ & 1. & $597585 E-02$ & -4. & $477520 E-02$ & 57 \\
\hline 58 & : & $\begin{array}{r}137070 E-02 \\
338708-01\end{array}$ & -3 & $365309 E-02$ & -4 & $715886 E-02$ & 9 & $082304 E-02$ & -3 & $121405 E-02$ & 2. & $410620 E-03$ & 60 \\
\hline & & $.3388798-01$ & -7 & $893953 E+02$ & -4 & $635114 E-02$ & 8 & $832398 E-02$ & -6 . & $.828166 E-04$ & 1. & $.271002 \mathrm{E}-01$ & 63 \\
\hline
\end{tabular}

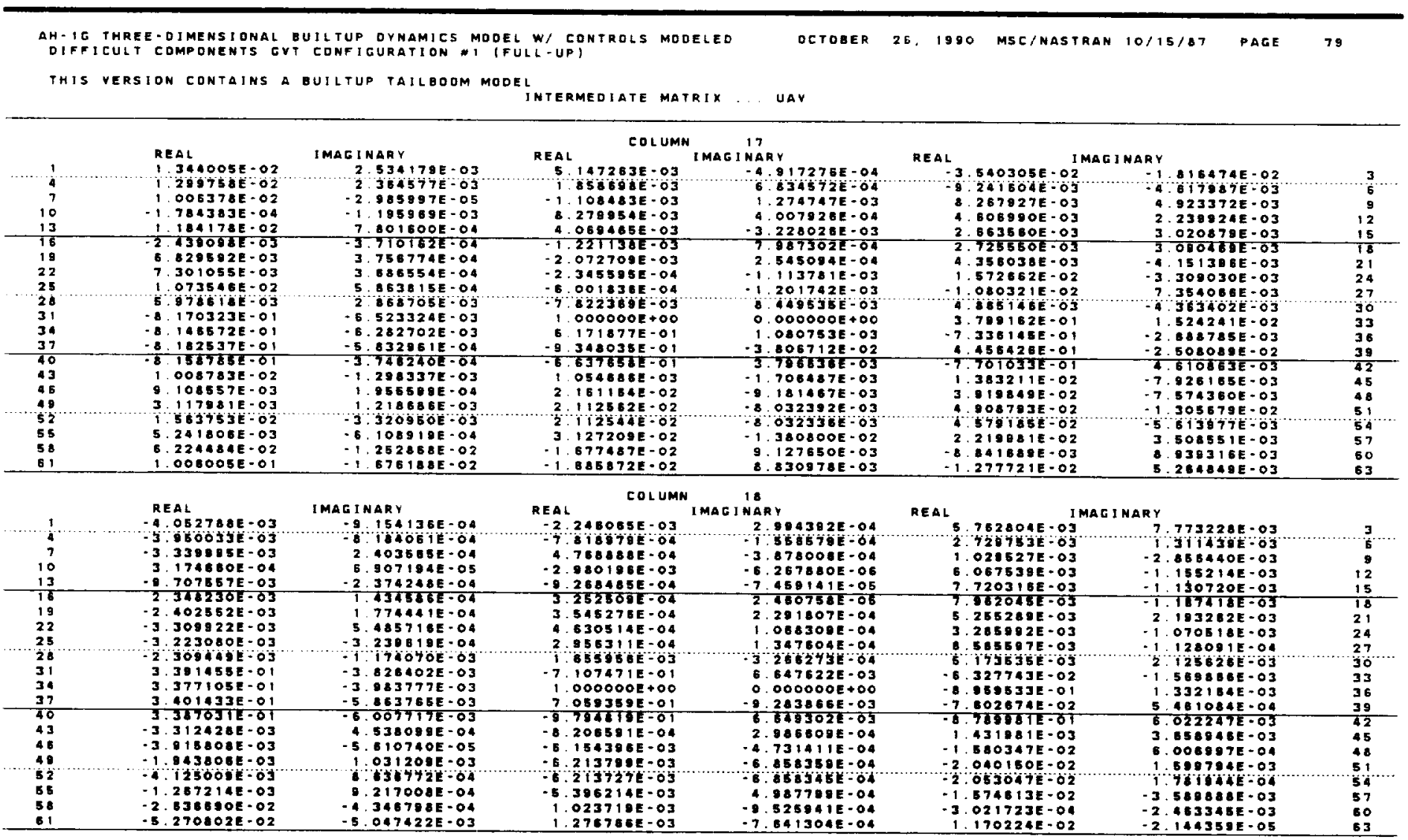




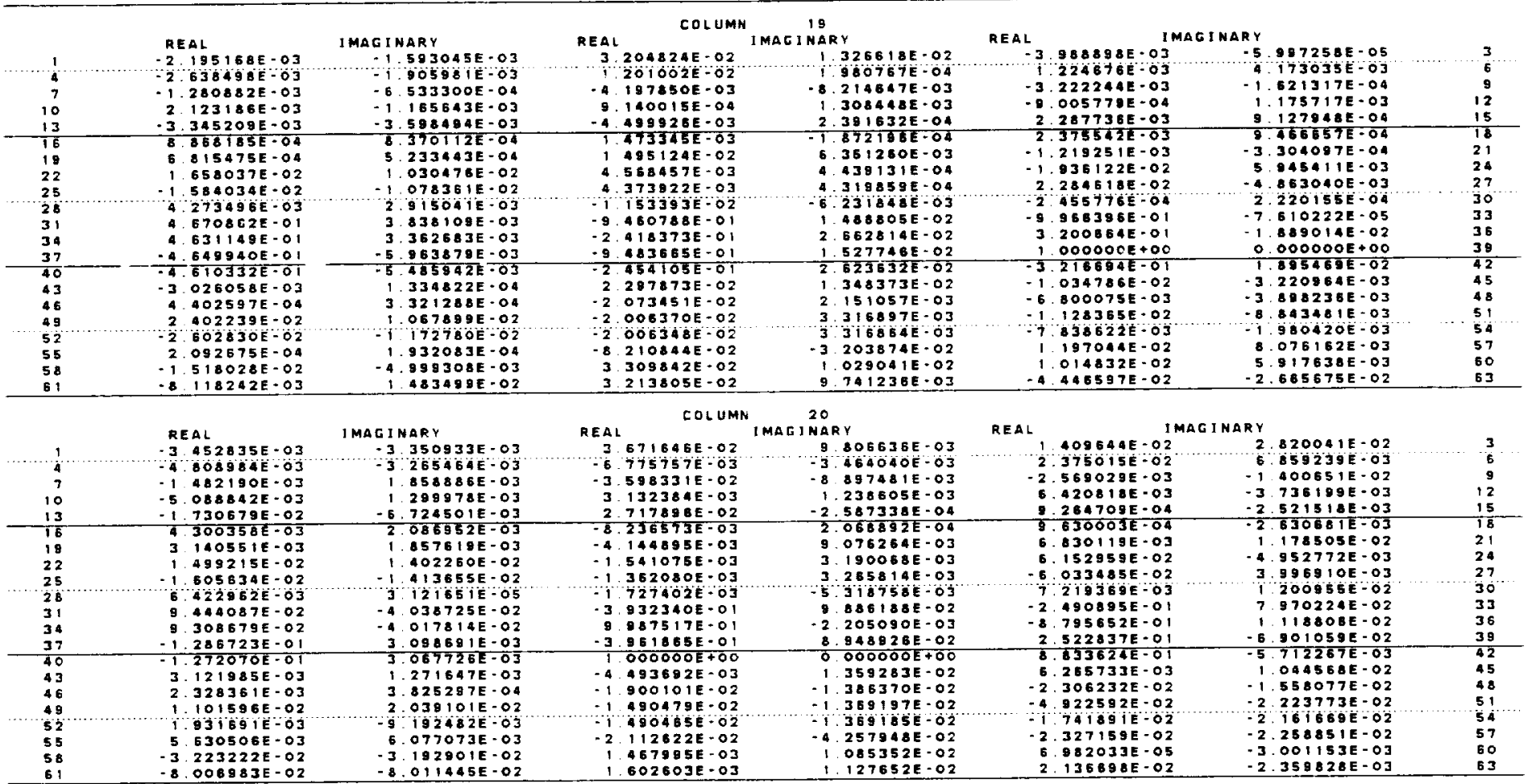

AH-1G THRE - DIMENSIONAL BUILTUP DYNAMICS MODEL W/ CONTROLS MOOELEO

DCTOBER 26, 1990 MSC/NASTRAM 10/15/87 PAEE

\begin{tabular}{|c|c|c|c|c|c|c|c|c|c|c|c|c|}
\hline $\begin{array}{r}1 \\
7 \\
7 \\
10 \\
13 \\
16 \\
19 \\
22 \\
25 \\
28 \\
31 \\
34 \\
37 \\
40 \\
43 \\
46 \\
49 \\
52 \\
55 \\
58 \\
61 \\
\end{array}$ & $\begin{array}{r}R E \\
1 \\
-1 \\
-1 \\
-1 \\
-7 \\
-2 \\
-9 \\
5 \\
-1 \\
4 \\
1 \\
1 \\
1 \\
1 \\
-1 \\
-1 \\
5 \\
-3 \\
-7 \\
-4 \\
2 \\
1 \\
\end{array}$ & 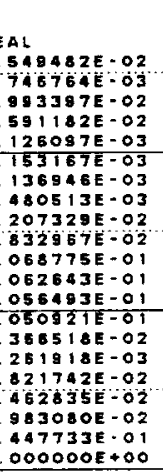 & 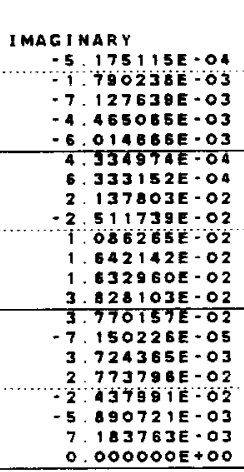 & $\begin{array}{r}\text { REAL } \\
5 \\
1 \\
-1 \\
4 \\
-7 \\
4 \\
-1 \\
-1 . \\
-6 \\
-1 \\
9 \\
1 . \\
11 . \\
3 . \\
1 \\
1 \\
1 \\
-4 \\
6 \\
5 \\
\end{array}$ & 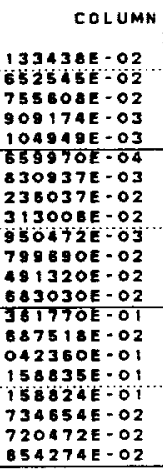 & 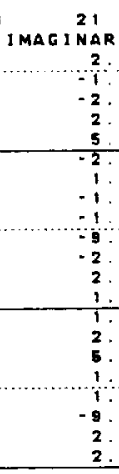 & 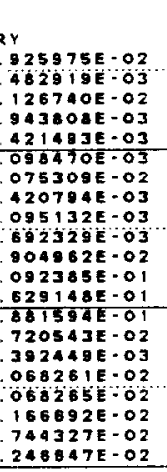 & 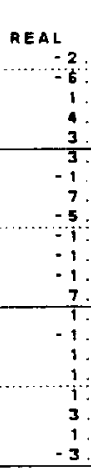 & 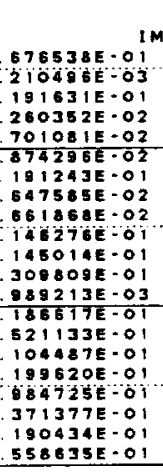 & 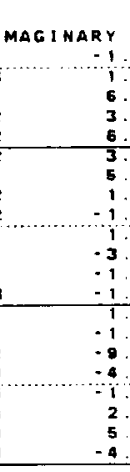 & 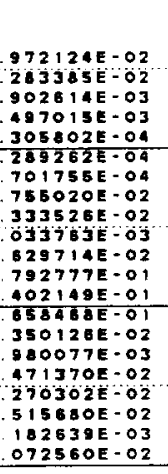 & $\begin{array}{r}3 \\
6 \\
9 \\
12 \\
15 \\
18 \\
18 \\
21 \\
24 \\
27 \\
30 \\
33 \\
38 \\
39 \\
42 \\
45 \\
48 \\
51 \\
54 \\
57 \\
60 \\
63 \\
\end{array}$ \\
\hline $\begin{array}{r}1 \\
7 \\
70 \\
13 \\
16 \\
18 \\
22 \\
25 \\
28 \\
31 \\
34 \\
37 \\
40 \\
43 \\
46 \\
49 \\
52 \\
55 \\
58 \\
61\end{array}$ & $\begin{array}{r}R \\
-3 \\
-1 \\
-2 \\
-1 \\
-1 \\
4 \\
3 \\
2 \\
-2 \\
-1 \\
-1 \\
3 \\
3 \\
2 \\
2 \\
3 \\
-2 \\
4 \\
-2 \\
-1 \\
\end{array}$ & 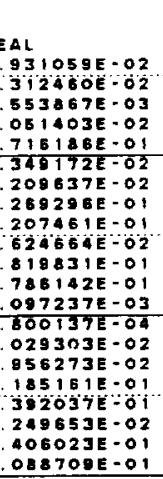 & 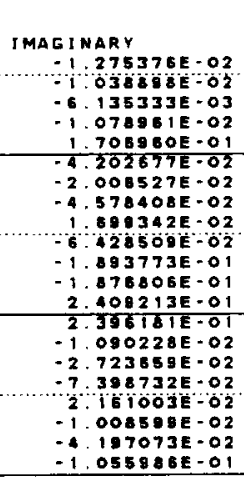 & $\begin{array}{r}\text { REAL } \\
2 \\
-2 \\
-1 \\
3 \\
-5 \\
1 \\
1 \\
2 \\
2 \\
2 \\
9 \\
-2 \\
1 \\
-2 \\
2 \\
-4 \\
-1 \\
-1 \\
-7 \\
2 \\
2 \\
\end{array}$ & 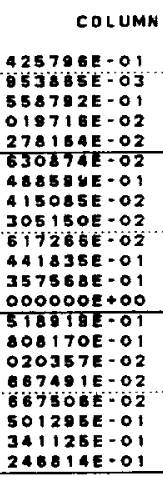 & 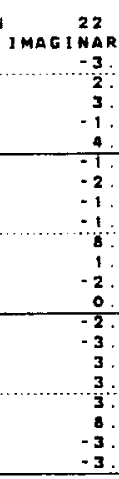 & 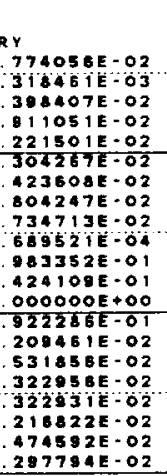 & $\begin{array}{r}\text { REAL } \\
1 \\
8 \\
-9 \\
1 \\
2 \\
2 \\
4 \\
2 \\
8 \\
4 \\
6 \\
3 \\
-6 \\
-3 \\
-4 \\
-1 \\
-3 \\
-1 \\
5 \\
1 \\
-4 \\
\end{array}$ & 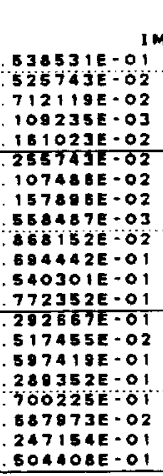 & $\begin{array}{r}\text { MAG INARY } \\
-1 \\
-3 \\
5 \\
-1 \\
-2 \\
-2 \\
2 \\
-2 \\
3 \\
2 \\
1 \\
1 \\
4 \\
-1 \\
6 \\
-7 \\
1 \\
-8 \\
-6 \\
-3 \\
4\end{array}$ & 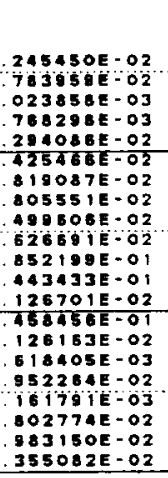 & $\begin{array}{r}3 \\
6 \\
12 \\
15 \\
15 \\
18 \\
21 \\
24 \\
27 \\
30 \\
33 \\
36 \\
39 \\
42 \\
45 \\
48 \\
51 \\
54 \\
57 \\
80 \\
63 \\
\end{array}$ \\
\hline
\end{tabular}




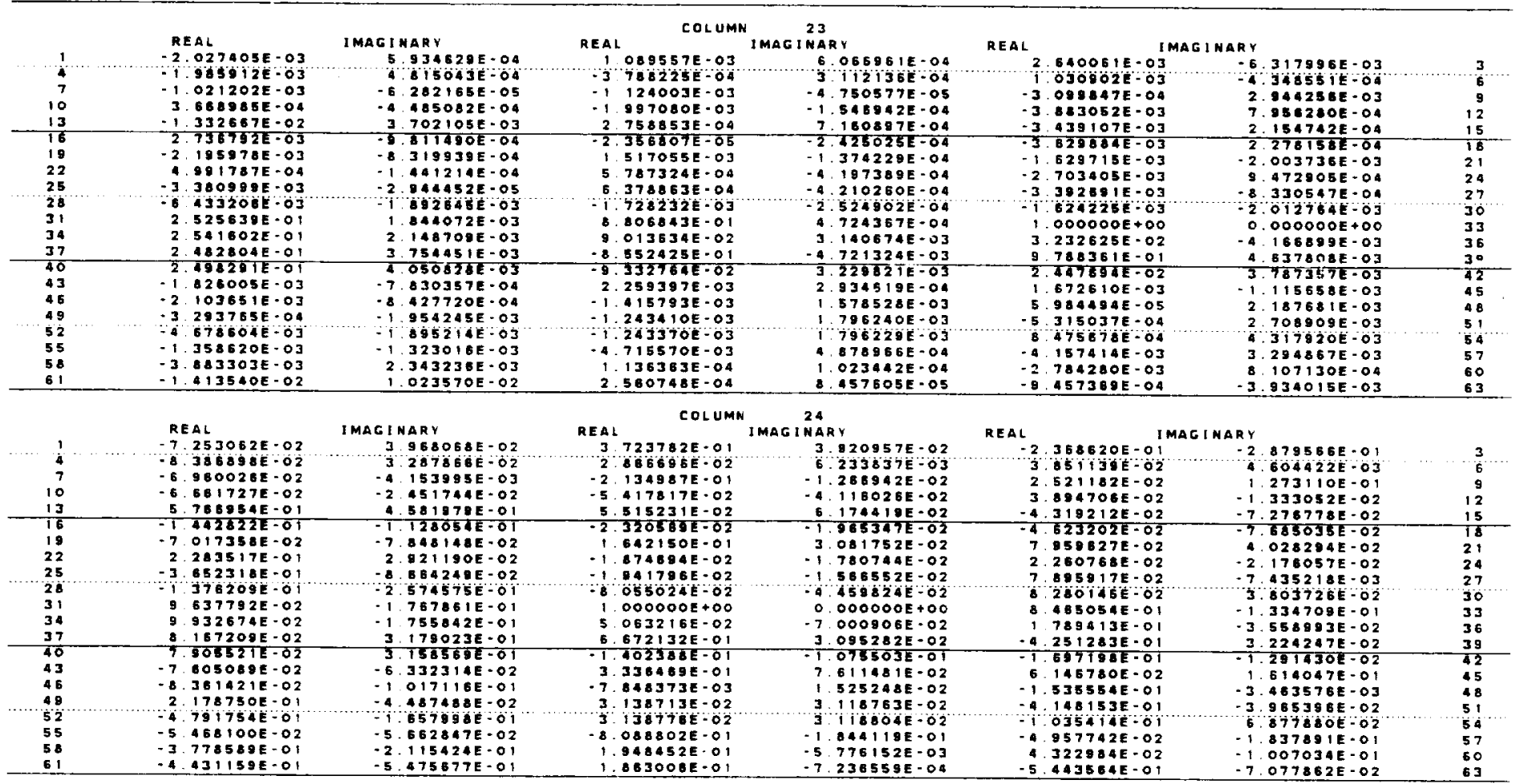

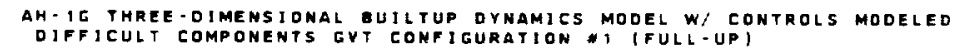

OCTOBER 26, 1990 MSC/NASTRAN 10/15/87 PAGE

83

THIS VERSION CONTAINS A BUILTUP TAILBOOM MODEL

INTERMEDIATE -MATRIX ... VAV

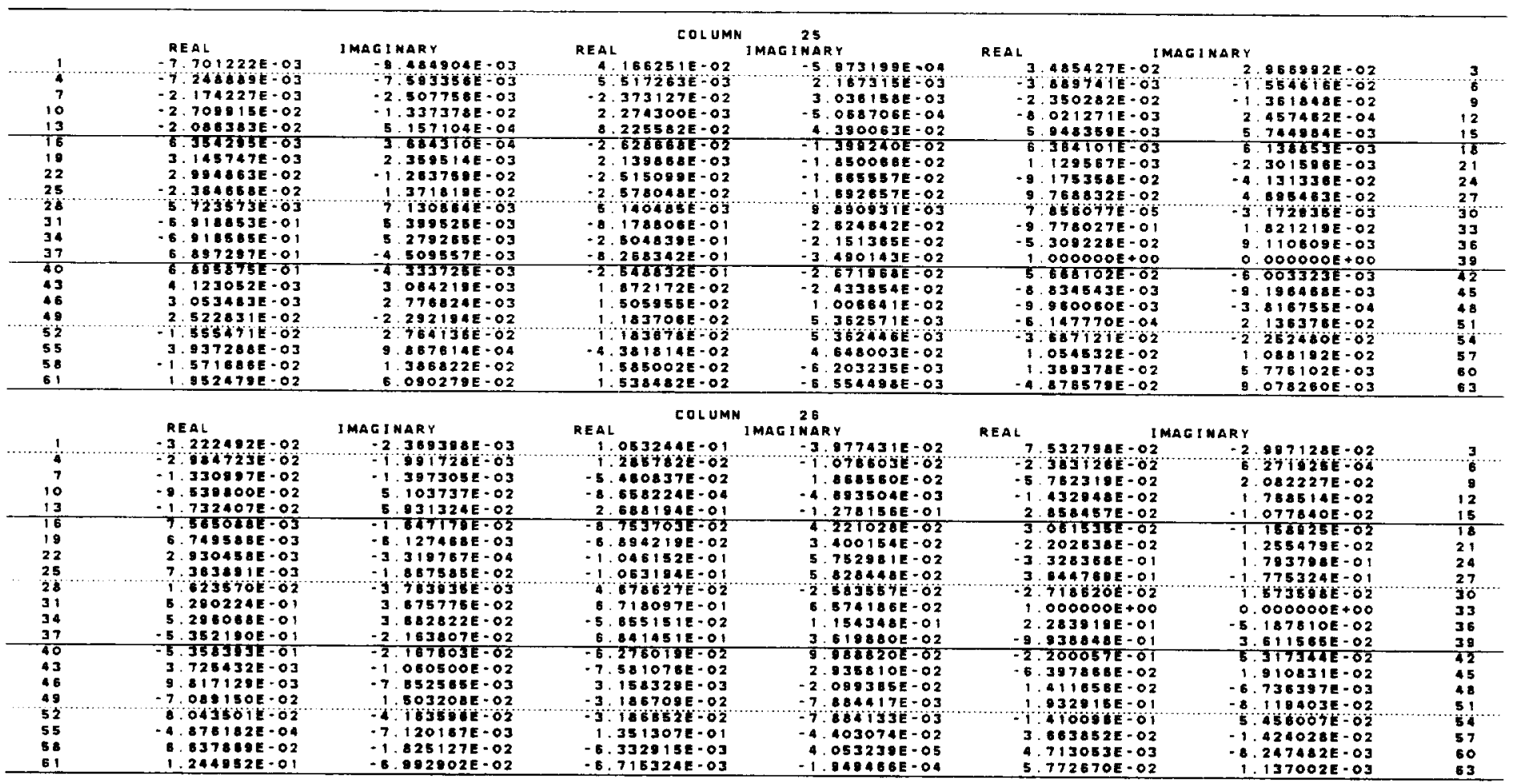




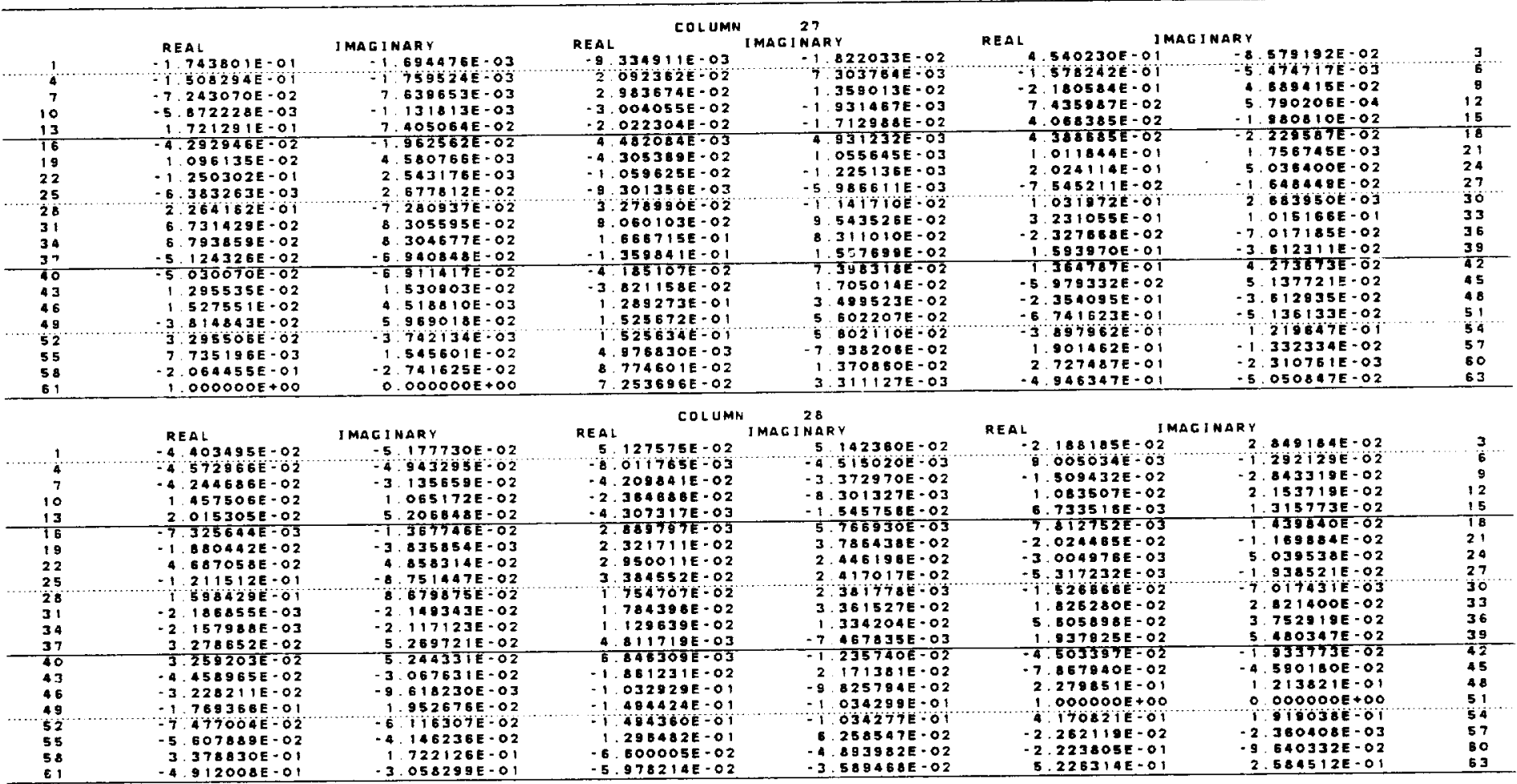

AH 1 G THREE-Dimensional BUILTUP OYNAMICS MODEL W/ CONTROLS MODELED

OCTOBER

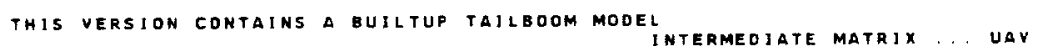

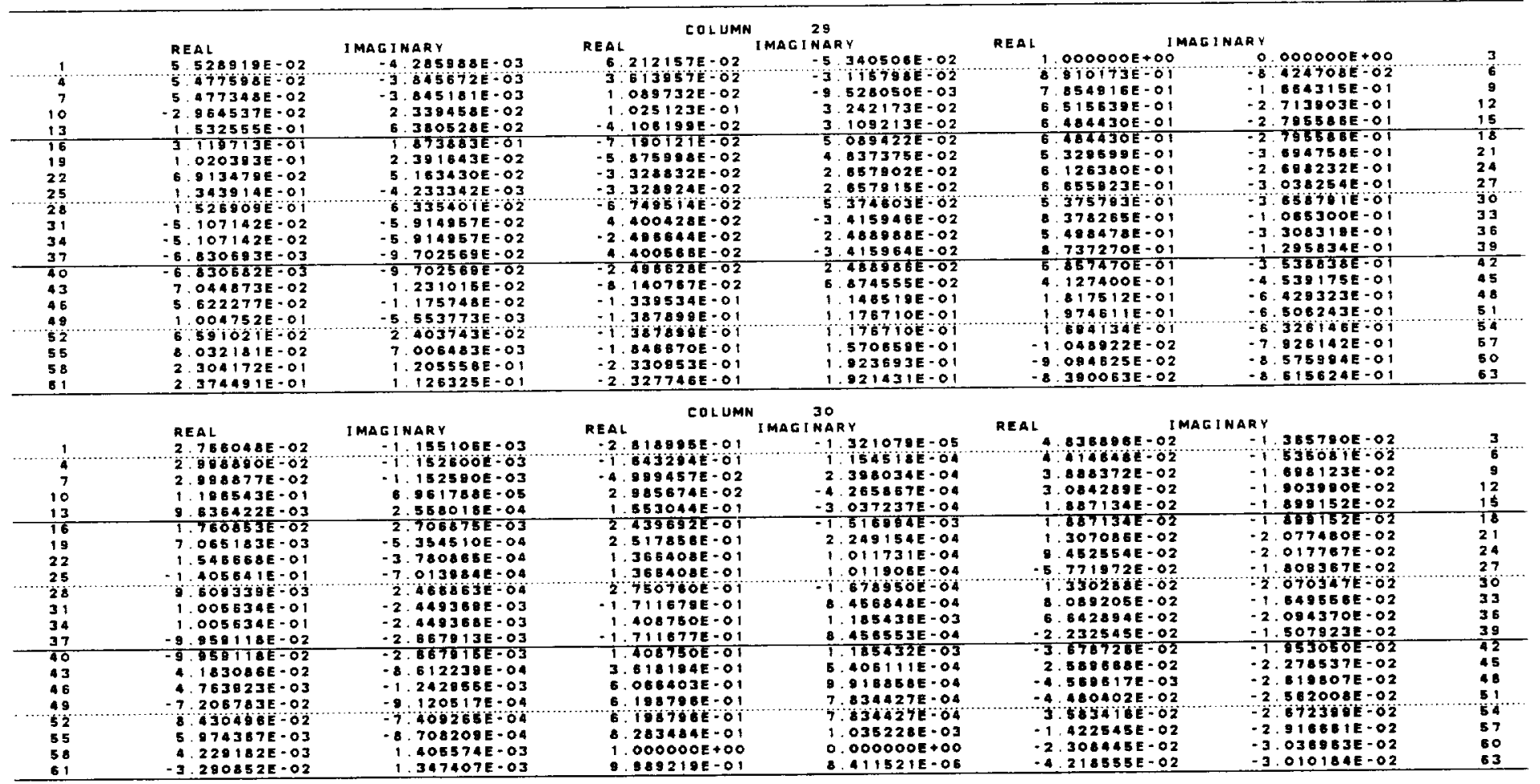


AH - IG THREE-DIMENSIONAL GUILTUP DYMAMICS MODEL W/ CONTROLS MODELEO

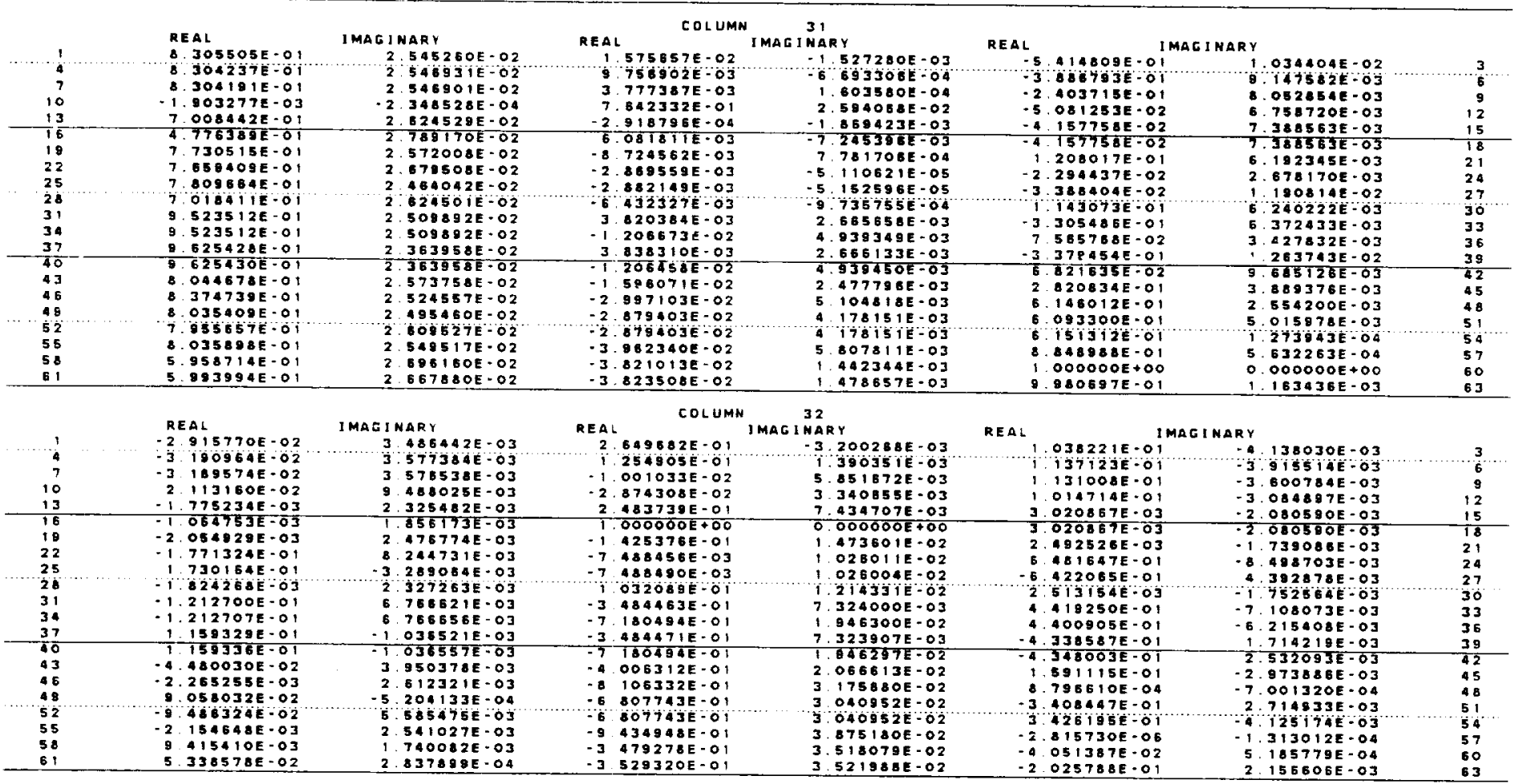

\footnotetext{
AM-IC THREE-DIMENSIONAL BUILTUP DYNAMICS MOOEL W/ CONTROLS MODELEO

THIS VERSION CONTAINS A BUILTUP TALLOOM MODEL
}

OCTDEE

26,1990 MSC / NASTRAN $10 / 15 / 87$

PAGE

MTERMEDIATE MATRIX ... UAV

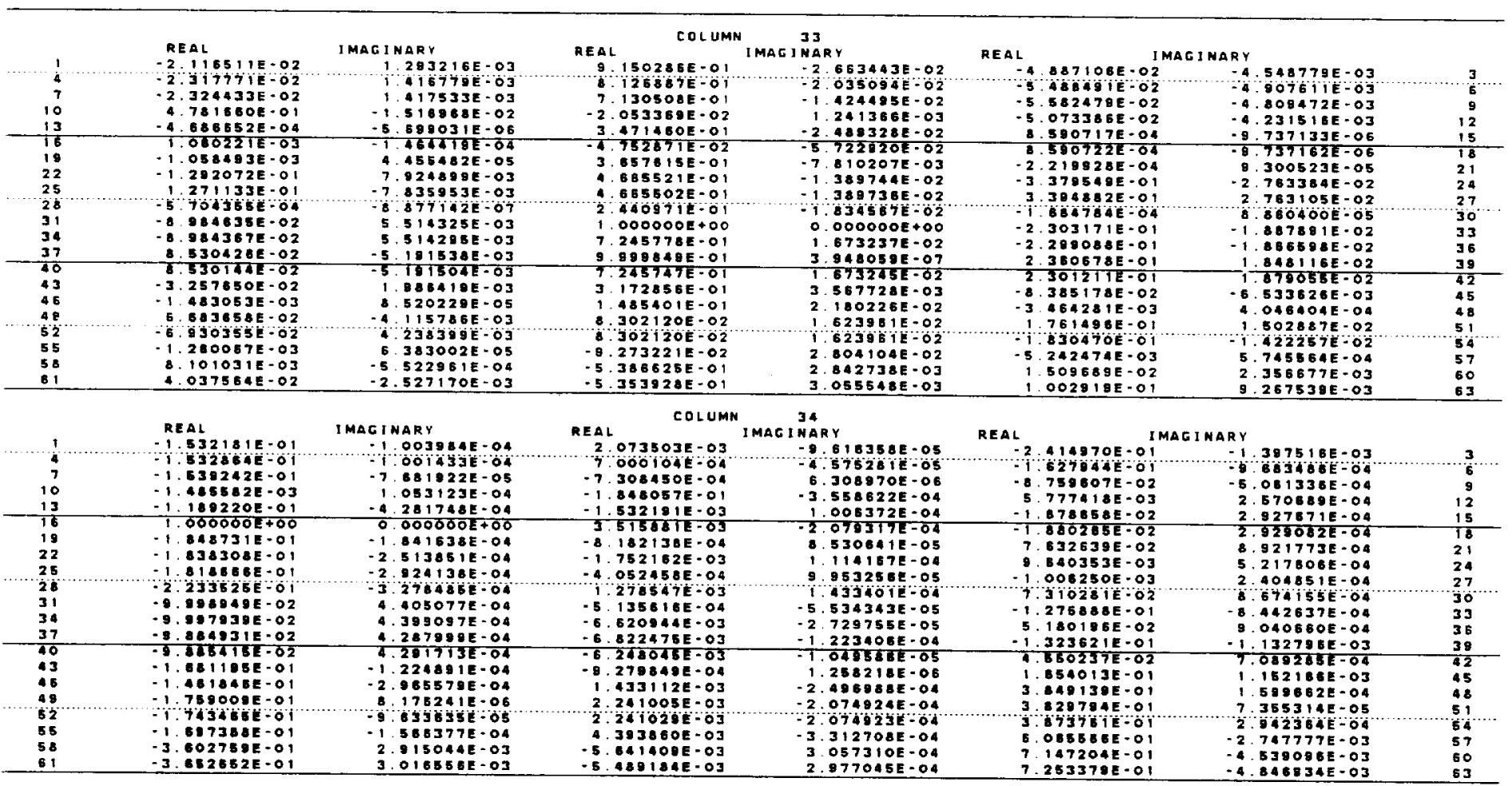


AH - : C THREE-DIMENS IONAL BUILTUP DYMAMICS MOOEL W/ CONTROLS MODELED

\begin{tabular}{|c|c|c|c|c|c|c|c|c|c|c|c|c|c|}
\hline & & $\Delta L$ & IMAG II & NARY & REAL & COLUMN & IMAG INAR & & PEAL & & MACINARY & & \\
\hline 1 & -2 & $318718 \mathrm{E}-02$ & 2 & $476131 E-04$ & 7 & $289581 E-02$ & -7 & $954096 E-03$ & 3 & A8979OE-OI & -1 & $565592 E-03$ & 3 \\
\hline 4 & -2 & $31724 E E-O 2$ & 3 & ZJZ3JZE-OA & $\therefore$ & 1OLSSTE OOL & $\because$ & BSBSZOE-OZ & $i$ & G IBS97E-OI & -8 & $544790 E-04$ & 6 \\
\hline 7 & -1 & $461925 E-02$ & B & $94656 O E-O 5$ & 1. & $075734 E-02$ & $-i$ & $697022 E-03$ & $\mathbf{5}$ & OT6582E-02 & -6. & $188659 E \cdot 04$ & g \\
\hline 10 & -2 & $136227 E-O Z$ & 2 & $190734 E-03$ & 1. & $95084 \mathrm{BE} \cdot 02$ & -6. & $370557 E-04$ & -7 & $336466 E-02$ & 4 & $046722 E-04$ & 12 \\
\hline 13 & 6 & $307161 E-02$ & -3 & $665822 E-04$ & -2. & $.365677 E-02$ & 2 & $548191 E-03$ & -7 & $.386148 E-02$ & -1. & $096570 E-04$ & 15 \\
\hline 16 & -3 & $903290 E-02$ & $\frac{5}{2}$ & $864689 E-03$ & 5 & $977786 E-03$ & -9 & $348399 E-04$ & -9 & $416806 \mathrm{E}-02$ & -1 & $.3828385-04$ & 18 \\
\hline 19 & 8 & $527147 E-03$ & -6 & $384715 E-04$ & -3 & $838160 E-02$ & 4 & $.327209 E-03$ & -1 & $372846 E-0$ & 1. & $322619 E-03$ & $2:$ \\
\hline 22 & 1 & $549548 E \cdot 03$ & 2 & $252125 E-03$ & -2 & $431332 E-02$ & 2. & $515302 E-03$ & -9 & $140052 \mathrm{E}-02$ & 2. & $O 87493 E-03$ & 24 \\
\hline 25 & 4 & $710857 E-02$ & -3 & $443518 E-03$ & -2. & $44185 O E-02$ & 2. & $.567821 E-03$ & -7 & $135372 E-02$ & -1. & $531128 E-03$ & 27 \\
\hline 28 & 2 & $704757 E-O 2$ & $\because \mathrm{i}$ & $278220 E=03$ & 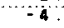 & $B S 5222 E-O 2$ & 5 & $368958 E-O J$ & $\because$ & 344 IBSE-OO & 1 . & 2OESEEE-OS & 30 \\
\hline 31 & -1 & $101642 E \cdot 01$ & 2 & $581789 E-04$ & 5 & $302858 E-03$ & -8 & $125889 E-03$ & $i$ & $370234 E-0$, & 7 & $759916 E-03$ & 33 \\
\hline 34 & -1 & $100491 E-01$ & 2 & $557641 \mathrm{E}-04$ & -1 & $160218 E-02$ & 3. & $.298036 E-03$ & -1 & $557181 E-O 1$ & B & $957260 E-04$ & 36 \\
\hline 37 & -7 & $518676 \mathrm{E} .02$ & -3 & $628933 E-03$ & 5 . & $674977 E-02$ & -1. & $.31596 I E-03$ & 1 & $568855 E-01$ & 3. & $501665 E-03$ & 39 \\
\hline 40 & -7 & $514172 E-62$ & -3 & $621495 E-03$ & -3 & $980109 E-02$ & 7 & $3280221-04$ & -1 & $.085356-01$ & 2 & $.251507 E=0 \overline{3}$ & 42 \\
\hline 43 & 1 & $394868 E-03$ & -9 & $080610 E-04$ & -4 & $076775 E-02$ & 4 & $585536 \mathrm{E}-03$ & $-i$ & $522826 E-01$ & 3 & 17433AE-OJ & 45 \\
\hline 46 & 3 & $300672 E \cdot 02$ & -1 & $424434 E-03$ & 2. & $053120 E-02$ & -3 & $525790 E-04$ & 7 & 667 y2E-OZ & 4. & $470507 E-03$ & 48 \\
\hline 49 & .4 & $703041 E .02$ & $i$ & $763844 E-03$ & 2. & $243044 E-02$ & -3 & $348821 E \cdot 04$ & 6 & $.899238 E-O 2$ & 5. & $029345 E-03$ & 51 \\
\hline 52 & $\mathrm{i}$ & $484136 E-O 2$ & $=3$ & $681785 E-03$ & 2 & $243 O A 3 E-O 2$ & 3 & उAB8/9E-OA & 8 & $330418 E-02$ & 4. & $793038 E-O 3$ & 54 \\
\hline 55 & 2 & $834098 E-03$ & -1 & $814372 E-03$ & 1. & $308087 E-01$ & -1 & $050322 E .02$ & 6 & $045728 E-01$ & -5 & $344832 E-03$ & 57 \\
\hline 58 & .5 & $825554 E-C 1$ & 8 & $071732 E-03$ & 2 & $345750 E-01$ & -3 & $258082 E-02$ & 9 & $372224 E-01$ & -8 & $661572 E-03$ & 60 \\
\hline B 1 & -6 & $791679 E-01$ & 8 & $72043 \mathrm{BE}-03$ & 2. & $345759 E-01$ & .3 & $250688 E \cdot 02$ & $i$ & $.000000 E+00$ & $\circ$. & $.000000 E+00$ & 63 \\
\hline
\end{tabular}

AH- 1 G THREE-DIMENSIONAL BUILTUP OYNAMICS MODEL W/ CONTROLS MOdELED

OCTOOER

26, 1990 MSC/NASTRAN 10/15/87

THIS VERSION CONTAINS A BUILTUP TALLBOOM MOOEL

COMPLEX EIGENYALUE = -1.199085E-02, -2.397042E-12

\begin{tabular}{|c|c|c|c|c|c|c|c|c|c|}
\hline POINT ID. & TYPE & Ti & $T 2$ & & 13 & & R 1 & R 2 & R 3 \\
\hline $100 \%$ & 6 & $\begin{array}{l}=111565 E-03 \\
-90094 E-13\end{array}$ & $\begin{array}{l}\because 19261 B E-03 \\
-2.781579 E-13\end{array}$ & $\begin{array}{l}-3 \\
-1\end{array}$ & $\begin{array}{l}336734 E-01 \\
521826 E-11\end{array}$ & 0 & & 0.0 & $\begin{array}{ll}0 & 0 \\
0 & 0\end{array}$ \\
\hline 1003 & G & $\begin{array}{r}-1210582 E-02 \\
=-956850 E-13\end{array}$ & $\frac{-6.902715 E-04}{-1.568207 E-13}$ & -2 & $\frac{.01 B 108 E-01}{370330 E-11}$ & $\frac{0}{0}$ & & 0.0 & 0.0 \\
\hline 1004 & G & $\begin{array}{r}-1.210551 E-02 \\
-9.576290 E-13\end{array}$ & $\begin{array}{l}-2.040782 E-04 \\
-3.918825 E=14\end{array}$ & $\because 7$ & $\begin{array}{l}322523 E-O 2 \\
223451 E-1 !\end{array}$ & $:$ & & $\begin{array}{ll}0 & 0 \\
0 & 0\end{array}$ & $\begin{array}{r}0.0 \\
0: 0\end{array}$ \\
\hline 1006 & G & $\begin{array}{l}-6.446625 E-02 \\
-1.561756 E-12\end{array}$ & $\begin{array}{l}\text { a. } 750125 E-04 \\
1.828658 E-13\end{array}$ & -1 & $\begin{array}{l}021787 E-02 \\
037256 E-11\end{array}$ & $:$ & & $\begin{array}{l}-2.009296 E-03 \\
-3.213142 E-14\end{array}$ & $\begin{array}{l}\text { 1. } 089763 E-05 \\
2.583406 E-15\end{array}$ \\
\hline 1007 & $\bar{c}$ & $\begin{array}{l}-6.297481 E-02 \\
-1.56431 E-12\end{array}$ & $\begin{array}{l}1.629545 E-03 \\
3.177732 E-13\end{array}$ & $\begin{array}{l}2 \\
-8\end{array}$ & $\begin{array}{l}392004 E-01 \\
698318 E-12\end{array}$ & 0. & O & $\begin{array}{l}0.0 \\
0.0\end{array}$ & $0: 0$ \\
\hline 1012 & $c$ & $\begin{array}{r}-4.038792 E-02 \\
\because 1.382696 E-42\end{array}$ & $\begin{array}{r}2.849248 E-03 \\
6771571 E-13\end{array}$ & 6 & $\begin{array}{l}535745 E-01 \\
95758 E-12\end{array}$ & $\begin{array}{l}-2 \\
-3\end{array}$ & $\begin{array}{l}451785 E-05 \\
866127 E-15\end{array}$ & $\begin{array}{r}-2.809339 E-03 \\
-3.215703 E-14\end{array}$ & $\begin{array}{r}1,070350 E-05 \\
2.586487 E-15\end{array}$ \\
\hline 1013 & $\sigma$ & $\begin{array}{l}-3.971895 E-02 \\
-1.221594 E-12 \\
\end{array}$ & $\begin{array}{l}2.849248 E-03 \\
6771571 E-13 \\
\end{array}$ & $\begin{array}{r}5 \\
-3 \\
\end{array}$ & $\begin{array}{r}651069 E-01 \\
712188 E-12 \\
\end{array}$ & $\begin{array}{l}-2 \\
-3 \\
\end{array}$ & $\begin{array}{l}451767 E-05 \\
765615 E-15 \\
\end{array}$ & $\begin{array}{l}-2.09359 E-03 \\
-3.215703 E-14 \\
\end{array}$ & $\begin{array}{r}1.070354 E-05 \\
2.57 B 684 E-15 \\
\end{array}$ \\
\hline 1017 & G & $\begin{array}{l}-6.327218 E-02 \\
-1.712820 E-12\end{array}$ & $\begin{array}{l}948327 E-04 \\
1.85889 E-13\end{array}$ & $\begin{array}{r}1 \\
-1\end{array}$ & $\begin{array}{l}086351 E-01 \\
040058 E-11\end{array}$ & $:$ & & $\because: 0$ & 0.0 \\
\hline $101 \mathrm{a}$ & $\epsilon$ & $\begin{array}{l}-6.200858 E-02 \\
-1.408350 E-12\end{array}$ & $\begin{array}{l}\text { 9 } 346 B d B E-O 4 \\
1 \text { BSDOEE- } 13\end{array}$ & -9 & $\begin{array}{l}115285 E-01 \\
947311=12\end{array}$ & $\because$ & & 0.0 & 0.0 \\
\hline 1021 & $a$ & $\frac{-1.252867 E-01}{-2.277212 E-12}$ & $\frac{1.38291 E-03}{2739222 E-13}$ & $=9$ & $\frac{.873263 E-02}{.030365 E-11}$ & $\frac{.2}{.3}$ & $\frac{451947 E-05}{839338 E-15}$ & $\frac{-2.809341 E-03}{-3.213164 E-12}$ & $\frac{1.080620 E-05}{2.581563 E-15}$ \\
\hline 1022 & c & $\begin{array}{r}-3.183446 E-01 \\
-4 \quad 48018 E-12\end{array}$ & $\begin{array}{l}323283 E-03 \\
5 \quad 379889 E-13\end{array}$ & $\begin{array}{r}0 \\
-1.2\end{array}$ & $\begin{array}{l}.873263 E-02 \\
.030350 E-11\end{array}$ & $\begin{array}{l}-2 \\
-3\end{array}$ & $\begin{array}{l}451972 E-05 \\
844425 E-15\end{array}$ & $\begin{array}{r}-2.809339 E-03 \\
-3.215221 E-14\end{array}$ & $\begin{array}{r}1069530 E-05 \\
2581043 E-15 \\
\end{array}$ \\
\hline 1023 & G & $\begin{array}{l}9.679386 E-02 \\
\text { 3. } 618885 E-13\end{array}$ & $\begin{array}{l}-1,458858 E-03 \\
-2.615802 E-13\end{array}$ & $\because 1$ & $\begin{array}{l}5255+4 E-01 \\
3034,7 E-11\end{array}$ & $\begin{array}{l}-2 . \\
-3 .\end{array}$ & $\begin{array}{l}452060 E-05 \\
843891 E-15\end{array}$ & $\begin{array}{l}-2.809217 E-03 \\
-3.21173 \mathrm{BE}-14\end{array}$ & $\begin{array}{l}\text { 1. } 089756 E-05 \\
2.580565 E-15\end{array}$ \\
\hline 1024 & $G$ & $\begin{array}{l}\text { G. } 393802 E-02 \\
1.850354 E-13\end{array}$ & $\begin{array}{l}-1.457132 E \cdot 03 \\
-2.614436 E-13\end{array}$ & $\because 1:$ & $\begin{array}{l}\text { BतE132E-01 } \\
.334215 E-11\end{array}$ & $\begin{array}{l}-2 . \\
-3 .\end{array}$ & $\begin{array}{l}452058 E-05 \\
847580 E-15\end{array}$ & $\begin{array}{l}-2.809218 E-03 \\
-3.211846 E-14\end{array}$ & $\begin{array}{l}1.070235 E-05 \\
2.572 B 4 E-15\end{array}$ \\
\hline 1025 & 5 & $\begin{array}{r}9.57938 B E-02 \\
3.613432 E-13\end{array}$ & $\begin{array}{r}-1225532 E-04 \\
6.135320 E-14\end{array}$ & $=6$ & $\begin{array}{l}978143 E-01 \\
027698=12\end{array}$ & $\begin{array}{l}-2 . \\
=3 .\end{array}$ & $\begin{array}{l}451780 E-05 \\
622074 E=15\end{array}$ & $\begin{array}{r}-2.809222 E-03 \\
-3.21337 b E-16\end{array}$ & $\begin{array}{l}1.070022 E-05 \\
2=611018 E-15 \\
10\end{array}$ \\
\hline 1026 & G & $\begin{array}{r}993802 E-02 \\
1.549607 E-13 \\
\end{array}$ & $\begin{array}{r}-1.225842 E-04 \\
6.001758 E-14 \\
\end{array}$ & $\begin{array}{r}1 . \\
-9\end{array}$ & $\begin{array}{l}958531 E-01 \\
.335130 E-12 \\
\end{array}$ & $\begin{array}{l}-2 \\
-3 \\
\end{array}$ & $\begin{array}{l}451811 E-05 \\
861045 E-15 \\
\end{array}$ & $\begin{array}{l}-2.009232 E-03 \\
-3.214428 E-14 \\
\end{array}$ & $\begin{array}{l}1.009754 E-05 \\
2.562033 E-15 \\
\end{array}$ \\
\hline 1028 & G & $\begin{array}{l}-2.15 A 429 E-01 \\
-3.35641 E-12\end{array}$ & $\begin{array}{l}5.585519 E-03 \\
1.222888 E-12\end{array}$ & .5 & $\begin{array}{l}991562 E-01 \\
183610 E-14\end{array}$ & $\begin{array}{l}-2 \\
-3\end{array}$ & $\begin{array}{l}451808 E-05 \\
787523 E-15\end{array}$ & $\begin{array}{l}-2.009350 E-03 \\
-3.217482 E-14\end{array}$ & $\begin{array}{l}1.070243 E-05 \\
2.576267 E-15\end{array}$ \\
\hline
\end{tabular}




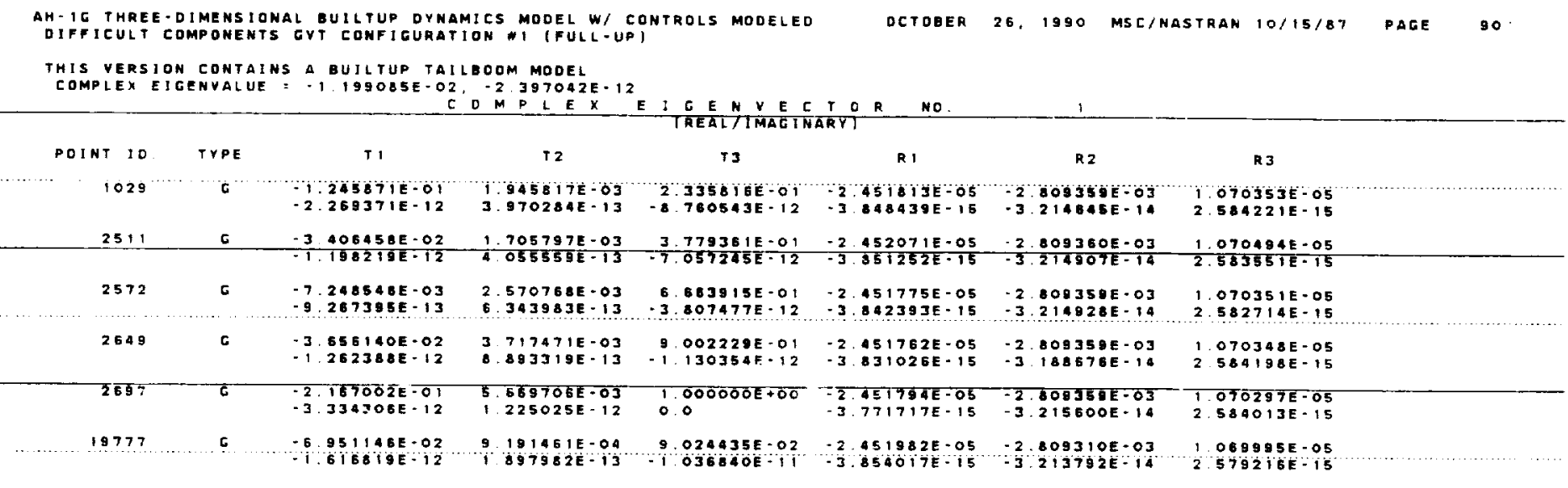

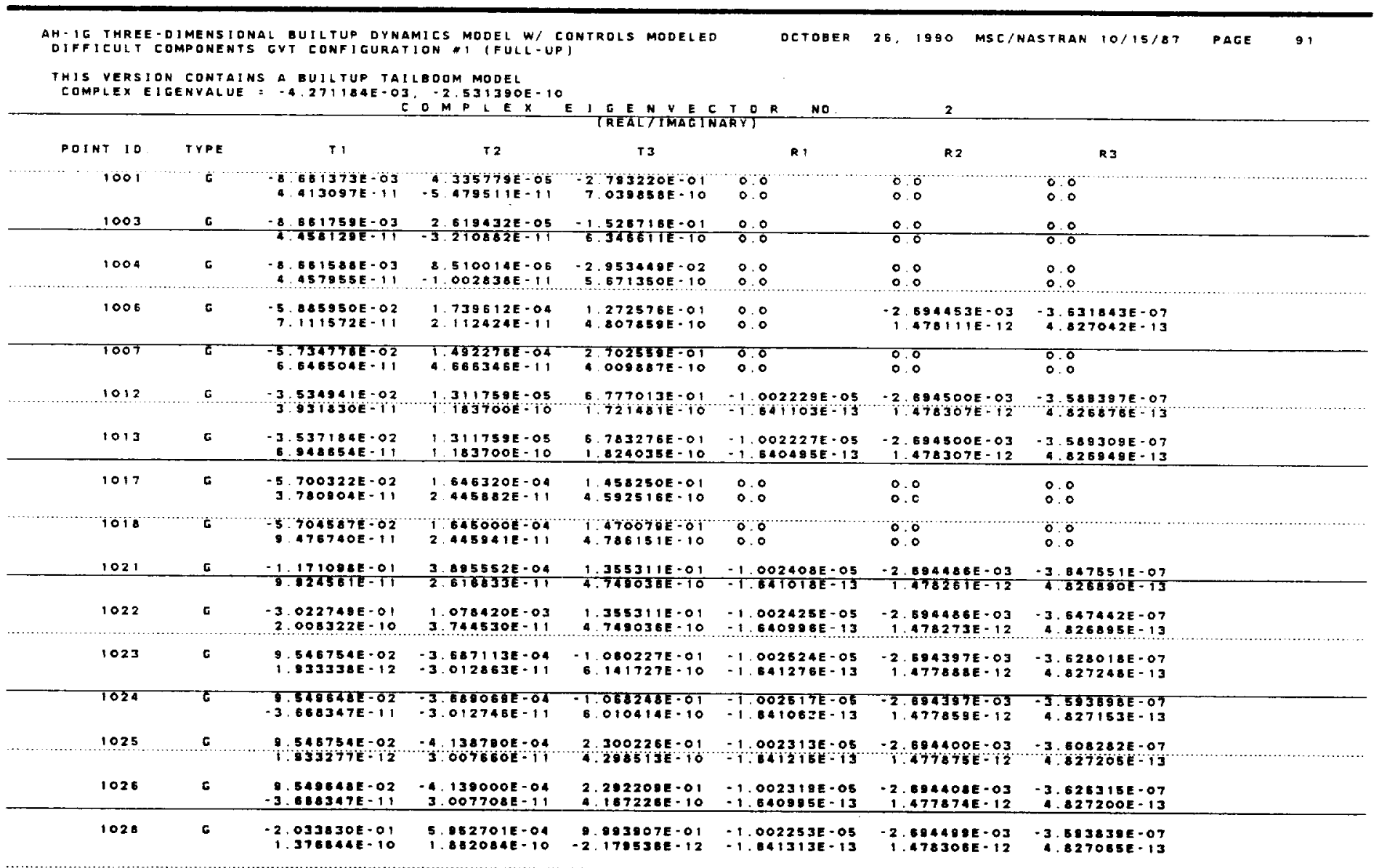


AH-iG THREE-DIMENSIONAL BUILTUP DYMAMICS MODEL W/ CONTROLS MDOELED

DIFEICULT COMPONENTS GVT COHFIGURATION I IFULL-UPI

THIS VERSION CONTAINS A BUILTUP TAILBOOM MOOEL

COMPLEX EIGENVALUE = $-4.2711 B 4 E-03,-2.531390 E \cdot 10$

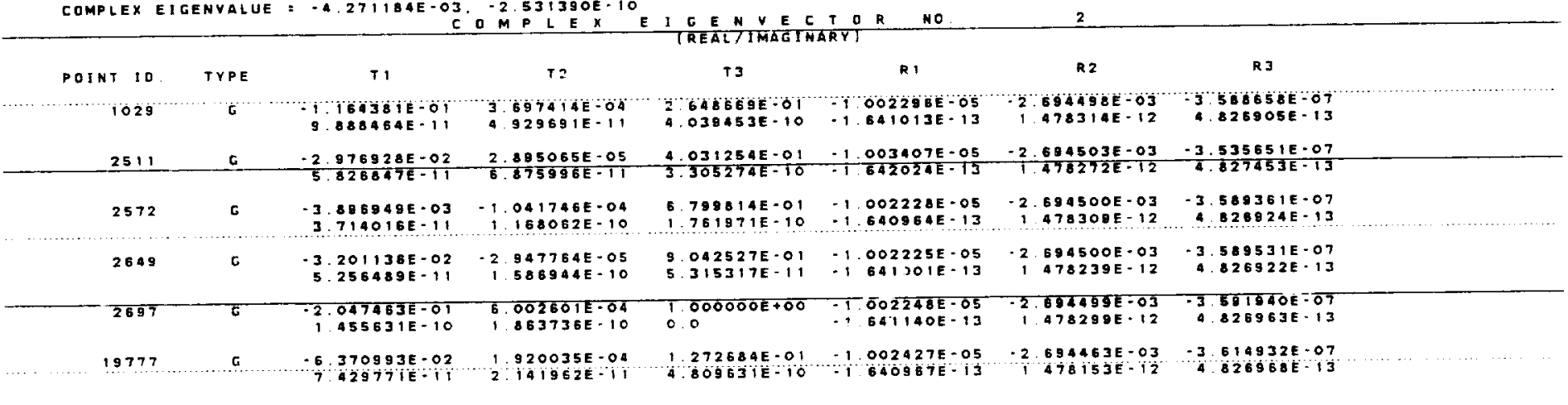

AH-1G THREE-DIMENSIONAL BUILTUP OYMAMICS MOOEL W/ CONTROLS MOOELEO

THIS VERSION CONTAINS A BUILTUP TAILBOOM MODE

COMPLEX EITENYALUE = -1.500943E-03, -1.678274E-02

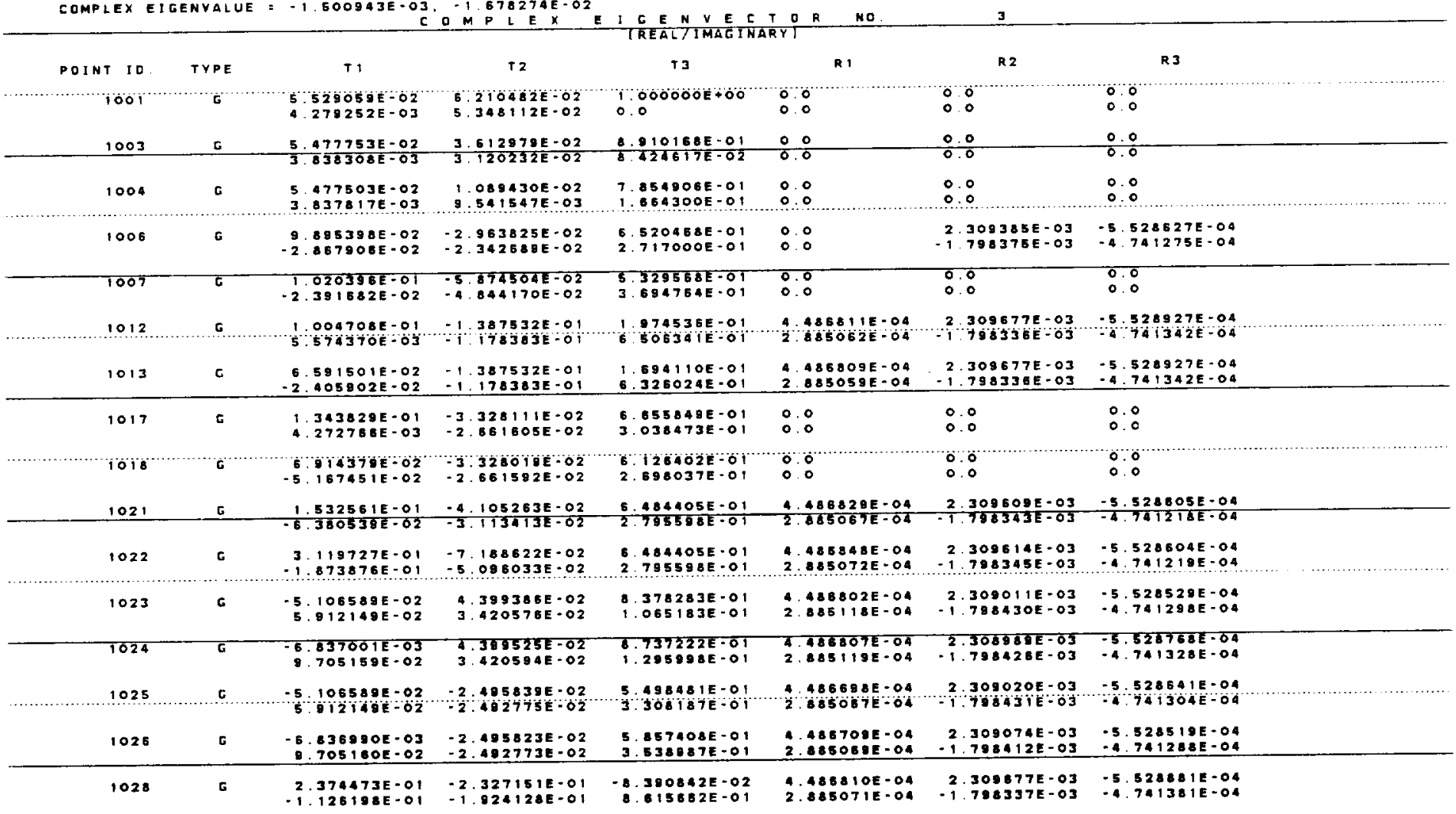




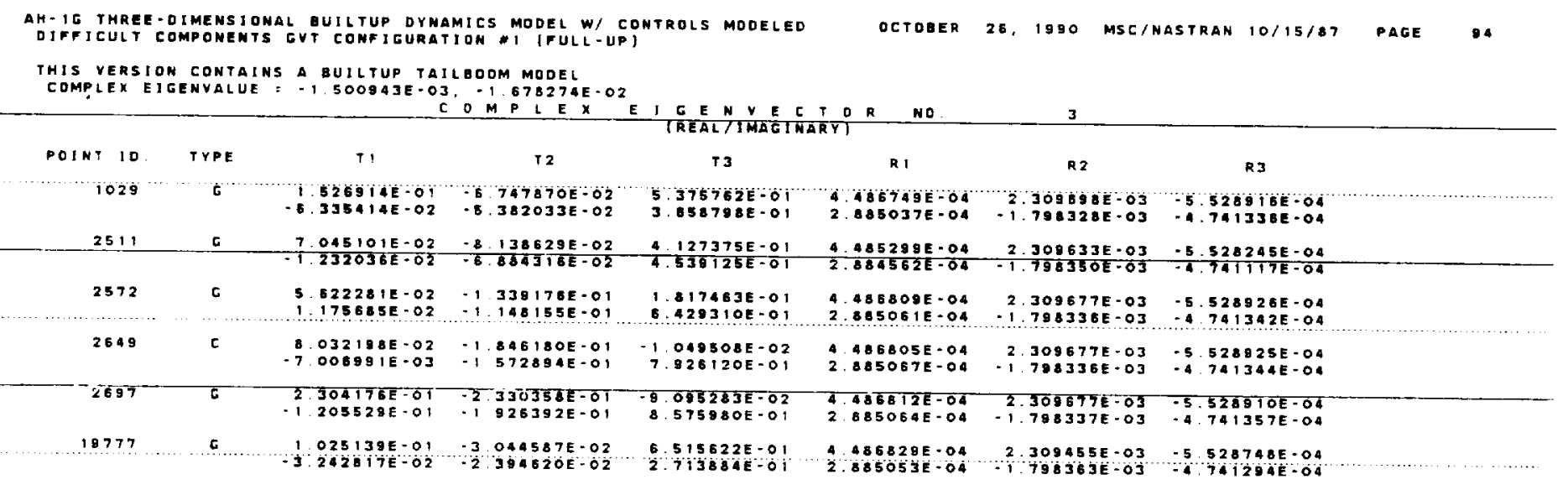

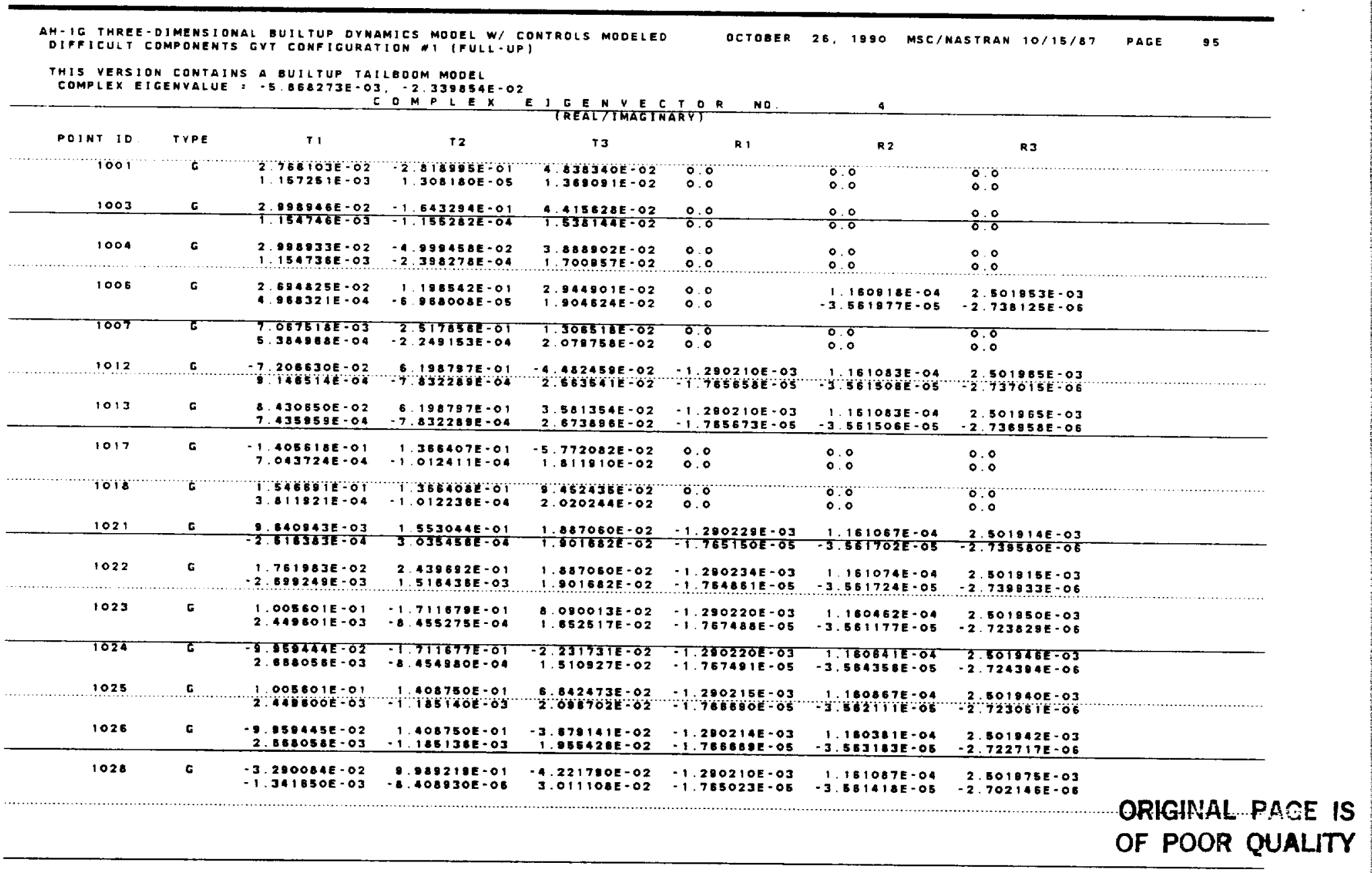



AH-1G THREE-DIMENSIONAL BUILTUP OYNAMICS MODEL W/ CONTROLS MODELED
DIFFICULT COMPONENTS GVT CONFIGURATION IFULL-UP)

TMIS VERSION CONTAINS A BUILTUP TAILBOOM MOOEL

COMPLEX EIGENVALUE: -5.868273E-03, -2 339854E-02

POINT IO TYPE

$1029 . \cdots+1 . \cdots$

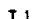

9. $613836 E-03$ $2.425351 E-a_{4}$

$2511 \quad$ E $\quad 183218 E-02$

$2572 \quad 6$

4. $764301 E-03$
$1.245001 E-03$

$2649 \quad G \quad \begin{array}{r}575775 E-03 \\ 8.733928 E-04\end{array}$

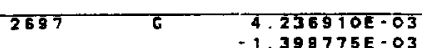

19777 G $2.885931 E-02$
1 GENYYETYOR NO

COMPLEX EIGEN YECY OR M

12

2. $750760 \mathrm{CO}$

T 3

A 1

R 2

$\begin{array}{lll}1.329741 E-O 2 & -190208 E-03 & 1\end{array}$

$\begin{array}{rrrrr}3.518194 E-01 & 2.588614 E-02 & -1.290220 E-03 & 1.151053 E-04 & 2.501959 E-03 \\ -5.40495 E-04 & 2.280558 E-02 & -1.966065 E-05 & -3.561625 E-05 & -2.735485 E-06\end{array}$

6. $063403 E-01$

$-590284 E-03 \quad-1.280210 E-03$

1. $161082 E-04$

2. $501965 E-03$
$-2736801 E-06$

Q. $283484 E-O)$

$283484 E-O T$
$1.034914 E-O 3$

2. $917753 \mathrm{E} \cdot 02$

$-1.200211 E-03$

$1.00000 \bar{E}+00$

$-2.311682 \mathrm{E}-02$

$2.885931 E-02 \quad 1,219766 E-01$
$4.297615 E-O 4=3789878 E-O 5$

$3.094240 E-02$

$-1.765883 \mathrm{E}-05$

1. $1610878-04 \quad 2.501966 E \cdot 03$

3. $561475 E-05-2735428 E \cdot O$

$\begin{array}{rr}1.161090 E-04 & 2.301969 E-03 \\ -3.561322 E-05 & -2.723694 E-06\end{array}$

$084244 E-02$
$906530 E-O 2$

. $1290216 E \cdot 03$

1. $160849 E-04$

2.501954E-03

AH- IG THREE-DIMENSIONAL BUILTUP DYNAMICS MODEL W/ CONTAOLS MODELEO

DCTOBER 25. 1990 MSC/NASTRAN $10 / 15 / 87$

PAGE

97

THIS VERSION CONTAINS A BUILTUP TALLBODM MODEL

COMPLEX EIGENYALUE: - - O O92172E-02,-1.262680E-01

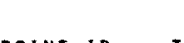

\begin{abstract}
T 1
\end{abstract}
ioo i

1003

1003

$1004 \quad 0$

1005

1007

101

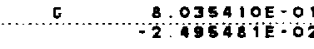

$1013 \quad$ G $955858 E-01$

7. $809664 E-O 1$

2. $464074 E-02$

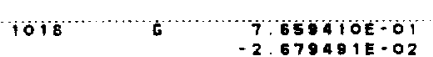

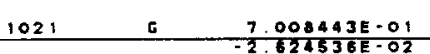

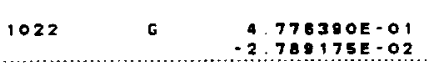

$9.523513 E-01$
$-2.509845-02$

$\begin{array}{rr}1024 & 9.625428 E-0 T \\ & -2.383983 E-02\end{array}$

$\begin{array}{rr}1025 & 0 \\ 1 & 523513 E-01\end{array}$

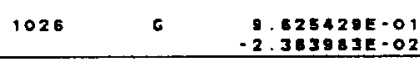

1028
C.

T 2

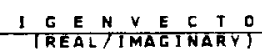
$\begin{array}{ll}575651 E-02 & -5 \text { A1ABIOE-OI } \\ 526840 E-03 & -1.034478-02\end{array}$

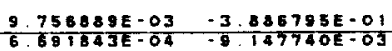

3. $777419 E-03$

-1. $603162 E-04$

$\begin{array}{ll}1.903396 E-03 & -5.091278 E-02 \\ 2348083 E-04 & -6.84340 E-03\end{array}$

S. OISA3E-O3 $177201 E-03$

2. $882254 E-03$

2 $10653 \mathrm{E}=\mathrm{O}$

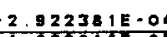

6. $080683 E-03$

7. $243788 E-03$

$3.82082 I E-03$
$26505 B E-03$

$3.6387 A 7 E-03$
$-2.66553 \mathrm{EE}-03$

$-1.205617 E-02$
$-4.03 B 23 E-03$

1. $20 B 402 E-02$

$\begin{array}{r}1.208402 E-02 \\ -1.938337 E-03 \\ \hline\end{array}$

S. 203904E-O
$2.687094 E-02$

$-3.823549 E-02$
$-1.478305 E-03$

6.821679E-02
T3

$-2.403716 E-01$

-8.053020E-O3

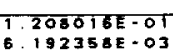

6. $093303 E-01$

B. $151309 E \cdot 01$

$-3.388339 E-02$

$1.190711 E-02$

$-2.2945048-02 \cdots$

$2.678204 E \cdot 03 \quad 0.0$

R 1

0

$\stackrel{0.0}{0}$

0.0

$0: 0$

0.0

$-9.279822 E-05$

7. $20179 E-O S$

$-9.281686 E-05$

$\circ .0$

0.0

-

$-4.157759 E-02$
$-7.3885865-03$

$-3.3054018-01$

$-3.305491 E-01$
$-6.373119 E-03$

$-9.273686 E-O 5$

$-9.259309 E-05$

$-7.824308 E-05$

$-3.379449 E-01$-9.262246E-05

1.263672E-02

$7.58722 E-02 \quad-9.280825 E-05$
$-3.43640=03 \quad-721567 E-05$

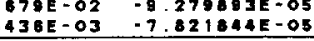

$9.00688-01$
$1.163177 E-03$

2

0.001000

0.0

0.0

$-3.251556 E-03$

$2.379891 E-05$

$\begin{array}{ll}0.0 & 0.0 \\ 0.0 & 0.0\end{array}$

$-3.247474 E-03 \quad-1.275141 E-04$
$-2392136 E-05$

$.3 .247474 E-03 \quad-1.275910 E-04$ $2.382136 E-05 \quad-1.824869 E-05$

$\begin{array}{ll}0.0 & 0.0 \\ 0.0 & 0.0\end{array}$

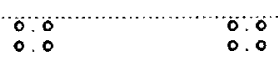

$-3.248231 E-03-1.289378 E-04$
$-2.392887 E-05-1.827462 E-05$

$-3.247918 E-02 \quad-1.269207 E-00$

$-3.257081 E-03 \quad-1.273093 E-04$

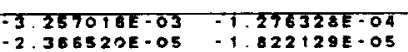

$-356714 E-03-1275015 E-04$
$-2.36382 E-O S \cdots$

$-3258025 E-03 \quad-1.273387 E-04$ $-2.387173 E-05 \quad-1.822673 E-05$

J.247480E-03 -1.276150E-04

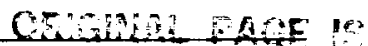


AH - IG THREE-OIMENSIONAL BUILTUP DYMAMICS MODEL W/ CONTROLS MODELED

THIS VERSION CONTAINS A BUITUP TAILBOOM MOOEL

COMPLEX EIGENYALUE = - 1 O92172E-02,-1,262630E-01

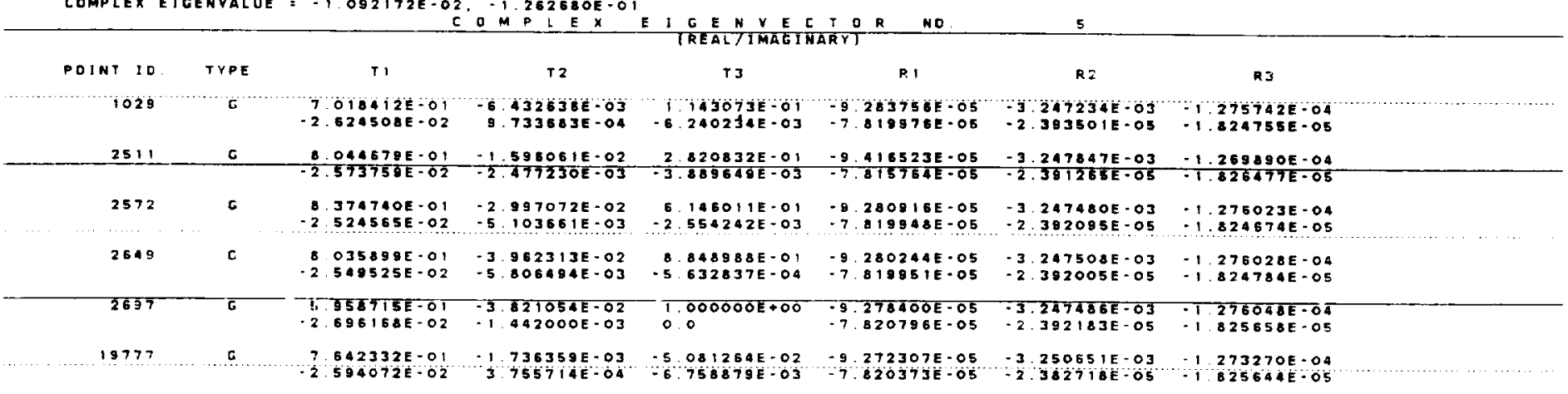

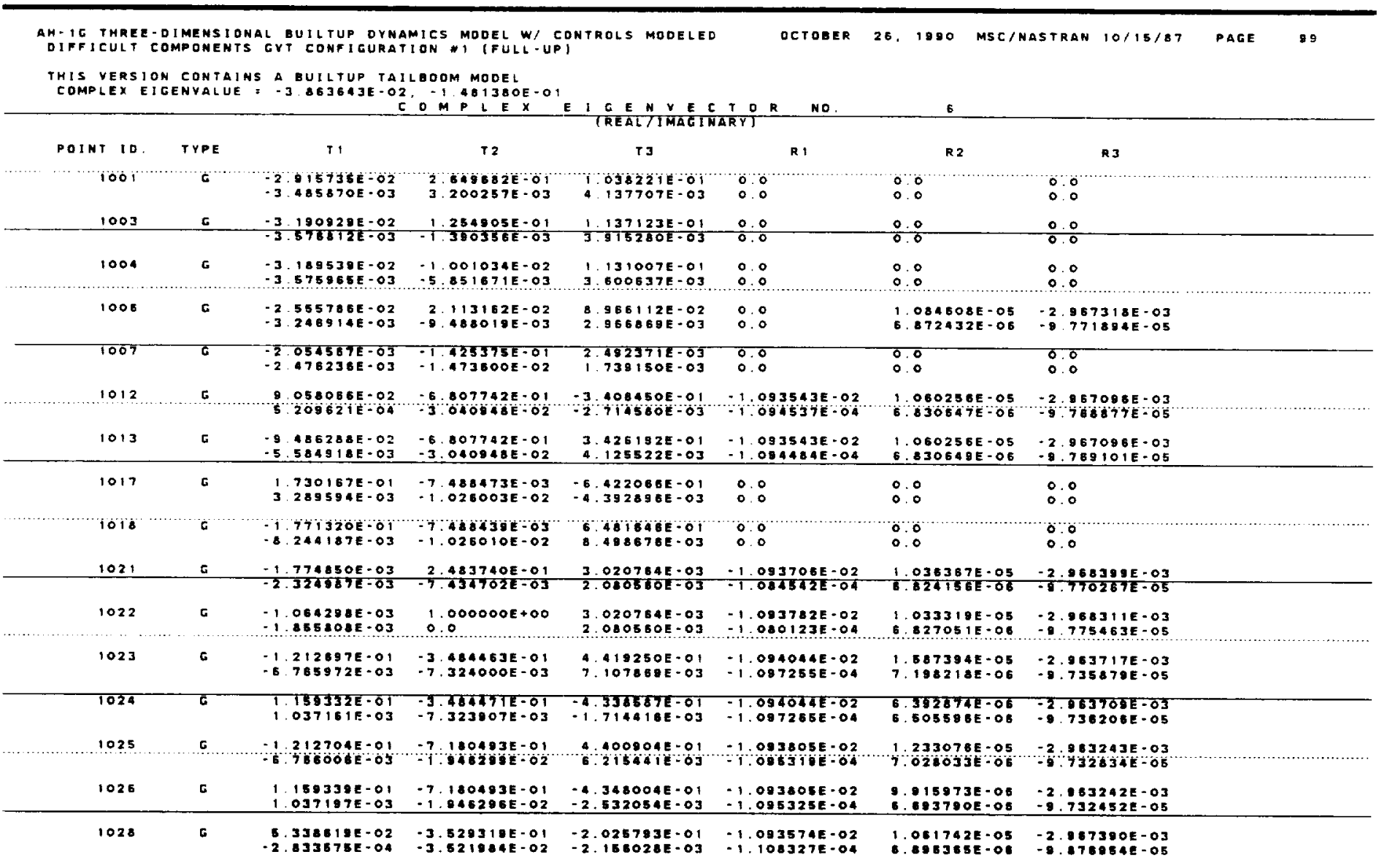


AH - IC THREE - DIMENSIOMAL SUILTUP OYMAMICS MODEL W/ CONTROLS MODELED DIFF ICULT COMPONENTS GVT COMFIGURATIDN I [FULL-UP]

THIS VERSION CONTAINS A BUILTUP TAILBDOM MODEL

COMPLEX EIGENVALUE $=-3.863643 E-02$.

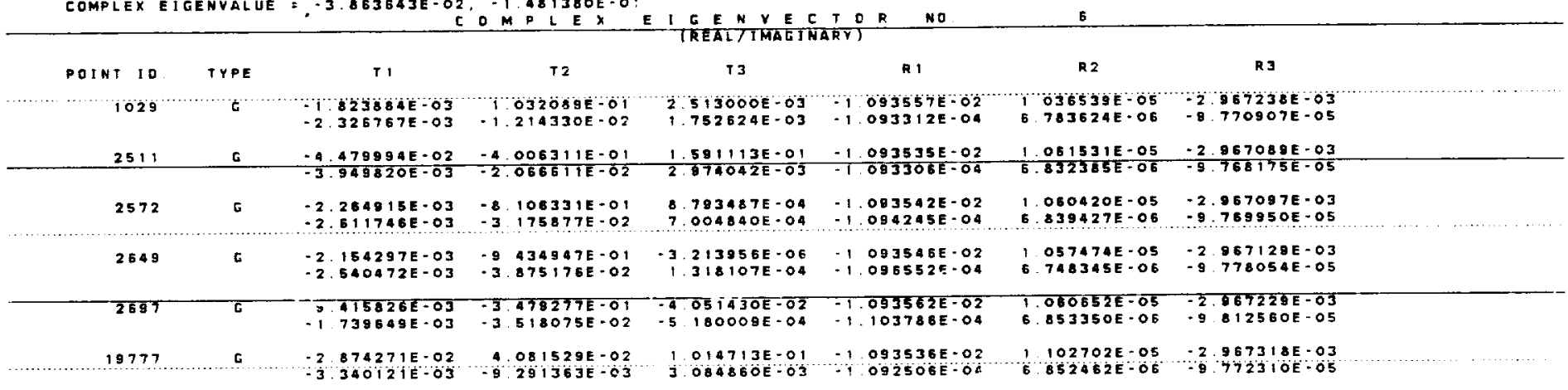

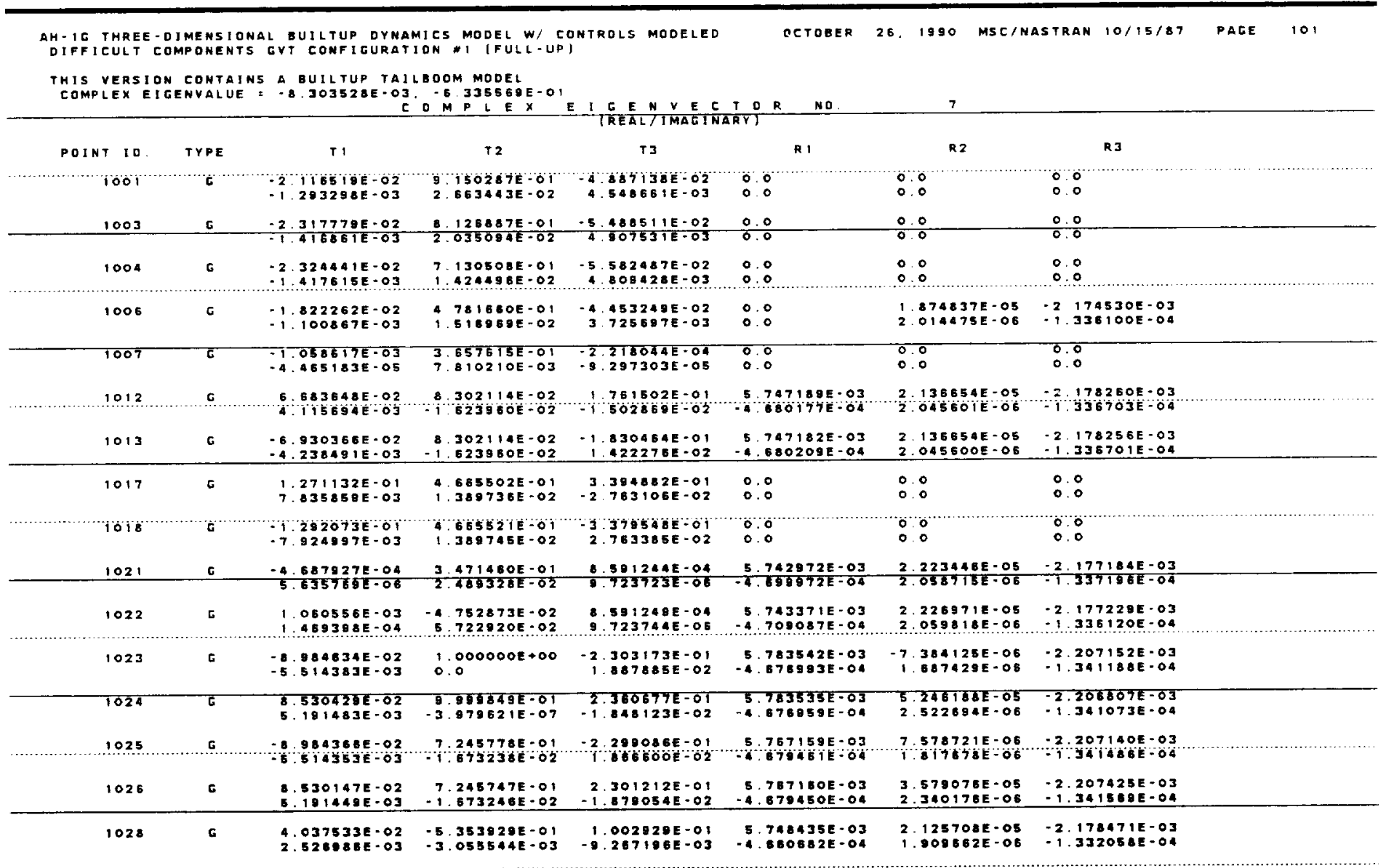


AH- IG THREE-DIMENSIDNAL GUILTUP DYMAMICS MODEL W/ CONTROLS MOdELEO DIFFICULT COMPONEWTS GVT CONFIGURATION 1 (FULL-UP)

THIS VERSION CONTAINS A BUILTUP TAILBOOM MODEL

COMPLEX EIGENYALUE = - - 303528E-03,-6.335569E-01

C. OMPLEX

POINT IO TYPE

$1029 . \cdots$,

$2511 \ldots$

20

257

2649

2657

19777

c
$T$

$5.7061606=04$

$\frac{-3.257650 E-02}{1.986508 E-03}$

$-1.483122 E-03$

- $528078 E-05$

1. $280166 \mathrm{E}-03$

$6.391983 E-05$

12

2. C4097IE-OI - TEB2952E-04

R:

$\frac{17.172855 E \cdot 01}{3.567727 E-03}$

$1485401 E-0$

$2180226 E-02$

$973228 E-02$

2. $804104 E-02$

5. $386525 E-01$
2. $42734 E-03$

5. $521141 \mathrm{~L}-0 \mathrm{a}$

$-2.053382 E-02$
$-1.24163 E-03$

4. $578298 E-01$
i $601271 E-02$

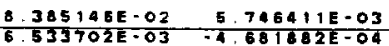

$-3.453671 E-03$
$-4.044601 E-04$ -4.747086E-03

5. $241606 E \cdot 03$ 5.74755TE-0J

5. $742689 E \cdot 04$

$1.509788 E \cdot 02$
$2.356338 E \cdot 03$

$5.073380 E-02$
$4.231516 E-O 3$

$5,74836 \overline{B E}=\overline{03}$

5. $741949 E \cdot 03$

$-4.683502 E-04$
R 2

2 $17589 E-O S$ 2 17SBSBE-OJ

2.131882E-05 - $2.178052 E-03$

$2.138573 E-05$
$2.2178219 E-03$

2. $125333 E-05-2.178338 E-03$

2. $172048 E-O E-1.335835 E-04$

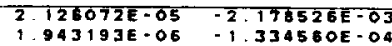

$2.045075 E-05 \quad-2174081 E-03$

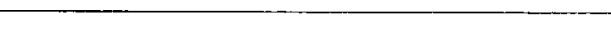

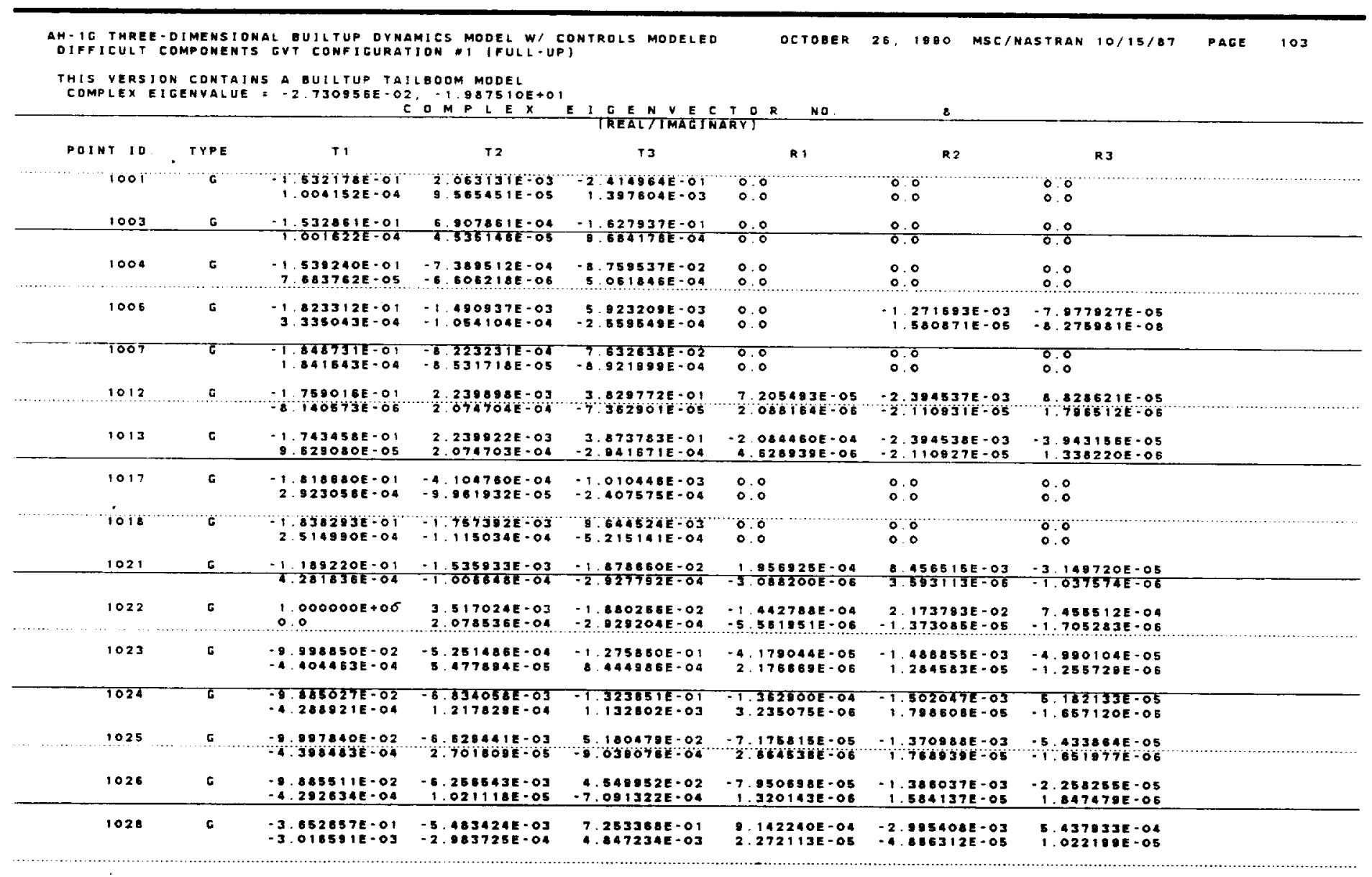


AH-15 THREE-DIMENSIONAL BUILTUP OYMAMICS MOOEL W/ CONTROLS.MODELED

DIFFICULT COMPONENTS GVT CONFIGURATION I (FULL-UP)

THIS VERSION CONTAINS A BUILTUP TAILBOOM MODEL

COMPLEX EIGENYALUE $=-2.730956 E-02,-1.987510 E+01$

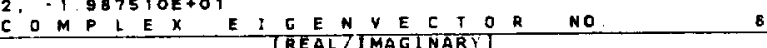

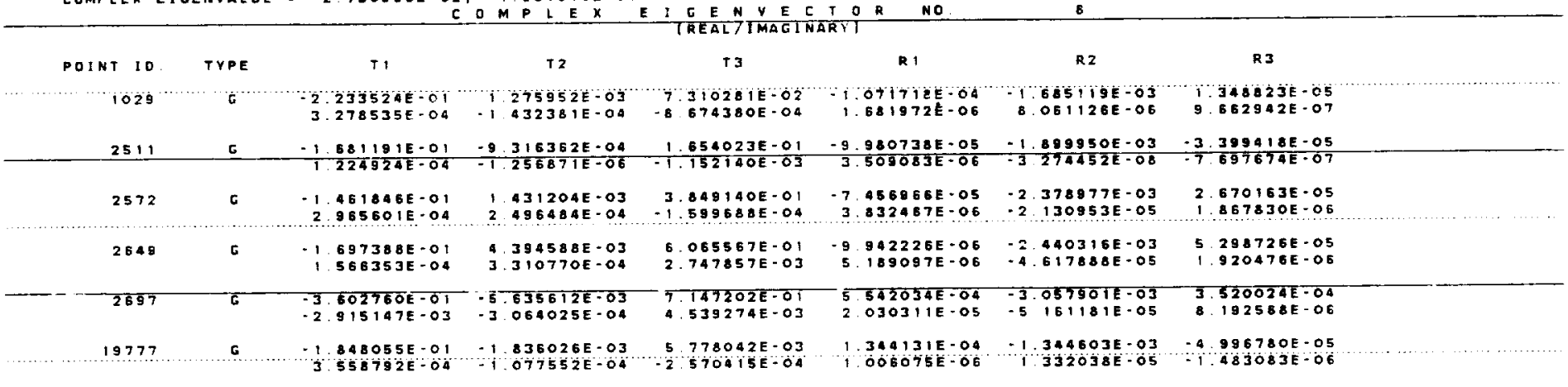
DIFFICULT COMPONENTS GVT CONFIGURATION I (FULL-UP)

OCTOBER 25, 1990 MSE/NASTRAN 10/15/87 PAGE 105

THIS YERSION CONTAINS BUILTUP TAILBOOM MOOEL

COMPLEX EIGENYALUE = - $1.262188 E-01,-2447568 E+01$

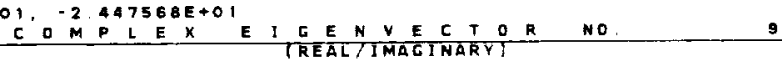

\begin{tabular}{|c|c|c|c|c|c|c|c|}
\hline & DE & TI & 12 & T 3 & & & \\
\hline 10001 & 6 & $\begin{array}{l}\text { POOE } \\
2171001 E-02\end{array}$ & $\begin{array}{l}-7.374458 E-02 \\
-3.538177 E-03\end{array}$ & $\begin{array}{r}1 \quad 442138 E=01 \\
5 \quad 061335 E-04\end{array}$ & $0: 0$ & $\begin{array}{l}0.0 \\
0.0\end{array}$ & 0.0 \\
\hline 1003 & E & $\frac{1.460826 E-02}{2.364281 E-04}$ & $\begin{array}{r}2.231037 E \cdot 03 \\
-2.548703 E-03\end{array}$ & $\begin{array}{r}-1.645372 E-01 \\
-3.055672 E-04\end{array}$ & $\div 0$ & $\frac{0.0}{0.0}$ & $\div 0: 0$ \\
\hline 1004 & $G$ & $\begin{array}{r}1.381936 E-02 \\
1.948579-04\end{array}$ & $\begin{array}{r}702385 E-02 \\
-1.688911 E-03\end{array}$ & 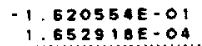 & 0.0 & $\begin{array}{l}0.0 \\
0.0\end{array}$ & $\therefore$ \\
\hline
\end{tabular}

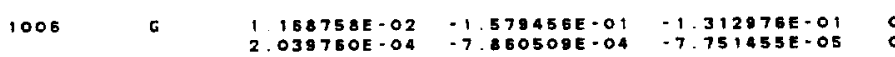

1009 G $7.083323 E-04-8.493840 E-02-3.823185 E-03 \quad 0.0$

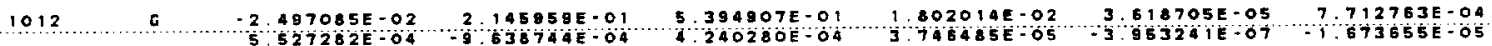

\begin{tabular}{|c|c|c|c|c|c|c|c|}
\hline 1009 & 6 & $\begin{array}{l}7.0833238-04 \\
1.178711 E-04\end{array}$ & $\begin{array}{l}-8.493840 E-02 \\
-3.725174 E-04\end{array}$ & $\begin{array}{l}-3.923185 E-03 \\
-3.014043 E=-04\end{array}$ & $\because: 0$ & $\because: 0$ & $\begin{array}{l}0.0 \\
0.0\end{array}$ \\
\hline 1012 & $a$ & $\begin{array}{r}-2497085 E-02 \\
-3527282 E=04\end{array}$ & $\begin{array}{r}2.145959 E-01 \\
-963874 q E=04\end{array}$ & $\begin{array}{r}594907 E-01 \\
4.240280 E=004\end{array}$ & $\begin{array}{l}1.802014 E-02 \\
3 .+46485 E-06\end{array}$ & 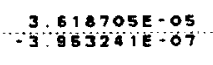 & $\begin{array}{r}7.712763 E-0 Q \\
\hdashline 79355 E-05 \\
\hdashline 73655\end{array}$ \\
\hline 1013 & G & $\begin{array}{r}2.343940 E-02 \\
-4.767028 E-04 \\
\end{array}$ & $\begin{array}{r}2.1459598-01 \\
-9.538736 E-04 \\
\end{array}$ & $\begin{array}{r}-5.779833 E=01 \\
-2.073500 E-03 \\
\end{array}$ & $\begin{array}{r}1.802555 E-02 \\
3.842983 E-05 \\
\end{array}$ & $\begin{array}{r}3.615928 E-05 \\
-3.949381 E-07 \\
\end{array}$ & $\begin{array}{r}7.711950 E-04 \\
.7758035 E-05 \\
\end{array}$ \\
\hline 1017 & G & $\begin{array}{l}-7.678298 E-02 \\
-4.607493 E-04\end{array}$ & $\begin{array}{l}-1.379429 E-01 \\
-7.128601 E-04\end{array}$ & $\begin{array}{l}1.000000 E+00 \\
0.0\end{array}$ & 0.0 & $\because 0$ & 0.0 \\
\hline 1018 & 6 & $\begin{array}{l}7.833304 E-O 2 \\
7246569 E-04\end{array}$ & $\begin{array}{l}-1.379475 E-01 \\
-7.07944 E-04\end{array}$ & $\begin{array}{r}-8.976126 E=01 \\
-1.617118 E-04\end{array}$ & $\begin{array}{l}0.0 \\
0.0\end{array}$ & $\begin{array}{lll}0.0 & 0 \\
0.0 & 0\end{array}$ & $0: 0$ \\
\hline 1021 & $E$ & $\frac{2.977527 E-03}{1367138 E-04}$ & $\frac{-3 \cdot 140482 E-01}{6.323010 E-05}$ & $\begin{array}{r}2.005981 E-03 \\
.203081 E-05\end{array}$ & $\frac{-5.788957 E-03}{-8.756022 E \cdot 05}$ & $\begin{array}{r}5.89223348-05 \\
-2.7060168-06\end{array}$ & $\frac{1.428623 E-03}{1.224712 E-05}$ \\
\hline 1022 & $G$ & $\begin{array}{r}-260853 E-03 \\
-4 \quad 089472 E-04\end{array}$ & $\begin{array}{l}5.130880 E-01 \\
7.17268 E-03\end{array}$ & $\begin{array}{r}2.008638 E-03 \\
-6.210825 E=05\end{array}$ & $\begin{array}{r}-1.858848 E-02 \\
-1.299552-04\end{array}$ & $\begin{array}{r}-9.528788 E-05 \\
-9.125358 E-05\end{array}$ & $\begin{array}{r}2.929075 E-03 \\
1.800269 E-05\end{array}$ \\
\hline 1023 & $\mathrm{G}$ & $\begin{array}{l}4.985265 E-02 \\
3.397780 E-04\end{array}$ & $\begin{array}{r}7.675277 E-01 \\
-2.889535=03\end{array}$ & $\begin{array}{r}-7.370909 E-01 \\
.251775 E-03\end{array}$ & $\begin{array}{r}1.838 B 50 E-02 \\
-1.850320 E-05\end{array}$ & $\begin{array}{r}-1.381488 E-03 \\
2.352923 E-05\end{array}$ & $\begin{array}{r}-2.267090 E-04 \\
3.013447 E-05\end{array}$ \\
\hline 1024 & $\bar{G}$ & $\begin{array}{l}-8.276593 E-02 \\
-6.011638 E-04\end{array}$ & $\begin{array}{r}9.063127 E-01 \\
-2.877710 k-03\end{array}$ & $\begin{array}{l}\text { P. B32229E-O1 } \\
-6.835700-04\end{array}$ & $\begin{array}{r}1.876580 E-02 \\
-1.628731 E-05\end{array}$ & $\begin{array}{r}1.792430 E \cdot 03 \\
-1.515812 E-05\end{array}$ & $\begin{array}{r}-1.800218 E-04 \\
2.990084 E-05\end{array}$ \\
\hline 1025 & 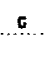 & $\begin{array}{r}492253 E-02 \\
350050 E=04\end{array}$ & $\begin{array}{r}800511 E-01 \\
-6.05423 E=04\end{array}$ & $\begin{array}{r}-7.000149 E-01 \\
7.27234 \text { IEE }\end{array}$ & $\begin{array}{r}825095 E-02 \\
-129208 E=05\end{array}$ & $\begin{array}{r}535366-04 \\
\hdashline-2134765=-06\end{array}$ & $\begin{array}{r}2.130581 E-03 \\
7.8764001=06\end{array}$ \\
\hline 1026 & a & $\begin{array}{r}-5.273929 E-02 \\
-5.983418 E-04 \\
\end{array}$ & $\begin{array}{r}8.889389 E-01 \\
-8.144859=04 \\
\end{array}$ & $\begin{array}{r}6.848917 E-01 \\
-4.418783 E-04 \\
\end{array}$ & $\begin{array}{r}1.828880 E-02 \\
-1.307122 E-05 \\
\end{array}$ & $\begin{array}{r}-3.181332 E-04 \\
8.850540 E-06 \\
\end{array}$ & $\begin{array}{r}2.122804 E-03 \\
7.260633 E-06 \\
\end{array}$ \\
\hline 1028 & G & $\begin{array}{l}-1.084563 E-02 \\
-3.146788 E-03\end{array}$ & $\begin{array}{l}-9.7751998-01 \\
-1.744610 E-02\end{array}$ & $\begin{array}{l}\text { 3. } 727258 E-01 \\
8.002545 E-03\end{array}$ & $\begin{array}{l}2.084738 E-02 \\
4.708500 E-04\end{array}$ & $\begin{array}{l}-2.940222 E-04 \\
-3.44174 E-05\end{array}$ & $\begin{array}{r}-1.0118282 E-04 \\
1.329212 E-04\end{array}$ \\
\hline
\end{tabular}

7.678298E-02-1.379429E-01 $1.000000 E+00$

0.0

$\begin{array}{lll}0 & 1.76935 E-04 & 335465 E-03 \\ 0 & 470303 E-06 & 1.0133985-05\end{array}$ 


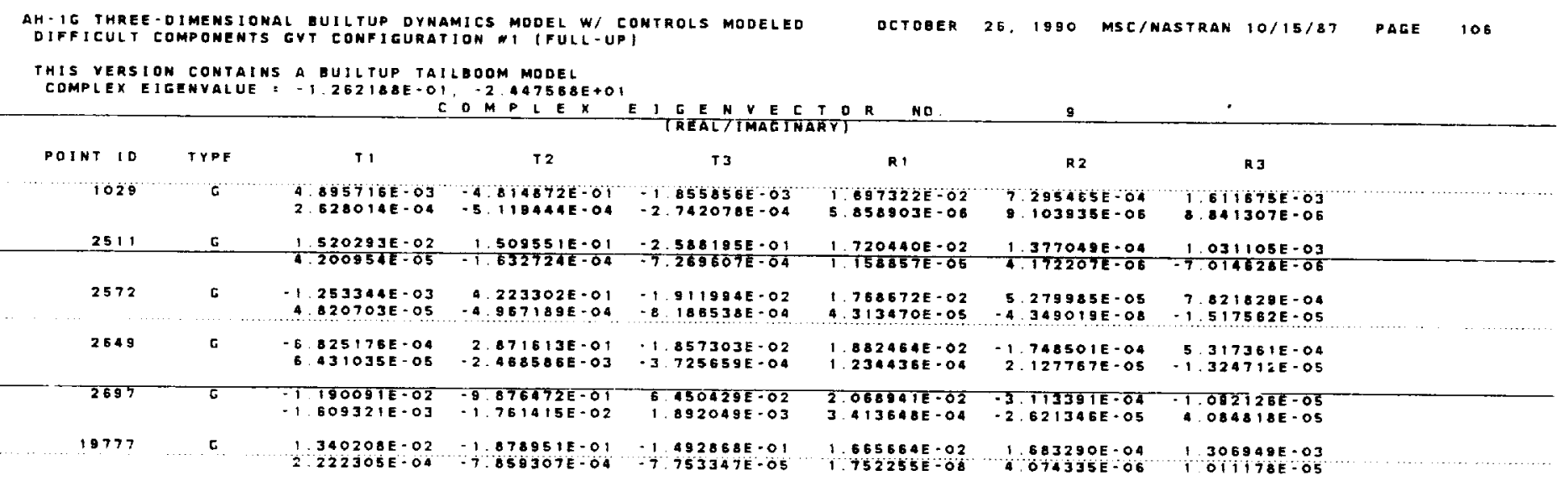

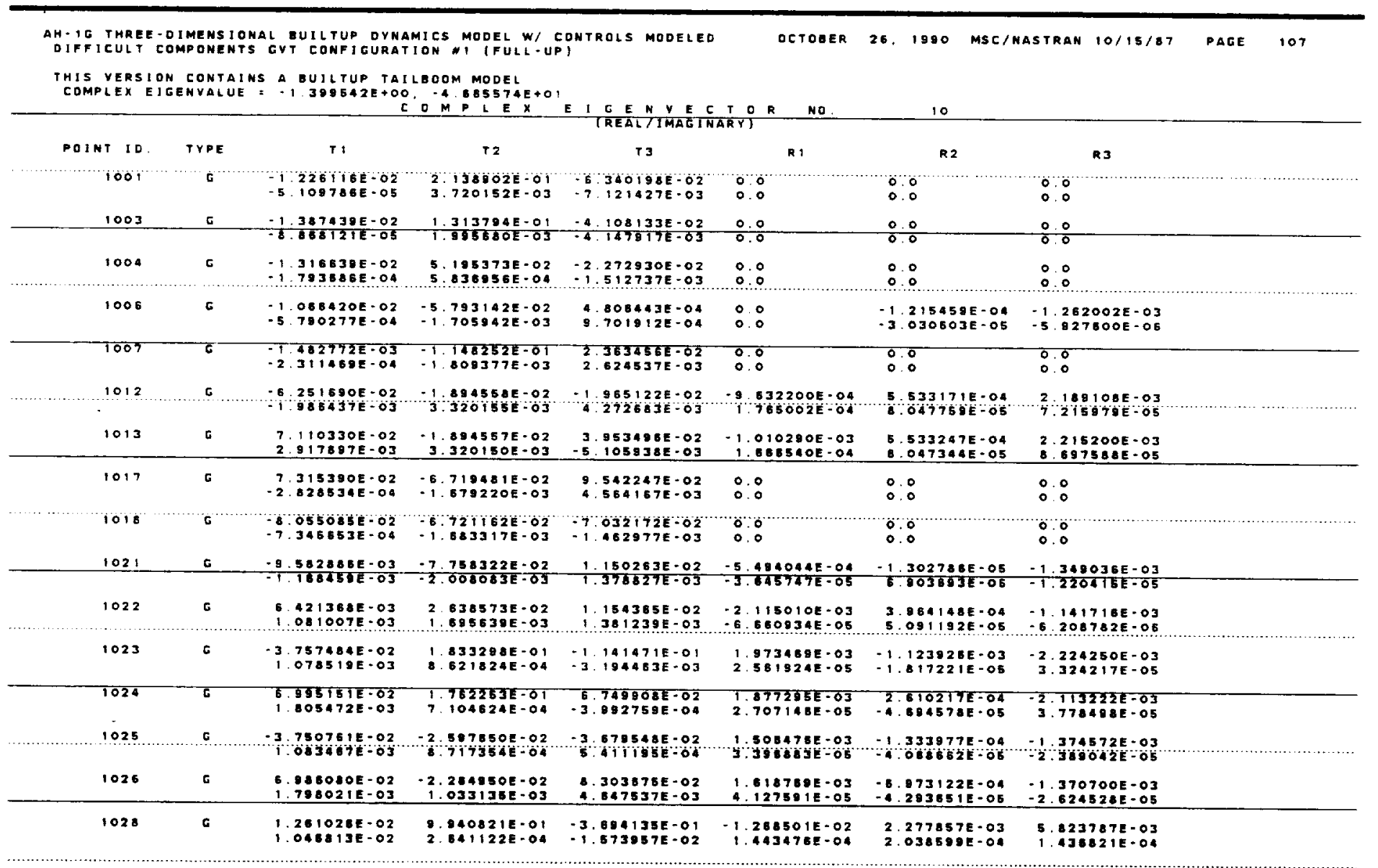


THIS YERSION CONTAINS A BUILTUP TAILBOOM MODEL

COMPLEX E1GENVALUE = $+1.399542 E+00,-4.6 B 5574 E+0$

CC PLEX E I TEENVECTOR NO 10

POINTIO TYPE T1 T2

1029
G
$-6258840 E-04$

$2511 \quad 6 \quad 6.213132 E-03 \quad 1.263818 E-01 \quad 2080758 E-02$

$2572 \quad C \quad-2.345707 E-03 \quad-2.802502 E-02$

C $\quad-5,334741 E-04$

2649

269

$19797+20$
$1.851322 E-03$
$-4833657 E-05$

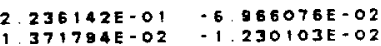

$1.000000 E+00$

$972770 E-02$
$1.233108 E-02$

$1.255305 E-02$
$-432003 E-04$

1.863 IA6E-O

$-5.045883 E-02 \quad-1.025280 E-03$
R 1 $220264 E-03$
$6.095 B 56 E-05$

$7.547030 E-04$

-8. $879911 E-04$

$5.058698 E+03$

2. $369995 E \cdot 04$

1. $231534 \bar{E} \cdot 0.2$

$395309 E-03$
R 2

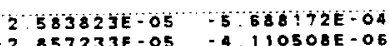

$\frac{1.020715 E-04}{5.753426 E-06} \quad \frac{9.390314 E-04}{5.255266 E-05}$

5. $416934 \mathrm{E} \cdot 04$

$5.941212 \mathrm{E}-05$

$2.043839 E-03$

$.636074 E-05$

1. $489626 \mathrm{E} \cdot 03$

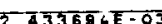

3. $240170 E \cdot 03$

5. $327951 E-03$

\begin{tabular}{l}
$1.306690 E-03$ \\
6. \\
\hline $1182 E-06$
\end{tabular}

AH-1G THREe-DIMENSIONAL BHLTUP DYMAMICS MODEL W/ CONTROLS MODELED

OCTOBER 26,1990 MSE/NASTRAN 10/15/8

THIS VERSION CONTAINS A BUILTUP TAILBOOM MODEL

COMPLEX E1GENVALUE $=-2.305214 E+00,-5.082168 E+01$

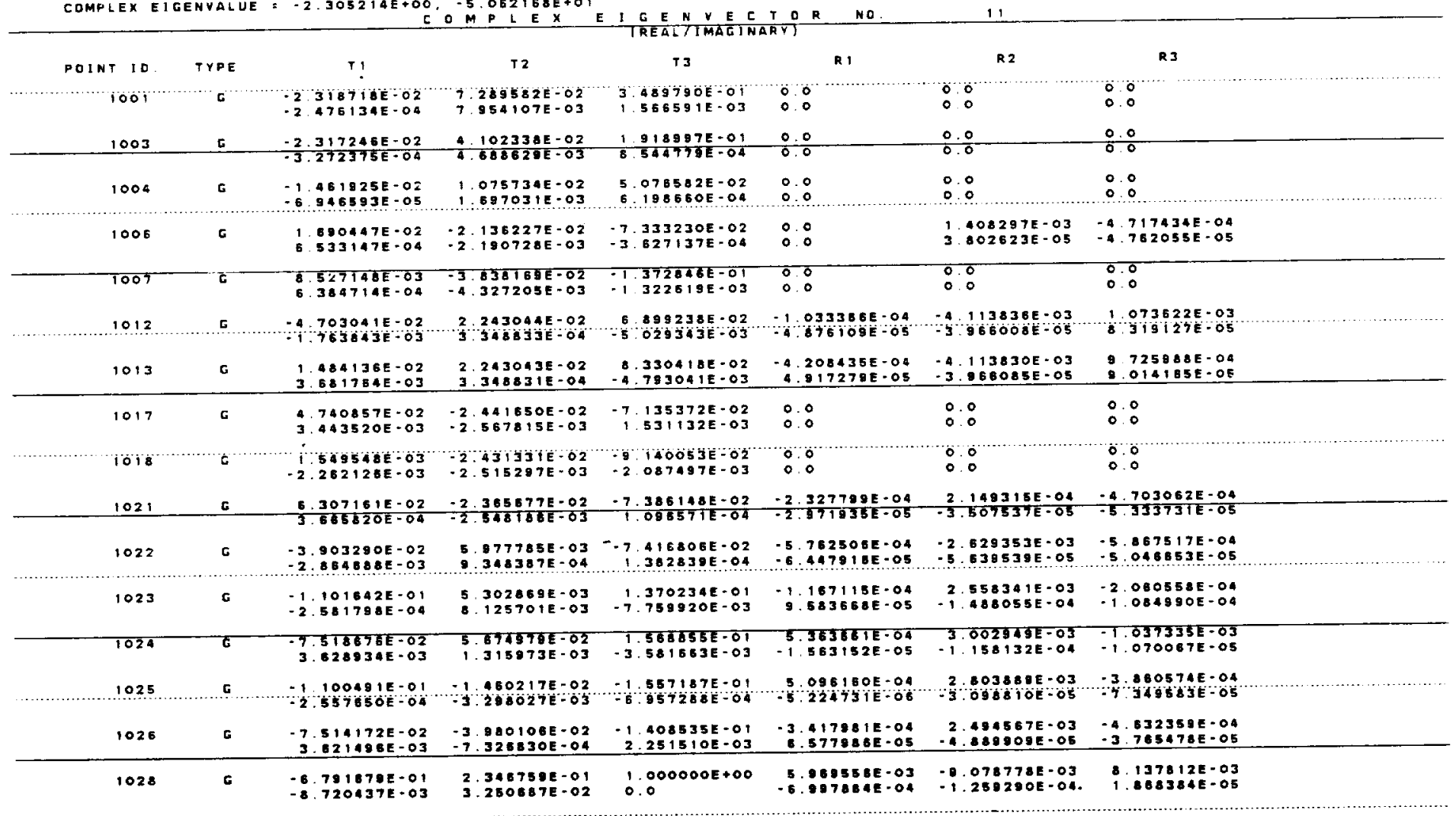


AH- IG THRE - DIMENSIONAL UUILTUP OYNAMICS MODEL W/ CONTROLS MODELED

THIS VERSION CONTAINS A BUILTUP TAILBOOM MODEL

COMPLEX EIGENYALUE = $-2.305214 E+00,-5.052168 E+0$

COMFLEX ET GENUECTOR NO

POINT IO TYPE

$1029 \quad$ G

$2572 \quad G \quad 3.300672 E \cdot 02$

$2649 \quad\left[\begin{array}{l}2.34099 E-03 \\ 1.214371 E-03\end{array}\right.$

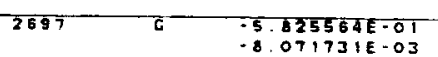

$19777 \quad G \quad G \quad 950846 E-02$
12

T3 $\begin{array}{lll}278220 E-03 & -4.885221 E-02 & -1.344169 E-01 \\ & -5.36855 E-03 & -1.286566-03\end{array}$ $\begin{array}{lll}394868 E-03 & -4.076775 E-02 & -1.522826 E-01\end{array}$

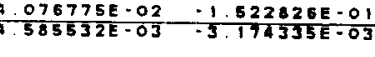

$053120 E-02$

-4.470507E-03

$1.308087 E-01$

2.345750E $=0$
3. $258081 \mathrm{E} \cdot 02$

2. $137403 E-02$

$2,137403 E-02$
$2,260088 E-O B$
$8.372224 E-01$
$8.66157 \mathrm{E}-03$

$933656 E-02$
$4.046727 \%=04$
R T

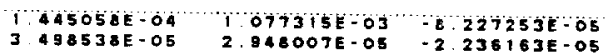

1.30562E-04 -5,754433E-0Q $2010332 E-04$

$-1737728 E-04 \quad-4.162583 E-03 \quad 9.581670 E-04$

$1.076532 E-05$
$-4.728378 E-05 \quad 8 \quad 724354 E-05$

$821271 E-04$
$1.4 .657681 E-03 \quad 1.710141 E-03$

$2466035 E_{-03}-9690790.03$ 5.

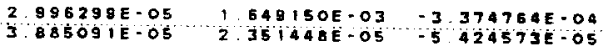

AH- IE THREE-DIMENSIONAL BUILTUP DYNAMICS MODEL W/ CONTROLS MOOELED

DCTOGER 26, 1990 MSC/NASTRAN 10/15/87 PAGE 111

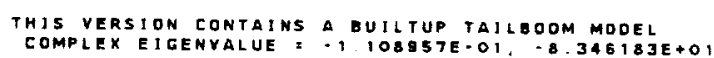

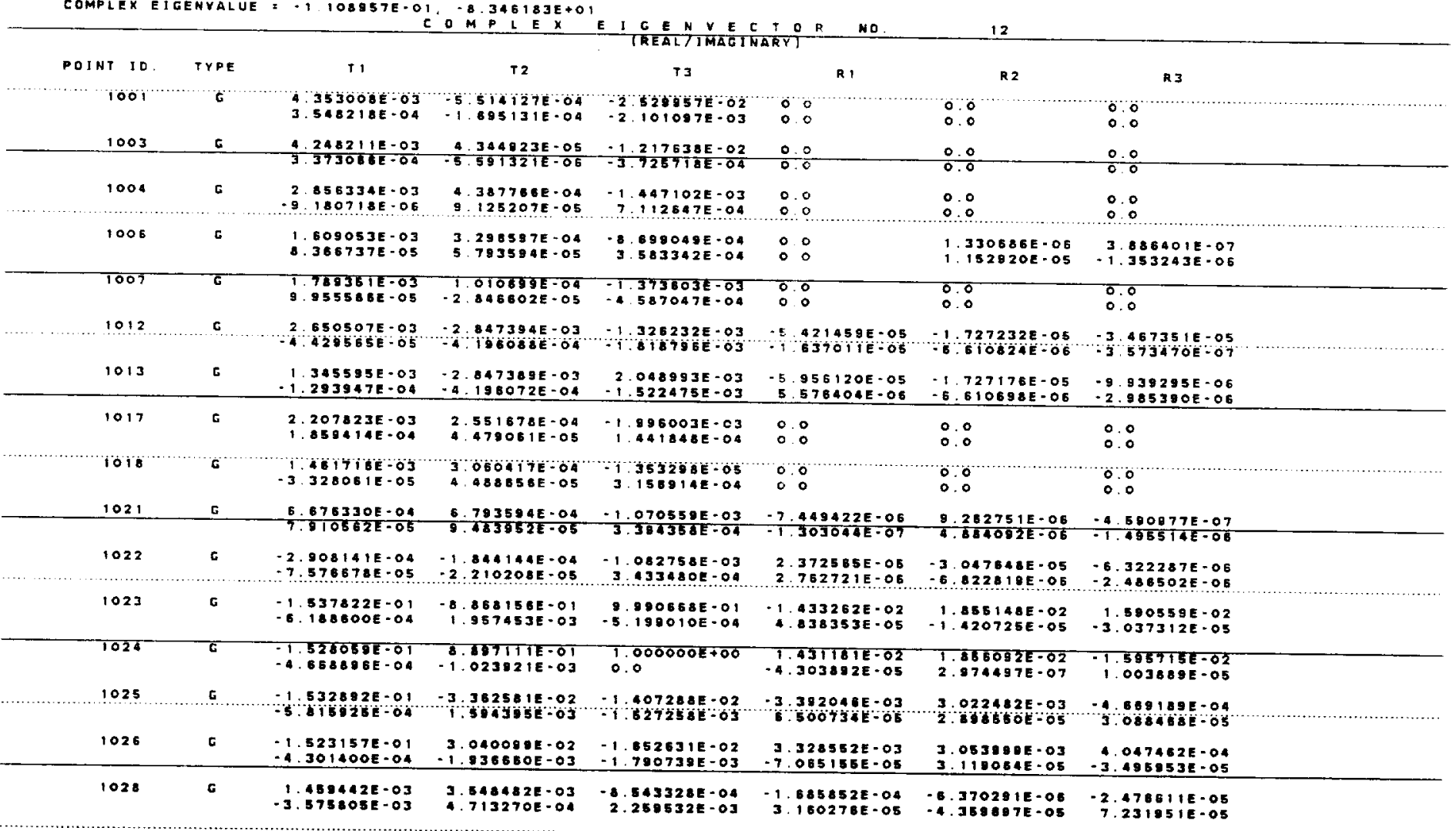


AH- IG THREE-Dimensiomal BUILTUP DYMAMICS MODEL W/ CONTROLS MDOELED

THIS VERSION CONTAINS A BUILTUP TAILBOOM MODEL

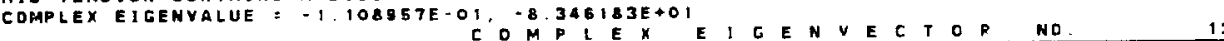

\begin{tabular}{|c|c|c|c|c|c|c|c|c|c|c|}
\hline POINT ID & TYPE & & $T_{1}$ & & 72 & & 13 & $R_{1}$ & R2 & R3 \\
\hline Tozg. & $\mathrm{c}$ & $i$ & $\begin{array}{l}830692 E-03 \\
645197 E-04\end{array}$ & i: & $\begin{array}{l}512277 E \cdot O 3 \\
663202 E \cdot 05\end{array}$ & $\begin{array}{l}\cdots \\
-4\end{array}$ & $\begin{array}{l}\text { OO9O76E-O3 } \\
312320 E-04\end{array}$ & $\begin{array}{l}-2.675572 E=05 \\
-1.990063 E-06\end{array}$ & $\begin{array}{r}=1.636047 E-05 \\
1.385665 E-05\end{array}$ & $\begin{array}{l}\because 2.144962 E-O 5 \\
-2.545388 E-O G\end{array}$ \\
\hline 2511 & E & $\frac{1}{-8}$ & $\frac{602477 E-03}{.55446 E-05}$ & -8 & $\frac{527689 E-04}{653597 E-04}$ & $\frac{-5}{-1}$ & $\frac{741716 E-04}{O 88118 E-03}$ & $\frac{-3.339998 E-05}{9.506558 E-07}$ & $\frac{-8.236060 E-06}{9.419827 E-06}$ & $\begin{array}{l}-1.592000 E-05 \\
-7.374727 E-06\end{array}$ \\
\hline 2572 & c & $\begin{array}{l}2 \\
1\end{array}$ & $\begin{array}{l}018316 E-03 \\
209989 E-05\end{array}$ & $\begin{array}{l}-3 \\
-4\end{array}$ & $\begin{array}{l}129734 E=03 \\
697474 E-04\end{array}$ & $\begin{array}{r}3 \\
-1\end{array}$ & $\begin{array}{l}424387 E-04 \\
514127 E-03\end{array}$ & $\begin{array}{r}5.123220 E-O 5 \\
-3747291 E-O 5\end{array}$ & $\begin{array}{l}-2.327100 E-05 \\
-6.047772 E-06\end{array}$ & $\begin{array}{l}-1.854008 E-05 \\
-9843449 E-07\end{array}$ \\
\hline 2649 & G & $\begin{array}{l}1 \\
3\end{array}$ & $\begin{array}{l}837798 E-03 \\
574303 E-05\end{array}$ & $\begin{array}{l}-4 \\
-3\end{array}$ & $\begin{array}{l}180949 E-03 \\
778845 E-04\end{array}$ & 3. & $\begin{array}{l}848164 E-03 \\
238637 E-04\end{array}$ & $\begin{array}{l}-9118873 E-05 \\
-1118815 E-05\end{array}$ & $\begin{array}{l}-4.027988 E \cdot 05 \\
-4.630417 E-06\end{array}$ & $\begin{array}{r}-1997616 E-05 \\
3389188 E-O 5\end{array}$ \\
\hline 2697 & C & & $\begin{array}{l}187609 E-03 \\
773572 E-03\end{array}$ & 4 & $\begin{array}{l}622063 E \cdot 03 \\
697362 E \cdot 04\end{array}$ & $\frac{1}{1}$ & $\begin{array}{l}600731 E-03 \\
924322 E \cdot 03\end{array}$ & $\begin{array}{r}-1.615712 E-04 \\
4593385-05\end{array}$ & $\begin{array}{l}-2.6757 \overline{38 E}-06 \\
-5.099536 E-05\end{array}$ & $\begin{array}{r}-1.267241 E-05 \\
3.966180 E-05\end{array}$ \\
\hline 19777 & c & 9 & $\begin{array}{r}638616 E-03 \\
314847 E-05\end{array}$ & 3 & $\begin{array}{l}598697 E-04 \\
\text { GT7TAQE-OS }\end{array}$ & $\begin{array}{r}-8 \\
3\end{array}$ & $\begin{array}{l}519923 E-04 \\
594509 E=04\end{array}$ & $\begin{array}{l}-1.658577 E-05 \\
\because \quad 033969 E-O 6\end{array}$ & $\begin{array}{r}\text { 1. } 683889 E-05 \\
9.84385 I E-O 6\end{array}$ & $\begin{array}{r}-619030 E-07 \\
-20+2062 E-06\end{array}$ \\
\hline
\end{tabular}

AH- IF THREE-DIMENSIONAL BUILTUP OYNAMICS MODEL W/ CONTROLS MOOELED

OCTDBER 25, 1990 MSC/NASTRAN 10/15/87 PAGE 113

DH - TH THRE - DIMENSIOHAL BUILTUP OYNAMICS MOOEL W/ CONT

THIS VERSION CONTAINS A BUILTUP TAILBOOM MODEL

THMPLEX EIGENYALUE =-1.650773E+00,-9.683298E+01

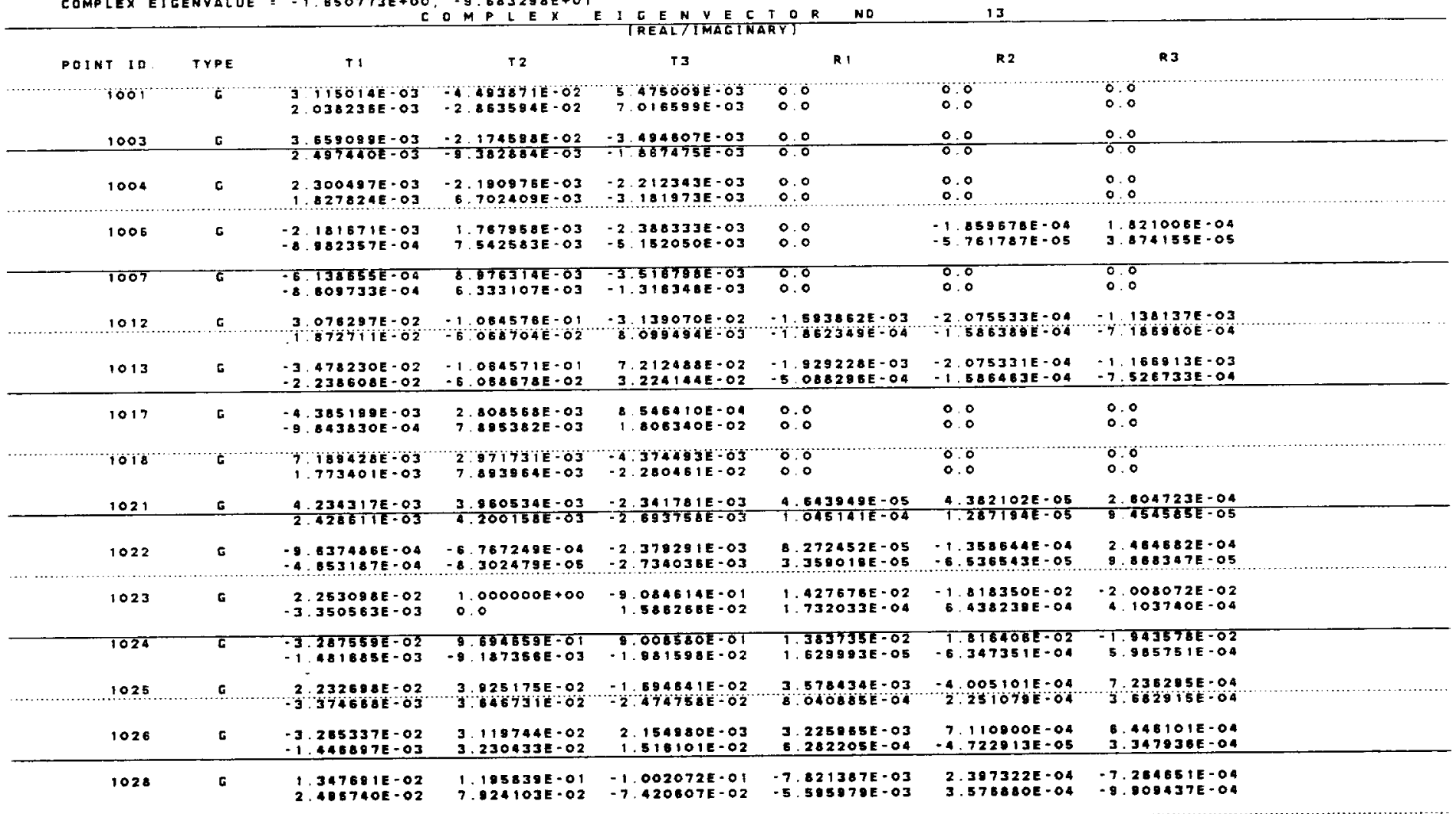


AH-1G THREE-DIMENSIONAL BUILTUP OYNAMICS MODEL W/ CONTROLS MODELED DIFFICULT COMPONENTS GVT CONFIGURATION IFULG-UPI

THIS VERSION CONTAINS A GUILTUP TAILBOOM MODEL

COMPLEX EIGENYALUE: $-1.550773 E+00,-9.683298 E+0$

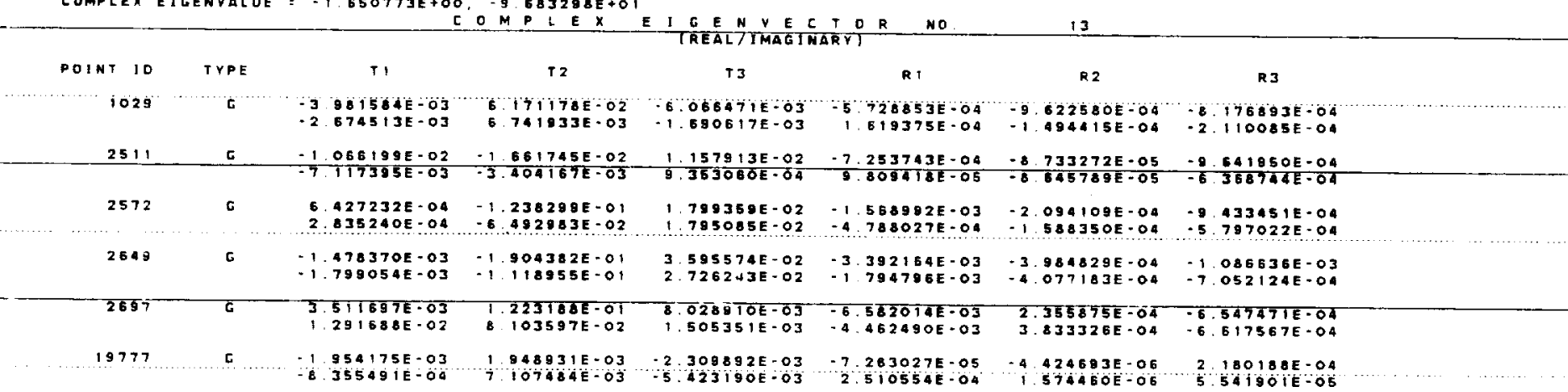

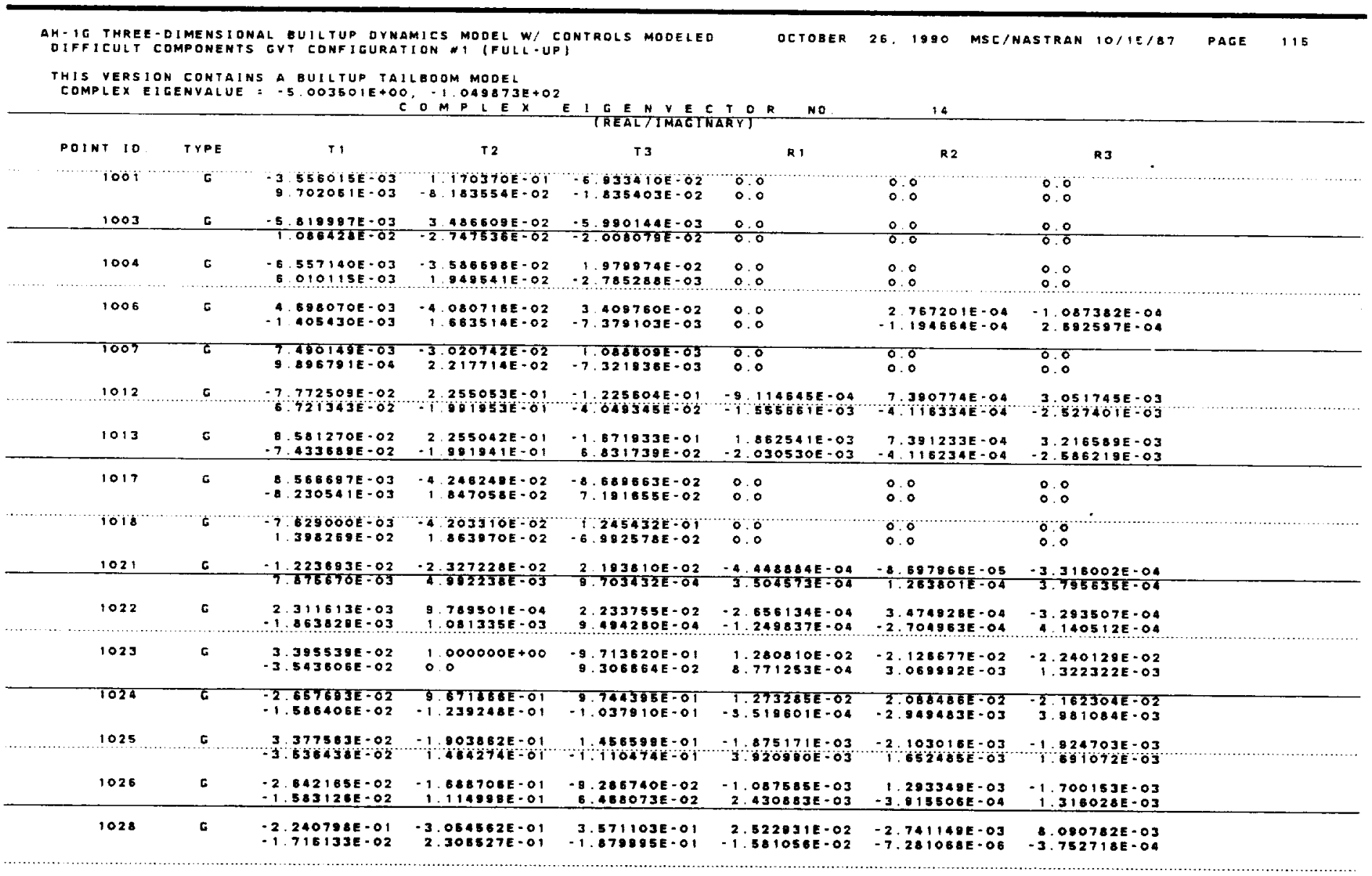




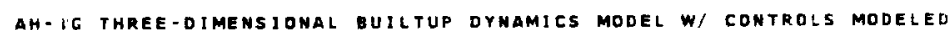

DIFF ICULT CDMPONENTS GYT CONFIGURATION \# 1 (FULL-UP)

THIS VERSION CONTAINS A GUILTUP TAILBOOM MODEL

COMPLEX EIGENYALUE = $.5 .003501 E+00,-1.049873 E+02$

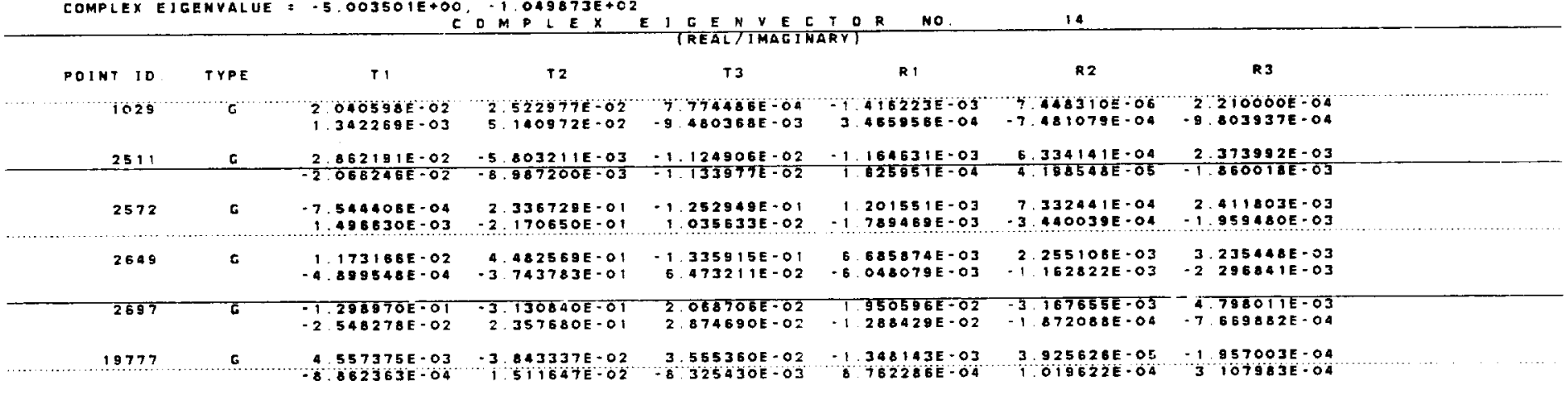



AH-IG THREE-OIMENS IONAL BUILTUP DYMAMICS MOOEL W/ CONTROLS MODELED
DIFFICULT COMPONENTS CVT COMFIGUATION I (FULL-UP)

THIS VERSIOM CONTAINS AUILTUP TAILBOOM MODEL

COMPLEX EIGENVALUE = - - O47928E+00, - $1.075832 E+02$

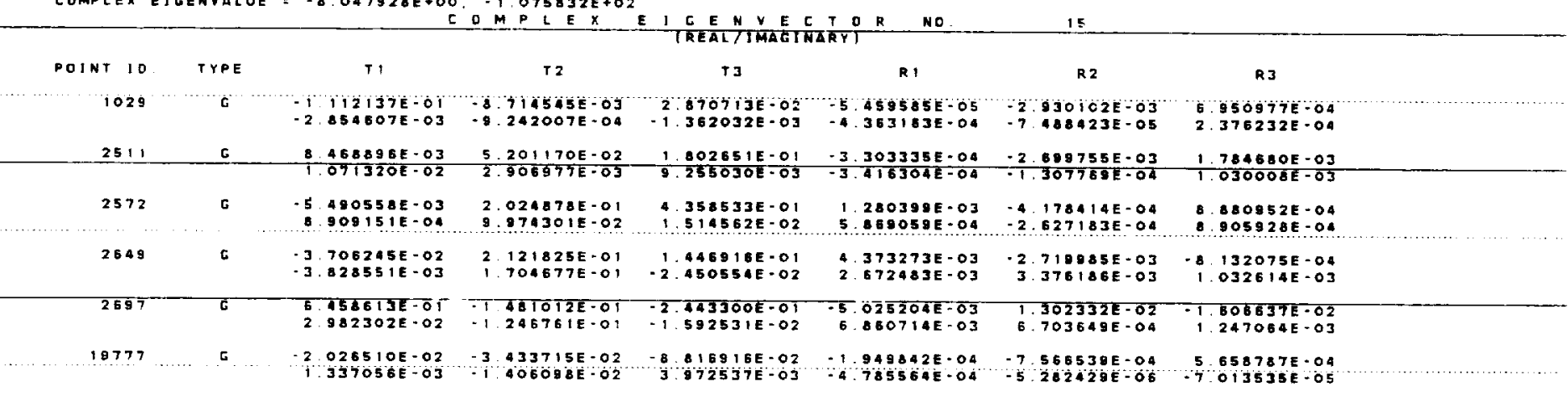

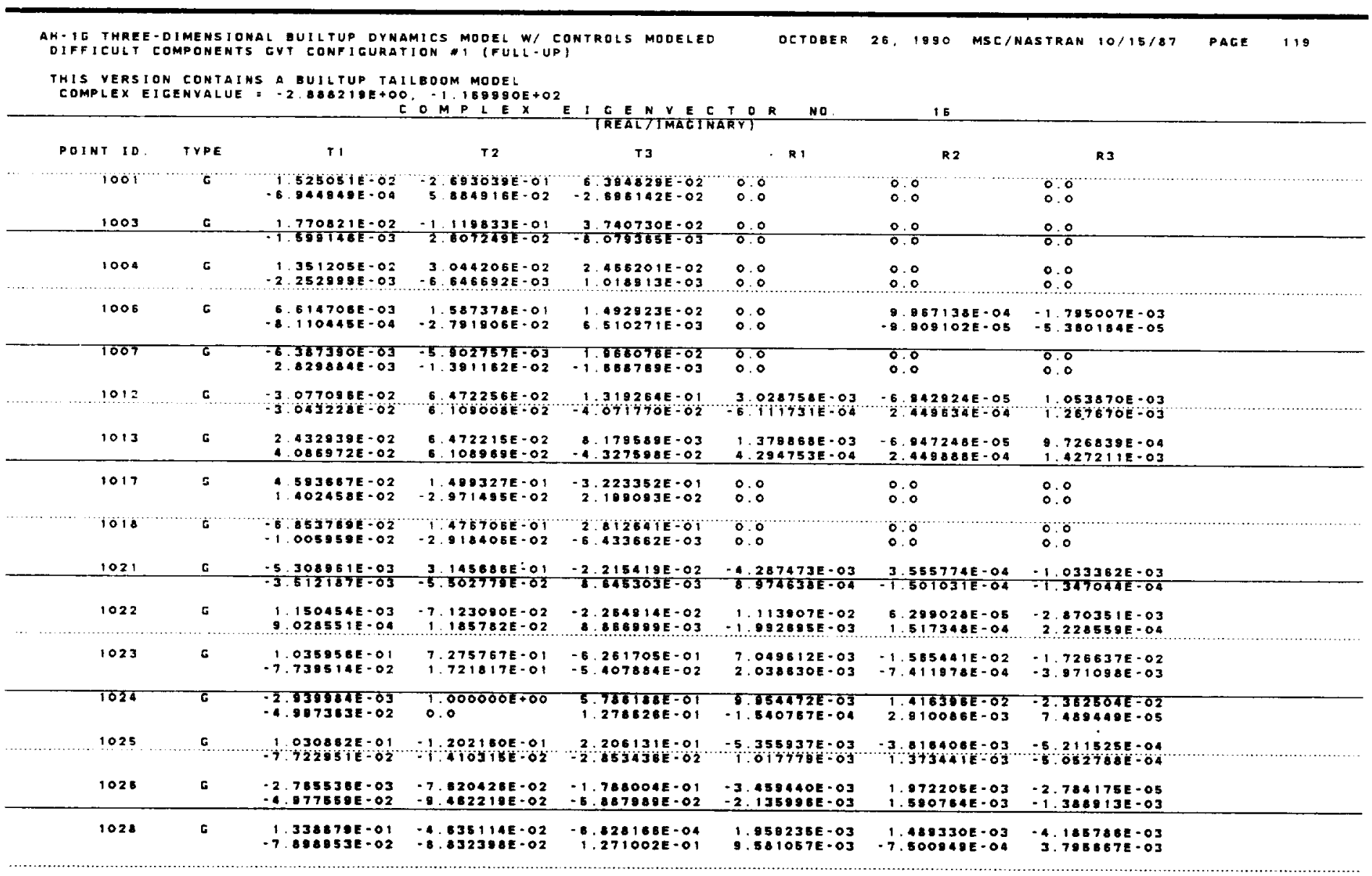


AH- IG THREe-dimens IOHAL BUILTUP DYMAMICS MODEL W/ CONTROLS MOdeled OIFF IEULT COMPONENTS GVT CONFIGURATION, 1 (FULL-UP)

DIFFICULT COMPONENTS CVT CONFIGURATION MI TRULL

COMPLEX EIGEHVALUE = -2.88219E+00.

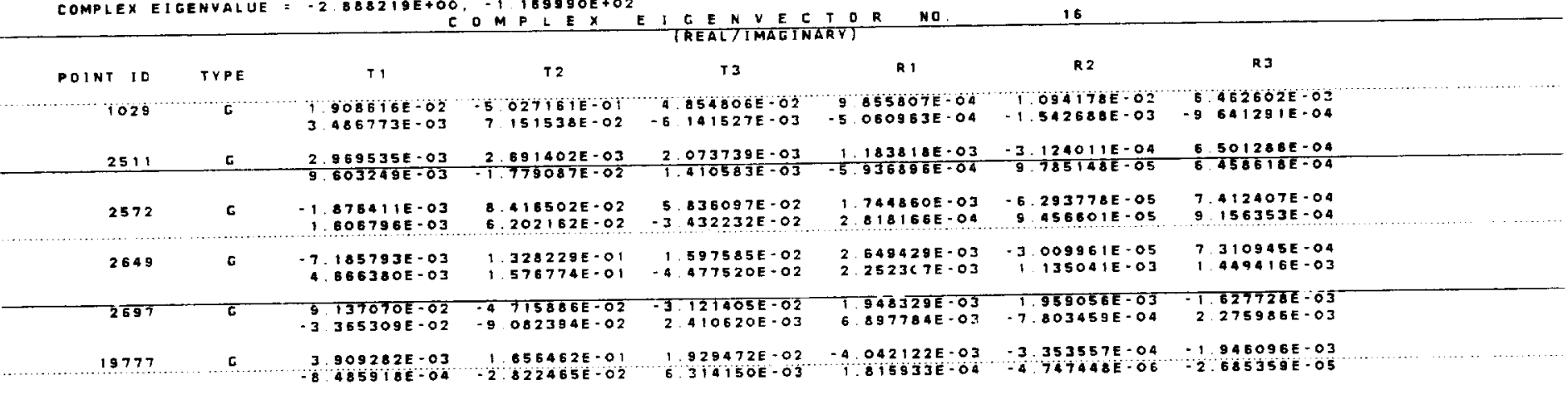

Am- ig three-dimensional builtup ornamics model W/ CONTRols modeled

DCTOBER 26, 1990 MSC/NASTRAN 10/15/87 PAGE 121

AM-IG THREE-DIMENSIONAL BUILTUP OYNAMICS MODEL W/ CONT
DIFFI CULT COMPONENTS GVT CONFIGURATION I IFULL-UPI

THIS VERSION CONTAINS A BUILTUP TAILBOOM MODEL

COMPLEX EIGENVALUE = $-2.451561 E-01,-1.181006 E+02$

\begin{tabular}{|c|c|c|c|c|c|c|c|c|}
\hline PDINT ID. & TYPE & $T 1$ & 12 & T 3 & & R 1 & R2 & a 3 \\
\hline $100 \%$ & ז' & $\begin{array}{l}1.344005 E-02 \\
2.534178 E-03\end{array}$ & $\begin{array}{l}5.147263 E-O 3 \\
-4.917276 E-O 4\end{array}$ & $\begin{array}{l}=3.540305 E-02 \\
-1.816474 E-O 2\end{array}$ & 0.0 & o & $\begin{array}{l}0 \\
0.0\end{array}$ & 0.0 \\
\hline 1003 & $G$ & $\frac{1.299758 E-02}{2.364579 E-03}$ & $\frac{1.058698 E-03}{6.834572 E-04}$ & $-9.241604 E-03$ & 0.0 & & $\frac{0.0}{0.0}$ & $\frac{0.0}{0.0}$ \\
\hline 1004 & $\sigma$ & $\begin{array}{r}1.006378 E-02 \\
-2.085997 E-05\end{array}$ & $\begin{array}{r}1.108483 E-03 \\
1.274747 E-03\end{array}$ & $\begin{array}{r}8.267927 E \cdot 03 \\
4.923372 E-03\end{array}$ & $\begin{array}{l}0.0 \\
0: 0\end{array}$ & & $\begin{array}{l}0.0 \\
0.0\end{array}$ & $\begin{array}{ll}0 & 0 \\
0 & 0\end{array}$ \\
\hline 1006 & G & $\begin{array}{l}\text { B. } 200438 E-03 \\
\text { 2. } 484495-04\end{array}$ & $\begin{array}{l}-1.784383 E-04 \\
-1.195968 E-03\end{array}$ & $\begin{array}{l}\text { 4. } 421457 E-03 \\
2.321869 E=03\end{array}$ & 0.0 & & $\begin{array}{l}7.001815 E-05 \\
7.086014 E-05\end{array}$ & $\begin{array}{r}-467444 E-05 \\
1.203441 E-05\end{array}$ \\
\hline 1007 & E & $\begin{array}{l}629592 E-03 \\
3.756774 E-04\end{array}$ & $\begin{array}{r}-2.072709 E-03 \\
2.545094 E-04\end{array}$ & $\begin{array}{r}4.3560380-03 \\
-4.15 i 396 E-03\end{array}$ & 0.0 & & $\begin{array}{l}0.0 \\
0.0\end{array}$ & $\begin{array}{l}0.0 \\
0.0\end{array}$ \\
\hline 1012 & c & $\begin{array}{r}317981 E-03 \\
1.218866 E-03\end{array}$ & $\begin{array}{r}2 \cdot 112562 E-02 \\
-83232 E-03\end{array}$ & $\begin{array}{r}4.908793 E-02 \\
=1.305679 E-02\end{array}$ & $-\frac{5}{2}$ & $\begin{array}{l}210007 E-04 \\
509249 E-04\end{array}$ & $\begin{array}{r}-1.242589 E-04 \\
-6018826 E-05\end{array}$ & $\begin{array}{r}1.230196 E-04 \\
-6200999 E=05\end{array}$ \\
\hline 1013 & $\mathrm{G}$ & $\begin{array}{r}1.563753 E-02 \\
-3.320850 E-03 \\
\end{array}$ & $\begin{array}{r}2112544 E-02 \\
-8,032336 E-03 \\
\end{array}$ & $\begin{array}{r}578185 E-02 \\
-5.813977 E-03 \\
\end{array}$ & $\begin{array}{l}-5.2 \\
-3.7 \\
\end{array}$ & $\begin{array}{l}298415 E-04 \\
740872 E-05 \\
\end{array}$ & $\begin{array}{l}-1.242574 E-04 \\
-6.918342 E-05 \\
\end{array}$ & $\begin{array}{r}3.44439 E-04 \\
-9.707820 E-05 \\
\end{array}$ \\
\hline 1017 & c & $\begin{array}{l}1.073546 E-02 \\
5.053815 E-04\end{array}$ & $\begin{array}{l}-6.001836 E-04 \\
-1.201742 E-03\end{array}$ & $\begin{array}{r}\because .080321 E=02 \\
7.354086 E=03\end{array}$ & 0.0 & & $: 0$ & 0.0 \\
\hline 1018 & $\bar{E}$ & $\begin{array}{l}7.301055 E-03 \\
3.006554 E-04\end{array}$ & $\begin{array}{l}-2.345565 E-O 4 \\
-1.113781 E-03\end{array}$ & $\begin{array}{l}\text { T.S72562E-O2 } \\
-3.300030 E-03\end{array}$ & 0.0 & & $\begin{array}{ll}0 & 0 \\
0 . & 0\end{array}$ & 0.0 \\
\hline 1021 & G & $\frac{1.184178 E-02}{7.801600 E-04}$ & $\begin{array}{r}4.069455 E-03 \\
-3.228026 E-03\end{array}$ & $\frac{2663560 E-03}{3.020879 E-03}$ & $\frac{-1.5}{6}$ & $\frac{228315 E-04}{160291 E-05}$ & $\begin{array}{l}1.187401 E=04 \\
3.830018 E=05\end{array}$ & $\frac{-4.599199 E-05}{5.543556 E-06}$ \\
\hline 1022 & G & $\begin{array}{r}-2.439098 E-03 \\
-3.710162 E-04 \\
\end{array}$ & $\begin{array}{r}1.221138 E-03 \\
7.983025-00\end{array}$ & $\begin{array}{l}2.725550 E-03 \\
3.080489 E-03\end{array}$ & $\begin{aligned} 1 \cdot \\
-1 \cdot 2\end{aligned}$ & $\begin{array}{l}858507 E-04 \\
259533 E-04\end{array}$ & $\begin{array}{r}-3.9092465-04 \\
-5.053855 E-05\end{array}$ & $\begin{array}{r}1 \\
2107079 E-04 \\
2270368 E-05\end{array}$ \\
\hline 1023 & c & $\begin{array}{l}-8.170323 E-01 \\
-6.523324 E-03\end{array}$ & $\begin{array}{l}1.000000 E+00 \\
0.0\end{array}$ & $\begin{array}{l}\text { 3. } 709162 E-01 \\
1.524241 E-02\end{array}$ & $\begin{array}{r}1 \\
-3\end{array}$ & $\begin{array}{l}\text { S13289E-O2 } \\
\text { B222BGE-O5 }\end{array}$ & $\begin{array}{l}1.257672 E-02 \\
3.252897 E-04\end{array}$ & $\begin{array}{r}-2.277318 E-02 \\
5.076740 E-05\end{array}$ \\
\hline $10 \overline{4}$ & 5 & $\begin{array}{l}-8.182537 E-04 \\
-5.832961 E-04\end{array}$ & $\begin{array}{l}-3.348035 E-01 \\
-3.805712 E-02\end{array}$ & $\begin{array}{r}4.4564265-01 \\
-2.508089 E-02\end{array}$ & $\begin{array}{l}-1 . \\
-4 .\end{array}$ & $\begin{array}{l}470901 E-02 \\
748082 E-04\end{array}$ & $\begin{array}{r}1.124467 E-02 \\
-5.993301 E-04\end{array}$ & $\begin{array}{l}2.1047 \overline{21 E-02} \\
8.488314 E-04\end{array}$ \\
\hline 1025 & C & $\begin{array}{l}-8.148572 E-01 \\
=6.282702 E=03\end{array}$ & $\begin{array}{r}5-171877 E-01 \\
1.080753 E=03\end{array}$ & $\begin{array}{r}-7.336145 E-01 \\
\because 2.686785 E-63\end{array}$ & $\frac{2}{4}$ & $\begin{array}{l}432523 E-02 \\
56025 \text { TIE-OS }\end{array}$ & $\begin{array}{l}2 \cdot 186752 E-02 \\
8.011310 E=05\end{array}$ & $\begin{array}{r}6.991421 E-03 \\
-3.345536 E-05\end{array}$ \\
\hline 1026 & c & $\begin{array}{l}-8.158785 E-01 \\
-3.746240 E-04 \\
\end{array}$ & $\begin{array}{r}6.037858 E-01 \\
3.788 B 38 E-O 3 \\
\end{array}$ & $\begin{array}{r}-7.701033 E-01 \\
4.10863 E-03 \\
\end{array}$ & $\begin{array}{r}-2 \\
8 \\
\end{array}$ & $\begin{array}{l}551078 E-02 \\
038980 E-05 \\
\end{array}$ & $\begin{array}{r}2.234442 E-02 \\
-6.557023 E-05 \\
\end{array}$ & $\begin{array}{r}7.028773 E-03 \\
2.088561 E-05 \\
\end{array}$ \\
\hline 1028 & c & $\begin{array}{r}1.000005 E-01 \\
-1.076183 E-02\end{array}$ & $\begin{array}{r}-1.85872 E-02 \\
830978 E-03\end{array}$ & $\begin{array}{r}-1.277721 E-02 \\
5.284849 E-03\end{array}$ & $\begin{array}{l}-4 \\
-1\end{array}$ & $\begin{array}{l}290889 E-04 \\
950081 E-04\end{array}$ & $\begin{array}{r}\text { B. } 104929 E-04 \\
-1.784836 E-04\end{array}$ & $\begin{array}{r}-3.588485 E-03 \\
3.883401 E-04\end{array}$ \\
\hline
\end{tabular}


AH - IG THRE - OIMENSIONAL BUILTUP DYNAMICS MODEL W/ COMTROLS MODELED DF JCULT COMPONENTS GVT CONFIGURATION "1 IFULL-UPI

THIS VERSIOH CONTAINS A BUILTUP TAILBDOM MODEL

COMPLEX EIGENVALUE = $=451561 E \cdot 01,-1181000 E+02$

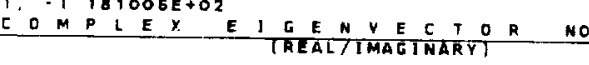
17
POINT ID TYPE
T 1
T 2
13

R 1

R2

83

\begin{tabular}{|c|c|c|c|c|c|c|c|c|c|c|c|}
\hline 1029 & $\mathbf{G}$ & & $\begin{array}{l}978618 E-03 \\
858705 E-03\end{array}$ & $\begin{array}{r}-7 \\
8\end{array}$ & $\begin{array}{r}822369 E-O 3 \\
449535 E-03\end{array}$ & -4 & $\begin{array}{l}665146 E-03 \\
353402 E-03\end{array}$ & $\begin{array}{l}-9 . \\
-1\end{array}$ & $\begin{array}{l}O 42134 E-O 5 \\
239122 E-O S\end{array}$ & $\begin{array}{r}1.240061 E-04 \\
-6.320322 E-05\end{array}$ & $\begin{array}{r}1661924 E-O A \\
-1.3083 B 2 E-O A\end{array}$ \\
\hline 2511 & $\mathbf{C}$ & & $\frac{008783 E-02}{298337 E=03}$ & $\frac{1}{-1}$ & $\frac{054686 E \cdot 03}{.706489 E-03}$ & & $\frac{.383211 E-02}{926165 E-03}$ & $\frac{-1}{3}$ & $\frac{454387 E-04}{108750 E-06}$ & $\frac{-1.990233 E-04}{5.113235 E-05}$ & $\begin{array}{r}2.571104 E-04 \\
-8.061137 E-05\end{array}$ \\
\hline 2572 & C & & $\begin{array}{l}108557 E-03 \\
955599 E-04\end{array}$ & $\begin{array}{r}2 \\
-9\end{array}$ & $\begin{array}{l}161154 E-02 \\
181467 E-03\end{array}$ & $\begin{array}{r}3 \\
-7 \\
-7\end{array}$ & $\begin{array}{l}919849 E-02 \\
574360 E-03\end{array}$ & $\begin{array}{r}7 \\
-8 \\
\end{array}$ & $\begin{array}{l}242432 E-05 \\
538870 E-05\end{array}$ & $\begin{array}{r}-1.667751 E-04 \\
-1128220 E-04\end{array}$ & $\begin{array}{r}1.50085 E-04 \\
-6484761 E-05\end{array}$ \\
\hline 2649 & $\sigma$ & $\begin{array}{r}5 \\
-6\end{array}$ & $\begin{array}{l}241806 E-03 \\
108019 E-04\end{array}$ & $\begin{array}{r}3 \\
-1\end{array}$ & $\begin{array}{l}127209 E-02 \\
380800 E-02\end{array}$ & 2. & $\begin{array}{l}219901 E-02 \\
508551 E-03\end{array}$ & $\begin{array}{r}5 \\
-\frac{2}{4}\end{array}$ & $\begin{array}{l}132090 E-04 \\
539033 E=04\end{array}$ & $\begin{array}{r}-5.285618 E-04 \\
2.110328 E-O 4\end{array}$ & $\begin{array}{r}2.622481 E-05 \\
-6.182785 E-05\end{array}$ \\
\hline 2697 & $\sigma$ & $\therefore$ & $\begin{array}{l}224484 E \cdot 03 \\
252868 E \cdot 02\end{array}$ & $\begin{array}{r}-1 \\
9\end{array}$ & $\begin{array}{l}874489 E=02 \\
127650 E-03\end{array}$ & 8 & $\begin{array}{l}841689 E-03 \\
939316 E-03\end{array}$ & $\begin{array}{l}-2 \\
-3\end{array}$ & $\begin{array}{l}053534 E-04 \\
296038 E-04\end{array}$ & $\begin{array}{r}1.143097 E=03 \\
-1.763033 E=04\end{array}$ & $\begin{array}{r}-1.636861 E=03 \\
1.79781 O E-04\end{array}$ \\
\hline 1978 & c & 8 & $\begin{array}{l}279954 E-03 \\
\text { OOTI26E-OQ }\end{array}$ & 11 & $\begin{array}{r}183082 E-04 \\
330472 E-03\end{array}$ & $\frac{4}{2}$ & $\begin{array}{l}606990 E-03 \\
233924 E-03\end{array}$ & -1, & $\begin{array}{l}717896 E-04 \\
587531 E-05\end{array}$ & $\begin{array}{r}5.907661 E-05 \\
792201 E-O 5\end{array}$ & $\begin{array}{r}4 \quad 150235 E-05 \\
1.08787 E-05\end{array}$ \\
\hline
\end{tabular}

AH-IG THREE-DIMENSIONAL BUILTUP DYNAMICS MDOEL W/ CONTROLS MODELED

OETOEER 26, 1990 MSE/NASTRAN $10 / 15 / 87$

PACE 123

THIS VERSION CONTAINS A BUILTUP TAILBOOM MODEL

COMPLEX EIGENVALUE: -3.355287E-01,-1.379099E+O2

\begin{tabular}{|c|c|c|c|c|c|c|c|c|}
\hline 1001 & 6 & $\begin{array}{l}-452786 E-03 \\
-9.154136 E-04\end{array}$ & $\begin{array}{r}-2 \\
2\end{array}$ & $\begin{array}{r}2460056-03 \\
294392 E-04\end{array}$ & $\begin{array}{l}5.782804 E-03 \\
7.773228 E-03\end{array}$ & $\begin{array}{ll}0 & 0 \\
0 & 0\end{array}$ & 0.0 & $\begin{array}{l}0.0 \\
0.0\end{array}$ \\
\hline 1003 & G & $\frac{-3.950033 E-03}{-8.184061 E-04}$ & -7 & $\frac{.818979 E-04}{.556578 E-04}$ & $\frac{2.729753 E-03}{1.311439 E-03}$ & 0.0 & $\frac{0.0}{0.0}$ & 0.0 \\
\hline 1004 & c & $\begin{array}{r}-3.338995 E-03 \\
2.403585 E-04 \\
\end{array}$ & -3 & $\begin{array}{l}788888 E-04 \\
878008 E-04 \\
27\end{array}$ & $\begin{array}{r}1.029527 E-03 \\
-2.856440 E-03 \\
\end{array}$ & $\begin{array}{l}0.0 \\
0.0\end{array}$ & $\begin{array}{ll}0 & 0 \\
\therefore & 0\end{array}$ & $\begin{array}{r}0.0 \\
0.0\end{array}$ \\
\hline 1005 & c & $\begin{array}{r}-2.884502 E-03 \\
5.573139 E-05\end{array}$ & 3 & $\begin{array}{l}174660 E-04 \\
907194 E-05\end{array}$ & $\begin{array}{r}5,151892 E-03 \\
-1,152839 E-03\end{array}$ & 0.0 & $\begin{array}{l}-2.588328 E-O 5 \\
-4.173881 E-O 5\end{array}$ & $\begin{array}{r}-4.762744 E-O 6 \\
8.700032 E=08\end{array}$ \\
\hline 1007 & G & $\begin{array}{r}-2.402552 E-03 \\
1.774441 E-04\end{array}$ & 2 & $\begin{array}{l}.545276 E-04 \\
291807 E-04\end{array}$ & $\begin{array}{l}5.285265 E-03 \\
2.193262 E-03\end{array}$ & 0.0 & 0.0 & 0.0 \\
\hline 1012 & G & $\begin{array}{r}-1.943806 E=03 \\
1031209 E=03\end{array}$ & -5 & $\begin{array}{l}213799 E-03 \\
85359=04\end{array}$ & $\begin{array}{r}-2.040150 E-02 \\
1.9904 E-03\end{array}$ & $\begin{array}{r}-3.162175 E-04 \\
4.86321 E=08\end{array}$ & $\begin{array}{r}367739 E-04 \\
5.136425 E-05\end{array}$ & $\begin{array}{r}506596 E=06 \\
-2.393735 E=05\end{array}$ \\
\hline 1013 & G & $\begin{array}{r}-1.125009 E-03 \\
8.836772 E=04 \\
\end{array}$ & $\begin{array}{l}-6 \\
-6 \\
\end{array}$ & $\begin{array}{l}213727 E-03 \\
858345 E-04 \\
\end{array}$ & $\begin{array}{r}-2.053047 E-02 \\
1.781944 E-04 \\
\end{array}$ & $\begin{array}{r}3.293191 E-04 \\
2.2886985-05 \\
\end{array}$ & $\begin{array}{r}1.367730 E-04 \\
5.136166 E-05 \\
\end{array}$ & $\begin{array}{r}-839515 E-05 \\
1.381344 E-05 \\
\end{array}$ \\
\hline 1017 & G & $\begin{array}{l}-3.223080 E-O 3 \\
-3.239618 E=04\end{array}$ & 2 & $\begin{array}{l}958311 E-04 \\
347604 E-04\end{array}$ & $\begin{array}{r}0.585597 E-03 \\
-1.128091 E=04\end{array}$ & $\begin{array}{ll}0.0 \\
0.0\end{array}$ & $\begin{array}{l}0.0 \\
0.0\end{array}$ & 0.0 \\
\hline 1018 & E' & $\begin{array}{r}-3.306022 E-03 \\
5.485718 E-04\end{array}$ & 4 & $\begin{array}{l}\text { E3OOSICE } \\
\text { OBS3OSE-OA }\end{array}$ & $\begin{array}{r}\text { 3. } 265682 E=03 \\
-1.070518 E-03\end{array}$ & $\begin{array}{l}0.0 \\
0.0\end{array}$ & $\begin{array}{l}0.0 \\
0.0\end{array}$ & $\begin{array}{l}0.0 \\
0.0\end{array}$ \\
\hline 1021 & E & $\frac{-9.707557 E \cdot 03}{-2.944248 E \cdot 04}$ & $\frac{-8}{-7}$ & $\frac{268485 E-04}{45914, T E=05}$ & $\begin{array}{r}7.720316 E \cdot 03 \\
1.730720 E \cdot 03\end{array}$ & $\frac{6.061252 E-05}{1.775513 E-06}$ & $\frac{-2.045410 E-04}{-2.355051 E-05}$ & $\frac{2.374374 E-06}{9.310348 E-06}$ \\
\hline 1022 & G & $\begin{array}{l}2.348230 E=03 \\
1.4345806=04\end{array}$ & 3 & $\begin{array}{l}252508 E-04 \\
480758 E-05\end{array}$ & 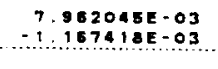 & $\begin{array}{r}-5.402861 E-05 \\
-3.350144 E-05\end{array}$ & $\begin{array}{r}4.034838 E-04 \\
2.261689 E-05\end{array}$ & $\begin{array}{r}\text { A.800039E -OS } \\
1.248779 E-O 5\end{array}$ \\
\hline 1023 & c & $\begin{array}{r}3.391455 E-01 \\
-3.826402 E-03\end{array}$ & -7. & $\begin{array}{l}107471 E-01 \\
647622 E-03\end{array}$ & $\begin{array}{l}-6.327743 E-02 \\
-1.589866 E-03\end{array}$ & $\begin{array}{r}9.101972 E-03 \\
2.648228 E-05\end{array}$ & $\begin{array}{l}\text { C. } 340223 E-05 \\
2.658200 E-05\end{array}$ & $\begin{array}{r}2.249292 E-02 \\
-1.742552 E-04 \\
\end{array}$ \\
\hline 1024 & $\bar{E}$ & $\begin{array}{r}3.401435 E-01 \\
-5.863785 E-03\end{array}$ & -9.5 & $\begin{array}{l}050359 E-01 \\
283666 E-03\end{array}$ & $\begin{array}{r}-7.602674 E=02 \\
5.61084 E-04\end{array}$ & $\begin{array}{r}-9.266704 E=03 \\
5.069294 E-05\end{array}$ & $\begin{array}{r}-2.930481 E-04 \\
4661314 E-05\end{array}$ & $\begin{array}{r}-2,230430 E-02 \\
2.762452 E-04\end{array}$ \\
\hline 1025 & G & $\begin{array}{r}3.377105 E-01 \\
-3.08347 t E-03\end{array}$ & 1.0 & $000000 E+00$ & $\begin{array}{r}-859533 E-01 \\
1.332164 E=03\end{array}$ & $\begin{array}{r}3.638193 E-02 \\
-1.605 B 16-03\end{array}$ & $\begin{array}{r}1.180140 E-02 \\
-3.354036 E=06\end{array}$ & $\begin{array}{r}1.891492 E-02 \\
-2.250470 E=06\end{array}$ \\
\hline 1026 & G & $\begin{array}{r}3.387031 E-01 \\
-6.007717 E-03 \\
\end{array}$ & $\begin{array}{r}-9.7 \\
8 .\end{array}$ & $\begin{array}{l}754819 E-01 \\
649302 E-03 \\
\end{array}$ & $\begin{array}{r}-0.720081 E-01 \\
6.022247 E-03 \\
\end{array}$ & $\begin{array}{r}-3.870282 E-02 \\
2.27305 B E-04 \\
\end{array}$ & $\begin{array}{r}1.180284 E-02 \\
-5.401923 E-05 \\
\end{array}$ & $\begin{array}{r}-1.8 B 8415 E-02 \\
1.304015 E-04 \\
\end{array}$ \\
\hline 1020 & G & $\begin{array}{l}-5.270802 E-02 \\
-5.047422 E-03\end{array}$ & $\begin{array}{r}1.2 \\
-7.8\end{array}$ & $\begin{array}{l}276785 E-03 \\
641304 E-04\end{array}$ & $\begin{array}{r}1.170224 E-02 \\
-2.144359 E-08\end{array}$ & $\begin{array}{l}0.853830 E-04 \\
1.838523 E-04\end{array}$ & $\begin{array}{l}-3.703985 E-04 \\
-1.423451 E-05\end{array}$ & $\begin{array}{l}2.121808 E-03 \\
4.213142 E-04\end{array}$ \\
\hline
\end{tabular}


THIS VERSION CONTAINS A BUILTUP TAILBOOM MODEL

COMPLEX EIGENVALUE = -3.355287E-01, -1.371099E+02 CDMPLEK E TEENYECTTO O R R N 18

POINT ID TYPE ion 9 ic $2511 \quad[\quad-3.312428 E \cdot 03 \quad-8 \cdot 205591 E-04$ $2572 \quad G \quad-3.915808 E-03 \quad-6 \cdot 154398 E-03$

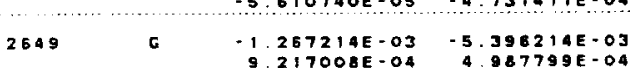

$2697-2.6365905-02$
$19777 \quad$ E $\quad-2980196 E-03$
$1.0235196-03$
$-9.525941 E-04$ $1.639810 E-04$
T3

5. $173535 E-03$ $21256265-03$

1.431981E-03

$-1.580347 E-02$
$6008997-04$

$1.574613 \mathrm{E}-02$
$\therefore .58988 \mathrm{E}-03$

$3.021723 E-04$
$2.463345=03$

$6.057539 E-03$

\section{R 1}

$2.432513 E-05-233583 E-05$

3.775509E.05 $000895 E-04$ $-8.574517 E-06-5.604600 E-06$ $-650672 E-06 \quad 1.559296 E-04$
$1272871 E-05 \quad 7893921 E-05$

$9.233746 E-05$ A $701340 E-04$ $\begin{array}{r}.517803 E-05 \\ \hline\end{array}$

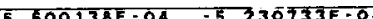
$198463 E-04-5.265372 E-05$

$7.810490 E-05 \quad-4 \quad 740559 E-05$
4 t3 $3231 E-05$ . $454593 E-05$

$-2.735000 E-05$
$-6.827502 E-07$

8. $153066 \mathrm{E}-05$ 2. $085449 E-05$

$104533 E-03$

$-9.596193 \mathrm{E}-06$

AH - IG THRE - DIMENSIOMAL DUILTUP OYNAMICS MOOEL W/ CONTROLS MODELEO octoBer 26,1990 mse/nastaAn 10/15/87 Page 125 AH- IG THRE - DIMENSI ONAL BUILTUP OYNAMICS MOOEL W/ CD
DIFF I CULT COMPONENTS GVT CONFIGURATION I (FULL-UP)

THIS VERSION CONTAINS A BUILTUP TAILOOOM MODEL

COMPLEK EIGENVALUE $=-7.666564 E-01,-1,500125 E+02$

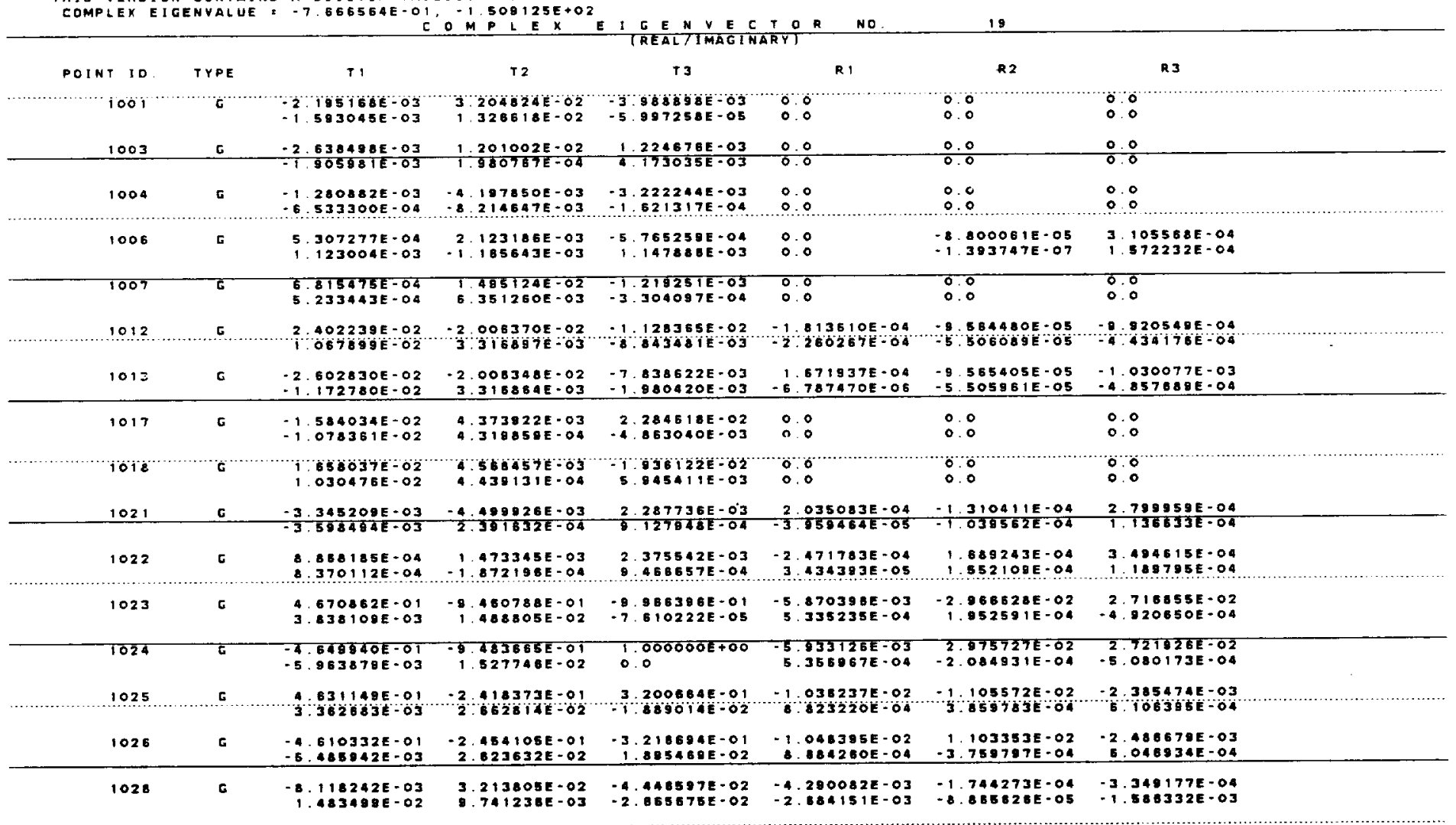



AH-IG THRE - DIMENSIONAL BUILTUP OYNAMICS MODEL W/ CONTROLS MODELED
DIFFICULT COMPOHENTS GVT CONFI GURATION I (FULL-UP)

THIS VERSION CONTAINS A BUILTUP TAILBOOM MOOEL

COMPLEX EIGENVALUE = $-7,66554 E-01,-1,509125 E+02$

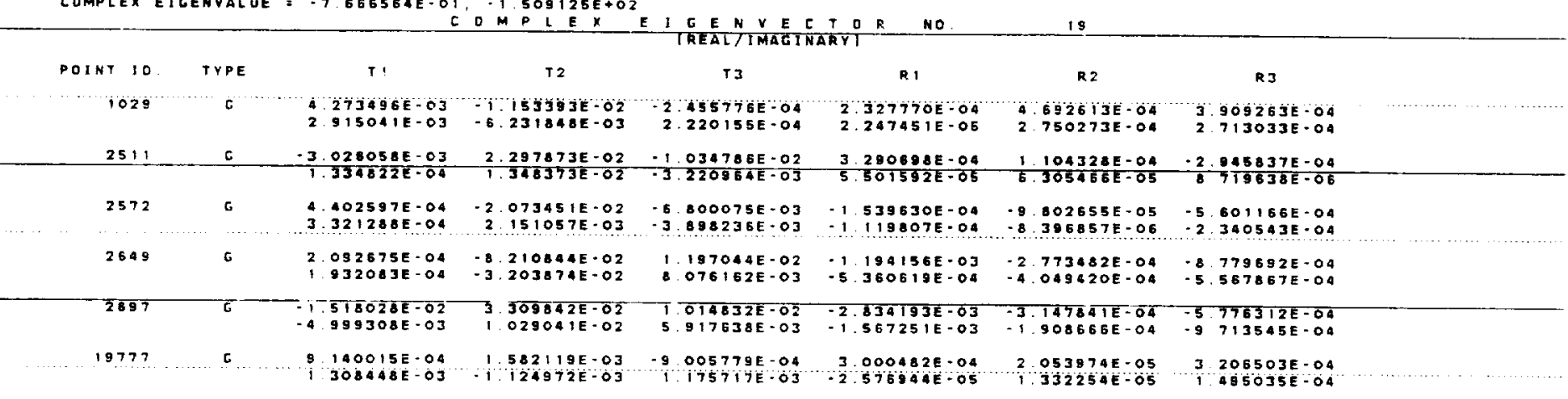

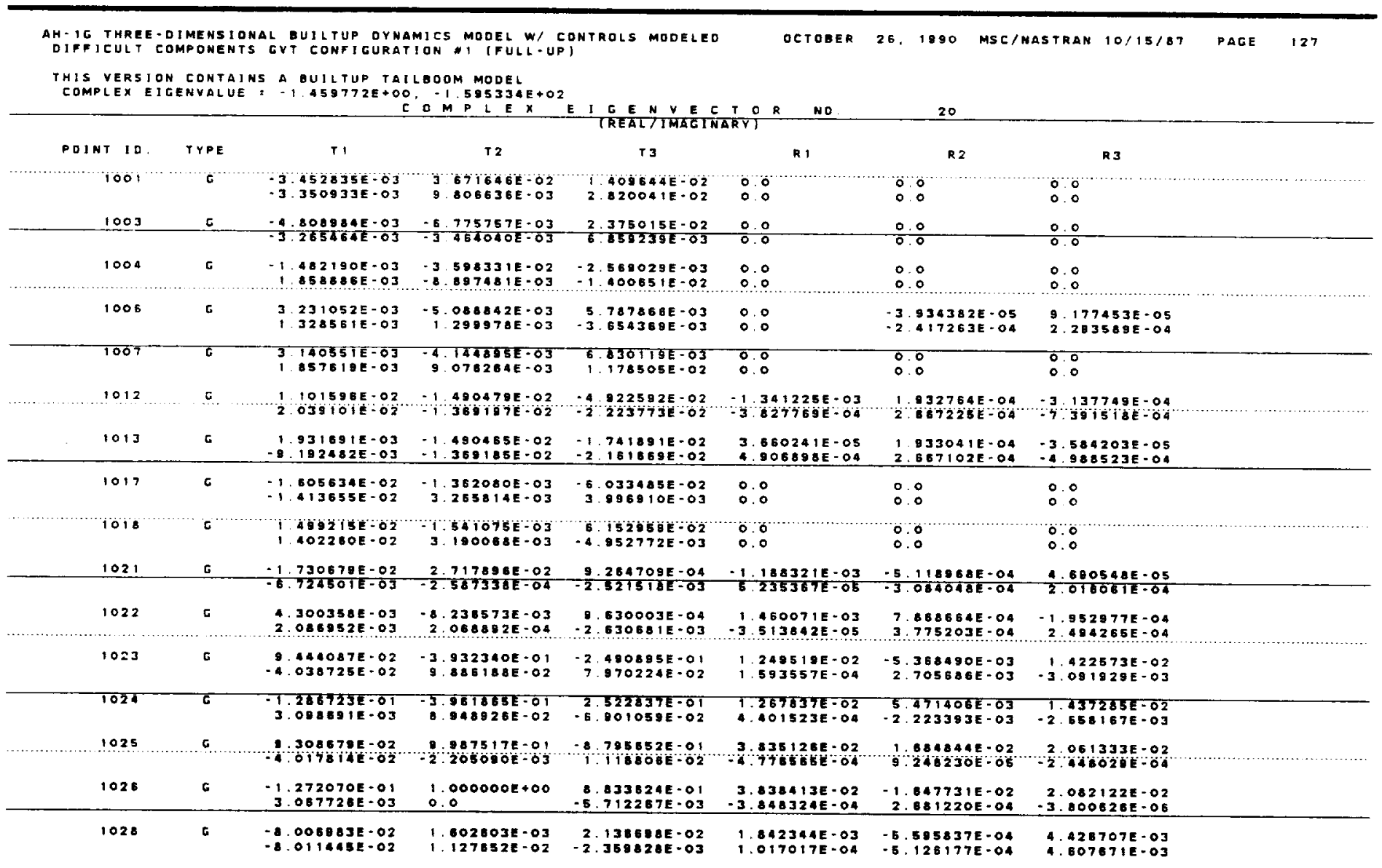


AH- I THREE-DJMENSIOMAL BUILTUP DYNAMICS MODEL W/ CONTROLS MOOELED

DCTOBER 26, 1990 MSC/MASTRAN 10/15/87 PAGE 128

AH- IG THREE-DIMENSIONAL BUILTUP DYNAMICS MODEL W/ CONTH
DIFF ICULT COMPONENTS GYT CONFIGURATION I (FULL-UP)

THIS VERSION CONTAIMS A BUILTUP TAILEODM MOOEL

COMPLEX EIGENYALUE $=-1.459772 E+00,-1.595336 E$

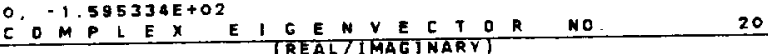

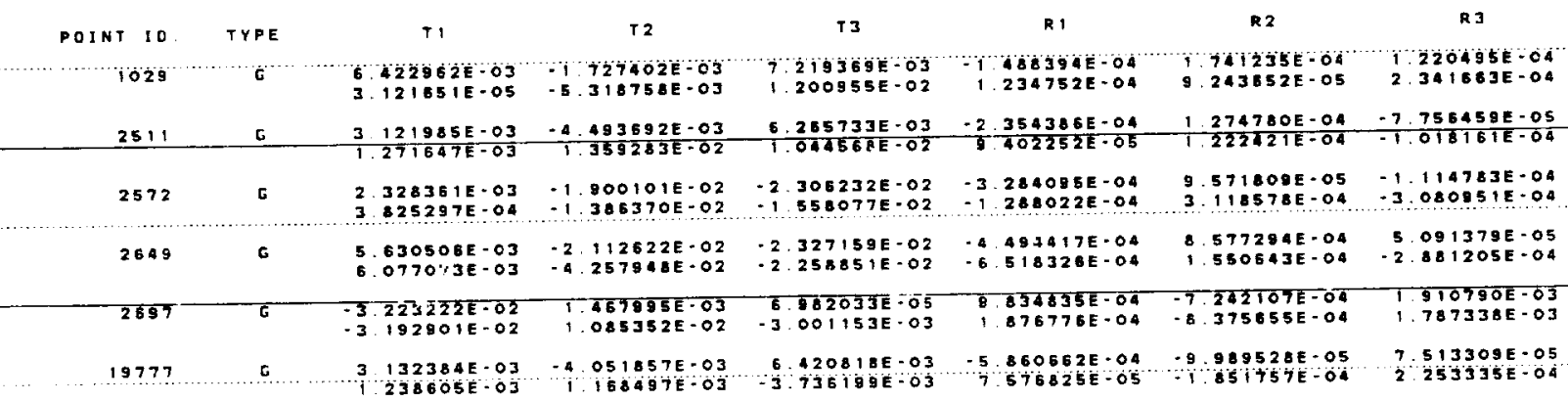

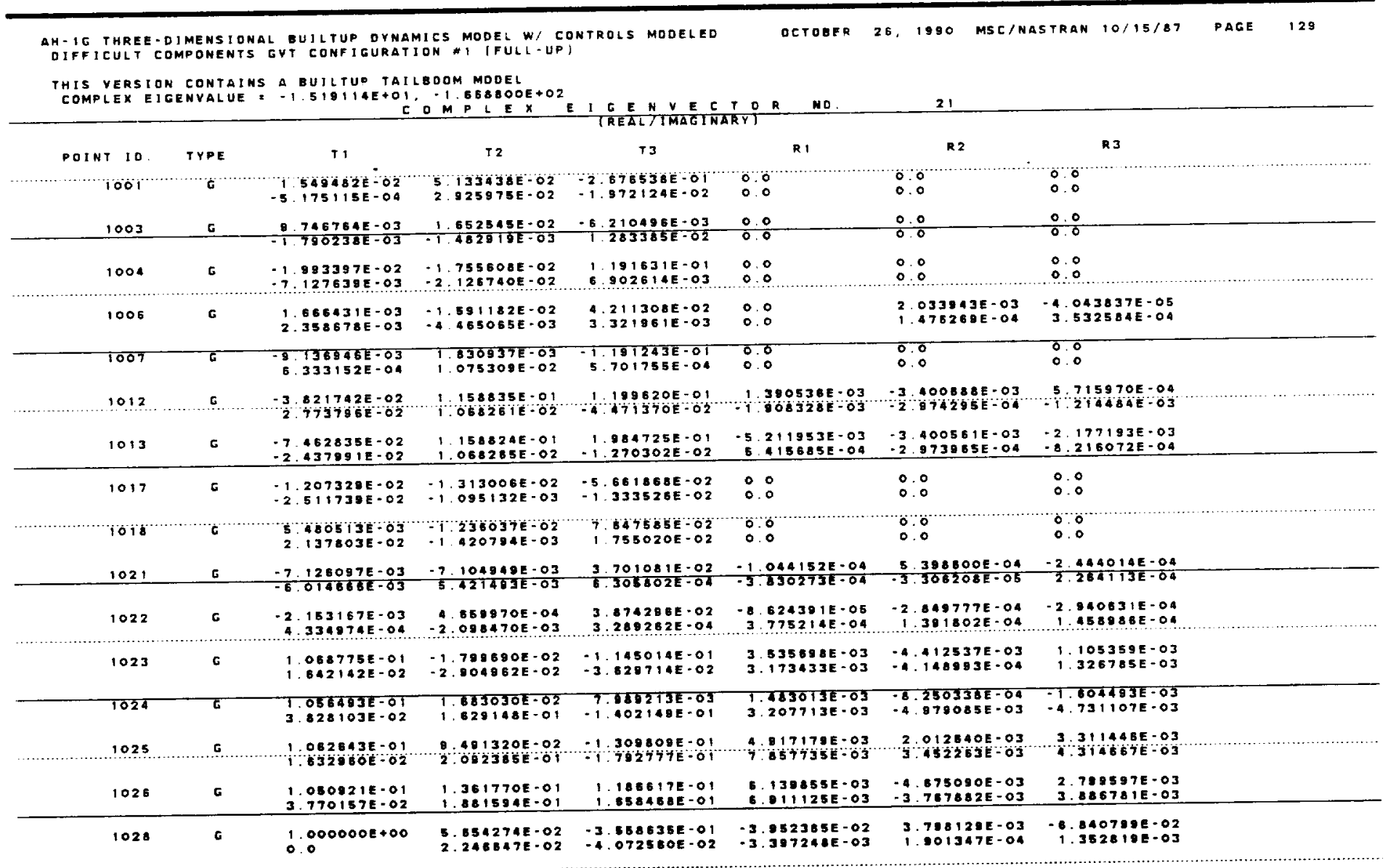


AH - IC THREE-DIMENSIONAL BUILTUP DYMAMICS MOOEL VI/ CONTROLS MODELEO DIFFICULT COMPONENTS GVT CONFIGURATION 1 (FULL-UP)

THIS VERSION CONTAINS A BUILTUP TAILBOOM MODEL

COMPLEX EIGENVALUE: - $1.519114 E+01,-1.868000 E+02$

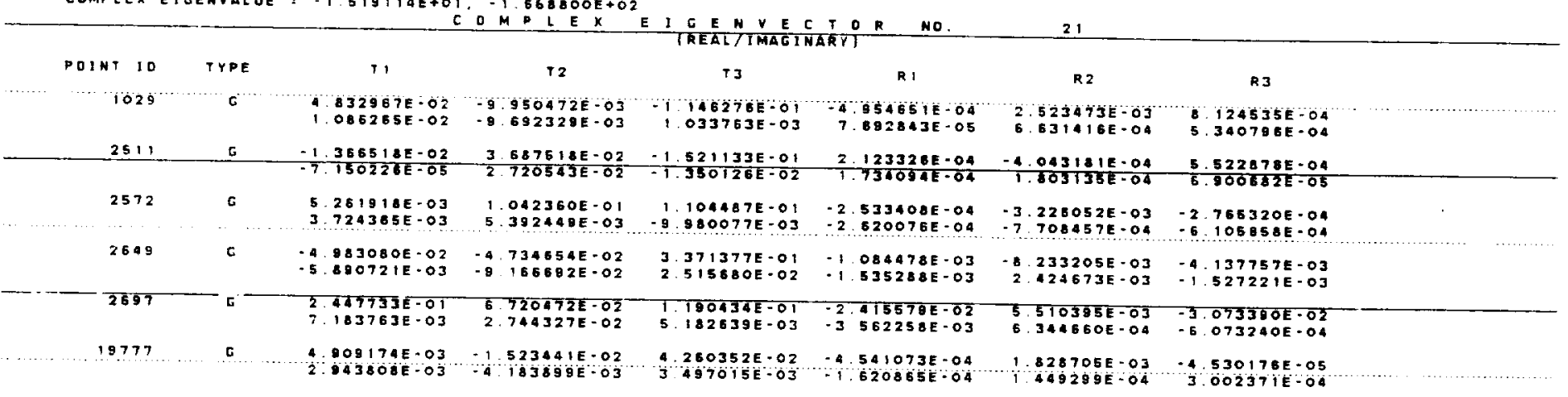

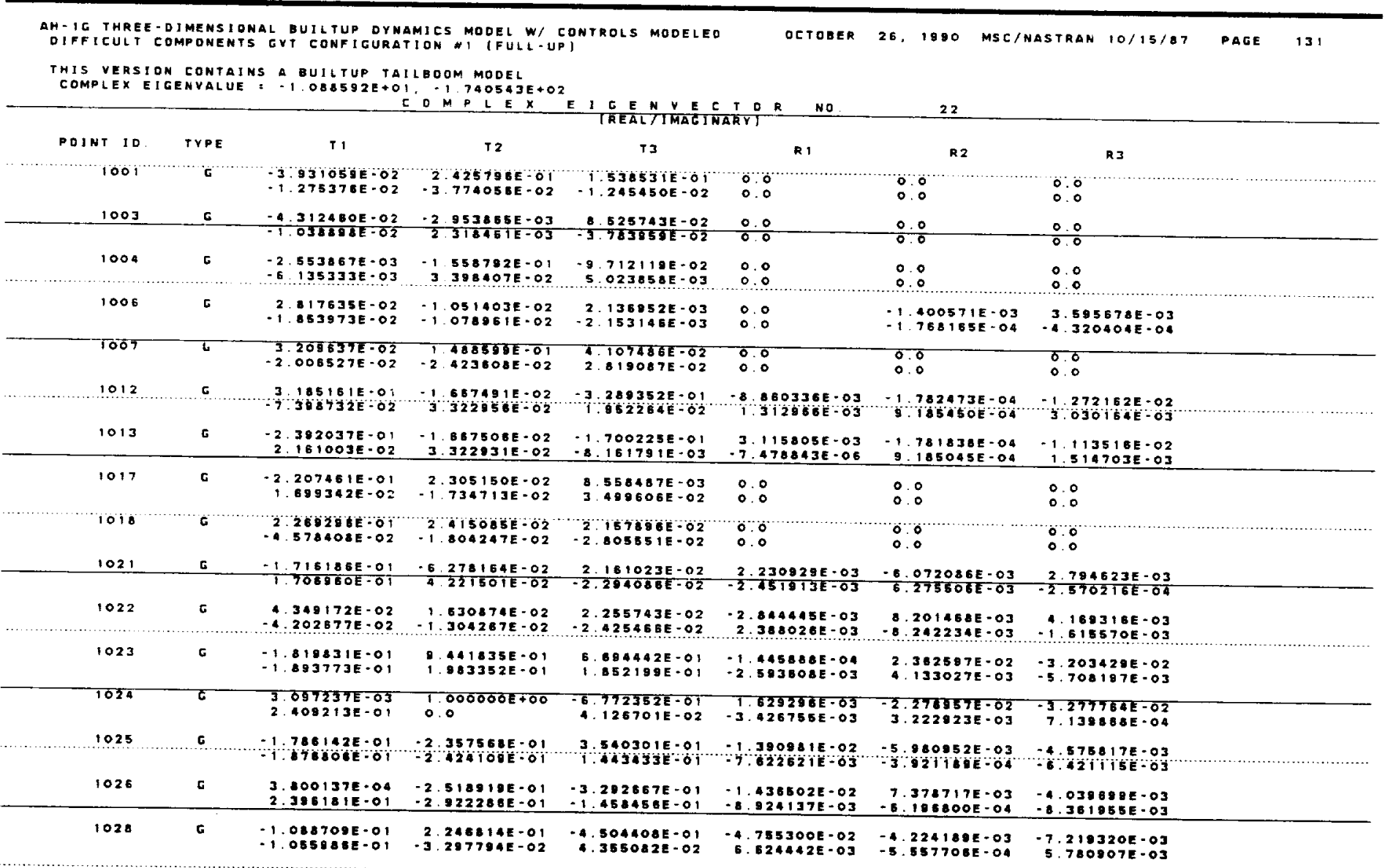


AH-IG THREE-DIMENSIONAL BUILTUP DYMAMICS MODEL W' CONTROLS MODELED

THIS VERSION CONTAINS A BUILTUP TAILBOOM MODEL

COMPLEX EI GENYALUE $=-1$. OB8592E+0, $-1740543 E+02$

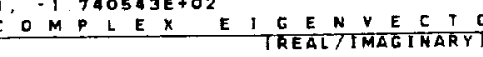

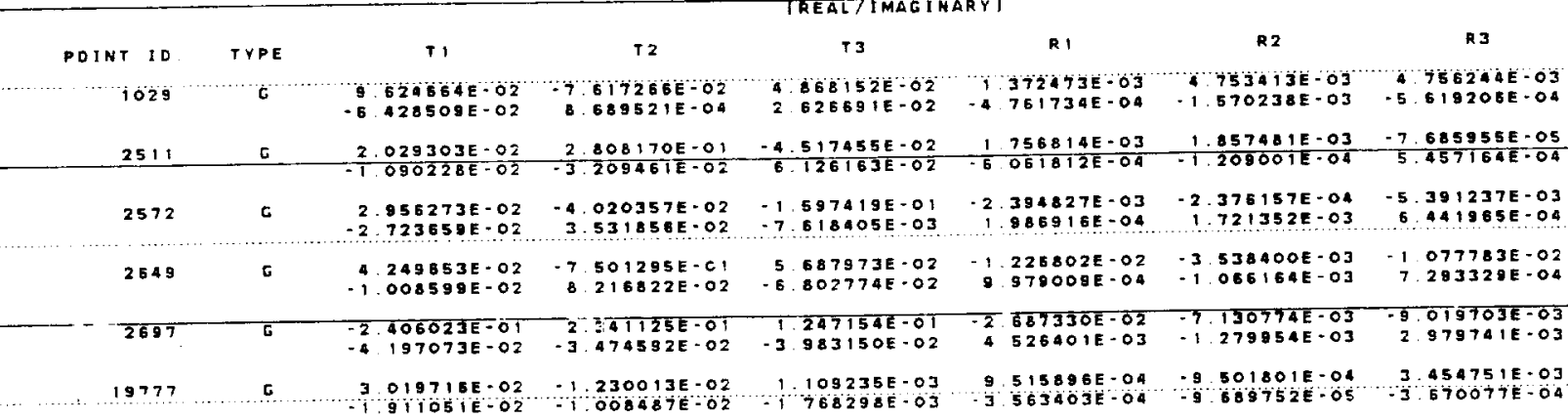

AH-IC THREE-DIMENSIONAL BUILTUP DYMAMICS MODEL W/ CONTROLS MODELED OCTOBER 26, 1990 MSC/MASTRAN 10/15/87 PAGE

THIS VERSION CONTAINS A BUILTUP TAILBOOM MOOEL

COMPLEX EIGENYALUE: $-1,183284 E-01 .-1.80224 B E+02$

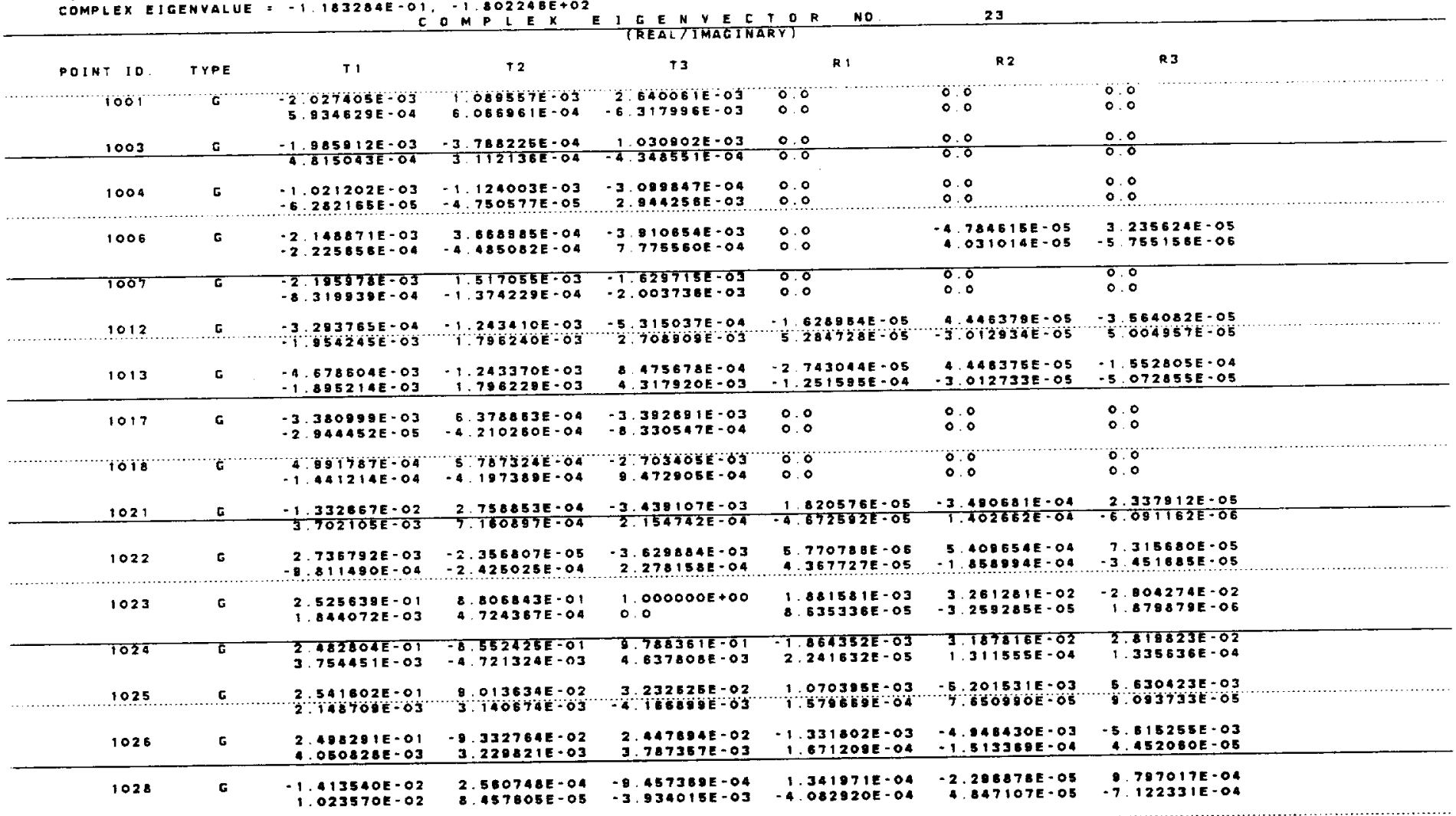


AM- IG THAEE-DIMENSIONAL BUILTUP DYMAMICS MDDEL W/ CONTROLS MOOELED DIFFICULT COMPONENTS GVT CONFIGURATION I (FULL-UP)

THIS VERSION CONTAINS A BUILTUP TAILBOOM MOOEL

COMPLEX EIGENYALUE = - 1 183284E-01, - $1.02246 E+02$

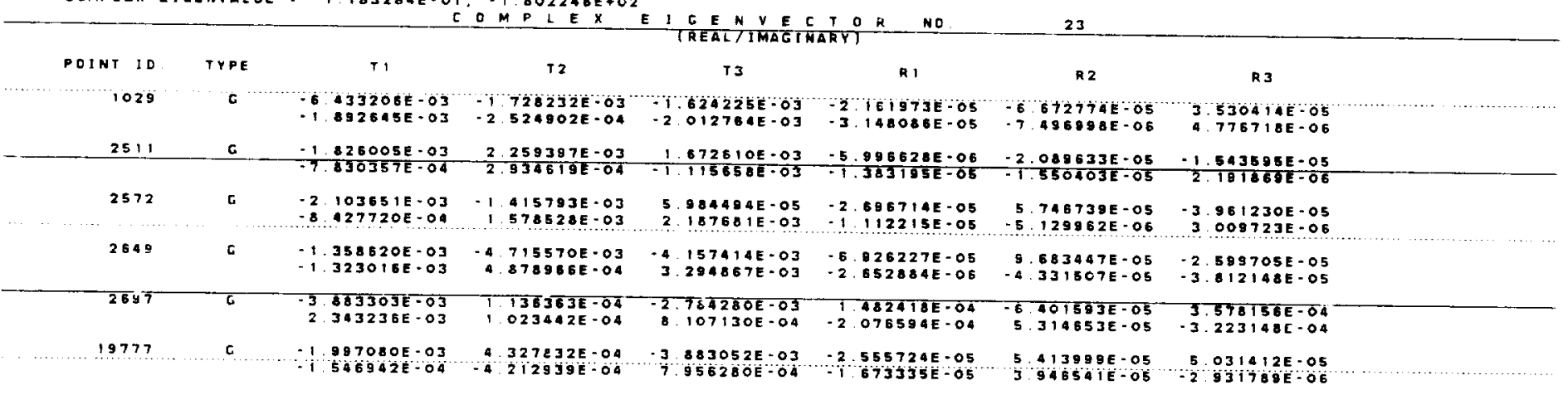

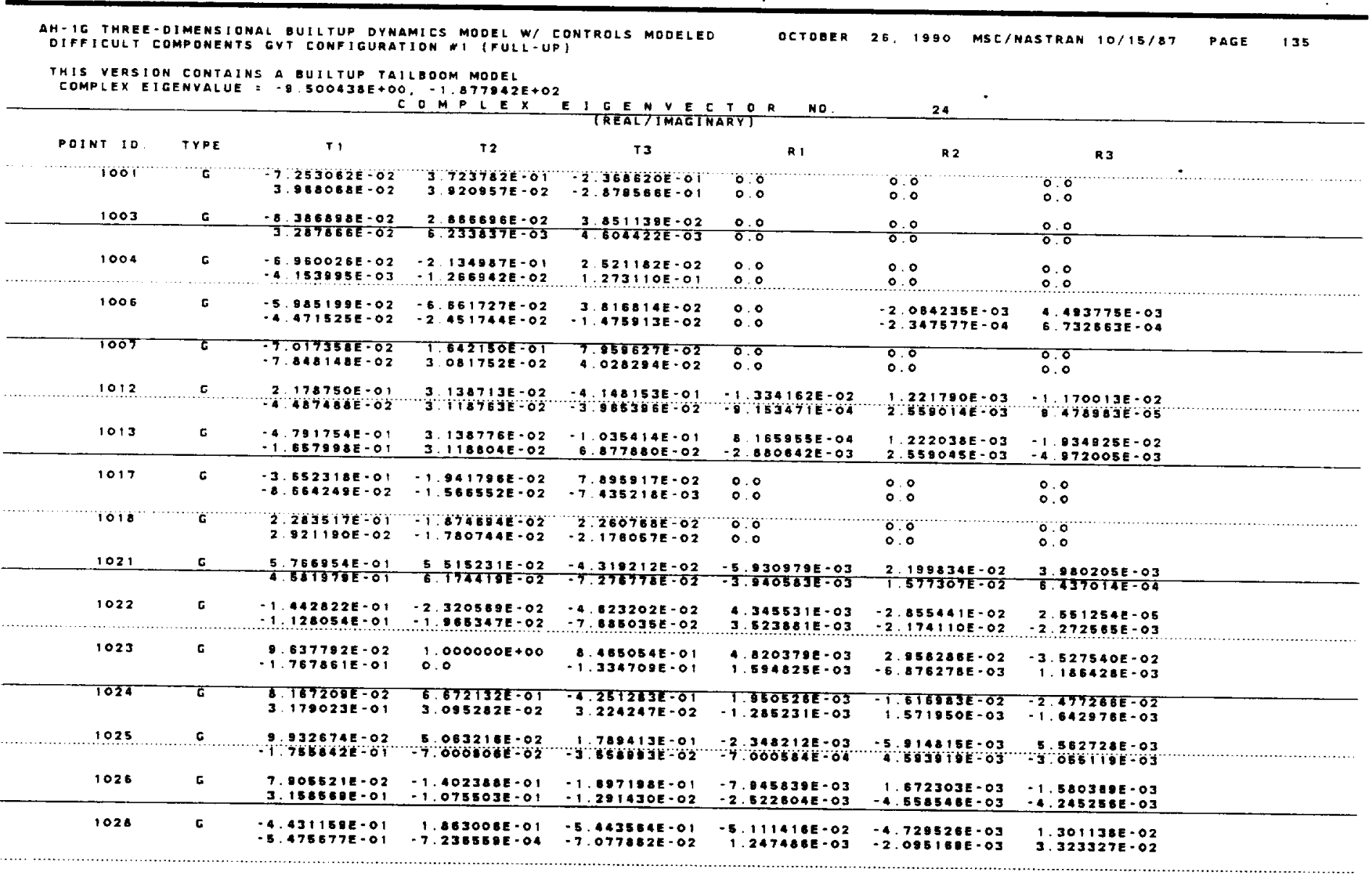


AH-ig THRE - DIMENSIDNAL BUILTUP DYNAMJCS MODEL W/ CONTROLS MOdeled

THIS VERSION CONTAINS A UILTUP TAILBOOM MOOEL

COMPLEX EIGENVALUE = -9500430E+00.- $977942 E+02$

\begin{tabular}{|c|c|c|c|c|c|c|c|c|c|c|}
\hline POINT ID. & TYPE & 11 & & 12 & & $T 3$ & & R 1 & $R 2$ & $R_{3}$ \\
\hline 1029 & $\mathrm{c}$ & $\begin{array}{l}=1.376209 E-09 \\
-2.574575 E \cdot 01\end{array}$ & $=6$ & $\begin{array}{l}O 55024 E-O Z \\
459224 E-02\end{array}$ & 3 & $\begin{array}{l}280145 E=02 \\
803726 E-02\end{array}$ & $=6$. & $\begin{array}{l}568836 E-04 \\
049338 E \cdot 03\end{array}$ & $\begin{array}{l}216536 E-O 3 \\
-3.290320 E-03\end{array}$ & $\begin{array}{l}\text { 5. 30TS2IE-03 } \\
1.071406 E-03\end{array}$ \\
\hline 25,11 & c & $\begin{array}{l}-7.605089 E-02 \\
-6.332314 E-02\end{array}$ & $\frac{3}{7}$ & $\frac{336469 E-01}{611481 E-02}$ & $\frac{6}{1}$ & $\frac{146780 E-02}{614047 E-01}$ & $\frac{7}{-1}$ & $\frac{292881 E-04}{473002 E-03}$ & $\frac{1.821873 E-03}{-2.234872 E-04}$ & $\frac{-4.065400 E-04}{1.127091 E-04}$ \\
\hline 2572 & c & $\begin{array}{l}-8.3614216-02 \\
-1.017116 E-01\end{array}$ & $\begin{array}{r}-7 \\
1\end{array}$ & $\begin{array}{l}048373 E-03 \\
525243 E-02\end{array}$ & $\begin{array}{l}-1 \\
-3\end{array}$ & $\begin{array}{r}535554 E-01 \\
453576 E-03\end{array}$ & $\begin{array}{l}-3 \\
-1 \\
-1\end{array}$ & $\begin{array}{l}709941 E-03 \\
707858 E-03\end{array}$ & $\begin{array}{r}1.6260675-03 \\
4.7367165-03\end{array}$ & $\begin{array}{r}-5.976284 E-03 \\
-1.3616175-03\end{array}$ \\
\hline 2649 & $c$ & $\begin{array}{l}-5.468100 E \cdot 02 \\
-5.662847 E-02\end{array}$ & $\begin{array}{l}-8 \\
-1\end{array}$ & $\begin{array}{l}088802 E-01 \\
044119 E-01\end{array}$ & $\begin{array}{l}-4 \\
-1\end{array}$ & $\begin{array}{l}957742 E-02 \\
837891 E-01\end{array}$ & $\begin{array}{l}-1 \\
-3\end{array}$ & $\begin{array}{l}368087 E-02 \\
755224 E-03\end{array}$ & $\begin{array}{l}3.619710 E-03 \\
5.801049 E-03\end{array}$ & $\begin{array}{l}-1.201062 E-02 \\
-2.514207 E-03\end{array}$ \\
\hline 2697 & $\bar{G}$ & $\begin{array}{l}-3.798582 E-01 \\
-2.115424 E-01\end{array}$ & $\begin{array}{r}1 \\
-5\end{array}$ & $\begin{array}{l}948452 E=01 \\
776152 E-03\end{array}$ & $\begin{array}{r}4 \\
.1\end{array}$ & $\begin{array}{l}322984 E-02 \\
007034 E-01\end{array}$ & $\begin{array}{r}-2 \\
4\end{array}$ & $\begin{array}{l}4006+3 E-02 \\
592135 E-03\end{array}$ & $\begin{array}{l}-8,980910 E-03 \\
-5.127948 E-03\end{array}$ & $\begin{array}{r}-3.364247 E-03 \\
1.147607 E-02\end{array}$ \\
\hline 19777 & G & $\begin{array}{l}-5477817 E-02 \\
-4 \text { 1TOOZE }\end{array}$ & $\begin{array}{l}-5 \\
-2\end{array}$ & $\begin{array}{l}531613 E-02 \\
191698 E-02\end{array}$ & 3 & $\begin{array}{l}894705 E-02 \\
333052 E-02\end{array}$ & $\begin{array}{l}-7 \\
-1\end{array}$ & $\begin{array}{l}212191 E-04 \\
322783 E=03\end{array}$ & $\begin{array}{r}913727 E-05 \\
1.260340 E-03\end{array}$ & $\begin{array}{r}5.096643 E-03 \\
1.0261 E-03\end{array}$ \\
\hline
\end{tabular}


THIS VERSION CONTAINS A BUILTUP TAILBOOM MODEL

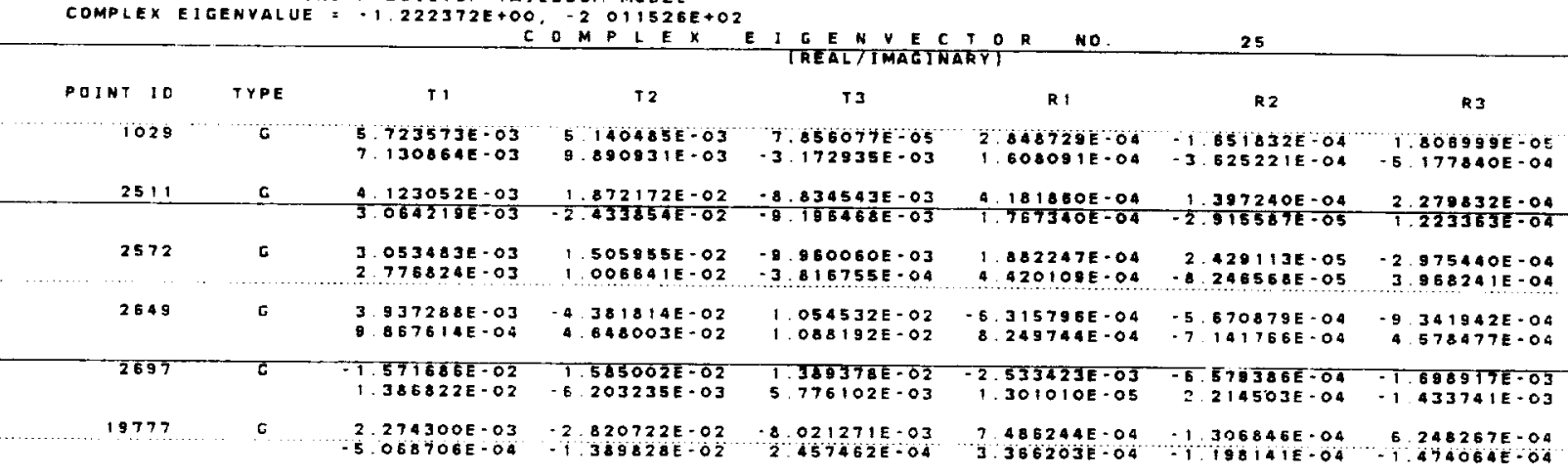

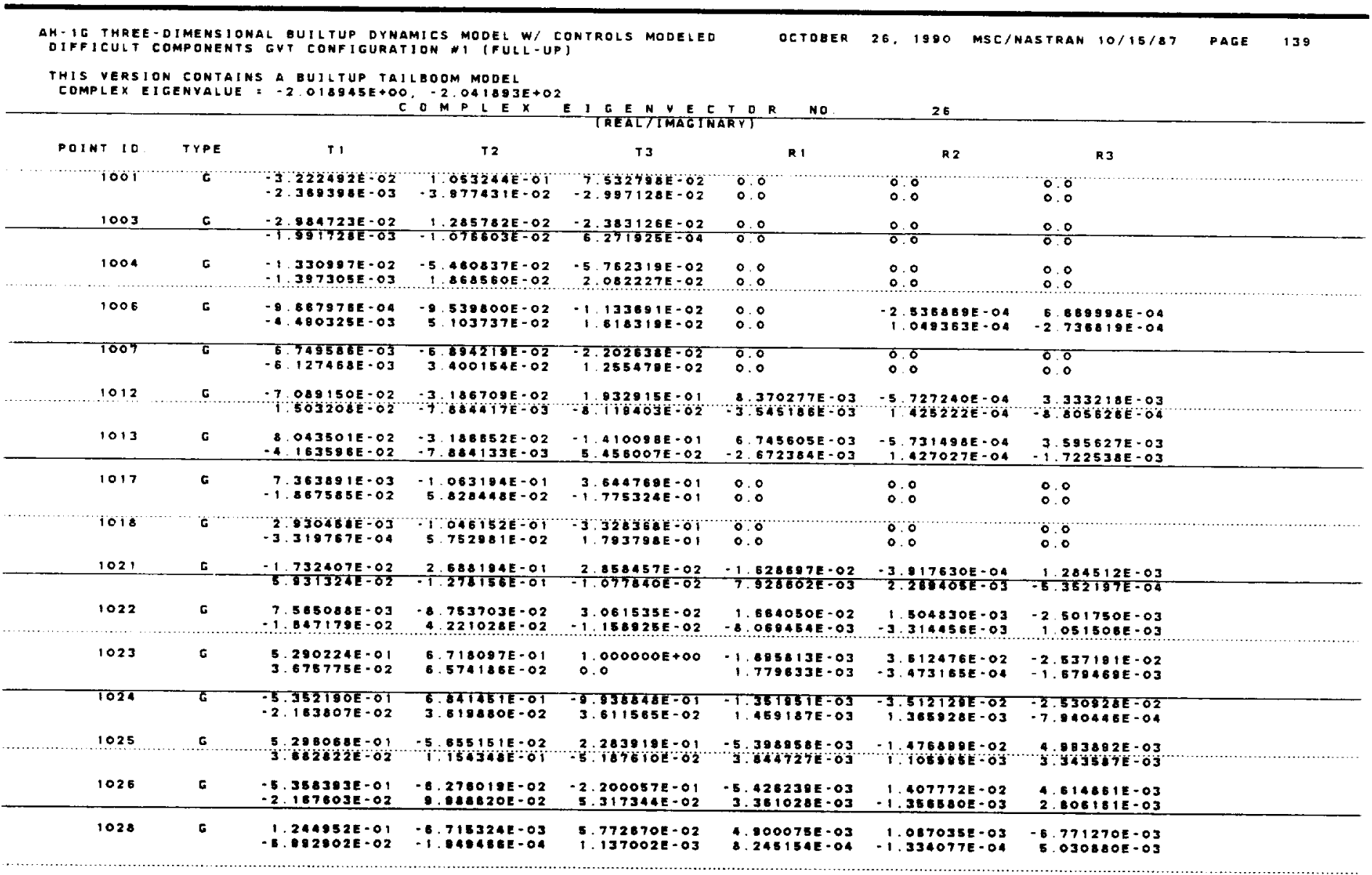


AH- IC THREE-DIMENSIONAL BUILTUP DYNAMICS MODEL W/ CONTROLS MODELED

THIS VERSION CONTAINS DUILTUP TAILBOOM MODEL

THIS VERSION CONTAINS A BUILTUPTAJLOOM MOOEL

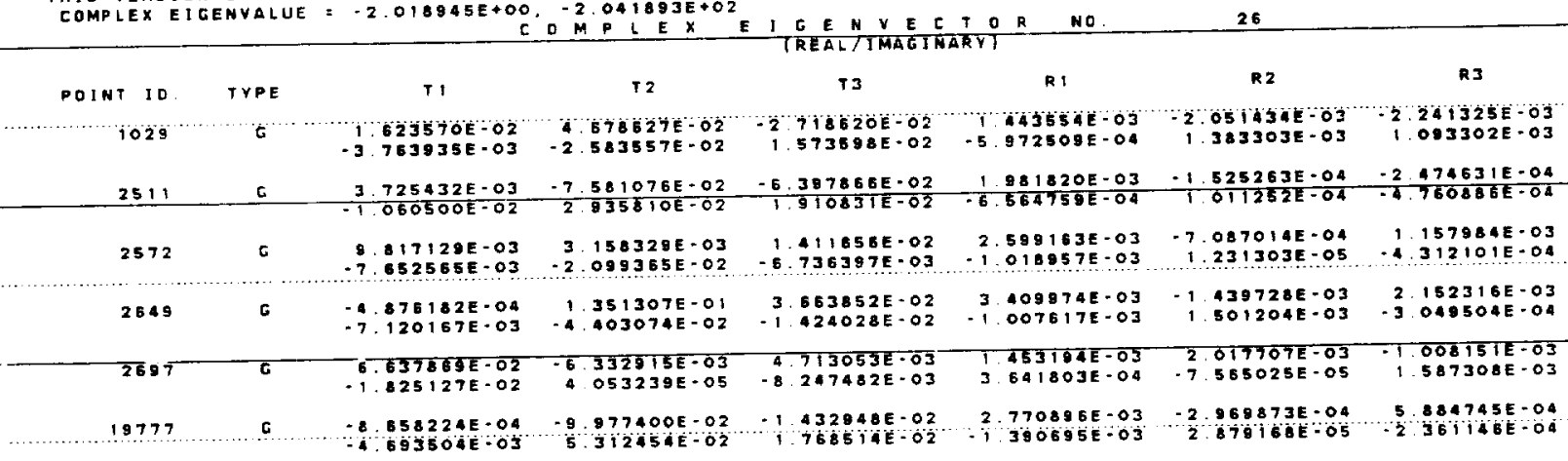

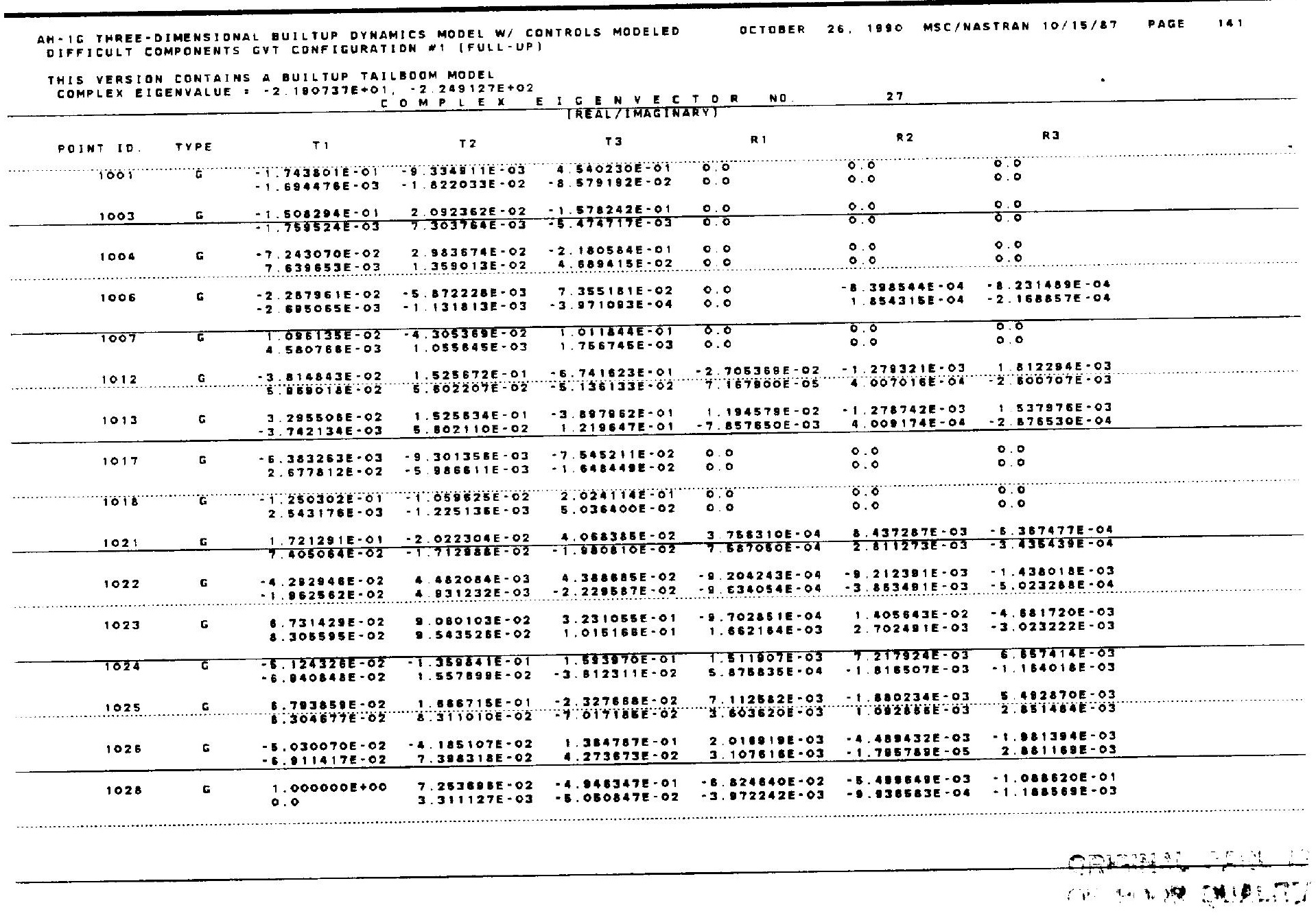


AH- IC THREE-OIMENSIONAL OUILTUP OYNAMICS MODEL W/ CONTROLS MODELED DIFFICULT COMPONENTS GVT CONFIGURATION "I (FULL-UP)

THIS VERSION COMTAINS A BUILTUP TAILBOOM MODEL

COMPLEX EIGENVALUE: - $2.190737 E+01,-2,249127 E+02$

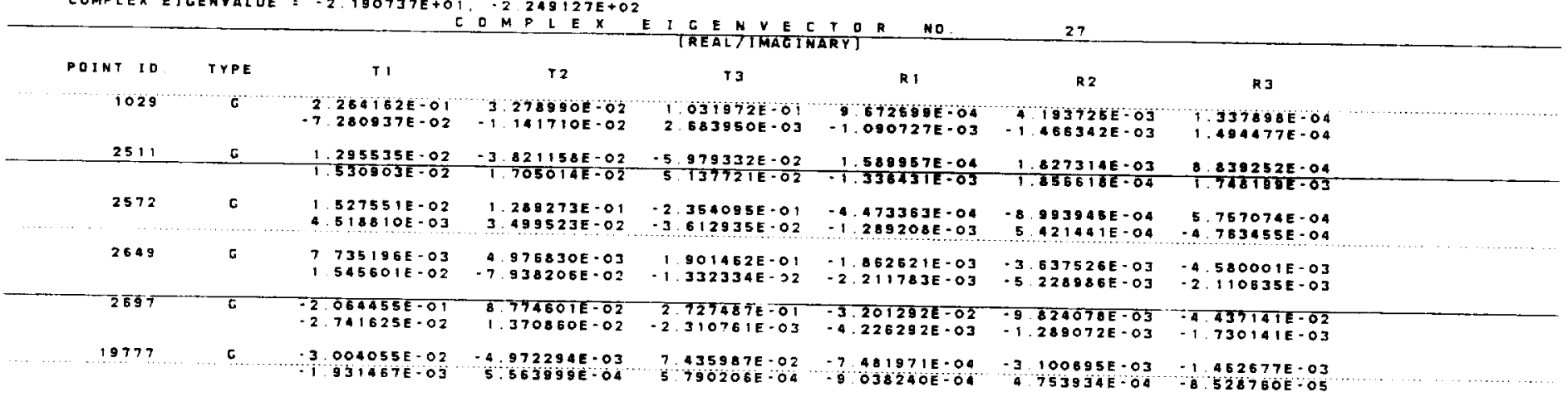

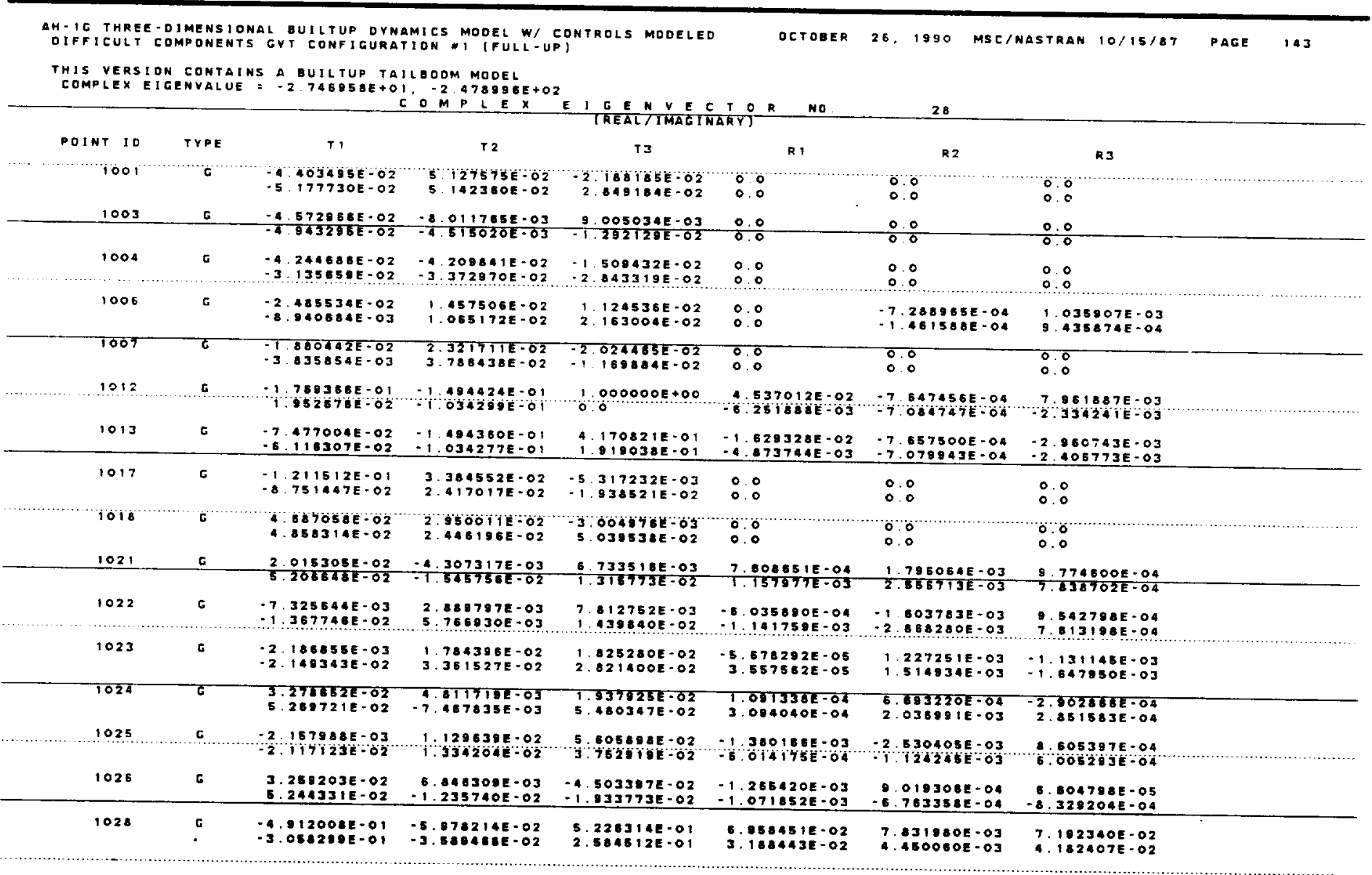


AH- IG THREE-OIMENSIONAL BUILTUP DYNAMICS MODEL W/ CONTROLS MODELEO

THIS VERSION CONTAINS A BUILTUP TAILBOOM MODEL

COMPLEX EIGENYALUE:-2,745958E+01, -2.47896E+02

CDMPLEK EIGEN YETT DR NO .

\begin{tabular}{|c|c|c|c|c|c|c|c|c|c|c|c|}
\hline POINT ID. & TYPE & & $T 1$ & & 12 & T3 & & RI & & R 2 & R 3 \\
\hline 1029 & 6 & $i$ & $\begin{array}{l}568429 E-O 1 \\
B 79875 E-02\end{array}$ & 2 & $\begin{array}{l}754707 E-O 2 \\
381778 E-03\end{array}$ & $\begin{array}{l}-1.52 B 65 B E-O 2 \\
-7.017431 E-03\end{array}$ & 5 & $\begin{array}{l}762957 E-O 3 \\
722476 E-O A\end{array}$ & 2 & $\begin{array}{l}277 O 71 E-02 \\
855971 E-03\end{array}$ & $\begin{array}{l}4.391909 E-O 4 \\
8.686146 E-04\end{array}$ \\
\hline 2511 & $E$ & $\frac{-4}{-3}$ & $\begin{array}{l}458965 E-02 \\
.057631 E-02\end{array}$ & $\frac{-1}{2}$ & $\frac{861231 E-02}{171381 E-02}$ & $\begin{array}{l}-7.867940 E \cdot 02 \\
-4.590180 E \cdot 0 \overline{2}\end{array}$ & $\frac{1}{6}$ & $\frac{716394 E-03}{942398 E-04}$ & $=1$. & $\frac{081338 E-03}{859830 E-04}$ & $\frac{2.626896 E-03}{-2.330043 E-03}$ \\
\hline 2572 & G & $\begin{array}{l}-3 \\
-\mathbf{9}\end{array}$ & $\begin{array}{l}228211 E-02 \\
518230 E-03\end{array}$ & $\begin{array}{l}-1 \\
-9\end{array}$ & $\begin{array}{l}032929 E-01 \\
825794 E-02\end{array}$ & $\begin{array}{l}2.27985 I E-O 1 \\
1213821 E-O 1\end{array}$ & 2 & $\begin{array}{l}723948 E-03 \\
921005 E-04\end{array}$ & $\begin{array}{r}4 \\
-1 \\
\end{array}$ & $\begin{array}{l}130790 E-05 \\
898283 E-03\end{array}$ & $\begin{array}{r}5.375501 E-04 \\
-3.192907 E-05\end{array}$ \\
\hline 2649 & c & $\begin{array}{l}-5 \\
-4\end{array}$ & $\begin{array}{l}607889 E-02 \\
146236 E-02\end{array}$ & c. & $\begin{array}{l}2964825-01 \\
258547 E-02\end{array}$ & $\begin{array}{l}-2.262119 E-02 \\
-2.360408 E-03\end{array}$ & 2 & $\begin{array}{l}906888 E-03 \\
038452 E-03\end{array}$ & $\begin{array}{r}-2 \\
1\end{array}$ & $\begin{array}{l}766755 E-03 \\
082597 E-02\end{array}$ & $\begin{array}{l}5.63884 B E-03 \\
3564684 E=03\end{array}$ \\
\hline 2897 & $\bar{G}$ & 3 & $\begin{array}{l}378830 E-01 \\
722126 E-01\end{array}$ & $\begin{array}{l}-6 \\
-4\end{array}$ & $\begin{array}{l}600005 E-0 \overline{2} \\
893982 E-02\end{array}$ & $\begin{array}{l}-2,223805 E-01 \\
-9.640332 E-02\end{array}$ & 2 & $\begin{array}{l}4 \overline{69632 E-02} \\
5207 B 1 E-02\end{array}$ & 8 & $\begin{array}{l}489770 E-02 \\
437937 E-03\end{array}$ & $\begin{array}{l}316 \overline{4595 E}=02 \\
1714987 E-02\end{array}$ \\
\hline 19777 & G & $\begin{array}{c}-2 \\
-8\end{array}$ & $\begin{array}{l}364586 E-02 \\
301327 E-03\end{array}$ & $\mathrm{i}$ & $\begin{array}{l}372306 E-02 \\
030179 E-02\end{array}$ & $\begin{array}{l}1.083507 E \cdot 02 \\
2.153719 E .02\end{array}$ & 3 & $\begin{array}{l}798953 E-04 \\
5971717-05\end{array}$ & $\therefore$ & $\begin{array}{l}522585 E-05 \\
685 T 44 E-04\end{array}$ & $\begin{array}{l}194342 E-03 \\
8729545 E-04\end{array}$ \\
\hline
\end{tabular}

AM- 1 T THREE-DIMENSIONAL BUILTUP OYNAMICS MODEL W/ CONTROLS MODELED
OIFFICULT COMPONEATS GVT CONFIGURATION I (FULL-UP)

DCTOEER 26, 1990 MSC/NASTRAN 10/15/87 PAGE 145

THIS VERSION CONTAINS A BUILTUP TAILBDOM MODE

COMPLEX EIGENVALUE : - $1500951 E-03,1.678272 E-02$

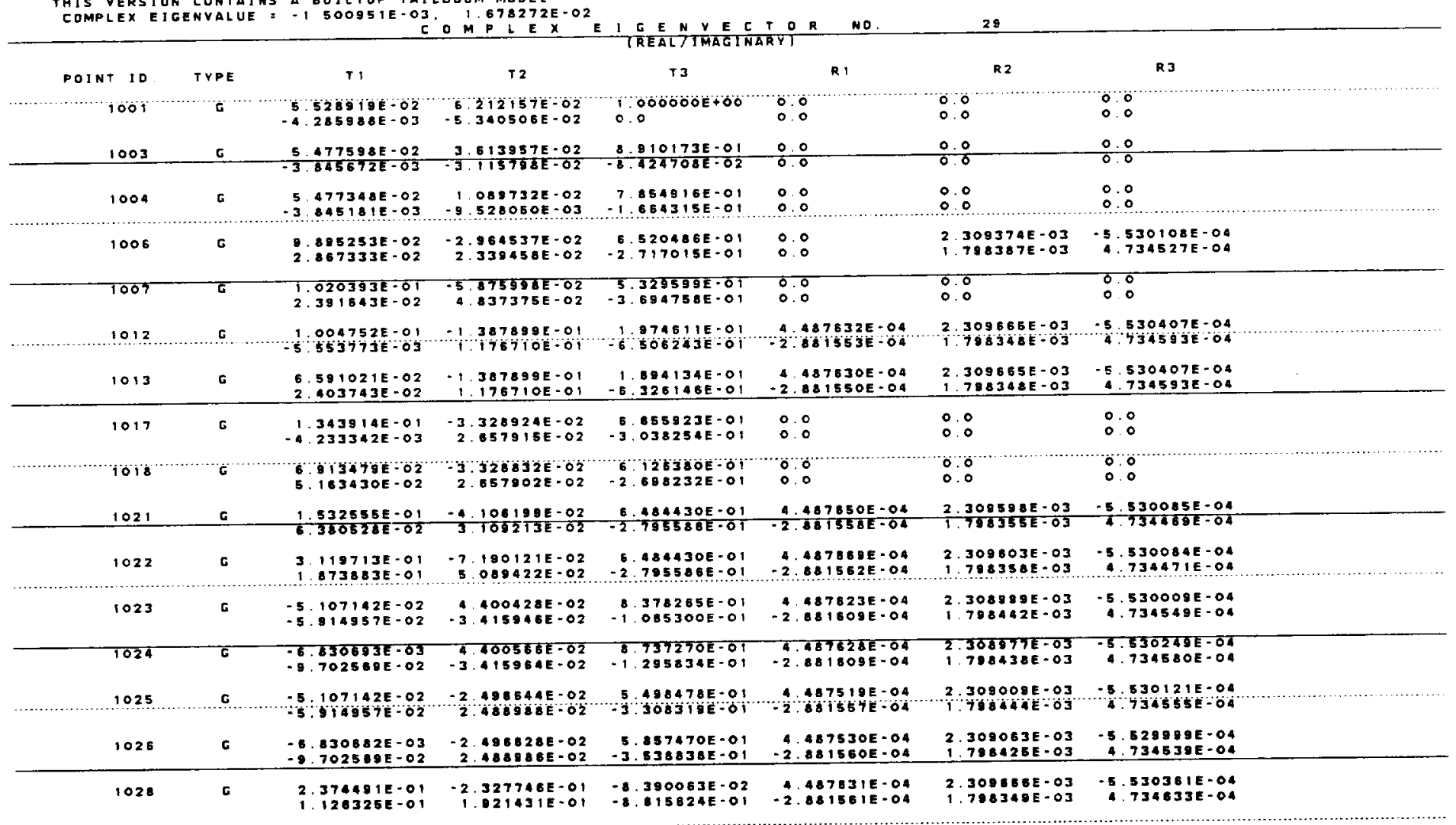


AH-IE THAEE-DIMENS IONAL QUILTUP DYMAMICS MODEL W/ CONTROLS MOOELEO
OIFFICULF COMPONENTS GYT CONFIGURATION \# (FULL-UP)

THIS VERSION CONTAINS A BUITUP TAILEOOM MOOEL

COMPLEX EIGENVALUE: -1.500851 E.03. 1. B $78272 E-02$

\begin{tabular}{|c|c|c|c|c|c|c|c|c|c|c|c|}
\hline & & & & & & & TREAL TIMAE & बरि & & & \\
\hline POINT ID. & TYPE & & $T 1$ & & T2 & & T 3 & & R 1 & R2 & R3 \\
\hline 1029 & $\mathrm{c}$ & & $\begin{array}{l}525909 E-O 1 \\
335401 E-02\end{array}$ & $\begin{array}{r}-6 \\
5\end{array}$ & $\begin{array}{l}729514 E=O 2 \\
374603 E \cdot 02\end{array}$ & $\cdot 5$ & $\begin{array}{l}375793 E-01 \\
658791 E-01\end{array}$ & $\begin{array}{r}4 \\
-2\end{array}$ & $\begin{array}{l}\text { QB } 5570 E-04 \\
B 81528 E-04\end{array}$ & $\begin{array}{l}2.309687 E-03 \\
1.79834 \text { IE-O3 }\end{array}$ & $\begin{array}{r}5 \\
-730396 E-O 4 \\
-734587 E-O 4\end{array}$ \\
\hline 2511 & $c$ & -7 & $\frac{044873 E-02}{231015 E-0 \overline{2}}$ & $\frac{-8}{6}$ & $\frac{140767 E-02}{874555 E-02}$ & $\begin{array}{r}4 \\
-4\end{array}$ & $\frac{127400 E-01}{1539175 E-01}$ & $\frac{4}{-2}$ & $\frac{188120 E-04}{.881052 E-04}$ & $\frac{2.309621 E-03}{1.7883 B 3 E-03}$ & $\frac{-5.529725 E-04}{4.734368 E-04}$ \\
\hline 2572 & c & $\begin{array}{r}5 \\
-1 \\
-1\end{array}$ & $\begin{array}{l}822277 E-02 \\
175748 E-02\end{array}$ & $\begin{array}{r}-1 . \\
1\end{array}$ & $\begin{array}{l}339534 E-01 \\
145519 E-01\end{array}$ & $\begin{array}{r}1 \\
-6 \\
\end{array}$ & $\begin{array}{l}817512 E-01 \\
429323 E-01\end{array}$ & $\begin{array}{r}4 \\
-2 \\
-2\end{array}$ & $\begin{array}{r}487630 E-04 \\
881532 E-04\end{array}$ & $\begin{array}{r}\text { 2. 309885E-03 } \\
1798348 E-03\end{array}$ & $\begin{array}{r}-5.530407 E-04 \\
4734593 E-04\end{array}$ \\
\hline 2649 & c & 8. & $\begin{array}{l}032181 E-02 \\
006483 E-03\end{array}$ & -1 & $\begin{array}{l}845670 E \cdot 01 \\
573559 E \cdot 01\end{array}$ & $\begin{array}{l}-1 \\
-7\end{array}$ & $\begin{array}{l}048922 E \cdot 02 \\
826142 E-01\end{array}$ & -4 & $\begin{array}{l}187526 E-D A \\
881557 E-04\end{array}$ & $\begin{array}{l}\text { 2. } 309666 E-03 \\
1.798348 E-C 3\end{array}$ & $\begin{array}{r}-5.530406 E-04 \\
4.734595 E=04\end{array}$ \\
\hline 2697 & $\bar{E}$ & $\begin{array}{l}2 \\
1\end{array}$ & $\begin{array}{l}304172 \bar{E}-01 \\
205556 E-01\end{array}$ & $\begin{array}{c}-2 \\
1\end{array}$ & $\begin{array}{l}330553 E-01 \\
923693 E-01\end{array}$ & $\begin{array}{l}-9 \\
-8\end{array}$ & 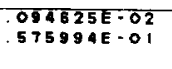 & .2 & $\begin{array}{l}487633 E-04 \\
881555 E-04\end{array}$ & $\begin{array}{l}2.309666 E=03 \\
1.798349 E=03\end{array}$ & $\begin{array}{r}-5.530390 E-04 \\
4.734508 E-O 4\end{array}$ \\
\hline 19777 & c & $\frac{1}{3}$ & $\begin{array}{l}025: 23 E-01 \\
242173 E-02\end{array}$ & -3 & $\begin{array}{r}045314 E-02 \\
391325 E-02\end{array}$ & $2:$ & $\begin{array}{l}515639 E-01 \\
.713003 E=01\end{array}$ & 2 & $\begin{array}{l}487650 E-04 \\
821544 E-04\end{array}$ & $\begin{array}{r}2.309443 E-03 \\
798375 E-03\end{array}$ & $\begin{array}{r}530228 E-04 \\
454545 E-O A\end{array}$ \\
\hline
\end{tabular}

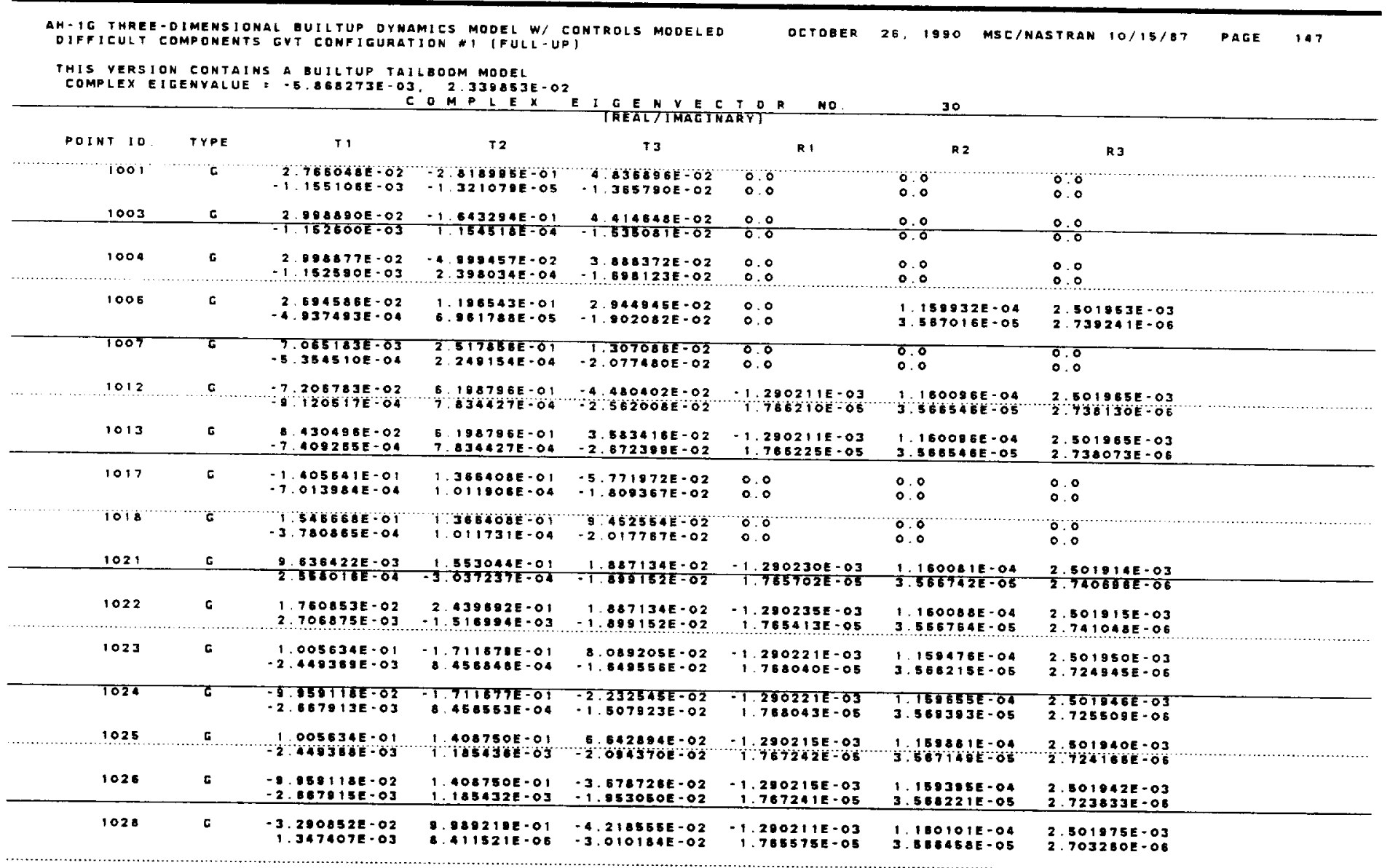


AH- 16 THREE-Dimensional BUILTUP DYMAMICS MODEL W/ CONTRDLS MOdeled

DIFFICULT COMPONENTS GYT CONF IEURATION I (FULL-UP)

THIS VERSION CONTAINS A BUILTUP TAILBOOM MODEL

COMPLEX EIGENVALUE = -5.868273E-03, $2.339853 E+02$

\begin{tabular}{|c|c|c|c|c|c|c|c|c|c|c|c|}
\hline POINT IO & \multirow{2}{*}{$\begin{array}{r}\text { TYPE } \\
\mathrm{G}\end{array}$} & \multicolumn{2}{|r|}{ TI } & \multicolumn{2}{|r|}{$T 2$} & \multicolumn{2}{|r|}{13} & \multicolumn{2}{|r|}{ RI } & R 2 & RI \\
\hline 1029 & & 2 & $\begin{array}{l}609339 E=03 \\
466863 E-04\end{array}$ & 2 & $\begin{array}{l}750750 E-O 1 \\
B 78950 E-O A\end{array}$ & -2 & $\begin{array}{l}330288 E-02 \\
070347 E-02\end{array}$ & $=1$ & $\begin{array}{l}290209 E-03 \\
766303 E-05\end{array}$ & $\begin{array}{l}1 \\
3.566604 E-05\end{array}$ & $\begin{array}{r}2.801965 E-O 3 \\
2739401 E-O 6\end{array}$ \\
\hline 2511 & G & $\frac{4}{-8}$ & $\frac{183086 E-02}{612239 E-04}$ & & $\frac{618194 E-01}{406111 E-04}$ & $\frac{2}{-2}$ & $\frac{589668 E-02}{278537 E-02}$ & $\frac{-1}{1}$ & $\begin{array}{l}290220 E-03 \\
766617 E-05\end{array}$ & $\frac{1.180067 E-04}{3.565665 E-05}$ & $\frac{2.501968 E-03}{2735602 E-06}$ \\
\hline 2572 & G & $\begin{array}{r}4 \\
-11 \\
-2\end{array}$ & $\begin{array}{l}763923 E-03 \\
242955 E-03\end{array}$ & 9. & $\begin{array}{l}086403 E-01 \\
918558 E-04\end{array}$ & $\begin{array}{l}-4 \\
-2 \\
2\end{array}$ & $\begin{array}{l}569617 E-03 \\
619807 E-02\end{array}$ & $\begin{array}{r}-1 \\
-1 \\
2+1\end{array}$ & $\begin{array}{l}290211 E-03 \\
766292 E-05\end{array}$ & $\begin{array}{r}160096 E-04 \\
3.566574 E-05\end{array}$ & $\begin{array}{r}2.501965 E-03 \\
2737916 E-06\end{array}$ \\
\hline 2649 & 6 & $\begin{array}{r}5 \\
-8\end{array}$ & $\begin{array}{l}974367 E-03 \\
708209 E-04\end{array}$ & 1 . & $\begin{array}{l}283484 E-01 \\
035228 E-03\end{array}$ & $\begin{array}{l}-1 \\
-2\end{array}$ & $\begin{array}{l}422545 E-02 \\
916561 E-02\end{array}$ & -1. & $\begin{array}{l}290212 E-03 \\
786435 E-05\end{array}$ & $\begin{array}{l}160101 E \cdot 04 \\
3 \quad 568516 E-05\end{array}$ & $\begin{array}{l}2501965 E-03 \\
2735543 E-O B\end{array}$ \\
\hline 2697 & $\bar{E}$ & 1 & $\begin{array}{l}229182 \bar{E}-03 \\
405574 E-03\end{array}$ & 1 & $\begin{array}{l}000000 E+00 \\
0\end{array}$ & $\begin{array}{l}-2 \\
-3\end{array}$ & $\begin{array}{l}308445 E-0 \overline{2} \\
036963 E-02\end{array}$ & $\begin{array}{r}-1 . \\
1\end{array}$ & $\begin{array}{l}290212 E=03 \\
765625 E-05\end{array}$ & $\begin{array}{l}1.160104 E-04 \\
3.566362 E-05\end{array}$ & $\begin{array}{l}2.501969 E-03 \\
2.724809 E-06\end{array}$ \\
\hline 19777 & 5 & 2 & $\begin{array}{l}985674 E-02 \\
265867 E=04\end{array}$ & $\begin{array}{l}1 \\
3\end{array}$ & $\begin{array}{l}219766 E-01 \\
782764 E-05\end{array}$ & $\because 3$ & $\begin{array}{l}084289 E-02 \\
903980 \mathrm{E}-02\end{array}$ & $\begin{array}{c}-1 \\
11\end{array}$ & $\begin{array}{l}290217 E-03 \\
766103 E-05\end{array}$ & $\begin{array}{l}1.159863 E \cdot 04 \\
3.5668 \mathrm{IEE}-05\end{array}$ & $\begin{array}{r}2.501954 E-03 \\
3.739513 E-06\end{array}$ \\
\hline
\end{tabular}

AH-1 1 THREE-OIMENSIONAL BUILTUP OYNAMICS MODEL W/ CONTROLS MODELED
OIFFICULT COMPONENTS GVT CONFIGURATION 1 IFULL-UP)

OCTOBER 26, 1990 MSC/NASTRAN 10/15/87 PAEE 149

THIS VERSION CONTAINS A BUILTUP TAILBOOM MDDEL

COMPLEX EIGENVALUE: - $1.092172 E-02$. $1.262880 E-01$

\begin{tabular}{|c|c|c|c|c|c|c|c|c|c|}
\hline PDINT ID. & TYPE & $T 1$ & $T 2$ & & 13 & & RI & R2 & R3 \\
\hline iool & 'c. & $\begin{array}{l}\text { B. } 305505 E=01 \\
2.545260 E-02\end{array}$ & $\begin{array}{l}1575657 E-O 2 \\
-1.527280 E-03\end{array}$ & -5 & $\begin{array}{l}A I G 809 E-O I \\
034404 E-02\end{array}$ & 00 & $\because$ & 0.0 & 0.0 \\
\hline 1003 & G & $\begin{array}{r}\text { 8. } 304237 E-01 \\
2.546931 E-02\end{array}$ & $\frac{9.756902 E-03}{.6 .693308 E-04}$ & $\frac{-3.8}{9 .}$ & $\frac{086793 E-01}{145882 E-03}$ & $\frac{0.0}{0.0}$ & $\div$ & $\frac{0.0}{0.0}$ & $\div 0$ \\
\hline 1004 & c & $\begin{array}{l}304191 E-01 \\
2.545901 E-02\end{array}$ & $\begin{array}{r}3.777387 E-03 \\
1.503580 E-04\end{array}$ & -2. & $\begin{array}{l}403715 E-01 \\
052854 E-03\end{array}$ & $\begin{array}{l}0.0 \\
0.0\end{array}$ & $\begin{array}{l}0 \\
0\end{array}$ & $\begin{array}{l}0 . \\
0: 0\end{array}$ & $: 0$ \\
\hline 1006 & G & $\begin{array}{l}7.702219 E-01 \\
2.587807 E-02\end{array}$ & $\begin{array}{l}-1.903277 E-03 \\
-2.348528 E-04\end{array}$ & $\begin{array}{r}-5 . \\
6 .\end{array}$ & $\begin{array}{l}091268 E \cdot 02 \\
843180 E \cdot 03\end{array}$ & $\because .0$ & : & $\begin{array}{r}-3.251556 E-03 \\
2.379910 E-05\end{array}$ & $\begin{array}{r}-1.271607 E-04 \\
1.226437 E-05\end{array}$ \\
\hline 1007 & $\tau$ & $\begin{array}{l}7.930515 E-01 \\
2.572008 E-02\end{array}$ & $\begin{array}{r}-8.724562 E=03 \\
7.781706 E=04\end{array}$ & 1.2. & $\begin{array}{l}2080+7 t-01 \\
192345 E-03\end{array}$ & 0.0 & \% & 0.0 & 0.0 \\
\hline 1012 & c. & $\begin{array}{r}035409 E-01 \\
2 \quad 05460 E-02\end{array}$ & $\begin{array}{r}-2.879403 E-02 \\
4178151 E=03\end{array}$ & 5. & $\begin{array}{l}093300 E-01 \\
0159+6 E=03\end{array}$ & $\begin{aligned}-9.2 \\
77.8\end{aligned}$ & $\begin{array}{l}280942 E-05 \\
820926 E-05 \\
821626 E-05\end{array}$ & $\begin{array}{r}-3.247474 E \cdot 03 \\
2.392155 E \cdot 05\end{array}$ & $\begin{array}{r}-1.276151 E-04 \\
1.826411 E-05\end{array}$ \\
\hline 1013 & G & $\begin{array}{l}7.855557 E-01 \\
2.008527 E-02 \\
\end{array}$ & $\begin{array}{r}-2.879403 E-02 \\
4 \quad 178151 E-03 \\
\end{array}$ & 6. & $\begin{array}{r}151312 E-01 \\
273943 E-04 \\
\end{array}$ & $\begin{array}{r}-9.2 \\
7.8 \\
\end{array}$ & $\begin{array}{l}282806 E-05 \\
821583 E-05 \\
\end{array}$ & $\begin{array}{r}-3.247474 E-03 \\
2.392155 E-05 \\
\end{array}$ & $\begin{array}{r}1.275920 E-04 \\
1.825280 E-05 \\
\end{array}$ \\
\hline 1017 & G & $\begin{array}{l}\text { 7. } 00654 E-01 \\
2.06042 E-02\end{array}$ & $\begin{array}{l}-2.8 B 2149 E-03 \\
-5.152598 E-05\end{array}$ & -3. & $\begin{array}{l}\text { 3884OAE-O2 } \\
19 O 814 E-O 2\end{array}$ & $: 0$ & $:$ & $: 0$ & $\because 0$ \\
\hline 1018 & 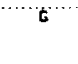 & $\begin{array}{l}1.550409 E-O 1 \\
2.670508 E-02\end{array}$ & $\begin{array}{l}-2.695516-O 3 \\
-5.110621 E-O 5\end{array}$ & -2 & $\begin{array}{l}29637 E=02 \\
678170 E-03\end{array}$ & 0.0 & \% & 0.0 & 0.0 \\
\hline 1021 & ᄃ & $\begin{array}{l}7.008442 E .01 \\
2.624529 E .02\end{array}$ & $\frac{-2.913798 E-04}{-1.869423 E-03}$ & -4 & $\frac{157758 E-02}{3865632 \cdot 03}$ & -9.2 & $\begin{array}{l}275595 E-05 \\
822855 E-05\end{array}$ & $\frac{-3.248231 E-03}{2.392706 E-05}$ & $\frac{-1.289388 E-04}{1.827874 E-05}$ \\
\hline 1022 & G & $\begin{array}{r}776388 E-01 \\
2.780170 E-02\end{array}$ & $\begin{array}{r}5.081811 E-03 \\
-7.245398 E-03 \\
\end{array}$ & -4 & $\begin{array}{l}187758 E \cdot 02 \\
38853 E-03\end{array}$ & $\begin{array}{r}-9.2 \\
7.8\end{array}$ & $\begin{array}{l}274806 E-05 \\
823142 E-05\end{array}$ & $\begin{array}{r}-3.247918 E-03 \\
2.397936 E-05\end{array}$ & $\begin{array}{r}-1.269217 E-04 \\
\text { S28131E-OS }\end{array}$ \\
\hline 1023 & $c$ & $\begin{array}{l}9.523512 E-01 \\
2.509032 E-02\end{array}$ & $\begin{array}{l}\text { 3. } 820384 E-03 \\
\text { 2. } 865558 E-03\end{array}$ & $\begin{array}{r}-3 \\
6\end{array}$ & $\begin{array}{l}305436 E-01 \\
372433 E-03\end{array}$ & -9.2 & $\begin{array}{l}260429 E=05 \\
826056 E=05\end{array}$ & $\begin{array}{r}-3.257081 E-O 3 \\
2.359580 E-O 5\end{array}$ & $\begin{array}{r}-1.273103 E-04 \\
1.823473 E=05\end{array}$ \\
\hline 1024 & $\bar{G}$ & $\begin{array}{l}.625428 E-01 \\
2.363958 E-02\end{array}$ & $\begin{array}{l}3.838310 E-03 \\
2.866133 E-03\end{array}$ & -3 & $\begin{array}{l}378454 E-01 \\
263743 E-02\end{array}$ & 7.8 & $\begin{array}{l}263367 E-O 5 \\
825576 E-O 5\end{array}$ & $\begin{array}{r}-3.2570161-03 \\
2.368540 E-05\end{array}$ & $\begin{array}{r}-1.276338 E-04 \\
1.822540 E-05\end{array}$ \\
\hline 1025 & c. & $\begin{array}{l}9.523512 E-01 \\
2.509092 E=02\end{array}$ & $\begin{array}{r}-1 \cdot 208 B 73 E-02 \\
-939360 E=03\end{array}$ & 7. & $\begin{array}{l}585768 E-02 \\
427832 E-03\end{array}$ & -92 & $\begin{array}{l}281945 E-05 \\
823514 E-05\end{array}$ & $\begin{array}{r}-3.258714 E-03 \\
2: 363401 E=06\end{array}$ & $\begin{array}{r}275025 E-04 \\
\therefore 822653 E=05\end{array}$ \\
\hline 1026 & c & $\begin{array}{l}9.828430 E-01 \\
2.383958 E-02 \\
\end{array}$ & $\begin{array}{r}-1.205458 E-02 \\
1.930450 E-03 \\
\end{array}$ & $\begin{array}{l}6 . \\
\text { g. }\end{array}$ & $\begin{array}{l}821635 E-02 \\
885128 E-03 \\
\end{array}$ & $\begin{array}{r}-9.2 \\
7.2 \\
\end{array}$ & $\begin{array}{l}281013 E-05 \\
123892 E-05 \\
\end{array}$ & $\begin{array}{r}-3.256025 E-03 \\
2.387192 E-05 \\
\end{array}$ & $\begin{array}{r}-1.273387 E-04 \\
1.823084 E-05 \\
\end{array}$ \\
\hline 1028 & G & $\begin{array}{l}5.083904 E-01 \\
2.657800 E-02\end{array}$ & $\begin{array}{r}-3 . \$ 23508 E-02 \\
1.478657 E-03\end{array}$ & 9. & $\begin{array}{l}980597 E-01 \\
163436 E-03\end{array}$ & $\begin{array}{r}-9.2 \\
7.2\end{array}$ & $\begin{array}{l}277141 E-05 \\
823258 E-05\end{array}$ & $\begin{array}{r}-3.2474898-03 \\
2.302038 E-05\end{array}$ & $\begin{array}{r}-1.276180 E-04 \\
1.27514 E-05\end{array}$ \\
\hline
\end{tabular}


THIS VERSION CONTAINS A BUILTUP TAILEOOM MODEL

COMPLEX EIGENYALUE = - 1 O92172E-02, 1.262600E-OI

\begin{tabular}{|c|c|c|c|c|c|c|c|c|c|c|}
\hline & & & & $2 . n$ & 1 & TREALT/MAGT & $\frac{T}{\mathrm{ARV}}$ & No & 31 & \\
\hline POINT ID. & IYPE & is & & T 2 & & $T 3$ & & R & R2 & R3 \\
\hline 1029 & $\tilde{\mathbf{G}}$ & $\begin{array}{l}\text { T.O1841 IE-OI } \\
\text { 2. } 624501 E-02\end{array}$ & $\begin{array}{l}-6 \\
-9\end{array}$ & $\begin{array}{l}132327 E-03 \\
735755 E-04\end{array}$ & & $\begin{array}{l}\text { MOBOZ3E-O } \\
\text { 240222E-O3 }\end{array}$ & $\begin{array}{r}-9.2 \\
7.2\end{array}$ & $\begin{array}{l}284876 E-05 \\
621723 E-05\end{array}$ & $\begin{array}{r}-3.24734 E-O 3 \\
2.393520 E-O 5\end{array}$ & $\begin{array}{r}275752 E-O 4 \\
1.25167 E-05\end{array}$ \\
\hline 2511 & c & $\frac{8.044678 E \cdot 01}{2.573758 E-02}$ & $\frac{-1}{2}$ & $\frac{595073 E-02}{-477565-05}$ & $\frac{2}{3}$ & $\frac{20834 E-01}{883376 E-03}$ & $\frac{-8.4}{9.8}$ & $\frac{417643 E-05}{817511 E-05}$ & $\frac{-3.247847 E-03}{2.301286 E-05}$ & $\frac{-1.260800 E \cdot 04}{1.826885 \cdot 05}$ \\
\hline 2572 & $\mathbf{G}$ & $\begin{array}{l}\text { E } 374739 E-01 \\
2.524557 E-02\end{array}$ & $\begin{array}{r}-2 \\
5 \\
\end{array}$ & $\begin{array}{l}997103 E-02 \\
105818 E-03\end{array}$ & & $\begin{array}{r}146012 E-01 \\
554200 E-03\end{array}$ & $\begin{array}{r}-82 \\
7: 8\end{array}$ & $\begin{array}{l}282036 E-05 \\
821695 E-05\end{array}$ & $\begin{array}{r}-3.247480 E-03 \\
2.3921145-05\end{array}$ & $\begin{array}{r}-1.275033 E-04 \\
1.825085 E-05\end{array}$ \\
\hline 2649 & c & $\begin{array}{l}\text { B. } 035888 E-01 \\
2.549517 E-02\end{array}$ & $\begin{array}{r}-3 \\
5\end{array}$ & $\begin{array}{l}962340 E-02 \\
807811 E-03\end{array}$ & $\begin{array}{l}8 \\
5\end{array}$ & $\begin{array}{l}\text { BAQ9AEE-O1 } \\
\text { B32263E-O4 }\end{array}$ & $\begin{array}{r}-9.2 \\
7.8\end{array}$ & $\begin{array}{l}281364 E-05 \\
821698 E-05\end{array}$ & $\begin{array}{r}-3.247508 E-03 \\
2.392024 E-05\end{array}$ & $\begin{array}{r}-1.276037 E-04 \\
1.825196 E-05\end{array}$ \\
\hline 2897 & $\mathrm{C}^{-}$ & $\begin{array}{l}\text { 5. } 958774 E-01 \\
2.596160 E-02\end{array}$ & $=3$ & $\begin{array}{l}821013 E-05 \\
442344 E+03\end{array}$ & $\begin{array}{l}T \\
0\end{array}$ & $0^{000000 E+00}$ & $\begin{array}{r}-9.2 \\
7.8\end{array}$ & $\begin{array}{l}279519 E-05 \\
822543 E-05\end{array}$ & $\begin{array}{l}\text {-3.345886E-03 } \\
2.39202 E-05\end{array}$ & $\begin{array}{r}-1276058 E=04 \\
1.26070 E=05\end{array}$ \\
\hline 19777 & 6 & $\begin{array}{l}\text { T. } 642332 E-01 \\
2.594068 E-O 2\end{array}$ & -1 & $\begin{array}{l}736220 E-03 \\
75647 \text { IE-OA }\end{array}$ & $\begin{array}{r}-5 \\
-6\end{array}$ & $\begin{array}{l}081253 E-02 \\
758720 E-03\end{array}$ & $\begin{array}{r}-9.2 \\
7.8\end{array}$ & $\begin{array}{l}273427 E-O S \\
82212 O E-O S\end{array}$ & $\begin{array}{r}-3,250651 E-03 \\
\text { Z.382737E-OS }\end{array}$ & $\begin{array}{r}-1.273280 E-04 \\
1.26056 E-05\end{array}$ \\
\hline
\end{tabular}

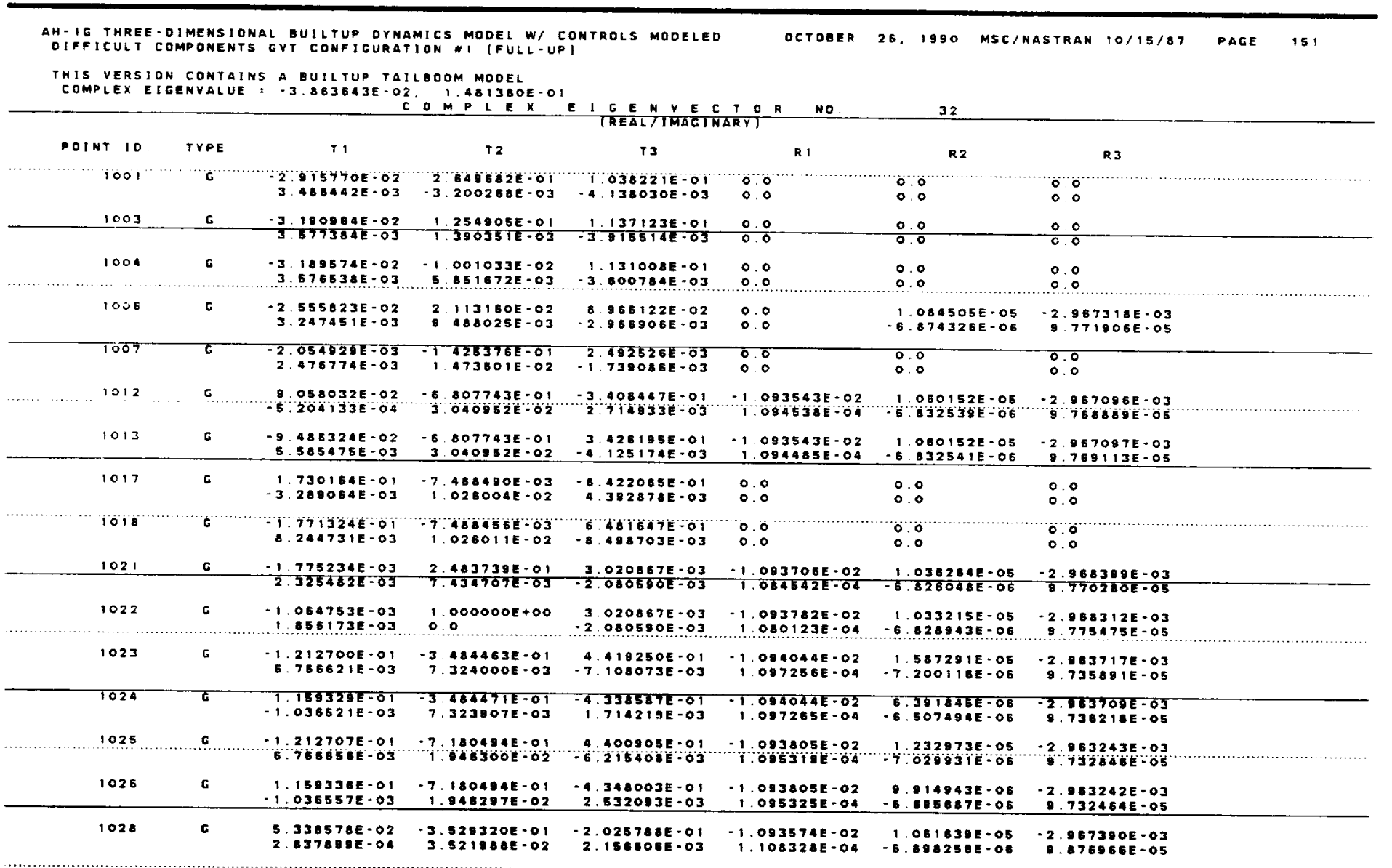


AH-IG THREE-DIMENSIONAL BUILTUP DYNAMICS MDOEL W/ CONTROLS MDOELED

OIFFI CULT COMPOMENTS GVT CONFIGURATION W (FULL-UP)

THIS YERSION CONTAINS A BUILTUP TAILBOOM MDOEL

COMPLEX EIGENYALUE $=-3.863643 E-O Z$.

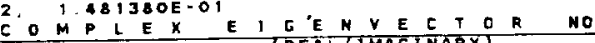

32

POINT 10. TYPE TI T2 TI

\begin{tabular}{|c|c|c|c|c|c|c|c|}
\hline POINT ID. & YPE & 11 & & & & & \\
\hline 1029 & 6 & $\begin{array}{r}1024268 E-03 \\
2.327263 E-03\end{array}$ & $\begin{array}{l}032089 E-01 \\
1.214331 E-02\end{array}$ & $\begin{array}{l}2.513154 E-03 \\
-1752564 E-03\end{array}$ & $\begin{array}{r}1.093557 E-02 \\
1.093312 E-04\end{array}$ & $\begin{array}{r}036435 E-0 S \\
-6.735515 E-05\end{array}$ & $\begin{array}{r}2.967230 E-03 \\
9770919 E-05\end{array}$ \\
\hline 2511 & G & $\frac{-4.480030 E-02}{3.950378 E-03}$ & $\frac{-4.006312 E-01}{2.056613 E-02}$ & $\frac{1.591115 E-01}{-2.973866 E-03}$ & $\frac{-1.093535 E \cdot 02}{1.09330 B E \cdot 04}$ & $\begin{array}{r}1.061428 E-05 \\
-6.834299 E-O B E\end{array}$ & $\frac{-2.987089 E-03}{9.768187 E-05}$ \\
\hline 2572 & c & $\begin{array}{r}-2.255255 E-03 \\
2.612321 E-03\end{array}$ & 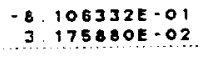 & $\begin{array}{r}9.798610 E-04 \\
-70013200-04\end{array}$ & $\begin{array}{r}-1.093542 E-02 \\
1.094246 E-04\end{array}$ & $\begin{array}{r}1.080317 E-05 \\
-8.1318 E-06\end{array}$ & $\begin{array}{r}-2.987098 E-03 \\
9769962 E-05\end{array}$ \\
\hline 2649 & c & $\begin{array}{r}-2.154648 E-03 \\
2.541027 E-03\end{array}$ & $\begin{array}{r}-9.434948 E-O 1 \\
3.875130 E-02\end{array}$ & $\begin{array}{l}-2.815730 E-06 \\
-1.313 C 12 E-04\end{array}$ & $\begin{array}{r}1.093546 E-02 \\
1.096533 E-04\end{array}$ & $\begin{array}{r}1.057370 E-05 \\
-6.750236 E-06\end{array}$ & $\begin{array}{r}-2.987129 E-03 \\
9778066 E-05\end{array}$ \\
\hline 2697 & $\bar{G}$ & $\begin{array}{l}9.415410 E^{-03} \\
1.740082 E^{-03}\end{array}$ & $\begin{array}{r}-3.479278 E=01 \\
3518079 E=02\end{array}$ & $\begin{array}{r}-4.051 \overline{387 E-02} \\
5.185779 E-04\end{array}$ & $\begin{array}{r}1.093582 E-02 \\
1.103786 E-04\end{array}$ & $\begin{array}{r}1.060546 E=05 \\
.8 .85242 E-06\end{array}$ & $\begin{array}{r}-2.967229 E \cdot 03 \\
9.812573 E-05\end{array}$ \\
\hline $1977 ?$ & c & $\begin{array}{r}-2.874308 E-02 \\
\hdashline 3.340555 E-03\end{array}$ & $\begin{array}{r}4081528 E-02 \\
928369 E-03\end{array}$ & 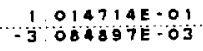 & $\begin{array}{r}-1093536 E-02 \\
092506 E-04\end{array}$ & 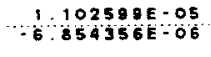 & $\begin{array}{r}295318 E-03 \\
977232 E-05\end{array}$ \\
\hline
\end{tabular}

QH- I THRE - DIMENSIONAL BUILTUP OYNAMICS MODEL W/ CONTROLS MDOELED

OCTOBER 26, 1990 MSC/NASTRAN 10/5/87 PALE

THIS YERSION CONTAINS A BUILTUP TAILBODM MODEL

THIS YERSION CONTAINS A BUILTUP TAILBODM MODEL
COMPLEX EICENYALUE = -8.303530E-O3, E. 335569E-OI

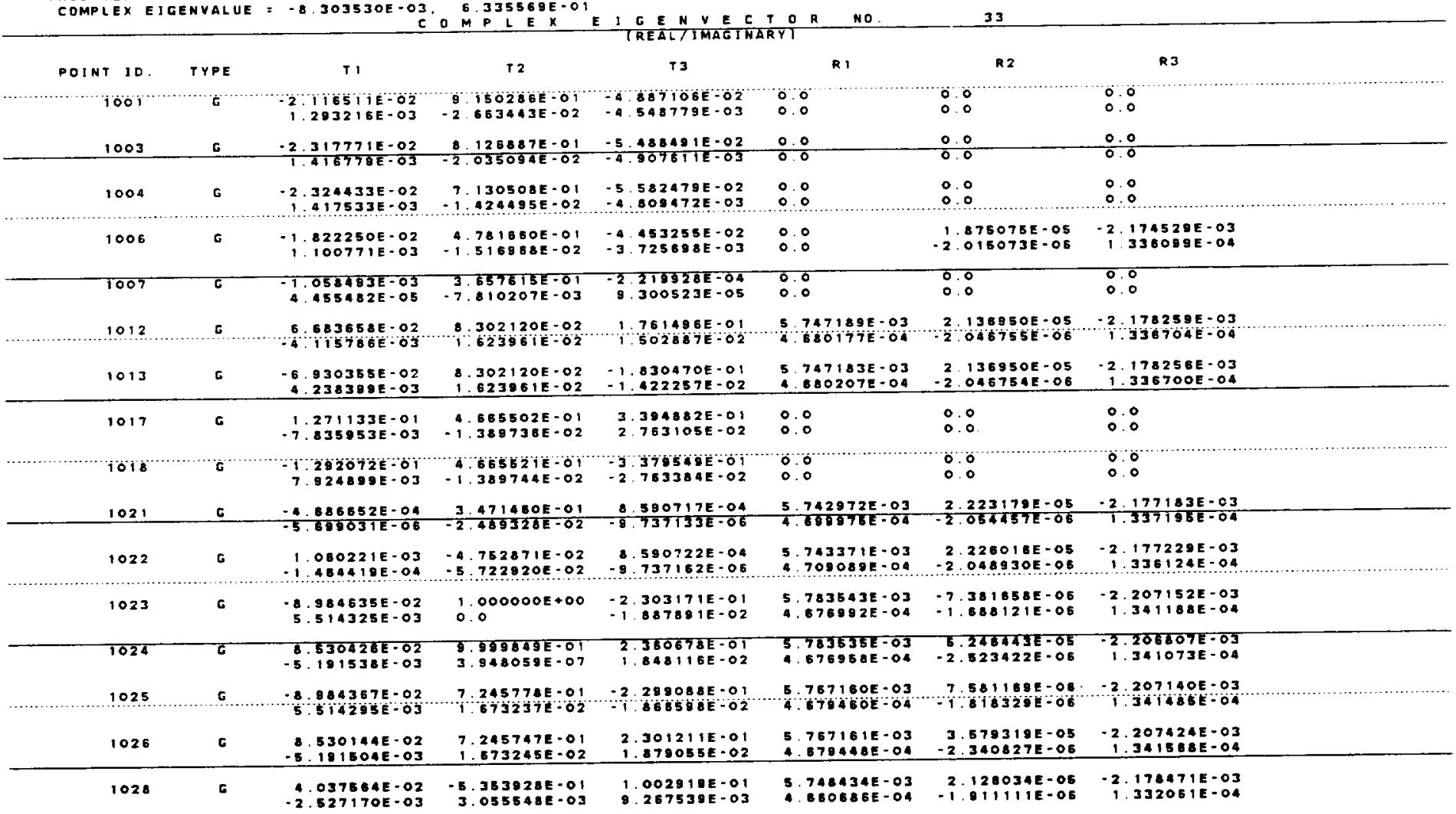



AH-IC THREE-DIMENSIONAL BUILTUP DYMAMICS MODEL W/ CONTROLS MODELED
DIFFICULT COMPONENGS GVT CONFIGURATION 1 (FULL-UP)

THIS VERSION CONTAINS A BUILTUP TAILBOOM MODEL

COMPLEX EJGENVALUE = -8.303530E-03. E.3355EgE-O

\begin{tabular}{|c|c|c|c|c|c|c|c|c|c|c|c|}
\hline & & & & & $x$ & E I & $\begin{array}{l}\text { GE N Y E C } \\
\text { TAEAL IMAEIN }\end{array}$ & $\frac{10}{R V I}$ & No & 33 & \\
\hline \multirow{2}{*}{$\begin{array}{r}\text { POINT IO } \\
1029\end{array}$} & TYPE & & TI & & 12 & & $T 3$ & & RI & R2 & R 3 \\
\hline & $\mathrm{c}$ & $\begin{array}{l}-5 \\
-8\end{array}$ & $\begin{array}{l}704355 E-04 \\
877142 E-07\end{array}$ & $\begin{array}{l}2 \\
-1\end{array}$ & $\begin{array}{l}\text { AOQ7TE-OI } \\
\text { SJ4567E-OZ }\end{array}$ & -1 & $\begin{array}{l}684784 E-O A \\
.860400 E-05\end{array}$ & $\begin{array}{l}5 \\
4\end{array}$ & $\begin{array}{l}747758 E-O 3 \\
682030 E-04\end{array}$ & $\begin{array}{l}2.417830 E-05 \\
-2.120284 E-06\end{array}$ & $\begin{array}{r}-2.135838 E-03 \\
1.336211 E-04\end{array}$ \\
\hline 2511 & ᄃ & $\frac{-3}{1}$ & $\frac{257650 E-02}{.685419 E-03}$ & 3. & $\frac{172856 E-01}{567728 E-03}$ & -8. & $\frac{345178 E-02}{.333626 E-03}$ & 5. 7 & $\frac{746411 E-03}{587880 E-04}$ & $\frac{2.132153 E-O 5}{.2 .04915 \mathrm{IE}-O \mathrm{OL}}$ & $\frac{-2.178051 E-03}{1.336772 E-04}$ \\
\hline 2572 & c & $\begin{array}{r}-1 \\
8\end{array}$ & $\begin{array}{r}483053 E-03 \\
520229 E-05\end{array}$ & 2 & $\begin{array}{l}485401 E \cdot 01 \\
180225 E-02\end{array}$ & $\begin{array}{r}-3 \\
4\end{array}$ & $\begin{array}{l}\text { T64281E-O3 } \\
046404 E-04\end{array}$ & $\begin{array}{l}\text { 5. } 7 \\
4.6\end{array}$ & $\begin{array}{l}747085 E-03 \\
680308 E=04\end{array}$ & $\begin{array}{r}21388 B 9 E-05 \\
-2 \quad 043899 E-05\end{array}$ & $\begin{array}{r}-178219 E-O 3 \\
1 \quad 336513 E-O A\end{array}$ \\
\hline 2649 & c & $\begin{array}{r}-1 \\
6\end{array}$ & $\begin{array}{l}260067 E-03 \\
383002 E-05\end{array}$ & $\begin{array}{r}-9 \\
2\end{array}$ & $\begin{array}{l}273221 E-02 \\
804104 E-02\end{array}$ & $\begin{array}{r}-5 \\
5\end{array}$ & $\begin{array}{l}242474 E-03 \\
745564 E-04\end{array}$ & $\begin{array}{l}\text { 5. } 7 \\
\text { a. }\end{array}$ & $\begin{array}{l}747557 E-03 \\
6751 B O E=04\end{array}$ & $\begin{array}{r}2,125630 E-O 5 \\
\cdot 2 \quad 173219 E-O 6\end{array}$ & $\begin{array}{r}-278338 E-03 \\
1335835 E-04\end{array}$ \\
\hline 2697 & E & $\begin{array}{r}8 \\
-5\end{array}$ & $\begin{array}{l}101031 E-03 \\
522961 E-04\end{array}$ & $\begin{array}{r}-5 \\
2\end{array}$ & $\begin{array}{l}386 \overline{525 E-01} \\
842738 E-03\end{array}$ & $\frac{1}{2}$ & 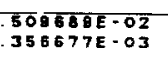 & $\begin{array}{l}5 \cdot 7 \\
4.6\end{array}$ & $\begin{array}{l}768360 E=03 \\
657654 E-04\end{array}$ & $\begin{array}{r}2.126451 E-05 \\
-1.944673 E \cdot 06\end{array}$ & $\begin{array}{r}-2.178528 E-03 \\
1.334561 E-04\end{array}$ \\
\hline 19777 & 0 & -2 & $\begin{array}{l}053369 E-02 \\
241366 E-03\end{array}$ & 4 & $\begin{array}{l}678298 E-O 1 \\
601270 E=O Z\end{array}$ & $\begin{array}{l}-5 \\
-4\end{array}$ & $\begin{array}{l}073386 E-02 \\
231516 E-03\end{array}$ & $\begin{array}{l}5 ? \\
46\end{array}$ & $\begin{array}{l}741949 E-03 \\
6 B 3502 E-04\end{array}$ & $\begin{array}{r}2.045317 E-05 \\
-2.054143 E-06\end{array}$ & $\begin{array}{r}-2 \quad 174080 E-03 \\
1.336044 E=04\end{array}$ \\
\hline
\end{tabular}

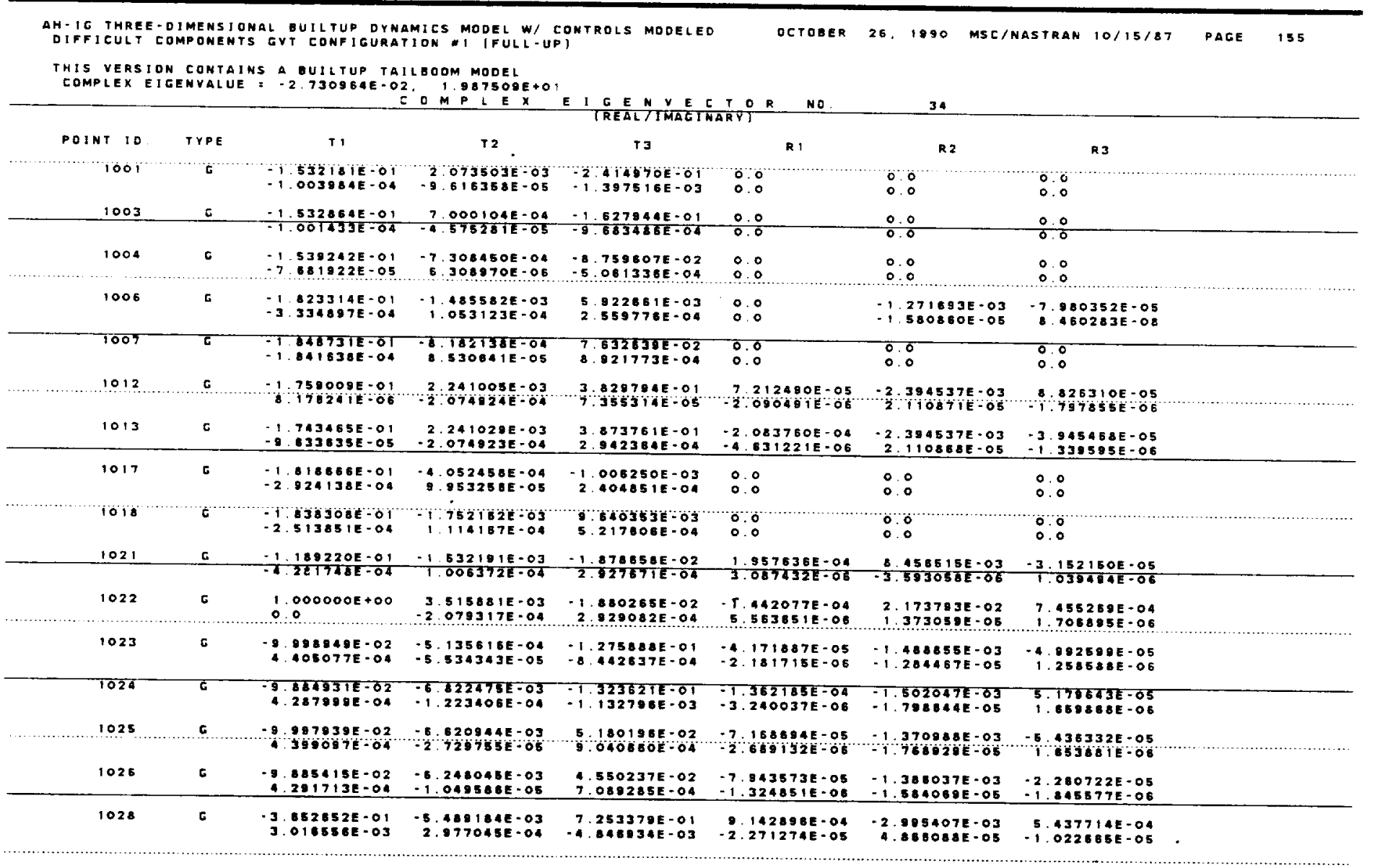


AH-IG THREE-DIMENSIONAL BUILTUP OYMAMICS MOOEL W/ CONTROLS MODELED

THIS VERSION CONTAINS A BUILTUP TAILEDOM MOOEL THIS VERSION CONTAINS A BUILTUP TAILBOOM MOOEL
COMPLEX EIGENVALUE: - 2 , $7309 B 4 E-02$. COMPLEX ETCENYETTOA NO.

\begin{tabular}{|c|c|c|c|c|c|c|c|c|}
\hline \multirow{2}{*}{ POINY ID. } & \multirow[b]{2}{*}{ TYPE } & \multirow[b]{2}{*}{$T 1$} & & & & & \multirow[b]{2}{*}{ R2 } & \multirow[b]{2}{*}{ R3 } \\
\hline & & & & $T 2$ & T3 & RI & & \\
\hline G & c & $\begin{array}{l}-2.233525 E=61 \\
-3.273485 E-04\end{array}$ & $i$ & $\begin{array}{l}278567 \mathrm{E}-O 3 \\
433401 E-04\end{array}$ & $\begin{array}{l}7.310281 E=02 \\
8.674155 E-04\end{array}$ & $\begin{array}{l}-1 \text { OJ10OgE-OA } \\
-1 \quad 586207 E-O E\end{array}$ & $\begin{array}{l}-1.685119 E-03 \\
-8.061212 E-06\end{array}$ & $\begin{array}{l}1.346421 E-O 5 \\
-9.651158 E-O T^{\prime}\end{array}$ \\
\hline 2511 & $\Xi$ & $\frac{-1.681195 E-01}{-1.224391 E-04}$ & $\frac{-9}{1 .}$ & $\frac{279849 E-04}{258218 E-06}$ & $\frac{1.054013 E-01}{1.152166 E-03}$ & $\frac{-9.973688 E-05}{-3.512907 E-06}$ & $\frac{-1.899949 E-03}{3.280308 E-08}$ & $\frac{-3.401773 E-05}{4.695694 E-07}$ \\
\hline 2572 & $\mathbf{G}$ & $\begin{array}{r}-1.461848 E-01 \\
-2.965579 E-04\end{array}$ & $\begin{array}{r}1 \\
-2\end{array}$ & $\begin{array}{l}433112 E-03 \\
495980 E-04\end{array}$ & $\begin{array}{l}3.849139 E-01 \\
1.599662 E-04\end{array}$ & $\begin{array}{l}-7.448960 E-05 \\
-3.834807 E-O 5\end{array}$ & $\begin{array}{r}-2.378977 E-03 \\
2.130806 E-05\end{array}$ & $\begin{array}{r}2.667846 E-05 \\
+1.869041 E-06\end{array}$ \\
\hline 2649 & G & $\begin{array}{l}-1.697388 E-01 \\
-1.566377 E-O 4\end{array}$ & $\begin{array}{r}4 \\
-3\end{array}$ & $\begin{array}{l}393860 E-03 \\
312708 E \cdot 04\end{array}$ & $\begin{array}{r}\text { 6. } 065566 E-01 \\
-2.747777 E-03\end{array}$ & $\begin{array}{l}-8.873806 E-06 \\
.5 .187701 E-06\end{array}$ & $\begin{array}{r}-2.440315 E-03 \\
4.617742 E-05\end{array}$ & $\begin{array}{r}5.296446 E-05 \\
.1 .822802 E-0 E\end{array}$ \\
\hline 2697 & $\bar{G}$ & $\begin{array}{r}-3.602759 E-01 \\
2.15044 E-03\end{array}$ & $\begin{array}{r}-5 \\
3\end{array}$ & $\begin{array}{l}641408 E-03 \\
057310 E \cdot 04\end{array}$ & $\begin{array}{l}.143206 E-01 \\
.4539096 E-03\end{array}$ & $\begin{array}{r}5.542691 E-04 \\
.2 .029510 E-05\end{array}$ & $\begin{array}{r}-3.057900 E \cdot 03 \\
5.180941 E \cdot 05\end{array}$ & $\begin{array}{r}3.519802 E-04 \\
-8.196752 E-0.0\end{array}$ \\
\hline 18777 & G. & $\begin{array}{r}-1.948057 E-01 \\
\because 3 \quad 558622 E=04\end{array}$ & $\therefore$ i. & $\begin{array}{l}830790 E-03 \\
\text { OT6E49E-OA }\end{array}$ & $\begin{array}{l}5777418 E-03 \\
2570 B 89=04\end{array}$ & $\begin{array}{r}344840 E-04 \\
=010444 E-06\end{array}$ & $\begin{array}{r}-1344603 E-03 \\
\because 1332015 E-05\end{array}$ & $\begin{array}{r}-4.999206 E-05 \\
1.484971 E-O 6\end{array}$ \\
\hline
\end{tabular}

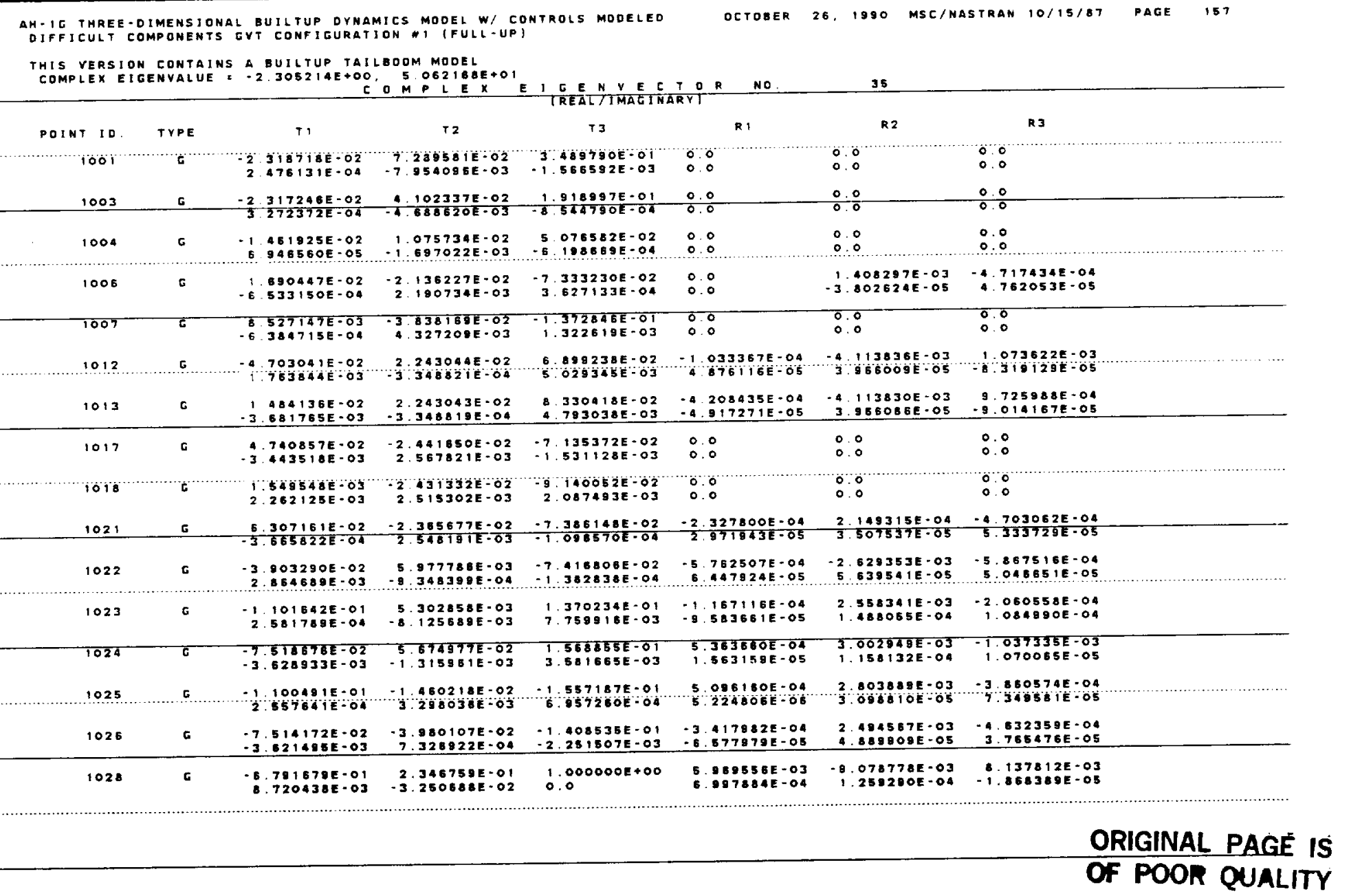


AH- 1 G THRE -DIMENSIOMAL QUILTUP DYNAMICS MODEL W/ CONTROLS MODELED

DIFFICULT COMPONENTS GYT CONFIGURATION I IFULL-UP)

THIS YERSION CONTAINS A BUILTUP TAILOOOM MOOEL

COMPLEX EIGENUALUE. - 2.305214E+OQ. 5 OB2168E+O

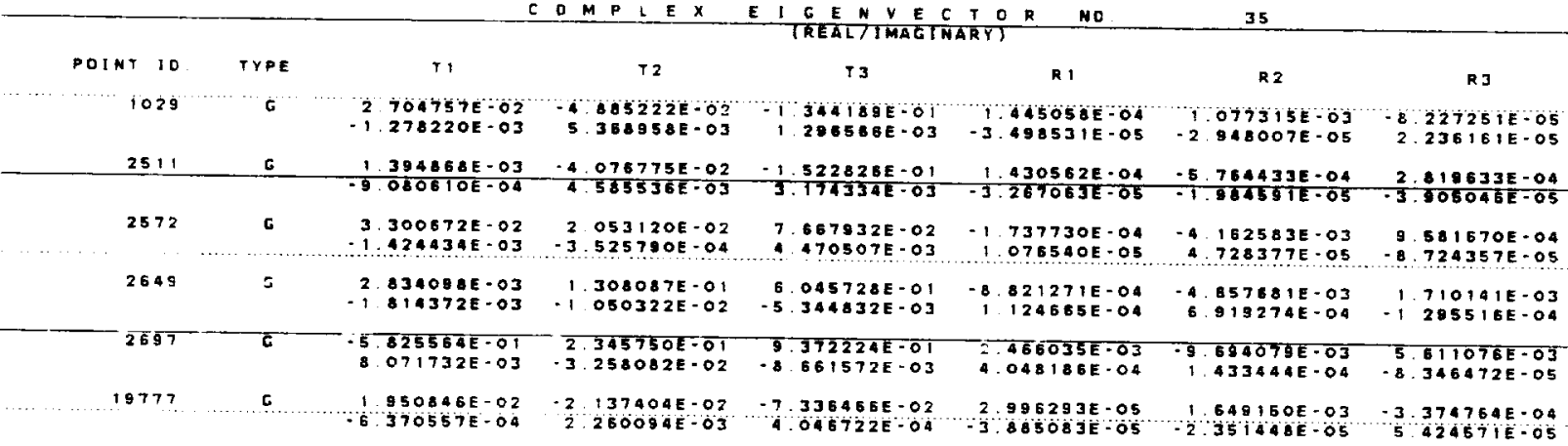

AH-IG THREE-DIMENSIONAL GUILTUP DYNAMICS MODELW/ CONTROLS MOOELEO

DIFFICULT COMPONENTS GVT CONFIGURATION, I IFULL-UP)

THIS VERSION CONTAINS A BUILTUF TAILEODM MODEL
DCTOBER 26,1990 MSC/NASTRAN $10 / 15 / 87$ PAGE 150 


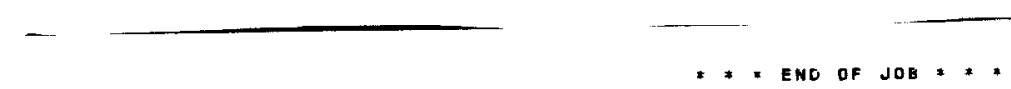

* Eno of job* *

$\underline{.}$

$\cdots$

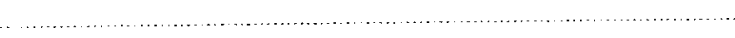




$\begin{array}{ll}\vdots & \\ i & \vdots \\ \vdots & \vdots\end{array}$

